
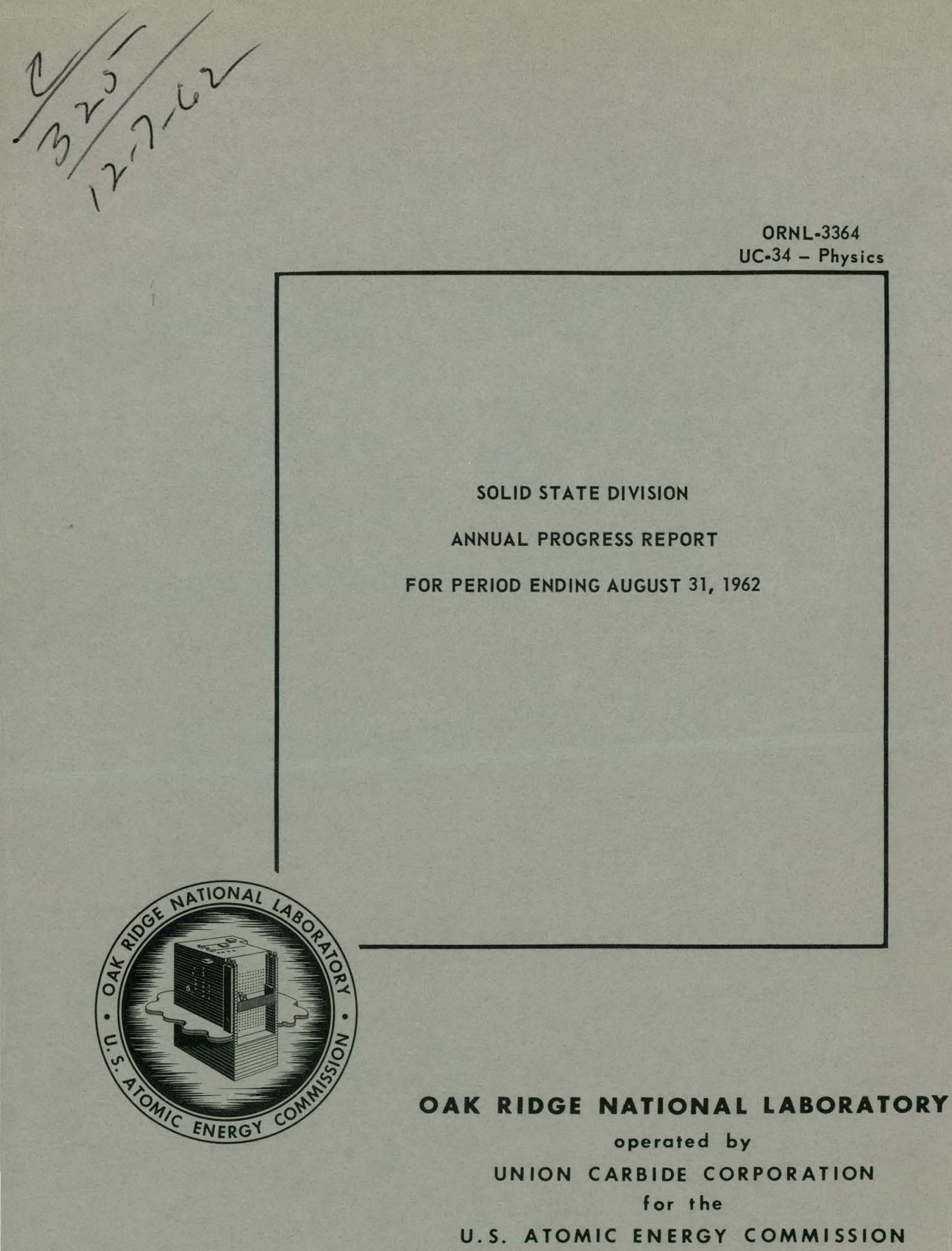

ORNL-3364

UC.34 - Physics

SOLID STATE DIVISION

ANNUAL PROGRESS REPORT

FOR PERIOD ENDING AUGUST 31, 1962

OAK RIDGE NATIONAL LABORATORY

operated by

for the

U.S. ATOMIC ENERGY COMMISSION 


\section{DISCLAIMER}

This report was prepared as an account of work sponsored by an agency of the United States Government. Neither the United States Government nor any agency Thereof, nor any of their employees, makes any warranty, express or implied, or assumes any legal liability or responsibility for the accuracy, completeness, or usefulness of any information, apparatus, product, or process disclosed, or represents that its use would not infringe privately owned rights. Reference herein to any specific commercial product, process, or service by trade name, trademark, manufacturer, or otherwise does not necessarily constitute or imply its endorsement, recommendation, or favoring by the United States Government or any agency thereof. The views and opinions of authors expressed herein do not necessarily state or reflect those of the United States Government or any agency thereof. 


\section{DISCLAIMER}

Portions of this document may be illegible in electronic image products. Images are produced from the best available original document. 
Printed in USA. Price: $\$ 3.00$ Available from the

Office of Technical Services

U. S. Department of Commerce

Washington $25, \mathrm{D} . \mathrm{C}$.

\section{LEGAL NOTICE}

This report was prepared as an account of Government sponsored work. Neither the United States, nor the Commission, nor any person acting on behalf of the Commission:

A. Makes any warranty or representation, expressed or implied, with respect to the accuracy, completeness, or usefulness of the information contained in this report, or that the use of any information, apparatus, method, or process disclosed in this report may not infringe privately owned rights; or

B. Assumes any liabilities with respect to the use of, or for damages resulting from the use of any information, apparatus, method, or process disclosed in this report.

As used in the above, "person acting on behalf of the Commission" includes any employee or contractor of the Commission, or employee of such contractor, to the extent that such employee or contractor of the Commission, or employee of such contractor prepares, disseminates, or provides access to, any information pursuant to his employment or contract with the Commission, or his employment with such contractor. 
ORNL-3364

UC.34 - Physics

TID-4500 (18th ed.)

Contract No. W-7405-eng-26

SOLID STATE DIVISION

\section{ANNUAL PROGRESS REPORT}

For Period Ending August 31, 1962

D. S. Billington, Director

J. H. Crawford, Jr., Associate Director

DATE ISSUED

NoV 26 196?

OAK RIDGE NATIONAL LABORATORY

Oak Ridge, Tennessee

Operoted by
UNION CARBIDE CORPORATION

for the

U. S. ATOMIC ENERGY COMMISSION 
THIS PAGE

WAS INTENTIONALLY LEFT BLANK 


\title{
Table of Contents and Summary
}

\author{
PART I. THEORY
}

\section{STUDIES OF PENETRATION OF MOVING ATOMS IN SOLIDS AND INTERATOMIC POTENTIALS}

\subsection{STOPPING POWER AS A BASIS FOR HARD-SPHERE APPROXIMATION}

O. S. Oen, M. T. Robinson, and D. K. Holmes..

By use of the hard-sphere approximation formalism of Holmes and Leibfried, ranges of energetic atoms in solids have been calculated with a hard-sphere radius defined so that the hard-sphere stopping power matches the "Bohr" stopping power at every energy. These calculated ranges are found tn be in much better agreement with the Monte Carlo results than those found by using a hard-sphere radius equal to the distance of closest approach in head-on collision.

\subsection{MACHINE STUDIES OF THE SLOWING DOWN OF ENERGETIC ATOMS IN SOLIDS}

M. T. Robinson and O. S. Oen.

Ranges of energetic atoms in solids have been studied theoretically by means of a mathematical model which incorporates the full latrice symmetry, as contrasted with earlier work in which the lattice was randomized to facilitate computation. The most striking effect noted is the possibility that an atom may become "channeled" along certain directions in the lattice (such as the $\langle 110\rangle$ in an fcc lattice) and move past many hundreds or thousands of lattice atoms with only slight energy loss.

\subsection{HARD-SPHERE SCATTERING APPROXIMATIONS}

E. J. Lee and M. T. Robinson

The relationship between the impact parameter and the energy transferred is studied in the hard-sphere applusiniation for two different choices of the hard-sphere radius and is compared with exact results.

\subsection{MONTE CARLO CALCULATIONS OF THE RANGES OF ENERGETIC ATOMS IN SOLIDS}

M. T. Robinson, D. K. Holmes, and O. S. Oen.

Abstract of paper presented at the Intemational Colloquium on Ion Bombardment, Bellevue, France, Dec. 4-8, 1961 (to be published). The slowing down of energetic atoms in solids has been investigated through the use of Monte Carlo methods on a large digital computer. It is pointed out in the paper that Victor Hugo has said: "Qui donc peut calculer le trajet d'une molecule?" The exponentially screened Coulomb potential assumed for atomic interactions in the elastic-collision range of interest is shown to be unsatisfactory.

\subsection{RANGES OF ENERGETIC ATOMS IN SOLIDS}

O. S. Oen, D. K. Holmes, and M. T. Robinson.

Abstract of paper submitted for publication in the Joumal of Applied Physics. Monte Carlo techniques are applied to calculation of distributions of primary atoms which have slowed down by elastic collisions in a solid. The exponentially screened Coulomb (Bohr) potential is primarily used, and the results are compared with the hardsphere approximation and with pertiuent experimental data. 


\subsection{CALCULATION OF AVERAGES FOR PRIMARY RECOIL DISTRIBUTIONS}

G. Leibfried

Abstract of published paper: J. Appl. Phys. 33, 1933 (1962). The slowing down of an energetic particle in a random assembly of scattering centers is discussed. Averages over the distribution for physically interesting lengths, such as the total path length and the vector distance to the final resting position, can be calculated in a very general form. Simplifying but realistic assumptions allow for the explicit evaluation of many averages and, in the case of the total length, the distribution function itself.

\subsection{SIMPLE APPROXIMATION FOR CLASSICAL SCATTERING AT LARGE ANGLES}

G. Leibfried and $O$. S. Oen

Abstract of published paper: J. Appl. Phys. 33, 2257 (1962). The classical scattering for repulsive potentials is treated approximately by matching the actual potential with an approximating potential fitted to the value and slope at the distance of closest approach. The differential cross section and the stopping power are compared with the machine results for an exponentially screened Coulomb potential.

\subsection{HIGHER-ORDER AVERAGES OF PRIMARY RECOIL DISTRIBUTIONS}

G. Leibfried

Abstract of paper submitted for publication in Zeitschrift fuer Pbysik. Analytical methods are developed for calculating averages over the final spatial distribution of primary atoms which slow down in a solid by means of elastic collisions. Particularly emphasized are averages over spatial components in combinations of higher degree than the second (e.g., $\overline{x^{3}}, \overline{x y^{2} z}$, etc.).

\subsection{LONG-RANGE CHANNELING EFFECTS IN IRRADIATED CRYSTALS?}

C. Lehmann and G. Leibfried

Abstract of paper to be submitted for publication. The range distribution of energetic atoms traveling along channels between atomic chains in crystals is calculated analytically. Numerical results are given for copper atoms in copper.

\section{THEORY OF SPUTTERING}

\section{I A GEOMETRICAL MODEL OF MONOCRYSTAL SPUTTERING}

M: T. Robinson

Experimental studies of the sputtering of copper monocrystals by rare-gas ions in the kev range have demonstrated the dramatic dependence of the sputtering yield (the number of target atoms ejected per incident ion) on the orientation of the ion beam with respect to the crystallographic axes of the target crystal. Similar experiments on germanium with 1- to 5-kev $\mathrm{Ar}^{+}$ions reveal only a small dependence on orientation. Some success has been had in understanding the copper data on the basis of a simple, essentially geometrical, model, and studies are in progress, both to improve the calculations and to extend them to targets of other symmetries.

\subsection{EXAMINATION OF A THEORETICAL MODEL OF ANISOTROPIC SPUTTERING AT HIGH ENERGIES BASED ON FOCUSING CHAINS}

C. Lehmann

A procedure is given to calculate the energy dependence of sputtering yields of single crystals for any surface orientation. Explicit calculations have been made for $\mathrm{Cu}^{+}$bombardment of copper under normal incidence for a (100), a (110), and a (111) surface. 


\section{SEMICONDUCTOR THEORY}

\section{I STATISTICAL MECHANICS OF SEMICONDUCTORS}

J. W. Miller and H. C. Schweinler

A report (K-1526) has been prepared and issued describing an IBM 7090 program for computing the Fermi level in nonmetals.

\subsection{DENSITY FUNCTION MODIFYING MINORITY CARRIER CAPTURE PROBABILITY IN NONME TALLIC CRYSTALS}

H. C. Schweinler and J. W. Miller

When a flux of charged particles impinges on a charged capturing center, the capture probability is modified by Coulomb attraction or repulsion. A function which relates the experimentally observed capture probability to an atomic capture probability is defined and tabulated.

\section{CRITICAL SIMPLICES IN BRILLOUIN ZONES}

H. C. Schweinler

At certain points, lines, and (internal) planes in the reduced Brillouin zone, here called critical 0-, 1-, and 2-simplices, the energy as a function of propagation vector has an algebraic form more complicated than that of Wigner and von Neumann. The first few 0-simplices are given for the case of a square lattice of points.

\section{A POSSIBLE EXPERIMENT TO STUDY RADIATION DAMAGE BY THE MÖSSBAUER EFFECT}

J. H. Barrett.

An experiment is proposed whereby the Mössbauer effect might be observable following thermal-neutron capture in certain materials. It is pointed our that the atom cmitting the Mösshauer (recoilless) radiation may very well be in an interstitial position. In case it is so located, ways are discussed in which the interstitial position may affect the spectra of the Mössbauer radiation.

\section{ELECTRON CROSS SECTIONS FOR ATOMIC DISPLACEMENTS}

O. S. Oen

An IBM 7090 computer program has been written for calculating the number of primary displaced atoms produced by energetic electrons. The program evaluates numerically the Mott series and is good, therefore, for elements of arbitrary $Z$. It is being extemited to include the cascade of displacements produced by the primary knocle-on. 


\section{PART II. CRYSTAL PHYSICS}

\section{X-RAY DIFFRACTION}

\section{1 SINGLE CRYSTALS GROWN FROM SMALL DROPLETS}

M. C. Wittels, F. A. Sherrill, and J. O. Stiegler.

Abstract of paper submitted for publication in the Journal of Applied Physics. Small single-crystal droplets of $\mathrm{Au}, \mathrm{Cu}, \mathrm{Ag}, \mathrm{Pb}, \mathrm{In}, \mathrm{Cu}_{3} \mathrm{Au}$, and $\mathrm{Ge}$ have been crystallized from the melt. Two different techniques were successfully employed.

\subsection{RADIATION EFFECTS IN URANIUM-DOPED ZIRCONIA}

M. C. Wittels, J. O. Stiegler, and F. A. Sherrill.

Abstract of paper to be published in the Journal of Nuclear Energy. A study concerning the radiationinduced monoclinic-to-fec phase transition in $\mathrm{ZrO}_{2}$ is given, and special emphasis is placed on specific particle events.

\subsection{A VERSATILE LANG CAMERA}

M. C. Wittels, F. A. Sherrill, and G. F. Fielder.

A Lang camera utilizing a conventional $x$-ray source together with a portable crystal rocker is described.

\subsection{THE 1961 POSTANNE ALING OF GRAPHITE-STORED ENERGY IN THE ORNL GRAPHITE REACTOR}

M. C. Wittels

Thermal measurements indicate that significant amounts of graphite-stored energy exist in the unannealed corner regions of the moderator stack. The large central region of the moderator core contains no spontaneously releasable stored energy.

\section{$7.5 X$ X-RAY OBSERVATIONS OF NEARLY PERFECT COPPER SINGLE CRYSTALS}

M. C. Wittels, F. A. Sherrill, and F. W. Young, Jr.

Published paper: Appl. Phys. Letters 1, 22 (1962). Lang-diffraction topographs and single-crystal rocking curves reveal a high degree of. perfection in large copper single crystals. Individual dislocations have been easily resolved, and an upper value for the width at half-maximum intensity of the (111) $\mathrm{K} \alpha_{1}$ reflection was 34 sec.

\section{ELECTRON MICROSCOPY}

\subsection{FISSION-FRAGMENT TRACKS IN METAL FILMS}

T. S. Noggle and J. O. Stiegler

Abstract of published paper: J. Appl. Phys. 33, 1726 (1962). Variation of fission-fragment track structures with thickness of thin metal films is interpreted in terms of electron transport of energy from the fission spike.

\subsection{FISSION-FRAGMENT TRACKS IN THIN FILMS OF $\mathrm{UO}_{2}$}

T. S. Noggle and J. O. Stiegler.

Abstract of published paper: Am. Soc. Testing Mater. Spec. Tech. Publ. 306, 47-63 (1961). Special experimental configurations permitting identification of the fission-fragment loss, direction of travel, and depth below the free surface give improved correlation of track structures with these parameters and lead to a suggested mechanism by which ionization may be converted to lattice damage. 


\subsection{NITROGEN ION BOMBARDMENT OF THIN PLATINUM FILMS}

J. O. Stiegler and T. S. Noggle.

Published paper: J. Apgl. Phys. 33, 1894 (1962). Tracks from the passage of 25-Mev nitrogen ions through thin platinum films indicate that track registration occurs by a different mechanism than the basically thermal processes which account for the tracks produced by fission fragments. It is suggested that displacement of atoms at the free surface by direct-charge interaction with the energetic ions could lead to the observed track structure.

\subsection{TRANSMISSION ELECTRON MICROSCOPE STUDIES ON SINGLE CRYSTALS OF UO,}

T. S. Noggle

Preliminary observations on irradiated $\mathrm{UO}_{2}$ single crystals have indicated that fission-fragment tracks are confined to surface regions of the material. Reaction between the $\mathrm{UO}_{2}$ and the organic material occurs during irradiation in polyethylene vials. This reaction leads to extensive microcracking and indicates sensitivity of $\mathrm{UO}_{2}$ to the environment during irradiation.

\subsection{DISLOCATIONS IN UO,}

T. S. Noggle

Abstract of paper to be published in the Proceedings of 5 th International Congress for Electron Microscopy. Aug. 29-Sept. 5. 1962, Philadelphia, Pennsylvania. Optical and electron microscope observations of dislocations in $\mathrm{UO}_{2}$ indicate appreciable plasticity at elevated temperatures. Motion of dislocations was noted in the electron microscope under conditions of heating by the electron beam.

\section{SPIN RESONANCE}

\subsection{RELATION BETWEEN MAGNETIC SUSCEPTIBILITY, ELECTRON SPIN RESONANCE, AND OPTICAL ABSORPTION OF THE E', CENTER IN FUSED SILICA}

R. A. Weeks and E. Sonder

Abstract of paper to be published in the Proceedings of the 1st International Conference on Paramagnetic Resonance, July 16-20, 1962, Jerusalem, Israel. The correlation of optical absorption with the electron spin resonance and majnetic susceptibility of the $F_{1}^{\prime}$ center in fused silica was utilized in calculating an oscillator strength for the center.

\subsection{PARAMAGNETIC SPECTRA OF É2 CENTERS IN CRYSTALLINE QUARTZ}

R. A. Weeks.

Abstract of paper submitted to the Physical Review. The electron spin resonance spectra of the $E_{2}^{\prime}$ centers in crystalline quartz have been analyzed in terms of a spin Hamiltonian for a spin state $S=\frac{1}{2}$. The anisotropy of the $g$ tensor and the observed hyperfine intcractions are utilized in proposing a plausible model for the centers.

\subsection{ALTERNATING. AND DIRECT-CURRENT CONUUCTIVITY OF GLASSES}

R. A. Weeks and E. Lell

The ac conductivity of an irradiated lead silicate glass was observed to increase monotonically with decreasing frequency, and the dc conductivity of the glass could be expressed as a sum of four time-dependent exponential functions plus a constant. These polarization processes were characteristic of the bulk material. A possible mechanism for storage of charge and energy which can result in the discharge effect may be found in such bulk polarization of certain elasses. 


\section{SUPERCONDUCTIVITY}

\subsection{DEPENDENCE OF SUPERCONDUCTING CRITICAL CURRENT OF Nb-Zr ALLOYS ON MAGNETIC-FIELD ORIENTATION}

S. T. Sekula, R. W. Boom, and C. J. Bergeron...

The dependence on magnetic field and orientation of the superconducting critical current of several $\mathrm{Nb}-\mathrm{Zr}$ alloys has been studied in the ORNL 100-kilogauss magnet. Data on cold-worked and heat-treated materials are reported.

\subsection{SUPERCONDUCTING TRANSITION TEMPERATURE OF PURIFIED TECHNETIUM}

M. L. Picklesimer and S. T. Sekula.

Abstract of paper submitted for publication in the Pbysical Review Letters. A recent determination of the superconducting transition temperature of purified technetium gave a value of $8.22^{\circ} \mathrm{K}$, which is lower than that previously reported.

\section{- 10.3 MECHANICAL PROPERTIES OF Nb-25\% Zr WIRE AT LOW TEMPERATURES}

S. T. Sekula and J. K. Redman $4.2^{\circ} \mathrm{K}$.

The tensile properties of commercially available $\mathrm{Nb}-25 \% \mathrm{Zr}$ wire have been determined at 300,77 , and

\subsection{NEUTRON IRRADIATION OF SUPERCONDUCTING TIN}

S. T. Sekula and C. E. Klabunde

The effect of neutron irradiation at $3.5^{\circ} \mathrm{K}$ on the superconducting transition of tin has been investigated.

\subsection{INTERACTION BETWEEN IRR ADIATION-INDUCED DEFECTS AND MAGNETIC STRUCTURE}

A. I. Schindler, R. H. Kernohan, J. Weertman, P. G. Huray; and W. L. Harman ............................................ 56

Abstract of paper to be submitted for publication in "Crystal Lattice Defect Supplement," Journal of the Pbysical Society of Japan. The effect of neutron irradiation at several different temperatures on the magnetic properties of some commercial, magnetically soft nickel-iron alloys has been investigated.

\section{PART III. METALS}

\section{INVESTIGATIONS OF METAL SURFACES}

F. W. Young, Jr., U. Bertocci, L. D. Hulett, L. H. Jenkins, and J. R. Savage

11. 1 ANODIC DISSOLUTION OF COPPER IN SOLUTIONS CONTAINING COPPER IONS.

Effects of surface orientation and defect structure on the dissolution process are discussed.

11.2 ANODIC DISSOLUTION OF COPPER IN COPPER.FREE SOLUTIONS.

The role of current density, solution composition, and orientation of the electrode surface in the development and growth of etch pits at dislocations is examined.

\subsection{KINETICS OF ETCH.PIT GROWTH AND ASSOCIATED PHENOMENA ON (111) COPPER} SURFACES IN ACID HALIDES.

The rate of growth of pits at dislocations and other changes in. surface topography were measured. The results are explained on a kinetic and geometric basis. 
A method for producing crystals of low dislocation density by annealing crystals grown by a modified Bridgman, technique is described.

11.5 ETCH-PIT STUDIES OF DISLOCATIONS IN COPPER CRYSTALS DEFORMED BY BENDING:

1. ANNEALED CRYSTALS; II. IRRADIATED CRYSTALS.....

Abstract of paper submitted to the Joumal of Applied Physics. Dislocation multiplication and motion was studied by use of an etch-pit technique to observe dislocations.

\section{LOW-TEMPERATURE IRRADIATION STUDIES}

R. R. Coltman, C. E. Klabunde, D. L. McDonald, and J. K. Redman

12.1 REACTOR DAMAGE IN PURE METALS AT $4^{\circ} \mathrm{K}$.

Thermal- and fast-neutron damage effects have been studied separately in high-purity metals irradiated near $4^{\circ} \mathrm{K}$. Unmoderated fission neutron damage has been studied in copper.

\subsection{INTENSE DAMAGE IN CADMIUM.}

By use of the effect of the suppression of isochronal recovery due to thermal-neutron dose in cadmium, an estimate is made of the defect concentration within a damaged region resulting from a fast-neutron hit.

12.3 NEW LOW-TEMPERATURE IRRADIATION FACILITY FOR THE BULK SHIELDING REACTOR

Preliminary flux measurements of a new high-purity thermal-flux facility and the construction progress of an associated $4^{\circ} \mathrm{K}$ irradiation facility are reported.

\section{DISLOCATION INTERACTIONS}

\subsection{EVIDENCE FOR VACANCY CLUSTERING IN DISLOCATIONS IN COPPER}

D. O. Thompson.....

Measurements of the internal friction and Young's modulus of a copper single crystal have been performed in which it is apparent that the number of dislocation pinning points introduced by neutron bombardment at $100^{\circ} \mathrm{C}$ is not a constant when measured as a function of temperature subsequent to bombardment. Analysis of the pinningpoint behavior has been made in terms of the Granato-Lücke theory of internal friction. It has been found that after light bombardments the pinning-point behavior can be described by second-order equilibrium with a binding energy of about $0.3 \mathrm{ev.} \mathrm{After} \mathrm{larger} \mathrm{doses} \mathrm{the} \mathrm{results} \mathrm{are} \mathrm{more} \mathrm{complex.} \mathrm{It} \mathrm{is} \mathrm{thought} \mathrm{that} \mathrm{the} \mathrm{general} \mathrm{results} \mathrm{may} \mathrm{be} \mathrm{due}$ to vacancy clustering in dislocations.

\subsection{ANALYSIS OF INTERNAL FRICTION IN COPPER AT' MEGACYCLE FREQUENCIES}

V. K. Paré

Analysis of a previous experiment has been redone, taking into account a more accurate theory of oscillatory dislocation motion and correcting for the distortion of time scale caused by delay in migration of radiation-defect pinning points from their places of creation to the dislocations. The frequency dependence now indicates that probably only one dislucation component was important in this sample. It appears that an increasing fraction of pinning points is lost as irradiatiun procecds. 


\subsection{TEMPERATURE DEPENDENCE FROM 250 TO $370^{\circ} \mathrm{K}$ OF DISLOCATION PINNING IN COPPER SINGLE CRYSTALS BY RADIATION DEFECTS}

V. K. Paré and D. O. Thompson

Abstract of published paper: Acta Met. 10, 382 (1962).

\subsection{A THERMALLY ACTIVATED INTERNAL-FRICTION SPECTRUM IN COPPER FOLLOWING NEUTRON IRRADIATION}

D. O. Thompson

Abstract of published paper: Acta Met. 10, 327 (1962).

\section{Ar $^{+}$ION BOMBARDMENT OF METAL SURFACES}

A. L. Southern, M. T. Robinson, and D. R. Burrowbridge

Determinations were made of the argon retained in copper after bombarding polycrystalline copper with 4-kev argon ions. Atom ejection patterns were obtained for the three major planes of aluminum and the (0001) plane of zinc, and the sputtering yield is reported for the (111) plane of aluminum.

\section{PART IV. NONMETALS}

\section{SEMICONDUCTOR STUDIES}

\section{1 RADIATION-INDUCED RECOMBINATION AND TRAPPING CENTERS IN GERMANIUM:}

I. THE NATURE OF THE RECOMBINATION PROCESS; II. ANNEALING BEHAVIOR

O. L. Curtis, Jr., and J. H. Crawford, Jr

Abstracts of published papers: Pbys. Rev. 124, 1731 (1961); Pbys. Rev. 126, 1342 (1962). Extensive measurements on irradiated germanium indicate that previous analyses of the recombination process are incorrect. Observations of the annealing behavior of radiation-induced recombination centers have been made. A model which explains the recombination and annealing data is presented.

\subsection{ANNEALING OF IRR ADIATION-INDUCED DAMAGE IN GERMANIUM}

J. C. Pigg

The annealing properties of irradiation-induced carrier change in antimony-doped germanium have been studied by using both isochronal and isothermal annealing. The irradiations were conducted at liquid-nitrogen temperature by using $\mathrm{Co}^{60}$ gamma irradiation. A model which explains the observed annealing behavior is proposed:

\subsection{TRAPPING CENTERS IN ARSENIC-DOPED GERMANIUM}

O. L. Curtis, Jr., and C. C. Robinson

Direct-current photoconductivity measurements are being used in conjunction with transient measurements to determine the properties of traps previously observed in arsenic-doped germanium.

\subsection{THERMOELECTRIC POWER IN GERMANIUM}

O. E. Schow

Although $40 \%$ of the carriers were removed from a $2-0 h m-\mathrm{cm}$-type germanium sample by $\mathrm{Co}^{60}$ photon irradiation, the Seebeck coefficient remained relatively unchanged. A definitive experiment, using $0.2-0 h m-c m ~ n-t y p e$ germanium, is in progress which will establish the validity of the premise that the Seebeck coefficient is less sensitive in antimony-doped germanium to $\mathrm{Co}^{60}$ photons than the theory predicts. 


\subsection{RADIATION EFFECTS IN ZINC SULFIDE}

R. O. Chester and O. L. Curtis, Jr.

Preliminary optical studies of $\mathrm{Co}^{60}$ gamma-ray and reactor-neutron-irradiated single crystals of zinc sulfide indicate no observable change in the absorption spectrum. Measurements of electronic properties are expected to be a more sensitive indicator of radiation damage than are optical absorption data. Therefore, work is in progress to prepare suitable specimens for electrical measurements.

\subsection{EFFECT OF A PHASE TRANSFORMATION ON THE VAPOR PHASE GROWTH OF SINGLE- CRYSTAL HgS}

O. L. Curtis, Jr.

Abstract of published paper: J. Appl. Phys. 33, 2461 (1962). Attempts have been made to grow singlecrystal $\mathrm{HgS}$ by using a vapor phase growth technique. The lack of success led to the discovery of a phase transformation which occurs between the temperature of deposition and room temperature.

\subsection{GROWTH OF SINGLE-CRYSTAL HgS}

O. L. Curtis, Jr.

Small single crystals of cinnabar have been grown by utilizing the temperature dependence of the reaction $\mathrm{HgS}+\mathrm{I}_{2} \rightleftharpoons \mathrm{HgI}_{2}+\frac{1}{2} \mathrm{~S}_{2}$. Attempts are being made to grow crystals large enough for electrical measurements.

\subsection{A STUDY OF THE EFFECTS OF CO ${ }^{60}$ GAMMA RADIATION UPON EXTRINSIC Bi ${ }_{2} \mathrm{Te}_{3}$}

M. J. Smith.

There is considerable evidence to indicate that the effects of $\mathrm{Co}^{60}$ gamma-radiation damage upon the electrical properties of $\mathrm{Bi}_{2} \mathrm{Te}_{3}$ vary in a consistent manner with the atomic percent of tellurium in the specimen. A possible interpretation is that gamma radiation creates Frenkel pairs consisting of substitutional bismuth and tellurium atoms in p-type and $n$-type $\mathrm{Bi}_{2} \mathrm{Te}_{3}$ respectively. $\mathrm{A}$ variation in the anisotropy of scattering upon extended irradiation appears to be responsible for certain of the observed effects. It is believed that further analysis of the gamma-radiation damage of the material will lead to a more quantitative understanding of the doping mechanism and band structure in $\mathrm{Bi}_{2} \mathrm{Te}_{3}$.

\subsection{EXPERIMENTAL THRESHOLD IRRADIATION ENERGY REQUIRED FOR THE PRODUCTION OF DEFECT CLUSTERS}

J. W. Cleland and R. F. Bass.

A number of preliminary experiments have been considered or conducted in relation to their potential application toward ascertaining the experimental threshold energy required for the production of defect clusters (disordered regions) in materials.

\subsection{LATTICE DEFECT.PRODUCTION IN THERMAL-NEUTRON-SHIELDED MATERIALS}

J. W. Cleland, R. F. Bass, and J. H. C.rawford, Jr.

Abstract of paper to be published in the Journal of Applied Pbysics. Electrical property measurements have been employed to determine the nature and yield of radiation-induced lattice defects in semiconducting materials for (a) $\mathrm{Co}^{60}$ photons, (b) thermal-neutron $(n, y)$ recoil damage, and (c) cadmium gamma rays in thermalneutron-shielded samples. Reasonable agreement was obtained with the theory of Schweinler (recoils) and that of Oen and Holmes (atomic-displacement cross sections by gamma rays). It is shown that the relative effects of (b) and $(c)$ are comparable in semiconducting materials; hence, lattice defects produced by the action of capture gamma rays emanating from the shield material should be considered in all reactor irradiation experiments in which shielding against thermal neutrons is employed. 


\subsection{EFFECT OF DISLOCATIONS ON CO ${ }^{60}$ RADIATION-INDUCED DEFECTS IN GERMANIUM}

J. W. Cleland and R. F. Bass....

The apparent $p$-type carrier concentration and acceptor level position of $\mathrm{Co}^{60}$ photon-irradiated germanium are shown to be dependent on the initial dislocation content of the material.

\section{12 MONITORING TECHNIQUES FOR CAPTURE GAMMA RAYS}

J. W. Cleland...

Ceric sulfate reduction and ferrous sulfate oxidation techniques have been used to confirm the predicted flux of capture gamma rays inside a thermal-neutron shield.

\section{INSULATING CRYSTALS}

\section{1 F- AND M-BAND COLORING IN HEAVILY IRRADIATED HIGH-PURITY KCI}

E. Sonder and W. A. Sibley

Investigation of radiation coloring with special emphasis on the relation between $F$ - and $M$-centers in heavily colored $\mathrm{KCl}$ has shown the following: The $M$-center concentration is proportional to the square of the $F$-center concentration, with the constant of proportionality depending upon the purity of the $\mathrm{KCl}$ samples, and the intensity of ionization. The proportionality constant is not changed by plastic deformation of the sample, nor is it dependent upon the energy or type (electrons or gamma rays) of irradiation. After heavy coloring the ratio of $F$-to $M$-centers can be changed by a short reirradiation at a different ionization intensity. This process is reversible, indicating that $F$ - and $M$-centers are in equilibrium during irradiation at room temperature.

\subsection{GAMMA IRRADIATION OF DOPED KCI}

W. A. Sibley and C. T. Butler.

The effect of gamma irradiation on $\mathrm{KCl}$ samples doped with $\mathrm{Ca}, \mathrm{Sr}, \mathrm{Cd}$, and $\mathrm{Mg}$ was studied. The data apparently indicate that crystals containing $\mathrm{Mg}$ and $\mathrm{Cd}$ have relatively fewer free positive-ion vacancies than those doped with Ca or Sr.

\subsection{INVESTIGATIONS OF COLOR CENTERS IN ALKALI HALIDES AT ORNL: STRUCTURE SENSITIVITY OF COLOR CENTER PRODUCTION IN GAMMA-IRR ADIIATED KCI}

J. H. Crawford, Jr.

Abstract of paper presented at the International Conference on Crystal Lattice Defects, Kyoto, Japan, Sept. 7-12, 1962. The presence of divalent cation impurities enhances coloration by Co 60 gamma rays in $\mathrm{KCl}$ during the early stages, whereas coloration in the later stages is suppressed. Various mechanisms to account for this are examined.

\subsection{LUMINESCENCE OF ALKALI HALIDES}

C. T. Butler......

Luminescent spectra of several alkali halides have been taken during and immediately following gamma irradiation at $5 \times 10^{6} \mathrm{r} / \mathrm{hr}$. Several broad maxima were observed in some spectra, and one spectrum showed an intense impurity band in the ultraviolet region.

\subsection{EFFECT OF ENERGETIC SECONDARY ELECTRONS ON THE SCINTILLATION PROCESS IN ALKALI HALIOE CRYSTALS}

A. Meyer and R. B. Murray

Abstract of paper to be published in the Pbysical Review. The effect of delta rays on the scintillation process in alkali iodide crystals has been investigated theoretically, and the results are found to be compatible with experiment. 


\subsection{STUDIES OF THE SCINTILLATION PROCESS IN CSI(TI)}

R. Gwin and R. B. Murray

Abstract of ORNI-3354. An experimental investigation of the scintillation process in CsI(Tl) crystals of varying thallium content has been completed. Several features of a previously proposed model are confirmed; however, the experimental results are in direct contrast with the proposed activator saturation process for highly ionizing particles.

\subsection{SPECIFIC HEAT OF SINGLE-CRYSTAL $\mathrm{MnCl}_{2}$ IN APPLIED MAGNETIC FIELDS}

R. B. Murray

Abstract of paper submitted to the Pbysical Review. The specific heat of a single-crystal sample of antiferromagnetic $\mathrm{MnCl}_{2}$ has been measured, in the liquid-helium temperature region, in applied magnetic fields. Results permit evaluation of the magnetic $H-T$ phase diagram.

\section{PURE MATERIALS}

\subsection{INTRODUCTION}

J. W. Cleland.

An extensive program is being conducted to develop the techniques to produce high-purity research specimens of immediate and long-range interest.

\subsection{OPTICAL ABSORPTION STUDIES ON Cu,}

C. M. Nelsún, R. F. Bass, and J. W. Cleland.

A procedure was developed to obtain parallel polished faces on single-crystal specimens of $\mathrm{Cu}_{2} \mathrm{O}$. Preliminary results were obtained between 25,000 and $6000 \mathrm{~A}$ for the optical absorption at room temperature, at $80^{\circ} \mathrm{K}$, and as affecred by $\mathrm{Co}^{60}$, photon irradiation.

\subsection{POTASSIUM CHLORIDE SINGLE CRYSTALS}

C. T. Butler and J. R. Russell

A pulling furnace for $\mathrm{KCl}$ has been built. Crystals ranging to $3 \times 3 \times 10 \mathrm{~cm}$ have been grown.

\subsection{RESEARCH MATERIALS INFORMATION CENTER}

This report very briefly describes the purpose and operation of the Solid State Division's Research Materials Information Center, which will provide information on the methods of production, availability, need for, and characterization of high-purity solid-state research materials to both producers and users. Details of system automation and analysis for coding are not included.

\subsection{PURIFICATION OF $\mathrm{Bi}_{2} \mathrm{Se}_{3}$ CRYSTALS DURING AND AFTER GROWTH}

Some degree of purification of $\mathrm{Bi}_{2} \mathrm{Se}_{3}$ can be realized by control of the partial pressure of selenium over the crystal. The purification is controlled by self-diffusion of bismuth within the lattice, a process which has an activation energy of approximately $3 \times 10^{4} \mathrm{cal} / \mathrm{mole}$.

It appears that purification of $\mathrm{Bi}_{2} \mathrm{Se}_{3}$ can be considerably enhanced by regulation of the constituent-element vaper pressure nver the growing crystal. $\Lambda$ modified Teal-Little crystal-growing furnace has been constructed for this purpose. 


\section{PART V. RADIATION METALLURGY}

\section{FUNDAMENTAL STUDIES}

\section{1 THE ORY OF THE DEPENDENCE OF RADIATION-ENHANCED DIFFUSION ON DISPLACEMENT PRODUCTION RATE AND IRRADIATION TEMPERATURE}

J. H. Barrett

A discussion is given of the time dependence of the interstitial and vacancy concentration during irradiation when these defects anneal by the combined processes of diffusion to fixed sinks and interstitial-vacancy recombination. The dependence of the radiation-enhanced diffusion on the displacement production rate and the irradiation temperature is summarized for this annealing process and for the two simple constituent annealing processes considered separately.

\subsection{EFFECT OF RADIATION ON ATOMIC REARR ANGEMENTS IN CU-AI}

J. M. Williams, M. S. Wechsler, C. Bassani, and J. H. Barrett

An experimental investigation of the flux dependence (dependence on the displacement production rate) of radiation-enhanced ordering in $\mathrm{Cu}-15$ at. \% $\mathrm{Al}$ is conducted by measuring electrical resistivity during ordering at $100^{\circ} \mathrm{C}$ at various locations in the reactor. The neutron flux is determined by using a number of thermal, epithermal, and threshold detectors. The results indicate that the rate of reaction at a given temperature varies as the square root of the flux.

\subsection{STRESS RELAXATION IN IRON ALLOYS CONTAINING CARBON AND/OR NITROGEN}

J. T. Stanley

Apparatus is being developed for the measurement of stress relaxation and low-frequency internal friction during irradiation. A discussion is given of the application of these techniques to the study of the effect of radiation on the precipitation of carbides and nitrides in $a$-iron.

\subsection{ATOMIC REARR ANGEMENTS IN DEFORMED CU-Si AND CU-Si-Mn ALLOYS}

J. M. Williams, M. S. Wechsler, and H. M. Otte

Abstract of paper presented by H. M. Otte at International Conference on Crystal Lattice Defects, Kyoto, Japan, Sept. 7-12, 1962. The effect of room-temperature deformation and subsequent isochronal annealing on various physical properties of $\mathrm{Cu}-6.7$ at. $\% \mathrm{Si}$ and $\mathrm{Cu}-6.7$ at. $\% \mathrm{Si}-1.3$ at. $\% \mathrm{Mn}$ is being studied. The results are interpreted in terms of the redistribution of solute stimulated by the deformation-produced defects.

\section{ENGINEERING PROPERTIES}

\section{I IN-PILE STRESS RUPTURE EXPERIMENTS}

W. E. Brundage, W. W. Davis, N. E. Hinkle, O. Sisman, J. C. Zukas, and A. L. Johnson......

In-pile tube-burst experiments on various engineering alloys (Inconel, 304 stainless steel, Zircaloy-2, and $\mathrm{Nb}-1 \% \mathrm{Zr}$ ) are described. The chief effects of the irradiation are to shorten the rupture life at a given stress and to decrease the deformation at fracture, although in certain cases no change in property was detected.

\subsection{IMPACT TESTS ON IRRADIATED PRESSURE-VESSEL STEELS}

R. G. Berggren, L. D. Schaffer, M. S. Wechsler, and T. N. Jones.

The results of impact tests on pressure-vessel steel surveillance samples taken from the SM-1 reactor after 16.4 and 20.8 Mwyr of operation are reported. The increases in brittle-ductile transition temperature are somewhat in excess of those predicted by accelerated-irradiation tests. Several reasons for this observation are discussed. 


\section{PART VI. REACTOR MATERIALS}

\section{FUEL MATERIALS}

O. Sisman

\section{I POSTIRRADIATION EXAMINATION OF GRAPHITE MATRIX FUEL}

J. G. Morgan, M. F. Osborne, E. L. Long, Jr., and P. E. Reagan

Postirradiation evaluations have been performed on dispersions of coated and uncoated $\mathrm{UC}_{2}$ in graphite. The fission product gradient was measured across a graphite can containing the fuel specimens.

\subsection{COATED FUEL PARTICLES}

R. M. Carroll, J. G. Morgan, M. F. Osborne, P. E. Reagan, and T. W. Fulton 161

Pyrolytic-carbon-coated $\mathrm{UC}_{2}$ particles were examined after irradiation at elevated temperatures. Both columnar and laminar coatings were also tested for fission-gas retention during irradiation.

\subsection{IRRADIATION EFFECTS ON METAL.CLAD FUELS}

M. T. Morgan, D. F. Toner, E. L. Long, Jr., C. D. Baumann, J. G. Morgan, M. F. Osborne,

H. E. Robertson, J. W. Gooch, and A. L. Johnson.....

Pellets of $\mathrm{UO}_{2}$ clad in stainless steel were examined after irradiation. These EGCR-prototype fuel capsules had been irradiated in the ORR and the ETR. Beryllium-canned $\mathrm{UO}_{2}$ and fueled BeO were also included in the evaluations.

\subsection{CONTINUOUS RELEASE OF FISSION GAS DURING IRRADIATION OF UO}

R. M. Carroll, P. E. Reagan, and T. W. Fulton

Thin-plate specimens of fused-crystal and single-crystal $\mathrm{UO}_{2}$ were irradiated in a reducing atmosphere. The releases of xenon, krypton, and iodine are compared as a function of temperature, specimen structure, stoichiomerry, and ueutron flux.

Electrical resistivity measurements of the three types of $\mathrm{UO}_{2}$ were obtained during irradiation. Comparisons are made of the in-pile performance of $\mathrm{Pt} v \mathrm{vst}-10 \% \mathrm{Rh}$ thermocouples with $\mathrm{W}-5 \% \mathrm{Re}$ v $\mathrm{W}-26 \% \mathrm{Re}$ in a reducing atmosphere.

\subsection{MARITIME REACTOR FUEL}

J. G. Morgan and M. F. Osborne

Maritime reactor prototype fuel elements were examined after irradiation. Cold-swaged and vibratory-compacted $\mathrm{UO}_{2}$ were used in the tests to determine fuel-rod stability as well as fission-gas releasc.

\subsection{FUEL CYCLE PROGRAM}

J. G. Morgan and M. F. Osborne

Postirradiation evaluations continued un sintered and powdered $\mathrm{UO}_{2}-\mathrm{ThO}_{2}$. 


\section{POLYMERS}

W. W. Parkinson, R. M. Keyser, W. K. Kirkland, and O. Sisman

The investigation of differences in the effects on polystyrene of gamma radiation and mixed reactor radiation was extended to infrared and gas chromatographic analysis. Infrared spectrum measurements showed more rapid growth of certain bands in reactor-irradiated specimens than in specimens exposed to gamma radiation alone. The volatile products of gamma irradiation of polystyrene indicated by gas chromatography were hydrogen, $G\left(\mathrm{H}_{2}\right) \sim$ $1.5 \times 10^{-2}$ molecule per $100 \mathrm{ev}$, and benzene, $G\left(\mathrm{C}_{6} \mathrm{H}_{6}\right) \sim 5 \times 10^{-4}$ molecule per $100 \mathrm{ev}$.

A versatile gamma source comprising a $\mathrm{Co}^{60}$ assembly in a $7 \times 10 \mathrm{ft}$ shielded compartment has been designed for the irradiation of plastics and rubbers during mechanical te sting.

\section{CERAMICS}

C. D. Bopp, O. Sisman, and R. L. Towns.

Equipment is described which is suitable for thermal analysis of small samples of nonmetallic materials. Calibration by means of Peltier power makes the method quantitative below abour. $900^{\circ} \mathrm{C}$.

Irradiation in a reactor causes zircon ceramic to resemble metamict zircon in the changes undergone on heating. Heating produces partial annealing at relatively low temperature, decomposition at higher temperature, and re-formation of zircon at still higher temperature. Not all of these processes may occur, depending on the irradiation dose.

\section{RADIATION DOSIMETRY}

C. D. Bopp, O. Sisman, W. K. Kirkland, and W. W. Parkinson

The acid concentration produced by irradiation of solutions of $\mathrm{C}_{2} \mathrm{H}_{2} \mathrm{Cl}_{4}$ in $\mathrm{C}_{8} \mathrm{~F}_{16}$ and in $\mathrm{C}_{10} \mathrm{H}_{22}$ has been measured to determine the suitability of these systems as dosimeters. The $\mathrm{C}_{8} \mathrm{~F}_{16}$ solutions, to which 0.3 wt \% $\mathrm{C}_{10} \mathrm{H}_{22}$ had been added to provide an adequate source of hydrogen, showed inconsistent acid yields unless water in excess of saturation ( 0.2 wt \%) was present. The $\mathrm{C}_{10} \mathrm{H}_{22}$ solution showed a dependence on both the temperature and dose rate during irradiation.

\section{PUBLICATIONS AND PAPERS}


Part I. Theory

D. K. Holmes 
THIS PAGE

WAS INTENTIONALLY

LEFT BLANK 


\section{Studies of Penetration of Moving Atoms in Solids and Interatomic Potentials}

\section{I.I STOPPING POWER AS A BASIS FOR HARD-SPHERE APPROXIMATION}

O. S. Oen M. T. Robinson

D. K. Holmes

The "hard-sphere approximation" has had widespread use with much success in radiation-damage theory. The main advantage of this approximation, from a mathematical viewpoint, is that it allows a simplified analytical approach to extremely complex problems. A disadvantage is the difficulty in evaluating the errors associated with it. For focusing collisions the hard-sphere approximation has been found to be very good. ${ }^{1}$ On the other hand, its use in range calculations accounts for the general features of the more exact calculations ${ }^{2}$ but gives results which are 10 to $80 \%$ too high (Bohr-potential calculations). The reason for this overestimate is that the stopping power of the Bohr potential is $10 \%$ (at low energy) to $80 \%$ (at high energy) greater than that of the approximating hard-sphere potential. This increased stopping power tends to shorten the calculated ranges. These results suggested that a new criterion for the hard-sphcrc radius might be more appropriate for range calculations.

The hard-sphere radius is usually defined as the distance of closest approach between two atoms in a head-on collision. An altemative method is to definc it so that the hard-sphere stopping puwet matches the exact, calculated stopping power. This is done in Eq. (1), where, for simplicity, the

${ }^{1}$ C. Lehmann and G. Leibfried, L. Physik 162, 203 (1961).

${ }^{2}$. . T. Robinson, D. K. Holmes, and O. S. Oen, Solid Stute Div. Ann. Progr. Rept. Aug. 31, 1961 , ORNL-3213, P 3. masses of the primary atom and lattice atoms are assumed to be equal:

$$
\frac{n \pi R_{\text {s.p. }}^{2} E}{2}=n \int_{0}^{E} T K(T, E) d T,
$$

where the left-hand expression is the hard-sphere stopping power, and the right-hand expression is the calculated stopping power for the potential being approximated. In Eq. (1), $n$ is the density of atoms, $E$ is the primary energy, $K(T, E)$ is the differential cross section for transferring an energy $T$ to a lattice atom by a primary of energy $E$ and $R_{\text {s.p. }}$ is the hard-sphere radius chosen to make the two expressions equal. In order to compare the ranges obtained by the two methods of choosing the hard-sphere radius to approximate the Bohr potential, the results of Figs. 1.1 and 1.2 have been calculated using the hard-sphere analytical range formalism of Holmes and Leibfried. ${ }^{3}$ Figure 1.1 shows that the average penetration depth calculated with a radius chosen by matching the stopping powers gives very good agreement with the Monte Carlo results. This method also gives agreement to better than 5\% of the Monte Carlo calculations for the total path and vector range. However, as seen from Fig. 1.2, the perpendicular spread is represented somewhat better by using a radius defined by the distance of closest approach.

It is concluded that, for theoretical estimates of ranges, this new method of selecting the hard-core radius is superior to the usual method, at least for simulating the Bohr potential. On the other hand, to estimate the lateral spread of, for example, a damaged region initiated by a primary knock-on, the usual method of picking a hard-sphere radius seems to be better.

\footnotetext{
${ }^{3}$ D. K. Holmes and G. Leibfried, J. Appl, Pbys, 31, $1046(1960)$.
} 


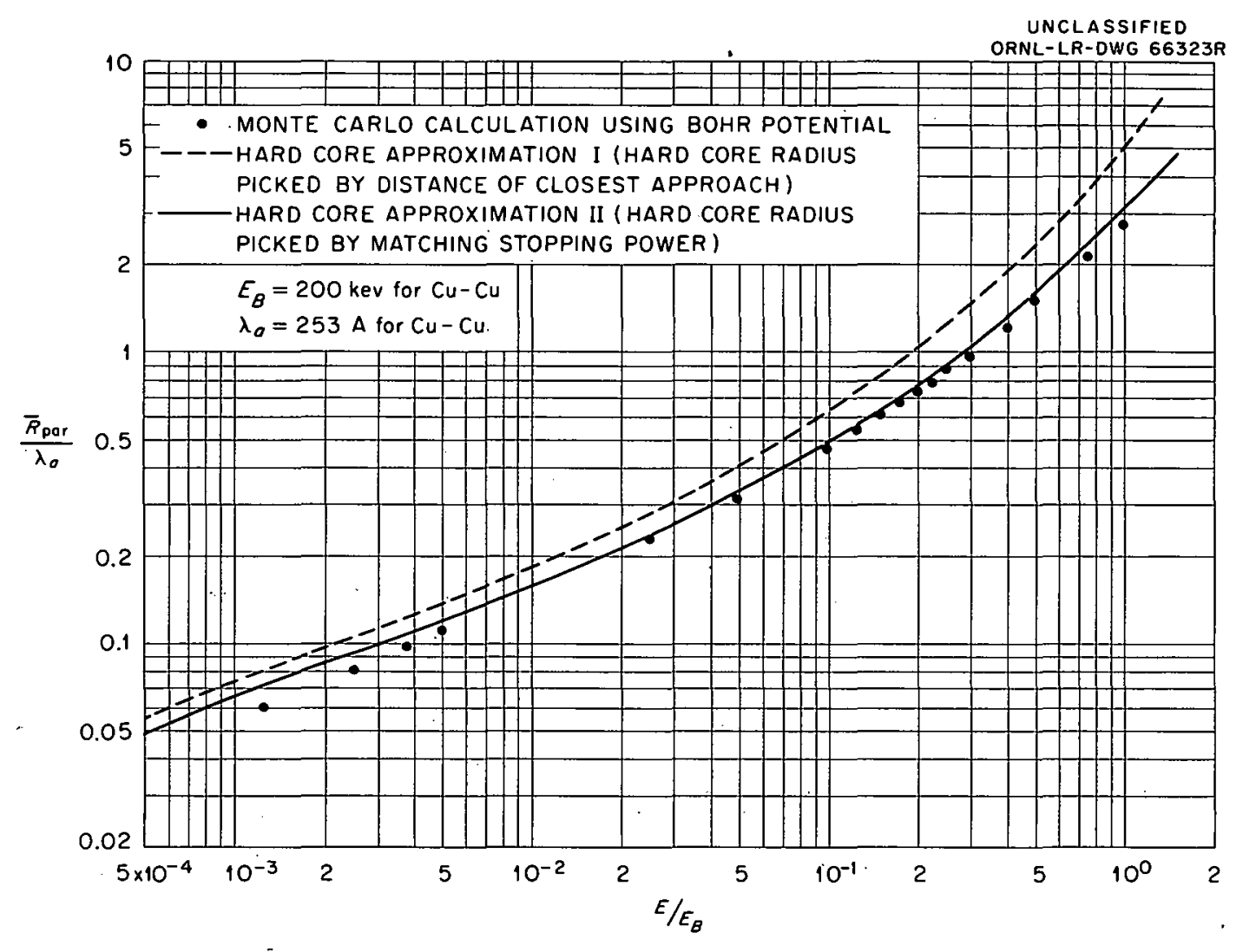

Fig. 1.1. Comparison of the Penetration Depth as a Function of Primary Energy for Two Choices of the HardSphere Radius and the Exact Calculation.

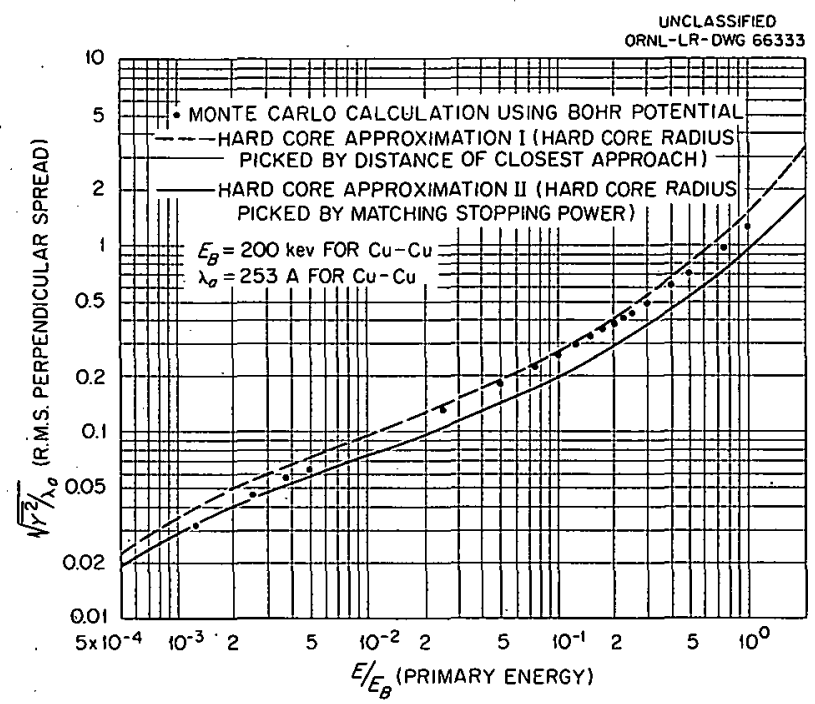

Fig. 1.2. Comparison of the "Spread" as a Function of Primary Energy for Two Choices of Hard-Sphere Radius and the Exact Calculation.
Frequently, the range of an energetic atom in a solid is calculated by integrating the reciprocal of the atomic stopping power. ${ }^{4.5}$ (In principle, energy losses by electron excitation and ionization can be included also. For simplicity, they are not included in this discussion.) As is well known, this method should be quite accurate, provided the atomic weight of the stopping material is much less than that of the projectile atom. This requirement is seldom met in radiation damage where interest usually lies in the range of an atom having the same atomic weight as that of the stopping material. However, even in this case, the range. found by integrating the reciprocal of the stopping power is expected to give the average total path

${ }^{4} \mathrm{~K}$. O. Nielsen, p 68 in Electromagnetically Enriched Isotopes and Mass Spectrometry, Academic Press, New York, 1956.

${ }^{5} \mathrm{~J}$. Linhard and M. Scharff, Phys. Rev. 124, 128 (1961) 
length traversed by the projectile. It is interesting to compare this "stopping power range" with the total path found by the discrete-energy-loss method for atoms of equal mass. As an example, the hard-sphere approximation to the Bohr potential will be discussed. The hard-sphere stopping power is

$$
-\frac{\Delta E}{\Delta L}=\frac{n \pi R^{2} E}{2}
$$

whare $R$ is now defined by

$$
E=\frac{E_{B}}{R / a} e^{-R / a}
$$

Here $E_{B}$ is the Bohr energy and $a$ is the screening length. The "stopping power range," $L^{*}$, is found to be

$$
L^{*}=2 \lambda,
$$

whereas the discrete-energy-loss method of Holmes and Leibfried gives a total path, $L$, equal to

$$
L=\lambda\left(3 / 2+R_{i} / a\right) .
$$

In the above formulas, $\lambda=1 / n \pi R_{i}^{2}$, where $R_{i}$ is the hard-sphere radius at the initial energy of the primary. It is noted that the "stopping power range" may be greater or less than that found by the discrete-energy-loss method, depending on whether $R_{i} / a$ is less or greater than $1 / 2$. This surprising result can be elucidated by examining the stopping power [Eq. (1)] shown as the solid line in Fig. 1.3.

When the range is determined by integrating the reciprocal of the stopping power, the particle is assumed to lose energy in a continuous manncr; thus it passes through all points of the $-\Delta E / \Delta L$ curve. On the other hand, in the method of discrete energy loss, the particle loses energy in arbitrary discrete amounts and therefore the "effective stopping power" is that shown by the dotted line (Fig. 1.3). It is seen that the stopping power may be greater or less than the "effective stopping power," which affects the calculated "ranges" in a corresponding manner.

The "stopping power range" using the exact, calculated stopping power of the Bohr potential has been evaluated and compared with the results of the Monte Carlo calculations. The results found were similar to those discussed above and could be interpreted in an analogous manner. For

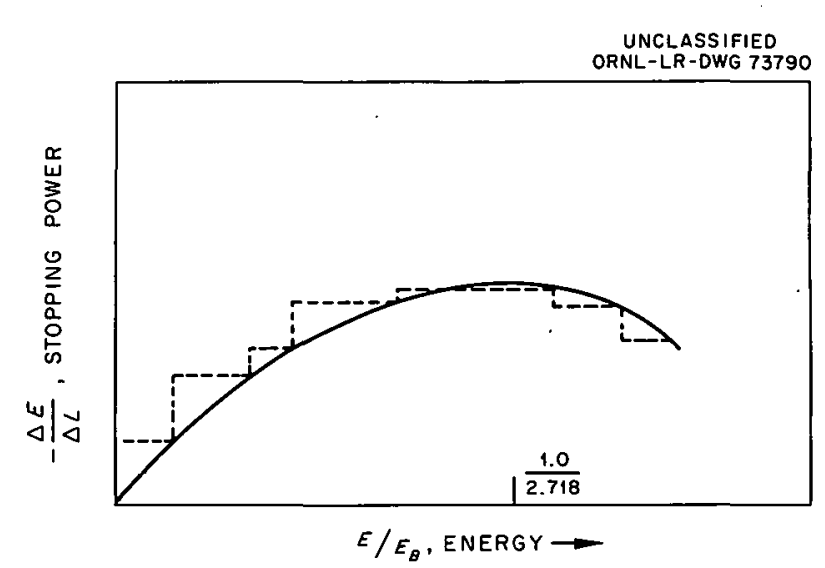

Fig. 1.3. Schematic Diagram of Hard-Sphere Stopping Power (Solid Line). The dotted line is the effective stopping power of a typical atom slowing down by discrete energy losses.

the Nielsen $\mathrm{C} / \mathrm{r}^{2}$ potential, the stopping power is a constant, independent of energy; therefore, it would be predicted that the two ranges would be equal, which, indeed, turns out to be true.

In conclusion, this method of examining the stopping power curve seems very useful in obraining an estimate of the accuracy of the "range" found by integrating the reciprocal of the stopping power.

\subsection{MACHINE STUDIES OF THE SLOWING DOWN OF ENERGETIC ATOMS IN SOLIDS}
M. T. Robinson
O. S. Oen

Several papers $6-10$ have been prepared describing various results from a series of machine calculations ${ }^{11}$ of the slowing down of energetic

${ }^{6}$ M. T. Robinson, D. K. Holmes, and O. S. Oen, Proceedings of the International Colloquium on lon Bomburdment, Paris, 1961 (to be published by Centre National de.la Recherche Scientifique).

${ }^{7}$ O. S. Oen, M. T. Robinson, and D. K. Holmes, Bull. Am. Phys. Soc, 7(2), 171 (1962).

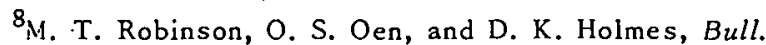
Am. Phys. Soc. 7(1), 171 (1962).

${ }^{9}$ D. K. Holmes, to be published in Proceedings of the International Atomic Energy Agency Symposium on Radiation Damage in Solids and Reactor Materials, Venice, 1962.

${ }^{10} \mathrm{O}$. S. Oen, D. K. Holmes, and M. T. Robinson, submitted to the Joumal of Applied Pbysics.

${ }^{11} \mathrm{M}$. T. Robinson, D. K. Holmes, and O. S. Oen, Solid Stute Div. Ann. Progr. Rept. Aug. 31, 1961. ORNI.-3213, p 3 . 
atoms in solids. The calculations are based on the assumption of purely elastic scattering encounters between atoms, so that the energy range of interest is below $100 \mathrm{kev}$. The histories of individual moving atoms are followed, using Monte Carlo techniques, as the atoms slow down in moving through the crystal. The potential of interaction on which most of the effort to date has been expended is the exponentially screened Coulomb or Bohr potential. In the Monte Carlo studies, the scattering angles are accurately calculated at each collision, using the classical scattering formulas. The results have been compared with approximate calculations in which the screened Coulomb potential has been replaced by a fitted hard-sphere potential or by a fitted inverse r-squared potential; neither of these gives completely acceptable results. The ranges of the atoms have been compared with experimental results, and it has been shown that the simple Bohr potential, using Bohr's screening radius, is inadequate in the sense that a stronger $Z$ dependence than that assumed by Bohr is needed to give adequate correspondence with experiment. With one exception, ${ }^{8}$ these papers dealt with calculations performed with an IBM 7090 program designated as RANGE I, in which the atoms of the solid were assumed to be randomly located in space. The correct density of the solid was maintained by choosing a constant "free flight" distance between collisions. A new program, RANGE II, has been written in which the atoms of the solid are correctly located on lattice sites, obviating the need to use the rather unsatisfactory concept of free flight. The opportunity was also taken to improve the treatment of the equations of motion of the particles involved in a collision and to elaborate the analysis of the results of the computations. In addition to distributions and central measures of various range quantities, the new program produces distributions of the final primary energy and of the final primary direction.

RANGE II permits the study of solids having any structure conveniently describable on orthorhombic coordinates (this includes hexagonal crystals, for example, but excludes many monoclinic and triclinic ones) and having as many as nine crystallographically distinct atoms (these may or may not be chemically different). A simple model of thermal vibrations is included, each kind of atom being permitted to execute simple harmonic motions about its lattice site with an appropriate rms amplitude. Other new features of the program include the possibilities of following the primary for a specified number of collisions (rather than through a specified energy range) and of truncating any histories exceeding a preset number of collisions. Various sorts of detailed output of individual histories are also permitted. The new program requires 50 to $150 \%$ more computer time than did RANGE I, the increase depending on which combination of several options is chosen.

Most of the results obtained with the RANGE II program refer to the following "standard problem": 10 -kev copper atoms are started isotropically from a lattice site in an infinite crystal of copper. They are permitted to lose energy in two-body interactions with the atoms of the crystal until their energies fall below $25 \mathrm{ev}$. Unless otherwise stated, this standard problem will be the source of the results cited below.

The most striking result of the calculations is that a small fraction of the primary particles $(\sim 1 \%$, depending on the potential chosen and the themal vibration amplitude) move exceedingly large distances with only very small losses in energy. Such events were anticipated for hard-core calculations, and a means was provided in the program for rejection of those primaries which traveled (at any energy) more than a preset distance. In the standard problem this distance was chosen as (approximately)

$$
a_{0}^{3} /[R(10 \mathrm{kev})]^{2}+25 \sqrt{6} a_{0} \approx 880 \mathrm{~A},
$$

where $a_{0}$ is the lattice constant of copper, $R(10$ kev) is the hard-core radius appropriate to the initial energy, and the second term represents the average distance for 100 collisions to occur. [The average distance which a primary moves between collisions in the fcc metals is approximately $\frac{1}{2}$ the unit translation in $a\langle 112\rangle$ direction, $(3 / 2)^{1 / 2} a_{0}$. This result is true of the calculations made for $A l, C u, A g$, and $A u$ with the RANGE I program as well as RANGE II.] In the standard program, $0.5 \%$ of the primaries were rejected on this score, the others all having ranges of ordinary magnitude. The surprising result was the presence of a similar group of particles in calculations made with the Bohr and with the Bom-Mayer potentials. The occurrence of these "wild" histories in the latter cases required numerous changes in the program to avoid excessive consumption of machine 
time and has temporarily interrupted the computational program because of their disastrous effects on many of the problems of interest.

A study of these penetrating events, which have become known as "channel" or "tunnel" events, reveals that the majority of them are moving very close to the $\langle 110\rangle$ directions of the lattice. A portion of a typical trajectory is shown in Fig. 1.4. The particle spirals leisurely down the channel in the [10i]] direction, its motion very largely restricted to a single plane. The stereographic triangle of Fig. 1.5 shows the directions of the 20 particles (out of 1009) in the standard problem which made over 250 collisions (the mean number of collisions in slowing down to $25 \mathrm{ev}$ was 57.6). Nine of these particles made over 1000 collisions; their directions after the 800th collision are also shown in the figure. It is evident that a sort of focusing is operative and that the $\langle 110\rangle$ direction is preferred. That this is not the only direction in which "channeling" is significant, however, is shown by a run in which $10-\mathrm{kev}$ copper primaries were normally incident on a (001) surface of copper. In this case, $75 \%$ of the primaries made more than 250 collisions; in fact, only 300 out of 1188 particles reached 25 ev before
AT THE ORIGIN OF THE COORDINATE SYSTEM, THE PROJECTILE ENERGY IS $387 \mathrm{eV}$ COLLISION NUMBERS ARE INOICATED ALONG THE TRAJECTORY. THE TOTAL NUMBER OF COLLISIONS IN THIS HISTORY WAS 10567.

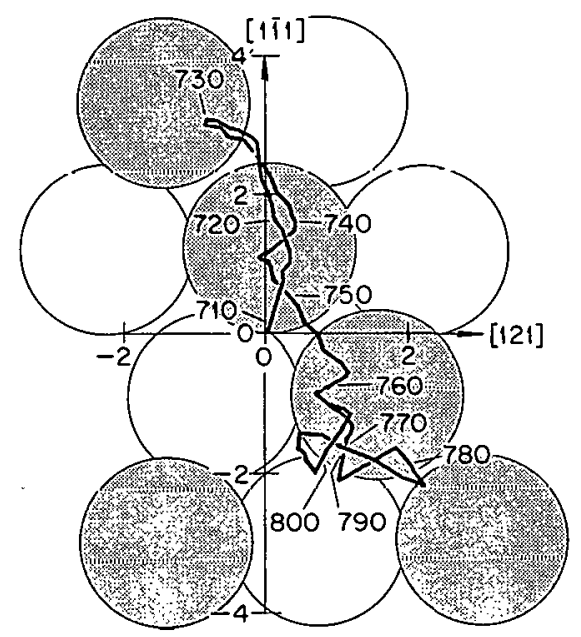

PROJECTION ONTO THE (1OT) PLANE SCALES IN ANGSTROMS CIRCLES SHOW LOCATIONS OF ATOMS IN THE PROJECTION

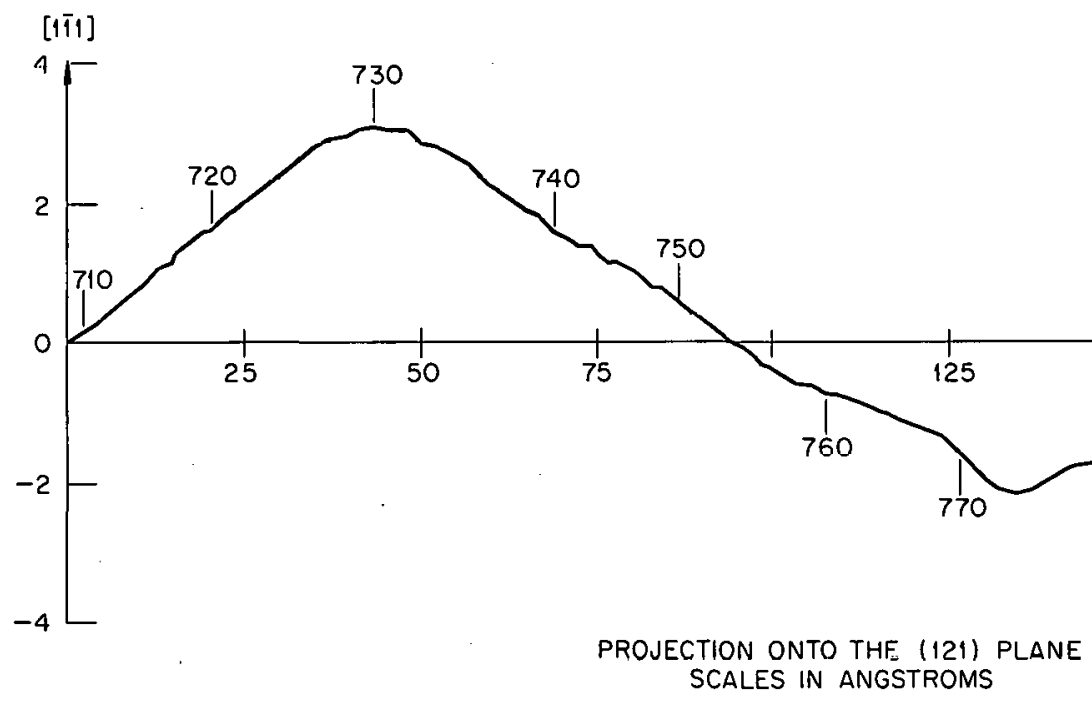

Fig. 1.4. Portion of a "Channel" Event. A 10-kev copper primary started from a lattice site. The Bohr potential $(k=i)$ was used. 
UNCLASSIFIED ORNL-LR-DWG 66797

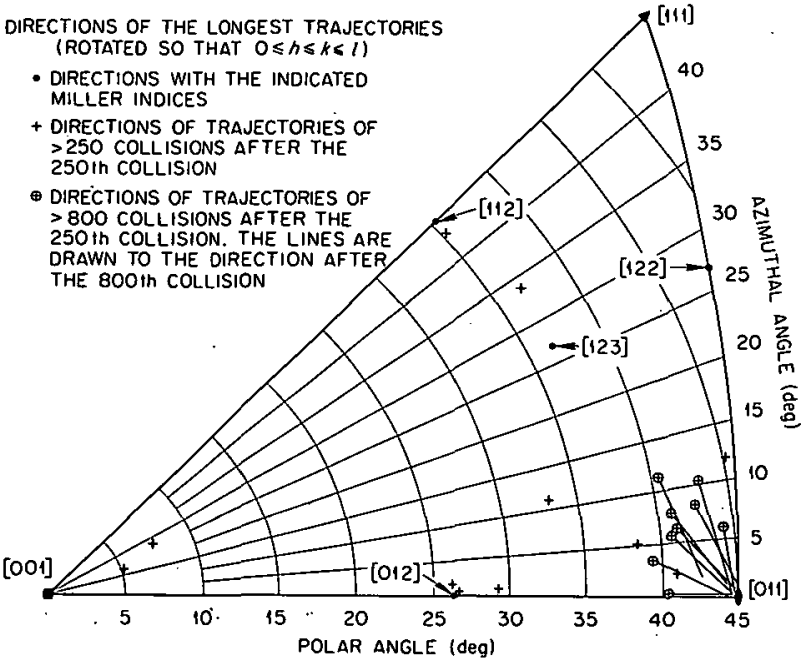

Fig. 1.5. Directions of the Longest Trajectories of 10-kev Copper. Primaries Slowing Down in Copper According to the Bohr Potential $(k=1)$. the computer run was arbitrarily terminated. All the very long trajectories were moving very nearly in the [001] direction, and only small energy losses had occurred.

If the "channel" events are ignored, comparisons of the lattice and random calculations can be made. These comparisons are given in Figs. 1.6 and 1.7 and in Table 1.1. With the use of the hard-core approximation (Fig. 1.6), the two solid models give results in quite close agreement. However, when the Bohr potential is employed (Fig. 1.7), the distribution of the vector range is seen to be significantly more skewed in the lattice model than in the random case. The shape of the distribution in the lattice case is more nearly like the experimental observations of Davies ${ }^{12}$ than is that in the random case. Whether the channel events are to be identified with the exponential tail observed by Davies is still a very open question.

\footnotetext{
${ }^{12}$ J. A. Davies et al., Can. J. Chem. 38, 1535 (1960).
}
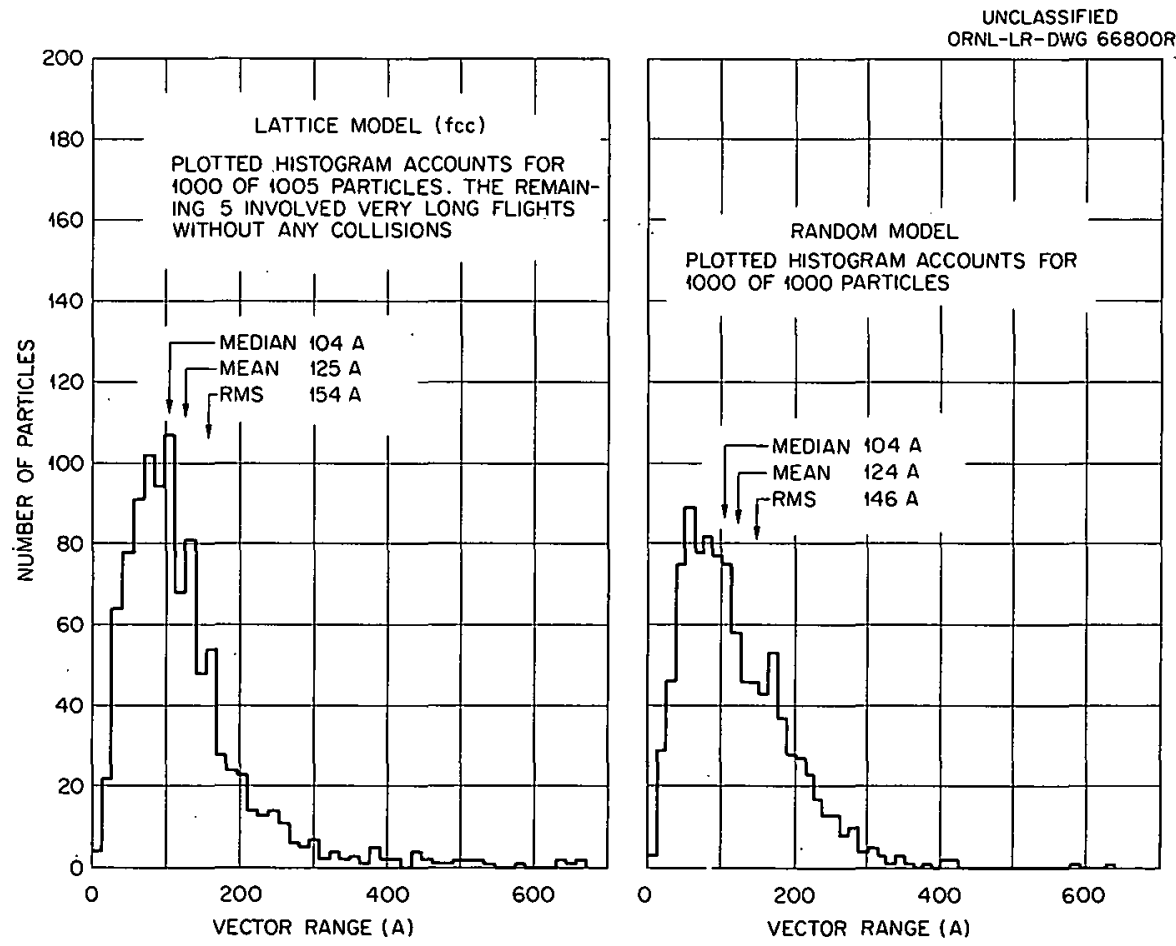

Fig. 1.6. Distribution of the Vector Range, 10-kev Copper Atoms Slowing Down to 25 ev in Copper. A comparison of the lattice and random models for hard-core interactions. 

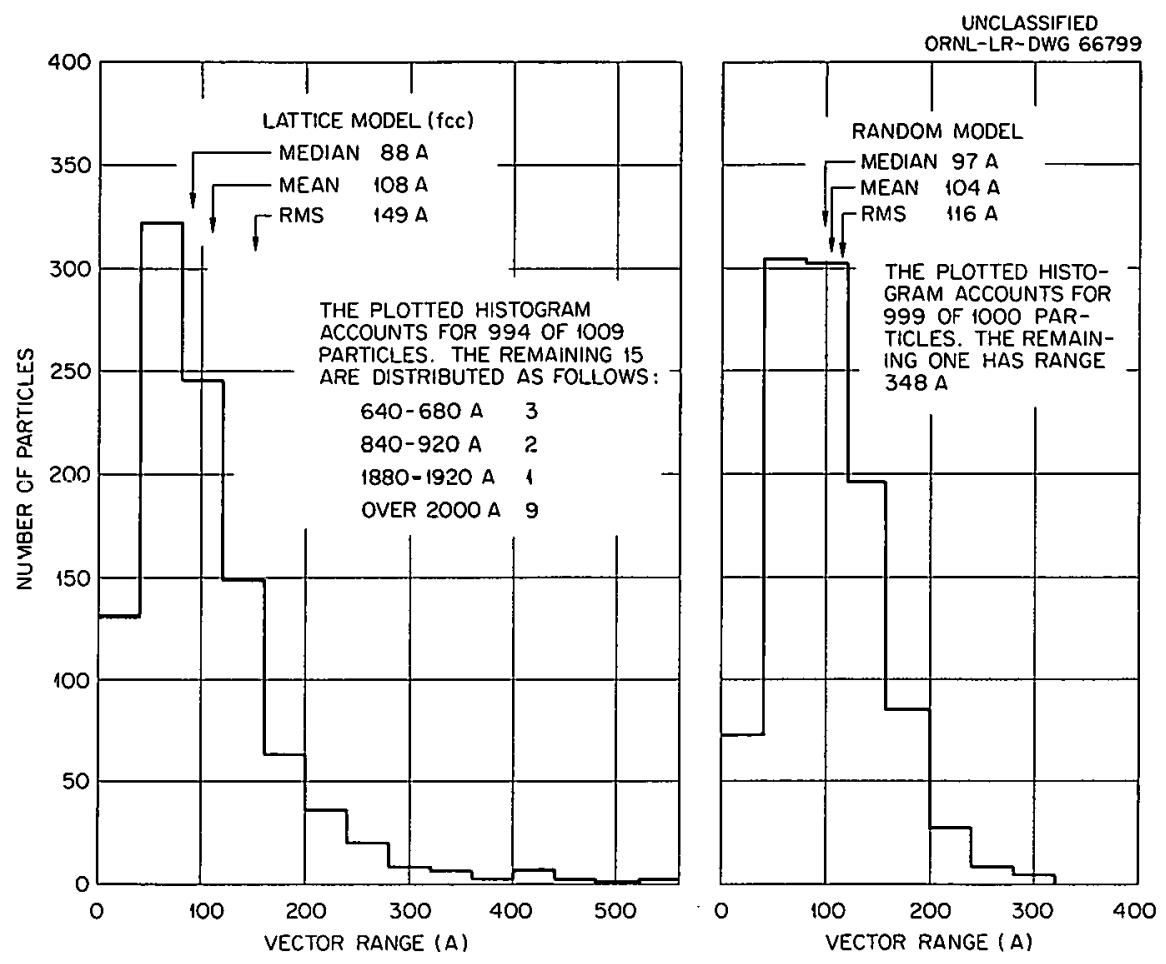

Fig. 1.7. Distribution of the Vector Range, 10-kev Copper Atoms Slowing Down to 25 ev in Copper. A comparison of the Lattice and random models for the Bohr potential.

The effects of thermal vibration on the vector range and the occurrence of channel events are shown in Table 1.2. The variations in the range quantities are probably due to statistical fluctuations, but the number of channel events decreascs to zero if a sufficiently large "temperature" is employed.

The channel events change in identity and number when superficially trivial changes are made in the potential, affecting only the potential at large separations. This fact suggests that much of what is seen in the calculations is due to unsatisfactory treatment of the scattering problem at large distances. For this reason, present efforts are being directed toward improvements in the computational procedure so that more satisfactory potentials may be used. The approach currently being investigated involves the developing of approximations to the scattering integrals for the interaction between two particles in the form

$$
T / T_{\max }=\frac{1+a_{1} b^{2}+a_{2} b^{4}+\ldots}{1+\beta_{1} b^{2}+\beta_{2} b^{4}+\ldots},
$$

where the $\alpha_{i}$ and $\beta_{i}$ are functions of energy and $b$ is the impact parameter. The well-known hardcore approximation may be represented by

$$
\left.\begin{array}{c}
\beta_{1}=\beta_{2}=\ldots=\alpha_{2}=\alpha_{3}=\ldots=0 \\
a_{1}=-1 / R^{2}
\end{array}\right\},
$$

where $R$ is the hard-core radius. The truncated Coulomb approximation ${ }^{13}$ may be represented by

$$
\left.\begin{array}{c}
a_{2}=a_{3}=\ldots=\beta_{2}=\beta_{3}=\ldots=0 \\
\beta_{1}=4 E^{*}\left(B+E^{*}\right) / A^{2} \\
a_{1}=-B^{2} / A^{2}
\end{array}\right\} \text {, }
$$

\footnotetext{
${ }^{13} \mathrm{G}$. Leibfried and O. S. Oen, J. Appl. Pbys. 33, 2257 (1962).
} 
Table 1.1. Comparison of Solid Models and Interatomic Potentials

(10-kev copper atoms slowing down to $25 \mathrm{ev}$ in copper)

\begin{tabular}{|c|c|c|c|c|c|}
\hline & \multicolumn{3}{|c|}{ Vector Range (A) } & \multirow{2}{*}{$\frac{\text { Median }}{\text { Mean }}$} & \multirow{2}{*}{$\frac{\text { RMS }}{\text { Mean }}$} \\
\hline & Median & Mean & RMS & & \\
\hline \multicolumn{6}{|c|}{ Bohr Potential $(k=1)$} \\
\hline Random & 97 & 104 & 116 & 0.93 & 1.12 \\
\hline Lattice & 88 & 108 & 149 & 0.81 & 1.38 \\
\hline \multicolumn{6}{|c|}{ Hard-Core Approximation } \\
\hline Random & 104 & 124 & 146 & 0.83 & 1.18 \\
\hline Lattice & 104 & 125 & 154 & 0.83 & 1.23 \\
\hline & \multicolumn{3}{|c|}{ Number of Collisions } & \multicolumn{2}{|c|}{ Total Path } \\
\hline & Median & Mean & RMS & \multicolumn{2}{|c|}{ Collision (A) } \\
\hline \multicolumn{6}{|c|}{ Bohr Potential $(k=1)$} \\
\hline Random & 59 & 61.9 & 67.8 & & \\
\hline Lattice & 50 & 57.6 & 74.2 & & \\
\hline \multicolumn{6}{|c|}{ Hard-Core Approximation } \\
\hline Random ${ }^{a}$ & & 7 & & & \\
\hline Lattice & 6 & 7.18 & 7.60 & & \\
\hline Theory (random) & & 6.99 & r. & & \\
\hline
\end{tabular}

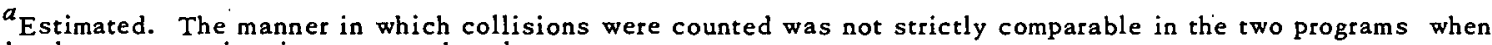
the hard-core approximation was employed.

Table 1.2. Effect of Thermal Vibrations on Vector Range

10-kev copper atoms slowing down to 25 ev in copper. Bohr potential $(k=1)$

\begin{tabular}{|c|c|c|c|c|c|c|}
\hline \multirow{2}{*}{$\begin{array}{l}\text { RMS Thermal } \\
\text { Vibration } \\
\text { Amplitude (A) }\end{array}$} & \multicolumn{3}{|c|}{ Vector Range (A) } & \multirow{2}{*}{$\frac{\text { Median }}{\text { Mean }}$} & \multirow{2}{*}{$\frac{\mathrm{RMS}}{\text { Mean }}$} & \multirow{2}{*}{$\begin{array}{c}\text { Channel } \\
\text { Events } \\
(\%)\end{array}$} \\
\hline & Median & Mean & RMS & & & \\
\hline 0 & 88 & 108 & 149 & 0.81 & 1.38 & 0.9 \\
\hline 0.025 & 84 & 107 & 150 & 0.79 & 1.40 & 0.4 \\
\hline $0.050^{a}$ & 88 & 113 & 152 & 0.78 & 1.35 & 0.1 \\
\hline 0.075 & 88 & 115 & 160 & 0.77 & 1.39 & 0.3 \\
\hline 0.100 & 88 & 109 & 143 & 0.81 & 1.31 & 0.0 \\
\hline $0.200^{b}$ & 86 & 108 & 133 & 0.80 & 1.23 & 0.0 \\
\hline Random model & 97 & 104 & 116 & 0.93 & 1.12 & \\
\hline
\end{tabular}

${ }^{a}$ Approximately $0^{\circ} \mathrm{K}$ for copper.

${ }^{b}$ Approximately the melting point of copper. 
where the potential is

$$
\begin{aligned}
& V_{T C}(r)=\frac{A}{r}-B \quad 0 \leqq r \leqq A / B, \\
& =0 \quad A / B \leqq r \leqq \infty \text {, }
\end{aligned}
$$

and $E^{*}$ is the reduced energy of the primary. Since the parameters, Eq. (3), give a very reasonable account of $T / T_{\max }$ for the Bohr potential, it is hoped that rather low-order polynomials will suffice to represent any interesting potential.

\subsection{HARD-SPHERE SCATTERING APPROXIMATIONS}

\section{E. J. Lee M. T. Robinson}

The Holmes-Leibfried energy-dependent hardsphere approximation ${ }^{14}$ has been compared with the approximation of Beeler and Besco ${ }^{15}$ in which the hard-sphere radius is dependent on both the incident particle en ergy and the impact parameter. In the Holmes-Leibfried approximation, the centerof-mass scattering angle, $\theta$, is given by $\cos \theta=$ $2(s / x)^{2}-1$, where $s$ is the impact parameter and $x$ is the distance of closest approach for head-on collision. In the Beeler-Besco approximation, the center-of-mass scattering angle is given by cos $\theta=2\left(s / R_{0}\right)^{2}-1$, where $K_{0}$ is the distance of closest approach for the given impact parameter, $s$. The two approximations and an accurate numerical solution obtained on an IBM 7090 computer are compared in Fig. 1.8 for an incident energy of $5 \mathrm{kev}$ in copper using the Bohr potential (exponentially screened Coulomb). The ratio of the energy transferred to the target particle to the maximum en ergy transferable, $T / T_{\max }=1 / 2(1-\cos$ $\theta$ ), is plotted as a function of impact parameter in Fig. 1.8. In the numerical solution the potential was truncated at a radius of 10.4942 screening radius units. Only a negligible error results from this truncation. The curves shown are typical and illustrate the overestimation of energy transfer

\footnotetext{
${ }^{14}$ D. K. Holmes and G. Leibfried, J. Appl. Phys. 31, 1050 (1960).

${ }^{15} \mathrm{~J}$. R. Beeler, Jr., and D. G. Besco, "Knock-On Cascades and Point Defect Configurations in Binary Materials," to be published in Intemational Atomic Energy Agency Symposium on Radialion Datnage in Sulids und Reactor Materials, Venice, May 7-11, 1962.
}

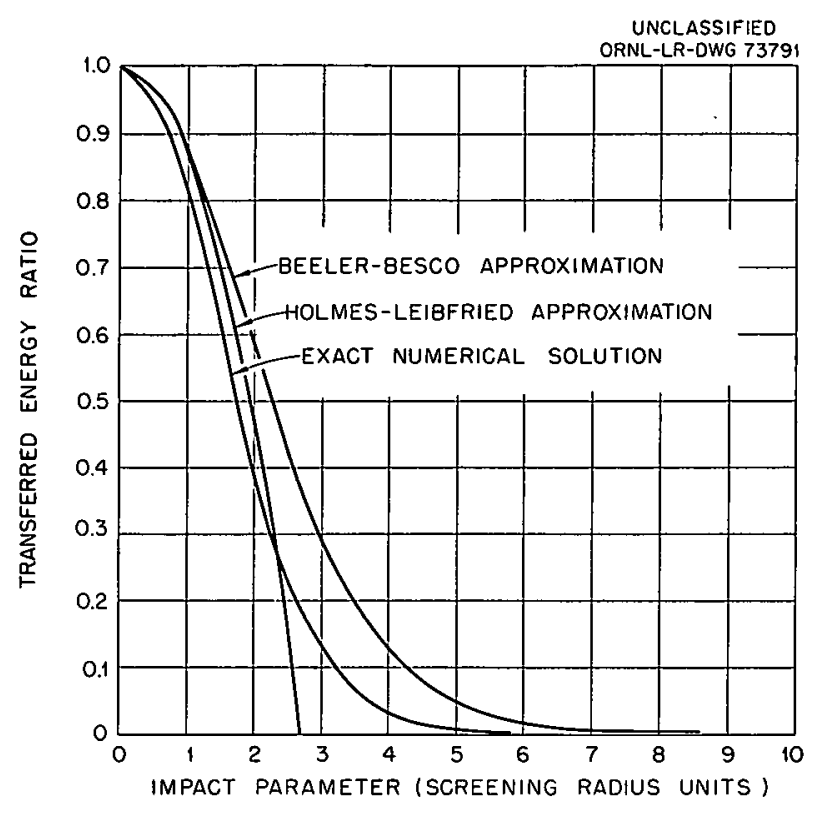

Fig. 1.8. Energy Transfer for Cu-Cu Collisions at 5 kev Incident Energy; Bohr Potential.

for all collisions by the Beeler-Bescn approximation. The disagreement increases at higher incident energies. For the closer collisions the Holmes-L eibfried a pproximation also overestimates the energy transfer, although to a lesser extent. On the other hand, it underestimates the energy transfer for more distant collisions. It is difficult to compare the detailed effect of the approximations on the range and other averages of interest because of the analytical complexity.

\section{4 MONTE CARLO CALCULATIONS OF THE RANGES OF ENERGETIC ATOMS IN SOLIDS ${ }^{16}$}
M. T. Robinson
D. K. Holmes

O. S. Oen

The slowing down of energetic atoms in solids has been investigated through the use of Monte Carlo methods on a large digital computer. Neither the hard-sphere approximation nor the inversesquare approximation affords a particularly satisfactory account of the exponentially screened

\footnotetext{
${ }^{16}$ Abstract of paper presented at the International Colloquium on lon Bombardment. Bellevue, France, Deccmber 4-8, 1961 (to bc published).
} 
Coulomb potential. It is found that, for copper, silver, and gold, slowing down in their respective solids, the screening radii must be, respectively, $1.15,1.6$, and 2.3 times greater than the values proposed by Bohr, in order to explain the available experimental data. It is apparent from the results of these calculations that modifications of the interatomic potential functions, especially for heavier elements, must be sought.

\subsection{RANGES OF ENERGETIC ATOMS IN SOLIDS ${ }^{17}$}
O. S. Oen
D. K. Holmes

M. T. Robinson

The ranges in solids of atoms having energies from 1 to $100 \mathrm{kev}$ have been calculated using Monte Carlo techniques. The model assumes that the movingatom loses all its energy. through binary elastic collisions with the atoms of the solid. The potential of interaction principally studied is an exponentially screened Coulomb (Bohr) potential, and the scattering angles are calculated explicitly. It is found that neither the hard-sphere approximation nor the inverse $r$-squared approximation to the Bohr potential is particularly good. To obtain correspondence with experimental results, the Bohr screening length must be increased as the atomic number of the interacting atoms increases.

\subsection{CALCULATION OF AVERAGES FOR PRIMARY RECOIL DISTRIBUTIONS ${ }^{18}$}

\section{G. Leibfried-}

The slowing down of an energetic particle in a random assembly of scattering centers is discussed. Certain averages for physically interesting quantities can be calculated in a very general form, including unequal masses of scattering and slowing down particles. The averages refer to quantities

\footnotetext{
${ }^{17}$ Abstract of paper submitted for publication in the Joumal of Applied Pbysics.

${ }^{18}$ Abstract of published paper: J. Appl. Phys. 33, 1933 (1962).
}

such as the total distance traveled $(L)$ and the vector distance to the final positions $\left(x_{i}\right)$ and powers thereof. Two assumptions simplify the evaluation of the general results considerably: ( $\alpha$ ) The mean free path $\lambda$ is a simple power of the energy $E: \lambda(E) \sim E^{\mu} ;(\beta)$ the distribution $g(E ; \epsilon)$ of energies $\epsilon$ after one collision with initial energy $E$ does not change its shape with $E: E g(E ; \epsilon)=$ $\kappa(\epsilon / E)$. A special distribution of this kind is the hard core distribution. Under assumptions $a$ and $\beta$ all calculations are reduced to the evaluation of an integral like $\int \kappa(\eta) \eta^{\mu} d \eta$. For equal masses and the hard core distribution, one can easily calculate averages of any power of $L$ and obtain the distribution function of $L$, this being proportional to $L^{1 / \mu} \exp (-L / \lambda)$. Under the assumption $\beta$ alone, with arbitrary $\lambda(E)$, results are given for special distributions and equal masses (including the hard core distribution). The evaluation of the averages now requires simple integrals over $\lambda$ for linear averages and double integrals for quadratic averages with known integral kernels. For unequal masses, approximations are discussed.

\subsection{SIMPLE APPROXIMATION FOR CLASSICAL SCATTERING AT LARGE ANGLES ${ }^{19}$}

$$
\text { G. Leibfried O. S. Oen }
$$

The classical scattering for repulsive potentials is treated approximately by a matching method. The matching potential is a cut-off potential which matches the value and the slope of the actual potential at the distance of closest approach. The method is thought to be successfully applicable to interatomic potentials of the screened Coulomb type. Comparison is made with machine results for an exponentially screened Coulomb potential. The differential cross section and the stopping power are compared for various energies. The agreement in the differential cross section is fair except for very low energy transfers. The stopping powers agree over a wide energy range with an error of only a few percent. Further comparison is made with another approximation given by Lehman and Shapiro.

\footnotetext{
${ }^{19}$ Abstract of published paper: J. Appl. Pbys. 33, 2257 (1962).
} 


\subsection{HIGHER-ORDER AVERAGES OF PRIMARY RECOIL DISTRIBUTIONS ${ }^{20}$}

\section{G. Leibfried}

One problem of interest in radiation damage theory is the slowing down of an energetic primary knock-on in its own lattice. The primary starts at the origin, slows down and comes to rest at a final vector distance, $r=\left(x_{s}\right)$. In this paper we study averages over the distribution $w(r)$ of the final positions. The slowing down is described here by a series of subsequent and independent two-body collisions, with the scattering centers located at random rather than in a lattice arrangement. Analytical expressions for the energydependent linear $\left(\bar{x}_{s}\right)$ and quadratic $\left(\bar{x}_{s} x_{t}\right)$ averages over $w(r)$ have been given in previous work. The purpose of this paper is to give a more elegant method which also is easily extended to higher order averages, $\left(\overline{x_{s} x_{t} x_{u}}\right),\left(\overline{x_{s} x_{t} x_{u} x_{v}}\right)$, and so on. These higher order averages improve the knowledge of the distribution, which, of course, would be the most desirable quantity to calculate.

\subsection{LONG-RANGE CHANNELING EFFECTS IN IRRADIATED CRYSTALS? ${ }^{21}$}
C. Lehmann
G. Leibfried

If an en ergetic atom, a primary, slows down in a lattice, it has the possibility of traveling long

\footnotetext{
${ }^{20}$ Abstract of paper submitted for publication in Zeitschrift fuer Pbysik.

21 Abstract of paper to be submitted for publication.
}

distances, without essential interaction, along almost force-free channels bordered by closepacked atomic chains. This effect has not been taken into account until recently, because, for the sake of simplicity, the lattice always has been replaced by a corresponding random arrangement. This "channeling" behavior has been found in machine calculations by Robinson, Holmes, and Oen, ${ }^{22}$ which take the lattice structure into account. In this paper the behavior of a primary moving along a channel bordered by the most densely packed directions is investigated analytically. Numerical results are given for a copper primary moving in a copper crystal, but the results can be easily extended to other cases. Two potentials are used: (1) an exponentially screened Coulomb potential after Bohr, used also in the machine calculations and thought to give an adequate description for relatively high energies and small interatomic distances; and (2) a purely exponential potential after Born-Mayer, better suited for relatively low energies and large atomic distances. The maximum ranges are very large; for $10 \mathrm{kev}$ they are of the order of $10^{3}$ lattice parameters for the Bom-Mayer potential and up to $10^{7}$ for the Bohr potential. Presumably, the Born-Mayer potential is a better description for these events. The investigation is confined to motion near the channel axis. Therefore, we can only obtain the range distribution near the maximum range. The probability of these events is such that they can play a role in high-energy sputtering from crystal surfaces and in radiation damage.

\footnotetext{
${ }^{22}$ To be published; $c \%$ also Bull. Am. Pbys. Soc. $7(2), 171(1962)$.
} 


\section{Theory of Sputtering}

\section{I A GEOMETRICAL MODEL OF MONOCRYSTAL SPUTTERING}

\section{T. Robinson}

Experimental studies ${ }^{1-5}$ of the sputtering of copper monocrystals by rare-gas ions in the kev range have demonstrated the dramatic dependence of the sputtering yield (the number of target atoms ejected per incident ion) on the orientation of the ion beam with respect to the crystallographic axes of the target crystal. Similar experiments 1,2 on germanium with $\mathrm{l}^{-}$to $5-\mathrm{kev} \mathrm{Ar}^{+}$ions reveal only a small dependence on orientation. Some success has been had in understanding the copper data on the basis of a simple, essentially geometrical, model, 1 and studies are in progress, both to improve the calculations and to extend them to targets of other symmetries.

The model assumes, following Rol, ${ }^{3}$ that the yield is determined to a sufficient degree by the first collision of the incident ion with a lattice atom: It may be mentioned, in support. of this assumption, that the range of an ion in a solid is also largely determined by the first flight, at least for not too low energies. ${ }^{6}$ The yield, $S$, is then as sumed to be given by

$$
S_{b k l}(E)=\alpha \tau_{b k l}(E) / \lambda_{b k l}(E),
$$

\footnotetext{
${ }^{I} A$. L. Southern, W. R. Willis, and M. T. Robinson, "Sputtering Experiments with 1 - to 5-kev $A_{r}+$ Ions," to be published in the Joumal of Applied Physics (see abstract in Part III of this report).

${ }^{2}$ A. L. Southern, M. T. Robinson, and D. R. Burrowbridge, Solid State Div. Ann. Progr. Rept. Aug. 31. 1961, ORNL-3213, p 49; "Ar ${ }^{+}$Ion Bombardment of Metal Surfaces," this report, Part III.

${ }^{3}$ P. K. Rol, Thesis, Amsterdam, 1960.

. ${ }^{4} \mathrm{O}$. Almén and G. Bruce, Nucl. Instr. Methods 11, 257 (1961).

${ }^{5} \mathrm{G}$. D. Magnuson, General Dynamics/Astronautics, private communication, 1962.

${ }^{6}$ D. K. Holmes and G. Leibfried, J. Appl. Pbys. 31, 1046 (1960).
}

where $E$ is the (laboratory) energy of the incident ion, $\alpha$ is a constant depending on the masses of the ions and lattice atoms, $\lambda_{b k}(E)$ is the mean free path of an ion incident in an $\langle b k l\rangle$ direction, and $\tau_{b k l}(E)$ describes the deposition of energy by the ion in its first collision. If Rol's original notion were followed, as was done in the first application of this theory to monocrystals, 1 we would write

$$
\tau_{b k l}^{(1)}(E)=2\left(\bar{T} / T_{\max }\right) E=2\left(\overline{\sin ^{2} \theta / 2}\right) E
$$

where $\theta$ is the center-of-mass scattering angle, $T$ is the transferred energy, and the overlines represent average values. The results of the original analysis of the copper data suggested that a better alternative might be to write

$$
\begin{aligned}
\tau_{b k l}^{(2)}(E)=(3 / 2)(\overline{\sin \theta / 2}) E^{1 / 2} \\
=(3 / 2)\left(|\bar{p}| /\left|p_{\max }\right|\right) E^{1 / 2},
\end{aligned}
$$

where $|p|$ represents the magnitude of the transferred momentum. Since convincingarguments with respect to a choice between these two altematives have not been developed, both will be retained for the present. Also, for the present, consideration will be restricted to hard-core scattering of the incident ions by the lattice atoms; the appropriate hard-core radius $R(E)$ will be chosen by any desired procedure. In hard-core scattering, if the impact parameter, $b$, could cover the entire range $0 \leqq b \leqq R$, there would be obtained

$$
\tau\left(\frac{1)}{b k l}(E)=E\right.
$$

and

$$
\tau_{b k l}^{(2)}(E)=E^{1 / 2}
$$

but, as pointed out before, ${ }^{I}$ the impact parameter range in a crystal is restricted by shadowing 
effects, which differ for different directions; therefore, crystallographic variability must be retained in the expressions for $\tau_{b k l}^{(1)}$ and $\tau_{b k l}^{(2)}$.

The target crystal is imagined to consist of an array of spheres of radius $R(E)$, arranged on an appropriate lattice, and the beam of ions is taken to be incident on it in an $\langle b k l\rangle$ direction. Consideration will be restricted to the case of normally incident ions. A fraction of the ions, $P_{b k l}(E)$, will undergo collisions between the surface of the crystal and a depth $t_{b k l}$, the wit translation parallel to $\langle h k l\rangle$, and in so doing will travel a mean distance $\bar{X}_{b k l}$. The remaining fraction of particles, $1-P_{h k l}(E)$, will travel indefinitely far without collision, because of the repeating structure of the crystal. This manifest difficulty may be treated in either of two ways: (1) As was done before, ${ }^{1}$ it may be assumed that those ions not colliding in $0 \leqq X \leqq$ $t_{h k l}$ have mean free paths, measured from the target surface, which are isotropic. Thus,

$$
\begin{aligned}
\lambda_{b k l}^{(1)}(E)=P_{b k l}(E) \bar{X}_{b k l}(E) & \\
& +\left[1-P_{b k l}(E)\right] \lambda_{0}(E),
\end{aligned}
$$

where

$$
\lambda_{0}(E)=\left\{n \pi[R(E)]^{2}\right\}^{-1},
$$

in which $n$ is the atomic density; the energy dependences indicated in Eq. (4) are all consequent on the energy dependence of the hard-core radius. (2) The other approach, which we will term the quasi-random, assumes that those particles penetrating beyond a depth $t_{b k l}$ may be regarded as uniformly distributed in space, thus leading to the result:

$$
\begin{aligned}
\lambda_{b k l}^{(2)}(E)=P_{b k l}(E) & \bar{X}_{b k l}(E) \\
& +\left[1-P_{b k l}(E)\right]\left[t_{b k l}+\lambda_{b k l}^{(2)}(E)\right]
\end{aligned}
$$

or

$$
\begin{aligned}
\lambda_{b k l}^{(2)}(E)= & \bar{X}_{b k l}(E) \\
& +\left[1-P_{b k l}(E)\right] t_{b k l} / P_{b k l}(E) .
\end{aligned}
$$

The sputtering yield may now be evaluated by inserting either Eq. (2) or Eq. (3) and either Eq. (4) or Eq. (6) into Eq. (1). The usefulness of the resulting expression is to be assessed by deter- mining $R(E)$ and a by comparison of the equation with experimental data.

An IBM 7090 program has been written to permit the rapid evaluation of Eqs. (2) to (6) and the associated yields for a variety of different crystals. These fall into three groups:

1. A face-centered cubic group employing four sets of positions of the space groups $F 43 \mathrm{~m}$. Any of the four sets of fourfold positions may be occupied completely or statistically by atoms of specified mass and radius; the atoms on the different sublattices may be different. If the $a$ sublattice alone is filled, the structure is that of the fcc metals; the $b$ sublattice represents the octahedral holes, and the $c$ and $d$ sublattices represent the tetrahedral holes. The structures of In $\mathrm{Sb}$ or germanium may be represented by filling the $a$ and $c$ sites, and that of $\mathrm{NaCl}$ is represented by filling the $a$ and $b$ sites.

2. A body-centered cubic group employing three sets of positions of space group $1 \mathrm{~m} 3 \mathrm{~m}$. The twofold $a$ sites are those of the bcc metals, the sixfold $b$ sites are the (distorted) octahedral holes, and the eightfold $c$ sites have tetrahedral symmetry if all $a$ and $b$ sites are occupied.

3. A hexagonal close-packed group based on three sets of positions of space group $\mathrm{PG}_{3} \mathrm{mc}$. The twofold $a$ sites are those of the hcp metals, the twofold $b$ sites are the octahedral holes, and the fourfold sites represent the tetrahedral holes. The fourfold sites are split, for convenience, into two twofuld sets. The axial ratio, $c_{0} / a_{0}$, of the hexagonal unit cell may be varied as desired.

The program has been debugged, and its application to sputtering in copper and germanium is in progress. Extension to zinc will be made soon.

\subsection{EXAMINATION OF A THEORETICAL MODEL OF ANISOTROPIC SPUTTERING AT HIGH ENERGIES BASED ON FOCUSING CHAINS}

\section{Lehmann}

Bombardment of single crystal surfaces with ions results in ejection of atoms along low-indexed crystallographic directions. Until recently, available theories ${ }^{7}$ of sputtering employed fitting parameters in order to explain experimental data and

\footnotetext{
${ }^{7}$ See literature citations in ref 8.
} 
did not account for the anisotropy of this effect. Thompson ${ }^{8}$ was the first to consider crystallographic dependence without the use of fitting parameters. This investigation is based on Silsbee-type 9 focusing collisions as the elementary event responsible for directional ejection of atoms from the solid.

A more refined and general theory which also is hoped finally to explain the emission ratio (yield) of atoms from single crystals as a function of surface orientation is being developed.

The events leading to ejections of atoms can be separated into two steps: (1) the history of the incoming ion, initiating focusing collision sequences at certain distances away from the surface, and (2) the history of an initiated focusing collision sequence toward the surface, eventually resulting in the ejection of an atom from the surface. The first step involves the question of the mean free path of a primary being shot onto an arbitrary.crystal surface. This question is closely related to the problem of channeling. ${ }^{10}$ At this time, neither reliable experimental data nor sufficient theoretical calculations are available about anisotropic penetration of an energetic primary into a solid. Therefore, the lattice is considered as random for the history of the incoming ion. This is assumed to be a good approximation since the anisotropy of the second step presumably is considerably higher.

It is assumed that the primary, of kinetic energy $E$, having normal incidence (all calculations can easily be extended to oblique incidence), collides with a lattice atom at a distance $x$ from the surface and transfers the energy $T$ to this stationary atom. By this energy release, a small zone (with negligible dimensions in comparison to the range of the focuson) of interstitials and vacancies is created, forming the origin of focusing collisions which start from this zone isotropically in all possible close-packed directions. The probability distribution of focuson ranges along close-packed directions has been discussed recently. ${ }^{11}$ In the

\footnotetext{
${ }^{8}$ M. W. Thompson, p 85 in Proceedings of the Fifth Conference on lonization Pbenomena in Gases, Munich, 1961. vol I, North Holland Publishing Company, Amsterdam.

${ }^{9}$ R. H. Silsbee, J. Appl. Pbys. 28, 1246 (1957).

${ }^{10} \mathrm{C}$. Lehmann and G. Leibfried, "Long Range Channeling Effects in Irradiated Crystals?" (this report; Part I).

${ }^{11}$ G. Leibfried, J. Appl. Phys. 30, 1388 (1959).
}

following, this formalism is used and applied, for reasons of simplicity, to copper ions on to copper.

The number of focusons starting in one of the 12 equivalent close-packed $\langle 110\rangle$ directions from a radiation-damage zone (in which the energy $T$ has been distributed) and having ranges $\geqq n D$ ( $D$ is the nearest neighbor distance) is

$$
\begin{array}{rlrl}
\nu & =1121.5 \frac{T}{2 E_{d}}\left[1-\frac{n}{n_{0}}\left(1-\ln \frac{n}{n_{0}}\right)\right], \\
n / n_{0} & \leqq 1, \\
& =0, & n / n_{0} & \geqq 1,
\end{array}
$$

where $E_{d}(\approx 25 \mathrm{ev})$ is the threshold energy for atomic displacement in the bulk, and $n_{0} D$ is the maximum range of a focuson along a close-packed direction. In copper, $n_{0}$ is $\approx 100$. Let us suppose that all the focusons start at a distance $x$ away from the surface (Fig. 2.1). The geometrical length of a close-packed chain intersecting the surface in a special direction, which includes the angle $\epsilon$ with the direction of incidence, is then $\alpha x$, where $a^{-1}=\cos \epsilon$. To account for the fact that the last atom in the chain has to have a certain minimum energy $E_{b}$ ("binding energy" or displacement energy for atoms in the surface) in order to be able to leave the surface, we formally increase the geometrical length $a x$ by a distance $\beta D$. This distance is chosen in such a way that the energy loss of the focuson after $\beta$ collisions equals $E_{b}$. Roughly, we set $\beta=E_{b} / \Delta E$, where $\Delta E$ is the energy loss per unit length $D$ along the chain. Thus the number of focusons in a special direction " $a$ " reaching the surface with an energy $\geqq E_{b}$ and therefore being able to leave the crystal is, according to Eq. (1),

$$
\begin{array}{rlrl}
\nu(\alpha, T) & =1 / 121.5 \frac{T}{2 E_{d}}[1-z(1-\ln z)], \\
& z \leqq 1, \\
& =0, & z \geqq 1,
\end{array}
$$

where $z=(\alpha x+\beta D) / 100 D$.

Now we have to take into account the distribution $W(x ; L)$ of collision lengths $x$ (which depends on the mean free path $L$ ) and also the distribution $g(T ; E)$ of the energy transfer $T$. From Eq. (2), we have the number of focusons in a direction $a$ 
UNCLASSIFIED

ORNL-LR-DWG 73792

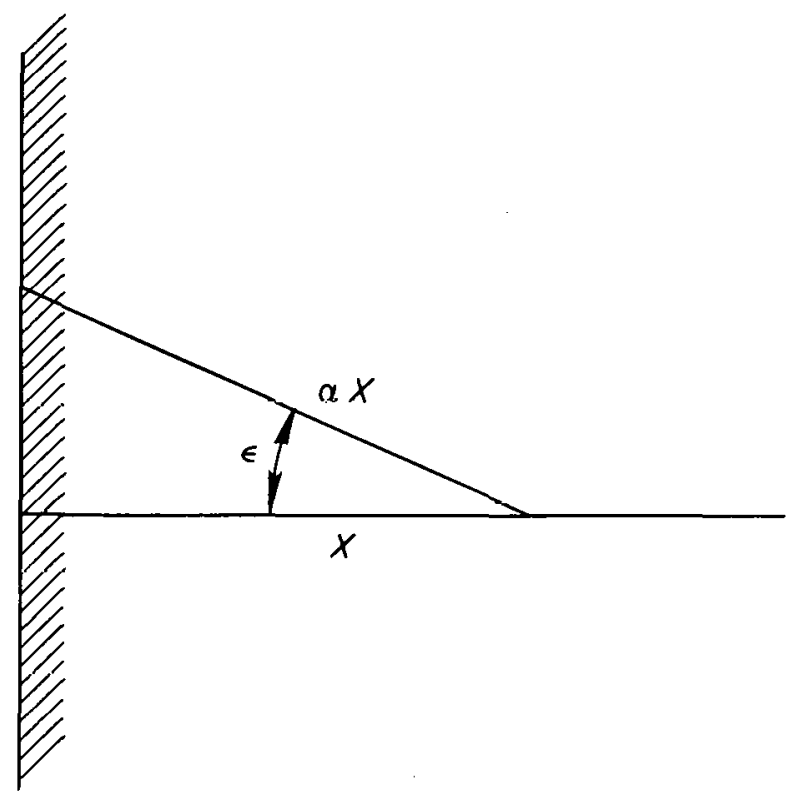

Fig. 2.1. Geometric Relation Between Angle and Length of a Close-Packed Atomic Row from a Cascade at Distance $x$ to the Surface.

which start from a radiation-damage zone of energy $T$ at a distance $x$ from the surface and result in ail ejection of a surface atom.

Averaging over the distributions $W$ and $g$ gives (for equal masses)

$\bar{\nu}(E ; \alpha, \beta)$

$=\int_{0}^{E} d T \int_{0}^{\infty} d x W(x ; L) g(E ; T) \nu(T, x ; E ; a, \beta)$

$=1 / 8 \frac{T}{2 E_{d}} \int_{0}^{\infty} \frac{e^{-x / L}}{L}[1-z(1-\ln z)] d x$,

where the average energy transfer

$$
\bar{T}=\int_{0}^{E} T g(E ; T) d T .
$$

These integrals can be evaluated easily for hardsphere interaction. Here we have

$$
W(x ; L)=\frac{e^{-x / L}}{L},
$$

where the mean free path $L$ is

$$
L(E)=1 / n_{0} \pi[R(E)]^{2},
$$

in which $n_{0}$ is the atomic space density of the lattice and $R$ is the hard-sphere radius. For the calculation of $L$, the mean free path in a randomized hard-core model is used, where the hard-core radius is the distance of closest approach. The employed interatomic potential is $V(r)=A e^{-r / a}$, with $A=$ $22.5 \mathrm{kev}$ and $a=D / 13$. The spectrum of energy transfer for hard spheres is

$$
\begin{aligned}
g(E ; T) & =\frac{1}{E}, & & 0 \leqq T \leqq E, \\
& =0, & & \text { otherwise } .
\end{aligned}
$$

With Eqs. (5) and (6), $\bar{\nu}$ in Eq. (4) becomes

$$
\begin{aligned}
\bar{\nu}(E ; a, \beta)=1 / 16 & \frac{E}{2 E_{d}}\left\{(1+\mu)+(1+\rho)\left(\ln \frac{\rho}{\mu}-1\right)\right. \\
& \left.-e^{\rho}[E i(-\rho)-E i(-\mu)]\right\}, \quad \text { (7) }
\end{aligned}
$$

where

$$
\begin{aligned}
\mu & =\frac{100 D}{a L}, \\
\rho & =\frac{\beta D}{\alpha L}, \\
-E i(-x) & =\int_{x}^{\infty} \frac{e^{-t}}{t} d l, m>x>0 \text {; see refs } 12 \\
& \text { and } 13 .
\end{aligned}
$$

To calculate finally the yield $S$ (number of sputtered atoms per incident primary), we have to sum Eq. (7) over all close-packed $\langle 110\rangle$ directions within an fcc lattice which intersect the surface ( $b k l)$ :

$$
S_{(b k l)}(E)=\sum_{i} \bar{\nu}\left(\alpha_{i} ; \beta ; E\right) .
$$

This has been done for a (110), a (100), and a (111) plane, considering: for the (110) surface four directions with chains of length $2 x$ and one direction with a chain of length $x$; for the (100)

\footnotetext{
${ }^{12}$ Eugene Jahnke, Fritz Emde, and Friedrich Lösch, Tables of Higher Functions, McGraw-Hill, New York, 1960.

${ }^{13}$ Tables of Sine, Cosine and Exponential Integrals, Federal Works Agency, Work Projects Administration, New York, 1940.
} 
surface - four directions with chains of length $\sqrt{2} \cdot x$; and for the (111) surface - three directions with chains of length $\sqrt{3 / 2} \cdot x$. The yield calculated after Eq. (8) is plotted in Fig. 2.2. For the binding energy $E_{b}$ of a surface atom, 5 ev (corresponding to $\beta=1$ ) has been chosen.

At the present stage of the theory, we have agreement with experimental data to within an order of magnitude, which supports the basic idea that focusing collisions are responsible for aniso- tropic sputtering. We do not yet $\mathrm{g}$. Wet the proper sequence $S^{111}>S^{100}>S^{110}$ indicated by experiments ${ }^{14}$ for energies above $1 \mathrm{kev}$. This is the goal of a more detailed investigation. Also, sputtering events will be included in which primaries differ from target materials.

\footnotetext{
${ }^{14} A$. L. Southern, W. R. Willis, and M. T. Robinson, "Sputtering Experiments with 1- to 5-kev Ar+ Ions," see abstract in Part III of this report.
}

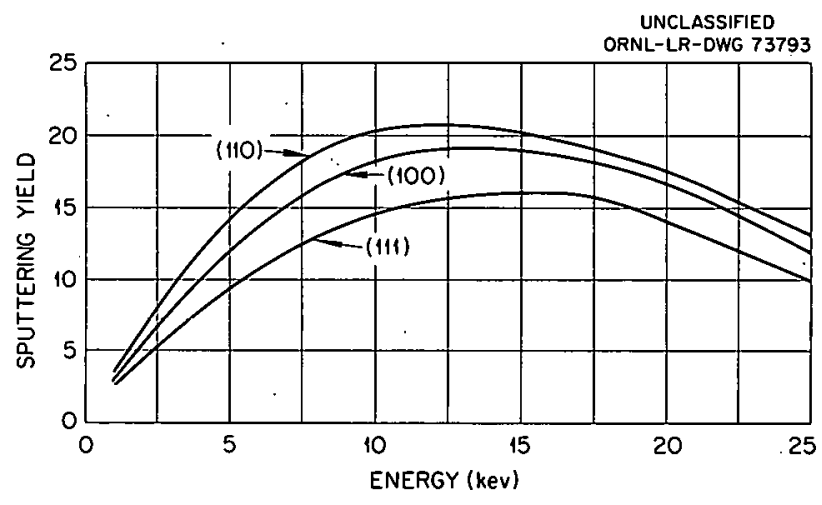

Fig. 2.2. Yield-Energy Relation for Sputtering from (110), (100), and (111) Surfaces for $\mathrm{Cu}^{+}$onto $\mathrm{Cu}$. 


\section{Semiconductor Theory}

\section{1 STATISTICAL MECHANICS OF SEMICONDUCTORS}
J. W. Miller ${ }^{1}$
H. C. Schweinler

The research reported in a preliminary way ${ }^{2}$ in this Report last year has been published as ORGDP Report K-1526, "An IBM 7090 Program for Computing the Fermi-Level in Non-Metals," by
J. W. Miller. Figures 4 and 5 from that report are reproduced here as Figs. 3.1 and 3.2. In them the position of the Fermi level as a function of temperature is given for silicon and germanium for a

\footnotetext{
${ }^{1}$ Central Data Processing Facility, Oak Ridge Gaseous Diffusion Plant.

2J. W. Miller and H. C. Schweinler, Solid State Div. Ann. Progr. Rept. Aug. 31, 1961, ORNL-3213, p 11.
}

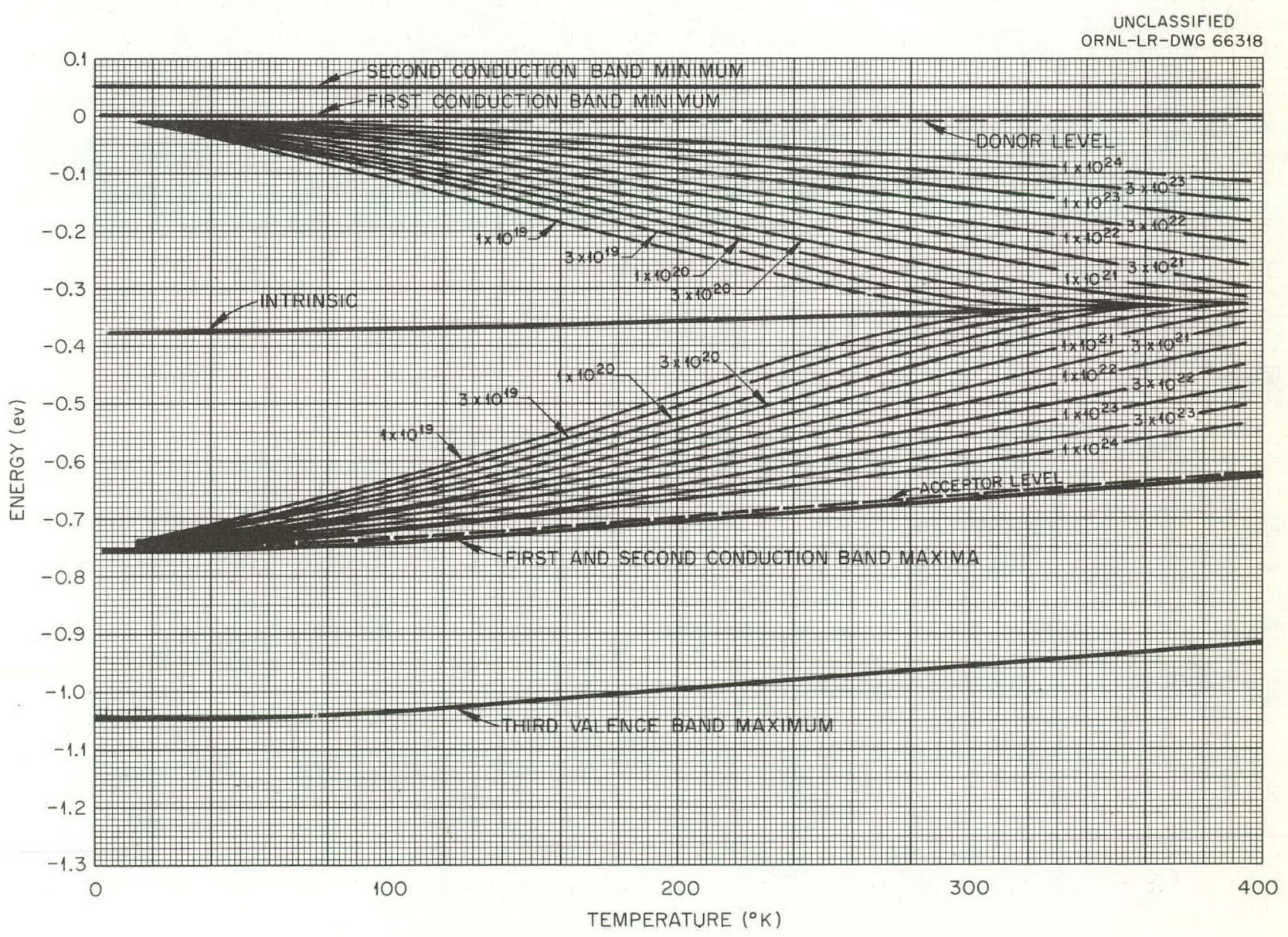

Fig. 3.1. Fermi Level in Germanium with Gallium and Antimony Doping as a Function of Majority Carrier Atoms per Cubic Meter. 


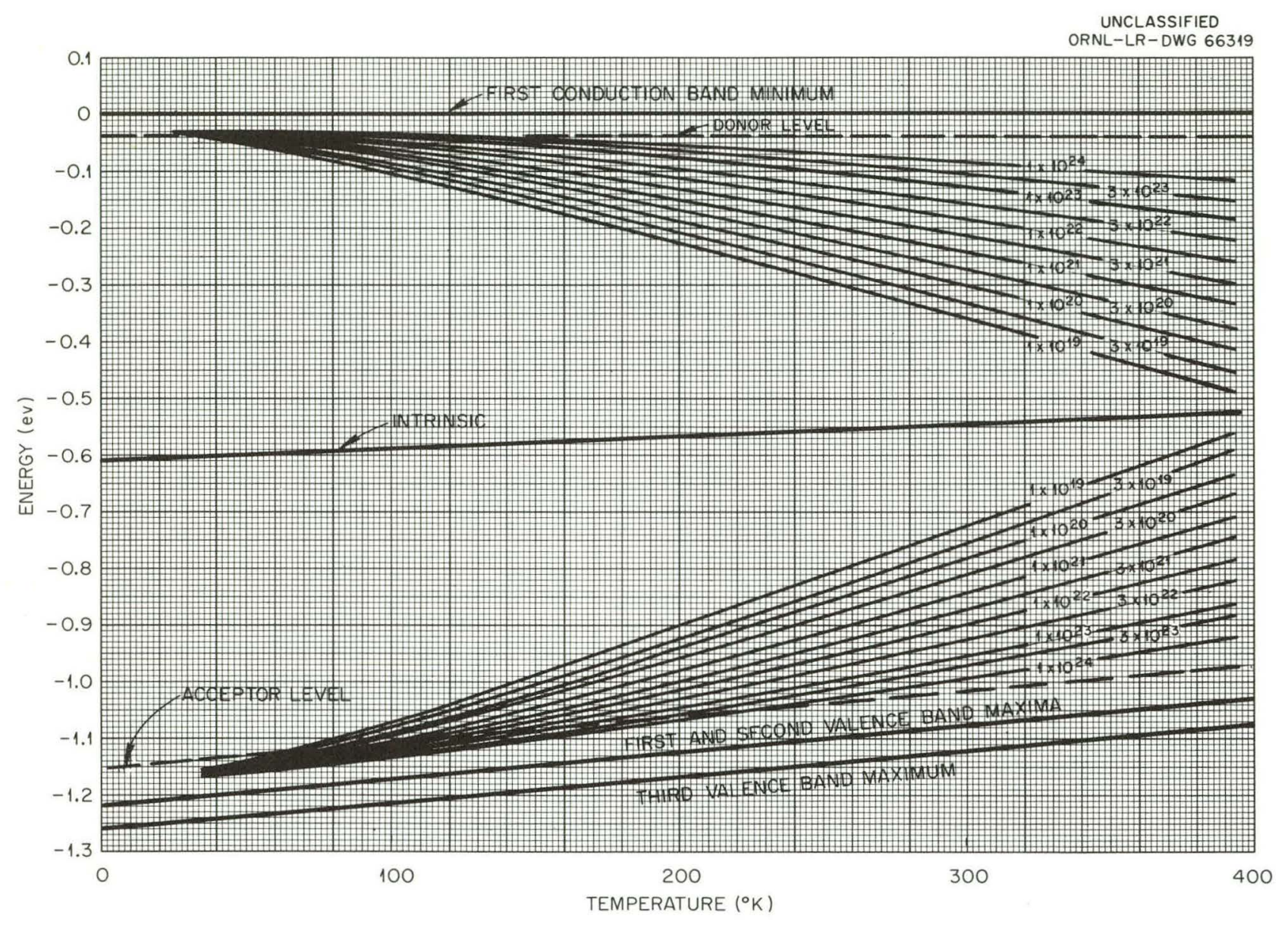

Fig. 3.2. Fermi Level in Silicon with Gallium and Antimony Doping as a Function of Majority Carrier Atoms per Cubic Meter.

wide range of donor and acceptor concentrations. A very useful summary rule which results from these detailed calculations is that the position of the Fermi level depends primarily on the temperature and the net number of electrons in the crystal and only secondarily on the combinations of numbers of donors and acceptors from which this net number of electrons is obtained. For further details the report referred to above should be consulted. Copies are available on request addressed to the Office of Technical Services, Department of Commerce, Washington 25, D.C.

\subsection{DENSITY FUNCTION MODIFYING CARRIER CAPTURE PROBABILITY IN NONMETALLIC CRYSTALS}
H. C. Schweinler
J. W. Miller ${ }^{1}$

In the theoretical expressions for the minority carrier lifetime in semiconductors, and in the theory of luminescence of insulating crystals, there enters an expression for the capture probability of a charged particle (electron or hole) at a defect. When the defect itself is charged, the 
capture probability may be augmented or reduced by a factor which depends on the increase or decrease of the charge density of the incident particle at the capturing center (as a result of Coulomb attraction or repulsion). The modified incident-particle density at the capturing center is assumed to be proportional to the capture probability. The particle density has been averaged with respect to a Maxwell velocity distribution of the bombarding particles, which is valid in the nondegenerate case.

The function so derived is given as

$$
D(\zeta)=\frac{2}{\sqrt{\pi}} \zeta \int_{0}^{\infty} \frac{e^{-w}}{e^{\zeta / \sqrt{w}-1}} d w
$$

and is presented in Table 3.1. It depends on the single variable

$$
\zeta=\frac{2 \pi z}{K} \sqrt{\frac{m^{* *}}{m} \frac{13.6 \mathrm{ev}}{k T}},
$$

involving the relative charge $z$ of the two charged points, the effective mass $m^{* *}$ of the moving carrier in the crystal, the dielectric constant $K$ of the crystal, and the absolute temperature $T$. The experimentally determined cross section may be expressed as this factor, $D(\zeta)$, multiplied by an atomic cross section. This determines (approximately) the variable $\zeta$, from which the relative charge of the capturing center can often be inferred.

Tablo 3. 1. Ratio of Particle Density at Capturing Center to Particle Density at Infinity

\begin{tabular}{clllll}
\hline$\zeta$ & \multicolumn{1}{c}{$\mathrm{D}(+\zeta)$} & $\mathrm{D}(-\zeta)$ & $\zeta$ & $\mathrm{D}(+\zeta)$ & $\mathrm{D}(-\zeta)$ \\
\hline 0 & 1.000000 & 1.000000 & 22 & $0.4176077(-4)$ & 24.824384 \\
1 & 0.5730042 & 1.7013834 & 24 & $0.1930218(-4)$ & 27.081120 \\
2 & 0.3329456 & 2.5897040 & 26 & $0.9058655(-5)$ & 29.337868 \\
3 & 0.1959816 & 3.5811192 & 28 & $0.4311674(-5)$ & 31.594622 \\
4 & 0.1167588 & 4.6302756 & 30 & $0.2079304(-5)$ & 33.851378 \\
5 & 0.07034540 & 5.7122414 & 32 & $0.1015067(-5)$ & 36.108135 \\
6 & 0.04282779 & 6.8131030 & 34 & $0.5012264(-6)$ & 38.364894 \\
7 & 0.02633057 & 7.9249850 & 36 & $0.2501667(-6)$ & 40.621651 \\
8 & 0.01633680 & 9.0433704 & 38 & $0.1261265(-6)$ & 42.878410 \\
9 & 0.01022344 & 10.1656362 & 40 & $0.6419729(-7)$ & 45.135168 \\
10 & $0.6449463(-2)$ & 11.290241 & 46 & $0.8918711(-8)$ & 51.905443 \\
11 & $0.4099585(-2)$ & 12.416271 & 50 & $0.2488133(-8)$ & 56.418960 \\
12 & $0.2624561(-2)$ & 13.543 .175 & 56 & $0.3861689(-9)$ & 63.189235 \\
13 & $0.1691612(-2)$ & 14.670622 & 60 & $0.1150708(-9)$ & 67.702752 \\
14 & $0.1097269(-2)$ & 15.798406 & 65 & $0.2615286(-10)$ & 73.344648 \\
15 & $0.7160575(-3)$ & 16.926404 & 70 & $0.6139716(-11)$ & 78.986544 \\
16 & $0.4699712(-3)$ & 18.054537 & 75 & $0.1484817(-11)$ & 84.628440 \\
17 & $0.3101412(-3)$ & 19.182756 & 80 & $0.3690431(-12)$ & 90.270336 \\
18 & $0.2057304(-3)$ & 20.311032 & 85 & $0.9407581(-13)$ & 95.912232 \\
19 & $0.1371460(-3)$ & 21.439342 & 90 & $0.2455284(-13)$ & 101.55413 \\
20 & $0.9185772(-4)$ & 22.567676 & 95 & $0.6550372(-14)$ & 107.19602 \\
& & & 100 & $0.1783875(-14)$ & 112.83792 \\
\hline
\end{tabular}

\footnotetext{
$a_{\text {The notation }} x(-y)$ means $x \cdot 10^{-y}$.
} 


\title{
4. Critical Simplices in Brillovin Zones
}

\author{
H. C. Schweinler
}

The behavior of the energy as a function of propagation vector in the vicinity of the Brillouin zone surface is well known from the work of Wigner and von Neumann. ${ }^{1}$ It leads to energy gaps and a vanishing group velocity at the Brillouin zone surface. The origin of these phenomena is the degeneracy of two states differing in propagation vector by $2 \pi$ multiplied by a reciprocal lattice vector.

${ }^{\mathrm{I}}$ E. P. Wigner and J. von Neumann, Physik. Z. 30, 467 (1929).
It is believed to be an essentially new discovery that there are other points and lines in the Brillouin zone for which (in the free-electron approximation) states of the same energy have propagation vectors differing by $2 \pi$ multiplied by a reciprocal lattice vector. The reduced propagation vectors to which these latter are congruent are called "critical simplices"; a critical 0-simplex is a point in the reduced Brillouin zone; a critical 1-simplex is a line in the reduced Brillouin zone; and a critical 2-simplex is a plane in (possibly entirely within) the reduced Brillouin zone.

Table 4.1. Critical 0-Simplices for a Square Lattice of Points

\begin{tabular}{|c|c|c|c|c|}
\hline $\begin{array}{l}\text { Critical } \\
\text { Simplex }\end{array}$ & Multiplicity & Congruent Points in Extended Zone & $\begin{array}{l}\text { Squared } \\
\text { Length }\end{array}$ & Symmetry $^{a}$ \\
\hline$(1 / 6,1 / 6)$ & 3 & $(7 / 6,1 / 6),(1 / 6,7 / 6),(-5 / 6,-5 / 6)$ & $50 / 36$ & $\sigma_{d}$ \\
\hline$(0,1 / 4)$ & 3 & $(-1,-3 / 4),(1,-3 / 4),(0,5 / 4)$ & $25 / 16$ & $\sigma_{\nu}$ \\
\hline$(0,1 / 3)$ & 3 & $(0,-5 / 3),(-1,4 / 3),(1,4 / 3)$ & $25 / 9$ & $\sigma_{\nu}$ \\
\hline$(1 / 4,1 / 4)$ & 3 & $(1 / 4,-7 / 4),(5 / 4,5 / 4),(-7 / 4,1 / 4)$ & $50 / 16$ & $\sigma_{d}$ \\
\hline$(3 / 14,5 / 14)$ & 3 & $(-11 / 14,-23 / 14),(-25 / 14,5 / 14),(17 / 14,19 / 14)$ & $650 / 196$ & $\epsilon$ \\
\hline$(3 / 10,3 / 10)$ & 3 & $(-17 / 10,-7 / 10),(-7 / 10,-17 / 10),(13 / 10,13 / 10)$ & $338 / 100$ & $\sigma_{d}$ \\
\hline$(1 / 6,3 / 6)$ & 4 & $(7 / 6,9 / 6),(-11 / 6,3 / 6),(7 / 6,-9 / 6),(-11 / 6,-3 / 6)$ & $130 / 36$ & $\sigma_{b}$ \\
\hline$(0,1 / 4)$ & 4 & $(1,-7 / 4),(2,1 / 4),(-1,-7 / 4),(-2,1 / 4)$ & $65 / 16$ & $\sigma_{v}$ \\
\hline$(1 / 14,3 / 14)$ & 3 & $(-27 / 14,-11 / 14),(15 / 14,-25 / 14),(29 / 14,3 / 14)$ & $850 / 196$ & $\epsilon$ \\
\hline$(1 / 10,1 / 10)$ & 4 & $\begin{array}{l}(-19 / 10,-9 / 10),(-9 / 10,-19 / 10),(1 / 10,21 / 10) \\
(21 / 10,1 / 10)\end{array}$ & $442 / 100$ & $\sigma_{d}$ \\
\hline$(0,1 / 8)$ & 3 & $(0,17 / 8),(-1,-15 / 8),(1,-15 / 8)$ & $289 / 64$ & $\sigma_{v}$ \\
\hline
\end{tabular}

\footnotetext{
${ }^{a}$ Schoenflies notation.
} 
In some cases, three and four states of the same energy in the free-wave approximation are congruent to the same critical 0 -simplex. This leads to a different form of secular equation and a different analytical form of the energy surface from that studied by Wigner and von Neumann. From the 0 -simplices as base, 1-simplices can be erected which have these same properties, now as a continuous function of the free coordinate of the propagation vector.

In the case of the square lattice of points in real space, leading also to a square lattice of points in reciprocal space, the critical simplex closest to the origin, other than those of Wigner and von Neumann, is $(1 / 6,1 / 6)$ and its symmetric equivalents. This simplex is congruent to the three wave vectors $(7 / 6,1 / 6),\left(\frac{1}{6}, 7 / 6\right)$, and $(-5 / 6$, $-5 / 6$ ); each of these is at squared distance $50 / 36$ from the origin in the extended Brillouin zone. The secular equation factors into a quadratic and a linear term when the wave vector (assumed to be close to the above critical 0-simplex) lies along the line $[1,1]$, but does not factor otherwise. It is evident from this brief discussion that a new algebraic structure of the energy surface in the vicinity of this point has been found.

The first 11 critical 0 -simplices, some of multiplicity 3 , as is that discussed above, and others of multiplicity 4 are given in Table 4.1. Hundreds of critical 0 -simplices have been located with the help of J. W. Miller of the ORGDP Central Data Processing Facility.

Each of these 0-simplices in the plane gives rise to a 1-simplex in space, and the intersection of 1-simplices gives certain 0-simplices in space. The space problem for simple cubic, body-centered cubic, and face-centered cubic lattices of points is under active investigation and numerical computation, but no results are available for publication. 


\title{
5. A Possible Experiment to Study Radiation Damage by the Mössbaver Effect
}

\author{
J. H. Barrett
}

Although the Mössbauer effect has recently found widespread use as a research tool in physics, ${ }^{1}$ this tool has had little application to the studies of defects in solids. Most methods of studying defects in solids, such as electrical resistivity measurements, are very insensitive to the detailed nature of the defects. A few methods of study; such as spin resonance; are sensitive to the'details of the structure: In the hope of adding another method of making detailed studies, the following experiment is suggested.

The general requirement for the existence of the Mössbauer effect for a given element is the existence of a stable. isotope. which has a low-lying nuclear level (less than: $100 \mathrm{kev}$ above the ground state) that can be suitably excited. : For the present experiment; one other requirement: must be added; namely, that the element must:also: have a'stable isotope with a mass number one unit lower. Among the elements that satisfy these two criteria are $\mathrm{Fe}, \mathrm{Ni}, \mathrm{Zn}, \mathrm{Ge}, \mathrm{Sn}$, and several rare earths. This group of elements includes one or more examples of all the simpler crystal structures: face-centered cubic, body-centered cubic, diamond, and hexagonal. Because the Mössbauer effect has been studied so extensively in $\mathrm{Fe}^{57}$, this isotope will be used as an example in the discussion below.

The proposed experiment would generate the Mössbauer effect by thermal-neutron capture:

$$
\begin{gathered}
\mathrm{Fe}^{56}+n \longrightarrow \mathrm{Fe}^{57 *} \longrightarrow \mathrm{Fe}^{57 m}+\text { high-energy } \gamma^{\prime} \mathrm{s} \\
\mathrm{Fe}^{57 m} \longrightarrow \mathrm{Fe}^{57}+14.4 \text {-kev } \gamma .
\end{gathered}
$$

\footnotetext{
${ }^{1}$ A recent review may, be found in: Hans Frauenfelder, The Mössbauer Effect, Benjamin, New York, 1962.
}

The 14.4-kev gamma ray is the one for which the Mössbauer effect is observed. As the higherenergy gamma rays are emitted, they will impart a recoil energy to the neutron-absorbing nucleus, causing it to be displaced from its original lattice position. ${ }^{2}$ The detailed nature of the defect structure produced may affect the energy of the $14.4 \mathrm{kev}$ gamma ray; which is emitted much later than the high-energy gamma rays: The following is an itemby-item analysis of the feasibility of observing the influence of the defect structure on the Mössbauer radiation.

1. A 14.4-kev gamma ray will have a very short mean free' path in iron. Within a depth below the surface equal to this mean free path, a $10=\mathrm{cm}^{2}$ iron foil. in a flux of $10^{7}$ thermal neutrons $\mathrm{cm}^{-2} \mathrm{sec}^{-1}$ will make:about $4 \times 10^{4}$ captures/sec:

2 . Recent work ${ }^{3}$ on the gamma-ray cascades following thermal-neutron capture by $\mathrm{Fe}^{56}$ indicates that $50 \%$ or more of these cascades will contain the 14.4-kev gamma ray. Items 1 and 2 will yield an acceptable counting rate.

3. In obtaining a thermal-neutron source near a nuclear reactor, there will be a very high gammaray background. The effects of the background can be minimized by using a very thin scintillation crystal, a krypton proportional counter, or possibly a semiconductor counter. The background problem can likely be overcome.

4. The high-energy gamma rays will be emitted in a time $\approx 10^{-13}$ sec. Following these highenergy gamma-ray emissions the average recoiling

\footnotetext{
${ }^{2}$ H. C. Schweinler, $\mathrm{p} 63$ in Chemical Elfects of Nuclear Transiormations, vol I, IAEA, Vienna, 1961.

${ }^{3}$ N. F. Fiebiger, W. R. Kane, and R. E. Segel, Pbys. Rev. 125, 2031 (1962).
} 
$\mathrm{Fe}^{57 m}$ nucleus will have a velocity $\approx 3.5 \times 10^{6}$ $\mathrm{cm} / \mathrm{sec}$. If its range is taken to be $\lesssim 50 A$, its flight time $\lesssim 3 \times 10^{-13} \mathrm{sec}$.

5. After the recoiling $\mathrm{Fe}^{57 m}$ nucleus has come to the end of its flight path, it will still have a few electron volts of energy. Treating this energy as a very localized high temperature and assuming a lattice thermal conductivity of $5 \times 10^{-3} \mathrm{w} \mathrm{cm}^{-1}$ $\left({ }^{\circ} \mathrm{C}\right)^{-1}$, one finds the local temperature will decay to within $10^{\circ} \mathrm{C}$ of the surroundings in a time $\approx 2 \times 10^{-10}$ sec. Also the occurrence of the Mössbauer effect in the usual way by electron capture of $\mathrm{Co}^{57}$ indicates that the thermalization time is less than the half-life of $\mathrm{Fe}^{57 m}, 10^{-7}$ sec. Items 4 and 5 indicate that the $\mathrm{Fe}^{57 m}$ nucleus will be stopped and thermalized before emitting the 14.4-kev gamma ray. Therefore, the Mössbauer effect should occur.

6. Since the recoiling $\mathrm{Fe}^{57 m}$ nucleus will have a few hundred electron volts of energy, ${ }^{2}$ it will probably create several interstitial-vacancy pairs. The rccoiling nucleus itself may wind up in an interstitial position, or it may undergo a replacement collision, leaving it at a regular lattice site. If the $\mathrm{Fe}^{57 m}$ nucleus comes to rest at a normal lattice site, the experiment will not yield any information about defects, although the Mössbauer effect should be observable. If it comes to rest at an interstitial position, the experiment should yield information about the nature of this defect. It is impossible to make a reliable estimate of the probability that the recniling $\mathrm{Fe}^{57 m}$ nucleus will end up in an interstitial position. An unfavorably low value of this probability seems to be the only factor that is likely to prevent. this type of experiment from yielding useful information about the nature of an interstitial. In order to have the $\mathrm{Fe}^{57 m}$ nucleus remain in the interstitial position until it emits the $14.4-\mathrm{kev}$ gamma ray, it may be necessary to have the neutron-absorbing foil at a low temperature.

7. This final item concerns the way in which the interstitial position of the $\mathrm{Fe}^{57 m}$ might alter the Mössbauer effect. Ludwig and Woodbury ${ }^{4}$ suggest on the basis of spin resonance studies that transition metals as interstitials in silicon transfer their $4 s$ electrons to the $3 d$ shell. When a transition element atom is squeezed, this seems likely to happen; it will be assumed to happen for an iron atom as an interstitial in iron. Detailed studies 5.6 of the Mössbauer radiation from $\mathrm{Fe}^{57}$ indicate that there is a chemical shift and a change in the hyperfine splitting if there is a $3 d^{8}$ electronic configuration around an $\mathrm{Fe}^{57 m}$ nucleus rather than the regular $3 d^{7} 4 s$ configuration of metallic iron. Also, the interstitial arrangement in iron might be a split interstitial similar to that of the facecentered cubic metals. If this is the arrangement, there would be a quadrupole interaction, which would be a further observable effect.

\footnotetext{
${ }^{4}$ G. W. Ludwig and H. H. Woodbury, Phys. Rev. 117, 1286 (1960).

${ }^{5}$ L. R. Walker, G. K. Wertheim, and V. Jaccarino, Pbys. Rev. Letters 6, 98 (1961). This is reprinted on p 236 of ref 1.

${ }^{6} \mathrm{G} . \mathrm{K}$. Wertheim and D. N. E. Buchanan, $\mathrm{p} 130$ in Proceedings of the 2nd International Conference on the Mössbauer Effect (ed. by D. M. J. Compton and Alan H. Schoen), Wiley, New York, 1962.
} 


\section{Electron Cross Sections for Atomic Displacements}

o. S. Oen.

In a previous report ${ }^{1}$ a calculational program was outlined for computing the number of atomic displacements produced by elastic collisions between fast electrons and atomic nuclei. At that time a FORTRAN program had been written for an IBM 7090 computer which allowed one to compute the primary displacement cross section using a sharp displacement threshold, for arbitrary electron energy, but limited to elements of low atomic number $(Z<27)$. This latter restriction resulted from using the McKinley-Feshbach version of the Mott series to describe the electronnucleus scattering. A new. FORTRAN program has been written which evaluates numerically the Mott series while computing the primary displacement cross section. The method used to calculate the Mott series is that of Doggett and Spencer. ${ }^{2}$ With the use of this new program; which: is good for arbitrary. $Z$; the primary displacement cross sections have be en calculated for $Z=29,47,50$, 79,82 , and 92 , using several sharp displacement threshold energies. For uranium, Fig. 6.1 shows that an error of a factor of 3 may arise from using the McKinley-Feshbach formula. For an element of low $Z$, such as copper, the two formulations differ by less than $10 \%$. An unexpected, interesting feature of the Mott formulation is the pronounced maximum found in the primary displacement cross section. This maximum occurs in a highly relativistic region and, therefore, cannot be explained within the framework of nonrelativistic mechanics,

${ }^{1}$ O. S. Den, Solid State Div. Ann. Progr. Rept: Aug. 31. 1961, ORNL-3213, p 9.

${ }^{2} \mathrm{~J}$. A. Doggett and L. V. Spencer, Phys. Rev. 103, 1597 (1956).

${ }^{3}$ W. D. Compton and G. W. Arnold, J. Appl. Pbys. $31,621(1960)$. as were the cases found by Compton and Arnold. ${ }^{3}$ In general, this relativistic-type maximum becomes less pronounced for elements of lower $Z$. It is planned to extend these calculations to include the cascade of displacements initiated by the primary knock-on.

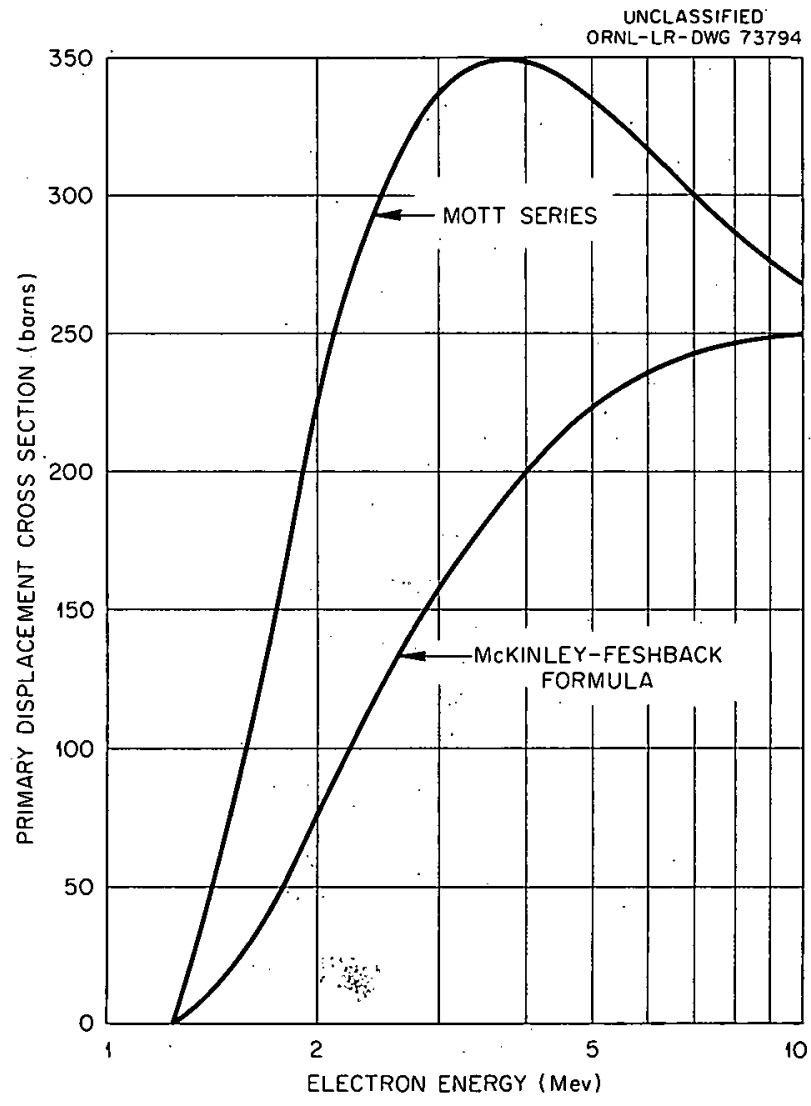

Fig. 6.1. Primary-Displacement Cross Section in Uranium as a Function of Energy; by using a Sharp Displacement Threshold of $25 \mathrm{ev}$. 
Part II. Crystal Physics

M. K. Wilkinson 
THIS PAGE

WAS INTENTIONALLY LEFT BLANK 


\section{X-Ray Diffraction}

\subsection{SINGLE CRYSTALS GROWN FROM SMALL DROPLETS ${ }^{1}$}

\author{
M. C. Wittels \\ F. A. Sherrill \\ J. O. Stiegler ${ }^{2}$
}

Single-crystal droplets of $\mathrm{Au}, \mathrm{Cu}, \mathrm{Ag}, \mathrm{Pb}, \mathrm{In}$, $\mathrm{Cu}_{3} \mathrm{Au}$, and $\mathrm{Ge}$ have been grown in vacuum by two simple methods. In each case graphite crucibles were employed and served as a point contact so that the droplets could solidify in the absence of a containment shell, free of mechanical and thermal stresses. The crystals approximate spheres 50 to $600 \mu$ in diameter and are thus ideally suited for $x$-ray diffraction studies in transmission. Although the surface-tension properties of the materials were important to the successful growth characteristics, it was also observed that exclusion of foreign nucleation catalysts and elimination of mechanical and thermal stresses were equally as significant.

\subsection{RADIATION EFFECTS IN URANIUM-DOPED ZIRCONIA $^{3}$}

$$
\text { M. C. Wittels J. O. Stiegler }{ }^{2}
$$$$
\text { F. A. Sherrill }
$$

A new study concerning the mechanism of the radiation-induced phase transformation, monoclinic to fcc, in $\mathrm{ZrO}_{2}$ is presented. The most important aspect of these experiments was the effort to separate the effects of fast neutrons, fission fragments, and uranium chemical impurities. This was accomplished by (1) carefully

\footnotetext{
${ }^{1}$ Abstract of article to be submitted for publication.

${ }^{2}$ Metals and Ceramics Division.

${ }^{3}$ Abstract of paper to be published in the Journal of Nuclear Energy.
}

doping chemically pure $\mathrm{ZrO}_{2}$ samples with selected concentrations of enriched and depleted uranium, (2) irradiating these samples in closely monitored thermal-neutron and fast-neutron environments and correlating the phase changes observed with the specific particle events, (3) irradiating $\mathrm{ZrO}_{2}$ that was obtained by oxidizing Zircaloy-2 which was free of fissionable impurities with a fast-neutron dose greater than $6 \times 10^{20}$ neutrons $/ \mathrm{cm}^{2}$, and (4) subjecting a large group of reactor-grade zirconium and its alloys, selected at random, to neutron activation analysis for $U$ and Th impurities.

The results of the investigation indicate that (1) fission fragment fluxes associated with $10^{15}$ $10^{16}$ fissions $/ \mathrm{cm}^{3}$ produce a monoclinic to fcc phase change in $\mathrm{ZrO}_{2}$, and the presence of $U$ in $\mathrm{ZrO}_{2}$ in concentrations less than 0.38 wt $\%$ does not act as a nucleation or stabilizing atom in the process, (2) the stability of the phases of $\mathrm{ZrO}_{2}$ under reactor irradiation depends entirely upon the action of a fission fragment flux of sufficient magnitude, and where conflicting data have been reported it is believed that an unknown presence of fissionable impurities would resolve these differences, (3) fast neutrons, solely, cannot induce the phase transition at doses up to $6.3 \times 10^{20}$ neutrons $/ \mathrm{cm}^{2}$, and (4) in 21 analyses for $U$ and Th in randomly selected reactor-grade zirconium and its alloys, fissionable impurities were found in 20 cases. Natural-uranium concentrations varied between 1.8 and $6.3 \mathrm{ppm}$, and thorium concentrations varied between 0.29 and $50.0 \mathrm{ppm}$.

Two different models are given to explain the mechanism of fission fragments in producing the phase transition. Both models require the collapse of small regions due to the pressures exerted by interstitials. In one case the action takes place at the beginning of the range of the fission fragment through an ionization displacement process, and in the other instance the process occurs near the end of the fission fragment where 
elastic collisions predominate. The effects of temperature are not clear and are not of prime importance, but the radiation stability of the fluorite structure of $\mathrm{ZrO}_{2}$, as in the case of $\mathrm{UO}_{2}$, appears to be significant.

\subsection{A VERSATILE LANG CAMERA}

$$
\text { M. C. Wittels F. A. Sherill }
$$

G. F. Fielder

Development work is being furthered in an effort to acquire a versatile $\mathrm{Lang}^{4}$ camera in which imperfections arising from several sources may be observed in nearly perfect crystals. It is believed that the instrument finally developed will permit one to observe as-grown dislocations, mechanically and thermally induced dislocations, foreign inclusions, small-angle tilts, and structural defects produced by various sources of radiation. The instrument being developed utilizes some of the aspects put forward by Webb ${ }^{5}$ and Schwuttke ${ }^{6}$ in their studies of lattice imperfections, but we have modified the instrumentation to include a portable crystal rocker for measuring rocking-curve line-widths of nearly perfect crystals.
Photographs of the apparatus are shown in Figs. 7.1-7.3. A conventional $x$-ray source (Ge-XRD-5) with a silver anode for thin-crystal transmission studies is utilized together with a diffractometer and scintillation counter for crystal alignment purposes. As shown, the anode-tocrystal distance has been increased to $92 \mathrm{~cm}$, and a slit $2.5 \mathrm{~cm} \times 2.5 \mathrm{~cm} \times 0.038 \mathrm{~mm}$ is employed to further collimate the $x$-ray beam. Ilford $x$-ray film or G-5-type nuclear plates are contained in the light-tight easily removable film casette that can be translated on a track along the line of the diffracted x-ray beam. Similarly, the direct-beam screen and scattering catcher has translatory movements with three degrees of freedom.

Conventional small-crystal goniometers are mounted on the center of the camera table and have a screw adjustment for vertical motion. For turning the crystal slowly about a vertical axis into the diffracting position, a gear reduction of

\footnotetext{
${ }^{4}$ A. R. Lang, Acta Met. 5, 358 (1957).

${ }^{5}$ W. W. Webb, Direct Observations of Imperfections in Crystals, pp 29-76, Interscience, New York, 1962.

${ }^{6}$ G. H. Schwuttke, J. Electrochem. Soc. 109, 27 (1962).
}

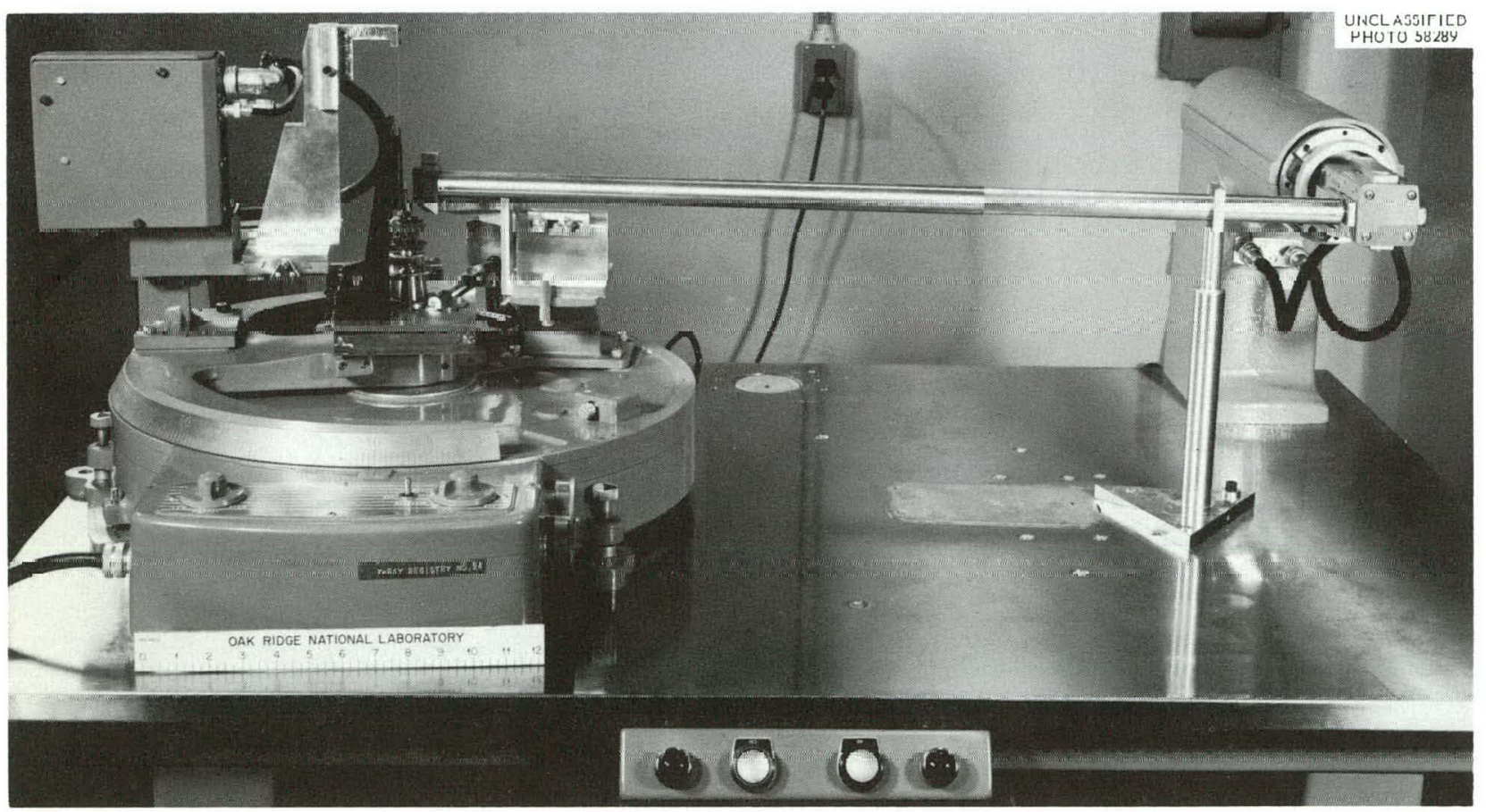

Fig. 7.1. Lang Camera with Crystal Rocker. 


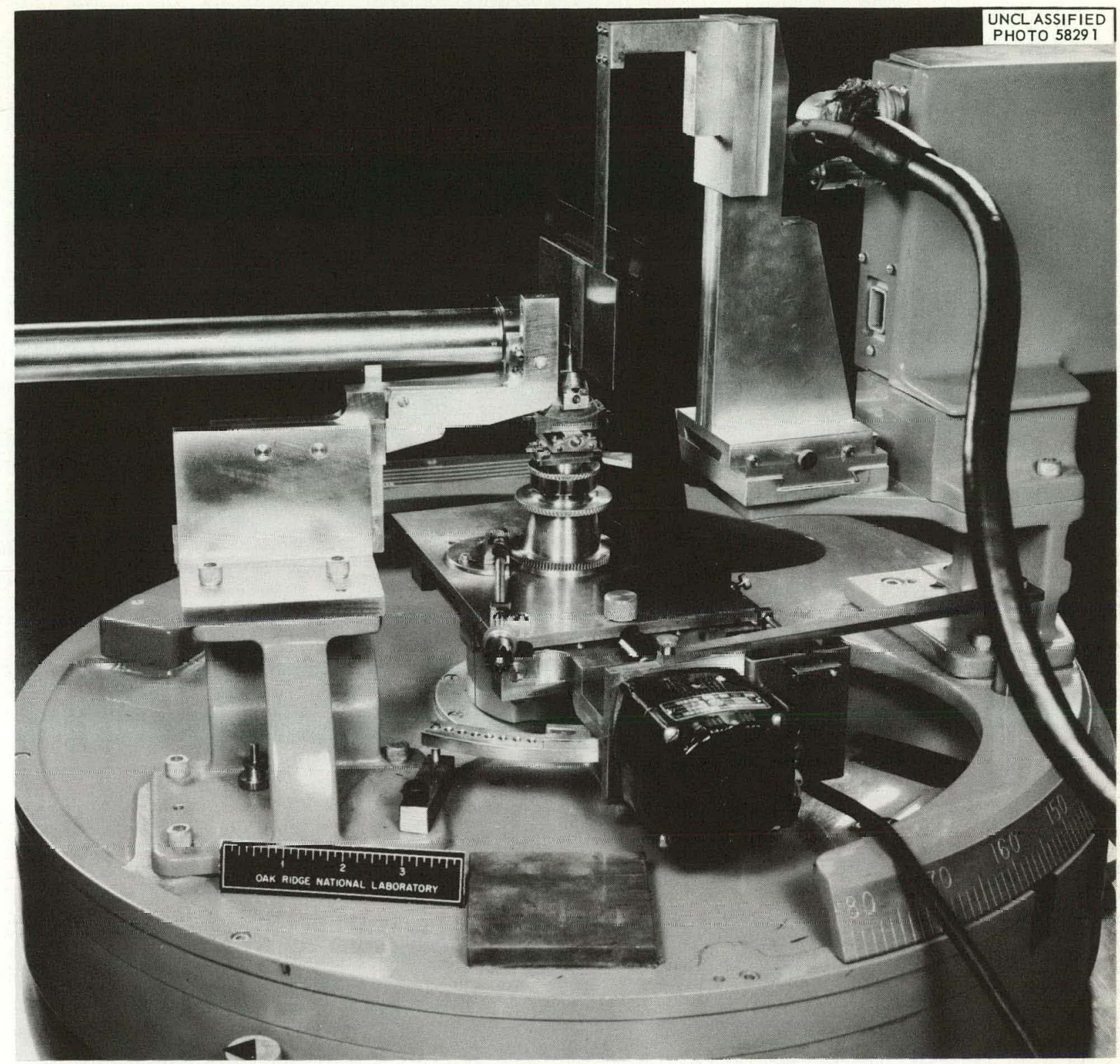

Fig. 7.2. Lang Camera Closeup Showing Screen and Film Holder.

$1800: 1$ is utilized, and this same gear system, when coupled with the synchronous 1-rph motor, permits an angular rock of $12 \mathrm{sec} / \mathrm{min}$. In nearly perfect crystals, where the half-width at maximum intensity at low index reflections is often less than $30 \mathrm{sec}$, it is desirable, if not necessary, to possess these slow motions. The rocking motor is portable and is decoupled from the table by means of a magnetic base mount when a translation motion is used.

The translating table is driven by a centermounted screw over a span of 2 in., and the table motion is reversed through microswitches attached to adjustable stops so that the translation span can be limited to the crystal width. 


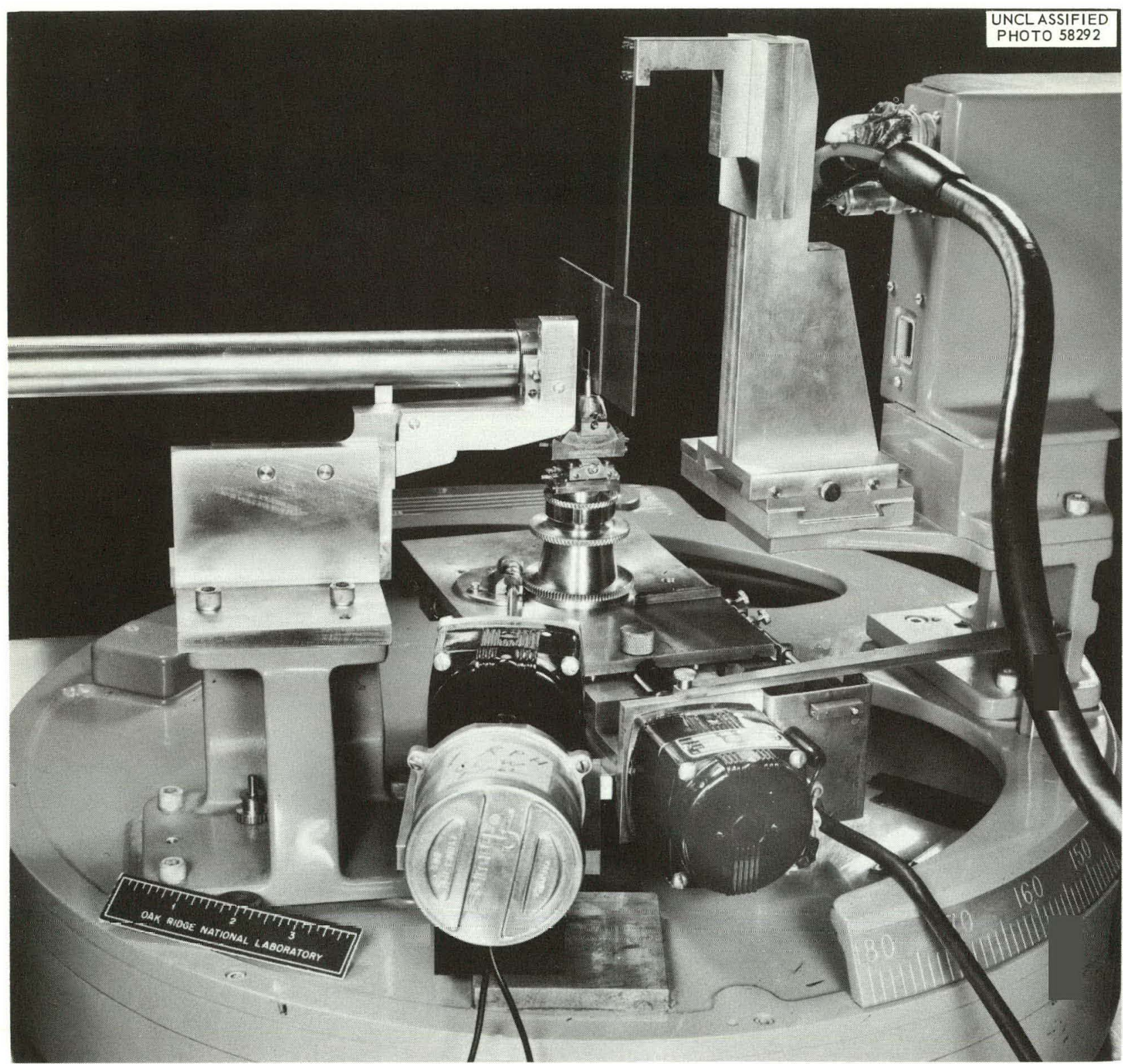

Fig. 7.3. Portable Crystal Rocker for Lang Camera.

The translation of the crystal is vital to the Lang technique in two respects: (1) the motion should be synchronous, and (2) the motion should be linear to within at least a few seconds of arc. Failure to obtain either or both of these features greatly hampers the effort to obtain clear diffraction topographs without obscurities in reasonable times. Present work with the development of the instrument is aimed at perfecting these features.

\subsection{THE 1961 POSTANNEALING OF GRAPHITE. STORED ENERGY IN THE ORNL GRAPHITE REACTOR}

\section{C. Wittels}

Following the September 1961 annealing of the ORNL Graphite Reactor, graphite cores were taken from the three main areas of interest in the moderator stack, and stored energies were measured 
by the radiation-calorimetric technique. The selection of these areas was predetermined by the 1960 discoveries of stored energy in the extreme corners of the fuel zone and in the first reflector rows, as well as by the peak temperatures reached during the September 1961 anneal.

The measured graphite stored energies are given in Table 7.1 and are largely self-explanatory. These data are also plotted in Fig. 7.4 as stored energy distributions along the channels; however, it should be noted that the energies released as graphically shown were measured to somewhat different peak temperatures (Table 7.1) and are therefore not strictly comparable; nevertheless, they are meaningful. Figure 7.5 shows (a) the $140^{\circ} \mathrm{C}$ isotherm reached during the 1961 annealing effort in a plane $7 \mathrm{ft} 6 \mathrm{in}$. west of the east graphite face, (b) the inner periphery in this plane of the preannealing stored energy region, and (c) the channels selected for stored energy determinations. The shaded area (Fig. 7.5) indicates the approximate area of successful annealing in this same plane achieved as a result of the 1961 annealing operation. Since the temperatures further toward the center of the reactor core were appreciably higher during the annealing operation $\left(>200^{\circ} \mathrm{C}\right)$, it is apparent that in the central region the integrated stored energy up to $250^{\circ} \mathrm{C}$ was reduced to near zero as a result of the 1961 operation.

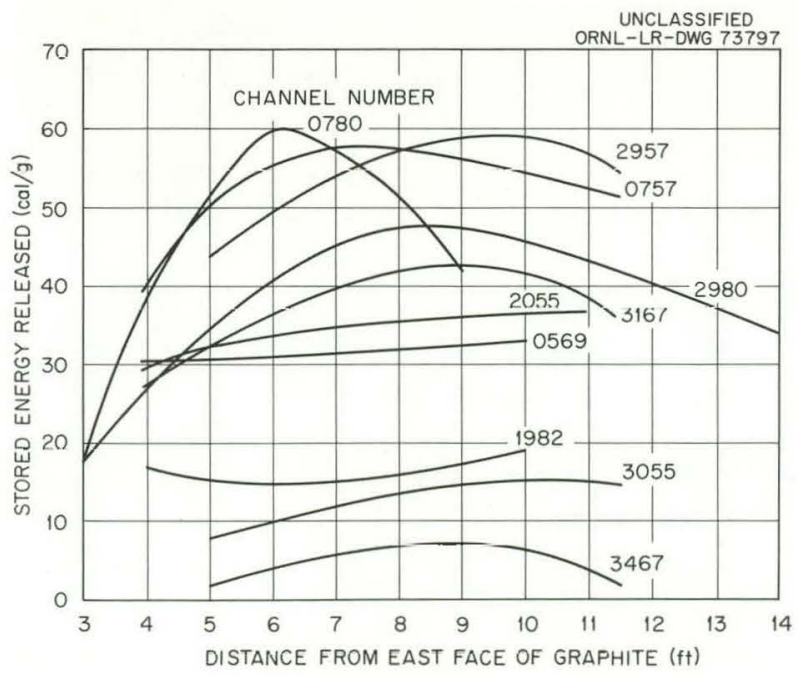

Fig. 7.4. Stored-Energy Distribution Along Fuel Channels.

Table 7.1. Stored-Energy Data - October 1961

\begin{tabular}{cccr}
\hline Channel & $\begin{array}{c}\text { Distance from } \\
\text { East Edge } \\
(\mathrm{ft})\end{array}$ & $\begin{array}{c}\text { Energy } \\
(\mathrm{cal} / \mathrm{g})\end{array}$ & $\begin{array}{r}\text { Maxim } \\
\text { Tempera } \\
\left({ }^{\circ} \mathrm{C}\right)\end{array}$ \\
\hline \multicolumn{4}{c}{} \\
2980 & Corner Fuel Channels \\
2980 & 3 & 17.2 & 242 \\
2980 & 6 & 40.7 & 290 \\
2980 & 10 & 45.4 & 296 \\
2957 & 14 & 33.9 & 268 \\
2957 & 5 & 43.7 & 293 \\
2957 & 9 & 58.6 & 315 \\
0757 & $11 \frac{1}{2}$ & 54.7 & 309 \\
0757 & 7 & 39.5 & 278 \\
0757 & $11 \frac{1}{2}$ & 56.9 & 316 \\
0780 & 3 & 50.4 & 301 \\
0780 & 6 & 16.8 & 244 \\
0780 & 9 & 59.8 & 296 \\
& & 41.4 & 287
\end{tabular}

Lateral Fuel Channels

3167

3167

3167

2055

2055

2055

0569

0569

0569

1982

1982

1982

3467

3467

3467

3567

3567

3567

3055

3055

3055

3255

3255

3255

3453

3453

3453
27.4

42.4

256

4
9

$11 \frac{1}{2}$

35.6

262

256

279

$\begin{array}{ll}34.7 & 273 \\ 36.3 & 273\end{array}$

11

4

30.4

266

267

269

249

247

250

\section{Reflector Channels}

$\begin{array}{ccc}5 & 2 & 237 \\ 8 & 7.0 & 239 \\ 11 \frac{1}{2} & 2 & 237 \\ 5 & 0 & 237 \\ 8 & 0 & 237 \\ 11 \frac{1}{2} & 0 & 237 \\ 5 & 7.9 & 237 \\ 8 & 13.0 & 238 \\ 11 \frac{1}{2} & 14.4 & 238 \\ 5 & 0 & 237 \\ 8 & 0 & 237 \\ 11 \frac{1}{2} & 0 & 237 \\ 5 & 0 & 237 \\ 8 & 0 & 237 \\ 11 \frac{1}{2} & 0 & 237\end{array}$


UNCLASSIFIED

ORNL - LR-DWG 73798

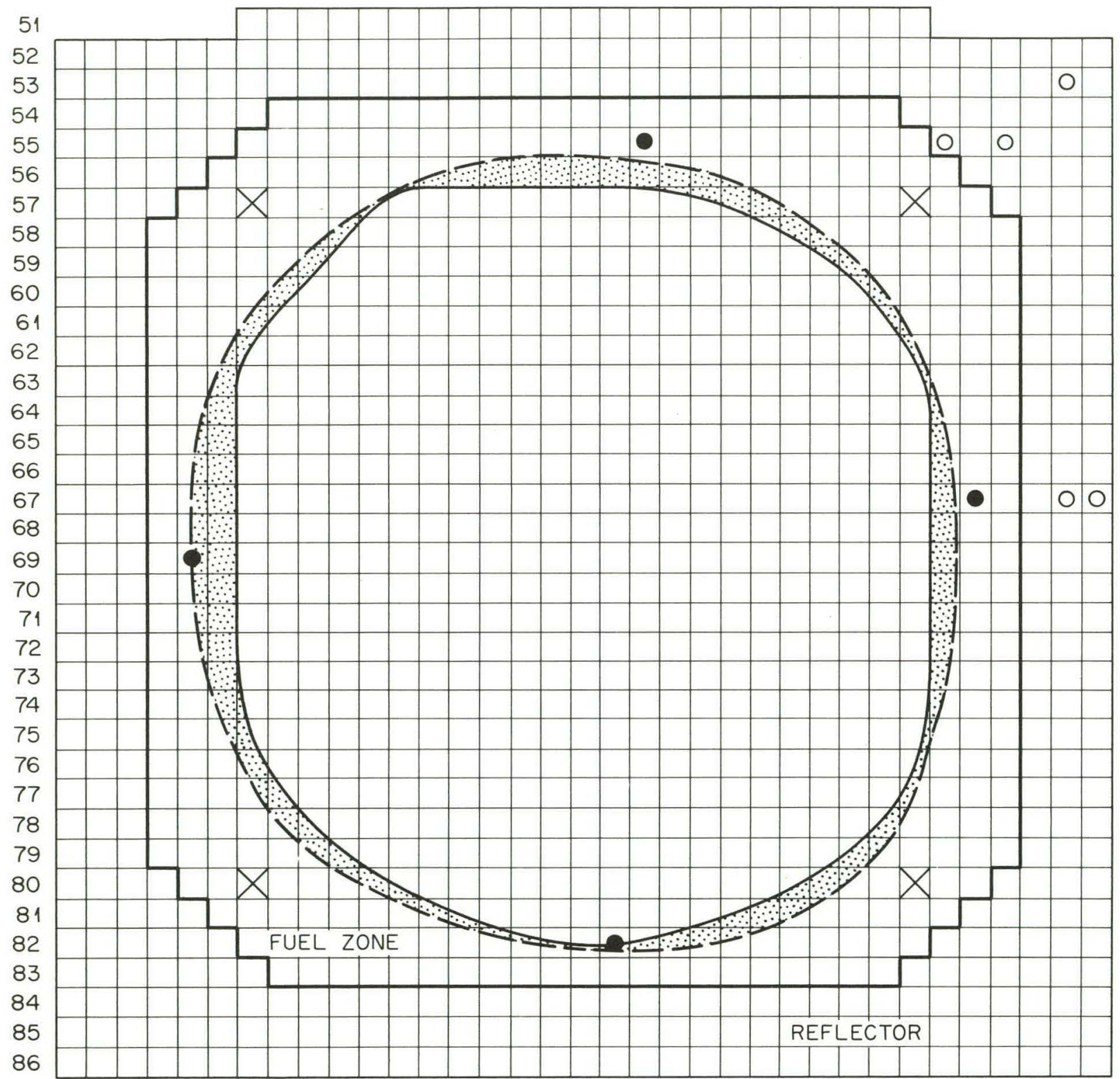

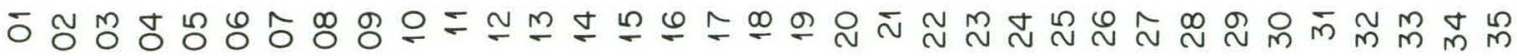

$--140^{\circ} \mathrm{C}$ ISOTHERM, $7 \mathrm{ft} 6 \mathrm{in.} \mathrm{FROM} \mathrm{EAST} \mathrm{GRAPHITE} \mathrm{FACE}$

INNER PERIPHERY 1960 STORED-ENERGY ZONE

$\because \because \because$ APPARENT 1961 ANNEALED REGIONS

$X$ CORNER FUEL ZONE CORES

- lateral fuel zONE cORES

O REFLECTOR ZONE CORES

Fig. 7.5. Front-Face View of Stored-Energy Status $7 \mathrm{ft} 6 \mathrm{in.}$ from East Graphite Face. 
It is clear from the stored-energy measurements in the lateral fuel channels close to the $140^{\circ} \mathrm{C}$ isotherm (Fig. 7.5) that the spontaneously releasable stored energy in the lateral areas has been largely reduced, so that the thickness of the areas is probably no greater than $12 \mathrm{in.}$ and may possibly approach zero on the south and bottom sides of the fuel zone.

The accumulation of stored energy in the comer fuel channels continues to grow at about $2 \frac{1}{2}$ cal $\mathrm{g}^{-1} \mathrm{yr}^{-1}$ to $250^{\circ} \mathrm{C}$, and at present is of a magnitude to cause an adiabatic rise in those regions to $350-375^{\circ} \mathrm{C}$, if the energy is released under ideal adiabatic condition. The total volume of graphite in these affected areas is about 7\% of the volume of the entire graphite stack, and therefore contains about $4.5 \mathrm{Mwhr}$ of adiabatically releasable stored energy at present.

In assessing the success of the 1960 and 1961 annealing operations the fact should not be overlooked that the predicted 1960 stored-energy region has been successfully annealed to a high degree. In addition, it has once again been shown that it was virtually impossible to heat the corner regions of the fuel zone to annealing temperatures, even under the contrived conditions of reverse airflow and blocked airflow with scrammed conditions from high temperature.

The growth of significant amounts of stored energy in the extreme corners of the fuel zone, even out to the very last slug rows, is still not adequately understood. For many winter months the temperatures in these channels is considerably lower than $30^{\circ} \mathrm{C}$, and presumably the damage rate at $10^{\circ} \mathrm{C}$ might be three times greater than the rate at $30^{\circ} \mathrm{C}$, the lowest temperature at which important amounts of data are available. Since the fast neutron flux $>1 \mathrm{Mev}$ in these extreme fuel corners is probably below an order of magnitude lower than that required by accepted radiation-damage theory to account for the damage produced, it appears that present radiation theory is inadequate in this regard. As a matter of speculation it is felt that some of the stored-energy problems in other aircooled graphite reactors (e.g., Windscale, BEPO, and $B R-1$ ) might have been due to stored-energy accumulations at the extreme edges of the fuel zones in these reactors, where it previously could have been easily overlooked.

Since the airflow in the reflector channels of the ORNL Graphite Reactor has been blocked, more coolant gas now passes through the entire fuel zone, and it therefore operates at a somewhat lower temperature during regular operation. Under these conditions stored energy would accumulate at a somewhat higher rate in the reactor during future years. Development work to provide a means of annealing the peripheral stored-energy regions of the reactor is proceeding satisfactorily, and it is hoped that the end result will furnish a flatter temperature distribution in the reactor core for normal operation as well as provide a sufficient thermal configuration for successful annealing.

\subsection{X-RAY OBSERVATIONS OF NEARLY PERFECT COPPER SINGLE CRYSTALS ${ }^{7}$}

$$
\begin{gathered}
\text { M. C. Wittels } \quad \text { F. A. Sherrill } \\
\text { F. W. Young, Jr. }
\end{gathered}
$$

It is well known that metal crystals are generally imperfect and that their dislocation densities usually exceed $\sim 10^{6} / \mathrm{cm}^{3}$, thereby making individual dislocations unresolvable when utilizing the Lang ${ }^{8}$ technique. However, recent etch-pit studies ${ }^{9}$ have indicated that large copper single crystals could be grown with a much higher degree of perfection than had been previously realized for any metal crystals except perhaps whiskers. This paper is a presentation of the initial x-ray diffraction studies of some of these highly perfect ${ }^{9}$ copper crystals.

We have utilized the Lang method for observing dislocation concentrations in bulk samples by employing techniques similar to those described by Webb ${ }^{10}$ and Schwuttke. ${ }^{11} \mathrm{As}$ an adjunct to the Lang camera, we have also adapted a portable crystal rocker which permits the measurement of line half-widths in seconds of arc.

The optimum contrast conditions for observing dislocations through the Lang technique require that $\mu t \cong 1$, where $\mu$ is the linear absorption

\footnotetext{
${ }^{7}$ M. C. Wittels, F. A. Sherrill, and F. W. Young, Jr., Appl. Phys. Letters 1, 22 (1962).

${ }^{8}$ A. R. Lang, Acta Met. 5, 358 (1957).

${ }^{9}$ F. W. Young, Jr., Bull. Am. Phys. Soc. 7, 215 (1962).

${ }^{10}$ W. W. Webb, Direct Observations of Imperfections in Crystals, PP 29-76, Interscience, New York, 1962.

${ }^{11} \mathrm{G}$. H. Schwuttke, J. Electrochem. Soc. 109, 27 (1962).
} 
coefficient, and $t$ is the crystal slice thickness. Since we used $\mathrm{Ag} K \alpha$ radiation, it was necessary to thin crystals to approximately $0.002 \mathrm{in}$. without introducing imperfections and strains. Needless to say, this is a very difficult task with a soft metal such as copper, and considerable caution must be exercised. This was successfully accomplished by first cutting wafers with an acid saw ${ }^{12}$ and then slowly electropolishing to the proper thickness. The orientation selected for the $\mathrm{x}$-ray transmission studies was a (110) face with diffraction from (111) planes which were perpendicular to the crystal surface.

Some of the Lang diffraction topographs obtained from these crystals are shown in Fig. 7.6. It is evident from these topographs that large areas of the crystals are completely free of dislocations. It is also apparent that dislocations which lie in or close to the plane of the wafer are strikingly evident and, moreover, that the strain field surrounding the dislocation core in copper can be as high as $50 \mu$. Etch-pit counts ${ }^{9}$ of dislocations in random slices from the same single-crystal ingot give a good correlation between the two methods, and confirm the proposal ${ }^{9}$ that crystals

${ }^{12} \mathrm{~F}$. W. Young, Jr., and T. R. Wilson, Rev. Sci. Instr. 32, 559 (1961). with dislocation concentrations as low as $5 \times 10^{2} / \mathrm{cm}^{3}$ are possible in copper.

As a further guide to the study of perfection of these copper crystals, rocking curves were run in reflection from (111) surfaces of large crystal slabs approximately $1 \times \frac{1}{4} \times \frac{1}{4}$ in. The same incident $x$-ray beam was utilized as for the Lang geometry, namely $\mathrm{Ag} K \alpha$ radiation collimated to give a total beam divergence of $15 \mathrm{sec}$. Under these conditions a clear separation of the $K a$ doublet was obtained, and the measured width of $K \alpha_{1}$ at half maximum intensity was $34 \mathrm{sec}$ (Fig. 7.7). Although this is not the classical method for rocking-curve measurerents, which requires strictly monochromatic radiation in the double-crystal spectrometer arrangement, ${ }^{13}$ it at least gives an upper limit of the absolute halfwidth. Furthermore, we feel it is probably quite close to the absolute half-width, since we have measured half-widths on germanium (111) as low as $23 \mathrm{sec}$ with the same geometry. The etch-pit determination of dislocations on this same crystal indicated the presence of $\sim 3 \times 10^{2}$ dislocations $/ \mathrm{cm}^{3}$. If this is the case, then probably some type of dislocation or imperfection other than these dis-

\footnotetext{
${ }^{13} \mathrm{R}$. W. James, The Optical Principles of the Diffraction of X-Rays, Bell, London, 1950.
}
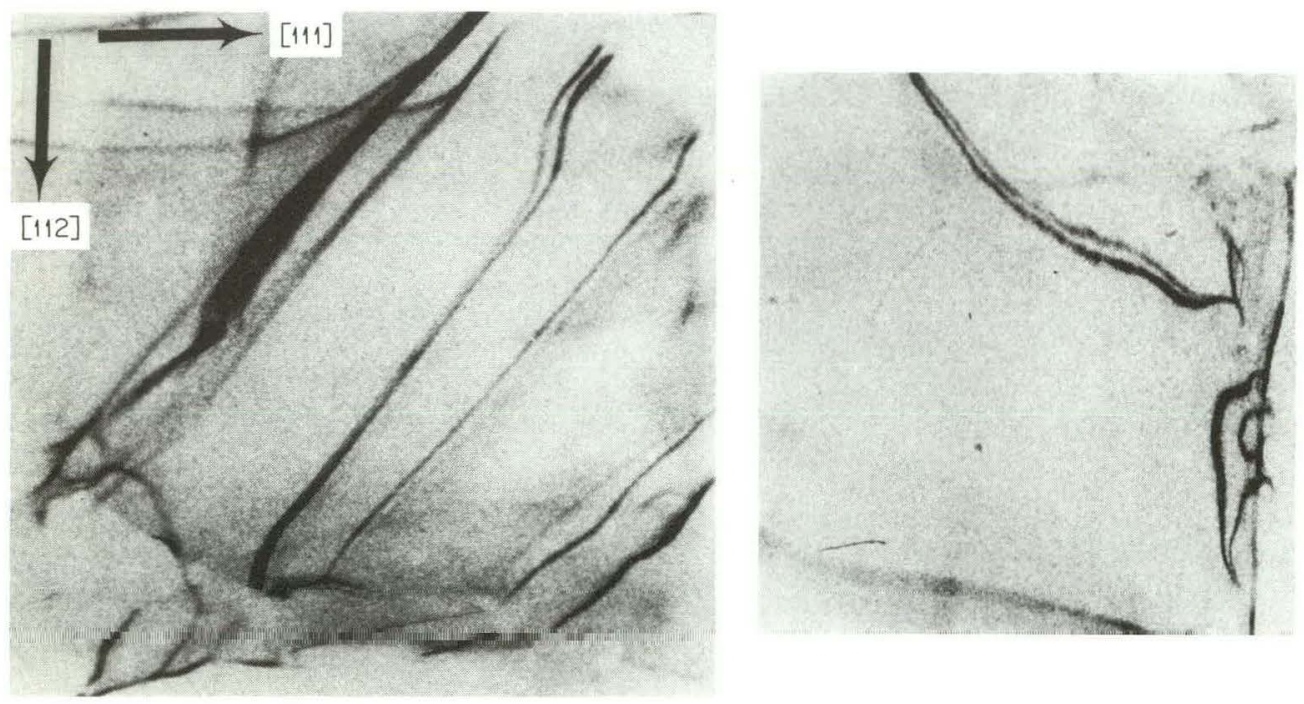

Fig. 7.6. Lang Diffraction Topographs of Three Copper Crystals. Orientation as shown. 10X. 
locations contributes to the line breadth observed in this crystal. It might possibly be due to small vacancy clusters or loops resulting from the collapse of such clusters.
It is hoped that further refinement in the use of the Lang technique as well as use of the doublecrystal spectrometer will aid in the determination of imperfections in copper.

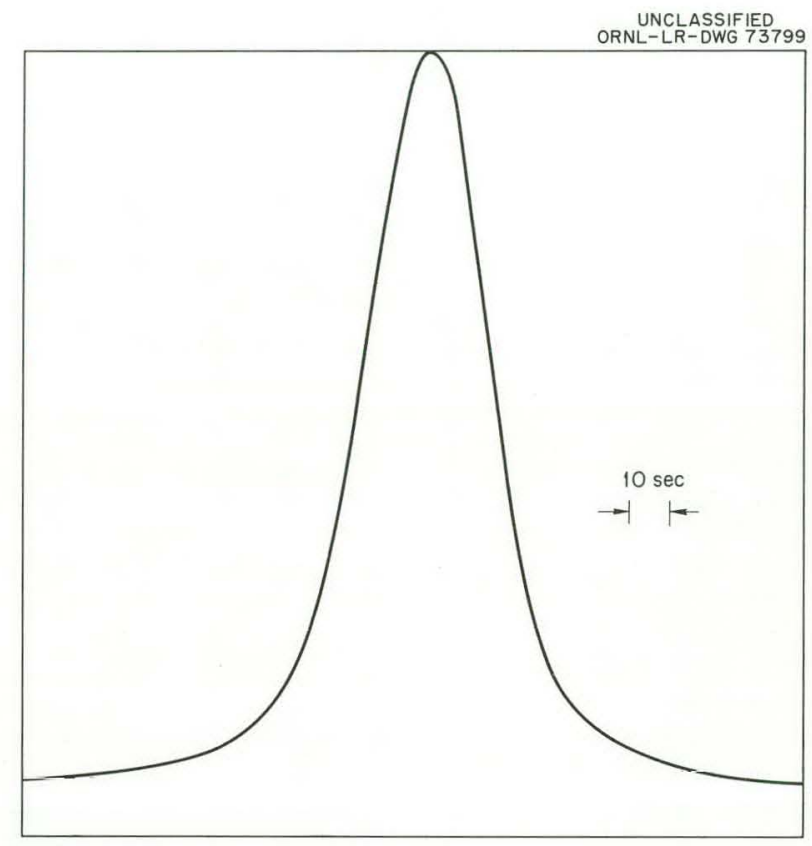

Fig. 7.7. Rocking Curve (Ag Ka $\alpha_{1}$ ) of Copper (111). Peak intensity approximately 8700 counts/min. 


\section{Electron Microscopy}

\subsection{FISSION-FRAGMENT TRACKS IN METAL FILMS ${ }^{1}$}

T. S. Noggle

$$
\text { J. O. Stiegler }{ }^{2}
$$

Fission-fragment tracks observed in electron microscope studies of platinum and palladium films are interpreted in terms of thermal processes involving melting and vaporization. The results require that the energy lost by the fragment be converted into thermal energy over the entire volume of the track in a time short enough that little of it is conducted away. A model in which free electrons disperse the energy over this volume is used to account for the widths and structures of the tracks.

\subsection{FISSION-FRAGMENT TRACKS IN THIN FILMS OF $\mathrm{UO}_{2}{ }^{3}$}

$$
\text { T. S. Noggle }
$$

$$
\text { J. O. Stiegler }{ }^{2}
$$

Surface effects in $\mathrm{UO}_{2}$ films produced by individual fission events which can be studied with the electron microscope offer promise of giving increased insight into the interaction of fission fragments with solids. Experimental techniques are described in which it is possible to identify the class of fragment giving rise to a particular length of track, the direction of travel of the fragment in producing the track, and the depth of the fragment below the free surface at points along the track. The results obtained with these techniques are correlated with the structures observed.

\footnotetext{
${ }^{1}$ Abstract of published paper: J. Appl. Phys. 33, 1726 (1962).

${ }^{2}$ Metals and Ceramics Division.

${ }^{3}$ Abstract of paper published in Am. Soc. Testing Mater. Spec. Tech. Publ. 306, 47-63 (1961).
}

The proposed mechanisms of energy loss of fission fragments are discussed and related to the studies of track structure. Results suggest that electron excitation and ionization energy loss are dominant in determining track registration. Finally, a new mechanism by which ionization energy may be converted into lattice damage is described.

\subsection{NITROGEN ION BOMBARDMENT OF THIN PLATINUM FILMS ${ }^{4}$}

$$
\text { J. O. Stiegler }{ }^{2} \quad \text { T. S. Noggle }
$$

Previous studies ${ }^{5}$ of thin, polycrystalline films of platinum and palladium irradiated with fission fragments revealed track structures that indicated material had been displaced, melted, or vaporized by the passage of the fragments through the films. The features of the tracks were explained ${ }^{5}$ in terms of a model developed by Ozeroff, ${ }^{6}$ in which free electrons disperse the energy lost by the fission fragment over the entire track volume before it appears as thermal energy.

In this model the fission fragment excites electrons in a cylindrical cloud about 50 to $75 \mathrm{~A}$ in diameter; this transfers an average of $\sim 90 \mathrm{ev}$ to each electron. The excited electrons move into the lattice, collide with conduction electrons, and reach conduction energies during the time they move $\sim 10 \mathrm{~A}$. With in an additional 100 to $200 \mathrm{~A}$ they reach equilibrium with the lattice. The energy lost by the fragment, therefore, appears as thermal energy over a region extending 100 to 200 A from the fission fragment's path.

\footnotetext{
${ }^{4} \mathrm{~J}$. O. Stiegler and T. S. Noggle, J. Appl. Phys. 33, 1894 (1962).

${ }^{5}$ T. S. Noggle and J. O. Stiegler, to be published in the Journal of Applied Physics.

${ }^{6} \mathrm{~J}$. Ozeroff, KAPL-205, pp 22-32 (1949).
} 
The results of observations of a series of palladium films ${ }^{5}$ varying in thickness between 25 and $400 \mathrm{~A}$ were in agreement with this model. In the thinnest films, tracks of light contrast were observed having widths of 200 to $400 \mathrm{~A}$, indicating that material had been removed from this region, probably due to vaporization. Films of intermediate thickness showed dark tracks, indicating melting followed by rapid cooling of the track region. Thicker films failed to show any evidence of tracks. These results were interpreted in terms of energy density within the track region. Reflection of the low-energy electrons by the potential barrier of the free surface confined them to the films and raised the energy density in the thinnest films high enough to cause complete vaporization. Only melting was possible in films $100 \mathrm{~A}$ thick, and in films greater than $200 \mathrm{~A}$ thick the energy density was not high enough to cause melting.

In order to test these ideas, a series of cyclotron irradiations of platinum films ( $<25 \Lambda$ thick) with 24-Mev nitrogen ions was carried out. The energy loss rate of $24-\mathrm{Mev}$ nitrogen ions in platinum is about 500 to $600 \mathrm{ev} / \mathrm{A}$, compared with $4000 \mathrm{ev} / \mathrm{A}$ for typical fission fragments. ${ }^{7}$ As a consequence, the energy density in the platinum films irradiated with the nitrogen ions should not be high enough to cause vaporization; however, uncertainty as to the exact film thickness leads to uncertainty as to whether melting should occur. On the basis of these considerations along with the observations and interpretation of the fission-fragment tracks in metal films, ${ }^{5}$ one would expect to see broad tracks indicative of melting if tracks can be observed as a result of the ion bombardment.

The platinum films were formed by evaporation onto the surfaces of carbon films (on the order of 200 A thick) which had previously been evaporated onto fresh cleavage surfaces of $\mathrm{NaCl}$. The irradiations were carried out in the ORNL 63-in. cyclotron. Films were irradiated on $\mathrm{NaCl}$ substrates positioned with the plane of the film

\footnotetext{
${ }^{7}$ Energy loss rates of nitrogen ions have been measured in nickel by H. L. Reynolds, D. W. Scott, and A. Zucker, Pbys. Rev. 95, 671 (1954), and energy loss rates of fission fragments in nickel and gold have been measured by C. B. Fulmer, ORNL 2320 (1D59). The figures used were determined by assuming that the loss ratc in platinum is about equal to that in gold, and that the ratio of loss rates in gold to nickel for fission fragments is approximately equal to that for nitrogen ions.
}

parallel to the direction of the ion beam, which was diffused by passing it through two thin nickel foils prior to striking the specimen. The initial energy of the beam was $28 \mathrm{Mev}$, and it was degraded about $2 \mathrm{Mev}$ in passing through each of the foils. After the irradiation the composite platinumcarbon film was removed from the $\mathrm{NaCl}$ and mounted on standard electron microscope grids for examination.

A transmission electron micrograph of a typical region of an irradiated film is shown in Fig. 8.1. For comparison a micrograph of an identical film after fission-fragment irradiation ${ }^{5}$ is shown in Fig. 8.2. The tracks in both cases are light, indicating that material has been removed from the track region. The tracks formed by fission fragments are of considerably higher contrast, suggesting that only partial removal of material occurred with the nitrogen ion irradiation. In addition, the tracks produced by the nitrogen ions are extremely narrow, 25 to $50 \mathrm{~A}$ wide, compared with fission-fragment tracks nearly 400 A wide. The occasional broad, contrasty tracks observed in the nitrogen-ion-irradiated film result from the superposition of two or more of the narrow, lowcontrast tracks.

The tracks produced by the nitrogen ion irradiation are completely different from the type predicted by the Ozeroff treatment in that they are narrow and light rather than broad and dark. Simple vaporization as a result of dispersal of the energy lost by the ion over the track volume by the free electron cloud cannot account for the observations. Since the volume over which electrons are excited by the nitrogen ions is less than that by fission fragments and also since the average energy transfer is less, one might expect a smaller volume to be ultimately affected by the ions. However, the major factor determining the width of the affected zone is the distance required for the slowed-down (conduction energy) electrons to come to equilibrium with the lattice. This distance is on the order of $100 \mathrm{~A}$ and should not vary for the two cases. The track widths, therefore, are too narrow to be explained on the basis of the Ozeroff theory.

These observations suggest that these tracks are not the result of a thermal effect. Quite possibly they occur through charge interaction between the moving ion and the surface layers of the film. If sufficient electrons are accelerated out of the film by the moving ion, a thin layer 


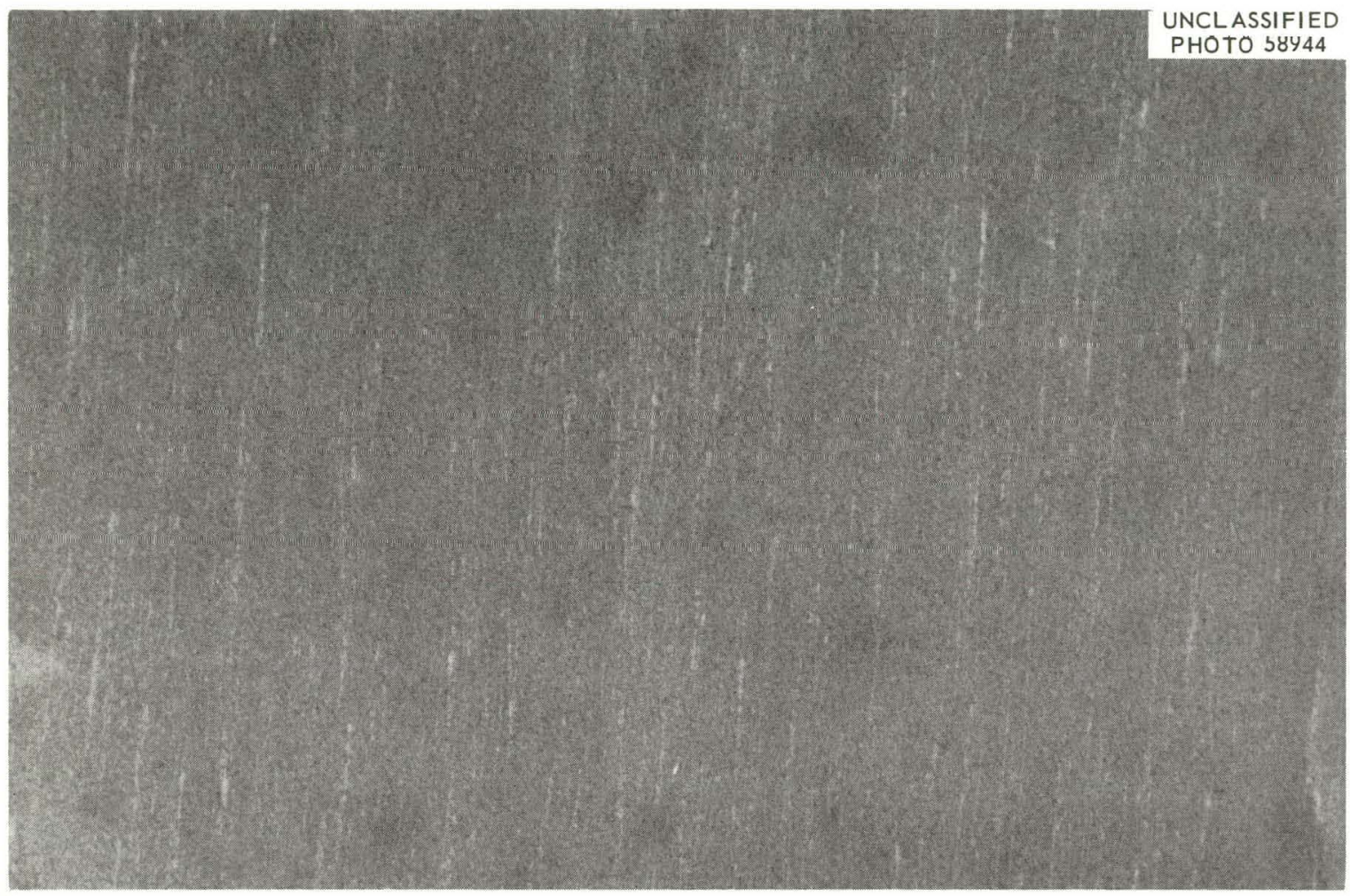

Fig. 8.1. Tracks Produced in a Platinum Film $<25$ A Thick by 24-Mev Nitrogen lons. 50,000X.

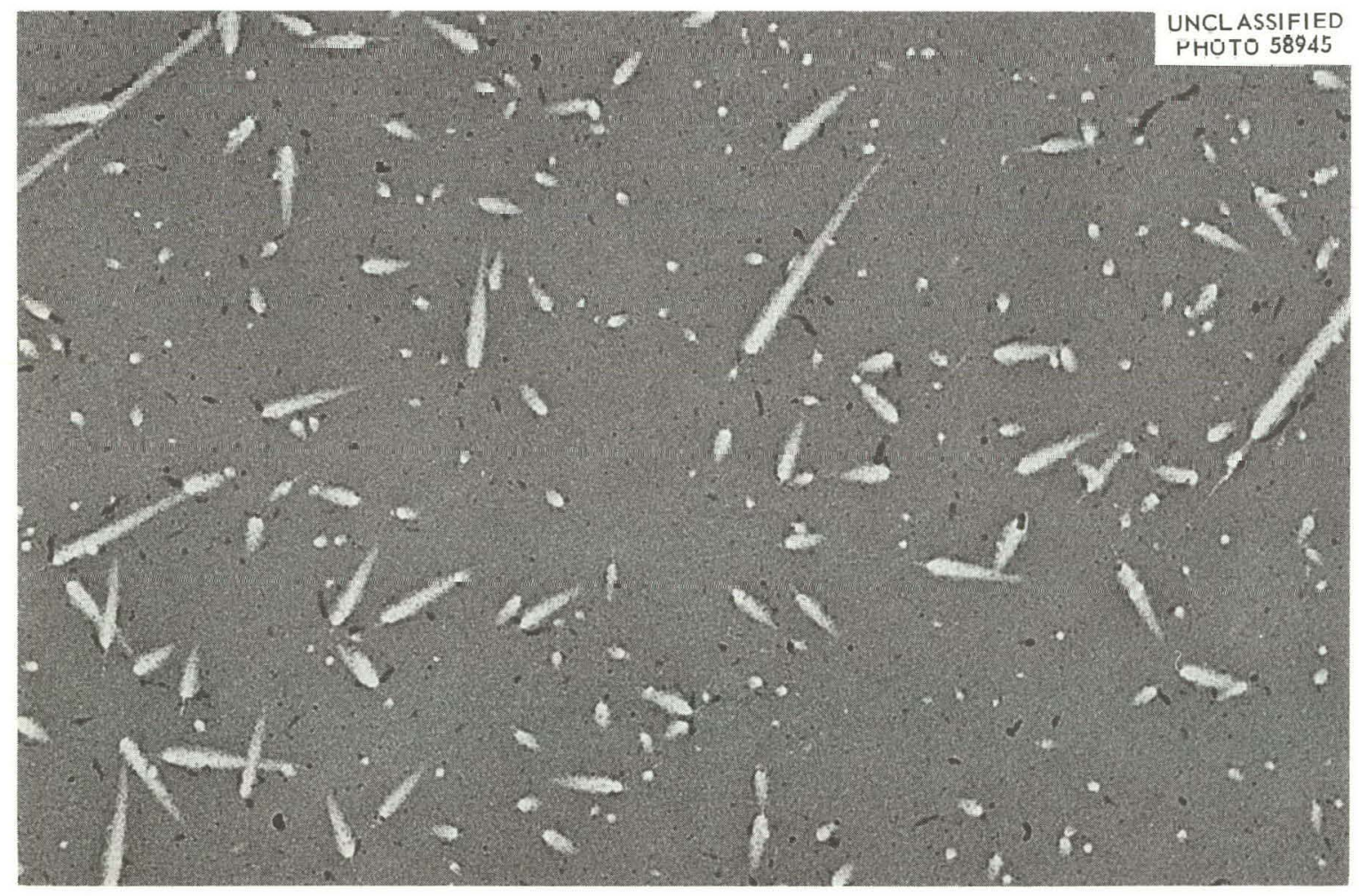

Fig. 8.2. Fission-Fragment Tracks in a Platinum Film $<25$ A Thick. 50,000X. 
at the surface will be left with a net positive charge. Free electrons will move into the region to neutralize the charge imbalance, but the separation of charge will probably be of sufficient duration that the electrostatic repulsion of the remaining positive ions will displace material at the surface, thus producing the light tracks. This process may occur to some extent in thicker films, but the relative disturbance would be too small to produce a detectable contrast effect. Only the broad tracks occurring from thermal effects when the ion is well within the interior of the film would be observed.

These results show that ions as light as nitrogen will leave tracks in very thin metal films. However, tracks left by $24-\mathrm{Mev}$ nitrogen ions in platinum films $<25 \mathrm{~A}$ thick are not the result of thermal processes responsible for fission-fragment tracks in thicker metal films. Direct charge interaction may displace sufficient material at a free surface to lead to a detectable contrast in very thin films.

The cyclotron irradiations were carried out under the direction of A. Zucker and M. Halbert of the Electronuclear Division.

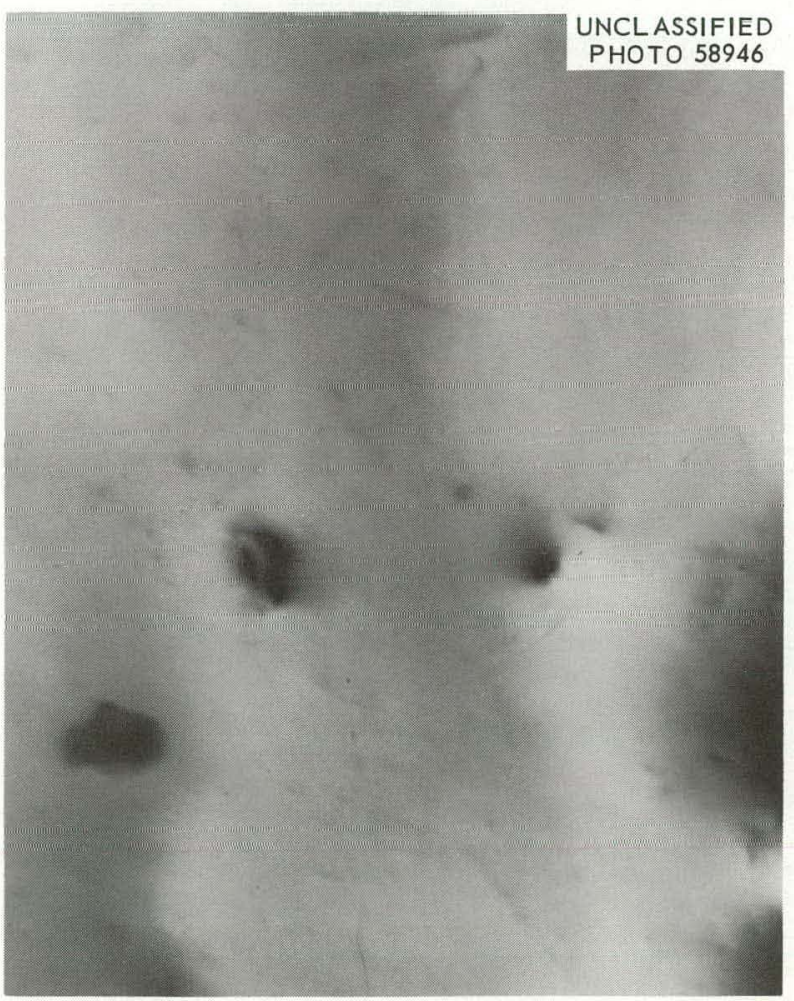

Fig. 8.3. Crystal of $\mathrm{UO}_{2}$ Irradiated in Vacuum to a Dose of $5 \times 10^{14}$ fissions $/ \mathrm{cm}^{3}$. 100,000X.

\subsection{TRANSMISSION ELECTRON MICROSCOPE STUDIES ON SINGLE CRYSTALS OF $\mathrm{UO}_{2}$}

\author{
T. S. Noggle
}

Preliminary observations have been made by transmission electron microscopy of fissionfragment tracks in single crystals of $\mathrm{UO}_{2}$. These tracks appear to be limited to the surface regions of the crystal and are not observed in specimens thinned subsequent to irradiation. No structural damage has been identified for the low irradiation doses employed to date; however, it has been found that some reaction occurs between $\mathrm{UO}_{2}$ and its environment when irradiated in air in a polyethylene capsule. Figure 8.3 shows a prethinned $\mathrm{UO}_{2}$ crystal irradiated in vacuum, and fission-fragment tracks are visible, but there are no other detectable changes from the unirradiated condition. Figure 8.4 shows a prethinned $\mathrm{UO}_{2}$ crystal irradiated in air in a polyethylene capsule. The marked change in structure reflects in good part the microcracking arising from a volume

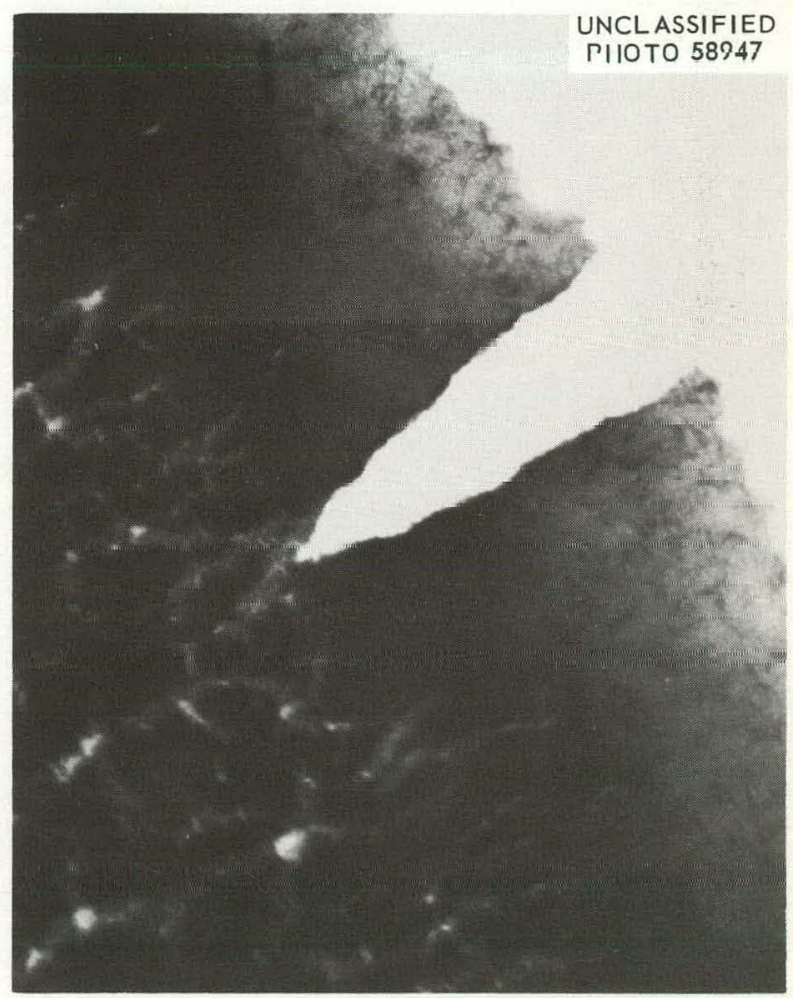

Fig. 8.4. Crystal of $\mathrm{UO}_{2}$ Irradiated in Air in a Polyethylene Capsule to a Dose of $5 \times 10^{14} \mathrm{fissions} / \mathrm{cm}^{3}$. $100,000 x$. 
decrease accompanying the reaction. Lower irradiation doses indicate that material from the environment initially reacts with the $\mathrm{UO}_{2}$ at discrete points on the surface, giving a coherent phase of higher density. Figure 8.5 shows such a specimen in which the small patches, believed to be reaction sites, show contrast effects associated with strain fields. Figure 8.6 shows several patches containing Moiré patterns, indicating that the material in the patch is crystalline and coherent with the $\mathrm{UO}_{2}$. These structures are limited to the surface regions of the crystal and are eliminated completely by a short etch in hot nitric acid. The new, thin regions present after etching show no trace of structures such as are present in Figs. 8.4-8.6, and it is estimated that the depth to which the reaction has progressed in Fig. 8.4 is of the order of $0.1 \mu$ or less.

A tentative explanation of these observations is that volatile organic material released by the polyethylene during irradiation reacts with the $\mathrm{UO}_{2}$ to form, in part at least, uranium monocarbide (UC). Moiré fringe spacings of $31.2 \mathrm{~A}$

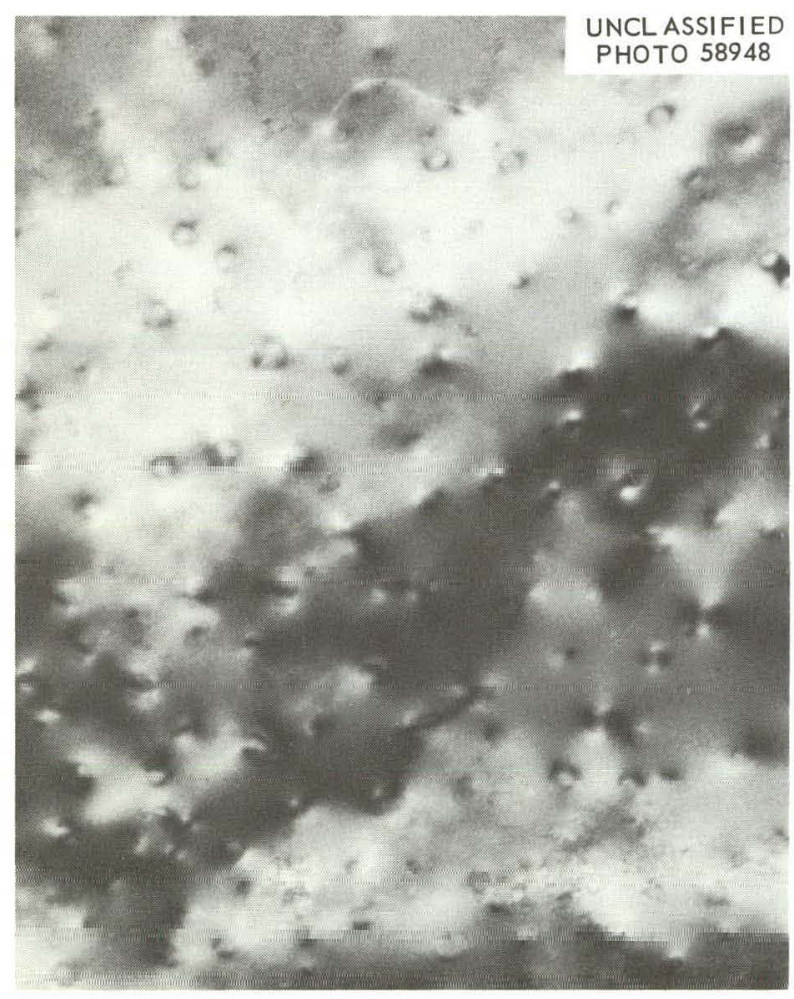

Fig. 8.5. Specimen of $\mathrm{UO}_{2}$ Irradiated to $5 \times 10^{13}$ fissions $/ \mathrm{cm}^{3}$ in Polyethylene Capsule. 50,000X.

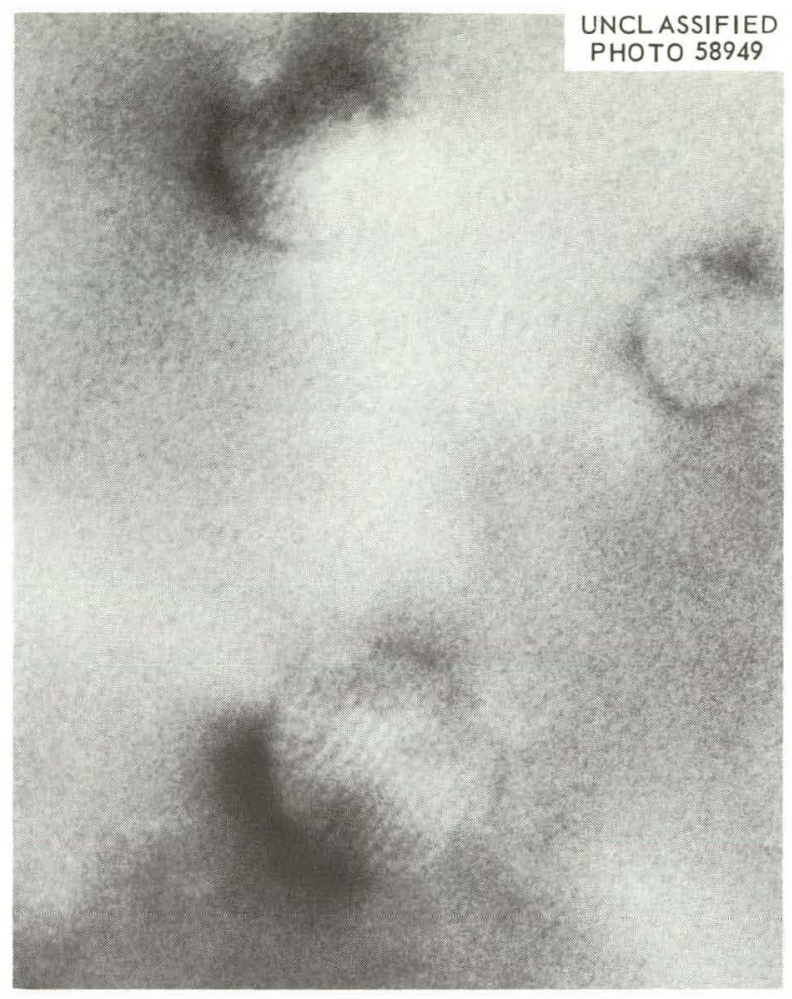

Fig. 8.6. Moiré Patterns in Reaction Sites on $\mathrm{UO}_{2}$ Irradiated to $5 \times 10^{13}$ fissions $/ \mathrm{cm}^{3}$ in Polyethylene Capsule. 400,000X.

have been observed in several instances and agree quite well with the spacing expected of the (111) reflection from UC in parallel orientation with $\mathrm{UO}_{2}$. The volume decrease associated with reduction and carburization would lead to cracking of the reactant layer in the latter stages of the process.

Although many details of this phenumenun need to be studied before the full significance of the nhservations will he known, these observations graphically illustrate the effect of environment on the surface integrity of $\mathrm{UO}_{2}$ during irradiation.

\subsection{DISLOCATIONS IN UO ${ }_{2}^{8}$}

$$
\text { T. S. Noggle }
$$

During the development of techniques for preparing single crystals of $\mathrm{UO}_{2}$ for elecuon

\footnotetext{
${ }^{8}$ To be published in the Proceedings of the 5 th International Congress for Electron Microscopy, Aug. 29-Sept. 5, 1962, Philadelphia, Pennsylvania.
} 
microscope studies of fission-fragment damage, observations have been made on dislocation structures which reflect an appreciable degree of plasticity at elevated temperatures.

Small platelets of $\mathrm{UO}_{2}$ on the order of $1 / 2 \mathrm{~mm}$ in diameter and 10 to $100 \mu$ thick were obtained from $\mathrm{UO}_{2}$ formed by hydrolysis of $\mathrm{UF}_{4}$ in a molten salt bath. These platelets were thinned by etching in hot fuming nitric acid to give areas suitable for transmission microscopy. During the course of thinning, a number of crystals developed arrays of etch pits delineating slip lines and slip bands as shown in Fig. 8.7. The orientation of all platelets showing these etch-pit patterns has proved to be (111), and the slip-line structures are in agreement with the slip system (100) [110] reported for fluorite. ${ }^{9}$ These dislocations were apparently introduced at elevated temperatures, probably during solidification of the molten salt in which they were grown. Electron microscope observations of dislocations in the etch pits as shown in Fig. 8.8 establish the relationship between etch pits and

${ }^{9}$ E. Schmidt and W. Boas, Kristall Plastizitat, Springer, Berlin, 1935.
Fig. 8.7. Optical Micrograph in Transmitted Light of (111) $\mathrm{UO}_{2}$ Crystal. $a-a^{\prime}$, etc., indicate pairing of rows of etch pits developed on the top and bottom surfaces respectively.
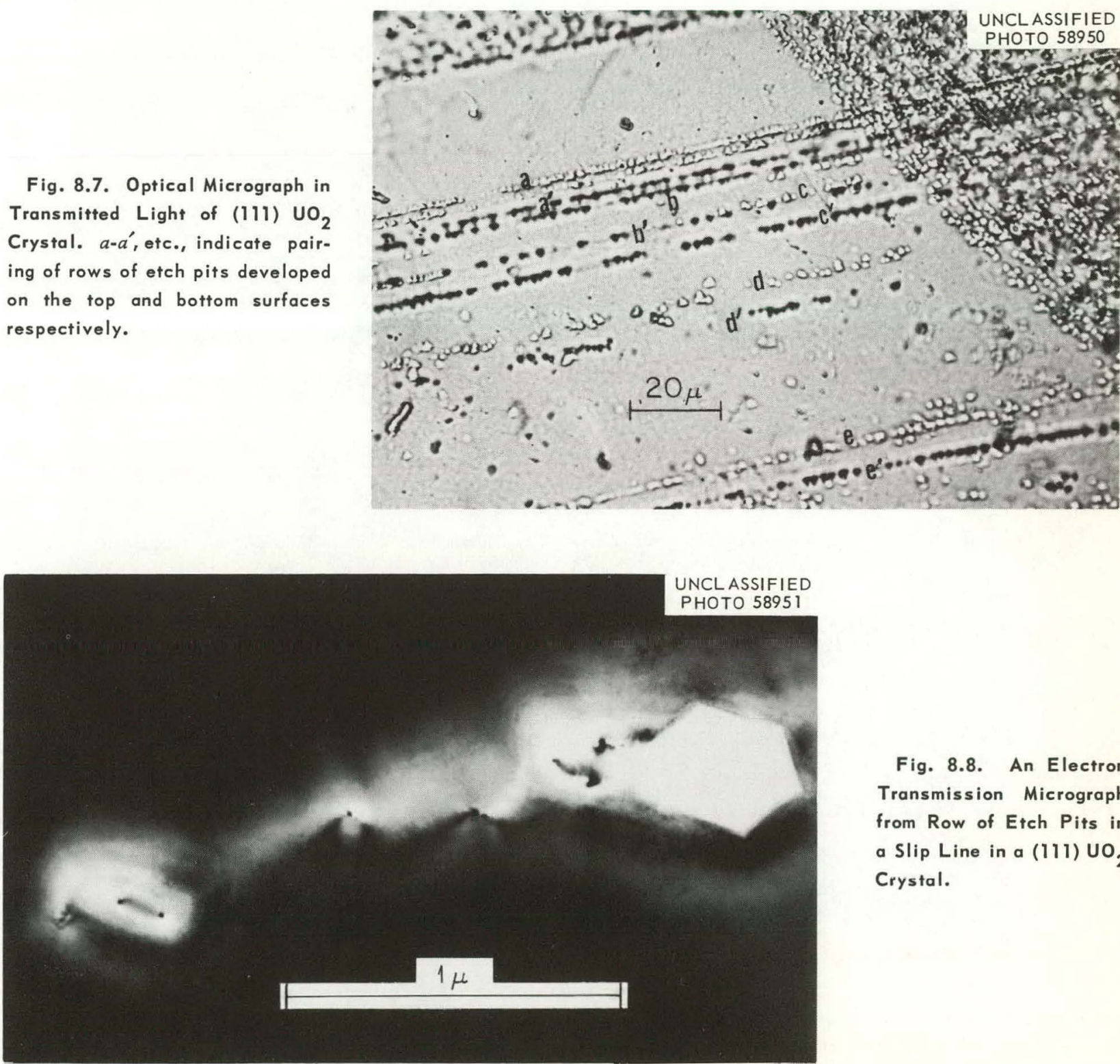

Fig. 8.8. An Electron Transmission Micrograph from Row of Etch Pits in a Slip Line in a (111) $\mathrm{UO}_{2}$ Crystal. 
dislocations in this system. In general, very few dislocations are observed in the electron microscope in (111) crystals, since the etch attack is accelerated at the dislocations terminating in the free surface (giving etch pits), and these regions are etched away before larger areas become thin enough for electron transmission. Dislocations are frequently observed in (110) crystals, which do not develop etch pits. Figure 8.9 shows an example of dislocations observed in a (110) crystal. The curvature of some of the dislocations seen here reflects the immobility of the dislocations at room temperature and indicates that the crystal was stressed during cooling from tem- peratures where the dislocations were mobile. Attempts to determine the Burger's vectors have been limited to diffraction dark-field observations of regions in which multiple reflections occur, and yield the result $h=k, l$ unspecified. This result is compatible with the [110] slip direction noted above.

Movement of the dislocations has not been observed under normal viewing conditions; however, during attempts to make observations in a region of marginal thinness, the beam was temporarily raised to a high value and rapid generation and motion of dislocations ensued. Figure 8.10 is from this area and shows dis-

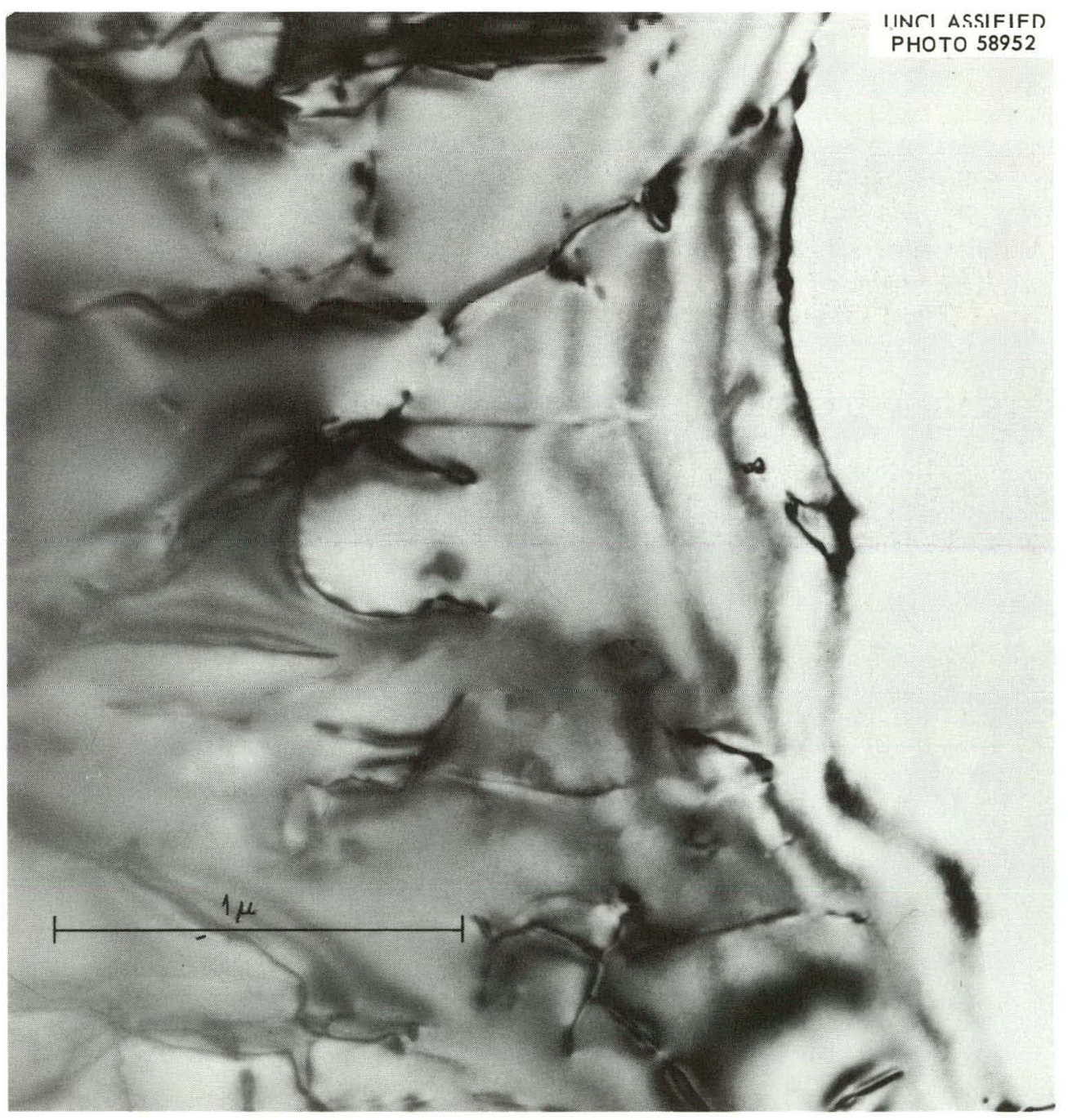

Fig. 8.9. Dislocations in a $(110) \mathrm{UO}_{2}$ Crystal Introduced During Growth or by Plastic Deformation During Cooling from Elevated Temperatures. 
locations and slip traces generated by their movement. Crystallographic analysis of the structures observed in this region is only in part compatible with a (100) [110] slip system and suggests that cross slip occurs in this material and that other slip systems may have been active under the ill-defined conditions established in this case.

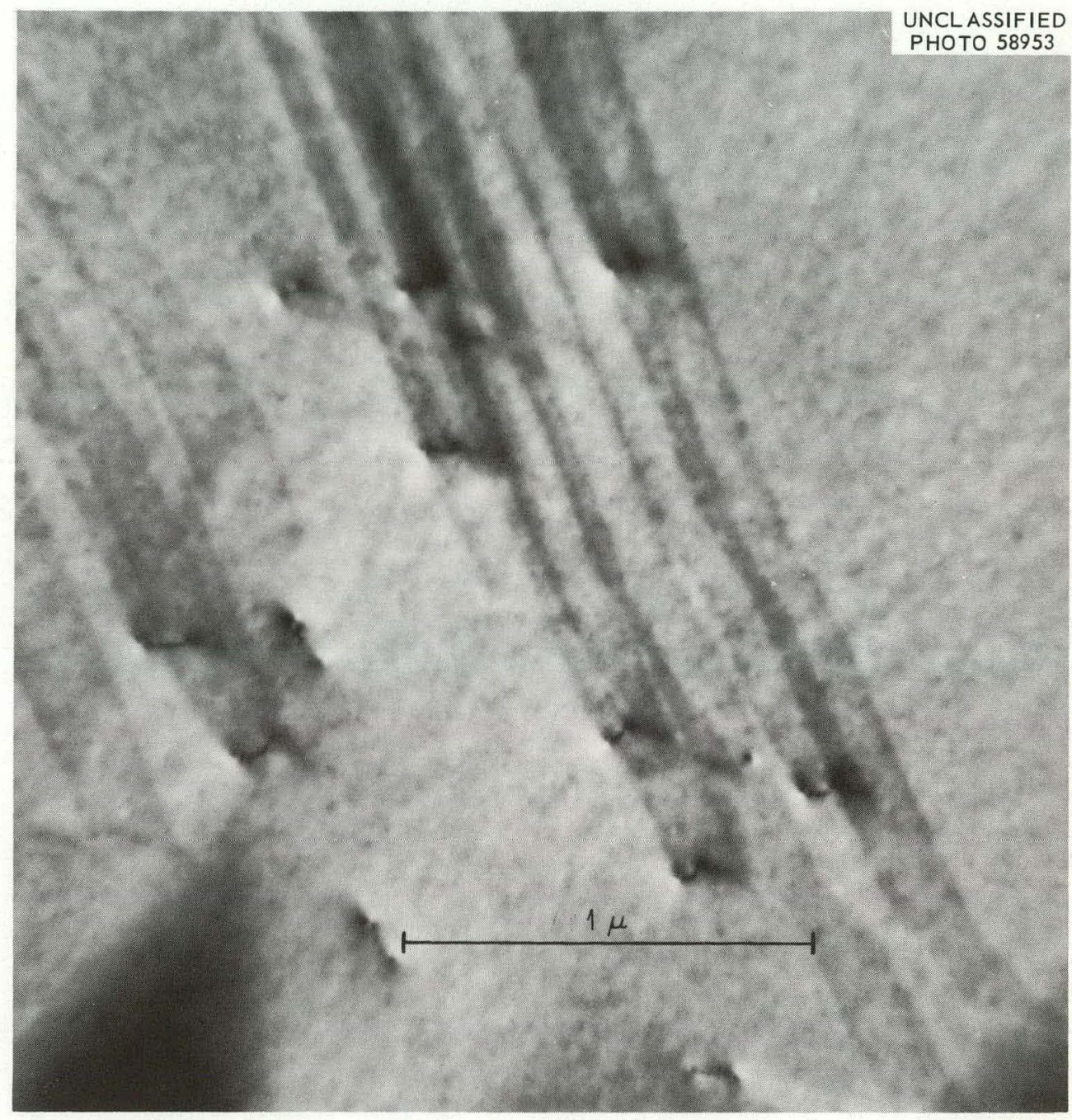

Fig. 8.10. Dislocations and Slip Traces Induced by Heating of the Electron Beam in a $(111) \mathrm{UO}_{2} \mathrm{Crystal}$ 


\section{Spin Resonance}

\subsection{RELATION BETWEEN MAGNETIC SUSCEPTIBILITY, ELECTRON SPIN RESONANCE, AND OPTICAL ABSORPTION OF THE $E_{1}^{\prime}$ CENTER IN FUSED SILICA ${ }^{1}$}

\author{
R. A. Weeks \\ E. Sonder
}

Through a series of irradiations with energetic particles followed by light bleaching and heat treatments of several varieties of fused silica, a one-to-one correspondence is found between an optical band at $2120 \mathrm{~A}$ and an electron spin resonance (ESR) line $g=2.0006$. A comparison is then made between the concentration of centers in this ESR line determined by an ESR comparison technique, and the total concentration is measured by a magnetic susceptibility method. The results of the comparison show that the primary paramagnetic center and the center which gives the ESR line $g=2.0006$ are the same. This center has been called the $E_{1}^{\prime}$ center. After appropriate irradiations and subsequent bleaching treatments, the concentration of spins in the $E_{1}^{\prime}$ center was measured by the susceptibility technique. The absorption coefficient and the width at half maximum absorption coefficient were found, and using Smakula's equation appropriate for a Gaussian function, the oscillator strength $f$ was found to be $f=0.14 \pm 0.04$.

\subsection{PARAMAGNETIC SPECTRA OF $E_{2}^{\prime}$ CENTERS IN CRYSTALLINE QUARTZ ${ }^{2}$}

\section{R. A. Weeks}

The electron spin resonance spectra of the $E_{2}^{\prime}$ center, a defect of the quartz structure, are in

\footnotetext{
${ }^{1}$ Abstract of paper to be published in the Proceedings of the 1st International Conference on Paramagnetic Resonance, July 16-20,1962, Jerusalem, Israel.

${ }^{2}$ Abstract of paper submitted to The Physical Review.
}

agreement with the spectra predicted by a spin Hamiltonian, $S=\frac{1}{2}$,

$$
\mathcal{H}=\sum_{i=1}^{6}\left(\vec{H} \cdot \overleftrightarrow{g_{1 i}} \cdot \overleftrightarrow{S}+\vec{H} \cdot \overleftrightarrow{g_{2 i}} \cdot \overleftrightarrow{S}\right) \text {. }
$$

The $i$ subscript refers to the symmetry operations of the crystal necessary to obtain the six components of each line observed for the magnetic field $H$ parallel to the $c$ axis of the crystal. The eigenvalues of the two $g$ tensors are:

$$
\begin{aligned}
& g_{1}\left(g_{\text {C axis }}=2.0008\right)\left[g_{\mid 1}=2.0015,\right. \\
& \left.g_{\perp}=2.0006\right] ; \\
& g_{2}\left(g_{C \text { axis }}=2.0010\right)\left[g_{1}=2.0022,\right. \\
& \left.g_{2}=2.0005, g_{3}=2.0007\right] .
\end{aligned}
$$

Hyperfine interactions are observed with nearby nuclei. On the basis of the intensity ratio of these lines to the unperturbed lines, it is suggested that the interaction is with $\mathrm{Si}^{29}, I=\frac{1}{2}$. Interaction of the defect electron with one $\mathrm{Si}^{2} 9$ produces a splitting of $A \approx 412$ gauss ( $c$ axis is parallel to the applied field). A comparison of $|\psi(0)|^{2}$ calculated from this interaction with the value of $\left|\psi_{3 S, S i^{3}} 3+(0)\right|^{2}$ indicates that the silicon atom is at the defect site. The remaining interactions are very weak; $A_{1}=1.0$ gauss, $A_{2}=0.8$ gauss, $A_{3} \approx 0.5$ gauss ( $c$ axis is parallel to the applied field). These lines are interpreted as being due to an $\mathrm{Si}^{29}$ in any one of three nearby sites. Assuming a dipole-dipole interaction, the separation of these sites from the defect is $\geqq 1.7 \mathrm{~A}$. A possible interpretation of these results in terms of plausible models is given, and a comparison is made with the magnetic characteristics of another defect of the quartz structure, the $E_{1}^{\prime}$ center. 


\subsection{ALTERNATING-AND DIRECT-CURRENT CONDUCTIVITY OF GLASSES}
R. A. Weeks
E. Lell ${ }^{3}$

Insulators which have been electrostatically charged have been observed to discharge, leaving in the insulator visible tracks along which the discharge took place. ${ }^{4}$ The electrostatic charging can be induced on the surface or in the bulk of an insulator by electron or gamma-ray irradiation. 5,6 Since these discharges have been observed in some glasses and not in others, there is some interest in the process by which charge and energy, supplied during irradiation, were stored in such insulators. Glasses with a high $\left(\sim 10^{19}\right.$ ohm $-\mathrm{cm})$ and some with a low $\left(\sim 10^{17}\right.$ ohm $\left.-\mathrm{cm}\right)$ intrinsic resistivity exhibit the discharge effect when irradiated with electrons. Since the discharge effect involves electrical processes, it seemed reasonable to investigate the electrical properties of the glasses of interest. The available data $4,7,8$ on the effect indicated that the decay time for its disappearance was quite long, and thus our interest was directed to a study of the low-frequency and dc conductivity of several glasses.

In Fig. 9.1 the loss tangent (ac conductivity) of a lead silicate glass is shown as a function of frequency and time after electron irradiation. There are two fearures of these measurements that should be noted: (1) there is a maximum in the loss tangent at some frequency below $50 \mathrm{cps}$, and (2) at least the high-frequency tail of this maximum disappears in a time of the order of $24 \mathrm{hr}$. It has also been observed that two glasses, exhibiting the discharge effect, also show the greatest increase in the loss tangent at $50 \mathrm{cps}$. Since this increase disappears in $\sim 24 \mathrm{hr}$, and the discharge effect can be observed after a time $>200 \mathrm{hr}$, the two phenomena are probably not directly related.

\footnotetext{
${ }^{3}$ Guest scientist from Bausch and Lomb, Inc., Rochester, N.Y.

${ }^{4}$ S. Whitehead, Dielectric Breakdown of Solids, p 170 , Oxford IIniversity Tress, London, 1951.

${ }^{5}$ B. Gross and P. V. Murphy, Nukleonik 2, 279 (1960).

${ }^{6}$ Cover picture of J. Appl. Pbys. 32 (1961).

${ }^{7} \mathrm{~V}$. Cullen, in Proc. Hot Lab. Equip. Conf., 7th, Cleveland, 1959.

${ }^{8} \mathrm{R}$. C. Hudson and R. A. Weeks, Solid State Div. Ann. Progr. Rept. Aug. 31, 1961, ORNL-3213, p 89.
}

The dc conductivity was measured in the lead silicate glass and in a high-purity silica. Within the range of specimen thickness and applied voltages, the observed final current was ohmic. The electrodes were silver paint.

An analysis of the decay of the current through the specimen, the polarization current, showed that it could be reasonably fitted to a function of the form

$$
i=\sum_{i=1}^{K} a_{i} e^{-t / \tau_{i}}+b i_{n},
$$

where $a_{i}$ is a constant appropriate to the characteristic time $\tau_{i}, b$ is a constant for a particular glass, and $i_{0}$ is the current observed after a time long compared with the longest $\tau_{i}$ and is presumed to be the intrinsic conductivity. In Fig. 9.2 a decay curve is shown of a lead silicate glass which had been given a $\mathrm{Co}^{60}$ gamma-ray dose of $10^{6} \mathrm{r}$. An analysis of the curve gave four characteristic times. The numbers in parentheses are the values of the $a_{i}$ 's which are the intercepts at $t=0$.

In Table 9.1 the $\tau_{i}$ 's found by this analysis are shown for two specimens subjected to gamma-ray irradiation and measured at about $25^{\circ} \mathrm{C}$ and at two higher temperatures. The average values of the

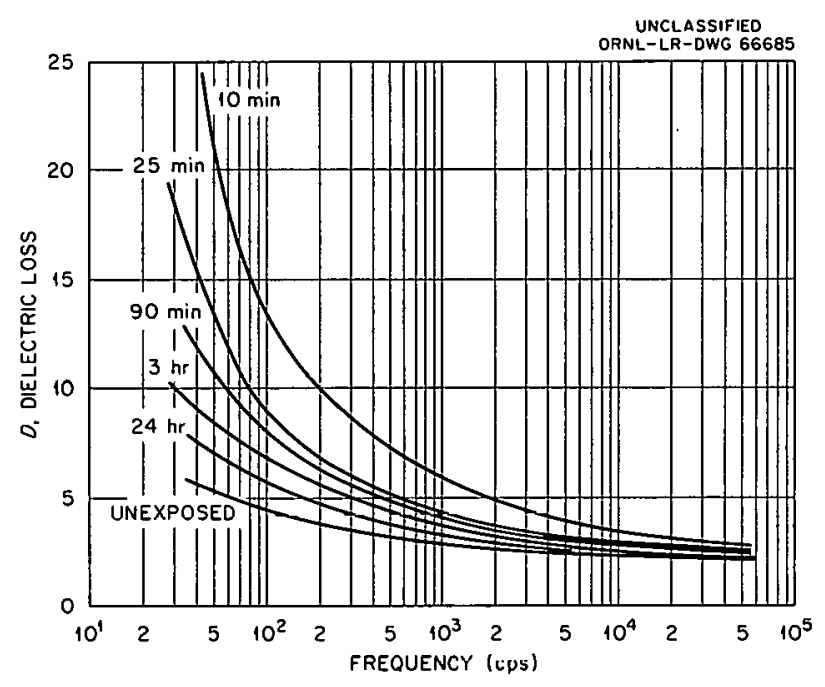

Fig. 9.1. Loss Tangent of a Lead Silicate Glass at Various Times After an Electron Iradiation with $1.5 \mu$ of 1.5-Mev Electrons for $5 \mathrm{~min}$. The temperature during irradlation was $\sim 30^{\circ} \mathrm{C}$ and during measurement was $23^{\circ} \mathrm{C}$. 


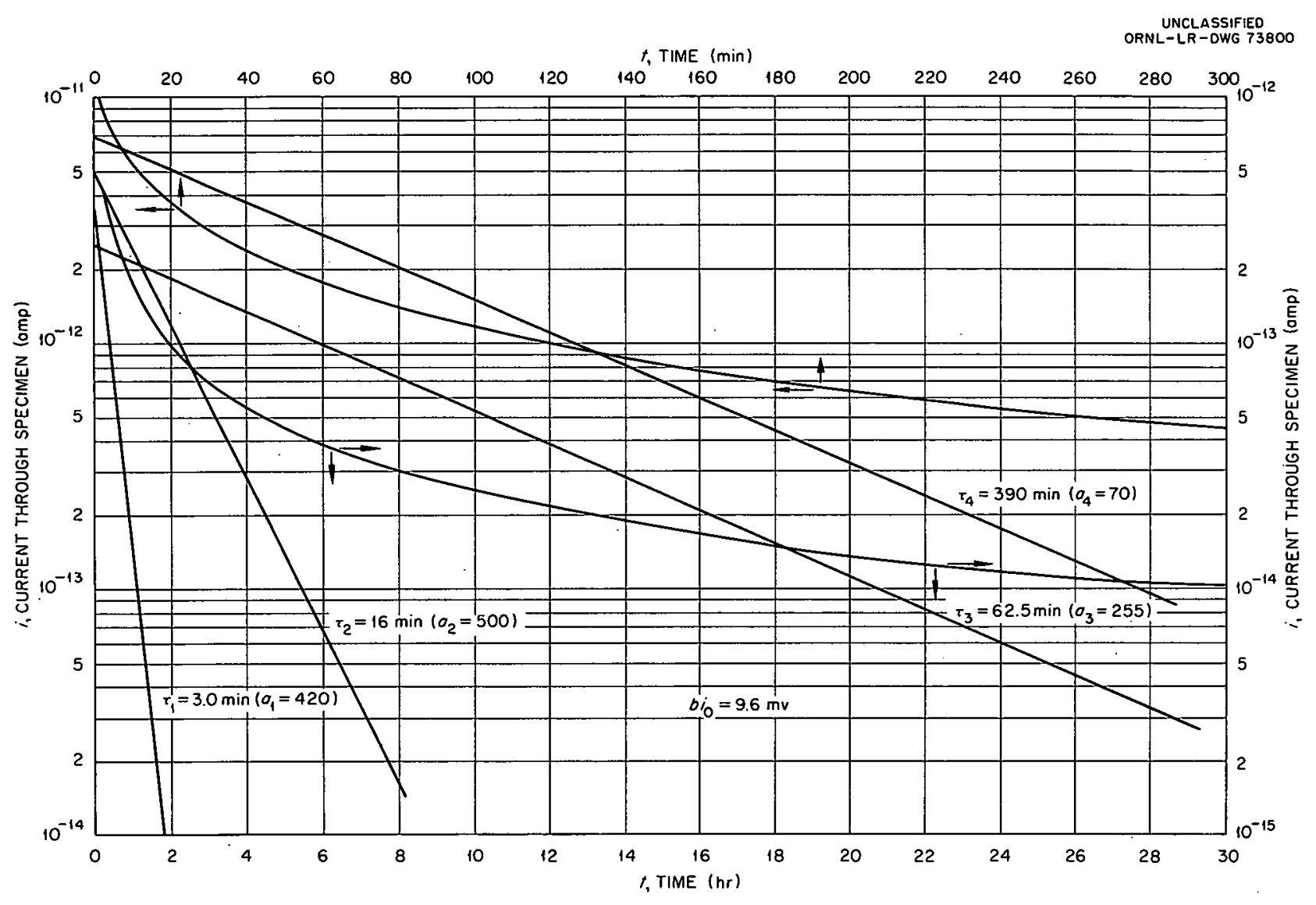

Fig. 9.2. Polarization Current Resolved into Four Processes, Each with a Characteristic Time. The specimen had been exposed to $10^{6} \mathrm{r}$ of $\mathrm{Co}^{60}$ gamma rays prior to the measurement. (The constant term bio was first subtracted, leaving the linear portion labeled os $\tau_{4}$, which wos then subtracted from the remainder, giving the linear portion labeled $\tau_{3}$, etc.)

$\tau_{i}^{\prime}$ 's are given for the unirradiated and irradiated conditions and the rms deviation calculated. At the elevated temperatures the $\tau_{i}^{\prime}$ 's exhibit a decrease that is larger than this rms deviation.

Although the $\tau_{i}$ 's showed only small changes with these treatments, the $a_{i}{ }^{\prime} s$ and the intrinsic conductivity $b i$ exhibited larger variations. These are shown in Table 9.2. Up to a dose of $10^{7} \mathrm{r}$, the $a_{i}$ 's and the $b i_{\circ}$ increased monotonically with increasing dose with the exception of $a_{1}$. The effect of elevated temperatures was to increase the $a_{i}$ 's with the exception again of $a_{1}$, for which there was $\bar{a}$ large decrease.

\section{Discussion}

Taylor has shown ${ }^{9}$ that the increase in the loss tangent at low frequencies (Fig. 9.2, curve for the unexposed case) is due to a distribution of relaxation times for sodium ion diffusion. The increase that we observed may have been due to the same mechanism. The increase in the loss tangent resulting from irradiation could be caused by a change in this distribution. However, Taylor's results showed that there was no change in the distribution with heating, and our $\tau_{i}$ 's derived from the dc measurements showed very little, if any, change with irradiation. It would be questionable on the basis of these results to relate the increase in loss tangent to a change in the distribution of relaxation times for ion diffusion. It is possible that irradiation displaces ions from deep traps and that while they are removed from the traps they can

\footnotetext{
${ }^{9}$ H. Taylor, Trans. Faraday Soc. 52, 873 (1956).
} 
Table 9.1. The DC Polarization Relaxation Times of a Lead Silicate Glass

\begin{tabular}{|c|c|c|c|c|}
\hline Treatment & $\tau_{1}$ & $\tau_{2}$ & $\tau_{3}$ & $\tau_{4}$ \\
\hline Specimen III (unexposed) & 3.1 & 13.8 & 62 & 366 \\
\hline One week & & $14.0^{a}$ & $62^{a}$ & $334^{a}$ \\
\hline Turned $^{b}$ & 3.1 & 14.5 & 62 & 336 \\
\hline $10^{5} \times \mathrm{Co}^{60}$ & 2.5 & 14.0 & 65 & 360 \\
\hline $2 \times 10^{5} \mathrm{r}$ & 2.8 & 13.8 & 68 & 375 \\
\hline $5 \times 10^{5} \mathrm{r}$ & 3.0 & 17.0 & 66 & 380 \\
\hline $1 \times 10^{6} \mathrm{r}$ & 3.0 & 15.0 & 65.5 & 375 \\
\hline \multicolumn{5}{|l|}{ Specimen IV } \\
\hline $5 \times 10^{5} \times \mathrm{Co}^{60}$ & 3.0 & 16.5 & 64 & 395 \\
\hline $1 \times 10^{6} \mathrm{r}$ & 3.0 & 16.0 & 62.5 & 390 \\
\hline Average value & $2.94 \pm 0.20^{c}$ & $15.0 \pm 1.4^{c}$ & $64.2 \pm 2.0^{c}$ & $368 \pm 20^{c}$ \\
\hline \multicolumn{5}{|l|}{ Specimen IV (unexposed) } \\
\hline $43^{\circ} \mathrm{C}$ & 2.3 & 12.2 & 57.5 & 345 \\
\hline $68^{\circ} \mathrm{C}$ & 2.2 & 12.2 & 51.0 & 330 \\
\hline
\end{tabular}

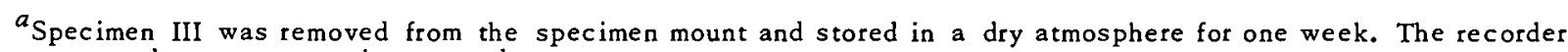
was not turned on soon enough to record $\tau_{1}$.

${ }^{b}$ Specimen III was removed and then reinserted in about $10 \mathrm{~min}$, reversed with respect to the applied voltage.

${ }^{c}$ The error is the rms deviation from the average value.

contribute to the ion diffusion and are observed as an increase in the loss tangent. Over a period of time thcse ions would be recrapped, thereby producing the observed decay in the irradiationenhanced loss tangent. Since the irradiation can also generate electrons and holes, these can alter the ionization states of such traps, and a fraction of the ions released by the irradiation would not be retrapped. These ions would then produce the enhancement of the intrinsic conductivity observed (Table 9.2).

The hypothesis that the time-dependent decay of the current through the specimen can be expressed as a sum of exponentials in time is based on the following facts:

1. The principle of superposition is obeyed. The observed decay curves can be resolved into linear sections which extend over two decades of current. The $\tau_{i}$ 's for several measurements under a variety of conditions have an rms deviation that is less than $\pm 9 \%$ for $\tau_{2}$ and less than $\pm 6 \%$ for the other three $\tau$ 's. These rms deviations indicate a reasonable agreement between the hypothesis of superposition and the observed curve. Other functions have not been analyzed, and it is possible that with four adjustable parameters a different function could be found which would fit the observed curve.

2. Ohm's law is applicable for the conditions of our experiment.

3. The final conductivity over a limited range of temperature obeys an Arrhenius equation but deviates at higher temperatures. This deviation could result from two independent processes.

4. The polarization currents found in other experiments on glasses 9,10 and in other materials ${ }^{11,12}$ exhibit an exponential time depend-

\footnotetext{
${ }^{10}$ R. J. Charles, J. Appl. Pbys. 32, 1115 (1961).

${ }^{11}$ R. W. Dreyfus, Pbys. Rev. 121, 1675 (1961).

$12 \mathrm{~J}$. R. Freeman et al., Rev. Mod. Pbys. 33, 553 (196i).
} 
Table 9.2. Values ${ }^{a}$ of the Intercepts of Each of the Lineor Portions of the DC Polarization Curves of a Lead Silicate Glass

\begin{tabular}{|c|c|c|c|c|c|}
\hline Treatment & $\tau_{1}$ & $\tau_{2}$ & $\tau_{3}$ & $\tau_{4}$ & $b i_{0}$ \\
\hline Specimen III & 260 & 51 & 25 & 5.8 & 1.6 \\
\hline One week & & $61^{b}$ & $18.8^{b}$ & $5.6^{b}$ & $1.4^{b}$ \\
\hline Turned $^{c}$ & 270 & 109 & 30.3 & 11.0 & 2.0 \\
\hline $10^{5} \times \mathrm{Co}^{60}$ & 22 & 195 & 111 & 40 & 5.0 \\
\hline $2 \times 10^{5} \mathrm{r}$ & 220 & 253 & 123 & 38 & 7.0 \\
\hline $5 \times 10^{5} \mathrm{r}$ & 450 & 345 & 210 & 59 & 8.0 \\
\hline $1 \times 10^{6} \mathrm{r}$ & 315 & 355 & 230 & 74 & 10.0 \\
\hline Specimen IV ${ }^{d}$ & & & & & 1.4 \\
\hline $5 \times 10^{5} \mathrm{r}$ & 570 & 420 & 220 & 53 & 11.0 \\
\hline $1 \times 10^{6} \mathrm{r}$ & 420 & 405 & 255 & 70 & 10.0 \\
\hline \multicolumn{6}{|l|}{ Specimen II } \\
\hline $1 \times 10^{7}=$ & 750 & 880 & 410 & 180 & 30 \\
\hline $1 \times 10^{8} \mathrm{r}$ & 2500 & 500 & 265 & 175 & 49 \\
\hline \multicolumn{6}{|l|}{ Specimen III } \\
\hline $43^{\circ}$ & 390 & 150 & 45 & 14 & 3.5 \\
\hline $68^{\circ}$ & 92 & 250 & 61 & 25 & 10.0 \\
\hline
\end{tabular}

${ }^{a}$ The numbers given in the table are the voltages in millivolts measured across the series resistor of the vibrating reed electrometer. Its value was $10^{12} \mathrm{ohms.}$

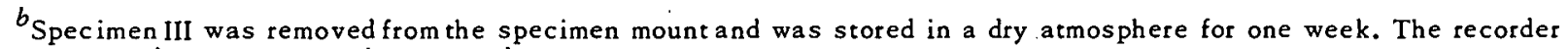
was not turned on soon enough to record $\tau_{1}$.

${ }^{c}$ Specimen III was removed and then reinserted in about $10 \mathrm{~min}$, reversed with respect to the applied voltage.

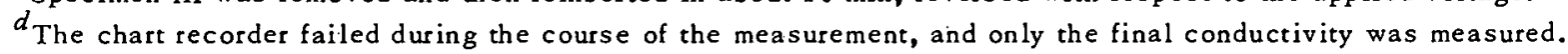

ence... The.fundamental assumption in these experiments is that there is a periodic array of porential wells in which charge carriers are trapped. In the absence of a field, these charge carriers and the associated charges of the structure creating the potential wells have a net dipole moment of zero. The application of an electric field changes the relative depth of the wells along the field direction, and these are preferentially filled by charge-carrier diffusion. The intrinsic conductivity of the glasses is due to such a process. $9,11,13$ Another process

${ }^{13}$ R. J. Maurer, Phys. Rev. 59, 691 (1941). closely related to this one and which exhibits an exponential dependence on time, is the reorientation of dipoles already present in the system. In this case neither charge component of the dipole diffuses away from the other, and there are several orientations possible, with each separated by a potential barrier. The $\tau$ 's that we have found may be the relaxation times of such a process.

One apparent contradiction between the experimental results and this hypothesis is that a thermal activation energy of $\sim_{1}$ ev should be observed. In the temperature range over which measurements have been made (Table 9.1), the $\tau$ 's appear to be temperature independent as 
compared with the intrinsic conductivity. The polarization process characterized by the $\tau$ 's may not be thermally activated in the temperature range which we have investigated.

\section{Summary}

The data do not support a space-charge hypothesis. There is agreement with the hypothesis that the polarization effects occur in the bulk of the glass.

The bulk polarization processes which have long, approximately temperature-independent relaxation times may furnish a mechanism by which charge and energy are stored. There does appear to be a correlation between large values of the $\tau^{\prime}$ 's and the discharge effect. 


\section{Superconductivity}

\subsection{DEPENDENCE OF SUPERCONDUCTING CRITICAL CURRENT OF Nb-Zr ALLOYS ON MAGNETIC-FIELD ORIENTATION}

\author{
S. T. Sekula \\ R. W. Boom ${ }^{1}$ \\ C. J. Bergeron ${ }^{2}$
}

There is a growing class of superconducting materials which exhibits zero resistance in magnetic fields much higher than the thermodynamic critical magnetic field. Such behavior is attributed to a "sponge" of fine filaments whose diameter is of the same order or less than the superconducting penetration depth $\left(\sim 10^{-6} \mathrm{~cm}\right) .^{3}$ These high-field filaments are variously associated with dislocations, highly strained regions, impurities, and other extended imperfections. This indeed is borne out to some extent by the observation that cold working enhances the critical current at high fields.

In a study of the critical current of cold-worked $\mathrm{Nb}-\mathrm{Z}_{\mathrm{r}}$ alloys of varying composition in a magnetic field, ${ }^{4}$ we have investigated the materials in both externally applied transverse and longitudinal fields in order to gain some insight as to the properties of these threads. Figure 10.1 shows data obtained on $\mathrm{Nb}-33 \% \mathrm{Zr}$ wires in a coldworked state and after a heat treatment in a hard vacuum, and is characteristic of a series of $\mathrm{Nb}-\mathrm{Zr}$ alloys. The critical-current curve of the coldworked material in the transverse configuration agrees quite well with other published data. The critical current of the longitudinal sample was

\footnotetext{
${ }^{1}$ Electronuclear Division.

${ }^{2}$ Consultant to Electronuclear Division from Louisiana State University, Baton Rouge.

${ }^{3}$ J. E. Kunzler, J. Appl. Phys. 33, 1042 (1962).

${ }^{4}$ Electronuclear Div. Ann. Progr. Rept. Dec. 31, 1961, ORNL-3257, p 46.
}

observed to increase with increasing field, and a maximum was found at 30 kilogauss. Heat treatment of this material at $800^{\circ} \mathrm{C}$ increased the critical current at low fields by a considerable amount in both configurations. In addition, the shape of the critical-current curves was greatly altered. X-ray analysis of cold-worked and heattreated material revealed no change in the preferred orientation of the wire. Metallographic

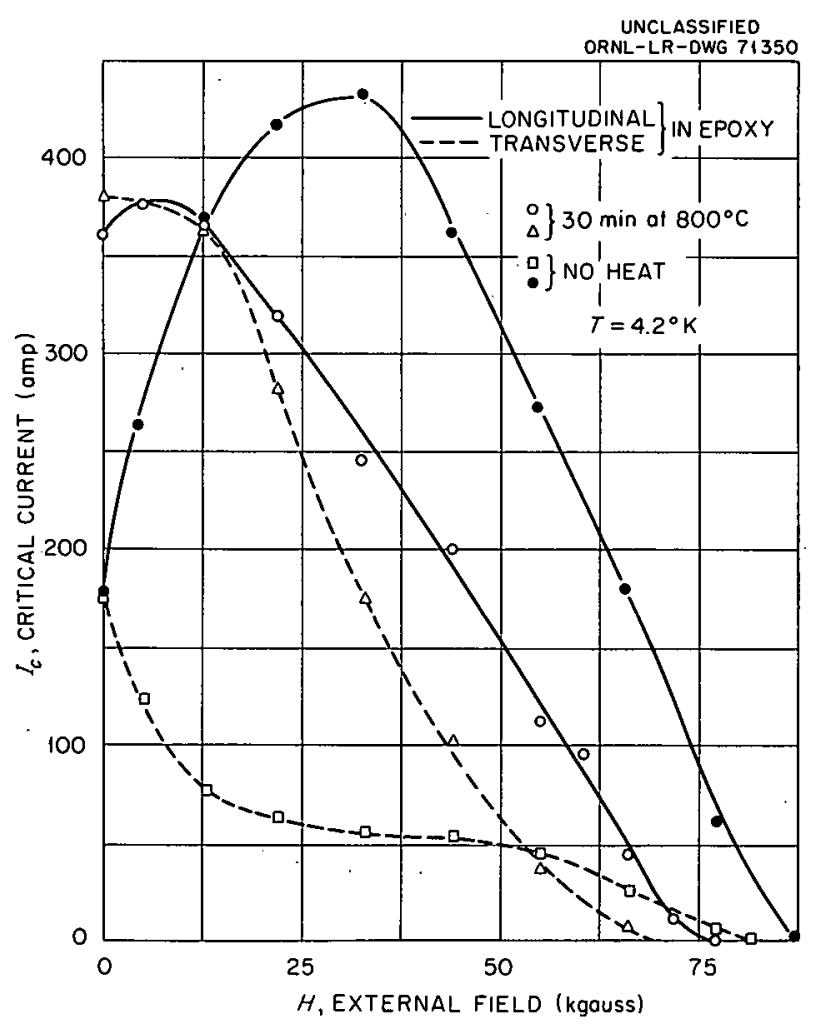

Fig. 10.1. Orientation Dependence of the Critical Current of $\mathrm{Nb}-33 \% \mathrm{Zr}$ Wires in a Magnetic Field. Data are shown for cold-worked and heat-treated material. 
examination ${ }^{5}$ of the cold-worked material showed that appreciable macro- and microsegregation existed across a diameter and a long a longitudinal section of the wire. Similar examination of the material heat-treated at $800^{\circ} \mathrm{C}$ for $30 \mathrm{~min}$ indicated that the microstructures were quite similar to those of "the cold-worked state, with the addition of a considerable amount of a precipitate, too fine to resolve optically, of unknown structure and composition. By inference from other studies of alloys of similar composition and structure, the heat treatment also resulted in a softening of the wire, indicating that recovery had occurred. There was no evidence that the material had recrystallized.

Similar peaking effects have been ubserved in highly oriented cold-rolled sheets of $\mathrm{Nb}-12 \% \mathrm{Zr}$ (ref 6) and $\mathrm{Nb}$ (ref 7) examined in a transverse field. It is interesting to note that critical-current dependence on magnetic field determined experimentally is not in agreement witheither the London or Pippard model of current-carrying filaments in magnetic fields. ${ }^{8}$

\subsection{SUPERCONDUCTING TRANSITION TEMPERATURE OF PURIFIED TECHNETIUM ${ }^{9}$}
M. L. Picklesimer ${ }^{10}$
S. T. Sekula

The superconducting transition temperature of technetium has been reported as $11.2^{\circ} \mathrm{K}$ for powder ${ }^{11}$ of greater than $99.9 \%$ purity and as $9.3^{\circ} \mathrm{K}$ for arc-melted material ${ }^{12}$ made from metal chips contaminated with oxide.

We found that hydrogen-reduced technetium powder presently available contains appreciable quantities of oxygen, both dissolved and as an

\footnotetext{
${ }^{5}$ Work done by M. L. Picklesimer, Metals and Ceramics Division.

${ }^{6}$ Hake, Berlincourt, and Leslie, IBM. J. Res. Develop. 6, 119 (1962).

${ }^{7}$ Leblanc and Little, p 362 in Proceedings of the $7 t h$ International Conference on Low Temperature Physics, University of Toronto Press, 1960.

${ }^{8}$ J. G. Daunt et al., Cryogenics 2, 212 (1962).

${ }^{9}$ Submitted for publication in Physical Review Letters.

${ }^{10}$ Metals and Ceramics Division.

$11 \mathrm{~J}$. G. Daunt and J. W. Cobble, Phys. Rev. 92, 507 (1953).

${ }^{12}$ V. B. Compton et al., Pbys. Reu. 123, 1567 (1961).
}

unreduced oxide. Microstructural examination of powder particles revealed that each particle was porous, like a sponge, with the dissolved and/or entrapped and unreduced oxide existing as a center between two surface layers of reduced metal.

Five-gram pressed powder pellets were inductionmelted under a vacuum of 1 to $5 \times 10^{-6}$ torr several times until no further evolution of vapor was detected. The resulting buttons were coldrolled to sheet approximately $1 / 16$ in. thick. Spectrographic analysis of this material indicated a purity of greater than $99.995 \%$ for metallic elements, the only impurity detected being 40 Ppm Na. The oxygen content was not determined.

A specimen approximately $1 / 16 \times 1 / 16 \times \frac{5}{8}$ in. was cut from the cold-rolled sheet, four platinum leads were spot-welded on, and the specimen and a portion of the leads were encased in cellulose acetate by dipping. The superconducting transition temperature in zero magnetic field was found to be $8.22 \pm 0.01^{\circ} \mathrm{K}$ by a dc resistance measurement using a current of $0.100 \mathrm{amp}$ and a voltage sensitivity of $1 \times 10^{-8} \mathrm{v}$.

The decreasing transition temperature of technetium with apparently increasing purity can be rationalized if it is postulated that dissolved oxygen raises the transition temperature. Thus it is likely that the superconducting transition temperature for pure technetium is not greater than $8.22^{\circ} \mathrm{K}$. Assurance that the correct value is not lower will have to await the development of analytical techniques for determining the oxygen content of technetium.

\subsection{MECHANICAL PROPERTIES OF Nb-25\% Zr WIRE AT LOW TEMPERATURES}
S. T. Sekula
J. K. Redman

Since the use of superconductors in solenoids is feasible for the generation of high magnetic fields, it is understandably necessary to know the tensile properties of these materials at low temperatures. In any serious design of a solenoid, the stresses that arise due to the interaction of magnetic field and current must be considered. The material investigated was 0.010-in.-diam $\mathrm{Nb}-25 \% \mathrm{Zr}$ wire, commercially made by the Wah Chang Corporation, and given to us by R. W. Boom and L. D. Roberts. 
Tensile tests were made on an Instron testing machine which applies a constant strain rate to the sample with simultaneous recording of the tension on the sample. Modified pin vises were used to grip the wires. Provisions were made to immerse the samples in either liquid nitrogen or liquid helium during the test. At a given test temperature, samples of various lengths were examined in order to eliminate errors in the modulus of elasticity due to strains in the system other than the sample.

The results at three temperatures are shown in Fig. 10.2. Since the statistics are meager, no significance as yet can be given to the decrease in the modulus in going from room temperature to liquid-nitrogen temperature. At room temperature the load at fracture was found to be $20.6 \pm 0.5 \mathrm{lb}$, while in liquid nitrogen the load was $25.7 \pm 1.0 \mathrm{lb}$. At liquid-helium temperature the scatter in the load at fracture was even greater, the value in this case being $29.0 \pm 2.0 \mathrm{lb}$. The moduli of elasticity for the three temperatures are given in the figure.

\subsection{NEUTRON IRRADIATION OF SUPERCONDUCTING TIN}
S. T. Sekula
C. E. Klabunde

Studies of the effect of radiation damage in superconductors at low temperatures $\left(4^{\circ} \mathrm{K}\right)$ have been limited to observations of the change in superconducting transition temperatures. ${ }^{13}$ Blanc et al. ${ }^{14}$ recently studied the magnetization curves

\footnotetext{
${ }^{13}$ Rinderer and Schmid, $\mathrm{p} 395$ in Proceedings of the $7 t h$ International Conference on Low Temperature Physics, University of Toronto, 1960.

${ }^{14}$ Blanc et al., $\mathrm{P} 393$ in Proceedings of the 7 th International Conference on Low Temperature Physics, University of Toronto, 1960.
}

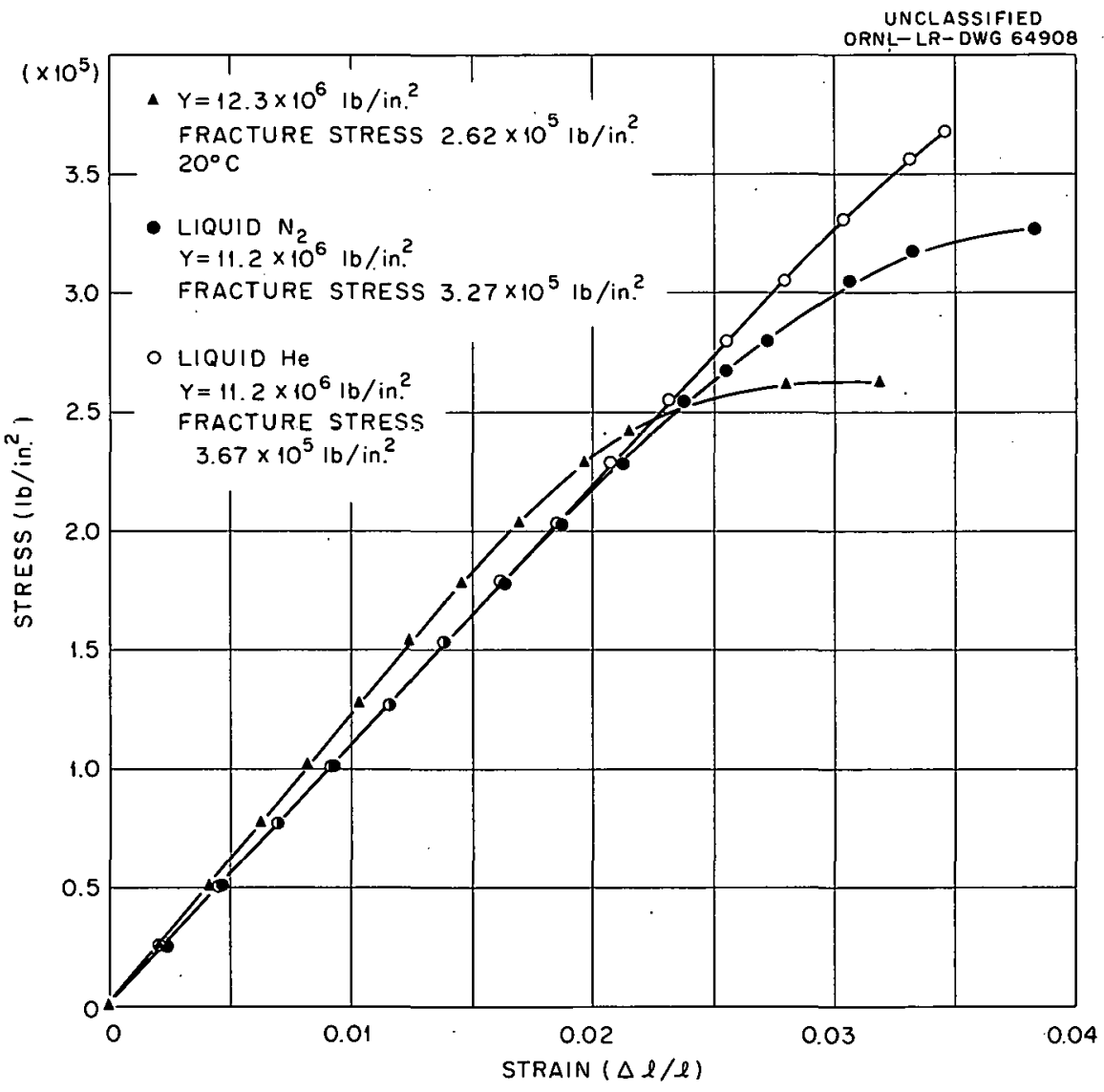

Fig. 10.2. Stress.-Strain Curves of 0.010 -in.-diam $\mathrm{Nb}-25 \%$. $\mathrm{Zr}$ Wires at 300,77 , and $4.2^{\circ} \mathrm{K}$. 
of tin and rhenium subsequent to neutron irradiation at $77^{\circ} \mathrm{K}$. However, since radiation-induced defects are quite mobile at $77^{\circ} \mathrm{K}$, it is desirable to irradiate and to examine the materials at low temperature and to anneal the material in a controllable fashion. With the introduction of point defects by low-temperature irradiation, one might expect a change in the superconducting transition temperature. ${ }^{13}$ Annealing at higher temperatures may result in a precipitation of point defects as extended imperfections, for example, dislocation loops, which would then be manifest in either a hysteresis in the magnetic transition ${ }^{14}$ or, as Shaw and Mapother ${ }^{15}$ have demonstrated in coldworked materials, a broadening of the superconducting-to-normal resistive transition.

As a pre liminary experiment the superconductingto-normal resistive transition of a pure tin wire as a function of current passing through the wire was examined at several tempcratures. Figure 10.3 shows the results obtaincd at $3.60^{\circ} \mathrm{K}$. These results are in good agreement with the London model of the intermediate state, which predicts

$$
\frac{\mathrm{R}}{\mathrm{R}_{n}}=\frac{1}{2}\left(1+\sqrt{1-\frac{\left(I_{c}\right)^{2}}{l}}\right), \quad I \geqq I_{c},
$$

where $K_{n}$ is the normal residual resistance, and $I_{c}$ is related to the radius of the wire $r$ and the thermodynamic critical field $H_{r}$ at a given temperature by the relation $H_{c}=2 l_{c} / r_{0}$. The superconducting-to-normal transition is characterized by a sharp rise in resistance at $I=I_{c}$ and an asymptotic increase with further increments of current.

A similar sample of tin was placed in the Hole 12 low-temperature facility for an exposure of one week, corresponding to a neutron dose of $5 \times 10^{17}$ neutrons $/ \mathrm{cm}^{2}$. Examination of the resistive transition in the reactor prior to irradiation revealed that the transition was smcared

\footnotetext{
${ }^{15}$ Shaw and Mapother, Pbys. Rev. 118, 1474 (1960).
}

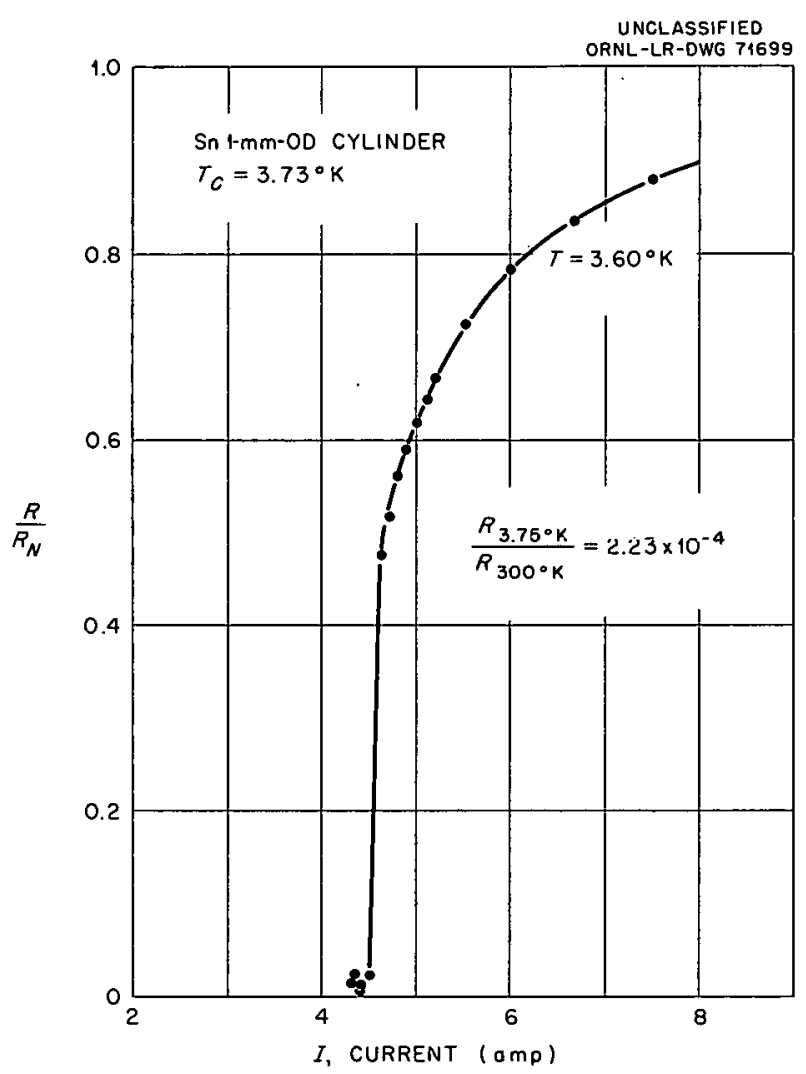

Fig. 10.3. Superconducting-to-Normal Transition of Tin. of $3.60^{\circ} \mathrm{K}$.

out. This is due to small uncontrollable temperature gradients caused by local gamma-ray heating. In addition, the vapor pressure thermometry was found to be inadequate for observing the small changes in the transition temperature one would expect after irradiation. No change was observed in the resistive transition after the irradiation or after subsequent 3 -min anneals at 25,70 , and $300^{\circ} \mathrm{K}$. Thus, within the limitations of the techniques, there was no effect of neutron irradiation on the superconducting tran sition of $t$ in.

Blanc et al. ${ }^{14}$ report a small decrease in the transition temperature and a small increase in the frozen-in moment in a tin sample after a neutron dose of $10^{18}$ neutrons $/ \mathrm{cm}^{2}$ at $77^{\circ} \mathrm{K}$. On the other hand, Shaw and Mapother ${ }^{15}$ found no significant change in the magnetic transition of tin severely cold worked at $4.2^{\circ} \mathrm{K}$. We hope to improve the techniques and extend the study to other materials. 


\subsection{INTERACTION BETWEEN IRRADIATION- INDUCED DEFECTS AND MAGNETIC STRUCTURE ${ }^{16}$}
A. I. Schindler ${ }^{17}$
J. Weertman ${ }^{17}$
R. H. Kemohan
P. G. Huray ${ }^{18}$
W. L. Haman 19

Hysteresis loop measurements have been conducted on sevéral commercial iron-nickel alloys

\footnotetext{
${ }^{16}$ To be submitted for publication in "Crystal Lattice Defect Supplement," Journal of the Physical Society of Japan.

${ }^{17}$ U.S. Naval Research Laboratory, Washington, D. C.

${ }^{18}$ Co-op student from the University of Tennessee, Knoxville.

${ }^{19}$ Co-op student from the Virginia Polytechnic Institute, Blacksburg.
}

during neutron irradiations at $90^{\circ} \mathrm{K}$ and at ambient reactor temperature in both zero and saturating magnetic field. Isochronal annealing tests were also performed on the alloys after irradiation. The low-temperature irradiation results indicated that magnetic properties are affected not by the point defects themselves but by their motion and eventual disposition. The radiation-produced changes appeared similar to those observed in these alloys upon magnetic annealing, that is, short-range atomic ordering. Further irradiation experiments on the alloys at temperatures from 70 to $175^{\circ} \mathrm{C}$ in a saturating magnetic field showed that the activation energy of the radiation-induced process was $0.3 \mathrm{ev}$. However, in all cases the radiation-induced changes were annealed out at temperatures below the usual ordering temperature. This recovery process appeared to be dependent upon the state of magnetization. 
's

Part III. Metals

F. W. Young, Jr. 
THIS PAGE

WAS INTENTIONALLY

LEFT BLANK 


\section{Investigations of Metal Surfaces}
F. W. Young, Jr.
U. Bertocci
L. D. Hulett
L. H. Jenkins
J. R. Savage

Electrochemical techniques offer a reasonable method of studying the reactivity of metal surfaces, since experimental conditions can be so adjusted that data can be obtained from systems in which the magnitude of either the current flowing or the potential measured at the electrode can be controlled. The electrical parameters observed for electrodes can, under certain conditions, be related to resultant changes in electrode surface structures, which are observed by optical and electron microscopy. While both crystal growth and dissolution may be examined profitably by such techniques, the emphasis in this work has been on the study of anodic processes under conditions of constant current.

Two possible conditions for the study of anodic prucesses are: (1) dissolution into solutions containing ions of the electrode metal, and (2) dissolution into solutions which initially contain few, if any, ions of the metal. Near equilibrium conditions, electrode potentials measured under condition (1) may be correlated sometimes with the energy required to remove material from sites related to the crystallographic orientation and/or the defect structure of the electrode surface. In case (2) the definition of an equilibrium condition with respect to oxidation of the metal is difficult and is of little practical interest. Also, concentration gradients at the electrode-solution interface play a predominant role in determining the emf values measured and the distribution of current densities over the metal surface. Since it is possible that dissolution mechanisms operative at the electrode surface may be affected by changes in such concentration gradients, systems of this type are also of interest.

\subsection{ANODIC DISSOLUTION OF COPPER IN SOLUTIONS CONTAINING COPPER IONS}

Studies of the galvanostatic dissolution of copper single crystals in oxygen-free $0.2 \mathrm{M} \mathrm{CuSO}_{4}$ ( $\mathrm{pH}$ adjusted to $\sim 1$ with $\mathrm{H}_{2} \mathrm{SO}_{4}$ ) have been continued. Observations on (100) oriented surfaces have been reported previously, ${ }^{1}$ and data are now being gathered on surfaces oriented (110) and (111). The latter orientations exhibit relationships of current vs potential similar to those observed for the (100) surface. However, it has not yet been possible to demonstrate that discontinuities in the curves resulting from a plot of overpotential vs current density are related to the defect structure of the electrode in the case of (110) and (111) orientations, as was shown for (100).

It has been observed that without application of an external emf, (110) and.(111) oriented surfaces, unlike the (100), develop a faceted structure within $24 \mathrm{hr}$ after exposure to the copper(II) sulfate solutions. Also, it has been demonstrated that the electrodes do not change weight under such conditions. The faceting must then result from the transfer of material from one area of the electrode to another; and, therefore, anodic and cathodic areas are localized over the electrode surface. It is reasonable to assume that such behavior prevents the correlation between measurable electrical parameters and the surface defect structure similar to that observed for (100) surfaces.

\footnotetext{
${ }^{1}$ L. H. Jcnkins and J. O. Stiegler, J. Electrochem. Soc. 109, 467 (1962).
} 
Current studies are directed to determining the conditions which control the development of facets on electrodes at chemical equilibrium, as well as establishing the experimental conditions necessary to demonstrate a relationship between electrical parameters and the defect structure of (110) and (111) surfaces in these solutions.

\subsection{ANODIC DISSOLUTION OF COPPER IN COPPER-FREE SOLUTIONS}

In an effort to determine the parameters affecting the formation of etch pits at dislocations, anodic dissolution experiments, by means of an external emf, have been carried out mostly on the (111) surface of copper single crystals.

It seems that in every case where etch pits are formed at dislocations, copper is dissolved in its univalent form. Thus, dislocation pits were observed in solutions of $\mathrm{HCl}, \mathrm{HCl}+\mathrm{HBr}, \mathrm{HBr}$, $\mathrm{HCl}+\mathrm{HI}$, and $\mathrm{NH}_{4} \mathrm{OH}+\mathrm{NH}_{4} \mathrm{Br}$. On the other hand, pits did not develop at dislocations in solutions of $\mathrm{HClO}_{4}, \mathrm{H}_{2} \mathrm{SO}_{4}$, and $\mathrm{CH}_{3} \mathrm{COOH}$, in which copper is anodically oxidized to the divalent state. No clear-cut results have been obtained for $\mathrm{KCN}$ or $\mathrm{NH}_{4} \mathrm{OH}$ solutions, although copper is oxidized to the univalent state and there is a tendency to form pits which apparently do not correspond to dislocations.

\section{Current Density and Concentration of Solutions}

The effect of current density upon dislocation pit formation has been investigated. For $\mathrm{HCl}$ and $\mathrm{HBr}$ solutions and their mixtures, the lowest current-density limit is set by the experimental method, since at current densities less than 0.1 $\mathrm{ma} / \mathrm{cm}^{2}$ the presence of oxygen in solutions makes the results unreliable. The concentration of components is also an important parameter; in $\mathrm{HCl}$ solutions the current-density range within which pitting is observed is rather narrow, but the addition of small quantities of $\mathrm{HBr}$ or $\mathrm{HI}$ extends this range considerably. A concentration of 0.1 mole of $\mathrm{HBr}$ per liter is sufficient to raise the upper limit for pitting from 5 to $80 \mathrm{ma} / \mathrm{cm}^{2}$. In the case of $\mathrm{HI}$ a concentration of $3 \times 10^{-4}$ mole/liter allows pitting up to about $30 \mathrm{ma} / \mathrm{cm}^{2}$. The presence of copper ions in $\mathrm{HCl}$ solutions, previously equilibrated with copper metal, has an adverse influence on pit formation at dislocations. A concentration of 0.05 mole of copper per liter is enough to prevent pit formation. However, if $\mathrm{HBr}$ is added to such solutions, pitting is again observed.

A lower limit of current density at which pits developed has been observed in solutions containing $0.3 \mathrm{M} \mathrm{NH}_{4} \mathrm{Br}$ in 1.5 to $5 \mathrm{M} \mathrm{NH} \mathrm{NH}_{4} \mathrm{OH}$. Dislocation pits are formed here only if the current density is more than 30 to $40 \mathrm{ma} / \mathrm{cm}^{2}$. This limit seems to shift toward higher values as the $\mathrm{NH}_{4} \mathrm{OH}$ concentration is increased.

In $\mathrm{HCl}$ solutions an upper limit of 5 to $6 \mathrm{ma} / \mathrm{cm}^{2}$ has been observed which seems to be independent of the $\mathrm{HCl}$ concentration in the range tested, 2 to $6 \mathrm{M}$. For $\mathrm{HBr}$ and $\mathrm{HCl}-\mathrm{HBr}$ mixtures, where $\mathrm{HBr}$ is not less than $0.1 \mathrm{M}$, the upper limit for pitting at dislocations is affected by stirring, by time, and by the precipitation of salt on the electrode; it is therefore dependent on concentration of the acids, as it affects the solubility of the cuprous salt. Current densities up to 150 $\mathrm{ma} / \mathrm{cm}^{2}$ could be used in the most concentrated solutions. The current density also affects the shape of the pits developed in such solutions. Generally, low current densities produce quasihexagonal pits as shown in Fig. 11.1a, while high current densities result in almost perfect triangles like that in Fig. 11.1b.

\section{Orientation Effects}

In the $\mathrm{HCl}-\mathrm{HBr}$ system the effect of bromide concentration and current density on the angular spread of the pitted area about the (111) pole on spherical crystals has been examined. The solutions investigated most thoroughly were $6 \mathrm{M}$ in $\mathrm{HCl}$, with bromide concentrations varying from 0.1 to $0.5 \mathrm{M}$. In such solutions, when current density increases, a maximum occurs in the angular spread of the pitted area. As bromide ion concentration is increased from $0.1 M$ to $0.5 \mathrm{M}$, the maximum in angular spread increases over the range 80 to $110 \mathrm{ma} / \mathrm{cm}^{2}$.

For current densities below the maximum, the ratio of the depth of an individual pit to its width likewise increases with current density. Also, the crystallography of the pits is independent of the orientation of the surface. A deviation of the surface from the (111) changes the section cut in the surface by the pyramid bounding the 

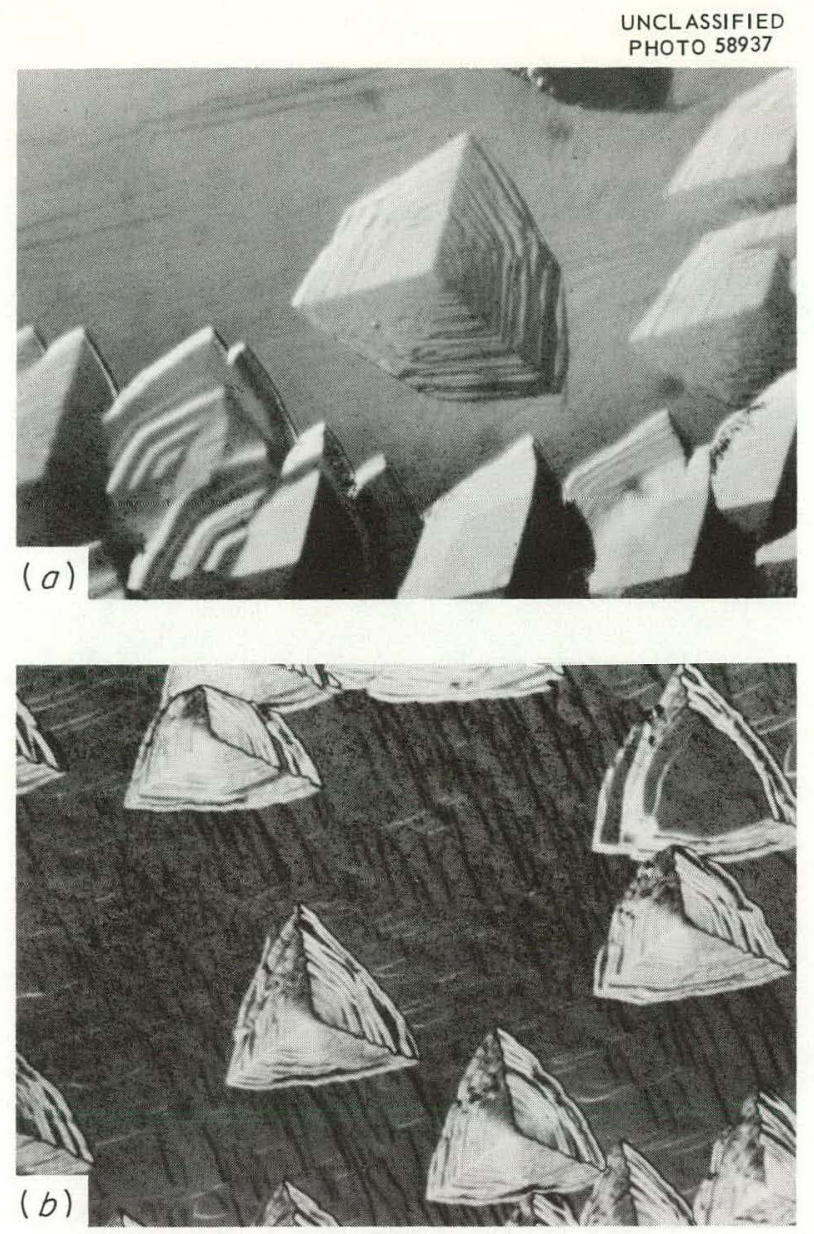

Fig. 11.1. Effect of Current Density on the Shape of Pits Developed in Acid Halide Solutions. (a) $5 \mathrm{ma} / \mathrm{cm}^{2}$. $550 X$. (b) $60 \mathrm{ma} / \mathrm{cm}^{2}$. $400 X$. Reduced $17 \%$.

pit. This effect is shown in Fig. 11.2, which is a diagram of the pitted area around a (111) pole. The symmetrical triangle in the center of the drawing represents the shape of a pit produced on perfectly oriented (111) surfaces. The distorted triangles represent the typical shapes of pits produced on surfaces slightly misoriented from the (111). A misoriented surface will have observable pits only if the sides of the pits are inclined to the (111) at angles greater than the angle of misorientaliun. Thus, an increase in current density by increasing the average depth/width ratio of the etch pits will increase the allowable angular spread of the pitted area.

In addition to experiments on the (111), some work has been done on (100) and (110) surfaces.

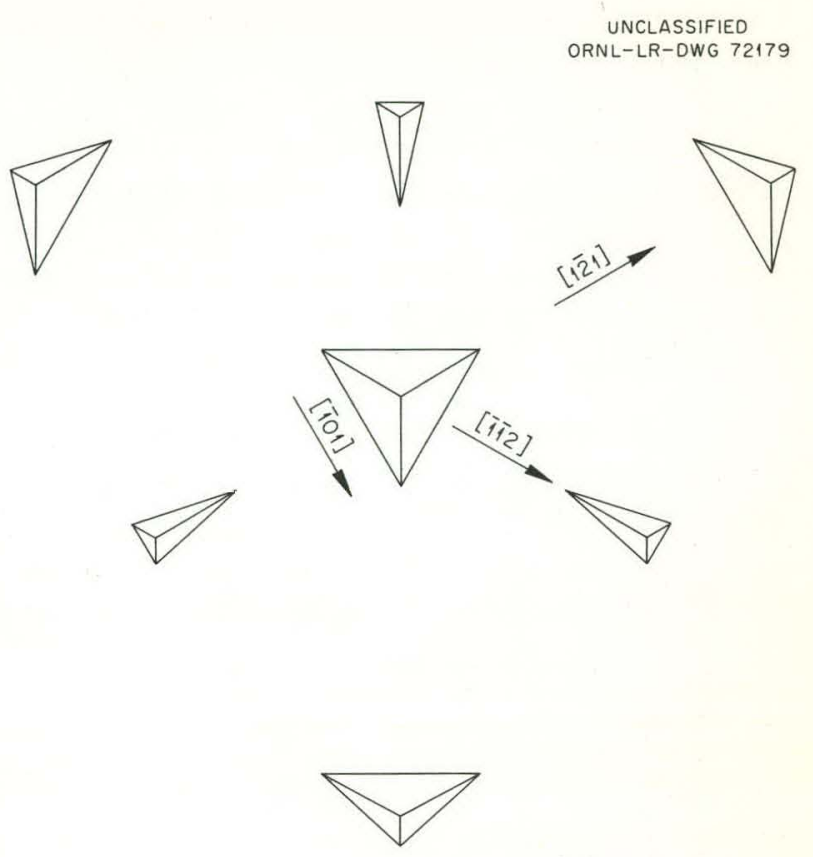

Fig. 11.2. Geometry of Pits as Affected by Slight Misorientations from the (111) Orientation.

Etch pits at dislocations can be developed by anodic attack on these surfaces also. Dissolution of (100) surfaces in an acid chloride-bromide solution produces etch pits at dislocations. However, the pit pattern has a large amount of random faceting that makes interpretation difficult. Subgrain boundaries are clearly delineated as rows of square pits, but isolated pits are sometimes difficult to distinguish from the surrounding square facets. Subgrain boundaries on the (110) can be displayed by anodic etching at low current densities, approximately $2 \mathrm{ma} / \mathrm{cm}^{2}$ in $\mathrm{HCl}$ solutions.

\subsection{KINETICS OF ETCH-PIT GROWTH AND ASSOCIATED PHENOMENA ON (111) COPPER SURFACES IN ACID HALIDES}

It has been determined that etch pits can readily be tormed at dislocation-surface intersections on the (111) face of copper by making the crystal the anode in an electrolytic cell, with various halide solutions as the electrolyte. The kinetics of the growth of the etch pits, and associated phenomena on the surface, is being studied by 
performing galvanostatic etching experiments under the optical microscope and by using time-lapse photomicrography. An example of the type of information obtainable by this technique is shown in Fig. 11.3, which is a sequence of photomicrographs taken at 10 -sec intervals of a (111) copper surface being electrochemically etched at a current density of $20 \mathrm{ma} / \mathrm{cm}^{2}$ in a $6 \mathrm{M} \mathrm{HCl}, 0.25 \mathrm{M}$ $\mathrm{HBr}$ solution. In the accompanying graph, Fig. 11.4, two dimensions of the etch pit, $X$ and $Z$ (the position of a ledge inside the pit and the position of ledges on the surface), are plotted as a function of time. The center of the pit is used as the locus for all measurements; therefore, only the slopes are significant for the ledges. Measurements are also being made, by the use of interferometric microscopy, of the depth $d$ of the pits as a function of their lateral dimensions and of the height of the ledges. A plot of $d / X$ vs time, for the pits formed under the same conditions as for Fig. 11.3 but in a separate experiment, is shown in Fig. 11.5. The relative depth of the pit decreases sharply during the first $30 \mathrm{sec}$ of etching; for longer times $d / X$ is approximately constant.

The dislocation density in this crystal was $2 \times 10^{3} / \mathrm{cm}^{2}$. If the pits are assumed to be symmetrical, volume $/$ pit $=1.3 \times 10^{-2} X^{3}$ after about $30 \mathrm{sec}$. Thus, while the size of the pit increases linearly with time, $X(\mathrm{~cm})=5.3 \times 10^{-5} t(\mathrm{sec})$, the volume increases cubically with time, $V\left(\mathrm{~cm}^{3}\right)=$ $1.9 \times 10^{-15} t^{3}$. From the current density, the rate at which copper is removed is equal to $w=1.4 \times 10^{-6} \mathrm{~cm}^{3} \mathrm{~cm}^{-2} \mathrm{sec}^{-1}$. Thus, during the course of the etching (Fig. 11.1), the relative amount of copper removed from the pits increases to only $\sim 10 \%$ of the total volume removed at $190 \mathrm{sec}$.

The velocity of the ledges on the surface is dependent on the position on the surface, as shown particularly in this experiment. In region $A$ (see Fig. 11.4) the density of ledges is $3.2 \times 10^{3} / \mathrm{cm}$ and the velocity of motion is $4.3 \times 10^{-5} \mathrm{~cm} / \mathrm{sec}$, while in region $B$ the density is $2.4 \times 10^{3} / \mathrm{cm}$ and the velocity is $3.6 \times 10^{-5} \mathrm{~cm} / \mathrm{sec}$. The flux of ledges, $\phi$, in region $A$ is $\phi_{A}=1.4 \times 10^{-1} / \mathrm{sec}$, while $\phi_{B}=0.9 \times 10^{-1} / \mathrm{sec}$. The difference in these values, though small, is thought to be significant. Whether the difference corresponds to small differences in ledge heights $L$ or to microscopic differences in current density is not resolvable at present. If a uniform current density
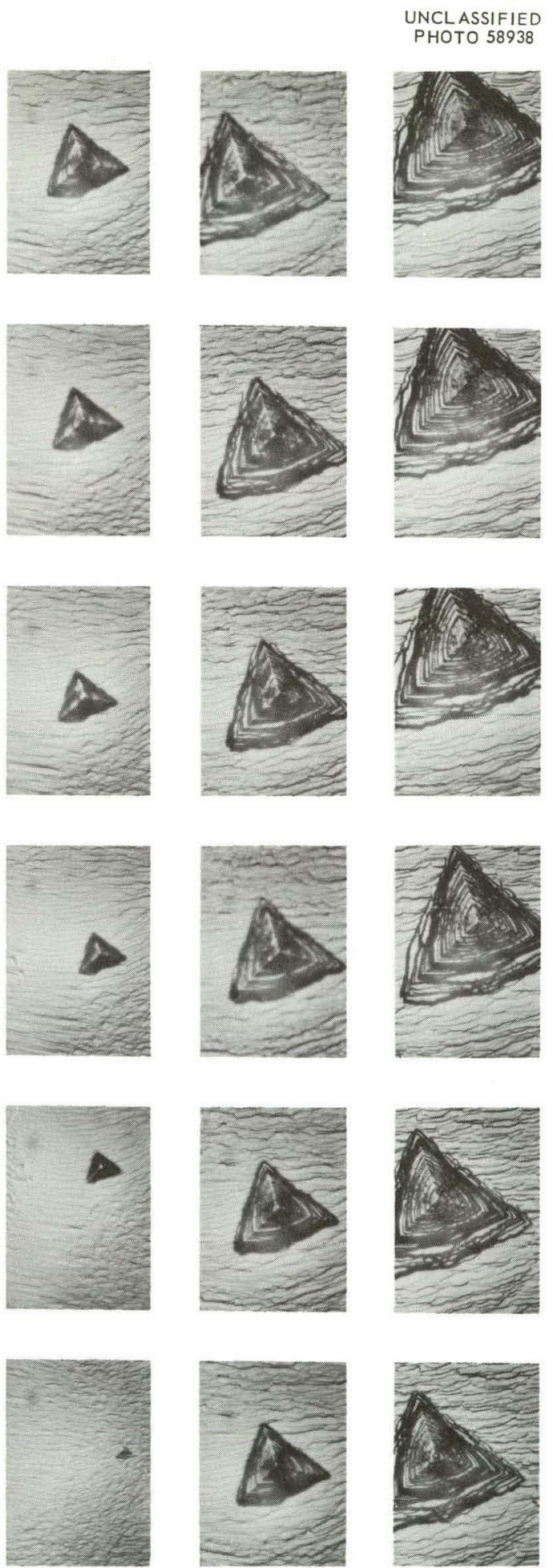

Fig. 11.3. Growth of Etch Pit $\left(20 \mathrm{ma} / \mathrm{cm}^{2} ; 6 \mathrm{M}\right.$ $\mathrm{HCl}, 0.25 \mathrm{M} \mathrm{HBr}$ ). First photograph after $20 \mathrm{sec}$; 10 sec intervals between subsequent photographs. 180X. Reduced $14 \%$. 


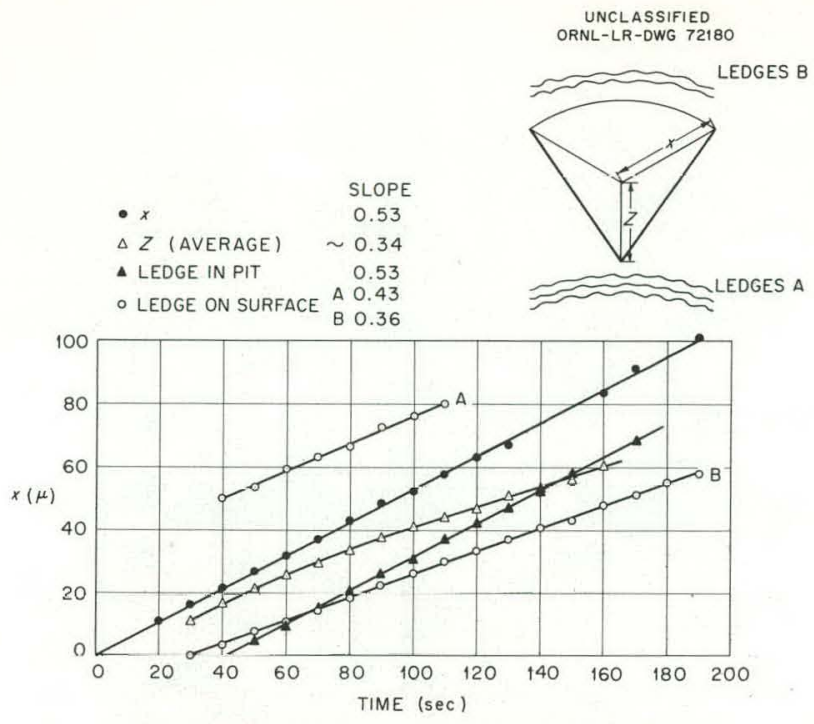

Fig. 11.4. Position vs Time for Several Features from Fig. 11.3 .
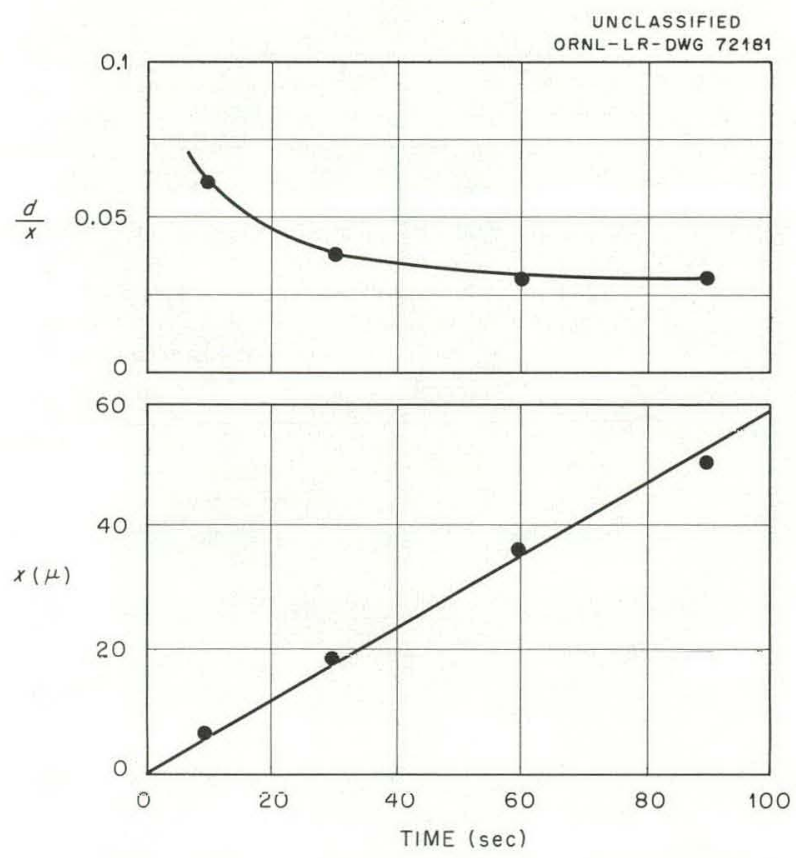

Fig. 11.5. Pit Size and Pit Depth vs Time. Same conditions as for Fig. 11.3.

is maintained microscopically from $\phi$ and $w, L$ is calculated to be $\sim 10^{-5} \mathrm{~cm}$, which agrees with interferometric measurements. On the other hand, by using the value $L \sim 10^{-5} \mathrm{~cm}$ and the measured values of the ledge density to calculate the corresponding misorientation, it can be shown that the ledges must have resulted from steps nucleated on the surface as well as ledges arising from slight misorientations of the surface. It is clear from the photographs that while the density of ledges does not change appreciably with time, the height does increase. And it should be remembered that the relative amount of copper being removed from the pits also increases with time. A possible explanation for these observations is that most of the copper is removed from monatomic steps during the early stages of the etching, that these steps bunch to form the multiatomic ledges, and subsequent removal occurs primarily at the ledges.

The side of the pit nearest ledges $B$ grows at exactly the same velocity as the ledges $B$ move. Since (from the geometry of a pyramidal pit with straight edges) the velocity of the side $\equiv \frac{1}{2} v_{X}$, the fact that for this experiment the velocity of the side $>\frac{1}{2} v_{X}$ is just a measure of the bowing out of this side. In a similar way the bowing in of the other sides is apparent and explicable. Since the ledges are moving in the $Z$ direction, it is apparent that $Z$ should be $<X$.

The velocity of a ledge inside the pit is the same as $v_{X}$ in this experiment. Since the surface is retreating (via ledge and step motion) as the pit grows, it would be expected that the leading ledge of the pit would periodically be removed, and thus $v_{X}$ would be less than the velocity of a ledge inside the pit. It would seem that the only way these two velocities could be equal is that the distance between the ledges in the pit increases near the edge of the pit. That this is true is apparent both from examination of the photomicrographs and from interferometric measurements.

One of the important observations is that the dislocations are not the sources of the ledges on the surface; evidently they arise both from surface nucleation and from slight misorientations of the crystal surface.

The kinetics of pit growth and of ledge motion are being studied in the manner described above as a function of the variables current density, concentrations of $\mathrm{Cl}^{-}$and $\mathrm{Br}^{-}$, dislocation density, and orientation. 


\subsection{GROWTH OF COPPER CRYSTALS WITH LOW DISLOCATION DENSITY}

A study is in progress to determine the factors controlling dislocation density in copper crystals grown from the melt. Copper of $99.999 \%$ purity (supplied by ASAR Company) is used as the stock material and is carefully cleansed by etching so as to avoid the introduction of impurities. The crystals, 1 in. in diameter by 6 in., are grown in a graphite (chlorinated C-18, National Carbon Company) crucible by use of a Bridgman technique at a rate of $1 \mathrm{in} . / \mathrm{hr}$. Approximately 125 nonseeded crystals have been grown, and for apparently identical conditions of growth the orientations of the growth direction were scattered over the stereographic triangle. The crystals were sliced to obtain (111) or (100) faces, via acid sawing and acid polishing, ${ }^{2}$ and the dislocation densities throughout the crystals were determined by etch-pit techniques. A definite correlation between growth direction and crystal perfection was observed, ${ }^{3}$ the dislocation density being an inverse function of the angular deviation of the growth direction from the (111) zone. Crystals with a dislocation density of $\sim 10^{3} / \mathrm{cm}^{2}$ and with no subboundaries were grown.

Attempts to grow seeded crystals, (111) growth direction, with low dislocation density have been unsuccessful to date. Apparently, during the seeding process the dislocation density is greatly increased; and although the growth direction is good, the dislocations are propagated during growth into the new crystal. These studies are being continued.

It has been found that subsequent annealing of the crystal slices for one week at $1075^{\circ} \mathrm{C}$ serves to reduce the dislocation density, and a number of samples with a dislocation density of $10^{2} / \mathrm{cm}^{2}$ have been obtained (see Fig. 11.6). The mechanism of this annealing process is not clear, but the dislocation density has been reduced from $5 \times 10^{4}$ to $\sim 5 \times 10^{2} / \mathrm{cm}^{2}$ on a reasonably routine basis.

In order to determine the dislocation densities in copper by independent methods, specimens of low dislocation density are being prepared for transmission $\mathrm{x}$-ray and electron microscopy. Thin

\footnotetext{
${ }^{2}$ F. W. Young, Jr., and T. R. Wilson, Rev. Sci. Instr. 32,559 (1961).

${ }^{3}$ F. W. Young, Jr., Bull. Am. Phys. Soc. 7, 215 (1962).
}
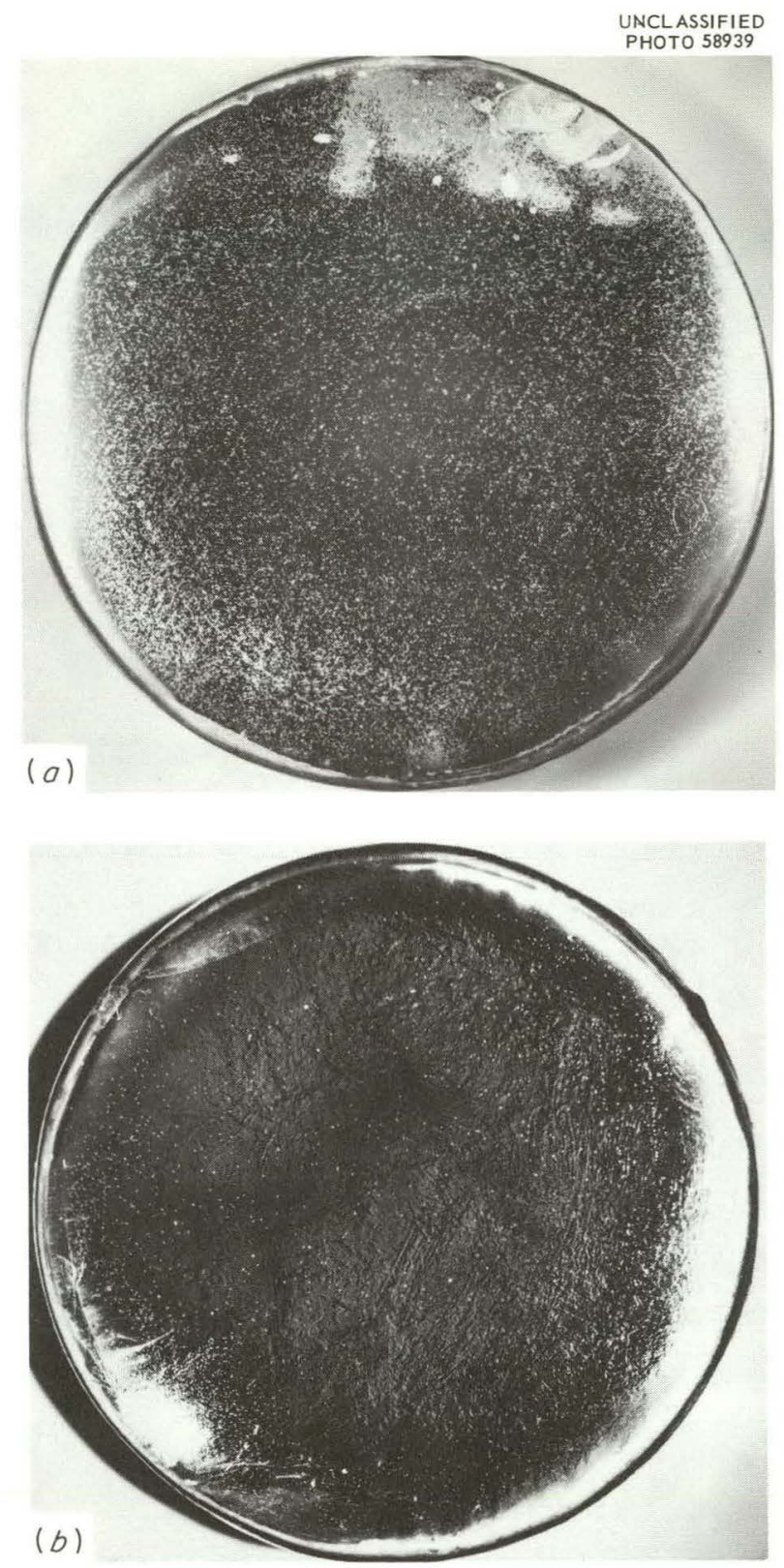

Fig. 11.6. Etch Pits at Dislocations on Copper Crystals. 3X. (a) As grown. (b) After annealing 1 week at $1075^{\circ} \mathrm{C}$. Reduced $24 \%$.

plates are cut with the acil saw, acid polished, and finally thinned by electropolishing. Since copper is so soft, it is very difficult to avoid accidental deformation of these plates, but preliminary results ${ }^{4}$ suggest that specimens suitable for $x$-ray microscopy can be prepared.

\footnotetext{
${ }^{4}$ M. C. Wittels, F. A. Sherrill, and F. W. Young, Jr., "X-Ray Observations of Nearly Perfect Copper Single' Crystals," see abstract this report.
} 


\subsection{ETCH-PIT STUDIES OF DISLOCATIONS IN COPPER CRYSTALS DEF ORMED BY BENDING: I. ANNEALED CRYSTALS; \\ II. IRRADIATED CRYSTALS 5}

Annealed and irradiated copper crystals of low initial dislocation density were deformed by bending, and dislocation multiplication and motion were studied by use of an etch-pit technique for observing the dislocations. For the annealed crystals there was dislocation motion and multiplication prior to macroscopic yielding, and the percentage of grown-in dislocations moved by the stress increased with the stress. The dislocations were hindered in the motion by subboundaries and by interaction with other dislocations. Relaxation effects were observed and were much greater for stresses less than the yield stress than for higher stresses. For the neutron-irradiated crystals there was no motion or multiplication of dislocations prior to yielding. The slip traces were long, with dislocations closely spaced in the trace. Although not conclusive, the evidence apparently was best interpreted in terms of source hardening. A cuberoot dependence of the yield stress on neutron dose was observed. No hardening resulted from irradiation with $10^{18} / \mathrm{cm}^{2}$ cobalt gamma rays.

\footnotetext{
${ }^{5}$ Abstract of paper submitted to Journal of Applied Pbysics.
} 


\section{Low-Temperature Irradiation Studies}

R. R. Coltman

\author{
C. E. Klabunde
}

\subsection{REACTOR DAMAGE IN PURE METALS AT $4^{\circ} \mathrm{K}$}

By relocating the fuel in the vicinity of a liquidhelium cryostat located in the ORNL Graphite Reactor, it has been possible to separate the thermal and fast components of the reactor neutron flux. Studies of the radiation damage effects arising from each type of flux have been made. It has been found that an appreciable fraction of the reactor damage in several metals arises from thermal neutrons. The effect results from the recoil of an atom from the $(n, \gamma)$ reaction at the time of thermal-neutron capture. The low-temperature recovery of thermal-neutron damage is greater and shows more annealing peak structure than the recovery of fast-neutron damage. Thermalneutron damage concentration studies have been made on cadmium; and pronounced suppression of the annealing is found as the concentration is increased. The mean primary recoil energy from a thermal-neutron capture event has been calculated for several elements. Values range from about $50 \mathrm{ev}$ for the heavier elements to several hundred electron volts for the lighter elements. The relative effects of atomic recoils from decay events and decay beta-atom collisions are estimated and found to be small compared with $(n, \gamma)$ recoil effects. Damage from fission neutrons has been studied in copper, and it was found that the recovery of this damage is suppressed compared with that obtained from reactor fast neutrons.

\footnotetext{
${ }^{1}$ Guest scientist from the Australian Atomic Energy Commission.
}

\author{
D. L. McDonald ${ }^{1}$ \\ J. K. Redman
}

The summary above describes the major portion of the work performed by the group during the past year. A detailed report of this work has been accepted for publication in the Joumal of Applied Physics.

\subsection{INTENSE DAMAGE IN CADMIUM}

In the temperature range 4 to $40^{\circ} \mathrm{K}$ the isochronal annealing behavior of thermal-neutron-irradiated cadmium shows a well-behaved suppression of the recovery as a function of neutron dose. Changes in residual resistivity were used to observe this effect. The mean recoil energy of a cadmium atom at the time of thermal-neutron capture ( $n, \gamma$ reaction) is calculated to be $130 \mathrm{ev}$. Although the threshold displacement energy for cadmium is not known, one may use similar results for copper, silver, and nickel to estimate that each capture event in cadmium might make between one and two Frenkel pairs. Thermal-neutron damage in cadmium, then, should be uniformly distributed as contrasted with fast-neutron damage, which is believed to occur in localized regions with a high damage concentration. The isochronal recovery of fast-neutron damage in cadmium is considerably suppressed compared with the recovery from various modest doses of thermalneutron damage. It is believed that if the thermalneutron damage concentration could be increased to the point where its recovery resembled that of fast-neutron damage in the temperature range 4 to $40^{\circ} \mathrm{K}$, then one might make a rough estimate of the defect concentration resulting from a typical fast-neutron hit. To achieve such a high concentration, the proper amount of $\mathrm{Cd}^{113}$ isotope was added to natural cadmium to produce a specimen 
with twice the capture cross section of natural cadmium. The specimen was then bombarded at $4.2^{\circ} \mathrm{K}$ for two weeks to an integrated thermalneutron flux of $6.5 \times 10^{17}$ nvt. The average concentration of capture events in this specimen was 0.2 at. \%, which might produce between 0.2 and 0.4 at. \% of Frenkel pairs. In Fig. 12.1 we see the isochronal recovery of this specimen along with that obtained after a substantially pure fastneutron bombardment. (The recovery from a thermal-neutron dose one-half as great is also shown to illustrate the suppression effect due to dose.) It can be seen that the recovery from this high thermal-neutron dose closely approached that obtained from a fast-neutron irradiation. From this it is estimated that the defect concentration in a damaged region resulting from a fast-neutron hit is between 0.3 and 1.0 at. \% Frenkel pairs.

\subsection{NEW LOW-TEMPERATURE IRRADIATION FACILITY FOR THE BULK SHIELDING REACTOR}

From the work completed this year it is clear that thermal neutrons make a significant contribution to the total reactor flux damage in many elements. The study of pure thermal-neutron damage is of particular basic interest for a large number of elements, since the primary atom recoil energies are relatively small. For the present work, thermal-neutron damage studies have been made by retracting the fuel in the vicinity of the liquid-helium cryostat located in the ORNL Graphite Reactor. This causes a substantial reduction in the fast-neutron flux, while the thermal-neutron flux remains unchanged. It is apparent, however, that for the detailed study of thermal-neutron

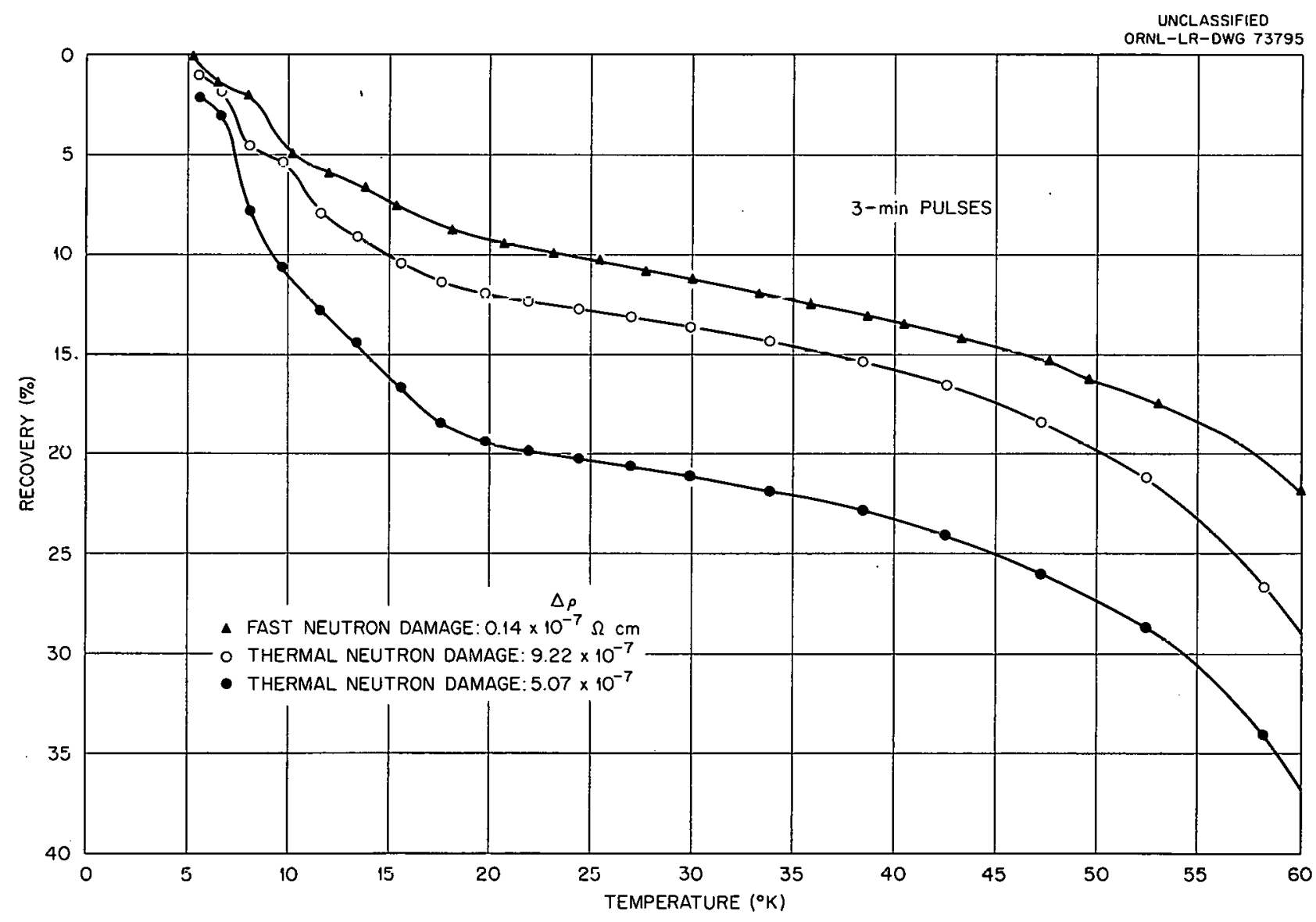

Fig. 12.1. Isochronal Recovery of Irradiated Cadmium. 
damage in many elements, a further reduction of the fast-neutron flux is necessary. The present fuel configuration around the cryostat (a 44-in.diam fuelless sphere) is the terminal fuel retraction that can be made. Any additional fuel retraction would make the reactor inoperable. To extend the thermal-neutron damage studies and to obtain virgin fission neutrons by the use of unmoderated $U^{235}$ converters, construction of a low-temperature irradiation facility to be associated with the BSR has begun. The initial effort will consist in placing a rectangular tank of heavy water between a face of the reactor and a liquid-helium cryostat. The tank will be 12 in. thick and will cover the entire reactor face.

In order to obtain an idea as to what damage effects might be expected in such a facility, an existing $\mathrm{D}_{2} \mathrm{O}$ tank, differing from the one above in that it did not quite cover the entire reactor face, was installed. M. S. Wechsler and J.M. Williams made some preliminary flux determinations adjacent to the tank, and J. W. Cleland and R. F. Bass obtained a solid-state characterization of the fast flux by measuring the removal rate of conduction electrons in $n$-type germanium. The monitors used in the flux determination were $\mathrm{Co}^{59}$, $\mathrm{U}^{238}, \mathrm{Ni}^{58}$, and $\mathrm{S}^{32}$. The techniques are discussed elsewhere in this report (see "Fundamental
Studies,". Part V). Table 12.1 shows the values of the various measured fluxes behind the $\mathrm{D}_{2} \mathrm{O}$ tank compared with values of the same quantities at the face of the BSR and at two positions in the ORNL Graphite Reactor.

The removal rate of conduction electrons in $n$-type germanium has been determined as 3.2 per incident fast (damaging) neutron, wherein it has been assumed that the uranium doughnut fission chamber in Hole 51 of the ORNL Graphite Reactor had an interior fast (damaging) flux of $8 \times$ $10^{11} \mathrm{~cm}^{-2} \mathrm{sec}^{-1}$. Two $n$-type germanium samples were irradiated for $1 \mathrm{hr}$ outside the 12 -in. tank of $\mathrm{D}_{2} \mathrm{O}$. The power level was $1 \mathrm{Mw}$. The samples were contained inside a $3 / 8$-in. boron carbide impregnated plastic container that has been shown to eliminate any effect from either thermal neutrons or capture gamma rays. The total conduction electron removal was 5.2 and $6.0 \times 10^{14} / \mathrm{cm}^{3}$ for the two samples. If one assumes a removal rate of 3.2 per incident fast (damaging) neutron, as above, then the total fast (damaging) neutron flux exterior to the tank of $\mathrm{D}_{2} \mathrm{O}$ was $4.9 \times 10^{10} \mathrm{~cm}^{-2}$ $\mathrm{sec}^{-1}$ in this experiment. It is believed that this value is in reasonable agreement with the threshold detectors indicated above, in that the germanium samples are probably sensitive to all neutrons above $1 \mathrm{kev}$ neutron energy.

Table 12.1. Neutron Flux Behind $D_{2} \mathrm{O}$ Tank in BSR Compared with Flux at the Face of BSR and Flux in Two Positions in the Graphite Reactor

\begin{tabular}{|c|c|c|c|c|c|}
\hline \multirow[b]{2}{*}{ Monitor } & \multirow{2}{*}{ Measured Flux } & \multicolumn{2}{|c|}{ Bulk Shielding Reactor } & \multicolumn{2}{|c|}{ Graphite Reactor, Hole $C^{a}$} \\
\hline & & $\begin{array}{l}\text { Face of } \\
\text { Reactor }\end{array}$ & $\begin{array}{c}\text { Behind } \\
\mathrm{D}_{2} \mathrm{O} \text { Tank }\end{array}$ & Center & $\begin{array}{c}\text { Reflector } \\
12 \text { in. from Fuel }\end{array}$ \\
\hline $\mathrm{Co}^{59}$ & $\begin{array}{l}\text { Thermal flux, } \\
\text { neutrons } \mathrm{cm}^{-2} \mathrm{sec}^{-1}\end{array}$ & $1 \times 10^{13^{b}}$ & $9.1 \times 10^{11}$ & $8.5 \times 10^{11}$ & $4.2 \times 10^{11}$ \\
\hline $\mathrm{Co}^{59}$ & Ratio, cobalt/cadmium & & 120 & 8 & 21 \\
\hline $\mathrm{U}^{238}$ & $\begin{array}{l}\Phi(E>1.6 \mathrm{Mev}) \\
\text { neutrons } \mathrm{cm}^{-2} \mathrm{sec}^{-1}\end{array}$ & $2.1 \times 10^{12^{c}}$ & $9.9 \times 10^{9}$ & $3.4 \times 10^{10}$ & $3.2 \times 10^{9}$ \\
\hline $\mathrm{Ni}^{58}$ & $\begin{array}{l}\Phi(E>2.9 \mathrm{Mev}) \\
\text { neutrons } \mathrm{cm}^{-2} \mathrm{sec}^{-1}\end{array}$ & $9.2 \times 10^{11^{c}}$ & $5.8 \times 10^{9}$ & $1.5 \times 10^{10}$ & $1.9 \times 10^{9}$ \\
\hline$s^{32^{\circ}}$ & $\begin{array}{l}\Phi(E>3.0 \mathrm{Mev}) \\
\quad \text { neutrons } \mathrm{cm}^{-2} \mathrm{sec}^{-1}\end{array}$ & $8.6 \times 10^{11^{c}}$ & $5.4 \times 10^{9}$ & $\therefore 1.3 \times 10^{10}$ & $1.4 \times 10^{9}$ \\
\hline
\end{tabular}

\footnotetext{
${ }^{a}$ M. S. Wechsler et al., "The Effect of Radiation on Atomic Rearrangements in Cu-Al," this report.

${ }^{b}$ F. C. Maienschein et al., Attenuation by Water of Radiations from a Swimming Pool Type Reactor, ORNL-1891, p 10 (1955).

${ }^{c}$ Estimated from neutron spectrum given by F. C. Maienschein in Third Radiation Effects Symposium 2, 31 (1958).
} 
The results shown above indicate that the scheme proposed for the BSR will give a higher absolute value of thermal-neutron flux as well as a higher ratio of thermal to fast neutron flux than can be attained in the ORNL Graphite Reactor. The use of a larger heavy-water tank (construction completed), which will completely cover a reactor face, and of a large void space between the cryostat and the $\mathrm{D}_{2} \mathrm{O}$ tank should further improve both the thermal flux and the ratio of thermal to fast neutrons.
An addition to the south end of the BSR building which will house the refrigeration equipment is about $50 \%$ complete. Helium compressor skids, vacuum jacketing for a new heat exchanger, and some instrumentation and controls have also been completed. The heat capacity of the new refrigeration system is estimated to be about $25 \mathrm{w}$ at about $4.5^{\circ} \mathrm{K}$. A new cryostat also under construction will have a sample chamber 1 in. in diameter and 9 in. long. 


\section{Dislocation Interactions}

\subsection{EVIDENCE FOR VACANCY CLUSTERING IN DISLOCATIONS IN COPPER}

\author{
D. O. Thompson
}

One of the problems in the study of radiation damage in metals is concerned with the interaction of radiation-produced point defects with existent defects, such as dislocations. It has been shown previously that these interactions are sufficient, even at extremely small neutron doses, to effect rather drastic reductions in the internal friction and modulus defect of pure copper crystals. ${ }^{1}$ From the fundamental viewpoint, part of this problem may be associated with the interaction between defects after they are trapped by the dislocations. For example, Coulomb and Friedel have discussed the growth of voids around dislocations due to excess vacancy concentrations. ${ }^{2}$ This process would be expected to contribute to source hardening of an irradiated material, since the interaction force between a defect and a dislocation is expected to be proportional to the misfit parameter of the defect. A part of the work this year, therefore, has been spent in an effort to determine whether sensitive internal-friction techniques could be used to learn something of defect-defect interactions in existent dislocations after light neutron bombardments with the aid of the GranatoLücke theory of dislocation internal friction. Thus, the work to be reported is an attempt at a systematic extension of some preliminary results given earlier in which it was suggested that perhaps the neutron-produced dislocation pinning-

${ }^{1}$ D. O. Thompson and D. K. Holmes, J. Appl. Phys. 27, 713 (1956).

${ }^{2} \mathrm{P}$. Coulomb and J. Friedel, "On the Formation of Cavities Along Dislocations," p 555 in Dislocations and Mechanical Properties of Crystals (ed. by J. C. Fisher et al.), Wiley, New York, 1956. point distribution was temperature dependent for a given concentration of defects. ${ }^{3}$

All measurements included in this set of experiments were made upon a single crystal of highpurity copper in an internal-friction apparatus described elsewhere [D. O. Thompson and F. M. Glass, Rev. Sci. Instr. 29, 1034-40 (1958)]. Once the sample was placed in the bombardment facility, no handling of it took place until. the experiments were over. A beam hole (HB-2) of the ORR served as the bombardment facility. The beam holes are equipped with stopcock-type shutters so that the neutron beam may be stopped independently of the reactor's operation. A series of five bombardments in all were given in this set of experiments. These were chosen to give neutron doses of about $3 \times 10^{10}, 6 \times 10^{10}, 12 \times 10^{10}, 24 \times 10^{10}$, and $48 \times 10^{10} n v t$. Between each of these bombardments the neutron beam was extinguished, the sample was aged at $100^{\circ} \mathrm{C}$ for at least $24 \mathrm{hr}$ so as to stabilize the crystal against any further thermal migration of radiation-produced defects to dislocations, and then the modulus defect and the amplitude-independent and amplitude-dependent internal friction were measured from about $200^{\circ} \mathrm{K}$ up to the postbombardment aging temperature.

The results of measurements of the amplitudeindependent decrement taken at a strain of about $5 \times 10^{-8}$ as a function of temperature after the first three bombardments are given in Fig. 13.1. Also shown in the upper portion of the figure is the temperature dependence of the preirradiation decrement taken at the same strain amplitude. From this figure it. is apparent that successive amounts of irradiation lower the overall values of the decrement and, further, that the temperature dependence of the decrement is altered.

\footnotetext{
${ }^{3}$ D. O. Thompson, Acta Met. 10, 327 (1962).
} 


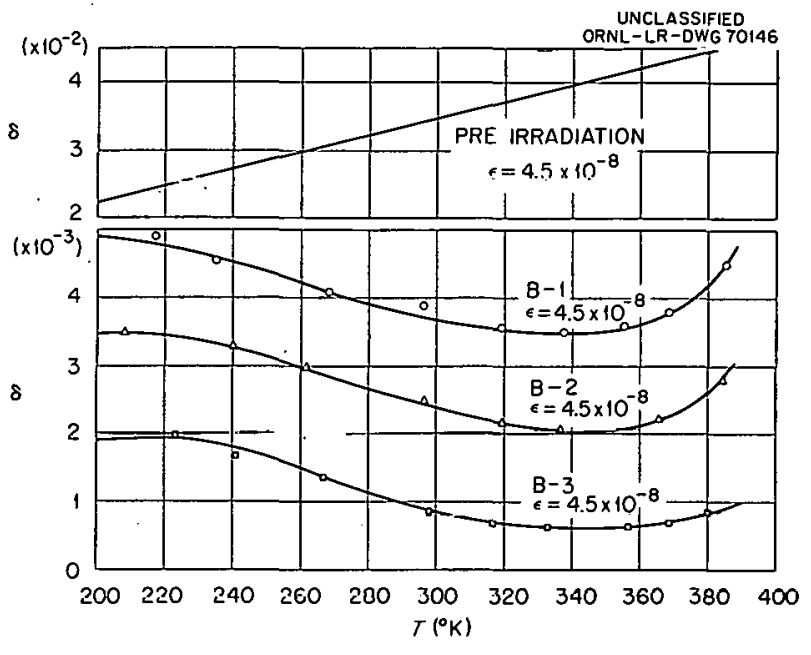

Fig. 13.1. Temperature Dependence of Small-Amplitude Decrement Before Irradiation and After the First Three Bombordments.

An example of the dependence of the decrement upon the strain amplitude over this temperature range is given in Fig. 13.2. These data were obtained after B-3, the third bombardment in the sequence. The interesting feature of these curves is that they cross at higher strains as the temperature is changed. Thus, with increasing temperature the small strain decrement decreases, but the strain range available before the decrement increases sharply is shortened.

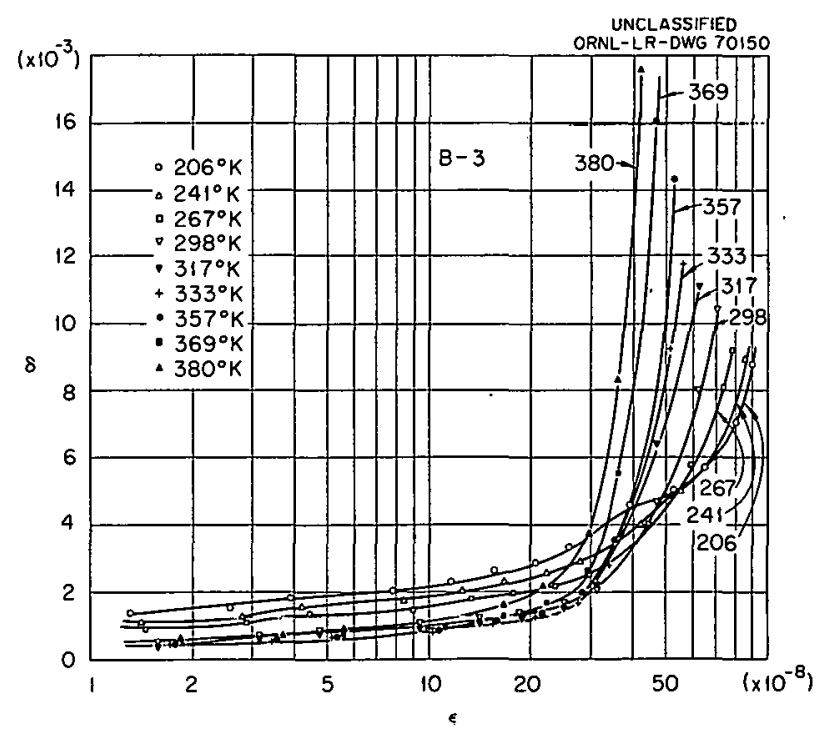

Fig. 13.2. Logarithmic Decrement vs Strain Amplitude After the Third Bumbordment at Vorious Temperatures.
The behavior of the decrement in this temperature range changed as a function of further dose. In Fig. 13.3 is shown a temperature cycle of the small-amplitude decrement after bombardment B-5, the largest dose in this set of experiments. From a low value of about $1 \times 10^{-4}$ near $330^{\circ} \mathrm{K}$ following the bombardment, the decrement increased upon cooling as shown in the figure by the open circles. Both time and temperature effects were apparent in the process. Upon warming, the decrement followed the upper path given by the solid circles. A series of 20 -hr isothermal anneals in the 360 to $400^{\circ} \mathrm{K}$ range was sufficient to restore the decrement to the $1 \times 10^{-4}$ value measured before the low-temperature traverse. An interesting feature to note is that the temperature dependence of the decrement during the warming portion of the cycle is again approximately linear, as in the preirradiated state.

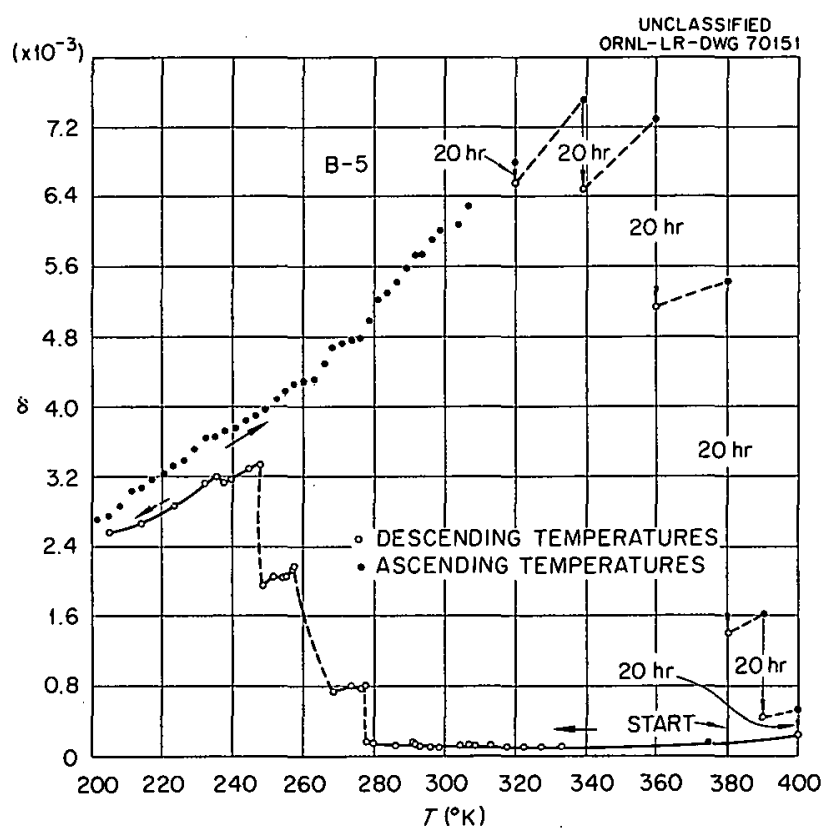

Fig. 13.3. Temperature Cycle of Small-Amplitude Decrement After the Fifth Bombardment. Values are given at the beginning and end of the isothermal onneal.

A change in the decrement-strain amplitude curves accompanies the type of transition shown in Fig. 13.3. In Fig. 13.4 are shown two sets of 


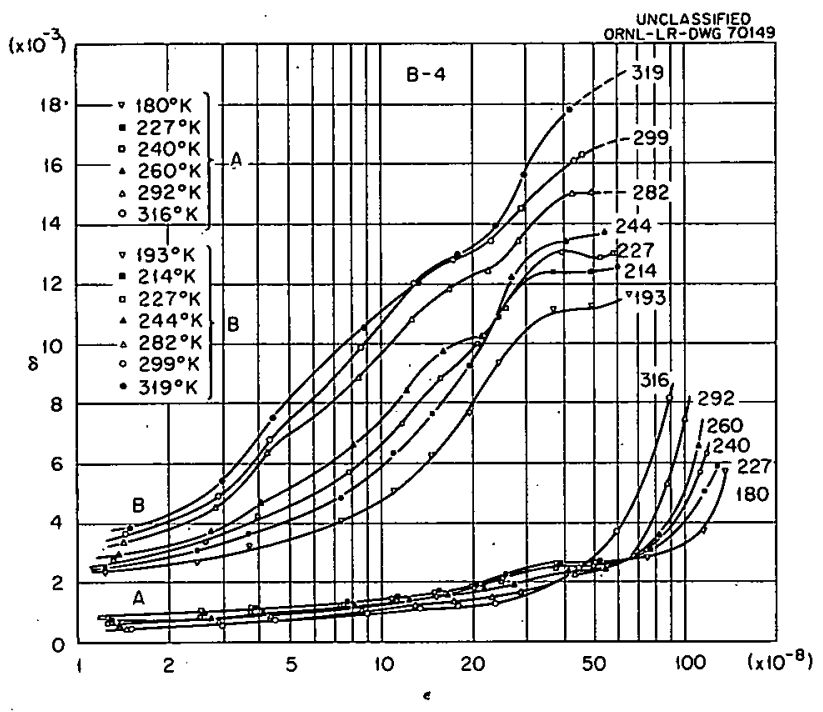

Fig. 13.4. Logarithmic Decrement vs Strain Amplitude After the Fourth Bombardment at Various Temperatures.

data obtained after B-4, the fourth bonbardment: The lower set of curves was obtained in the first set of measurements following the bombardment. After these were obtained, the sample was allowed to remain at room temperature for several days and was remeasured in the low-temperature range with no intervening high-temperature cycle and with the addition of no more irradiation. The lower set is like those obtained after the first three bombardments, while the upper set is like that obtained after B-5. It will be seen that they are indeed quite different sets of curves: (1) The upper set shows the "normal" temperature dependence, while the lower, or first, set is "inverted." (2) No crossing of the curves occurs in the upper set as it does in the lower. (3) The decrement reaches a plateau as a function of strain in the upper set, which is not the case in the lower set.

An interpretation of these results has been made in terms of the Granato-Lücke theory of internal friction due to dislocations. ${ }^{4}$ The basic experimental results indicate, in terms of this theory, that dislocation pinning points are lost as the temperature is lowered, and are recovered by suitable annealing treatments as it is raised. The recovery is true for all bombardments made

\footnotetext{
${ }^{4}$ A. Granato and K. Lücke, J. Appl. Phys. 27, .583 (1956).
}

to date, but the recovery temperatures are higher after B-5 than after the early bombardments. Even though it is clear that the simple GranatoLücke theory is not adequate to explain completely the results, but should probably be amplified to include the effects of at least two kinds of dislocations upon the internal friction and modulus defect, it has been used to calculate the number of pinning points as a function of temperature for constant amounts of irradiation. This has been done with both the amplitude-independent decrement results in Fig. 13.1 and the modulus defect results. The results of such calculations are given in Fig. 13.5 in terms of normalized curves for bombardments 1 and 2. The parameters $Y^{-1 / 2}-1$ and $Z^{-1 / 4}-1$ are, theoretically, equal to the ratio of the dislocation pinning points added by irradiation to those present before irradiation, ${ }^{5}$ and $f$ is the fraction of pinning points at temperature $T$ divided by the number observed at the temperature of maximum density. Thus, the maximum number derived from the decrement occurs at $360^{\circ} \mathrm{K}$, while the maximum number obtained from the modulus data occurs at approximately $320^{\circ} \mathrm{K}$. According to the simple GranatoLücke theory, these curves should be coincident; it is apparent that they are not. It seems probable that the noncoincidence is associated with the existence of more than one dislocation component whose contributions to the total decrement and modulus defect are not equal.

${ }^{5}$ D. O. Thompson and V. K. Paré, J. Appl. Phys. 31, 528 (1960).

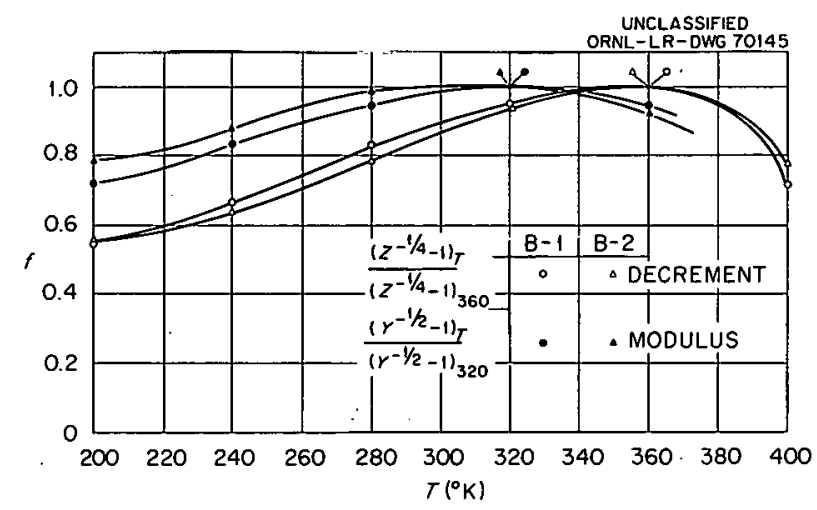

Fig. 13.5. Fraction of Pinning Points as a Function of Temperature Relative to the Maximum Number Observed. 
A logically consistent physical interpretation of the results can be made in terms of vacancy clustering and the onset of void formation around dislocations, as suggested by Coulomb and Friedel. Previous work has indicated that the primary radiation-produced defect responsible for dislocation pinning in this temperature range migrates to the dislocations with an energy of motion of about $1.0 \mathrm{ev}$ in the lattice. ${ }^{6}$ This motion energy is thought to be characteristic of the single vacancy in copper. At the $100^{\circ} \mathrm{C}$ aging temperature following bombardment, the dislucations become supersaturated with vacancies, if it is assumed that thermal equilibrium is attained before bombardment. When the temperature is lowered, some form of precipitation of the excess vacancy concentration is to be expected. Internalfriction experiments are apparently sensitive to this process through the number of pinning points present, so long as the pinning points remain relatively immobile and the stresses arc kept small enough so that dislocation breakaway docs not occur. Thus, if two isolated vacancies at one temperature precipitate to form a divacancy at another temperature, the internal-friction experiments would count a change of a factor of 2 in the pinning-point ratio between those temperatures.

In Fig. 13.6 are shown the results of fitting the fractional pinning-point decrement curves of Fig. 13.5 to a theoretical second-order process. The

${ }^{6}$ V. K. Paré and D. O. Thompson, Acta Met. 10, 382 (1962).

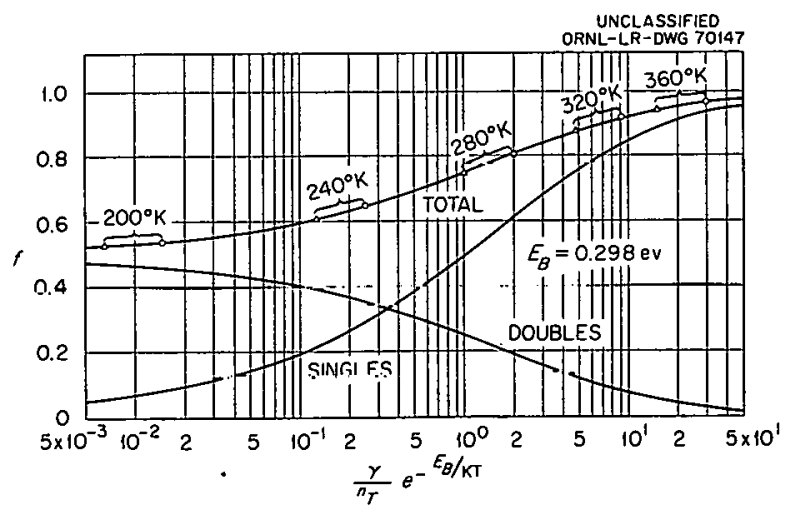

Fig. 13.6. Fit of Decrement Fraction in Fig. 13.5 to Theoretical Second-Order Curves. upper curve represents the normalized total number of pinning points as a function of the "universal" parameter $\gamma / n_{T} e^{-E_{B} / k T}$, and the two other curves are the component numbers of single and double pinning points. $\gamma$ is a constant, $n_{T}$ is the defect concentration per lattice site, $E_{B}$ is the binding energy of the double pinning point, $k$ is Boltzmann's constant, and $T$ is the absolute temperature. Since $n_{T}$ is expected to be proportional to the neutron dose, it is evident that either increasing the dose or lowering the temperature, or both, corresponds to moving to the left on the abscissa and to smaller values of the parameters. This procedure evidently favors the formation of doubles at the expense of singles. The points shown on the upper curves are the fractional decrement points of Fig. 13.5 fitted to the theoretical curve. There is an arbitrariness in the fitting procedure due to the fact that the pinning-point distribution is known nowhere as to its absolute composition. However, it can be shown that if a second-order curve is to be fitted, the relative fraction of $55 \%$ at $200^{\circ} \mathrm{K}$ in Fig. 13.5 fixes the lower limit of the absolute ordinate in Fig. 13.6 to $91 \%$, corresponding to a lower abscissa limit of about 8 . Having selected the coordinates for the initial point, however, all the remaining points of Fig. 13.5 are fixed in Fig. 13.6 by the value of the binding energy. The best fit of the data to the theoretical curve is obtained with starting coordinates at $360^{\circ} \mathrm{K}$ for the first bombardment of $97 \%$ and 30 , and a binding energy of $0.298 \mathrm{ev}$. Open circles are the first bombardment results, and triangles are the results of the second. It is to be noted that, while any one run is obtained as a function of temperature at constant dose, results obtained after increasing doses must fit on the same curve with the same selection of initial points and binding energy. This is the case because of the dose-proportional $n_{T}$ in the abscissa parameter. Thus, doubling the neutron dose corresponds to halving the abscissa value for the same temperature. It will be seen that the agreement of theory and experiment is indeed excellent. The modulus data can be fitted to the same kind of curve with a somewhat smaller value for the binding energy. Tests of uniqueness have not been performed, so that it is not known whether the results will fit only a second-order curve. Results from the third bombardment do not fit so well as do the first two, and show a systematic deviation at lower temperatures. Results after 
the fourth and fifth doses are not even close, as is evident. Thus, the second bombardment is approaching the limit of the simple, second-order behavior in this work.

It should be possible to evaluate the entropy change associated with the second-order process from the values of the abscissa. With the above binding energy of about $0.3 \mathrm{ev}$ and a reasonable value for the average dislocation loop length of $2.5 \times 10^{-4} \mathrm{~cm}$, so that $n_{T} \sim 1 \times 10^{-4}$ per lattice site, it follows that the change in entropy is 4.4k. While this is not a particularly meaningful number at present, it is felt that its magnitude is of the right order.

It is thought that the results after larger doses may indicate the formation of larger vacancy clusters in which the divacancy may act as a building block as the excess vacancy concentration is increased by bombardment. Analysis of the isothermal anneals indicated in Fig. 13.3 in terms of pinning-point numbers yields an associated activation energy of about $0.95 \mathrm{ev}$. The physical meaning of this energy is not readily apparent at present, nor is it. known as yet whether this energy depends upon the neutron dose and, consequently, upon the excess vacancy concentration.

An analysis of the strain-amplitude-dependent decrement curves has not been completed in terms of the Granato-Lücke theory for amplitude-dependent damping. It is thought, however, that the behavior of these curves is in at least qualitative agreement with the interpretation given above. After small doses, the strain range before dislocation breakaway occurs is decreased with increasing temperature from $200^{\circ} \mathrm{K}$, and the smallamplitude decrement is decreased. The combination may indicate that as the temperature is raised, a greater number of "soft" pinning points become available at the expense of fewer "hard" ones. This result would be consistent with the secondorder behavior given above. After the larger bombardments, there is no longer any "inversion" of the amplitude-independent decrement, but neither is there any crossing of the amplitude-dependent curves. The "saturation" character of these later curves may indicate that complete breakaway is achieved. Thus, the higher-dose, low-temperature-induced pinning-point distribution appears to be. formed with a larger binding energy per pinning point than does the one for the small dose; it consequently requires a higher range of temperatures $\left(\sim 400^{\circ} \mathrm{K}\right)$ to restore it to the prelow-temperature distribution.

While these experiments are still rather preliminary, they have served to demonstrate the usefulness of internal-friction techniques in the study of details of dislocation-vacancy interactions in a rather quantitative way. It must be realized that the Granato-Lücke theory does not describe completely adequately the internal friction of most copper crystals studied to date, probably due to the presence of two or more dislocation components. The results given here are deduced on the basis of the simple theory, and as such are subject to refinement. This is the best that can be done at present; on the other hand, the fact that reasonable results are obtained may indicate that the internal friction from one dislocation component is several times larger than that from the others.

Many experiments are indicated as a consequence of the present work. Among them is an extension of the work to higher doses to try to determine whether the annealing, as observed after the fourth and fifth bombardments in the present series, is dependent upon dose. A more complete study of the strain-amplitude curves is also anticipated, as well as completion of the analysis of present ones. These should, in terms of existing theory, enable an evaluation of the relative interaction forces of the various defect clusters with the dislocations. An analysis is difficult, for the validity of existing theory is not certain. Present results indicate, however, that the interaction force of the clustered defect is larger than that of the single, as would be expected.

\subsection{ANALYSIS OF INTERNAL FRICTION IN COPPER AT MEGACYCLE FREQUENCIES}

\section{K. Paré}

An experiment was previously reported ${ }^{7}$ in which the internal friction of a copper crystal was measured at $10,30,50,70,90$, and $100 \mathrm{Mc} / \mathrm{sec}$ by the ultrasonic pulse method, while the sample was

${ }^{7}$ D. O. Thompson, V. K. Paré, and F. W. Young, Jr., Solid State Div. Ann. Progr. Rept. Aug. 31, 1960. ORNL-3017, p 40. 
being irradiated at room temperature. The irradiation facility was beam hole HB-2 of the ORR; the fast-neutron flux above $0.75 \mathrm{Mev}$ was about $1.2 \times 10^{8}$ neutrons $/ \mathrm{cm}^{2} / \mathrm{sec}$.

Although an analysis of the data has been theoretical and experimental results have since become available which make it possible to give a more meaningful treatment. Methods have been developed for applying the results of Oen, Holmes, and Robinson ${ }^{8}$ to the data, and quantitative information has been obtained ${ }^{9}$ on the distribution of delay times required for defects, created by fast-neutron hits, to diffuse to the dislocations and become pinning points. The lattcr consideration is important, since it implies that the irradiation time is not ordinarily a linear measure of the number of radiation defect pinning points which have arrived at the dislocation lines.

The basic purpose of the experiment is to test the validity of the vibrating-string model of dislocation motion, developed by Koehler ${ }^{10}$ and by Granato and Lücke. ${ }^{11}$ In this model the dislocations are imagined to be held by randomly spaced, fixed pinning points. The free lengths are assumed to oscillate under the influence of the stresses associated with the internal-friction measurement, and the energy loss associated with this motion is calculated by the use of the theory of a vibrating string in a viscous damping medium. The form of the dependence of internal friction on frequency depends on the damping; the situation is analogous to the question of critical damping of a simple harmonic oscillator. The experimental results show clearly that in pure, annealed copper crystals the damping of the dislocation loops (though not of the sample) is greater than "critical." In this case the damping force prevents excitation of the ordinary vibrating-string resonance; ${ }^{11}$ the logarithmic decrement of the sample is proportional to frequency at low frequencies, where the dislocation motion is limited by line

${ }^{8}$ O. S. Oen, D. K. Holmes, and M. T. Robinson, Solid State Div. Ann. Progr. Rept. Aug. 31, 1960, ORNL$3017, \mathrm{p} 3$.

${ }^{9}$ D. O. Thompson and V. K. Paré, Solid State Div. Ann. Progr. Rept. Aug. 31, 1961, ORNL-3213, p 24; Acta Met. 10, 382 (1962).

${ }^{10} \mathrm{~J}$. S. Koehler, Imperfections in Nearly Fer/ect Crystals, p 197, Wiley, New York, 1952.

11 A. Granato and K. Lücke, J. Appl. Phys. 27, 583, $789(1956)$. tension, and inversely proportional at high frequencies, where the damping force limits the motion. These regions are connected by a peak which becomes very broad when one takes into account the assumed random spacing of the pinning points. In treating this case theoretically, one can neglect the inertia of the dislocation line; this has been done by Oen, Holmes, and Robinson. ${ }^{8}$ The effect of irradiation, which introduces additional pinning points, is to simultaneously reduce the magnitude of the peak and move it upward in frequency, in such a way that the asymptotic behavior at high frequency, where the pinning points do not limit the motion, is not affected.

In the study ${ }^{9}$ of delayed pinning by radiation defects, the semiautomatic, elastic constantinternal-friction spectrometer, ${ }^{12}$ operating in the neighborhood of $15 \mathrm{kc}$, was used to study the time dependence of pinning after brief irradiations of a copper crystal at various temperatures from 280 to $370^{\circ} \mathrm{K}$. It was found that the results could be interpreted if the existence of both delayed and undelayed pinning points was assumed. The former were found to predominate by a factor of about 150, and their cumulative delay-time distribution, covering a factor of $10^{6}$ in delay time, was derived from the data. The temperature dependence of the delay time was found to be represented by a single activation energy, ahout $1.0 \mathrm{ev}$.

With the above information it is possible to calculate, for any irradiation schedule, the actual time dependence of the relative number of pinning points which have arrived at the dislocation lines. The calculation involves integrating over the delay-time distribution; for this purpose the distribution was represented analytically by a piecewise continuous set of power law functions, and the integration was done on the IBM 7090 computer at the Oak Ridge Central Data Processing Facility, with a FORTRAN program written for the purpose. Since it is not yet possible to obtain absolute pinning-point densities, the results are expressed in terms of the equivalent time $\tau$ for which, if only the undelayed defects were available, the sample would have to be irradiated to give the pinning-point density actually attained at clock

${ }^{12}$ D. O. Thompson and F. M. Glass, Rev. Sci. Instr. 29, 1034 (1958). 
time $t$. The quantity $\tau$ should then be a linear measure of the number of radiation defect pinning points which have arrived at the dislocation lines.

Figure 13.7 shows the frequency dependence of the internal friction at various equivalent times during the irradiation. Clearly, at the start of the experiment the logarithmic decrement is very near to being inversely proportional to frequency; this indicates that the range of measurements is above that of the broad peak. The theoretical function $^{8}$ has been fitted as well as the data permit at each equivalent time; the results are the dashed lines in Fig. 13.7. The passage of the peak through the range of measurement can

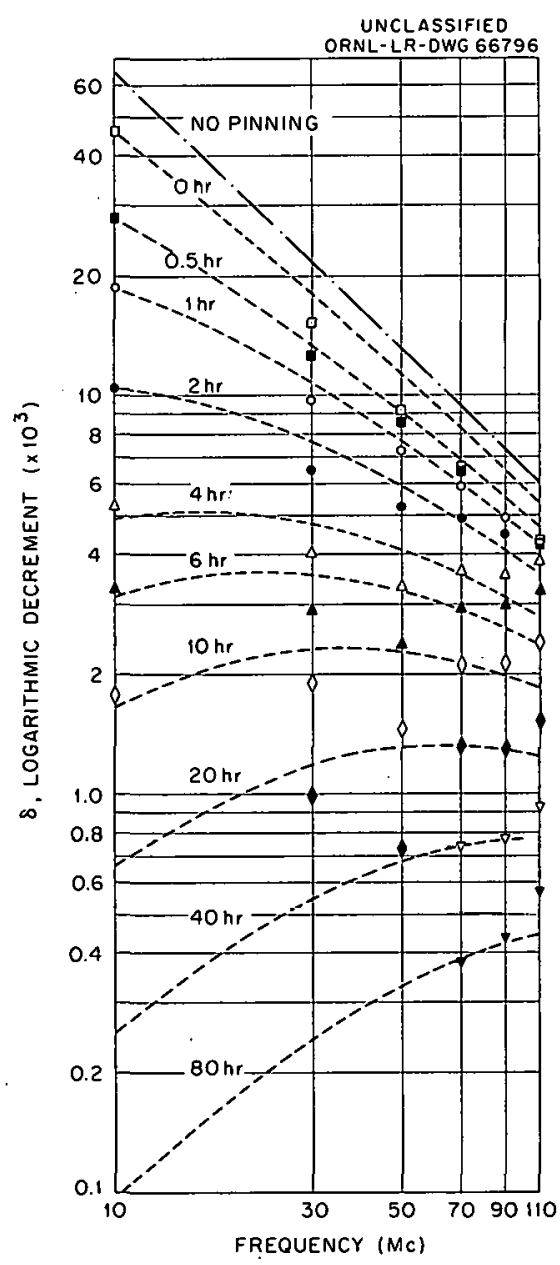

Fig. 13.7. Frequency Dependence of Internal Friction in Copper Crystal, af Various Equivalent Irradiation Times. Dashed lines are theoretical curves for a single dislocation component, fitted to the 10- and 70-Mc data. be seen clearly. Note that the theoretical curves cannot be drawn in with complete arbitrariness; they must all correspond to the same high-frequency asymptotic behavior.

It may be objected that the data appear as a double-humped peak which should be represented by two dislocation components; however, attempts to fit the data in this way have been unsuccessful. On the other hand, the single-component curves shown pass nearly through both the 10- and 70-Mc points. It is therefore felt that the double-hump behavior represents experimental difficulties.

The curves in Fig. 13.7 represent only part of the comparison of theory with experiment. It is also necessary to see whether the pinning-point density implied in the above fit is related in a reasonable way to the input of pinning points to the dislocation lines as indicated by the equivalent time $\tau$. From the theoretical results ${ }^{8}$ it is possible to use the data at a single frequency to obtain values of $n_{r} / n_{0}$, the ratio of the density of radiation defect pinning points to the density of original, preirradiation pinning points; however, in order to do so it is necessary to know $f_{m}$, the frequency at which the maximum decrement would have occurred in the preirradiation condition. For consistency a single value of $f_{m}$ was chosen for the analyses at all six frequencies. Selection was on the basis that the resulting curves of $n_{r} / n_{0}$ vs $\tau$ should be. linear, at least for small $\tau$ values. The curves obtained are shown in Fig. 13.8 .

The noncoincidence of some:- of the curves represents the same experimental difficulties which gave rise to the double-hump behavior in the frequency dependence. Note that the 10- and 70-Mc data may be combined to form one continuous curve; this corresponds essentially to the sequence of theoretical curves displayed in Fig. 13.7 .

The interesting aspect of the curves in Fig. 13.8 is that one would have expected a straight line of unit slope. Similar curvature has been observed previously ${ }^{13}$ in analogous curves obtained from data taken in the kilocycle range; the interpretation at that time was that there was pinning of two types of dislocations at different rates. The apparent inability to represent

${ }^{13}$ D. O. Thompson and V. K. Paré, J. Appl. Pbys. 31, 528 (1960). 


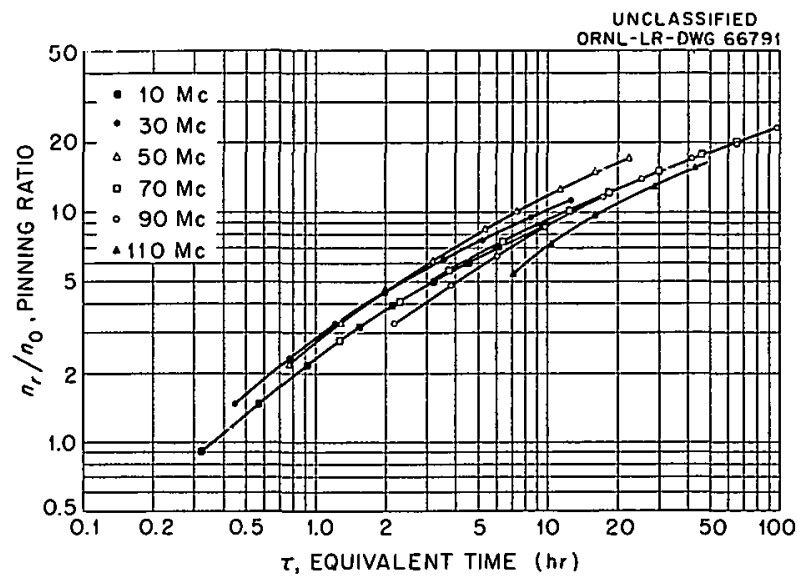

Fig. 13.8. Voriation of Relutive Pinning-Polnt Density with Equivalent Irradiation Time.

the data of Fig. 13.7 by other than one type of dislocation shows, however, that another explanation must be found in the present case. While it is not yet possible to construct a quantitative explanation, it may be noted that the results described in the accompanying article by $D$. $O$. Thompson indicate that radiation defect pinning points react with one another while confined to the dislocation lines. It is thus conceivable that some such reaction may tend to remove an increasing fraction of pinning points as the concentration increases, giving rise to the decreasing slope in Fig. 13.8.

The experiment offers the possibility of determining the dislocation parameters in the theory. The average dislucation density in the sample was determined from an etch-pit study by $F$. W. Young, Jr. From this and the high-frequency asymptotic decrement behavior one can obtain the dislocation damping constant; from a knowledge of $f_{m}$ and line-tension values derived from the work of DeWit and Koehler, ${ }^{14}$ the initial average loop length can be found. Lastly, from the bombarding flux and the initial slope coefficient on Fig. 13.8, one can estimate the number of pinning points per primary knock-on. The results are displayed in Table 13.1. It can be seen that it is somewhat more plausible that the dislocations are of edge type, since the initial

\footnotetext{
${ }^{14} \mathrm{G}$. DelWit and J. S. Koehler, Phys. Rev. 116, 1113 , 1121 (1959).
}

Table 13.1. Parameters Determined for Sample Used in Pulse-Echo Measurements

\begin{tabular}{lll}
\hline $\begin{array}{l}\text { Dislocation density, } \mathrm{cm}^{-2} \\
\begin{array}{l}\text { Damping constant, } \\
\text { dyne-sec/cm }\end{array}\end{array}$ & $\begin{array}{l}L=1 \times 10^{7} \\
\text { Network length, cm } \\
\text { (approximation) }\end{array}$ & $L^{-1 / 2}=1.5 \times 10^{-4}$ \\
$\begin{array}{l}\text { Frequency of preirradiation } \\
\text { maximum, kc }\end{array}$ & $f_{m}=354$ & \\
& & \\
Assumed dislocation type & Screw & $\mathrm{Edge}$ \\
Line tension, dynes & $6.4 \times 10^{-4}$ & $6.4 \times 10^{-5}$ \\
Initial loop length, cm & $1.2 \times 10^{-3}$ & $3.6 \times 10^{-4}$ \\
Total pinning points per & 3.6 & 12 \\
primary knock-on & & \\
\hline
\end{tabular}

loop length required for screws is greater than a rough estimate of the network length. The value of the damping constant agrees closely with the theoretical estimate of G. Leibfried (quoted by Granato and Lücke ${ }^{11}$ ).

\subsection{TEMPERATURE DEPENDENCE FROM 250 TO $370^{\circ} \mathrm{K}$ OF DISLOCATION PINNING IN COPPER SINGLE CRYSTALS BY RADIATION DEFECTS ${ }^{15}$}

$$
\begin{array}{ll}
\text { V. K. Paré } & \text { D. O. Thompson }
\end{array}
$$

Measurements of internal friction and modulus defect at $15 \mathrm{kc}$ have been used to study, in the neighborhood of room temperature, the pinning of dislocations in copper crystals by fast-neutroninduced defects. By measuring at various constant temperatures the delayed pinning following a short irradiation, a delay-time distribution for the pinning defects was obtained, and the process was found to be governed by a single activation energy, $1.0 \mathrm{ev}$. Although this energy is reasonable for the diffusion of vacancies to dislocations, the shape of the delay-time distribution does not agree with theory, and the apparent number of jumps at the end of the process is anomalously low.

\footnotetext{
${ }^{15}$ Abstract of published paper: Acta Met. 10, 382
} 
13.4 A THERMALLY ACTIVATED INTERNALFRICTION SPECTRUM IN COPPER FOLLOWING NEUTRON IRRADIATION ${ }^{16}$

D. O. Thompson

Measurements of the internal friction and Young's modulus of a neutron-irradiated copper single crystal as a function of temperature indicate that some conversion of the primary dislocation pinning defect in the range 270 to about $400^{\circ} \mathrm{K}$ takes place in the dislocation neighborhoods. This conclusion

${ }^{16}$ Abstract of published paper: Acta Met. 10, 327 (1962). is based upon the observation that, with the cessation of bombardment and a presently unspecified incubation period, a thermally activated internalfriction spectrum is developed, which is characterized by an energy of about $0.56 \mathrm{ev}$. These experiments differ from previous work in this field in that an effort is made to allow the dislocation-defect distribution to reach complete thermal equilibrium without the steady-state addition of more defects to the dislocations by continuing bombardment. It is felt that the incubated spectrum may be divacancy-controlled and that the activation energy of $0.56 \mathrm{ev}$ may be interpreted as the motion energy of the divacancy near the dislocations. 


\title{
14. $\mathrm{Ar}^{+}$Ion Bombardment of Metal Surfaces
}

\author{
A. L. Southern \\ M. T. Robinson \\ D. R. Burrowbridge ${ }^{1}$
}

Sputtering yields and atom ejection studies previously reported ${ }^{2,3}$ have been continued with copper, aluminum, and zinc for the better understanding of focusing collisions as postulated by Silsbee 4 and correlation to the fundamental radiation damage processes. Some of the previous results have been prepared for publication. An abstract of the paper follows. 5

\section{Sputtering Experiments with 1- to $5-\mathrm{kev} \mathrm{Ar}^{+}$Ions}
A. L. Southern
W. R. Willis ${ }^{6}$

M. T. Robinson

Sputtering yiclds have been determined with 1- to 5-kev $\mathrm{Ar}^{+}$ions normally incident upon targets of type 304 stainless steel, three different types of polycrystalline copper, a wide variety of copper monocrystals, and monocrystals of silicon and germanium. Ejection patterns have been recorded from these targets and from a monocrystal of InSb. The sputtering yield of polycrystalline copper depends on the source of the metal, apparently due to variations in preferred orientation. The yield from copper monocrystals is strongly dependent on orientation, the effect becoming more

\footnotetext{
${ }^{1}$ Co-op student from Virginia Polytechnic Institute, Blacksburg.

${ }^{2}$ A. L. Southern et al., Solid State Div. Ann. Progr. Rept. Aug. 31, 1960, ORNL-3017, p 52.

${ }^{3}$ A. L. Southern et al., Solid State Div. Ann. Progr. Rept. Aug. 31, 1961, ORNL-3213, p 49.

${ }^{4}$ R. H. Silsbee, J. Appl. Pbys. 28, 1246 (1957).

${ }^{5}$ Abstract of paper to be published in the Journal of Applied Physics.

${ }^{6}$ Professor of Physics, West Virginia Wesleyan College, Buckhannon; consultant.
}

pronounced as the energy is increased. A simple model is presented which accounts for this behavior in terms of the variation with direction of the initial mean free path of the incident ion. The ejection patterns leave little doubt that focusing collision chains are primarily responsible for the transport of momentum to the surfaces of close-packed metals.

To determine the number of argon atoms that are interstitial in copper due to argon ions bombarding the copper surface, the following experiment was performed. Samples of polycrystalline $99.999 \%$ copper were cut from rolled sheets produced by American Smelting and Refining Company, were etched with nitric acid, and then bombarded with 4-kev argon ions at the same current density. Two samples were bombarded such that $0.396 \mathrm{mg}$ of copper was sputtered from one sample and $1.04 \mathrm{mg}$ from the other. The argon content was determined by vacuum melting and spectrographic analysis of the evolved gas. Only the upper limit of the argon gas could be determined; it was $0.02 \mu \mathrm{g}$ in the first case and $0.1 \mu \mathrm{g}$ in the second case. If one considered the argon to be contained only in the volume which is defined by the area of the bombarded spot and the range of argon in copper, then for the first case there would be approximately one argon atom for every ten copper atoms. The different value for the amount of argon contained in the second sample suggests that the argon is not contained within such a volume. Possible explanations would be that the argon diffuses into the undisturbed lattice or that some of the ions lose their energy in a direction with a component parallel to the surface.

Aluminum has been investigated in order to compare the sputtering yield with copper, since the ratio of atomic diameter to lattice spacing 


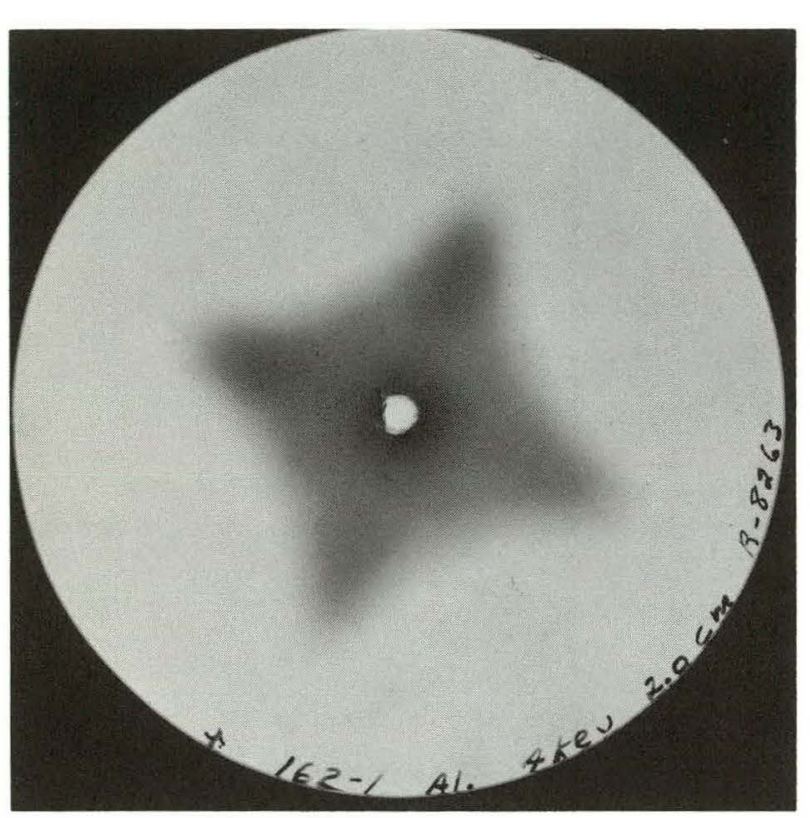

(a) $\langle$ 100〉 AT $4 \mathrm{kev}$

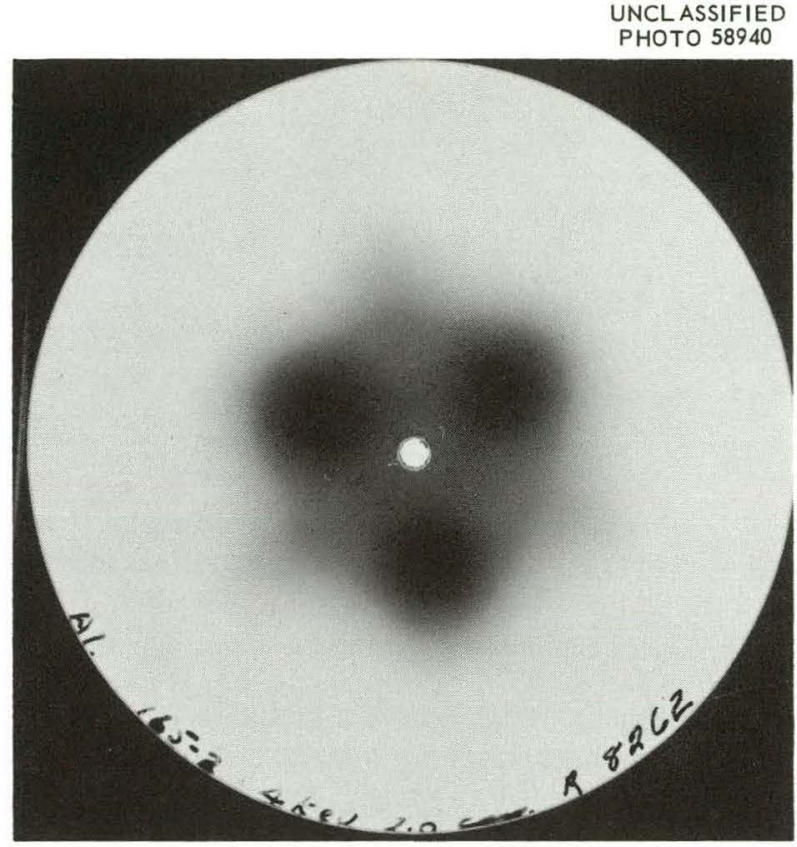

(b) $\langle 111\rangle$ AT $4 \mathrm{kev}$

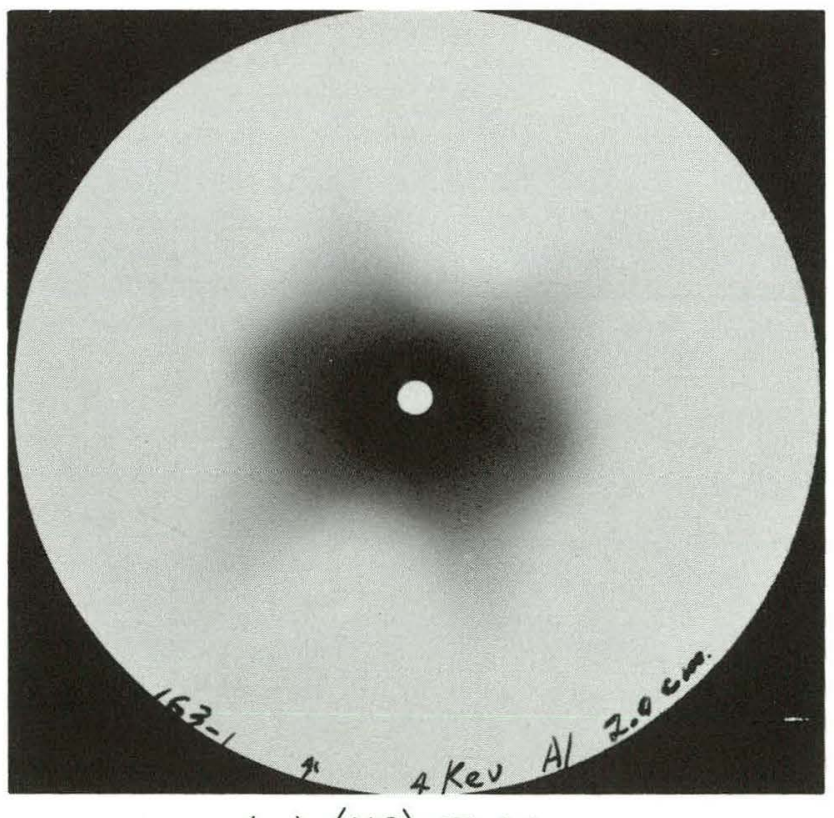

(c) $\langle 110\rangle$ AT $4 \mathrm{kev}$

Fig. 14.1. Ejection Pattern Obtained from Some Aluminum Monocrystals Under Normally Incident Ar ${ }^{+}$lon Bombardment. 
of aluminum is smaller than that of copper. According to Silsbee, ${ }^{4}$ the efficiency of the focusing process in this case would be less than in copper. Therefore, the sputtering yield would be less, and one would expect the atom ejection spots to be larger. The atom ejection pattern of the (111) plane of aluminum shows the spots to be the base of a cone with a small open angle of $\sim 18^{\circ}$, whereas in copper this angle was $\sim 12^{\circ}$. Figure 14.1 shows the ejection patterns from the (100), (111), and (110) planes of aluminum bombarded normal to the surface. The center of the spots corresponds to an ejection angle as expected from the crystal geometry. Preliminary values of the sputtering yield as a function of energy for the (111) plane of $99.99 \%$ aluminum, bombarded normal to the surface, are shown in Fig. 14.2. The crystal was electropolished ${ }^{7}$ prior to sputtering. At $5 \mathrm{kev}$ the (111) plane of copper has a yield of 9.3 atoms/ion, and the (111) plane of aluminum has a yield of 2.54 atoms/ion. This value is lower by $60 \%$ than that expected from the decrease in focusing efficiency as measured by the spread of the ejected spots. This difference might result from the oxide film on aluminum.

The (0001) plane of zinc was studied to compare the patterns of an hcp crystal that has a staggered chain of atoms with fcc crystals which have close-packed chains of atoms. The atom ejection pattern shows faint spots on the corners of a hexagonal pattern which has an evenly distributed background. This pattern is energy-dependent and has the best focus at a bombarding ion energy of less than 1100 ev. From the crystal lattice spacing and assuming a chain of two atoms only, the ejected atom should escape from the surface at an angle $32^{\circ}$ to the normal. With a bombarding ion energy of less than $1100 \mathrm{ev}$, the outer edges of the pattern were $33^{\circ}$ to the normal. Figure 14.3

\footnotetext{
${ }^{7}$ R. D. Heidenreich, J. Appl. Pbys. 20, 993 (1949).
}

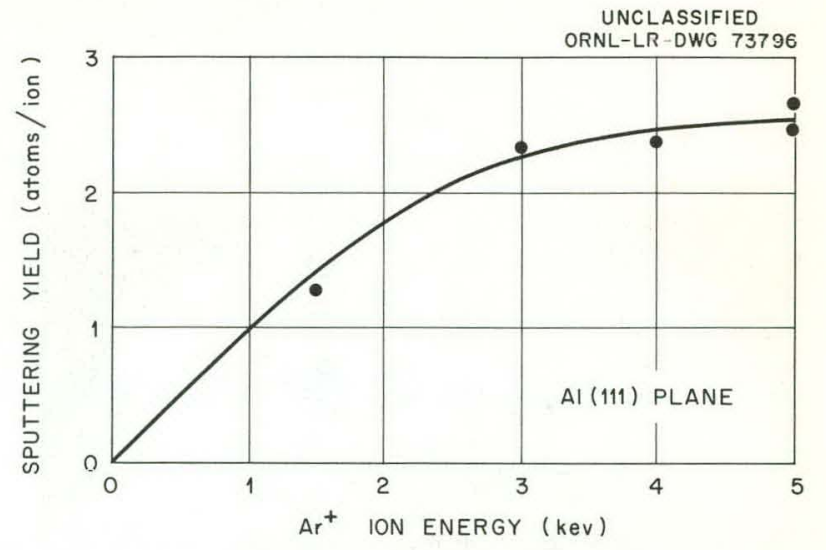

Fig, 14.2. Sputtering Yield of an Aluminum (111) Plane as a Function of the Normally Incident $\mathrm{Ar}^{+}$Ion Energy.

shows patterns at different bombarding energies for the (0001) plane of zinc. Figure 14.4 shows patterns of (0001) zinc taken at $2 \mathrm{~cm}$ from the target for the same bombarding energy at different bombarding times. Spots are detectable in the direction $\langle 2 \overline{1} \overline{1} 0\rangle$. The six points appear to be composed of two triangles which can be explained from the zinc model by assuming that each triangular pattern results from atoms ejected from alternate layers. ${ }^{8}$ The background, which can be seen particularly in Fig. 14.4, is due to radiation damage of the crystal.

Experiments are in progress for determining the yields and ejection patterns of ordered and disordered crystals of $\mathrm{Cu}_{3} \mathrm{Au}$; also, the sputtering yields and ejection patterns of gold are being determined.

${ }^{8}$ C. Lehmann, private communication. 


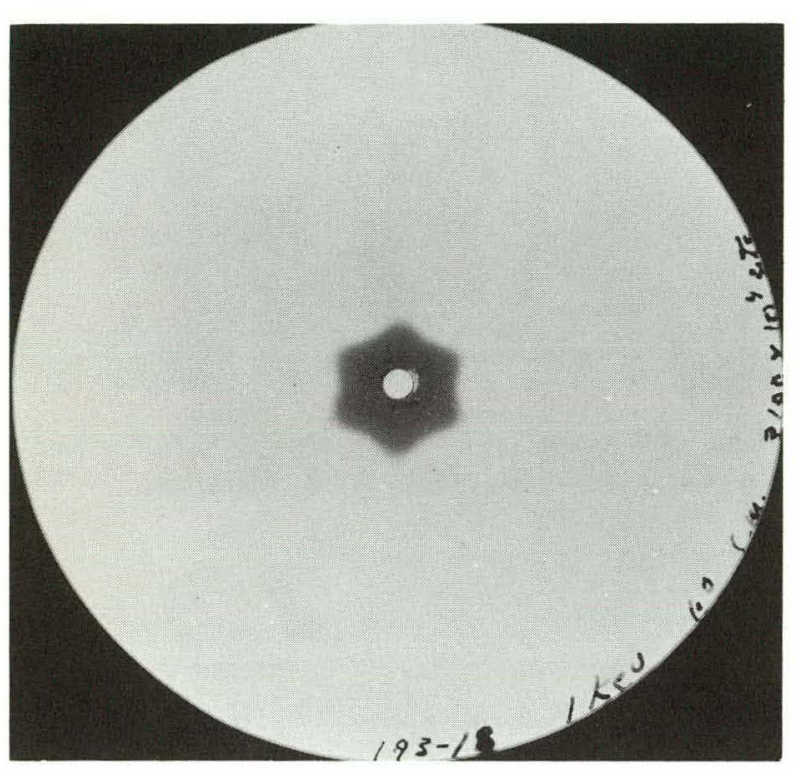

(a) $1 \mathrm{kev}$

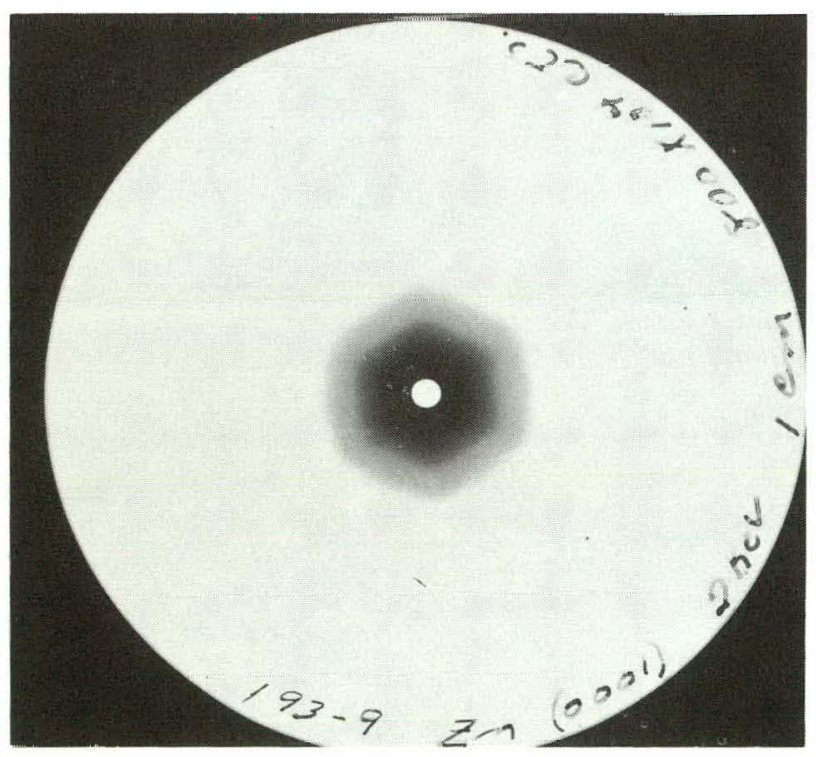

(c) $2 \mathrm{kev}$

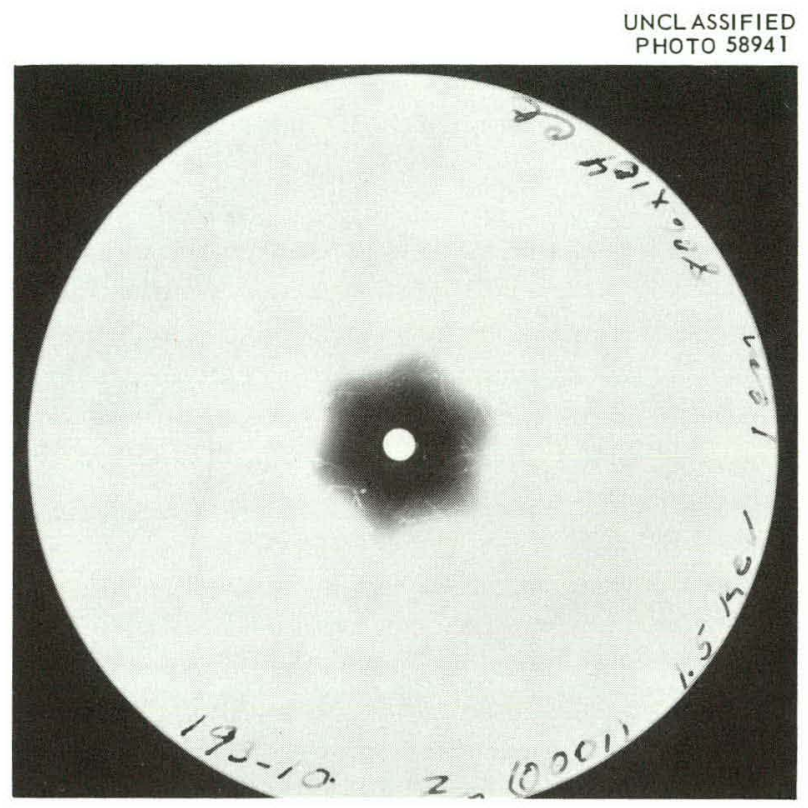

(b) $1.5 \mathrm{kev}$

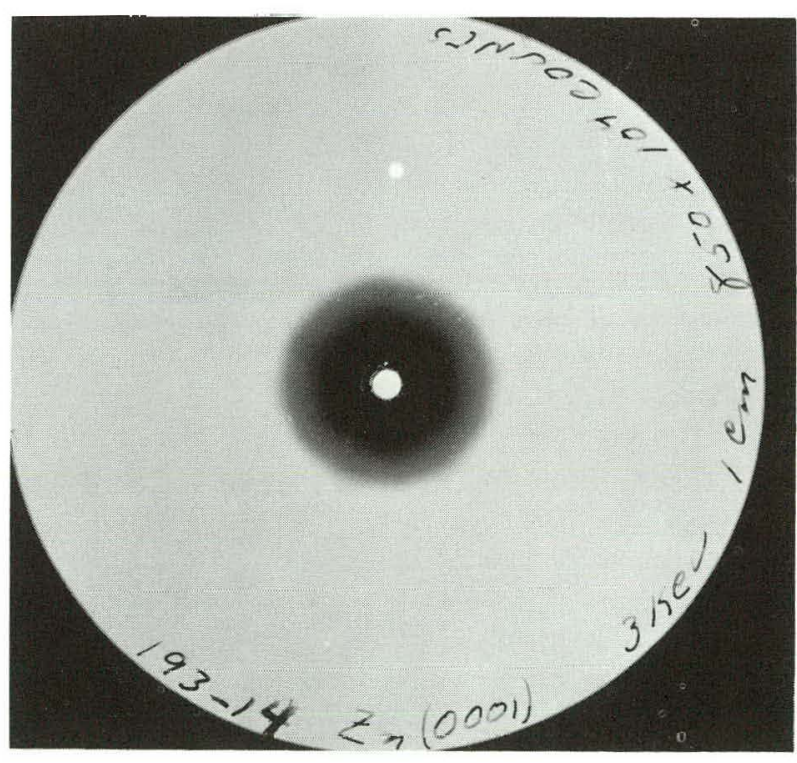

(d) $3 \mathrm{kev}$

Fig. 14.3. Atom Ejection Pattern for a Zinc (0001) Plane Under Normally Incident $\mathrm{Ar}^{+}$Ion Bombardment as a Function of Ion Energy. 

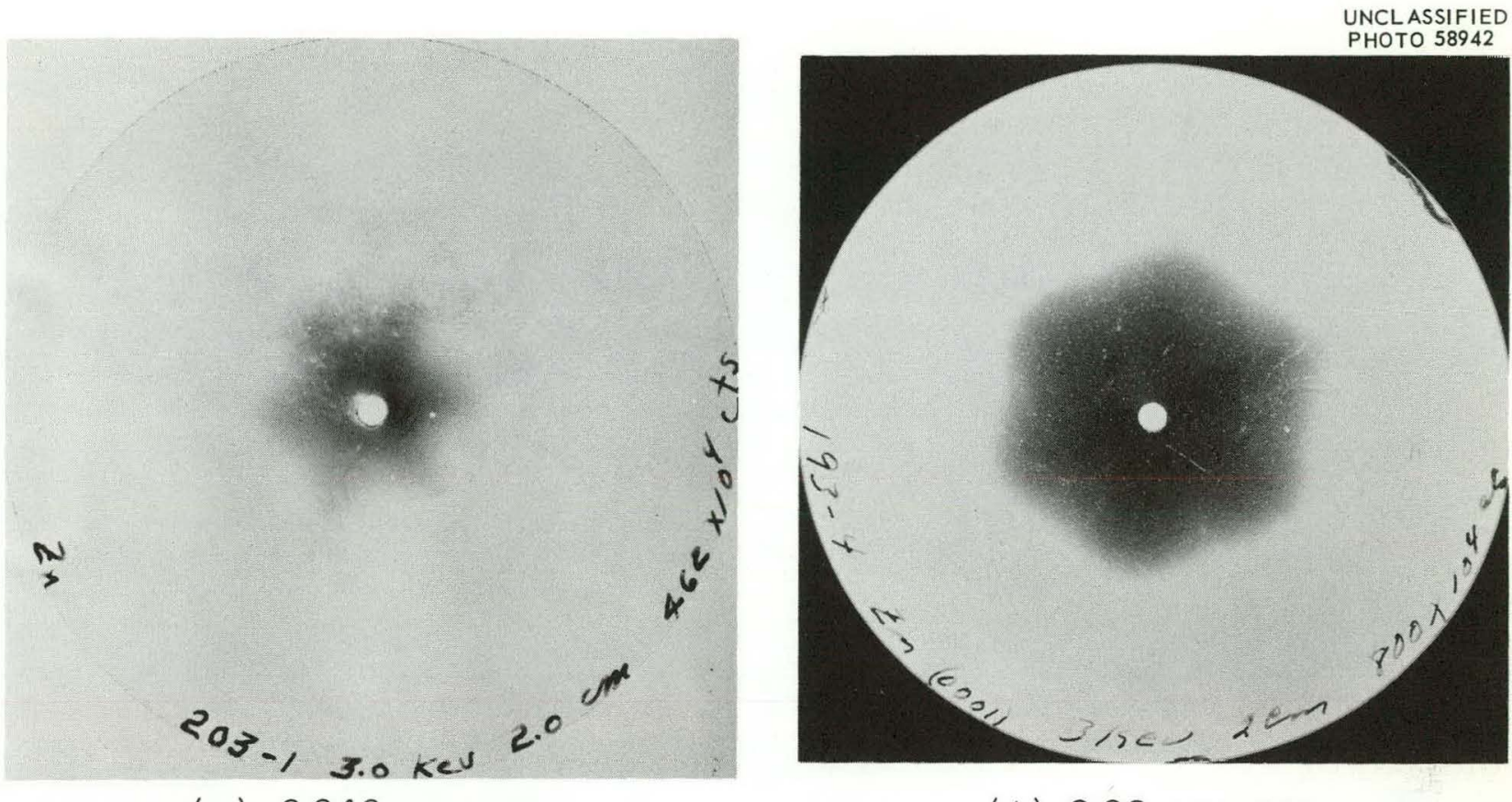

\section{(a) $0.046 \mathrm{amp}-\mathrm{sec}$}
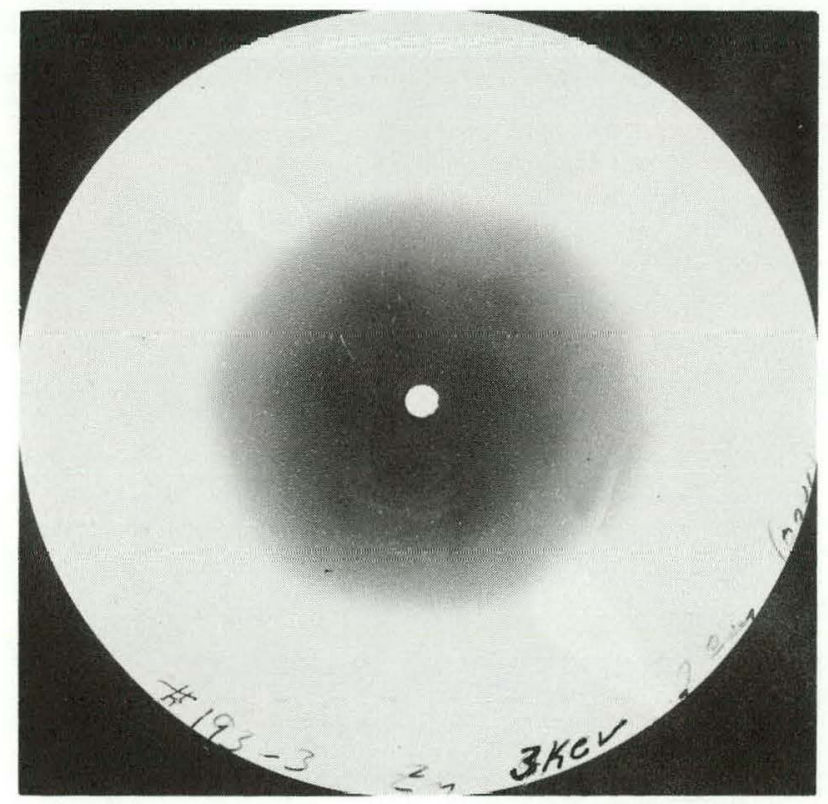

(c) $0.124 \mathrm{amp}-\mathrm{sec}$

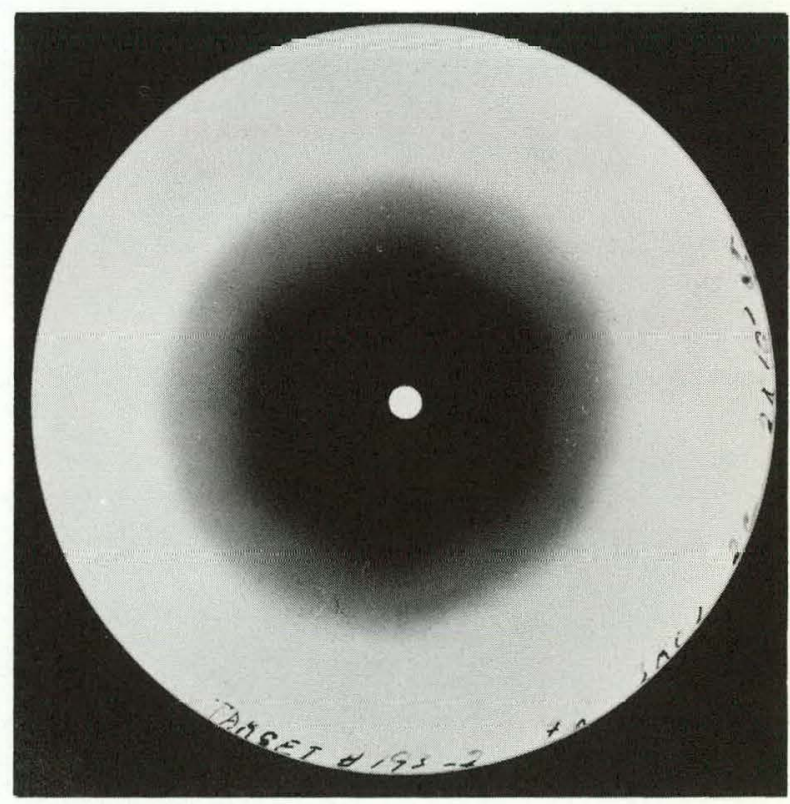

(d) $0.2 \mathrm{amp}-\mathrm{sec}$.

Fig. 14.4. Atom Ejection Pattern for a Zinc (0001) Plane Under Normally Incident Ar ${ }^{+}$lon Bombardment at 3 kev as a Function of Bombardment Time. 
THIS PAGE

\section{WAS INTENTIONALLY LEFT BLANK}


Part IV. Nonmetals

J. H. Crawford, Jr. 
THIS PAGE

WAS INTENTIONALLY

LEFT BLANK 


\section{Semiconductor Studies}

\subsection{RADIATION-INDUCED RECOMBINATION AND TRAPPING CENTERS IN GERMANIUM}

\section{The Nature of the Recombination Process'}

$$
\text { O. L. Curtis, Jr. J. H. Crawford, Jr. }
$$

Extensive measurements on irradiated germanium indicate that previous analyses of the recombination process are incorrect. A model which explains the observations in both $n$ - and p-type material is presented. According to this model, the recombination level lies $0.36 \mathrm{ev}$ above the valence band in gamma-irradiated $n$-typc germanium. Presumably, due to the extensive perturbation caused by neutron irradiation, the level lies slightly lower in neutron-irradiated material. Trapping levels which are not present in antimonydoped germanium occur in arsenic-doped material $\sim 0.17 \mathrm{ev}$ above the valence band. Other trapping levels are observed only in high-resistivity antimony-doped samples. It has not been possible, from the data presented, to determine the values of hole and electron capture cross sections associated with the recombination level; however, the ratio $\sigma_{p} / \sigma_{n}$ has been determined. Its value is $\sim 1000$.

\section{Annealing Behavior ${ }^{2}$}

$$
\text { O. L. Curtis, Jr. J. H. Crawford, Jr. }
$$

An investigation has been made of the annealing properties of radiation-induced recombination

\footnotetext{
${ }^{1}$ O. L. Curtis, Ir., and J. H. Crawford, Jr., Phys. Rev. 124, 1731 (1961).

${ }^{2}$ O. L. Curtis, Jr.; and J. H. Crawford, Jr., Phys.
}

centers in germanium. The annealing behavior of antimony-doped germanium is grossly different from that of arsenic-doped material. The experimental observations are explained on the following basis: The recombination level at 0.36 ev above the valence band belongs to the vacancy. In antimony-doped material the vacancy disappears by association with an antimony atom. This process does not occur in arsenic-doped material, and higher temperatures are required to produce annealing. It is thought that a trapping level, present only in arsenic-doped material, located $0.17 \mathrm{ev}$ above the valence band might be due to an arsenic-interstitial pair.

\subsection{ANNEALING OF IRRADIATION-INDUCED DAMAGE IN GERMANIUM}

$$
\text { J. C. Pigg }
$$

The annealing properties of the radiation-induced carrier change in anrimony-doped germanium have been investigated. The irradiations were conducted at liquid-nitrogen temperatures with Co 60 gamma irradiation. A model which explains the observed behavior has been proposed. On the basis of this model, the observed annealing consists in vacancy diffusion simultaneously to impurity sites and annihilation centers. An anal$y$ sis of the activation energy for the annealing process yields values in agreement with vacancy motion but which cannot be resolved into unique components. The complex activation energy is explained by the model in terms of the impurity concentration. It is observed that the change in carrier concentration saturates before complete annealing is achieved. This saturation is stable for further annealing at higher temperatures. The saturation behavior is also explained in terms 
of the model. The vacancies are considered to diffuse to annihilation centers, such as dislocation lines, and to the site adjacent to an antimony atom. Those which zo to annihilation centers are removed from the system. Those which go to an antimony atom are trapped. The antimony-vacancy complex can break up to supply vacancies back to the system or can trap an additional vacancy, producing an antimonyvacancy complex. The antimony-divacancy reaction comes into equilibrium very quickly when compared with the annihilation process. As this equilibrium is established, a new energy level associated with the antimony-vacancy complex appears in the forbidden band at $0.09 \mathrm{ev}$ below the conduction band.

A detailed discussion of this work will be issued as a separate report.

\subsection{TRAPPING CENTERS IN ARSENIC-DOPED GERMANIUM}
O. L. Curtis, Jr.
C. C. Robinson

Recent studies ${ }^{3,4}$ have demonstrated the existence of trapping levels in arsenic-doped germanium which are not observed in material doped with antimony. On the basis of recombination data alone, it is not possible to determine trap concentrations; however, we have been able to obtain this quantity from dc photoconductivity measurements at low temperature. At low temperature the photoconductivity for a given light intensity is increased because the trapping of holes greatly increases the electron lifetime. However, at sufficiently high excitation levels, the traps become filled, and any additional holeelectron pairs quickly recombine. Thus, one expects a break in the photoconductivity vs light intensity curve when the light intensity is just sufficient to fill the traps. Since, as a condition for the experiment, the trapping time is long compared with the recombination time, it can be assumed that all the photoconductivity is contributed by electrons. The number of excess electrons at the light intensity corresponding to

\footnotetext{
${ }^{3}$ O. L. Curtis, Jr.; and J. H. Crawford; Jr., Pbys. Rev. 124, 1731 (1961).

${ }^{4}$ O. L. Curtis, Jr., and J. H. Crawford, Jr., Phys. Rev. 126, 1342 (1962).
}

the break in the photoconductivity vs light intensity curve is then equal to the number of hole traps.

Experimentally, a constant current is passed through the specimen and a change in voltage, $\Delta V$, corresponding to the photoconductivity is measured. At low excitation levels, while the traps are being filled, $\Delta V / V \approx \Delta n / n$, where $n$ is the electron concentration in the dark and $\Delta n$ is the change produced by illumination. (In order to prevent contact effects, only the middle half of the specimen is illuminated. The value $\Delta V / V$ refers only to this central portion.) The value at the break of the curve $(\Delta V / V)^{\circ}$, is to a good approximation equal to $N_{t} / n$. Figure 15.1 illustrates that the expected behavior is observed. Relative intensity is measured by observing the photoconductivity of an unirradiated sample placed beside the test specimen. (The response of the unirradiated sample was determined to be linearly dependent on light intensity in the region of interest by using calibrated light attcnuators.) The condition for which a curve such as that in Fig. 15.1 would be expected can be predicted on the basis of a plot similar to that in Fig. 15.3 of the preceding annual prozress report (ORNL3213). From the data of Fig. 15.1, the rate of production of hole traps in arsenic-doped germanium is found to be $\sim 3 \times 10^{-4} \mathrm{gamma} / \mathrm{cm}$. This value must be considered tentative since

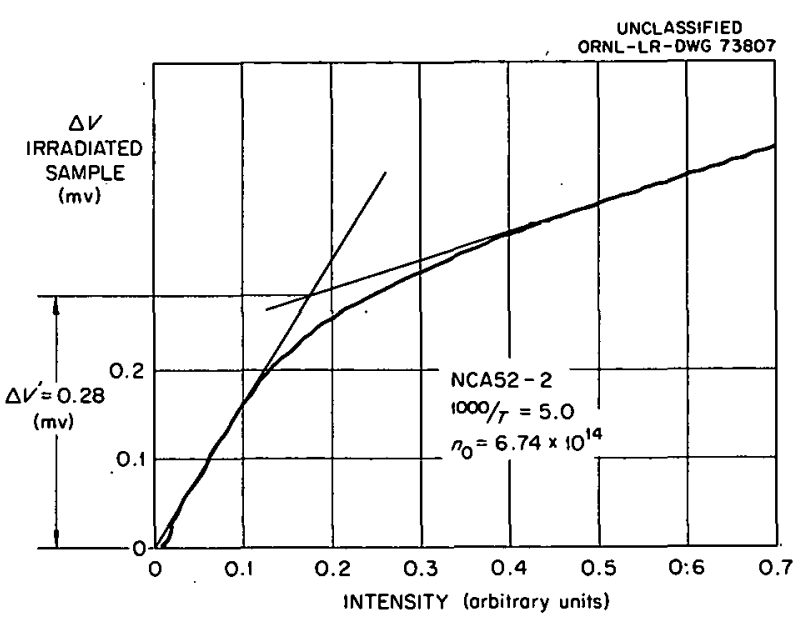

Fig. 15.1. Photoconductivity as a Function of Intensity for an Arsenic-Doped Germanium Specimen Irradiated with $2 \times 10^{16} \mathrm{gammos} / \mathrm{cm}^{2}$. The total valtage drop across the somple was $68 \mathrm{mv}$. 
it is a result of only one measurement. A check will be made to determine whether the number of traps is directly proportional to the total irradiation and whether the trap concentration depends on the amount of arsenic present. Transient measurements will be used to determine capture probability for these centers. Annealing studies are also being performed.

\subsection{THERMOELECTRIC POWER IN GERMANIUM}

\section{O. E. Schow}

An n-type 2-ohm-cm germanium thermoelectric sample and a Hall plate, which were cut from the same ingot, were measured as a function of photon irradiation at ambient temperature in a Co ${ }^{60}$ gamma field. The experimental conditions were described previously. ${ }^{5}$

A plot of the results of the experiment is shown in Fig. 15.2. The Seebeck coefficient decreased approximately $4 \%$ as a result of the first irradiation and then retumed to the approximate preirradiation value as a result of the two latter exposures. Robinson ${ }^{6}$ made some fast-neutron

${ }^{5}$ O. E. Schow, Solid State Div. Ann. Progr. Rept. Aug. 31, 1961, ORNL-3213, pP 59-60.

${ }^{6} \mathrm{C}$. C. Robinson, Solid State Div. Ann. Progr. Rept. Aug. 31, 1959, ORNL-2829, pP 129-31.

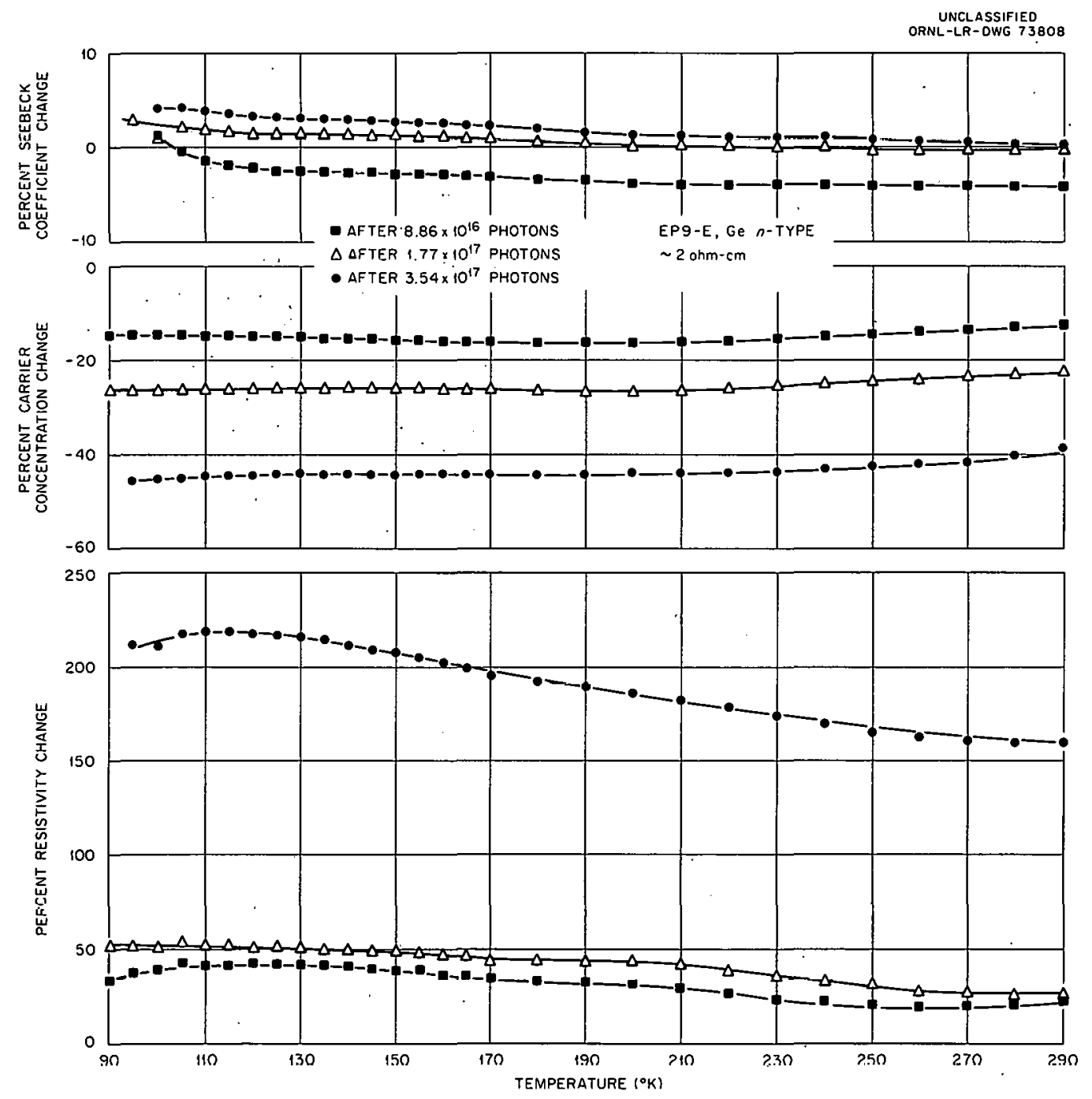

Fig. 15.2. Change of Sample Parameters due to $\mathrm{Co}^{60}$ Photon Irradiation as a Function of Temperafure. 
irradiations on materials from this same ingot and found a similar behavior of the Seebeck coefficient as a function of irradiation.

Figure 15.3 shows a comparison of the measured Seebeck coefficients of three unirradiated arsenicdoped germanium samples as reported by Geballe and $\mathrm{Hull}^{7}$ and the measured Seebeck coefficients of a single antimony-doped germanium sample in which the carrier concentration has been decreased in steps by $\mathrm{Co}^{60}$ photon irradiation. The significance of the data is that the Seebeck coefficient should have increased $3.3 \%$ for a $40 \%$ decrease in the carrier concentration. The measured Seebeck coefficient increased only $0.2 \%$. Since the experimental errors are about $0.2 \%$, it is questionable that the sample changed at all.

In view of the experimental relation between carrier concentration and the magnitude of the Seebeck coefficient, which predicts an increase in the latter, the present observations are difficult to understand.

${ }^{7}$ T. H. Geballe and G. W. Hull, Phys. Rev. 94, 1134 (1954).

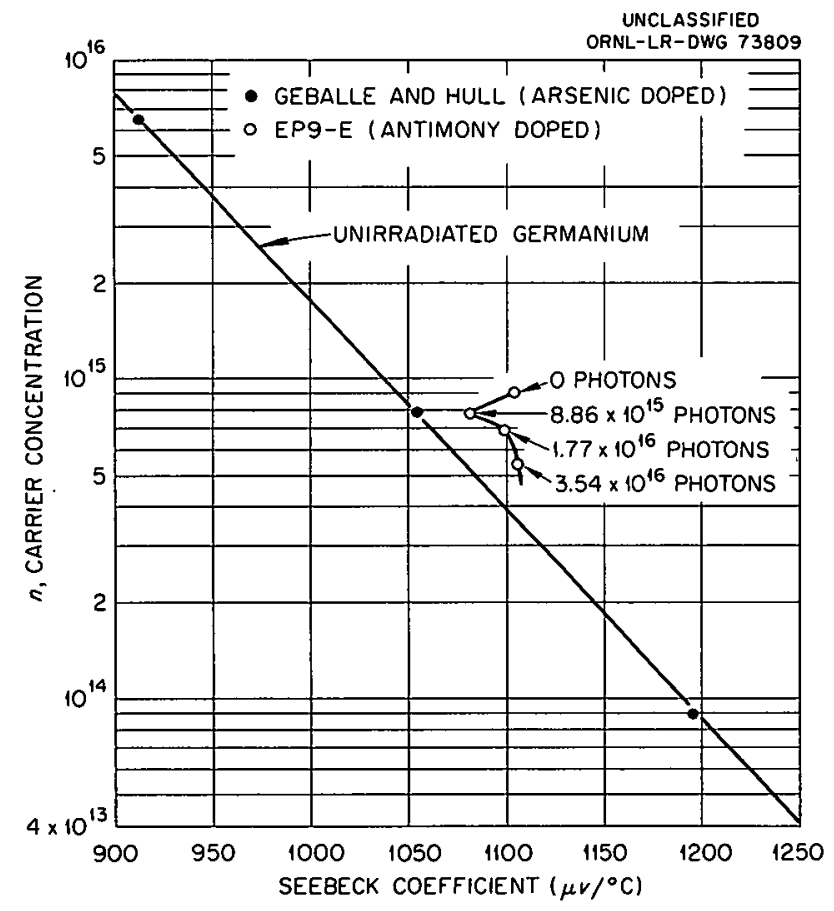

Fig. 15.3. Comparison of the Seebeck Coefficient of Irradiated and Unirradiated n-Type Germanium as a Function of Carrier Concentration of $280^{\circ} \mathrm{K}$.
Since the changes were smaller than expected, the above experiment was scaled up by a factor of 10. The total damage should be approximately 15 times that produced by the $2-0 h m-c m$ irradiation. An analog computer has been constructed to calculate a continuous $X-Y$ plot of the Seebeck coefficient as a function of the sample temperature. Because of the small changes involved in this experiment, a program of rigorous standards has been established.

The materials are now being irradiated.

\subsection{RADIATION EFFECTS IN ZINC SULFIDE}

$$
\text { R. O. Chester }{ }^{8} \quad \text { O. L. Curtis, Jr. }
$$

A study of the effects of irradiation of zinc sulfide by reactor neutrons and by $\mathrm{Co}^{60}$ gamma rays is being undertaken.

Single crystals have been obtained from the Harshaw Chemical Company. Spectrographic analysis indicates copper ( $20 \mathrm{ppm})$ as the major impurity present. Luminescence spectra ${ }^{9}$ verify the presence of copper. Debye-Scherrer diffraction patterns of powder made from the single crystals show only the low-temperature, cubic phase. Laue patterns on single crystals, however, indicate that twinning is present. ${ }^{10}$

Bulk resistivity of the crystals is of the order of $10^{10} \mathrm{ohm}-\mathrm{cm}$. Contacts to the sample were produced by applying indium with an ultrasonic soldering iron and heating the sample at $600^{\circ} \mathrm{C}$ for $\frac{1}{2} \mathrm{hr}$.

It has been suggested ${ }^{11}$ that the high bulk resistivity of zinc sulfide is due to compensation of donor and acceptor concentrations. Samples with resistivities of the order of $10^{2}$ to $10^{3} \mathrm{ohm}$ $\mathrm{cm}$ were prepared by Aven by heating zinc sulfide in the presence of molten zinc. It is argued that zinc acts as a getter for copper and other metallic impurities and removes zinc vacancies.

\footnotetext{
${ }^{8}$ On leave from the Instrumentation and Controls Division.

${ }^{9}$ We are indebted to C. T. Butler for his kind assistance in obtaining luminescence data.

${ }^{10}$ The x-ray studies were performed by M. C. Wittels and $F$. A. Sherrill.

${ }^{11} \mathrm{M}$. Aven, Electronics Div. Abstr. 11(1), 46 (May
} 
Aven verifies the change in copper concentration by observing a well-known ${ }^{12}$ change in the luminescence properties of the zinc sulfide.

We have attempted to duplicate Aven's technique. It is felt that zinc sulfide treated in this fashion is likely to be much more useful in radiation-effects studies than the high-resistivity material. Thin layers of the uncompensated material have apparently been produced, and an effort is being made to produce thicker specimens of the lower-resistivity material.

A 24-hr exposure in the $\mathrm{Co}^{60}$ source No. 2 (total photon flux of $2.4 \times 10^{16}$ per square entimeter) produced no observable change in the absorption spectrum of the zinc sulfide crystals. The same was true of a $570-\mathrm{hr}$ exposure in the slant animal tunnel of the ORNL Graphite Reactor (total neutron flux of $3.0 \times 10^{15}$ per square centimeter). These optical studies are to be followed by more sensitive electrical studies.

\subsection{EFFECT OF A PHASE TRANSFORMATION ON THE VAPOR-PHASE GROWTH OF SINGLE-CRYSTAL $\mathrm{HgS}^{13}$}

$$
\text { O. L. Curtis, Jr. }
$$

Attempts have been made to grow single-crystal $\mathrm{HgS}$ by a vapor-phase growth technique. The lack of success led to the discovery of a phase transformation which occurs between the temperature of deposition and toom temperature. The crystals were deposited in the cubic metacinnabar form and transformed to the hexagonal cinnabar form upon cooling, with a resultant, destruction of the deposited crystals. There is a large supercooling effect which seems to depend upon particle size. When cinnabar is heated in an atmosphere of air, the transformation to metacinnabar occurs at $335 \pm 10^{\circ} \mathrm{C}$. Possibilities for the use of other techniques in the growth of single-crystal $\mathrm{HgS}$ are considered.

\subsection{GROWTH OF SINGLE.CRYSTAL HgS}

$$
\text { O. L. Curtis, Jr. }
$$

Efforts are continuing toward the growth of single-crystal cinnabar. It has recently been demonstrated ${ }^{14}$ that various crystals can be grown at a temperature below their sublimation point by adding iodine to the starting material. In our case, the equation for equilibrium is

$$
\mathrm{HgS}+\mathrm{I}_{2} \rightleftharpoons \mathrm{HgI}_{2}+\frac{1}{2} \mathrm{~S}_{2} \text {. }
$$

At the warm $\left(\sim 420^{\circ} \mathrm{C}\right)$ end of the tube, the reaction proceeds toward the right. In the cooler $\left(\sim 300^{\circ} \mathrm{C}\right)$ portion of the tube, the reverse reaction takes place, releasing the iodine.

Since material is deposited below the phasetransformation temperature, ${ }^{15}$ single crystals should be produced. We have succeeded in obtaining small single crystals of $\mathrm{HgS}$. These crystals were bright red and transparent. Unfortunately, the size of the largest crystal $(\sim 2 \times 2 \mathrm{~mm})$ is inconvenient for electrical measurements. Various alterations in technique are being tried in the hope of producing larger crystals. For instance, the use of chlorine in place of iodine as a transport medium is being planned.

\subsection{A STUDY OF THE EFFECTS OF $\mathrm{Co}_{0} 60$ GAMMA RADIATION UPON EXTRINSIC $\mathrm{Bi}_{2} \mathrm{Te}_{3}$}

$$
\text { M. J. Smith }
$$

Since most of the properties of $\mathrm{Bi}_{2} \mathrm{Te}_{3}$ are extremely sensitive to constituent-element ratios, ${ }^{16}$ it is difficult to obtain any quantitatively reproducible data from one specimen to the next. Consequently, the band structure and extrinsic conductivity models, which have been proposed for $\mathrm{Bi}_{2} \mathrm{Te}_{3}$, have been based on qualitative trends. In order to study inherent properties of extrinsic $\mathrm{Bi}_{2} \mathrm{Te}_{3}$, it is desirable to introduce lattice defects in a specimen, by some controlled method, and to quantitatively evaluate the resultant changes in properties. This philosophy was employed by Schultz et al. ${ }^{17}$ in their work on heavy deformation and the annealing of $\mathrm{Bi}_{2} \mathrm{Te}_{3}$. However, most

\footnotetext{
12 W. . van Gool, Pbilips Res. Reports, suppl 3 (1961). ${ }^{13}$ O. L. Curtis, Jr., J. Appl. Phys. 33, 2461 (1962).

${ }^{11} \mathrm{R}$. Nitsche, H. U. Bolsterli, and M. Lichtensteiger, J. Phys. Chem. Solids 21, 199 (1961).
}

15 O. L. Curtis, Jr., J. Appl. Phys. 33, 2461 (1962).

${ }^{16}$ C. B. Satterthwaite and R. W. Ure, Jr., Phys. Rev. 108, 1164 (1957).

${ }^{17}$ J. M. Schultz, J. P. McHugh, and W. A. Tiller, Scientific Paper No. 929-8901-P1, Westinghouse Research Laboratories. 
of their results were obtained from polycrystalline materials and, because of the extremely anisotropic nature of $\mathrm{Bi}_{2} \mathrm{Te}_{3}$, do not necessarily apply as such to single crystalline material.

It has been shown in Groups IV and III-V semiconductors that $\mathrm{Co}^{60}$ gamma radiation causes point defects which result in a variation of the Fermi level. ${ }^{18}$ The highly reproducible damage of light gamma radiation in these semiconductors does not generally alter lattice parameters, bonding strength, effective mass, or overall band structure. The damage is due primarily to vacancies and interstitials which result in additional doping levels, trapping centers, and variation in current-carrier mobility.

The purpose of this endeavor is to describe and evaluate $\mathrm{Co}^{60}$ gamma radiation damage in $p$ and $n$-type $\mathrm{Bi}_{2} \mathrm{Te}_{3}$. The results will be used to contribute to the existing knowledge conceming the extrinsic conductivity and band structure of $\mathrm{Bi}_{2} \mathrm{Te}_{3}$.

Bismuth telluride crystallizes in a rhombohedral lattice, although, for convenience, it may be indexed as a hexagonal structure. ${ }^{19}$ The crystal lattice of the compound is constructed of layers (or leaves ${ }^{20}$ ) of atoms lying perpendicular to the major axis of the unit rhombohedral cell. Each layer is composed of atomic sublayers arranged in the sequence $\mathrm{Te}^{\mathrm{I}}-\mathrm{Bi}-\mathrm{Te}^{\mathrm{II}}-\mathrm{Bi}-\mathrm{Te}^{\mathrm{I}}$. The superscripts are used to distinguish types of differently bonded tellurium atoms. ${ }^{20}$ The bonding between tellurium atoms in adjacent layers is assumed to be of the Van der Waals ${ }^{21}$ type, indicating an electron density of effectively zero between these bonds. The $\mathrm{Bi}-\mathrm{Te}^{\mathrm{II}}$ bonds are assumed to be fully covalent, whereas there is some degree of ionicity in the $\mathrm{Bi}-\mathrm{Te}^{\mathrm{I}}$ bonds. ${ }^{20}$ It is assumed that each excess bismuth atom occupies a $\mathrm{Te}^{\mathrm{II}}$ site and behaves as a singly ionized acceptor, and each excess Tellurium atom substitutes for a bismuth atom and acts as a singly ionized donor. ${ }^{16}$ These donor and

${ }^{18}$ D. S. Billington and J. H. Crawford, Jr., Radiation Damage in Solids, Princeton University Press, Princeton, N.J., 1961.

${ }^{19}$ M. H. Franchombe, British J. Appl. Phys. 9, 415 (1958).

${ }^{20} \mathrm{~J}$. R. Drabble and C. H. L. Goodman, J. Pbys. Chem. Solids 5, 142 (1958).

21 J. Black et al., J. Phys. Chem. Solids 2, 240 (1957). acceptor levels lie in the conduction and valence bands, respectively, so that the defects are ionized at all temperatures. ${ }^{22}$ Airapetiants et al. ${ }^{23}$ propose that electron conduction proceeds along bismuth layers and that holes move within the tellurium sublattice. Schultz et al. ${ }^{17}$ report that bismuth and tellurium vacancies $\left(V_{\mathrm{Bi}}, V_{\mathrm{Te}}\right.$ ) are electrically active defects and are acceptor and donor types, respectively, whereas interstitials are effectively neutral.

Single crystals of $\mathrm{Bi}_{2} \mathrm{Te}_{3}$ were grown by the Bridgman technique. ${ }^{16}$ The use of any foreign doping element was avoided. Current-carrier type and resistivity were controlled by the excess or deficit of tellurium in the melt. ${ }^{16}$

Specimens for electrical measurements were cut by an ultrasonic cutter, and leads were soldered in place. Resistivity and Hall measurements were made before and after irradiation by employing the device of Ure. ${ }^{24}$ The method of Pinnow et al. ${ }^{25}$ was used to obtain thermal diffusivities. All radiations were made at $45^{\circ} \mathrm{C}$ in the Solid State Division gamma source No. 2.

The observable changes of the electrical properties of $p$ - and $n$-type $\mathrm{Bi}_{2} \mathrm{Te}_{3}$ on a $100 \mathrm{hr}$ radiation in the gamma source are surprisingly large for the high number of current carriers $\left(10^{18}\right.$ to $10^{19}$ per cubic centimeter) present in the samples.

The Hall coefficient of $n$-type material at $77^{\circ} \mathrm{K}$ increases by approximately $20 \%$, after $100 \mathrm{hr}$ irradiation. The percentage of change in the Hall coefficient of $n$-type material vs the initial Hall coefficient (Fig. 15.4) goes through a maximum at $R_{0}=2.5 \mathrm{~cm}^{3} /$ coulomb. The Hall mobility of $n$-type specimens at $77^{\circ} \mathrm{K}$ approaches a value of approximately $8.5 \times 10^{2} \mathrm{~cm}^{2} \mathrm{v}^{-1} \mathrm{sec}^{-1}$, after extended gamma radiation. For specimens with initial mobilities above this value, the mobility decreases; for specimens below this value, the mobility increases with time in the source. The resistivity of $n$-type $\mathrm{Bi}_{2} \mathrm{Te}_{3}$ at $77^{\circ} \mathrm{K}$ increases by as much as $21 \%$ over its preirradiation value after $100 \mathrm{hr}$ gamma radiation. The percentage of change in

\footnotetext{
${ }^{22}$ B. Yates, J. Electronics 6, 26 (1959).

${ }^{23} \mathrm{~S}$. V. Airapetiants et al., J. Tech. Phys. (USSR), to be published.

${ }^{24} \mathrm{R}$. W. Ure, Jr., Rev. Sci. Instr. 28, 836 (1957).

${ }^{25}$ D. S. Pinnow, Che-yu Li, and C. W. Spencer, Rev. Sci. Instr. 32, 1417 (1961).
} 


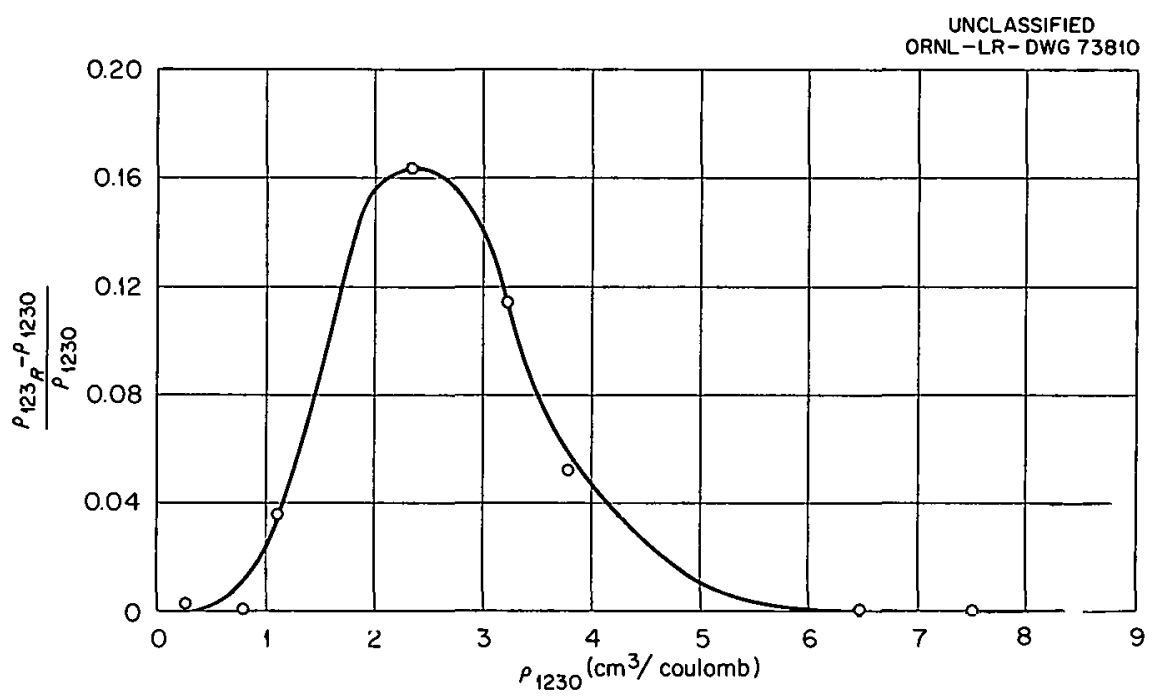

Fig. 15.4. Change in Hall Coefficient After $100 \mathrm{hr}$ in $\mathrm{Co}^{60}$ Gamma Source vs Initial Hall Coefficient for $n-T_{y p e ~ B i}{ }_{2} \mathrm{Te}_{3}$.

resistivity reaches a maximum for specimens of initial mobility of $R / \rho=1.5 \times 10^{3}$. There is no noticeable annealing of the radiation damage in $n$-type material below $150^{\circ} \mathrm{C}$.

Bismuth telluride of the $p$ type is exceedingly sensitive to gamma radiation. For $p$-type specimens with an initial Hall mobility at $77 \% \mathrm{~K}$ of $R / \rho \approx 1.5 \times 10^{3} \mathrm{~cm}^{2} \mathrm{v}^{-1} \mathrm{sec}^{-1}$, the conductivity, Hall coefficient, and Hall mobility go through a sharp minimum, apparently approaching zero, after approximately $100 \mathrm{hr}$ in the source. The crystal remains $p$ type after the minimum. The Hall mobilities of $p$-type materials approach a value of approximately $7.5 \times 10^{3} \mathrm{~cm}^{2} \mathrm{v}^{-1} \mathrm{sec}^{-1}$ at $77^{\circ} \mathrm{K}$ upon extended exposures to $\mathrm{Co}^{60}$ gamma radiation. There appears to be no appreciable annealing of radiation damage in $p$-type $\mathrm{Bi}_{2} \mathrm{Te}_{3}$ at liquid-nitrogen temperatures; however, annealing at room temperature is considerable. Figure 15.5 gives Hall mobility at $77^{\circ} \mathrm{K}$ vs time of anneal at $300^{\circ} \mathrm{K}$ for spccimen 0-24-121P. Hall mobility is an exponential function of annealing time. The curve reaches a minimum after $5 \mathrm{hr}$ at $300^{\circ} \mathrm{K}$ and then approaches the unirradiated value in less than $12 \mathrm{hr}$. All radiation damage appears to be removed upon a 24 -hr anneal at $150^{\circ} \mathrm{C}$.

Thermal diffusivity increases in $n$-type and decreases in $p$-type $\mathrm{Bi}_{2} \mathrm{Te}_{3}$ upon irradiation. In general, the thermal diffusivity is considerably more sensitive to radiation damage than the electrical properties.
It appears that the magnitude of the radiation effects on $p$ - and $n$-type $\mathrm{Bi}_{2} \mathrm{Te}_{3}$ depend, to a large extent, on the percentage of tellurium present. Radiation damage approaches zero for intrinsic material (i.e., tellurium $=60$ at. \%). This result indicaces that the damage involves primarily substitutional atoms ( $\mathrm{Te}_{\mathrm{Bi}}$ in $n$ type and $\mathrm{Bi}_{\mathrm{Te}}^{\mathrm{II}}$ in $p$ type). A Frenkel defect 16,17 involving one substitutional atom reduces the number of

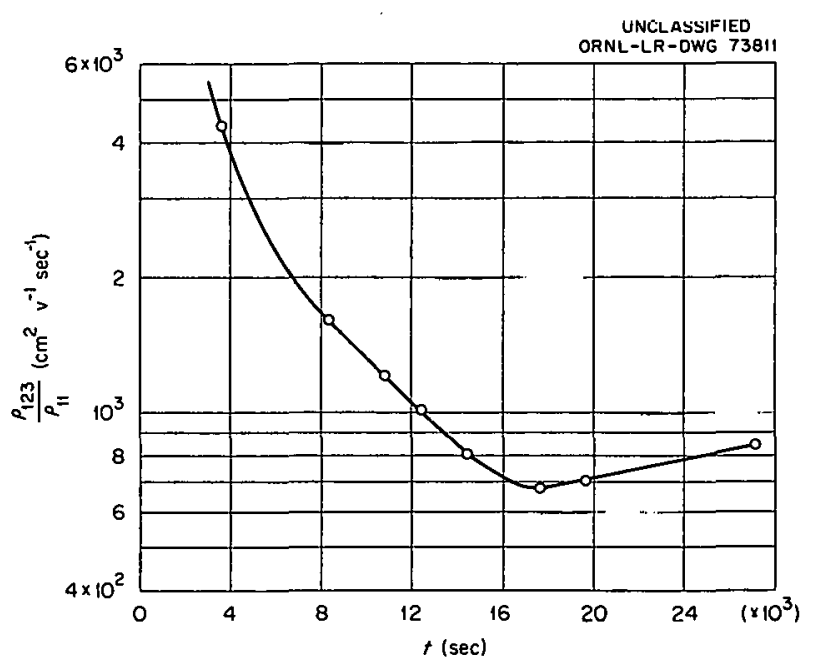

Fig. 15.5. Annealing Curve for Specimen 0-2.4-121P. 
electrons or holes by two in $\mathrm{Bi}_{2} \mathrm{Te}_{3}$. Furthermore, an effect upon the electrical properties resulting from a disturbance of the bismuth sublattice in $n$-type material and the tellurium sublattice in $p$-type material is consistent with the proposition of Airapetiants et al. ${ }^{23}$ The interatomic forces in $\mathrm{Bi}_{2} \mathrm{Te}_{3}$ are much more sensitive to change in the electronegative sublattice than in the electropositive. ${ }^{26}$ Therefore, the electrical properties are more sensitive to the tellurium vacancy created in $p$-type material than to the bismuth vacancy in n-type $\mathrm{Bi}_{2} \mathrm{Te}_{3}$. It is not understood why radiation damage causes a minimum in the Hall coefficient and electrical conductivity in p-type material. It may be that a significant amount of carrier compensation is creared by gamma radiation; however, more experimental verifications are necessary before any conclusion can be drawn.

Cobalt-60 gamma radiation damage in p-type $\mathrm{Bi}_{2} \mathrm{Te}_{3}$ anneals rapidly at rcom temperature. The Hall mobility appears to be an exponential function of time. There are two branches to the curve; therefore there are at least two mechanisms involved in the annealing of the damage. One of the mechanisms, perhaps the second branch, represents the diffusion of bismuth interstitials back to tellurium vacancies.

In $\mathrm{Bi}_{2} \mathrm{Te}_{3}$ crystals with initial Hall mobilities less than $8.5 \times 10^{2} \mathrm{~cm}^{2} \mathrm{v}^{-1} \mathrm{sec}^{-1}$, the Hall mobility increases with increasing doses of gamma radiation. The effect $c$ an be attributed to an alteration of the relaxation-time tensor for scattexing in the collision term of the kinetic equation. ${ }^{27}$ The anisotropy of scattering is a function of carrier density, ${ }^{26}$ and the relation between the Hall coefficient and carrier concentration is a function of the doping level. ${ }^{28}$ It is seen in Fig. 15.6 that gamma radiation changes the slope of the extrinsic portion of the $R$ vs $T^{-1}$ curve without altering the slope of $\rho$ vs $T^{-1}$.

\footnotetext{
${ }^{26} \mathrm{H}$. J. Goldsmid, J. Appl. Phys. 32, 2198 (1961).

${ }^{27}$ I. Ya. Korenblit, Soviet Phys.-Solid State 2, 2737 (1961).

${ }^{28} \mathrm{P}$. A. Walker, Proc. Pbys. Soc. (London) 76, 113 (1960).
}

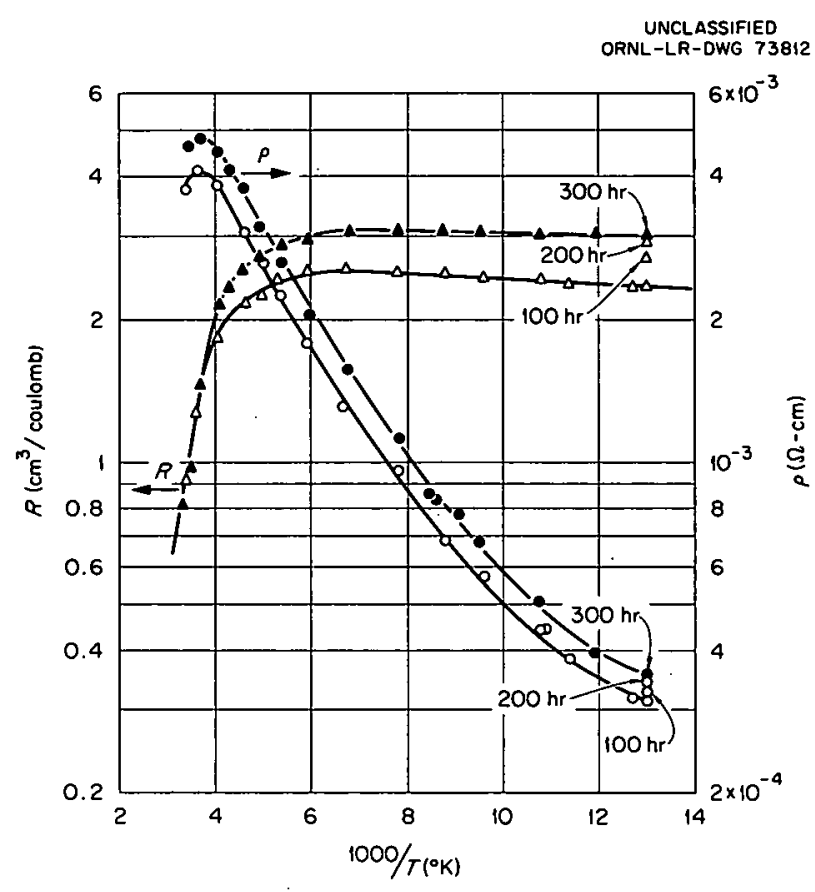

Fig. 15.6. Hall Coefficient and Resistivity Before and After 300-hr Gamma Radiation vs $1 / T$ for Specimen $0-85-88 N$.

\section{Magneto Resistance of $\mathrm{Bi}_{2} \mathrm{Te}_{3}$}

The fact that an increase in Hall mobility upon extended gamma irradiation of $\mathrm{Bi}_{2} \mathrm{Te}_{3}$ may be explained by an alteration of the anisotropy of scattering suggested that the galvanomagnetic experiment of Drabble et al. ${ }^{29}$ be repeated for irradiated $\mathrm{Bi}_{2} \mathrm{Te}_{3}$. Drabble and Wolfe ${ }^{30}$ suggested a "six tilted ellipsoids" model for both the conduction and valence bands. The 12 independent galvanomagnetic terns were derived by assuming that a scattering relaxation time $\tau$ exists and may be written in the form

$$
\tau=b E^{-\lambda},
$$

where the index $\lambda$ and the quantity $b$ depend on the scattering mechanism.

Korenblit ${ }^{27}$ repeated the derivation of Drabble and Wolfe but assumed that the collision term in

${ }^{29}$ J. R. Drabble, R. D. Graws, and R. Wolfe, Proc. Pbys. Soc. 71, 3 (1958).

$30 \mathrm{~J} . \mathrm{R}$. Drabble and R. Wolfe, Proc. Pbys. Sco. 69, $1.101(1.956)$. 
the kinetic equation is obtained for scattering described by a relaxation-time tensor, the components of which are independent of direction of the electron wave vector. The resulting galvanomagnetic coefficients involve an anisotropic scattering term which is not present in the expressions of Drabble and Wolfe.

The effect of irradiation on the magneto-resistance parallel to the cleavage plane is shown in Fig. 15.7 for $n$-type specimen $0-85-88 \mathrm{~N}$ and $p$-type specimen 0-24-121P. The value of $\Delta \rho / \rho$ increases with time of irradiation for $n$-type material and finally saturates after a change of approximately $10 \%$. For $p$-type material $\Delta \rho / \rho$ initially decreases and then increases continuously upon extended irradiation. If it is assumed that $\Delta \rho / \rho$ is proportional to the mobility squared, ${ }^{31}$ then (where the notation is the same as that of ref 14)

$$
\frac{\left(\rho_{123}\right)^{2}}{\left(\rho_{11} \rho_{1133}\right)}=K \text {, }
$$

$31_{1}$. Eastermann and A. Foner, Phys. Rev. 79, 365 (1950). where $K$ is a constant. Equation (2) was substantiated by Drabble et al. ${ }^{29}$ for $n$-type material over a rather limited range of doping. However, the results could not be repeated by Goldsmid ${ }^{26}$ on highly doped $n$-type crystals. Likewise, $K$ appears to be a slowly varying function of gamma radiation dose for $n$-type $\mathrm{Bi}_{2} \mathrm{Te}_{3}$. The value of $K$ changes by over one-half an order of magnitude following extended irradiation of $p$-type material. Therefore, preliminary results of the effect of $\mathrm{Co}^{60}$ gamma radiation upon the galvanomagnetic properties of $\mathrm{Bi}_{2} \mathrm{Te}_{3}$ indicate that anisotropic scattering must be considered in the derivation.

\subsection{EXPERIMENTAL THRESHOLD IRRADIATION ENERGY REQUIRED FOR THE PRODUCTION OF DEFECT CLUSTERS}
J. W. Cleland
R. F. Bass

It has long been recognized that the type of lattice defect created by irradiation depends critically on the type and energy of the incident
(In) $0-85-88 \mathrm{~N}$, PRE-IRRADIATION

(2n) $0-85-88 \mathrm{~N}, 100 \mathrm{hr}$ IRRADIATION

(3n) $0-85-88 \mathrm{~N}, 300 \mathrm{hr}$ IRRADIATION

(1p) 0-24-12IP, PRE-IRRADIATION
UNCLASSIFIED

ORNL-LR-DWG 73813

(2p) 0-24-121P, $100 \mathrm{hr}$ IRRADIATION

(3p) 0-24-121P, $200 \mathrm{hr}$ IRRADIATION

(4p) $0-24-121 \mathrm{P}, 300 \mathrm{hr}$ IRRADIATION

(5p) 0-24-121P, $500 \mathrm{hr}$ IRRADIATION

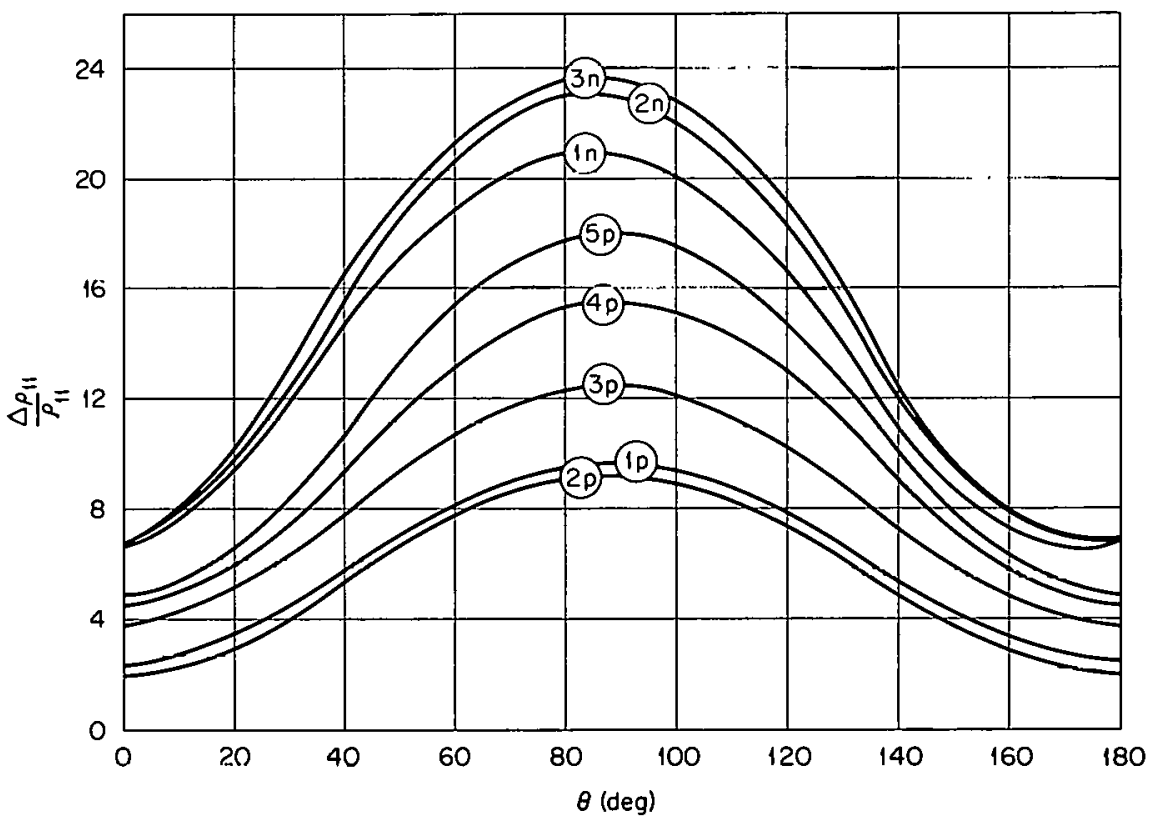

Fig. 15.7. Magnetoresistance vs Angle Between Current and Magnetic Field. 
bombarding particle. The threshold energy for displacement of a lattice atom to a stable interstitial position is about $15 \mathrm{ev}$ for germanium and silicon and 30 to $60 \mathrm{ev}$ for many metals. It has been suggested that the resultant defect is an isolated (point) Frenkel-type interstitial-vacancy pair. One can therefore hope to examine the separate effect of this type of latrice disorder through the use of $\mathrm{Co}^{60}$ photon or low-energy (<2-Mev) electron irradiation experiments. The effect of reactor neutrons, fission spectrum neutrons, and monoenergetic 14-Mev neutrons has been shown to be markedly different for certain experimental observations on semiconductors and metals. It has been suggested that the resultant damage is in the form of disordered regions or defect clusters. It would therefore seem important to attempt to ascertain the minimum incidentparticle energy or lattice-atom recoil energy required to produce defect clusters; to that end the following experiments have been conducted or are under consideration.

1. The effect of $\mathrm{Co}^{60}$ photon irradiation is to introduce defect states that act as acceptors 32 in $n$-type germanium, $n$ type is converted to $p$ type, and it is found that there is an apparent saturation in hole concentration with a limiting Fermi level of $0.24 \mathrm{ev}$ above the valence band after a relatively small total dose ( $10^{16}$ photons $/ \mathrm{cm}^{2}$ for high-purity specimens). Continued irradiation, to doses as high as $10^{19}$ photon $\mathrm{s} / \mathrm{cm}^{2}$, produces no further change in the hole concentration or Fermi-level position, whereas irradiation with $3 \times 10^{12}$ fission neutrons $/ \mathrm{cm}^{2}$ increases the hole concentration and lowers the Fermi level.

2 . The critical shear stress of copper single crystals was not increased ${ }^{33}$ as a consequence of irradiation with $10^{18} \mathrm{Co}^{60}$ photons $/ \mathrm{cm}^{2}$, whereas the critical shear stress of copper single crystals has been shown to have a cube-root dependence $e^{34}$ on the total incident flux of $10^{13}$ $10^{19}$ neutrons $/ \mathrm{cm}^{2}$ from a reactor.

The onset of increase in hole concentration and decrease in the apparent acceptor ionization energy in germanium, and the onset of increase

${ }^{32} \mathrm{~J}$. W. Cleland, J. H. Crawford, Jr., and D. K. Holmes, Phys. Rev. 102, 722 (1956).

${ }^{33} \mathrm{~F}$. W. Young, unpublished data.

${ }^{34} \mathrm{~T}$. H. 'Blewitt, to be published in Nuovo Cimento; F. W. Young, unpublished data. in critical shear stress in copper single crystals have, therefore, been selected as the criteria to be utilized to determine the threshold energy of a primary recoil for the production of defect clusters in these two types of materials.

3. It has recently been shown ${ }^{35}$ that lattice defects can be produced in semiconductors from the energetic capture gamma rays that are emitred by cadmium upon thermal-neutron capture. Approximately $25 \%$ of these gamma rays have energies in excess of $3 \mathrm{Mev}$, and these would produce energetic Compton electrons that would impart a primary recoil energy of $300 \mathrm{ev}$; however, the hole concentration and Fermi-level position of converted germanium is not affected by a total irradiation of $5 \times 10^{15}$ cadmium gamma rays $/ \mathrm{cm}^{2}$. The critical shear stress of copper single crystals is also not increased ${ }^{33}$ as a consequence of irradiation with capture gamma rays from cadmium.

4. Thermal-neutron-induced $(n, y)$ recoils have been employed ${ }^{36,37}$ to produce lattice defects in materials. One can calculate the maximum kinetic energy of recoil as a consequence of capture gamma-ray emission as $180 \mathrm{ev}$ in germanium, $400 \mathrm{ev}$ in copper, and $780 \mathrm{ev}$ in silicon. Note that these values are greatly in excess of the threshold energy for lattice-atom displacement; however, the end result of $(n, y)$ recoil in germanium and copper is apparently identical with that obtained from $\mathrm{Co}^{60}$ photons or cadmium gamma rays.

5. One cannot hope to use conventional radioisotope photoncutron sources in radiation-damage experiments, since the total available flux is too low; however, special sources have been constructed, or could be designed, to provide an intense source of fairly energetic photoneutrons.

(a) Hennelly 38 has described an SbBe source with a total output of $10^{10}$ neutrons/sec from

${ }^{35}$ J. W. Cleland, R. F. Bass, and J. H. Crawford, Jr., to be published in the Journal of Applied Pbysics: see also J. W. Cleland, R. F. Bass, and J. H. Crawford, Jr., "Lattice Defect Production in Thermal Neutron Shielded Materials," this report.

${ }^{36} \mathrm{~J}$. H. Crawford, Jr., and J. W. Cleland, "Transmutation Doping and Recoil Effects in Semiconductors Exposed to Thermal Neutrons," vol 1, p 269 in I.A.E.A. Conference on Radioisotopes, Copenhagen, 1960; Intern. J. Appl. Radiation Isotopes 9, 189 (1960); J. W. Cleland, to be published in Nuovo Cimento.

${ }^{37} R$. R. Coltman, to be published in the Journal of Applied Physics.

${ }^{38}$ E. J. Hennelly, Nucleonics 19(3), 104, 124 (1961). 
1000 curies of antimony, which gives a useful flux of $\sim 10^{8}$ neutrons $\mathrm{cm}^{-2} \mathrm{sec}^{-1}$. Approximately $70 \%$ of the $\mathrm{Sb}^{124}$ gamma rays have an energy of $1.74 \mathrm{Mev}$, and the $(\gamma, n)$ threshold of beryllium is $1.67 \mathrm{Mev}$; thus the maximum neutron energy from such a source is $70 \mathrm{kev}$ and the mean energy is 25 to $35 \mathrm{kev}$. Although the effect of gamma rays is expected to be much greater than that of the much smaller flux of photoneutrons from such a source, the $(y, n)$ cross section ${ }^{39}$ in the beryllium is only about $0.8 \times 10^{-27}$ barn, the qualitatively different effect of the latter should be detectable ${ }^{40}$ if defect clusters were produced. The maximum energy of a recoil in germanium or copper for an incident $25-\mathrm{kev}$ photoneutron is $\sim 600 \mathrm{ev}$. Therefore an SbBe source of the magnitude described by Hennelly ${ }^{38}$ might be employed to provide a lower-limit check on the minimum recoil energy that is required to produce disordered regions in germanium or increase the critical shear stress of copper. Such an experiment is under way at the present time with the Savannah River SbBe source.

(b) Wattenburg ${ }^{41}$ has employed a photoneutron source to measure total cross-section values from $30 \mathrm{kev}$ to about $1.0 \mathrm{Mev}$ for many materials. Metal rods $(1.25 \times 5 \mathrm{~cm})$ of manganese, gallium, indium, antimony, lanthanum, or fused $\mathrm{NaF}$ were activated in a flux of $10^{12}$ neutrons $\mathrm{cm}^{-2} \mathrm{sec}^{-1}$ and then placed inside a cylinder of beryllium or $\mathrm{D}_{2} \mathrm{O}$. The actual variation in photoneutron energy in $(\gamma, n)$ reactions is only $1 \%$; however, this particular experimental arrangement had a total energy variation of about $15 \%$, as measured by proton recoils in a cloud chamber, due to energy losses in the beryllium or $\mathrm{D}_{2} \mathrm{O}$. Representative $Q$ values (neutron emission rate per second) ranged from $2 \times 10^{7}$ to about $1.5 \times 10^{6}$ in these experiments; however, these $Q$ values might be incrcascd by a factor of 200 for the Oak Ridge Research Reactor and by a factor of 3000 to 5000 for the High Flux Isotope Reactor; hence, an irradiation facility of extreme versatility might be designed from these general considerations.

${ }^{39}$ E. Guth and M. Mullin, Pbys. Rev. 76, 234 (1949).

$40 \mathrm{~J}$. W. Cleland, D. Binder, and J. H. Crawford, Jr., Solid State Div. Ann. Progr. Rept. Aug. 30, 1956, ORNL-2188, p 7 .

$41_{A}$ Warrenherg, Phys. Re1l. 71, $497(1947)$ (c) Preliminary experiments have been conducted with an $\mathrm{NaF}-\mathrm{Be}$ source. Fused $\mathrm{NaF}$ was irradiated in an isotope capsule in the No. 1 hydraulic facility of the Oak Ridge Research Reactor in an estimated thermal flux of $2 \times 10^{14}$ neutrons $\mathrm{cm}^{-2} \mathrm{sec}^{-1}$ for $24 \mathrm{hr}$, after which the capsule, containing an estimated 280 curies of $\mathrm{NaF}$, was placed inside a $3 / 4$ in. ID $\times 13 / 4$ in. OD cylinder of beryllium for 24 to $42 \mathrm{hr}$ of radioactive decay. Ceric sulfate dosimeter ${ }^{42}$ techniques indicated an average value of $1.05 \times 10^{5} \mathrm{r} / \mathrm{hr}$, or $1.36 \times 10^{10}$ photons $\mathrm{cm}^{-2} \mathrm{sec}^{-1}$ for the geometry employed. The atomic displacement cross-section data of Oen and Holmes ${ }^{43}$ were used to calculate an expected number of lattice-atom displacements from the $2.76-$ and $1.38-\mathrm{Mev} \mathrm{Na}^{24}$ gamma rays $\left(1.04 \times 10^{14}\right.$ defects $/ \mathrm{cm}^{3}$ over $\left.24 \mathrm{hr}\right)$, and the $(\gamma, n)$ cross-section data ${ }^{39}$ for beryllium were used to estimate a total $0.82 \mathrm{Mev}$ photoneutron dose of $6 \times 10^{10} / \mathrm{cm}^{2}$ over $24 \mathrm{hr}$. High-purity singlecrystal n-type samples of germanium were exposed. Figure 15.8 is a plot of $\log \mathrm{Hall}$ coefficient and resistivity vs inverse temperature before irradiation (curves I) and after successive 24-hr activations and decays (curves II, $42 \mathrm{hr}$; curves III, $40 \mathrm{hr}$; curves IV, $24 \mathrm{hr}$; and curves V, $24 \mathrm{hr}$ ). The measured removal of conduction electrons, determined at $78^{\circ} \mathrm{K}$, for these exposures at the ambient temperature of the reactor pool were $4.22 \times 10^{13}, 2.66 \times 10^{13}, 2.59 \times 10^{13}$, and $2.28 \times 10^{13}$ per cubic centimeter, respectively. Corresponding data for a similar sample that was exposed to the $\mathrm{Na}^{24}$ gammas without the beryllium cylinder gave a measured removal of conduction electrons of $1.99 \times 10^{13}$ and $2.69 \times 10^{13}$ per cubic centimeter for two consecutive 24-hr activations and 25-hr decays. One high-purity $n$-type sample, $n_{0}=6.8 \times 10^{12}$ electron $/ \mathrm{cm}^{3}$ at $78^{\circ} \mathrm{K}$, was converted to $p$-type material after the first 42-hr decay and remained a high-resistivity $p$-type sample, and with an apparent $0.24-\mathrm{ev}$ activation energy, after a total of five successive activations that totaled $170 \mathrm{hr}$ of decay. It is evident from these data that we have constructed a copious gamma-ray source. The removal rate of conduction electrons agrees within about a factor

\footnotetext{
${ }^{42}$ We are indebted to $L$. C. Bate of the Analytical Chemistry Division for these measurements.

${ }^{43} \mathrm{O}$. S. Oen and D. K. Holmes, J. Appl. Pbys. 30, 1789 (1959).
} 


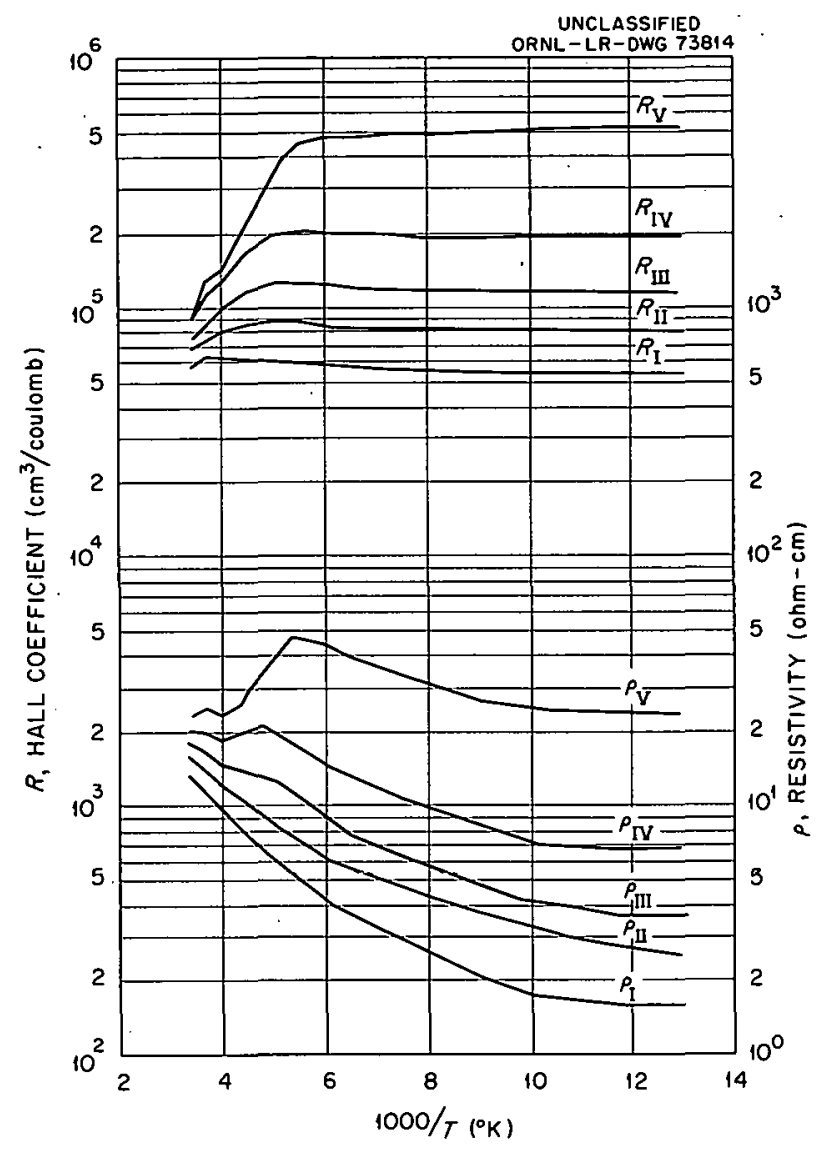

Fig. 15.8. Hall Coefficient and Resistivity vs Inverse Temperature for n-Type Germanium Before Irradiation and After Successive Exposures in an NaBe Source.

of 4 of that predicted by theory. ${ }^{43}$ This result is not disturbing in that experimental values are typically..low, compared with theoretical values, in most radiation-damage experiments. The gammaray effect is also not disturbing, in itself, in that these do not introduce defect clusters. If the calculated dose of photoneutrons is valid, then a total dose of $3.6 \times 10^{11}(0.82-\mathrm{Mev})$ neutrons $/ \mathrm{cm}^{2}$ would be expected in these experiments. No evidence of defect clusters was observed, whereas an estimated $3.2 \times 10^{12}$ neutrons $/ \mathrm{cm}^{2}$ (fission spectrum) converted $n$-type germanium to $p$-type and indicated the production of defect clusters (see paragraph 9 below).

(d) Molten alkali fluoride salt has been utilized as a heat-exchange medium in certain loop experiments, test reactors, and research or power reactors. The saturation activity of the sodium in sodium fluoride coolant in the intermediate heat exchanger of the North.American Aviation-Atomics Intemational SRE reactor is 0.28 curie $/ \mathrm{cm}^{3}$, and the gamma flux has been estimated as $2 \times 10^{5}$ $\mathrm{r} / \mathrm{hr}$. It is possible that an extremely potent source of $0.2-\mathrm{Mev}\left(\mathrm{D}_{2} \mathrm{O}\right)$ or $0.82 \mathrm{-Mev}$ (beryllium) photoneutrons could be obtained from such a facility; however, this type of radiation-effects installation has not been critically evaluated for use as a photoneutron source.

(e) Photoneutrons can also be produced from beryllium or $\mathrm{D}_{2} \mathrm{O}$ by converting the electrons from a linear accelerator to bremsstrahlung in a high- $Z$ converter (i.e., 30 mils of water-cooled gold) and then letting the gamma rays impinge on beryllium or $\mathrm{D}_{2} \mathrm{O}$. Data from Harwell ${ }^{44}$ would indicate a total output of $1.5 \times 10^{19}$ neutrons $/ \mathrm{kw}$ sec for 3.1-Mev electrons on beryllium, $2 \times 10^{9}$ neutrons $/ \mathrm{kw}-\mathrm{sec}$ for $3.1 \mathrm{-Mev}$ electrons on $\mathrm{D}_{2} \mathrm{O}$, and $5 \times 10^{10}$ neutron $\mathrm{s} / \mathrm{kw}-\mathrm{sec}$ for 6.0 -Mev electrons on beryllium. Commercial machines are available with an average beam power of 3 to $65 \mathrm{kw}$; however, the only electron accelerator at ORNL is the small $2.0-\mathrm{Mev}$ Van de Graaff in the Chemistry Division.

(f) It has been suggested that the energetic capture gamma rays from cadmium might be used in a thermal-neutron flux to produce photoneutrons from beryllium or $\mathrm{D}_{2} \mathrm{O}$. An altemative approach is that of Almqvist, ${ }^{45}$ who examined the $\mathrm{Li}^{6}(n, a) \mathrm{He}^{3}$ reaction in a thermal-neutron flux, and the subsequent $\mathrm{Li}^{6}(t, n) \mathrm{Be}^{8}$ reaction, which produced $2.7 \times 10^{-4}(15.3-\mathrm{Mev})$ neutron per triton. There is a fundamental limitation conceming photoneutron production in a reactor that should be considered in all radiation-effects experiments. The background fast -(damaging) flux in a thermalneutron locale can be estimated from the cadmium ratio. For example, the vertical graphite thimbles of the Argonne CP-5 reactor have a thermal flux in excess of $10^{12}$ neutrons $\mathrm{cm}^{-2} \mathrm{sec}^{-1}$ and a cadmium ratio in excess of $10^{4}$; however, these data would indicate that the background fast (damaging) flux from the reactor itself is about $10^{-3}$ that of the thermal-neutron flux, whereas the a vailable flux of photoneutrons from beryllium, $\mathrm{D}_{2} \mathrm{O}$, or the $\mathrm{Li}^{6}(t, n)$ reaction is only about $10^{-4}$ that of the thermal-neutron flux. The effect of

\footnotetext{
${ }^{44}$ B. J. Moyer, Nature 167, 346 (1951).

${ }^{45}$ E. Almquist, Can. J. Research 28A, 433 (1950).
} 
photoneutrons would therefore be totally masked by the fast-neutron background, unless a facility with a cadmium ratio in excess of $10^{5}$ were used. The cadnium ratio of the slant animal tunnel of the ORNL Graphite Reactor is in excess of $10^{5}$; however, the thermal flux in this locale is only $1.5 \times 10^{9}$ neutron $\mathrm{cm}^{-2} \mathrm{sec}^{-1}$.

6 . Some preliminary experimental results have been obtained with the $\mathrm{Li}^{7}(p, n) \mathrm{Be}^{7}$ reaction, using the 5.5-Mev Van de Graaff ${ }^{46}$ at ORNL. The neutron source is that of a $1 / 4$-in.-diam beam of protons that is incident on a rotating target coated with lithium. One must accept an inherent nonuniformity in the neutron flux, since the samples must be located about $1 / 2$ in. from the target to obtain a reasonable total dose. The lower limit on neutron energy was $0.7 \mathrm{Mev}$ because the machine was unstable for protons with energies lower than $2.42 \mathrm{Mev}$. The maximum flux of 0.7 Mev neutrons, as determined with a long counter, was about $5 \times 10^{7} \mathrm{~cm}^{-2} \mathrm{sec}^{-1}$ for a beam current of $7 \mu \mathrm{a}$. The energy spread was $100 \mathrm{kev}$. A total irradiation of $10 \mathrm{hr}$ was required to amass an estimated total dose of $1.8 \times 10^{12}$ neutrons $/ \mathrm{cm}^{2}$, which removed $1.6 \times 10^{13}$ electrons $/ \mathrm{cm}^{3}$ from a sample of $n$-type germanium. A total irradiation of 30 to $50 \mathrm{hr}$ under these conditions would be required to convert the purest known $n$-type germanium to $p$-type, and this irradiation time would be doubled or tripled for higher-energy neutrons, since the yield decreases with increasing proton energy. One p-type sample of germanium, which had previously been converted from n-type by a total dose of $10^{18} \mathrm{Co}^{60}$ photons $/ \mathrm{cm}^{2}$, was also irradiated with an estimated $1.8 \times 10^{12}(0.7-\mathrm{Mev})$ neutrons $/ \mathrm{cm}^{2}$. No evidence of defect clusters or disordered regions was observed.

7. The use of other conventional radioisntope neutron sources for radiation-damage experiments is also limited, at present, by low total fluxes. Such typical commercial sources as Po-Be $(90$ curies), $2 \times 10^{8}$ neutrons/sec (138-day half-life), or $\mathrm{Pu}-\mathrm{Be}$ ( 10 curies), $2 \times 10^{7}$ neutrons $/ \mathrm{sec}(24,000$ yr half-life), are not of sufficient intensity. The neutron energy spectrum of this type of source is complex in that the average neutron energy is 4.5 Mev and the maximum neitron energy is

\footnotetext{
${ }^{4} G_{W}$ e are indebted to P. H. Stelson and W. T. Newton of the High Voltage Laboratory for assistance in thesc experiments.
}

10.8 Mev. One can, however, demonstrate radiation-damage effects ${ }^{47}$ in germanium from an $\mathrm{Am}^{241}$-Be source (16 curies), and one can also predict that $\mathrm{Cm}^{242}-\mathrm{Be}$ and $\mathrm{Cf}^{252}-\mathrm{Be}$ sources will be of great value in certain radiation-effects experiments in the future.

8. High-purity $n$-type samples of germanium were exposed at reactor ambient temperatures $\left(40^{\circ} \mathrm{C}\right)$ in the slant animal tunnel of the ORNL Graphite Reactor $\left(1.5 \times 10^{9}\right.$ thermal neutrons $\left.\mathrm{cm}^{-2} \mathrm{sec}^{-1}\right)$ betwe en two $1 \times 1 \times \frac{1}{8}$ in. fission plates that contained 17.16 wt \% (2.5 at. \%) of $93.16 \%$ enricied $U^{235}$. The estimated flux of fission spectrum neutrons was $3.8 \times 10^{12} \mathrm{~cm}^{-2} \mathrm{hr}^{-1}$. Figure 15.9, which is a plot of $\log$ Hall coefficient and

${ }^{47} \mathrm{~J}$. W. Cleland and R. F. Bass, unpublished data.

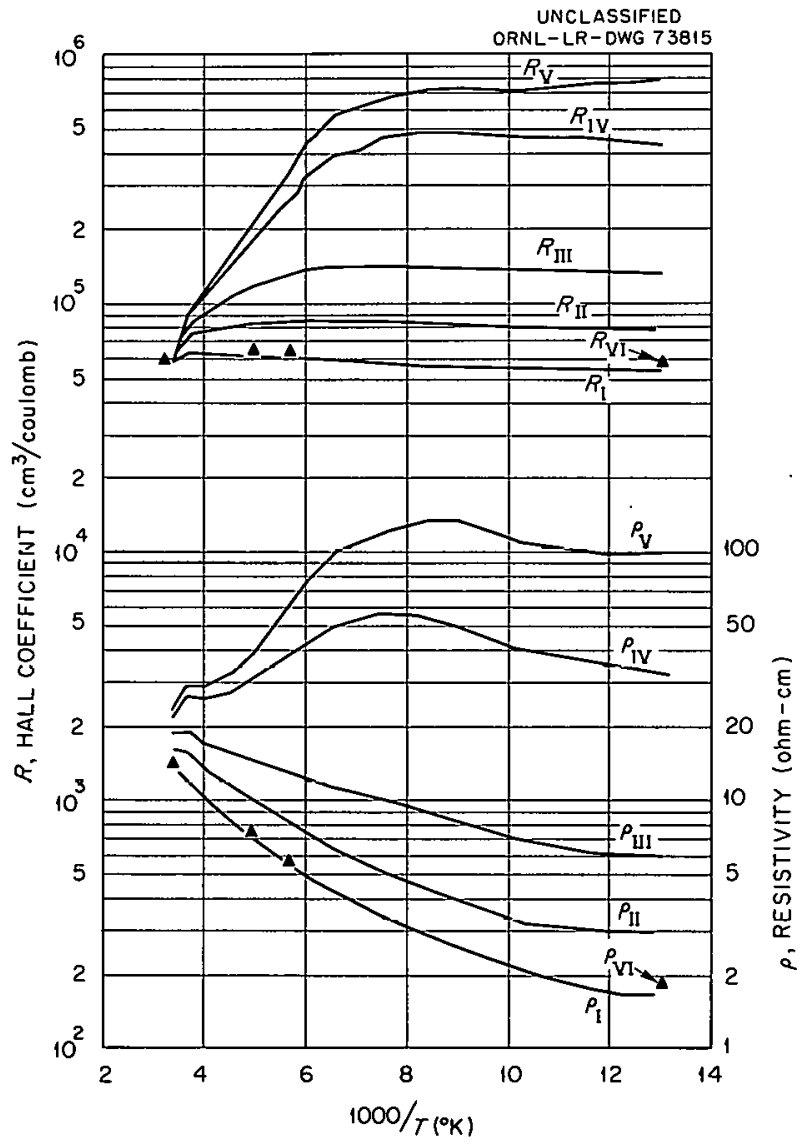

Fig. 15.9. Hall Coefficient and Resistivity vs Inverse Temperature for $n$-Type Germanium Before Irradiation, After Fission-Spectrum Neutron Irradiation, and After Annealing. 
resistivity vs inverse temperature, represents typical data before irradiation (curves I), after three successive 2 -hr irradiations (curves II, III, and IV), after a $1 / 2$-hr irradiation (curves V), and after a vacuum heat treatment of $20 \mathrm{hr}$ at $450^{\circ} \mathrm{C}$ (curves VI). The measured removal of conduction electrons as determined at $78^{\circ} \mathrm{K}$ was $4.18 \times 10^{13}$, $3.67 \times 10^{13}, 3.86 \times 10^{13}$, and $6.79 \times 10^{12}$ per cubic centimeter for the four irradiations, corresponding to removal rates of 5.5, 4.8, 5.1, and 3.6 per incident fission spectrum neutron. These values are in reasonable agreement ${ }^{48}$ with -3.2 electrons per neutron as obtained in the Hole 51 fission chamber doughnut of the ORNL Graphite Reactor for an estimated total fast (damaging) flux of $8 \times 10^{11}$ neutrons $\mathrm{cm}^{-2} \mathrm{sec}^{-1}$. Note that more than $95 \%$ of the radiation-induced defects were removed by the vacuum anneal. This value may well be $100 \%$, since the replacement of leads, chemical etch, and remeasurement of sample dimensions may account for the $5 \%$ discrepancy. Figure 15.10, which shows plots of log Hall coefficient and resistivity. vs inverse temperature, indicates the effect of fission spectrum neutron irradiation on a sample of high-purity $n$-type germanium with an initial electron concentration of $6.6 \times 10^{12} / \mathrm{cm}^{3}$ (at $78^{\circ} \mathrm{K}$ ) before irradiation (curves I), after three successive 1 -hr irradiations (curves II, III, and IV), and after one 2-hr irradiation (curves V). Note that the sample was converted to $p$-type after an estimated total dose of $3.2 \times 10^{12}$ fission spectrum neutrons $/ \mathrm{cm}^{2}$ and that the $p$-type hole concentration is increased and the apparent activation energy is decreased by successive irradiations. The measured rate of addition of p-type holes was $1.41 \times 10^{12}, 3.91 \times 10^{12}$, and $6.6 \times 10^{12} \mathrm{per}$ cubic centimeter, corresponding to an addition. rate of $0.37,1.03$, and 0.87 holes per fission spectrum neutron. These values are also in reasonable agreement ${ }^{48}$ with 0.8 hole per neutron as obtained in Hole 51 (see above).

9. High-purity n-type samples of germanium have also been. exposed to the monoenergetic (14-Mev) neutrons from a $\mathrm{D}(t, n) \mathrm{He}^{4}$ reaction. These data have been discussed in previous reports ${ }^{49}$

\footnotetext{
${ }^{48} \mathrm{~J}$. H. Crawford, Jr., and J. W. Cleland, Progress in Semiconductors, vol 2, p 67, Wiley, New York, 1957.

${ }^{49} \mathrm{O}$. L. Curtis, Jr., and J. W. Cleland, Solid State Div. Ann. Progr. Rept. Aug. 31, 1959. ORNL-2829, p 12.9; Solid Stale Div. Ann. Progr. Rept. Aug. 31. 1958, DRNL-2614, pp 4-6.
}

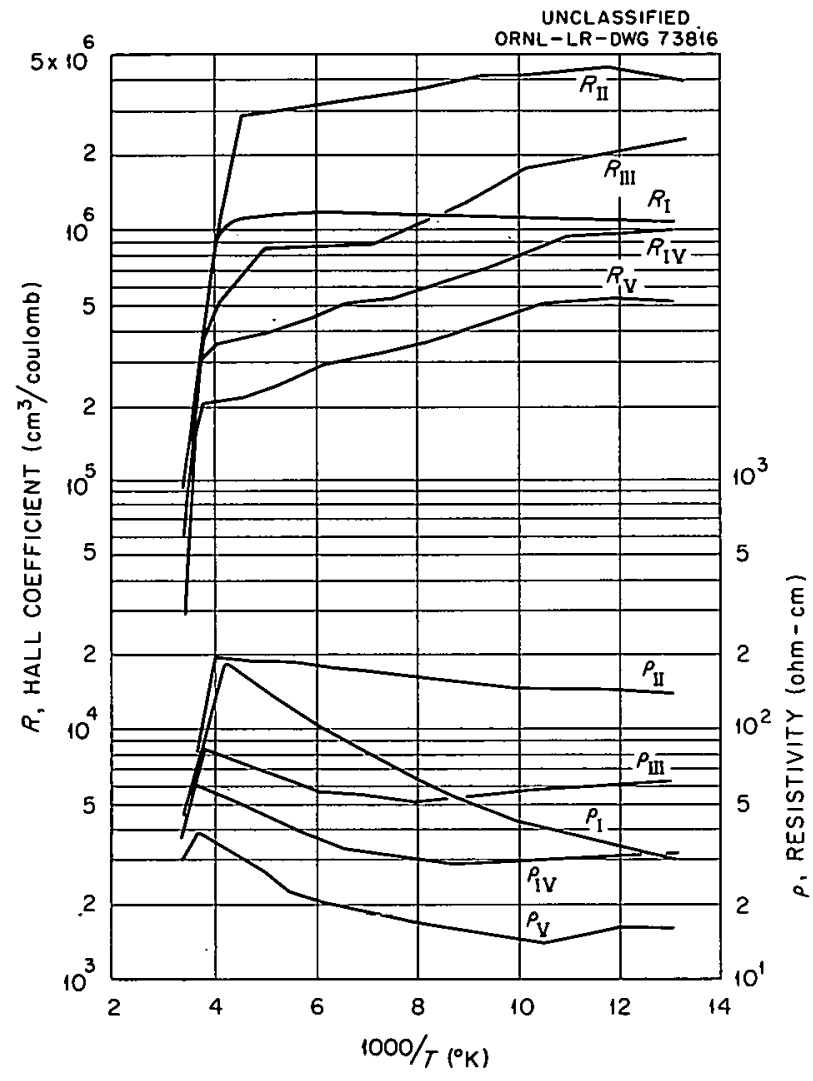

Fig. 15.10. Hall Coefficient and Resistivity vs Inverse Temperature for High-Purity n-Type Germanium Before Irradiation and After Fission-Spectrum Neutron Irradiation (Now $p$-Type).

and in the open literature, ${ }^{50}$ but not in connection with the current studies of irradiation energy vs nature of damage. Figure 15.11 shows plots of $\log$ Hall coefficient and resistivity vs inverse temperature, obtained on a sample identical to that used in Fig. 15.3 for a series of irradiations. Note that $2.6 \times 10^{12}(14-\mathrm{Mev})$ neutron $\mathrm{s} / \mathrm{cm}^{2}$ converted the material to $p$-type in a similar manner to that of $3.2 \times 10^{12}$ (fission spectrum) neutrons $/ \mathrm{cm}^{2}$ and that the p-type hole concentration is increased and the apparent activation energy is decreased by successive irradiations.

In summary, it has been indicated that defect clusters (disordered regions) are apparently introduced in germanium with a total irradiation of

50 O. L. Curtis, Jr., and J. W. Cleland, J. Appl. Pbys. 31, 423 (1.960). 


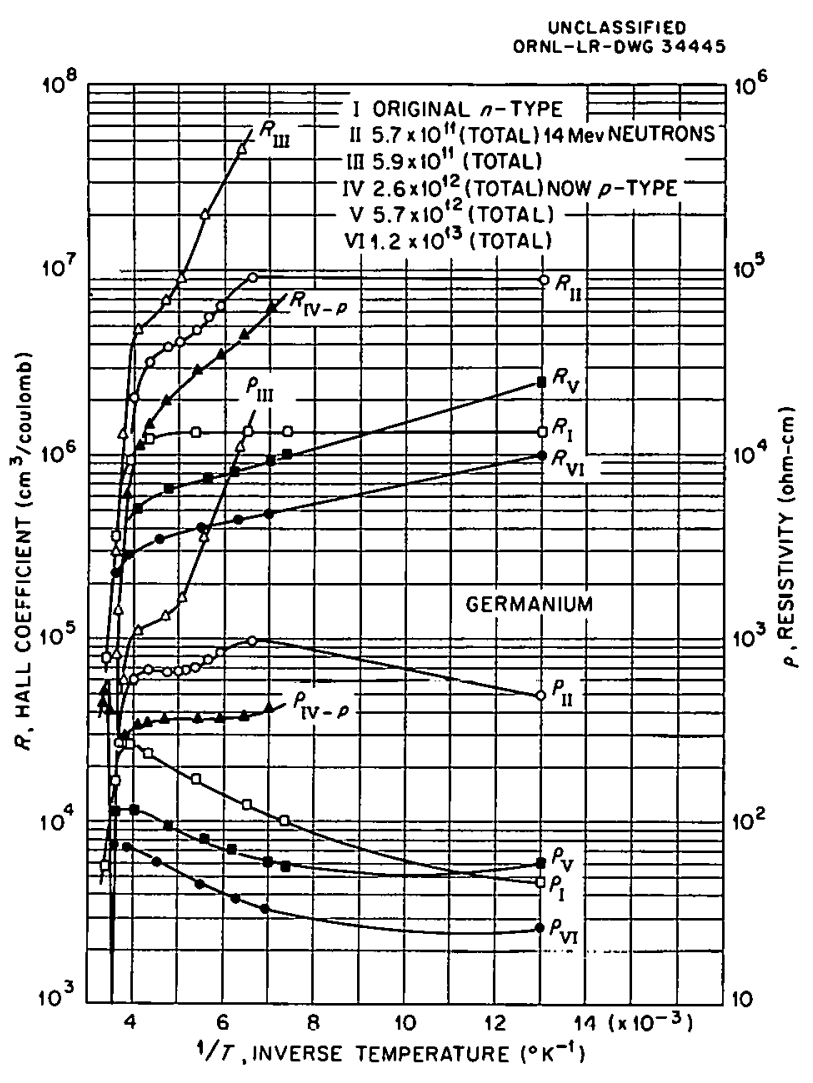

Fig. 15.11. Hall Coefficient and Resistivity vs Reciprocal Temperafure of Initially n-Type Germanium After Successive Irradiations of 14-Mev Neutrons.

$3 \times 10^{12}$ fission spectrum or $14-\mathrm{Me}$ n eutron $\mathrm{s} / \mathrm{cm}^{2}$, whereas no evidence for such defect clusters has been obtained for a total irradiation of $10^{19} \mathrm{Co}^{60}$ photons $/ \mathrm{cm}^{2}, 5 \times 10^{15}$ cadmium gamma rays $/ \mathrm{cm}^{2}$, $10^{16}(n, \gamma)$ recoils $/ \mathrm{cm}^{2}, 1.8 \times 10^{12}(0.7-\mathrm{Mev})$ photoneutrons $/ \mathrm{cm}^{2}$, or $3 \times 10^{11}(0.82-\mathrm{Mev})$ photoneutrons $/ \mathrm{cm}^{2}$. Therefore it must be concluded that a neutron energy in excess of 0.7 to 0.8 $\mathrm{Mev}$, which corresponds to a primary recoil energy of $20,000 \mathrm{ev}$, is required to produce defect clusters or disordered regions, unless a larger total dose is required for this range of neutron energies than has been experimentally available to date.

\subsection{LATTICE DEFECT PRODUCTION IN THERMAL NEUTRON SHIELDED MATERIALS 51}
J. W. Cleland
R. F. Bass
J. H. Crawford, Jr.

In connection with our studies of lattice displacements in silicon and germanium caused by recoil of nuclei upon thermal-neutron capture and capture gamma-ray emission, ${ }^{52}$ it was observed that a comparable amount of lattice damage, as indicated by a change in carrier concentration, was observed even when the specimens were shielded from thermal neutrons by cadmium foil. On the other hand, no detectable change in carrier concentration was observed when a boroncontaining shield was used. It is well known that cadmium emits energetic gamma rays upon neutron capture, whereas the dominant capture process of $8^{10}$ is an $(n, \alpha)$ reaction, with essentially all the $a$ 's being stopped in the shield.

Tabulations of the gamma-ray spectrum resulting from thermalneutron capture in cadmium have been given by Bartholomew and $\mathrm{Higgs}^{53}$ and Groshev et al., ${ }^{54}$ and these are summarized in Table 15.1. Over one-half the gamma rays emitted fall in the interval 1-5 Mev. Prior experience 55,56 has shown that $\mathrm{Co}^{60}$ gamma rays (1.3 Mev) are sufficiently energetic to displace lattice atoms through the agency of Compton electrons in both silicon and germanium. Moreover, the displacement cross section via this mechanism is expected to increase markedly with gamma-ray energy. This is reflected in the calculations of Oen and Holmes, ${ }^{57}$ whose results for aluminum $(A=27)$ and copper $(A=63)$, obtained for a displacement energy of $15 \mathrm{ev}$, are shown in Table 15.2. Because of the close similarity of atomic weights of these elements to those of silicon and germanium, the tabulated values should be reasonably representative for

${ }^{51}$ J. W. Cleland, R. F. Bass, and J. H. Crawford, Jr., to be published in the Joumal of Applied Pbysics.

$52 \mathrm{~J}$. H. Crawford, Jr., and J. W. Cleland, "Transmutation Doping and Recoil Effects in Semiconductors Exposed to Thermal Neutrons," vol 1, p 269 in I.A.E.A. Conference on Radioisotopes, Copenhagen, 1960 Intern. J. Appl. Radiation Isotopes 9, 189 (1960); J.W. Cleland, to be published in Nuovo Cimento.

${ }^{53} \mathrm{G}$. A. Bartholomew and L. A. Miggs, CRGP-784, (1958); see also Nucleonics 18(11), 171 (1960).

${ }^{54} \mathrm{~L}$. V. Groshev et al., Atlas of the Gamma Ray Spectra from the Radiative Capture of Thermal Neutrons, Pergamon, London and New York, 1959.

${ }^{55} \mathrm{~J}$. W. Cleland, J. H. Crawford, Jr., and D. K. Holmes, Phys. Rev. 102, 722 (1956); H. H. Crawford, Jr., and J. W. Cleland, J. Appl. Phys. 30, 1204 (1959).

$56 \mathrm{E}$. Sonder and L. C. Templeton, J. Appl. Phys. 31,1279 (1960).

${ }^{57} \mathrm{O}$. S. Oen and D. K. Holmes, J. Appl. Phys. 30, 1289 (1959). 
Table 15.1. Tabulation of Gamma-Ray Spectra from Thermal-Noutron Capture in Cadmium 53,54

\begin{tabular}{cc}
$\begin{array}{c}\text { Gamma-Ray Energy } \\
\text { (Mev) }\end{array}$ & $\begin{array}{c}\text { Photons per } 100 \\
\text { Captures }\end{array}$ \\
\hline $0-1$ & 135 \\
$1-2$ & 92 \\
$2-3$ & 96 \\
$3-5$ & 73 \\
$5-7$ & 17 \\
$7-9$ & 1 \\
9 & 0.1 \\
9.05 & 414.1 \\
Highest & Total \\
\hline
\end{tabular}

Table 15.2. Atomic Displacement Cross Sections in A.luminum and Copper by Gamma Rays 55

\begin{tabular}{|c|c|c|}
\hline $\begin{array}{c}\text { Gamma-Ray } \\
\text { Energy } \\
\text { (Mev) }\end{array}$ & $\begin{array}{c}\sigma_{d}^{C} \times 10^{-24} \mathrm{~cm}^{2} \\
(\text { Aluminum) }\end{array}$ & $\begin{array}{c}\sigma_{d}^{C} \times 10^{24} \mathrm{~cm}^{2} \\
\text { (Copper) }\end{array}$ \\
\hline 1.00 & 0.33 & 0.25 \\
\hline 1.25 & 0.50 & 0.50 \\
\hline 1.50 & 0.68 & 0.85 \\
\hline 1.75 & 0.85 & 1.20 \\
\hline 2.00 & 1.00 & 1.55 \\
\hline 2.50 & 1.30 & 2.30 \\
\hline 3.00 & 1.50 & 3.00 \\
\hline 4.00 & 1.90 & 4.10 \\
\hline 5.00 & 2.10 & 5.00 \\
\hline
\end{tabular}

the latter when calculated on the same basis. Therefore, simple theory predicts a higher rate of defect introduction for cadmium capture gamma rays as compared with $\mathrm{Co}^{60}$ gamma rays.

The observed rates of defect introduction in silicon and germanium by $\mathrm{Co}^{60}$ gamma rays, cadmium capture gamma rays, and thermal neutrons are compared in Table 15.3. These values were obtained from the experimental rates of carrier removal ascertained by Hall coefficient measurements in high-purity $n$-type silicon and germanium irradiated at $40^{\circ} \mathrm{C}$ under the assumption
Table 15.3. Lattice Defect Production in Semiconductors

\begin{tabular}{clcc}
\hline $\begin{array}{c}\text { Type of } \\
\text { Irradiation }\end{array}$ & Material & $\begin{array}{c}N_{D} / \phi, \\
\text { Theory }\end{array}$ & $\begin{array}{c}N_{D} / \phi, \\
\text { Observed }\end{array}$ \\
\hline Co ${ }^{60}$ photons & Silicon & 0.026 & 0.0007 \\
& Germanium & 0.023 & 0.0006 \\
Cadmium gamma & Silicon & 0.046 & 0.008 \\
rays & Germanium & 0.076 & 0.018 \\
Thermal neutrons & Silicon & & 0.014 \\
& Germanium & & 0.054 \\
\hline
\end{tabular}

that two carriers are removed per Frenkel pair. All the thermal-neutron and cadmium gamma-ray exposures were conducted in a thermal-neutron facility ${ }^{58}$ of the Oak Ridge Graphite Reactor with a cadmium ratio in excess of $10^{5}$. The flux of cadmium capture gamma rays was estimated from an expression which represents the flux at the center of a hollow sphere of shield material which is black to thermal neutrons: ${ }^{59}$

$$
\phi_{\gamma}=3 \bar{n} \phi_{\mathrm{th}} / 8
$$

where $\bar{n}$ is the average number of gamma rays emitted by $\mathrm{Cd}^{113}$ on thermal-reutron $(\bar{n}=4,1)$, and $\phi_{t h}$ is the thermal flux to which the enclosing shield is exposed. It is evident that the cadmium gamma rays are $\sim 11$ times more effective in producing defects in silicon and $\sim 30$ times more effective in germanium for exposures at $40^{\circ} \mathrm{C}$ than are $\mathrm{Co}^{60}$ gamma rays. It is also interesting to note from Eq. (1) that the effect per thermalneutron capture in the cadmium shield is comparable to the effect per thermal neutron absorbed in the specimen. Also shown in Table 15.3 are the defect introduction rates for the two types of gamma rays as calculated from the displacement cross sections of Oen and Holmes. The cross section employed for the cadmium capture gamma rays is the weighted average obtained from Tables 15.1 and 15.2; all photons with energies greater than $5 \mathrm{Mev}$, which account for less than $5 \%$ of the total spectrum, were neglected. It should be pointed out that the larger

\footnotetext{
${ }^{58}$ The authors are indebted to W. S. Lyon for his assistance in the determination of thermal flux values. ${ }^{59}$ O. S. Oen, private communication.
} 
theoretical production rates as compared to experimental values are not particularly surprising, since experimental values are typically low by as much as an order of magnitude in radiationeffects experiments. Moreover, at the irradiation temperature employed, appreciable annealing of damage would be expected during the irradiation periods of $100 \mathrm{hr}$ or more employed here. ${ }^{60}$ More significant is the fact that the more energetic capture gamma rays produce relatively much more damage, compared with $\mathrm{Co}^{60}$ gamma rays, than the simple theory predicts. This latter observation suggests that either (1) agreement between simple theory and experiment improves, the higher the energy of incident radiation is above the displacement threshold, or (2) the resultant defects from cadmium capture gamma rays are more resistant to thermal anneal than those from $\mathrm{Co}^{60}$ gamma rays at the temperature of irradiation. A similar result has been obtained in electronbombarded germanium, ${ }^{61}$ wherein it is observed that the greatest disparity between theory and experiment occurs for electron energies near the displacement threshold.

\subsection{EFFECT OF DISLOCATIONS ON Co 60 RADIATION-INDUCED DEFECTS IN GERMANIUM}

$$
\text { J, W. Cleland R. F. Rass }
$$

It has been previously observed ${ }^{62}$ that $\mathrm{Co}^{60}$ photons produce randomly distributed interstitials and vacancies that act as acceptors of conduction electrons in $n$-type germanium and that the effect of extended irradiation is to convert $n$-type matcrial to $p$ type. In urder to investigate the influence of preexisting imperfections, three specimens of widely different dislocation density, ${ }^{63}$ as determined by etch-pit counting, were irradiated as indicated in Fig. 15.12, which shows a plot

${ }^{60}$ W. L. Brown, W. M. Augustyniak, and T. R. Waite, J. Appl. Phys. 30, 1258 (1959).

$61 \mathrm{~W}$. L. Brown and W. M. Augustyniak, J. Appl. Phys. 30, 1300 (1959).

$62 \mathrm{~J}$. H. Crawford, Jr., and J. W. Cleland, J. Appl. Pbys, 30, 1204 (1959).

${ }^{63}$ We are indebted to A. G. Tweet, General Electric Co., for the dislocation-free material and to $R$. $A$. Logan, Bell Telephone Laboratory, for the control and plastically deformed specimens.

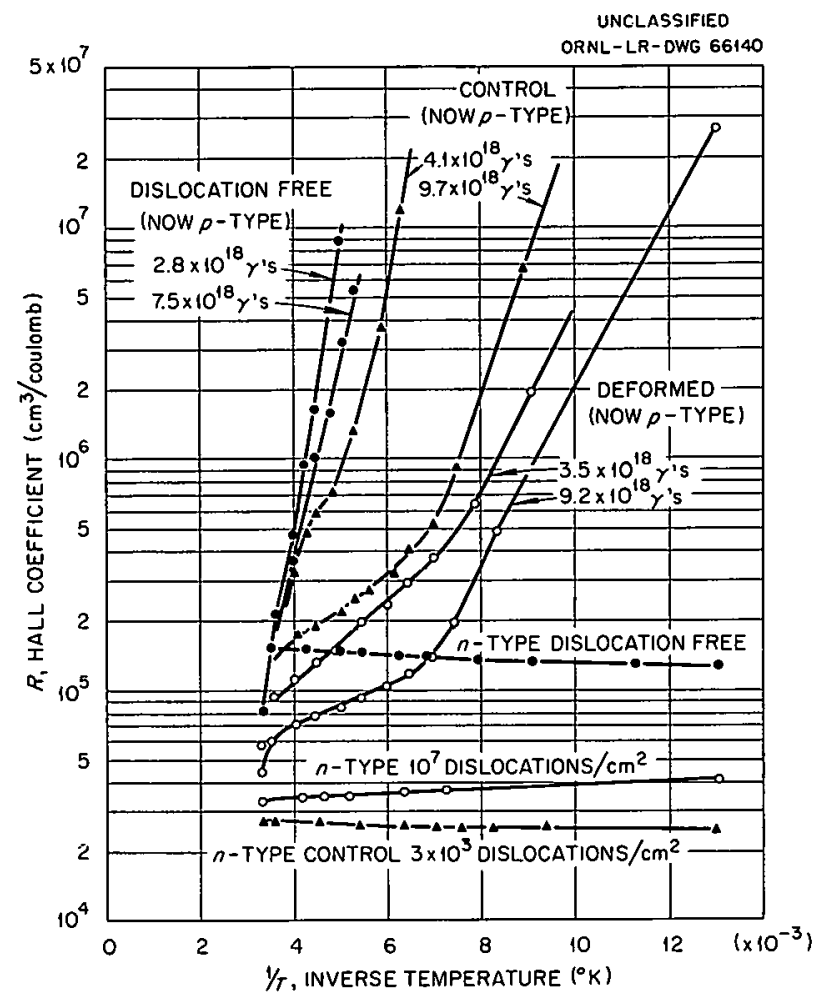

Fig. 15.12. Hall Coefficient and Resistivity vs Inverse Temperature for n-Type Germanium Before Irradiation and After Co ${ }^{60}$ Photon Irradiation.

of log Hall coefficient and resistivity vs inverse temperature. The removal rate of conduction electrons was identical, within about $10 \%$, for all three specimens on the $n$-type side, which would suggest that the existing imperfections ${ }^{64}$ had no effect on the irradiation-produced lattice defects. The hole concentration and apparent acceptor level position on the p-type side, however, is markedly affected by the initial dislocation density. The Hall coefficient curves saturated for each specimen as indicated, and no further increase in hole concentration or change in the apparent acceptor level was observed as a consequence of continued irradiation; therefore these data would suggest some mode of defect interaction with existing dislocations.

${ }^{64} \mathrm{~J}$. W. Cleland and J. H. Crawford, Jr., Solid State Div. Ann. Progr. Repl. Aug. 31, 1959, ORNL-2829, pp 134-36. 


\subsection{MONITORING TECHNIQUES FOR CAPTURE GAMMA RAYS}

\author{
J. W. Cleland
}

The Biological Thermal-Neutron Treatment Chamber, which is usually referred to as the slant animal tunnel, on top of the ORNL Graphite Reactor, has a themal flux of $1.5 \times 10^{9}$ neutrons $\mathrm{cm}^{-2} \mathrm{sec}^{-1}$ and a cadmium ratio of $10^{5}$, which corresponds to a background fast (damaging) flux of only about $10^{5}$ neutron $\mathrm{cm}^{-2} \mathrm{sec}^{-1}$.

The flux of capture gamma rays inside a hollow shield of material that is black to thermal neutrons has been estimated ${ }^{65}$ as $3 / 8 \bar{n} \phi_{t h}$, where $\bar{n}$ is the average number of gammas emitted per thermal-neutron absorption, and $\phi_{t h}$ is the thermalneutron flux. One would, therefore, expect $3 / 8(4.1)\left(1.5 \times 10^{9}\right)=2.3 \times 10^{9}$ gammas $\mathrm{cm}^{-2}$ $\mathrm{sec}^{-1}$ from cadmium, with a distribution of gammaray energies as indicated. ${ }^{65}$ One would also expect $3 / 8\left(1.5 \times 10^{9}\right)=5.6 \times 10^{8}(0.52-\mathrm{Mev})$

\footnotetext{
$65 \mathrm{~J}$. W. Cleland, R. F. Bass, and J. H. Crawford, Jr. "Lattice Defect Production in Thermal-Neutron Shielded Materials," this report.
}

gammas $\mathrm{cm}^{-2} \mathrm{sec}^{-1}$ from $\mathrm{B}_{4} \mathrm{C}$ (boron-impregnated plastic).

Ceric sulfate reduction techniques ${ }^{66}$ were employed to obtain an average value of 400 to 800 $\mathrm{r} / \mathrm{hr}$ without any shield material, $1860 \mathrm{r} / \mathrm{hr}$ inside $\mathrm{B}_{4} \mathrm{C}$, and $9200 \mathrm{r} / \mathrm{hr}$ in side cadmium. Ferrous sulfate oxidation techniques ${ }^{67}$ indicated an average value of $600 \mathrm{r} / \mathrm{hr}$ without any shield material and $7540 \mathrm{r} / \mathrm{hr}$ in side cadmium.

These values correspond to a flux of $3.2 \times 10^{8}$ photons $\mathrm{cm}^{-2} \mathrm{sec}^{-1}$ without shielding (assuming an average energy of $1.0 \mathrm{Mev}$ ), $1.0 \times 10^{9}$ for $\mathrm{B}_{4} \mathrm{C}$ (assuming a 0.52 -Mev gamma ray), and $3.4 \times 10^{9}$ for cadmium (assuming an average energy of 1.25 Mev). It is evident from these data that the rate of production of capture gamma rays is about as predicted and that chemical dosimetry techniques can be employed to determine the photon flux inside a thermal-neutron shield in the absence of fast neutrons.

\footnotetext{
${ }^{6} G_{W e}$ are indebted to L. C. Bate of the Nuclear Analysis Group of the Analytical Chemistry Division for these measurements.

${ }^{67}$ We are indebted to C. J. Hochanadel of the Chemistry Division for the se measurements.
} 


\section{Insulating Crystals}

\subsection{F. AND M-BAND COLORING IN HEAVILY IRRADIATED HIGH-PURITY KCI}

\author{
E. Sonder \\ W. A. Sibley
}

In the hope of elucidating the processes of radiation coloring in the alkali halides, a comprehensive study of gamma-ray and electron damage in $\mathrm{KCl}$ was initiated. The initial goals were (1) to determine the factors that accelerate or slow down the coloring rate, (2) to investigate the relation between $F$ - and $M$-centers, and ( 3 ) to look for any effect of direct knock-on damage by comparing gamma-ray and electron coloring. An abstract of the results of gamma irradiations, which will appear in the Pbysical Review, ${ }^{1}$ follows:

Measurements of $F$ - and $M$-band absorption at room temperature as a function of gamma irradiation are used to study the relation between $F$-centers and $M$-centers in a number of $\mathrm{KCl}$ crystals from a variety of sources. Although the F-center coloring curves exhibit a varicty of forms, presumably due to different trace impurities and imperfections in the crystals, the $M-c e n t e r$ density seems in all cases to be nearly proportional to the square of the $F$-center concentration. The ratio of the $M$-center concentration to the square of the $F$-center concentration appears to be related to trace impurity content. Zone rcfining decreases the relative $M$-center introduction, as does deliberate doping with calcium, whereas plastic deformation has no effect on the ratio. Bleaching increases the $M$ center concentration at the expense of $F$-centers; however, short reirradiation with gamma rays causes the ratio of M-center concentration to the square of $F$-center concentration to return to its pre-bleaching value. The results are thought to indicate that a radiation equilibrium exists between $F$ - and $M$-centers which is frozen-in when the crystals are removed from the source.

\section{Effect of Radiation Intensity}

The fact that the $M$-center concentration is approximately proportional to the square of the F-center concentration in a given sample of $\mathrm{KCl}$ has been demonstrated previously. ${ }^{1,2}$ Figure 16.1 shows clearly that the ratio or slope of the $a_{M}$ vs $\left[\alpha_{F}^{\prime}\right]^{2}$ curve, where $\alpha_{M}$ is the absorption coefficient at the maximum of the $M-b$ and and $a_{F}^{\prime}$ is the corrected absorption ${ }^{3}$ at the peak of the $F$-band, is quite strongly dependent upon the intensity of

\footnotetext{
${ }^{1}$ W. A. Sibley and E. Sonder, to be published in the Physical Review on October 15.

${ }^{2}$ B. J. Faraday, H. Rabin, and W. D. Compton, Phys. Rev. Letters 7, 57 (1961).

${ }^{3}$ F. Okamoto, Phys. Rev. 124, 1090 (1961).
}

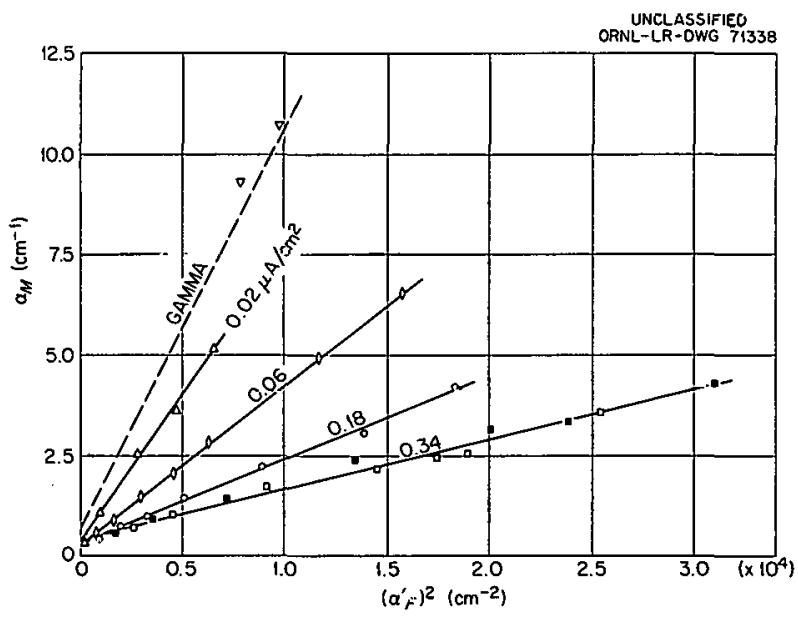

Fig. 16.1. Effect of Radiation Intensity on the $F$. and M-Center Relation. The dashed curve is for a sample gomma-irradiated in a $4.5 \times 10^{6}$ r source. The solid lines are for samples electron-irradiated with 1.5-Mev electrons and the current densities shown. 
irradiation. [In the work of Okamoto, ${ }^{3}$ dichroic absorption in the $F$ - and $M$-bands was measured and indicated that one-half to one times the $M$ absorption at $8250 \mathrm{~A}$ also appears as additional $M$-center absorption under the $F$-band. Correction for this was made by subtracting 0.8 times the height of the $M$-band from the height of the $F$-band; that is, $a_{F}^{\prime}=a_{F}$ (measured) $-0.8 a_{M}$.] Each solid curve in the figure is for one run, that is, for a series of absorption measurements performed after successive irradiations with a given electron current. The four samples were all from the same

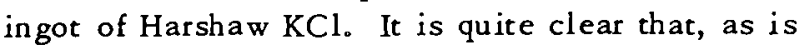
the case for gamma irradiation, straight lines are obtained; however, the slopes are smaller; that is, the relative $M$-center concentration is less for irradiations with higher currents. To compare these results with those on gamma-irradiated $\mathrm{KCl}$, a curve for a previously reported gamma-irradiated Harshaw $\mathrm{KCl}$ sample $^{2}$ is included as a dashed line.

A comparison between gamma and electron irradiations can be made by calculating the energy absorbed per second for the electron-'and gammairradiated samples. In the electron case it was assumed that all the energy lost by the slowing electrons caused ionization in the $\mathrm{KCl}$. In the gamma case a predominance of Compton absorption of the $1.2-\mathrm{Mev} \mathrm{Co}^{60}$ gamma rays was as sumed, so that simple conversion from the $4.5 \times 10^{6} \mathrm{r}$ calibration of the source was possible. In Table 16.1 are shown the values of energy absorbed per second as well as the slope for the samples shown in Fig. 16.1.

It might be noted that, as was the case in the gamma-irradiated $\mathrm{KCl}$, the $a_{M}$ vs $\left[a_{F}^{*}\right]^{2}$ curves do not pass through the origin. Since, at the irradiation fluxes used in these experiments, effects due to the early stages of coloring are all concentrated very near the origin of our graphs, it is tempting to attribute the excess $M$-center concentration observed to early-stage processes. The implication would be that some of these early-stage processes might take place in regions of anomalously high $F$-center concentrations, that is, near impurity clusters or dislocations where the tendency to form di-F-centers would be greater than in the bulk of the $\mathrm{KCl}$.

\section{Equilibrium Between $F$ - and $M$-Centers}

It has been reported previously that it is possible after bleaching a sample to cause it to return to its pre-bleaching, relative $F-$ and $M$ center concentrations with very little additional radiation. ${ }^{1,4}$ Since different intensities of irradiation caused different ratios of $\alpha_{M} /\left[\alpha_{F}^{-}\right]^{2}$ to be observed, a number of experiments were performed in which samples were irradiated at constant intensity unitil they were heavily colored; then, for short irradiation times, the intensity was changed. In Table 16.2 is presented the radiation history of three such runs, one in which the sample was first colored in the gamma source and two where the primary coloring was done by using the electron accelerator with 0.02 and $0.36 \mu \mathrm{a} / \mathrm{cm}^{2}$ respectively. The results are illustrated in Fig. 16.2. The curves of Fig. 16.1 are reproduced

${ }^{4}$ L. E. Silverman and L. I. Grossweiner, Phys. Rev. 121,1072 (1961).

Table 16.1. Rate of Energy Absorption and Slope of $\alpha_{M}$ vs $\left[\alpha_{F}^{\prime}\right]^{2}$ Plots

\begin{tabular}{|c|c|c|c|c|}
\hline Sample & Type of Irradiation & $\begin{array}{l}\text { Current Density } \\
\qquad\left(\mu \mathrm{a} / \mathrm{cm}^{2}\right)\end{array}$ & $\begin{array}{l}\text { Energy Absorbed } \\
\left(\text { Mev } \mathrm{cm}^{-3} \mathrm{sec}^{-1}\right)\end{array}$ & $\begin{array}{l}\text { Slope } \\
(\mathrm{cm})\end{array}$ \\
\hline $\mathrm{H} 4$ & $4.5 \times 10^{6} \mathrm{r}$ & Gamma source & $1 \times 10^{11}$ & $1.0 \times 10^{-3}$ \\
\hline $\mathrm{H} 27$ & 1.5 -Mev electrons & 0.02 & $5 \times 10^{11}$ & $7.3 \times 10^{-4}$ \\
\hline $\mathrm{H} 23$ & 1.5-Mev electrons & 0.06 & $1.5 \times 10^{12}$ & $4.0 \times 10^{-4}$ \\
\hline $\mathrm{H} 21$ & 1.5-Mev electrons & 0.18 & $4.5 \times 10^{12}$ & $2.0 \times 10^{-4}$ \\
\hline $\mathrm{H} 22$ & 1.5-Mev electrons & 0.34 & $9 \times 10^{12}$ & $1.2 \times 10^{-4}$ \\
\hline
\end{tabular}


Table 16.2. Change in $F$ - and M-Band Absorption upon Short Irradiations

\begin{tabular}{|c|c|c|c|c|c|c|c|}
\hline \multirow[t]{2}{*}{$\begin{array}{c}\text { Sample } \\
\text { Designation }\end{array}$} & $\begin{array}{c}\text { Type of } \\
\text { Irradiation }\end{array}$ & $\begin{array}{c}\text { Electron Current } \\
\text { Density } \\
\left(\mu \mathrm{a} / \mathrm{cm}^{2}\right)\end{array}$ & \multicolumn{2}{|c|}{$\begin{array}{l}\text { Irradiation } \\
\text { Time }\end{array}$} & $\begin{array}{l}\text { Absorbed } \\
\text { Energy } \\
\left(\mathrm{Mev} / \mathrm{cm}^{3}\right)\end{array}$ & $\begin{array}{l}\text { Corrected Absorption } \\
\text { at } F-\text { Band Maximum } \\
\qquad\left(\alpha_{F}^{\prime}\right)\end{array}$ & $\begin{array}{c}\text { Absorption at } \\
M-B \text { and Maximum } \\
\left(\alpha_{M}\right)\end{array}$ \\
\hline & & & & & $\times 10^{14}$ & & \\
\hline $\mathrm{H} 24$ & Electron & 0.33 & & $\min$ & 26.6 & 159.4 & 3.54 \\
\hline $\mathrm{H} 24$ & Electron & 0.05 & 10 & $\mathrm{sec}$ & 0.1 & 151.8 & 6.66 \\
\hline $\mathrm{H} 24$ & Electron & 0.05 & 15 & $\sec$ & 0.2 & 147.8 & 8.51 \\
\hline $\mathrm{H} 24$ & Electron & 0.33 & 10 & sec & 0.9 & 160.1 & 3.50 \\
\hline H26 & Gamma & & 20. & & 97.0 & 98.3 & 10.69 \\
\hline 1126 & Electron & 0.17 & 15 & sec & 0.7 & 115.4 & 2.30 \\
\hline H 26 & Electron & 0.17 & 10 & $\sec$ & 0.4 & 117.8 & 2.08 \\
\hline H 26 & Electron & 0.02 & 60 & sec & 0.4 & 112.1 & 5.86 \\
\hline H26 & Gamma & & 10 & $\min$ & 0.8 & 102.7 & 11.20 \\
\hline H27 & Electron & 0.02 & 90 & $\min$ & 26.2 & 80.5 & 5.14 \\
\hline $\mathrm{H} 27$ & Electron & 0.18 & 15 & $\mathrm{sec}$ & 0.7 & 90.3 & 1.89 \\
\hline H27 & Electron & 0.18 & 10 & sec & 0.4 & 92.7 & 1.99 \\
\hline $\mathrm{H} 27$ & Electron & 0.02 & 60 & $\sec$ & 0.3 & 88.0 & 4.80 \\
\hline $\mathrm{H} 27$ & Gamma & & 10 & $\min$ & 0.8 & 78.4 & 7.76 \\
\hline
\end{tabular}

lightly, and the heavy dashed lines and arrows show the changes in $\mathrm{F}$ - and $\mathrm{M}$-center concentration induced by the short irradiations. The irradiation time for each step is also shown. It is clear from the results that short irradiation at changed intensity will cause the ratio $\alpha_{M} /\left[\alpha_{F}^{\prime}\right]^{2}$ to shift to a position characteristic of that intensity. Increased radiation-energy flux causes a decrease in $M$-center and an increase in $F$-center concentrations, and a lowering of the flux causes the converse to take place. Moreover, there is a definite tendency after short irradiation at a given flux for the ratio $a_{M} /\left[a_{F}^{\prime}\right]^{2}$ wo approach the value it would have reached if the sample had been irradiated at the given flux all the time. This would seem to confirm that an equilibrium exists under irradiation conditions and that, except for the slow introduction of additional $F$ - and $M$ centers, the equilibrium is reversible. The time to reach equilibrium, as shown by the 10 - and $15-\mathrm{sec}$ irradiation of sample H24, seems to be of the same order as the time necessary to go through the first stage of coloration in these samples.

Similar measurements were performed on a number of samples from an ingot of chlorinetreated $\mathrm{KCl}$. Although the relative $M$-center concentration in all these samples was lower, the same variation of $a_{M} /\left[\alpha_{F}^{0}\right]^{2}$ with intensity and the reversibility with short irradiation at changed intensity were found.

\section{Comparison of Different $\mathrm{KCl}$ Samples}

In Figs. 16.3 and 16.4 are presented coloring curves and $a_{M}$ vs $\left[a_{F}^{\prime}\right]^{2}$ curves for a number of differently prepared samples of $\mathrm{KCl}$, all irradiated at the same electron current density. [Samples $\mathrm{H}, \mathrm{I}$, and $\mathrm{O}$ were obtained commercially from Harshaw, Isomet, and Optovac respectively. 


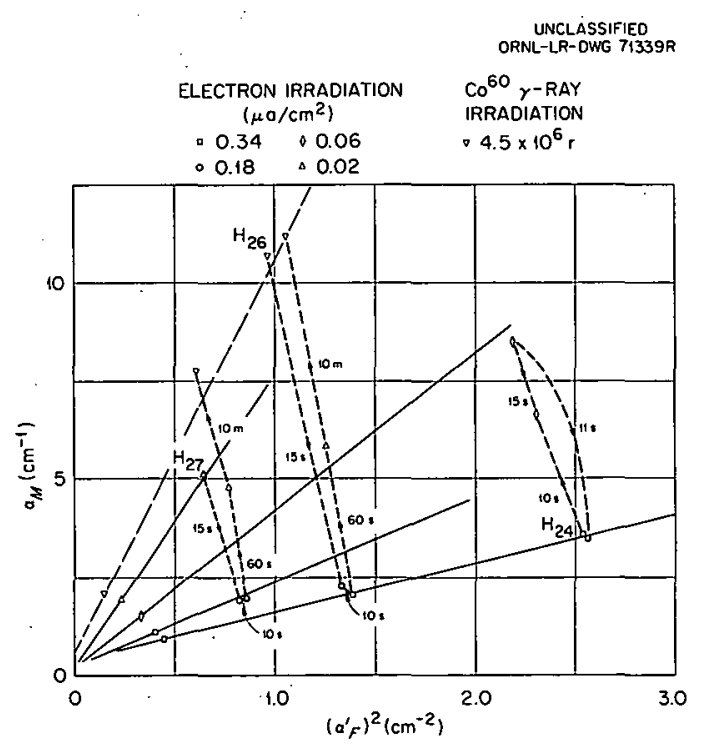

Fig. 16.2. Radiation Equilibrium Between $F$ - and $M$ Centers. The points connected by the heavy dashed lines show changes in $F$ - and $M$-center concentrations upon irradiation with different intensities.

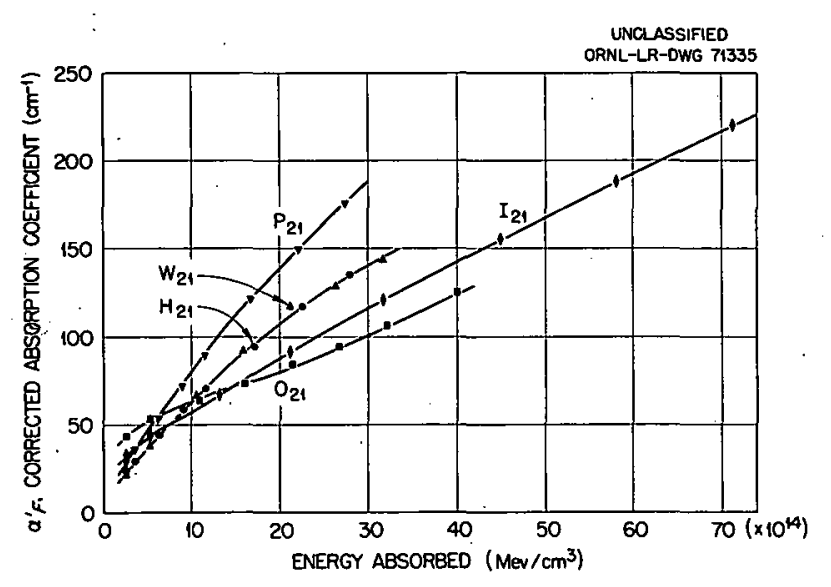

Fig. 16.3. Introduction of F-Centers by 1.5-Mev Elec. trons $\left(0.18 \mu a / \mathrm{cm}^{2}\right)$ in Differently Prepared Samples of $\mathrm{KCl}$.

Sample P21 was pulled from the melt after chlorine treatment, ${ }^{5}$ and sample $W 21$ was zone-refined in chlorine gas. ${ }^{6}$ Except for 121 , the samples were cleaved from the same ingots as samples with

${ }^{5}$ The chlorine-treated $\mathrm{KCl}$ was supplied by $\mathrm{R}$. Pohl and $\mathrm{J}$. Ashe of Cornell University.

${ }^{G}$ The zone-refined $\mathrm{KCl}$ ingot. was supplied by $\mathrm{J} . \mathrm{G}$. Castle and R. Warren of Westinghouse.

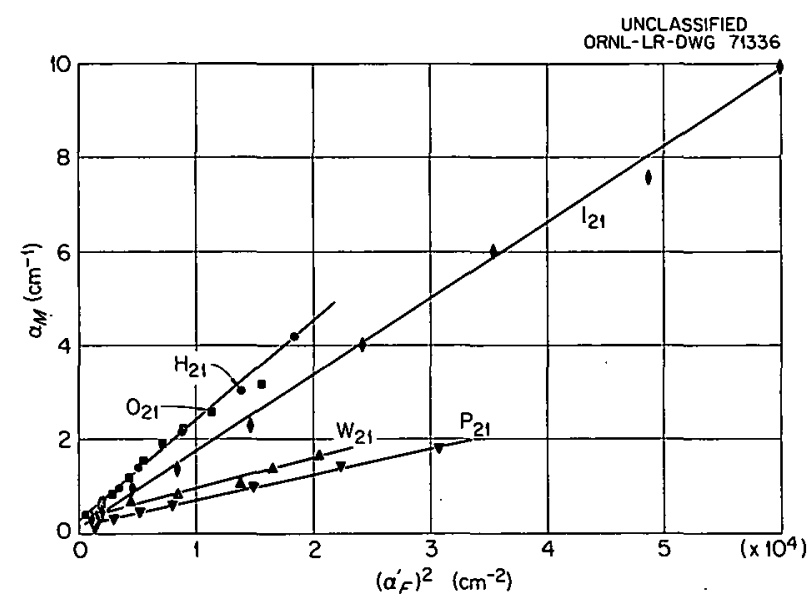

Fig. 16.4. Effect of Different Sample Preparation in the $F$. and $M$-Center Relationship.

similar letters that were gamma-irradiated. (See ref 2 and the abstract at the beginning of this section.)] The curves are meant to be compared with Fig. 1, ref 1 , in which information on preparation and source of ingots $P$ and $W$ is also given (see ref 1 , Table 1). The shapes of the curves are qualitatively the same for the gammaand electron-irradiation cases. This evidence, together with the fact noted earlier that when the actual energy absorbed per second is taken into account the rate of coloration under gamma rays is consistent with coloring rate for electrons, would indicate that the knock-on displacement type of damage is negligible, as compared with photolytic processes, ${ }^{7}$ during electron irradiation of $\mathrm{KCl}$.

\subsection{GAMMA IRRADIATION OF DOPED KCI}

$$
\text { W. A. Sibley C. T. Butler }
$$

The investigation of the effect of gamma irradiation on high-purity crystals was extended to some $\mathrm{KCl}$ samples which had been purposely doped with calcium, strontium, cadmium, and magnesium. These crystals were analyzed by flame photometry techniques in order to determine the concentration of divalent impurities present. Table 16.3 shows the results of these analyses. The experimental procedures utilized in the investigation were the same as those previously described for the high-purity-materials work (see Sec 16.1).

\footnotetext{
${ }^{7}$ J. H. O. Varley, Nature 174, 886 (1954).
} 
Table 16.3. List of Samples

\begin{tabular}{ccc}
\hline Sample & Impurity & $\begin{array}{c}\text { Concentration } \\
(\mathrm{ppm})\end{array}$ \\
\hline $\mathrm{KCl}: \mathrm{Sr}$ & $\mathrm{Sr}$ & 210 \\
& $\mathrm{Ca}$ & 30 \\
$\mathrm{KCl}: \mathrm{Cd}$ & $\mathrm{Cd}$ & $>300$ \\
& $\mathrm{Ca}$ & $<10$ \\
$\mathrm{KCl}: \mathrm{Mg}$ & $\mathrm{Mg}$ & 350 \\
& $\mathrm{Ca}$ & 30 \\
$\mathrm{Ca} 1$ & $\mathrm{Ca}$ & 110 \\
$\mathrm{Ca} 3$ & $\mathrm{Ca}$ & 280 \\
\hline
\end{tabular}

The F-band growth curves for the doped specimens are illustrated in Fig. 16.5. The increased first-stage coloring in the calcium- and strontiumdoped samples has been observed previously ${ }^{8.9}$ and is attributed to the increased number of positive-ion vacancies which must be present in order to preserve electrical neutrality within the crystal. Since the $F$-center is known to be a negative-ion vacancy with an associated electron, it is difficult to understand how an increase in the number of positive-ion vacancies in the lattice, which suppresse's the number of anion vacancies, increases the $F$-center production rate. Recently, Crawford and Nelson ${ }^{10}$ have shown that it is possible to transform a cation vacancy into an $F$-center by photolysis, thus resolving the difficulty. It should be noted, however, that the growth curves for the cadmium- and magnesiumdoped $\mathrm{KCl}$ do not exhibit this increased coloring in the first stage, even though considerably more impurity is present in these crystals. This indicates either that there is a difference in the way calcium and strontium, in the one case, and cadmium and magnesium in the other, enter the crystal as impurities or that the interaction of these impurities with positive-ion vacancies is

\footnotetext{
${ }^{8}$ H. W. Etzel, Phys. Rev. 87, 906 (1952).

${ }^{9}$. H. Crawford, Jr., paper presented at the International Conference on Crystal Lattice Deferts, Kyoto, Japan, Sept. 7-12, 1962.

${ }^{10}$ J. H. Crawford, Jr., and C. M. Nelson, Phys. Rev. Letters 7, 57 (1961).
}

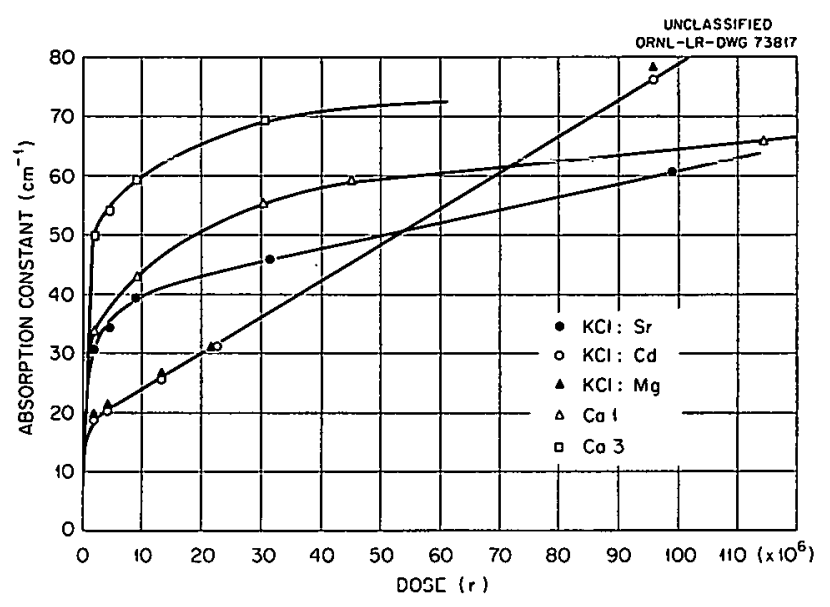

Fig. 16.5. F.Center Coloring Curves for KCl Crystals Containing Different Impurities.

different (assuming that only divalent impurities are responsible for the observed change in coloring properties).

If the number of free positive-ion vacancies is less for crystals doped with magnesium and cadmium and if this deficiency accounts for the depressed first stage in these samples, then it should be possible to freeze-in enough free vacancies by a quench from high temperatures to increase the first-stage coloring in these crystals. Blocks cleaved from calcium- and magnesium-doped ingots were held at $525^{\circ} \mathrm{C}$ for $10 \mathrm{~min}$ and then quickly dropped into cold acetone in an effort to quench in the high-temperature defect configuration. Specimens for irradiation were cleaved from the centers of these blocks. Figure 16.6 shows the

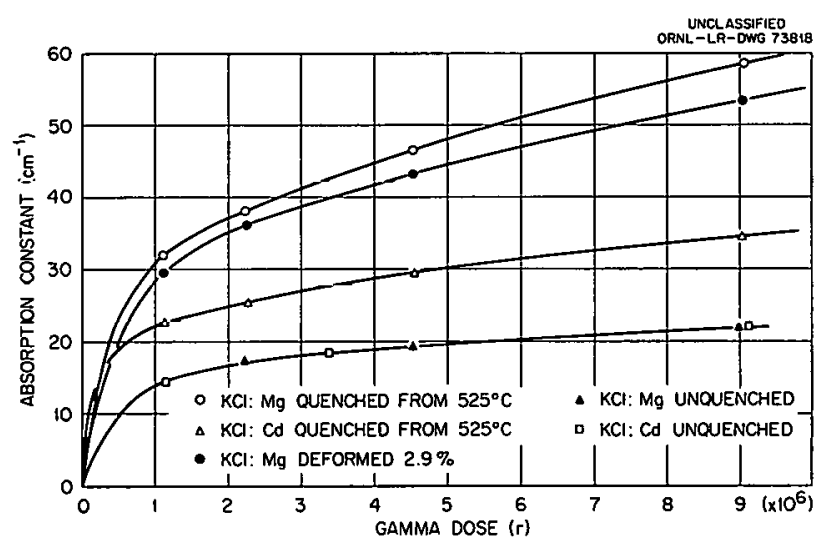

Fig. 16.6. F-Center Growth Curves of Treated $\mathrm{KCl}$ Crystals. 
result of coloring the quenched specimens. It is clear that there is an increase in the first-stage coloring of the quenched samples; however, thermal deformation could possibly cause the observed increase. A magnesium-doped crystal from the same ingot was deformed $2.9 \%$ in a $<100\rangle$ direction, in a toolmaker's vise. A specimen cleaved from this block was then irradiated. The results of this treatment are also shown in Fig. 16.6 and indicate that the themal deformation would have to be about $3 \%$ in order to explain the experimental results. This magnitude of thermal deformation is probably not possible. Also, cleavage cracks are always observed on the surface of the quenched blocks. These probably reduce the total stress on the center of the block from which the irradiation specimens are cleaved.

\subsection{INVESTIGATIONS OF COLOR CENTERS IN ALKALI HALIDES AT ORNL: STRUCTURE SENSITIVITY OF COLOR-CENTER PRODUCTION IN GAMMA-IRRADIATED KCI ${ }^{\prime \prime}$}

\author{
J. H. Crawford, Jr.
}

Investigations of the structure sensitivity of color center formation in $\gamma$-irradiated $\mathrm{KCl}$ at ORNL are reviewed. Both Stage I and Stage II coloration ranges are sensitive to the origin of the crystals studied. The presence of divalent cation $\left(\mathrm{M}^{++}\right)$ markedly enhances Stage I and suppresses Stage II relative to nominally pure crystals. The enhancement in Stage I can be explained by a model involving the conversion of an isolated cation vacancy into an $F$-center and a $\left[\mathrm{Cl}_{3}^{-}\right]$ion by a radiolytic reaction. Mechanisms by which $\mathrm{M}^{++}$ might suppress Stage II coloration are explored. These are: (a) Suppression of the concentration of electrons and holes produced by the radiation field by acting as electron-hole recombination centers, (b) poisoning dislocations as a source of lattice defects, and (c) alteration of the nature of quenched-in defects. None of these possibilities

\footnotetext{
${ }^{11}$ Abstract of paper to be presented at the International Conference on Crystal Lattice Defects, Kyoto, Japan, Sept. 7-12, 1962.
}

is wholly satisfactory to explain experimental observations.

\subsection{LUMINESCENCE OF ALKALI HALIDES}

$$
\text { C. T. Butler }
$$

Luminescence spectra of several alkali halides were recorded during and immediately following gamma irradiation at $5 \times 10^{6} \mathrm{r} / \mathrm{hr}$.

Figure 16.7 shows the spectra of Isomet $\mathrm{NaCl}$ and $\mathrm{KCl}$ as well as Harshaw CsI, LiF, and $\mathrm{KCl}$. The telescope used to focus light from the crystal into the spectrometer cut off just below $400 \mathrm{~m} \mu$. Thus, the apparent peaking of the luminescence of the various samples near $400 \mathrm{~m} \mu$ may not be real; indeed, Cerenkov radiation should produce a very high output below $400 \mathrm{~m} \mu$. The longer wavelength peaks, however, are more certain. Note that the CsI light output is roughly 1000 times that of the others. The curves have been corrected for instrument response, and all except the $\mathrm{NaCl}$ curve have been partially corrected for absorption under their $F$ bands.

Figures 16.8-16.10 present the afterglow spectra for these same specimens, as well as for two doped $\mathrm{KCl}$ samples. The glows decreased rapidly after removal from the gamma source; therefore, the curves which begin at about $10 \mathrm{sec}$ and run to about $35 \mathrm{sec}$ after removal do not present the true spectrum in each case. The half-life for decay of the 435 band in the KCl's was about 50 sec; it was not measured for the othermaterials. The records have been corrected for instrument spectral response. The spectrum of LiF is the only one needing any $F$-band correction. The $F$ band location is indicated.

The 435-m $\mu$ peak is dominant for Harshaw $\mathrm{KCl}$, whereas a many-line spectrum predominates in the Isomet $\mathrm{KCl}$ afterglow. The components of the Isomet ultraviolet band are separated by $0.24 \mathrm{ev}$ and possibly arise from cyanate, which is present in some of Isomet's alkali halides. This band could be reexcited with visible light after fading, but the ease with which it could be activated dropped with time after irradiation. It could not be excited at all beyond about $10 \mathrm{~min}$ after gamma irradiation. 


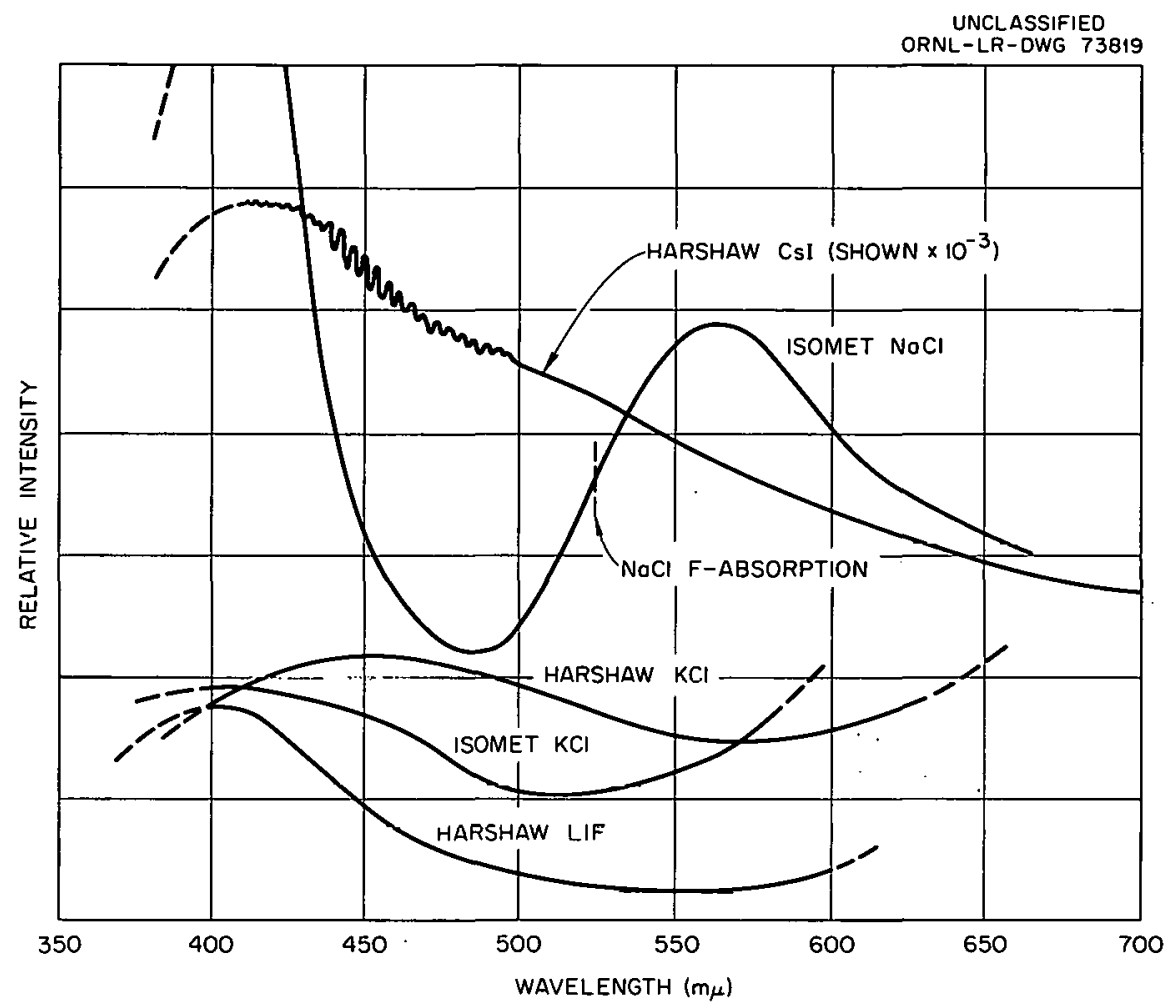

Fig. 16.7. Luminescence of Various Alkali Halides Under Gamma Irradiation of $5 \times 10^{6} \mathrm{r} / \mathrm{hr}$.

APPROXIMATE TIME AFTER REMOVING FROM $\gamma$-FLUX (sec)

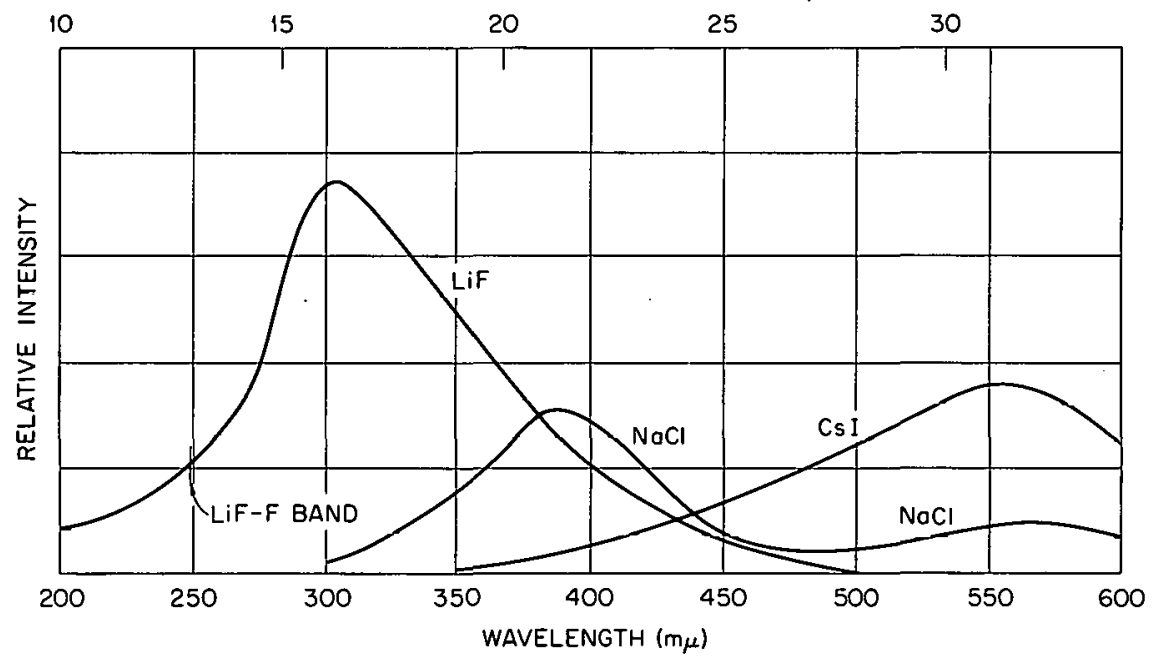

Fig. 16.8. Luminescent Afterglow of Several Alkali Halides After Gamma Irradiation at $5 \times 10^{6} \mathrm{r} / \mathrm{hr}$. 


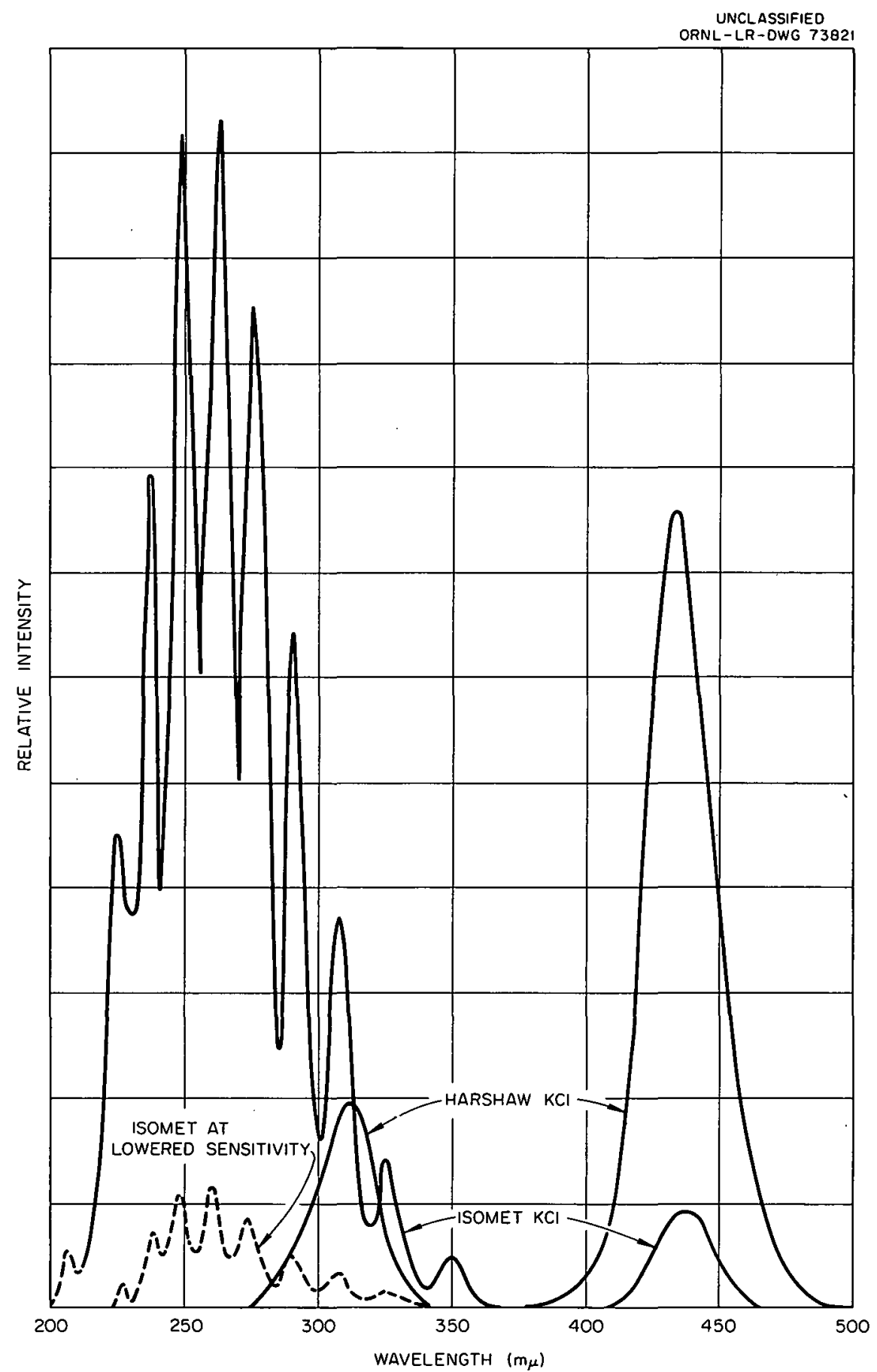

Fig. 16.9. Luminescent Afterglow of Gamma-Irradiated $\mathrm{KCl}$. (a) Isomet $\mathrm{KCl},(b)$ Isomet at lowered sensitivity, and $(c)$ Har show $\mathrm{KCl}$. 


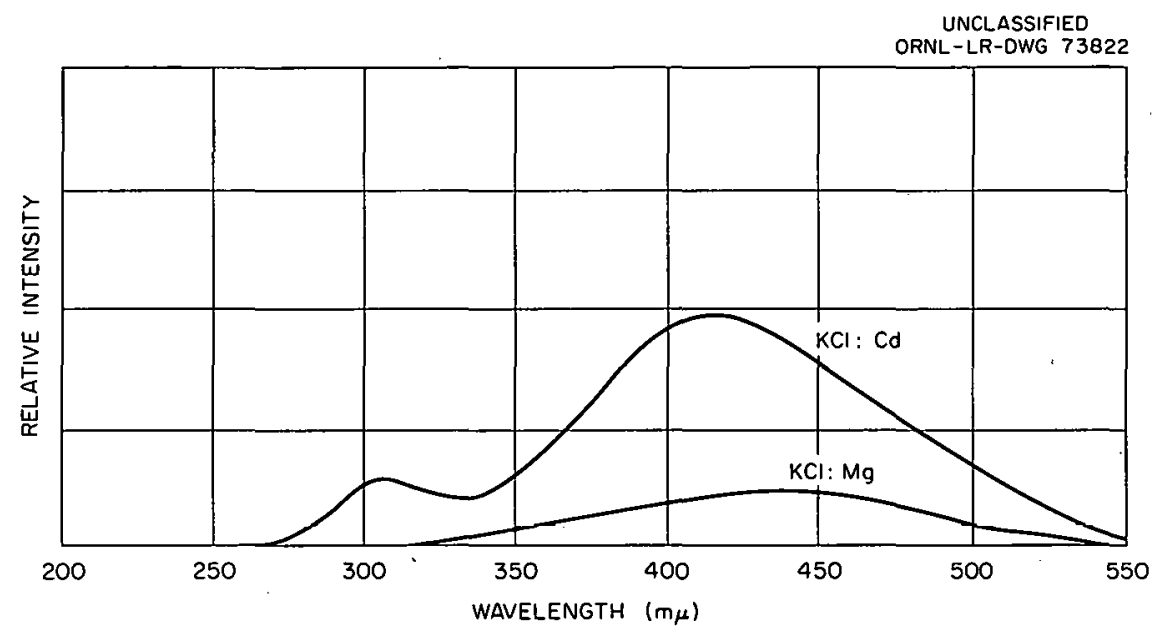

Fig. 16.10. Luminescent Afterglows of Gamma-Irradiated, Doped $\mathrm{KCl} .(a) \mathrm{KCl}: \mathrm{Cd},(b) \mathrm{KCl}: \mathrm{Mg}$.

\subsection{EFFECT OF ENERGETIC SECONDARY ELECTRONS ON THE SCINTILLATION PROCESS IN ALKALI-HALIDE CRYSTALS ${ }^{12}$}
A. Meyer
R. B. Murray

Experimental results have previously shown that the scintillation efficiency of alkali halide crystals to heavy particles is not a function of $d E / d x$ alone but is instead composed of a series of discrete functions, one for each incident particle.

This paper presents an analysis of these experimental results with attention to the effect of energetic secondary electrons (delta rays) produced by the primary particle. In this treatment the total light emitted per unit path length of the incident particle is the sum of two contributions: one from the highly ionized primary column, and one from those delta rays which escape the primary column and produce light with a high efficiency.

\subsection{STUDIES OF THE SCINTILLATION PROCESS IN CsI(TI) ${ }^{13}$}
R. Gwin ${ }^{14}$
R. B. Murray

An experimental investigation of the scintillation process in CsI(Tl) crystals has been made. The scintillation response of CsI(Tl) crystals, having various thallium contents, was measured for excitation of the crystals by monoenergetic gamma rays, protons, and alpha particles. The investigation was made to provide a test of some of the features of a theoretical model of the scintillation process in thallium-activated alkali iodides proposed by Murray and Meyer.

In order to insure that the results obtained in this program would provide a critical test of the scintillation model, special attention was paid to technical effects which could influence the interpretation of the experimental data. For example, the effect of the pulse-analysis time on the relative scintillation response of $\operatorname{CsI}(\mathrm{TI})$ to various charged particles was investigated. In addition, the emission spectra of the CsI(Tl) crystals were measured for excitation by $\mathrm{X}$-rays, protons, and alpha particles. A knowledge of the emission spectra of the crystals is necessary in order to be sure that only the luminescence characteristic of the thallium activator is considered in the analysis of the experimental data.

The results of the investigation have shown that the scintillation efficiency of CsI(TI) is a continuous function of $d E / d x$, as assumed in the scintillation model, within the accuracy of the

\footnotetext{
${ }^{12}$ Abstract of paper to be published in the Physical Review.

${ }^{13}$ Abstract of ORNL -3354 .

${ }^{14}$ Loanee from Neutron Physics Division.
} 
experiments when the effect of delta rays is considered. This leads to the conclusion that the light output of CsI(Tl) crystals is, in general, a nonlinear function of the energy of the particle. The shape of the $d L / d E$ versus $d E / d x$ curve for CsI(Tl) crystals has been shown, in this work, to be nearly independent of the thallium content of the crystal. This is in distinct contrast to the prediction of the scintillation model.

An examination of the emission spectra of CsI(Tl) crystals has shown that light is emitted in a band, centered at about $3300 \AA$, which is characteristic of the emission of the pure crystal, as well as the thallium luminescence. The relative intensity of this with respect to the thalliumactivator luminescence increases as the thallium content decreases. It has been suggested in this work that the emission band characteristic of pure CsI crystals is associated with the radiative decay of an iodine molecule ion of the type $\mathrm{I}_{2}$, which has trapped an electron. On this basis it has been suggested that thallium-activator luminescence results from the successive capture of an electron and a hole at a thallium center. The 3300- $\AA$ emission band of CsI overlaps the optical-excitation spectrum of CsI(TI) for thalliunactivator luminescence. Some thallium centers in
CsI(Tl) are therefore excited by optical emission in the $3300 \AA$ band.

\subsection{SPECIFIC HEAT OF SINGLE.CRYSTAL $\mathrm{MnCl}_{2}$ IN APPLIED MAGNETIC FIELDS ${ }^{15}$}

\author{
R. B. Murray
}

The specific heat of a single-crystal sample of $\mathrm{MnCl}_{2}$ has been measured, in the liquid helium temperature region, in applied magnetic fields extending up to 7.26 kilo-oersteds. In all cases the magnetic field was applied parallel to an $a$-axis of the hexagonal cell. The two specific heat maxima, which have been previously reported at $1.81^{\circ}$ and $1.96^{\circ} \mathrm{K}$ in zero field, are associated with antiferromagnetic transitions. Both transition temperatures are found to be field dependent. The upper transition temperature increases with applied field while the lower transition temperature decreases. These results are correlated with neutron diffraction studies of the magnetic structure transitions.

\footnotetext{
${ }^{15}$ Abstract of a paper submitted to the Pbysical Review.
} 


\section{Pure Materials}

\subsection{INTRODUCTION}

J. W. Cleland

It is the purpose of this program to produce research-quality materials that are needed for the AEC materials research program. An extensive program is being conducted to develop techniques to produce high-purity and controlled-impurity research specimens (mostly single crystals) of immediate and long-range interest to investigators at this laboratory, other AEC installations, and $A E C$ contractors. The acmal choice of research materials has been made, for the most part, on the basis of the potential gain to a fundamental understanding of solids; this should be realized through an intensive investigation of a given material by a number of research groups under contract to the AEC. Those materials that are commercially available, for the most part, are not being considered in this program.

The Pure Materials Program involves a number of research divisions at ORNL, and subcontracts have been negotiated with research groups outside ORNL to secure specialized skills relating to certain materials of interest. Three distinct phases are usually required for each material: (1) initial purification of the starting material, (2) crystal growth, and (3) evaluation of the end product. Special analytical techniques, such as mass spectral analysis, neutron activation analysis, and microspectroanalysis, supplied by the Analytical Chemistry Division, are important for the initial purification and for ascertaining the purity of the end product. Many of the actual crystal growth techniques are performed by groups in the Reactor Chemistry, Metals and Ceramics, and Solid State Divisions, and the experience of these groups is required for a final analysis of the crystal perfection.

Approximately $50 \mathrm{in}$. of $\frac{1}{4}$-in.-diam, high-purity, high-perfection tungsten single crystals have been obtained from the Linde Company, Indianapolis, Ind., under contract. These crystals were approximately 2 to $12 \mathrm{in.} \mathrm{long}$ and were equally divided between (100), (110), and (111) directions within 10 to $60 \mathrm{~min}$ of arc with essentially no lineage. Neutron activation analysis results performed at ORNL indicated $<0.7 \mathrm{ppm}$ copper, $<30 \mathrm{ppm}$ molybdenum, and $<5 \mathrm{ppm}$ tantalum. Low-temperature resistivity ratio measurements conducted in the Metals and Ceramics Division also indicated superior crystals. The tungsten contains $<50 \mathrm{ppm}$ total impurities, with $<5 \mathrm{ppm}$ each of carbon, oxygen, nitrogen, and sulfur.

The effects of initial surface preparation, crystal orientation, oxygen pressure and oxidation temperature have been critically examined as a part of a program to produce good-quality single crystals of $\mathrm{Cu}_{2} \mathrm{O}$ by the Virginia Institute for Scientific Research, Richmond, Va. Unidirectional oxidation has been achieved in specimens up to $1.5 \mathrm{~mm}$ thick, and test samples have been submitted to ORNL for further examination.

Large $(300-\mathrm{g})$ single crystals of high-purity $\mathrm{Li}^{7} \mathrm{~F}\left(99.99 \% \mathrm{Li}^{7}\right)$ have been produced by the Stockbarger technique. ${ }^{1}$ Small $\left(1 / 10 \times 1 / 10 \times \frac{1}{2} \mathrm{~mm}\right)$ strain-free single-crystal samples of $\mathrm{LiF}$ with $<100 \mathrm{ppm}$ magnesium have been grown from the gaseous phase. The compound $\mathrm{Na}_{2} \mathrm{~B}_{4} \mathrm{O}_{7}$ has been employed as a suitable solvent for $\mathrm{TiO}_{2}$ and $\mathrm{ZrO}_{2}$, and small needles of these materials have been produced. Optically perfect crystals of $\mathrm{ThO}_{2}, \mathrm{I} \mathrm{mm}$ on an edge, have also been grown. Lithium fluoride has been employed as a suitable solvent for $\mathrm{UO}_{2}, \mathrm{ThO}_{2}, \mathrm{ZrO}_{2}, \mathrm{TiO}_{2}$, and $\mathrm{MgO}$. Magnesium oxide crystals ( $1 \mathrm{~mm}$ on an edge) have been produced from $\mathrm{LiF}-\mathrm{NaF}$ and $\mathrm{LiF}-\mathrm{NaF}-\mathrm{MgF}_{2}$ eutectic mixtures.

${ }^{1}$ C. F. Weaver, B. J. Sturm, and R. E. Thoma, Reactor Chem. Div. Ann. Progr. Rept. Jan. 31, 1962, ORNL-3262, p 187. 
A pressurized Bridgman-Stockbarger furnace has been constructed by the Metals and Ceramics Division ${ }^{2}$ and will be used in an attempt to grow $\mathrm{Cu}_{2} \mathrm{O}$ crystals directly from the melt. Differential thermal analysis studies of part of the $\mathrm{MgO}-\mathrm{Li}^{2} \mathrm{O}$ $\mathrm{WO}_{3}$ system were performed in search of a suitable phase region for the growth of $\mathrm{MgO}$. The results are negative. Three modifications of the Verneuil process for crystal growth have been designed and assembled and are now being evaluated as an alternative method for the production of highmelting-point crystals, such as $\mathrm{MgO}$.

\subsection{OPTICAL ABSORPTION STUDIES ON $\mathrm{Cu}_{2} \mathrm{O}$}

$$
\begin{gathered}
\text { C. M. Nelson } \\
\text { J. W. Cleland }
\end{gathered}
$$

Samples of $\mathrm{Cu}_{2} \mathrm{O}$ were supplied under contract to the Solid State Division by the Virginia Institute for Scientific Research for a preliminary investigation of the optical absorption properties.

The following procedure was developed to obtain parallel, polished faces: A flat depression was machined in the end of a circular Bakelite plug to a depth greater than that of the samples. Careful machining was required to keep the face of the depression flat and perpendicular to the sides of the plug. The samples were glued around the edges with Duco cement, after which the faces were ground flat on a lapping machine. The mounted samples were then polished in a Syntron apparatus, using a water suspension of $0-0.3 \mu$ $\mathrm{Al}_{2} \mathrm{O}_{3}$ particles. Several samples were also polished using $0-0.1 \mu$ mesh; however, this additional step may not have been necessary. After one side was lapped and polished, the mounted sample was immersed in acetone. One or two hours was required to dissolve the cement, after which the sample was remounted and the other side was lapped and polished. One attempt was made to further reduce the thickness of an already polished sample by further polishing. It was found that the $0-0.3 \mu$ particles had apparently broken down after 2 or $3 \mathrm{hr}$, so that very little additional material was removed. Number 00 emery cloth was employed to decrease the sample

\footnotetext{
${ }^{2}$ G. W. Clark, Metals and Ceramics Div. Ann. Progr. Rept. May 31, 1962, ORNL-3313, p 181 .

${ }^{3}$ Consultant from Emory and Henry College, Emory, Va.
}

thickness (only a very few passes were required), after which the sample was repolished with $0-0.3 \mu$ $\mathrm{Al}_{2} \mathrm{O}_{3}$. Samples 0.24 to $0.38 \mathrm{~mm}$ thick were obtained by the above techniques.

Studies of the initial optical absorption indicated that the first samples submitted and labeled as (111) and (110) had very opaque regions in the interior of the material. The (110) sample was particularly opaque, as shown by the high apparent absorption in the infrared region. There are no resolvable absorption bands from 25,500 to about $6400 \mathrm{~A}$ (the apparent absorption edge) at room temperature. Some absorption is apparent, beginning around $12,000 \mathrm{~A}$ and increasing toward $6400 \mathrm{~A}$, but no definite bands.

Room-temperature optical absorption measurements were also made on two samples, labeled $A-2$ and A-3, which had been produced by oxidation from one side only. These samples did not exhibit an opaque region, and no absorption peaks were apparent near the absorption edge at room temperature.

One of the polished (111) samples was remounted, lapped, and polished, on one side only, to less than one-half its original thickness, in an effort to remove any central opaque region. The optical absorption of this sample $(0.38 \mathrm{~mm}$ thick) compared well with the absorption curve for $A-3$ $(0.345 \mathrm{~mm}$ thick). These data would suggest that one must remove the central region from samples originally oxidized on both sides before they can be employed in optical absorption studies.

Sample A.3 was mounted in a Dewar, with paper supports to guard against breakage. Mea surements were made after the absorption reached an apparent final value near $80^{\circ} \mathrm{K}(2 \mathrm{hr})$. A large drop in absorption and change in the edge from near 6400 to $6260 \mathrm{~A}$ was observed. There were some inflection points and changes in slope, but no peaks were apparent for this sample.

It was felt that a yet thinner sample was necessary to investigate absorption at lower temperatures; therefore, sample A-3 was reground and polished from 0.345 to $0.24 \mathrm{~mm}$, and the absorption was determined at room temperature and near $80^{\circ} \mathrm{K}$. The edge at room temperature was near $6400 \mathrm{~A}$. There was again a large drop in absorption and in position of the edge between room temperature and $80^{\circ} \mathrm{K}$. The most important features of the measurement near $80^{\circ} \mathrm{K}$ were the appearance of a sharp peak at $5827 \mathrm{~A}$ and the suggestion of another peak at $5787 \mathrm{~A}$. 
The A-2 sample, which initially had no opaque regions, was irradiated with $\mathrm{Co}^{60}$ photons (No. 1 source). Very little effect was noticed with $1.5 \mathrm{hr}$ of irradiation, but with $4.5 \mathrm{hr}$ and particularly after $20 \mathrm{hr}$ irradiation $\left(2 \times 10^{17}\right.$ photons $\left./ \mathrm{cm}^{2}\right)$, a decided change was observed near the absorption edge. All measurements were made at room temperature with proper precaution to prevent the sample from being exposed to ambient light after irradiation. There was a noticeable decrease in absorption starting near the edge and continuing as far as was possible with the Cary spectrophotometer $(6050 \mathrm{~A})$. An exposure to the tungsten lamp of the Cary model for a period of about $2 \mathrm{hr}$ restored the absorption near the edge and enhanced it below $6350 \mathrm{~A}$. No changes during any of these treatments were noticed in the region 25,000 to $6600 \mathrm{~A}$.

\subsection{POTASSIUM CHLORIDE SINGLE CRYSTALS}

\author{
C. T. Butler \\ J. R. Russell
}

The ultimate aim of the $\mathrm{KCl}$ pure materials program is to produce, on a routine basis, large single crystals having high chemical purity and low defect concentration. In the first phase of this program, high chemical purity is being sought. Sources for pure starting material, furnace materials, and analysis procedures are being investigated separately.

\section{Starting Material and Furnace-Charge Preparation}

While handling and preparation techniques are being developed, reagent-grade material is being used. It is known to contain several metallic and nonmetallic impurities, as well as dissolved gases, organic, and other particulate material. To remove the last two impurities, the $\mathrm{KCl}$ powder is recrystallized in quartz or Teflon laboratory ware, using doubly distilled water. It is baked overnight in vacuum before being melted under a dry gas for a pulling run.

In the near future the preparation procedures will be improved by including multiple distillation, zone purification, and/or ingot precasting in a dry $\mathrm{HCl}$ atmosphere.

\section{Puller and Zone Refiner}

A pulling furnace of the so-called Czochralski type has been constructed. It can be evacuated and can be filled with a static atmosphere. The pull rate is continuously variable from 0.01 to over $2 \mathrm{~cm} / \mathrm{hr}$. The vacuum and gas-handling system is stainless steel, whereas the header is nickel-plated stainless steel. The envelope and pull rod are quartz. A nickel pull rod is being made. The seed chuck is monel, nickel, or CA-18 graphite, and the crucibles are CA-18 graphite or platinum. It is too early to determine which materials are best suited to each job. The pulling mechanism is driven from a differential ball screw so that gross changes in seed height can be made without disengaging gears or stopping the slower pulling action.

A horizontal zone refiner has also been constructed. The envelope is of quartz, and the end plates and much of the gas-handling apparatus are of monel. The remainder of the apparatus is Teflon. These materials allow use of $\mathrm{HCl}$ gas as the process atmosphere. The crucibles are CA-18 graphite. The zoner will be used both to supply pure, crystalline $\mathrm{KCl}$ for experimentation and to purify starting material for the puller.

\section{Evaluation of . Boules}

Crystals ranging to $3 \times 3 \times 10 \mathrm{~cm}$ have been produced. Routine tests on boules include etchpit counts, flame photometry tests for calcium, strontium, and magnesium, and spectroscopic runs for many other impurities. Optical absorption spectra are measured from $1 \mu$ to the fundamental edge on most boules, and infrared runs are made on some boules to determine the presence of complex-ion impurities.

\subsection{RESEARCH MATERIALS INFORMATION CENTER}

T. F. Connolly

\section{Introduction}

Purpose. - The purpose of the Solid State Division's Information Center is to provide information on methods of production, availability of, 
need for, and characterization of high-purity solidstate research materials to both producers and users. This operation will involve two related but separable functions: (1) handling "availability" and "materials required" information and (2) handling scientific reports containing background information on methods and results of assay and characterization, uses, and other significant new developments. These functions will be discussed separately.

Scope. - The materials covered will be all high-purity solid-state research materials (e.g., metals, alloys, semiconductors, compounds, elements, solid-solution systems, laser and maser materials, and refractories). The only restriction is that they be research materials - as distinct, for example, from structural materials. Radioactive and stable isotopes (covered by the Isotopes Information Center) will not be included, although there will be some unavoidable overlap: pure charge materials for isotope-separation processes will be within the purview of the Research $\mathrm{Ma}$ terials Information Center (RMIC), as will be certain isotopically enriched pure materials. Material will be characterized according to all physical properties of interest (electrical, magnetic, thermophysical, etc.). An example of the difficulty of setting boundaries, of exactly defining the scope of the Center, is presented by the field of radiation effects. Some parts of this field will have to be included since radiation is used to provide structural information not otherwise available. Irradiation is also a method of chemical analysis (neutron activation) and of producing isotopically enriched materials. It might be argued that it would be desirable to accept the loss of some peripheral information in order to set an arbitrary boundary - to say, perhaps, that radiation effects will be included only when they are involved in the characterization or production of an ultrapure material. Such a boundary, however, is not quite so definite as it sounds (an important characteristic of a material might be how it. stands up under irradiation, or an unforeseen future use of some material might be conditional upon its radiation response). Under these circumstances. the limit of coverage must be defined by the list of materials itself; radiation effects on high-purity research materials become a necessary part of the collection. Again, the boundary line is at structural materials, which are dealt with by, among others, the Defense Materials Information Center of Battelle Memorial Institute.
Evaluation of Material. - Research personnel, active in their fields, will be depended on for reference and evaluation of reports and papers. A list of reviewers, composed of individuals active in the research-materials field, is being prepared, and individuals selected will be requested to evaluate preprints, papers, and data on available materials and to make recommendations concerning their inclusion in an automated information system. In view of the scientific information problem, this evaluation is a logical and necessary exten sion of the traditional journal-article reviewing system, an extension based on recognition of the fact that no scientific information system can be effective if the scientist disdains a part in it.

\section{Handling "Availability" and "Materials Required" Information}

A data sheet is being used for the listing of materials, noted as produced or requested. Copies of the sheet were sent out (the second week in "July) with form letters announcing the establishment of the Center to about 400 commercial industries, research laboratories, universities, govemment agencies, and other organizations. The response so far has been gratifying. There have been expressions of willingness to cooperate, requests for from 10 to 1000 data sheets, and requests for further information on (1) what is expected from participants in the program, (2) what participants can expect from the program, and (3) details of operation of the program.

Data Sheets and Reprints. - It is hoped, first, that participants will fill out the data sheets (for either produced or desired materials) as completely as possible, even if this involves a delay in response. If the sheets cannot be filled out, the information will be welcomed in any other form. References to pertinent reports or papers are urgently needed, whether they deal with methods of production, assay, tests of use, or evaluations obtained from studies of special physical characteristics. Reprints of such papers would be preferred (their analysis and coding by the RMIC will be discussed in the section on background material), but if they are unavailable, the references will suffice.

Exchange of Information. - Those desiring materials with special physical or chemical characteristics should, of course, describe their needs as fully as possible, with particular 
emphasis on the specific inadequacies of available materials. This information will be transmitted to those groups in a position to produce the materials as a guide to the developing needs of their potential users. Person-to-person exchanges or loans of materials will be facilitated through the simple device of tabbed "inverted" files - one for requesters and their requirements and one for materials, their characteristics, and their producers. The se files are now being prepared. Every material brought to the attention of the Center as being produced (commercially or in the course of research) or needed will be assigned a card. This card will be inserted in a visible file, and the bottom (or visible) portion will be marked with a colored strip bearing the name of the requester for a special form of the material. Any time an improved or changed material (with its source) is added to a card so tabbed, the information will be checked against the tabbed name or names in the file of requests. If the added material fits the specifications noted in the request file, the requester will be norified of the source. No purcbuses, loans, or exchanges will be arranged by the Center.

Data sheets will be organized, indexed, and stored on 100-ft reels of coded 16-mm microfilm. They will be retrievable through a microfilm viewer-printout combination in answer to specific requests or for inclusion in periodic bulletins.

\section{Handling Background Material}

Input. - The background or peripheral material will be taken from several sources, the most important of which are expected tobe (1) references , and reprints provided with the data sheets and (2) references from program participants which concern significant new developments that come to their attention in their own fields (here, again, reviewers of papers from other sources can be of assistance). Literature searches will be continued routinely by the Center, and special searches are being arranged through the cooperation of the AEC Division of Technical Information Extension at Oak Ridge; ORNL prepublication abstracts are being examined. The Center now receives reports in the categories of Metals and Ceramics, Solid State, Physics, and Reactor Chemistry from all AEC installations. All such material will be evaluated - sometimes merely by considering its source, and, where this is not definitive, by requesting the review described above. When such a review is requested, the reviewer will be asked, if in his opinion the work is not sufficiently novel, to inform the Center of work that supersedes it.

Coding and Filming. - Evaluated reports will be coded according to a key-term (coordinate-index) system and microfilmed by a special film-encoding camera. They will be retrieved by a codeinterpreting viewer-printer by the conventional method of appropriate combinations of assigned numerical equivalents of the key terms.

These terms will be controlled by a thesaurus and limited to items of interest to the program. A tentative list of terms, which is growing, is now at about 400, excluding the names and classes of materials. According to the rate of growth of the list so far and the scope of the Center as now envisioned, the list of terms should not exceed 600. The materials list is now at about 350, and no predictions can be made about its eventual size.

Each paper to be filmed will be analyzed for its key terms, and the numerical code equivalent of its set of terms will appear as coding marks on the film. The film storage and retrieval method may be regarded as a photoelectrically interpreted analog of conventional punched-card systems, with the important difference that repeated sorts of the collection are not necessary to establish a "match" between questions and answers. A typical question so answered would be a survey of the latest preparative procedures for a certain material or class of materials. Answers to questions will be in the form of complete reprints, abstracts, or references - reproduced as photoprints by an attachment to the automated film scanner. Review articles (as opposed to bulletins) are not planned; however, bibliographies produced by the machine should be good source material for such reviews. When bibliographies are used for this purpose, the user will be asked to provide copies of the review articles for reproduction and dissemination (at cost) to interested program participants.

\section{Present Status}

The Center is at its second stage, that of collecting and organizing a mass of information from a wide variety of sources. By October of this year, it should be possible to begin the 
feedback process of transmitting "availability" and "materials required" information between producers and users on a routine basis, with some scattered items on production methods.

Collection of documents and references that will form the store of background information for the preparation of surveys and bibliographies has already begun, but this material will not be organized, coded, and microfilmed until the spring of 1963.

The following is a list of program participants to date:

Battelle Memorial Institute

Oak Ridge National Laboratory

Brookhaven National Laboratory

Hanford Laboratories-General Electric Company

U.S. Naval Ordnance Test Station, Inyokern, China Lake, Calif.

U.S. Naval Ordnance Laboratory, White Oak, Silver Spring, Md.

Lewis Research Center, NASA

Cornell University:

Laboratory of Atomic and Solid State Physics

Materials Science Center

The Franklin Institute, Philadelphia, $\mathrm{Pa}$.

Virginia Polytechnic Institute

Iowa State University, Ames Laboratory

Massachusetts Institute of Technology, National Magnet Laboratory

University of Chicago, Institute for the Study of Metals University of North Carolina

University of California, Berkeley, Lawsence Radiation Laboratories

Catholic University of America

Brown University

University of Pennsylvania, Laboratory for Research on the Structure of Matter

Northwestern University, Materials Research Center

University of Wisconsin

University of Nebraska

Stanford Research Institute, Department of Solid State

Harvard University, Division of Engineering and Applied Physics

Stanford University, Department of Materials Science

Purdue University

Mellon Institute

Atomics International

Sandia Corporation

T. J. Watson Research Center, IBM

Ray theon Company

Sylvania Electric Products, Inc.

Bell and Howell Research Center

Bendix Research Laboratories

Virginia Institute for Scientific Research

Commercial producers such as Texas Instruments, Light and Company, Ltd., Eagle-Picher, Semi-Elements, etc.

\subsection{PURIFICATION OF $\mathrm{Bi}_{2} \mathrm{Se}_{3}$ CRYSTALS DURING AND AFTER GROWTH}

\author{
M. J. Smith
}

The extrinsic, electrical properties of chemically pure $\mathrm{Bi}_{2} \mathrm{Te}_{3}$ (ref 4) and $\mathrm{Bi}_{2} \mathrm{Se}_{3}$ (ref 5) may be attributed to deviations from stoichiometric proportions. The compound $\mathrm{Bi}_{2} \mathrm{Te}_{3}$ exists as a single phase over a range of composition through the inclusion of substitutional point defects. That is, excess bismuth atoms occupy tellurium sites and are singly ionized acceptors, and excess tellurium atoms occupy bismuth lattice sites and behave as singly ionized donors. ${ }^{4}$ A similar phenomenon ${ }^{5}$ is considered to be true in $\mathrm{Bi}_{2} \mathrm{Se}_{3}$. Selenium acts as an acceptor; however, limited solid solubility of selenium in $\mathrm{Bi}_{2} \mathrm{Se}_{3}$ prevents growth of $p$-type material from a melt containing excess selenium. ${ }^{6}$

The ability of a crystal to exist as one phase over a range of composition implies some thermodynamic consequences which are important in the preparation and purification of these materials. In $\mathrm{Bi}_{2} \mathrm{Te}_{3}$ and $\mathrm{Bi}_{2} \mathrm{Se}_{3}$ the crystal vapor has two phases and two components; thus, it has two degrees of freedom. Therefore, in principle, the composition and, hence, the electrical properties of the crystal can be controlled by regulating the temperature and the partial pressure of one component. In practice, however, it is necessary to work at those temperatures at which the crystalvapor equilibrium is established in a reasonable length of time.

It has been demonstrated previously that controlled selenium vapor pressures can be used to prevent loss of selenium from $\mathrm{Bi}_{2} \mathrm{Se}_{3}$ to the vapor at high temperatures. ${ }^{7}$ A device similar to that described in ref 7 was used to attempt vaporcrystal doping of $\mathrm{Bi}_{2} \mathrm{Se}_{3}$. The device employed the following modifications: the excess selenium was not held within the specimen chamber but was contained in an additional chamber attached to the specimen chamber by a connecting tube. The temperatures of the selenium chamber, connecting

\footnotetext{
${ }^{4}$ C. B. Satterthwaite and R. W. Ure, Jr., Phys, Rev. 108, 1164 (1957).

${ }^{5} \mathrm{~K}$. Hashimoto, Mem. Fac. Sci., Kyusyu Univ. B2, 141, 187 (1958); J. Phys. Soc. (Japan) 16, 1970 (1961).

${ }^{6}{ }_{\mathrm{M}}$. J. Smith, unpublished research at Cornell University, Ithaca, N.Y.

${ }^{7}$.M. J. Smith, E. S. Kirk, and C. W. Spencer, J. Appl. Pbys. 31, 1504 (1960).
} 
tube, and specimen chamber were controlled separately, thereby regulating the partial pressure of selenium in the system. Resistivity and Hall measurements were taken on a number of single crystals of $\mathrm{Bi}_{2} \mathrm{Se}_{3}$ at various specimen temperatures and selenium vapor pressures as a function of time.

It can be shown that the approach to equilibrium concentrations within a thin slab is expressed by the following relation: ${ }^{8}$

$$
\begin{aligned}
& \frac{n_{\mathrm{eq}}-\bar{n}}{n_{\mathrm{eq}}-n_{i}} \\
& \quad=\frac{8}{\pi^{2}} \sum_{v=0}^{\infty} \frac{1}{(2 v+1)^{2}} e^{-\left[(2 v+1)^{2} \pi^{2} / b^{2}\right] D t},
\end{aligned}
$$

where $n_{\text {eq }}$ is the equilibrium concentration in the crystal of hiickness $b$ at ambicnt temperature $T_{A}, \bar{n}$ is the average concentration in the crystal at some time $t, n_{i}$ is the initial concentration at $t=0$, and $D$ is the diffusion coefficient of the process at the temperature of the specimen.

For $t \times D$, sufficiently large, the first term in the series gives a good approximation:

$$
\frac{n_{\mathrm{eq}}-\bar{n}}{n_{\mathrm{eq}}-n_{i}} \approx \frac{8}{\pi^{2}} e^{-t D \pi^{2} / b^{2}} \text {. }
$$

If, in the particular case of $\mathrm{Bi}_{2} \mathrm{Se}_{3}, n_{0}$ is the concentration of current carriers and if this concentration of current carriers is, as proposed, controlled by the vapor pressure of selenium over the crystal,

$$
\frac{n_{0_{\mathrm{eq}}}-\bar{n}_{0}}{n_{0_{\mathrm{eq}}}-n_{0_{i}}}=\frac{n_{\mathrm{eq}}-\bar{n}}{n_{\mathrm{eq}}-n_{i}}=r \approx \frac{8}{\pi^{2}} e^{-\ell D \pi^{2} / b^{2}}
$$

For time sufficiently large, $r$ is independent of the vapor pressure over the crystal and depends only on the ambient temperature, $T_{A}$, of the crystal and the time of exposure to a particular vapor pressure of selenium. Hence, $r$ may be calculated as a function of time from Eq. (3) by obtaining several measurements of $\bar{n}_{0}$ at varying times and vapor pressures of selenium. The natural logarithm of $r$ for specimen CBS-2 is plotted in Fig. 17.1 as a function of time for

${ }^{8}$ W. Jost, Diffusion in Solids, Liquids, Gases, 3d ed., p 37, Academic Press, New York, 1960.

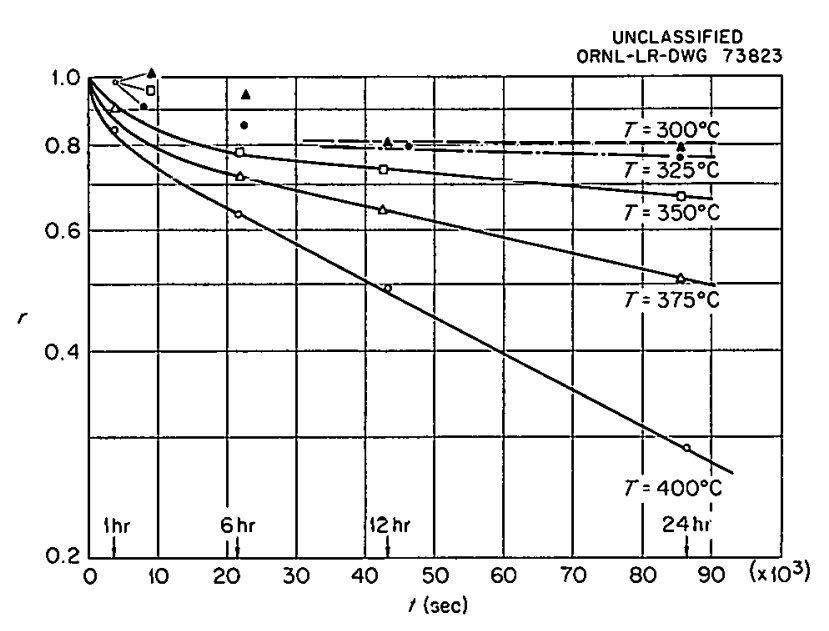

Fig. 17.1. Plot of $\left(n_{\text {eq }}-\bar{n}\right) /\left(n_{\text {eq }}-n_{i}\right)=r$ vs $t$ for Crystal CBS.2 of $\mathrm{Bi}_{2} \mathrm{Se}_{3}$ at Several Ambient Temperatures.

various ambient temperatures. The slopes of the straight-line portion of each curve give the diffusion coefficient for the process. Diffusion coefficients obtained on all other specimens of $\mathrm{Bi}_{2} \mathrm{Se}_{3}$ were identical at higher temperatures. Considerable scatter was encountered at lower temperatures, however, because of the in accuracy of Eq. (3) at these temperatures.

Figure 17.2 shows a plot of $\ln D$ vs $1 / T$. The activation energy of the diffusion process is $3 \times 10^{4} \mathrm{cal} / \mathrm{mole}$. The dependence of the diffusion coefficient on temperature may be expressed by

$$
D=3.5 e^{-3 \times 10^{4} / R T} \mathrm{~cm}^{2} / \mathrm{sec} .
$$

The self-diffusion coefficient of selenium in $\mathrm{Bi}_{2} \mathrm{Se}_{3}$ is reported ${ }^{9}$ to be expressed by

$$
D_{\mathrm{Se}_{\mathrm{B}} \mathrm{Bi}_{2} \mathrm{Se}_{3}}=8.5 \times 10^{-9} e^{-5.0 \times 10^{4} / R T} .
$$

The literature does not contain information on the self-diffusion of bismuth in $\mathrm{Bi}_{2} \mathrm{Se}_{3}$; however, values for the bulk diffusion of antimony in $\mathrm{Bi}_{2} \mathrm{Se}_{3}$ have been obtained. ${ }^{10}$ Antimony is similar to bismuth in size and electronic configuration:

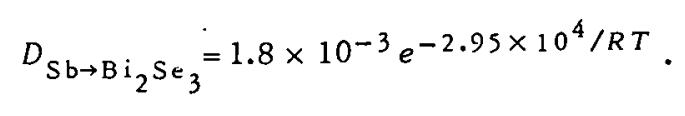

${ }^{9}$ A. A. Kuliev and G. B. Abdullaev, Soviet Pbys.Solid State 1, 545 (1959).

${ }^{10}$ B. I. Boltaks, Zh. Tekbn. Fiz. 25, 767 (1955). 


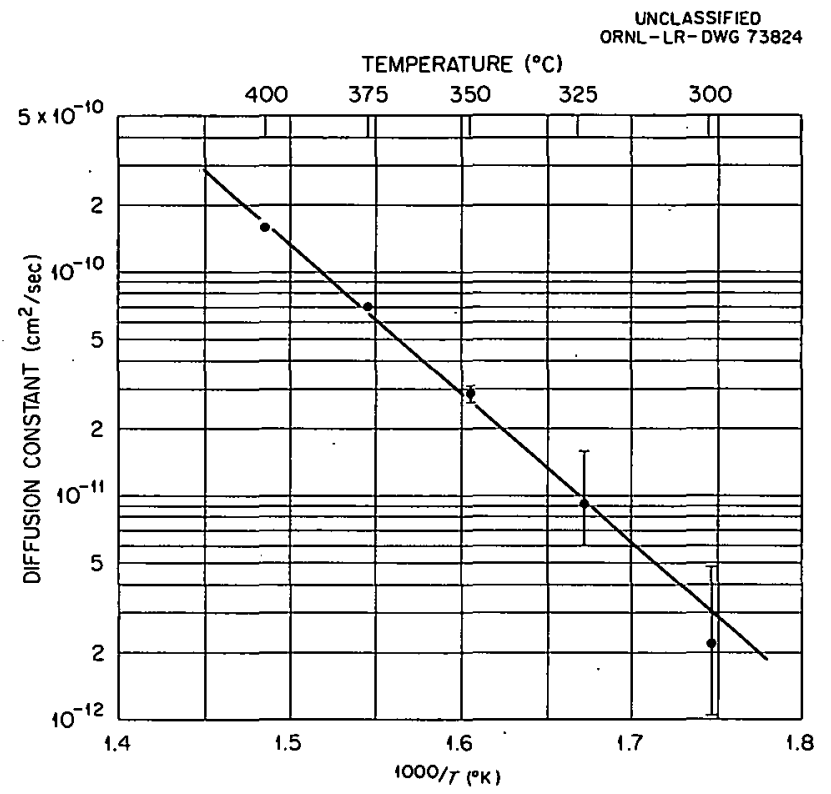

Fig. 17.2. Selenium Vapor-Crystal Diffusion Coefficient of $\mathrm{Bi}_{2} \mathrm{Se}_{3}$ Exposed to Various Vapor Pressures of Selenium vs 1000/T.

The activation energies of Eqs. (6) and (4) are essentially identical. These results indicate that, if the process is an atomic diffusion phenomenon, it is probably controlled by the diffusion of bismuth. rather than selenium. The extremely low diffusion coefficient of selenium would prevent any observable effect on electrical properties in a reasonable length of time. On the other hand, the extremely low solid solubility ${ }^{6}$ of bismuth in $\mathrm{Bi}_{2} \mathrm{Se}_{3}$ renders control of the electrical properties by bismuth vapor pressures impossible.

The maximum reduction of current carriers which is possible under the conditions studied (ambient temperature $T_{A}=400^{\circ} \mathrm{C}$; temperature of selenium container $T_{\mathrm{Se}}=400^{\circ} \mathrm{C}$ ) is only $33 \%$; that is,

$$
\frac{n_{i}-n_{e q}}{n_{i}}=0.33
$$

at $T_{A}=400^{\circ} \mathrm{C}$ and $T_{\mathrm{Se}}=400^{\circ} \mathrm{C}$. Therefore, it appears that a high degree of purification of $\mathrm{Bi}_{2} \mathrm{Se}_{3}$ is impossible by means of crystal-vapor doping. However, if the vapor pressure of selenium is controlled over liquid $\mathrm{Bi}_{2} \mathrm{Se}_{3}$ and a crystal is pulled very slowly from the melt, it is reasonable to predict a reduction in current carriers by several orders of magnitude.

Figure 17.3 is a schematic of a modified TealLittle crystal furnace designed to maintain varying vapor pressures of constituent elements over a liquid while the crystal is growing. The furnace consists of a flanged quartz pipe (1) which is mounted on a water-cooled base (8). The base supports an auxiliary heater (10). The quartz pipe can be evacuated through outlet (9). Inside the quartz pipe is a stainless steel, wire-wound heater (5). The stainless steel heater tube was machined and grooved, coated with $\mathrm{AlO}_{2}$, and wound with B\&S 24 Nichrome wire. A viewing window was cut in the heater. The heater element (6) was similarly prepared. Inside the two heaters is a quartz jar (2), which is flanged at the top. A stainless steel top (3) fits into the quartz jar. The stainless steel pulling rod and chuck (not shown) passes through the rod support assembly (11). The inside quartz jar is evacuated through this rod and is sealed to the outside when the stainless steel evacuating tube is lowered into the molten compound in the crucible (7). Furnace (4) maintains an excess of one of the constituent elements at controlled temperatures and thereby controls the vapor pressure of the system. 


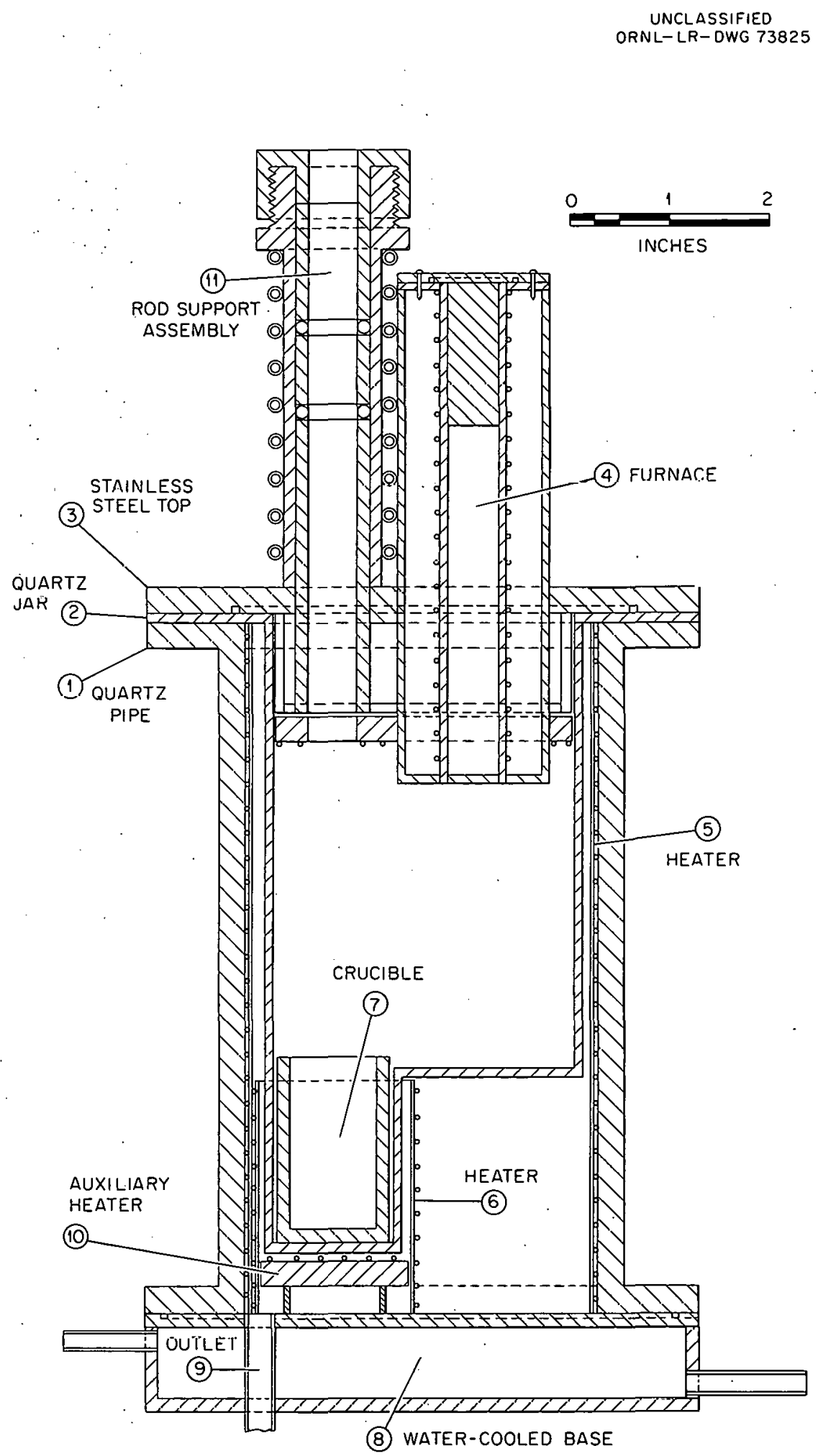

Fig. 17.3. Controlled Vapor-Pressure Crystal-Pulling Furnace. 
THIS PAGE

WAS INTENTIONALLY

LEFT BLANK 


\section{Part V. Radiation Metallurgy M. S. Wechsler}


THIS PAGE

\section{WAS INTENTIONALLY \\ LEFT BLANK}




\section{Fundamental Studies}

\subsection{THEORY OF THE DEPENDENCE OF RADIATION-ENHANCED DIFFUSION ON DISPLACEMENT PRODUCTION RATE AND IRRADIATION TEMPERATURE}

\section{J. H. Barrett ${ }^{1}$}

Unless experiments are done at very low temperatures, radiation-produced point defects usually anneal out during the irradiation. The most obvious annealing mechanisms are diffusion of the defects to fixed sinks (dislocations, grain boundaries, and surfaces) and recombination of interstitial-vacancy pairs. The equations for this combination of annealing processes are

$$
\begin{aligned}
& \frac{d v}{d t}=K-\beta_{\nu} \nu_{\nu}\left(v-v_{0}\right)-\nu_{i} v i, \\
& \frac{d i}{d t}=K-\beta_{i} \nu_{i} i-\nu_{i} \nu i,
\end{aligned}
$$

where $K$ is the displacement production rate (interstitial-vacancy pair production rate) per lattice site, $v$ and $i$ are the concentrations of vacancies and interstitials per lattice site, $\nu_{0}$ is the thermal equilibrium concentration of vacancies, $\beta_{\nu}=\alpha_{\nu} \lambda^{2}, \beta_{i}=\alpha_{i} \lambda^{2}, \lambda=$ jump distance of a defect, the $\alpha^{\prime} s$ are concentrations of fixed sinks, and the $\nu$ 's are jump frequencies.

Dienes and Damask ${ }^{2}$ have treated the steady state solutions of Eqs. (1) and (2); Lomer ${ }^{3}$ has treated both the steady state and the transient

\footnotetext{
${ }^{1}$ Theory Section, Solid State Division.

${ }^{2}$ G. J. Dienes and A. C. Damask, J. Appl. Pbys. 29, 1713 (1958).

${ }^{3}$ W. M. Lomer, Diffusion Coelficients in Copper Under Fast Neutron Irradiation, AERE-T/R-1540 (December 1954).
}

solutions of the se equations. The solutions below are essentially Lomer's, but they are given in a somewhat more tractable form.

As is the case in many metals, it is assumed here that $\nu_{i} \gg \nu_{\nu}$. In this case, the interstitials will quickly achieve a quasi-steady state. Equation (2) may be solved for $i$ to give

$$
i=\left(K-\frac{d i}{d t}\right) / \nu_{i}\left(\beta_{i}+v\right) \text {. }
$$

Except for a very brief initial transient period, it is assumed that $d i / d t<<K$. (A sufficient condition for this assumption is $K / \beta_{i}^{2} \nu_{i} \ll 1$.) Neglecting di/dt in Eq. (3) and substituting the result into Eq. (1) gives

$$
\frac{d v}{d t}=K \frac{\beta_{i}}{\beta_{i}+v}-\beta_{\nu} \nu_{v}\left(\nu-v_{0}\right) .
$$

On the assumption that $v=v_{0}$ at $t=0$, the solution to Eq. (4) is

$$
\begin{aligned}
\nu(t)=\nu_{0}+\left\{\left[\left(\frac{\beta_{i}+\nu_{0}}{2}\right)^{2}+\frac{K \beta_{i}}{\beta_{\nu} \nu_{\nu}}\right]^{1 / 2}\right. \\
\left.\quad-\frac{\beta_{i}+v_{0}}{2}\right\} \chi\left(\beta_{\nu} \nu_{\nu} t\right),
\end{aligned}
$$

where $\chi(\tau)$ is defined by

$$
\begin{aligned}
& (1-\chi)^{1+\rho}\left(1+\frac{1-\rho}{1+\rho} \chi\right)^{1-\rho}=e^{-2 \tau}, \\
& \rho=\frac{\beta_{i}+\nu_{0}}{2} /\left[\left(\frac{\beta_{i}+\nu_{0}}{2}\right)^{2}+\frac{K \beta_{i}}{\beta_{\nu} \nu_{\nu}}\right]^{1 / 2} .
\end{aligned}
$$


It may be noted that $0 \leqq \rho \leqq 1$. Limiting forms for $\chi(\tau)$ are

$$
\begin{array}{ll}
\chi=\sqrt{1-e^{-2 \tau}} & \text { for } \rho \approx 0, \\
\chi=1-e^{-\tau} & \text { for } \rho \approx 1 .
\end{array}
$$

For other values of $\rho$, Eq. (6) may be solved by numerical methods to give $\chi(\tau)$. Figure 18.1 shows a plot of $\chi(\tau)$ for the two limiting cases and one other case. For most radiation damage experiments, $K$ will be large enough to make $\rho \approx 0$.

In studies of radiation-enhanced diffusion, a quantity "of interest is the time $t(J)$ required for a certain number of jumps per lattice site to occur. The dependence of $t(J)$ on $K$ and on the absolute temperature $T$ may be expressed in the form

$$
t(J) \propto K^{p} e^{E / k T} .
$$

Values of $p$ and $E$ are given in. Table 18.1. For completeness the table also includes the simpler cases of either the pair-recombination or the

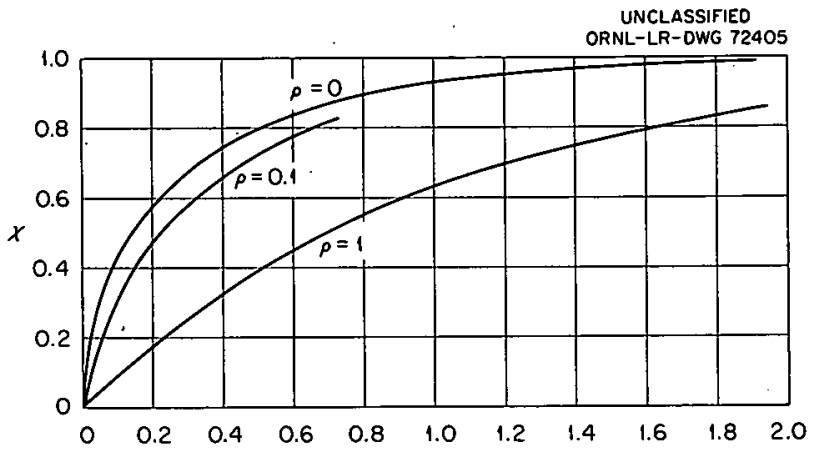

Fig. 18.1. Plot of $\chi$ vs $\tau$ for Various Values of $\rho$.

diffusion-to-fixed-sink annealing processes alone. These latter two processes have been treated by Dienes and Damask. ${ }^{2}$

It should be pointed out that the abuve analysis assumes that the point defects are randomly distributed upon irradiation and that they remain

Table 18.1. Values of $p$ and $E$ for the Three Annealing Mechanisms

\begin{tabular}{|c|c|c|c|c|}
\hline Annealing Process & Time Range & $\begin{array}{l}\text { Type of } \\
\text { Defect }\end{array}$ & $p$ & $E$ \\
\hline \multirow{4}{*}{$\begin{array}{l}\text { Diffusion to fixed } \\
\text { sinks alone }\end{array}$} & $t \ll\left(\beta_{\nu} \nu_{v}\right)^{-1}$ & $\nu$ & $-1 / 2$ & $\frac{1}{2} E_{\mathrm{mv}}$ \\
\hline & $t \ll\left(\beta_{i} \nu_{i}\right)^{-1}$ & $i$ & $-1 / 2$ & $\frac{1}{2} E_{\mathrm{mi}}$ \\
\hline & $t \gg\left(\beta_{\nu} \nu_{\nu}\right)^{-1}$ & $v$ & -1 & 0 \\
\hline & $t \gg\left(\beta_{i} \nu_{i}\right)^{-1}$ & $i$ & -1 & 0 \\
\hline \multirow{4}{*}{$\begin{array}{l}\text { Pair recombination } \\
\text { alone }\end{array}$} & $t \ll\left(K \nu_{i}\right)^{-1 / 2}$ & $v$ & $-1 / 2$ & $\frac{1}{2} E_{\mathrm{mv}}$ \\
\hline & & $i$ & $-1 / 2$ & $\frac{1}{2} E_{\mathrm{mi}}$ \\
\hline & $t \gg\left(K \nu_{i}\right)^{-1 / 2}$ & $v$ & $-1 / 2$ & $E_{\mathrm{mv}}-\frac{1}{2} E_{\mathrm{mi}}$ \\
\hline & & $i$ & $-1 / 2$ & $1 / 2 E_{\mathrm{mi}}$ \\
\hline \multirow{4}{*}{$\begin{array}{l}\text { Pair recombination } \\
\text { and diffusion to } \\
\text { fixed sinks }(\rho \approx 0)\end{array}$} & $\imath \ll 4\left(\beta_{\nu} \nu_{\nu}\right)^{-1}$ & $v$ & $-1 / 3$ & $\frac{2}{3} E_{\mathrm{mv}}$ \\
\hline & & $i$ & -1 & 0 \\
\hline & $t \gg 4\left(\beta_{\nu} \nu_{v}\right)^{-1}$ & $v$ & $-1 / 2$ & $1 / 2 E_{\mathrm{mv}}$ \\
\hline & & $i$ & $-1 / 2$ & $-\frac{1}{2} E_{\mathrm{mv}}$ \\
\hline
\end{tabular}

$E_{\mathrm{mv}}$ and $E_{\mathrm{mi}}$ are the activation energies for motion of a vacancy and interstitial, respectively. The other symbols are defined in the text. 
so during their annealing. Another possibly important factor, not embraced by the analysis, is that fixed sinks may be created during the irradiation by a process of vacancy aggregation in to small pores which collapse forming dislocation loops. Also, no attempt is made to take the effect of solute-vacancy or solute-interstitial pairing in to account. These processes may alter the solute jump frequencies in a significant way.

\subsection{EFFECT OF RADIATION ON ATOMIC REARRANGEMENTS IN CU-AI}
J. M. Williams
C. Bassani ${ }^{4}$
M. S. Wechsler
J. H. Barrett ${ }^{5}$

\section{Introduction}

It has been shown ${ }^{6-8}$ that the bombardment of $\mathrm{Cu}-\mathrm{Al}$ alloys with neutrons, electrons, or gamma rays results in a decrease in resistivity, provided the temperature is at once high enough for the radiation-produced defects to be mobile and low enough to be below the temperature where equilibrium order can be obtained by thermal diffusion alone. The decrease in resistivity saturates as the equilibrium arrangement is approached. The phenomenon is illustrated schematically in F.ig. 18.2. Curve $A B$ represents the $\rho$ vs $T$ curve observed for normal cooling at a given rate, and curve $A C$ represents the ideal equilibrium curve for an infinitely slow cooling rate. Below $T_{2}$ for the normal cooling, the atomic arrangement characteristic of point $A$ is frozen in. Upon isothermal irradiation below $T_{2}$ (provided the temperature is not too low), the resistivity decreases from a

\footnotetext{
${ }^{4}$ Now at CCR - Euratom, Ispra (Varese), Italy.

${ }^{5}$ Theory Section, Solid State Division.

${ }^{6} \mathrm{M}$. S. Wechsler and R. H. Kernohan, Phys. Chem. Solids 7, 307 (1958).

${ }^{7}$ R. H. Kernohan and M. S. Wechsler, Phys. Chem. Solids 10, 175 (1961).

${ }^{8}$ M. S. Wechsler and R. H. Kernohan, "The Effect of Radiation on Diffusion-Controlled Reactions in Copper-Base Alloys," paper SM-25/25, Symposium on Radiation Damage to Solids and Reactor Materials, May $7-11$, 1962, Venice, Italy.
}

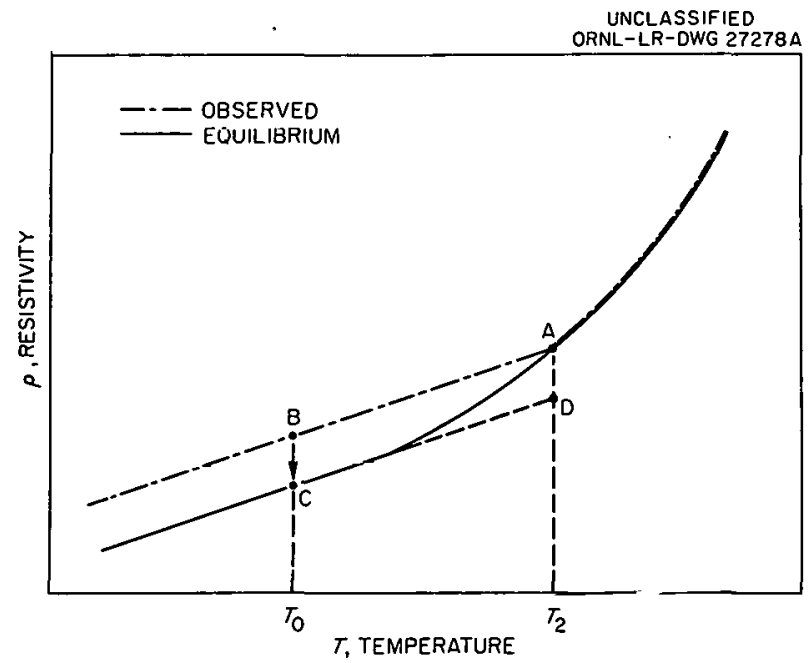

Fig. 18.2. Schematic Plot of Resistivity vs Tempera. ture.

point on $A B$ to a point on $A C$. For single cry stals of $\mathrm{Cu}-15$ at. \% Al cooled at $15^{\circ} \mathrm{C} / \mathrm{hr}, T_{2}$ is about $210^{\circ} \mathrm{C}$ and the decrease upon irradiation varies from zero at $210^{\circ} \mathrm{C}$ to about 0.18 microhm-cm at $50^{\circ} \mathrm{C}^{7}$

The above theoretical analysis (summarized in Table 18.1, Sec 18.1) shows that a study of the temperature and flux dependence of the rate of radiation-enhanced diffusion yields information conceming the mechanism of annihilation of the diffusion-enhancing defect. The temperature dependence for the reaction in $\mathrm{Cu}-15$ at. \% Al has been reported previously; ${ }^{7}$ it was found to correspond to an activation energy of $0.5 \mathrm{ev}$ or about one half the activation energy for motion of vacancies in $\mathrm{Cu}-17$ at. \% Al observed by $\mathrm{Li}$ and Nowick. $\quad$ This result suggests that $E=1 / 2 E_{\mathrm{mv}}$ in Table 18.1. Preliminary experiments dealing with flux dependence were reported earlier. ${ }^{10}$ This work has been extended in the past year. In addition, the effect of repeated irradiation and thermal cycling was studied.

\footnotetext{
${ }^{9} \mathrm{C}$ Y. Li and A. S. Nowick, Pbys. Keu. 103, 294 (1956).

${ }^{10} \mathrm{M}$. S. Wechsler et al., Solid State Div. Ann. Progr. Rept. Aug. 31, 1961, ORNL-3213, pp 32-42.
} 


\section{Neutron Flux Measurements and Calculation of Displacement Production Rate}

In the flux-dependen ce experiments, single cry stal samples of $\mathrm{Cu}-15$ at. \% Al were irradiated at $100^{\circ} \mathrm{C}$ at various fluxes (various displacement production rates, $K$ ) by using different positions in Hole C of the ORNL Graphite Reactor. The resistivity was measured during irradiation and the time, $\tau_{1 / 2}$, for half-completion of the decrease in resistivity was taken as a measure of the time, $t(J)$ [Eq. (7), Sec 18.1], for a certain number of atom jumps per site.

In order to determine $K$ for each of the experimental positions, various thermal, resonance, and threshold detectors were used to measure the neutron flux and spectrum as a function of distance into Hole $C$. The effective threshold energies and the corresponding effective cross sections for several threshold detectors are shown in Table 18.2. The threshold monitors used in Hole $\mathrm{C}$ were $\mathrm{Np}^{237}, \mathrm{U}^{238}, \mathrm{Ni}^{58}$, and $\mathrm{S}^{32}$. The $\mathrm{U}^{238}$ fission monitors contained only $2 \mathrm{ppm} \mathrm{U}^{235}$.

The detectors, $\mathrm{Co}^{59}, \mathrm{Mn}^{55}$, and $\mathrm{Na}^{23}$, were used in determining the themal and epithermal flux. Each monitor was irradiated both bare and enclosed in a 0.027-in. thickness of cadmium. The thermal flux is evaluated from the difference of the induced activities, $A_{\text {thermal }}=A_{\text {bare }}-A_{\mathrm{Cd}}$, using

$$
\Phi_{\text {thermal }}=\frac{A_{\text {thermal }}}{\sigma_{\text {th }}} \text {, }
$$

where $\sigma_{\text {th }}$ is the themal cross section. In order to find the epithemal flux from the activities of the cadmium-covered monitors, we follow a method similar to that given by Trice. ${ }^{11}$ Assuming a $1 / E$ spectral distribution in the epithermal region, we write the activation integral for the cadmiumcovered samples as

$$
\begin{aligned}
A_{\mathrm{Cd}} & =\Phi_{0}\left(\int_{E_{\mathrm{Cd}}}^{\infty} \frac{\sigma_{1 / \nu}}{E} d E+\int_{E_{\mathrm{Cd}}}^{\infty} \frac{\sigma_{r}}{E} d E\right) \\
& =\Phi_{0}\left(I_{1 / \nu}+I_{r}\right),
\end{aligned}
$$

where $\Phi_{0}$ is the flux per unit $\ln E$ interval, $E_{\mathrm{Cd}}$ is the cadmium cutoff energy, and $\sigma_{1 / \nu}$ and $\sigma_{r}$ are the $1 / v$ and resonance components of the cross sections respectively. The cutoff energy was determined for a cadmium thickness of 0.027 in. in

\begin{tabular}{|c|c|c|c|c|c|}
\hline $\begin{array}{l}\text { Detector } \\
\text { Element }\end{array}$ & - & Nuclear Reaction & $\begin{array}{c}E_{i}, \\
\text { Threshold } \\
\text { Energy } \\
\text { (Mev) }\end{array}$ & $\begin{array}{c}\sigma_{i} \\
\text { Effective } \\
\text { Cross Section } \\
\text { (barns) }\end{array}$ & Reference \\
\hline $\mathrm{Np}$ & & $\mathrm{Np}^{237}(n, n) \mathrm{Ba}^{140}$ & 0.6 & 1.7 & $a$ \\
\hline $\mathrm{U}$ & & $\mathrm{U}^{238}\left(n, n \mathrm{Ba}^{140}\right.$ & 1.6 & 0.58 & $b$ \\
\hline $\mathrm{Ni}$ & & $\mathrm{Ni}^{58}(n, p) \mathrm{Co}^{58}$ & 2.9 & 0.39 & $b$ \\
\hline $\mathrm{s}$ & & $\mathrm{S}^{32}(n, p) \mathrm{P}^{32}$ & 3.0 & 0.32 & $b$ \\
\hline $\mathrm{Fe}$ & & $\mathrm{Fe}^{56}(n, p) \mathrm{Mn} 56$ & 7.5 & 0.11 & $b$ \\
\hline$A 1$ & & $\mathrm{Al}^{27}(n, p) \mathrm{Mg}^{27}$ & 8.2 & 0.12 & $b$ \\
\hline
\end{tabular}

${ }^{11} \mathrm{~J}$. B. Trice, Nucleonics 16(7), 81 (1958).

Table 18.2. Detector Elements and Dosimetry Parameters for Several Threshold Reactions

$a_{\text {Based on the }} \mathrm{Np}^{237}$ fission cross section curve given in $\mathrm{H}$. W. Schmitt and R. B. Murray, Phys. Rev. 116, 1575 (1959), and the fission spectrum given in L. Cranberg, Phys. Rev. 103, 662 (1956).

${ }^{b}$ T. O. Passel and R. L. Heath, Nucl. Sci. Eng. 10, 308 (1961). 
an isotropic flux, and $I_{1 / \nu}$ was evaluated for each monitor. Table 18.3 shows $I_{r}$ values extracted from recent literature, along with the $I_{1 / v}$ values and thermal cross sections for the reactions that were used. The analysis of the cadmium-covered activity yields $T_{0}$.

The samples were analyzed by the Nuclear Analysis Group of the Analytical Chemistry Division. ${ }^{12}$ Gamma counting was used for all activities except for the $\mathrm{P}^{32}$ activity, which is due to $\beta$ emission. The gamma-ray spectrometer consisted of a $3 \times 3$ in. NaI crystal and a 200. channel analyzer. For fission monitors, the $\mathrm{Ba}^{140}$ activity was separated chemically before counting. The techniques involved in analyzing the samples are presented more fully elsewhere. ${ }^{13}$

The monitors were irradiated simultaneously for a period of about $24 \mathrm{hr}$ with the reactor at full power $(3400 \mathrm{kw})$. The samples were positioned in the hole by placing them in thin plastic bottles and setting these bottles into ports in a $1 / 2$-in.ID $\times 5 / 8$-in.-OD polyethylene tube which was inserted into the hole. The cadmium-covered detectors were separated from the nearest bare detectors by at least $4 \frac{1}{2}$ in. in all cascs. The standard error is about $\pm 6 \%$ for each of the monitors.

\footnotetext{
${ }^{12}$ We would particularly like to thank W. J. Hampton for his work in preparing and counting the monitors.

${ }^{13} \mathrm{P}$. Dragoumis, J. R. Weir, and G. W. Leddicotte, Fast-Flux Measurements in the ORR Core, ORNL3028 (1961).
}

Figure 18.3 shows the results of the study. It is seen that the flux-spectrum is fairly well determined for all positions in Hole $C$ from the center of the reactor out to about $2 \frac{1}{2} \mathrm{ft}$ into the reflector. The spectrum determined for a position $19 \mathrm{ft} 10 \mathrm{in}$. into the hole is compared with the theoretical spectrum of Robinson, Oen, and Holmes ${ }^{14}$ (ROH spectrum) in Table 18.4. The theoretical values ( $\Phi_{\text {the or }}$ ) were calculated from the ROH spectrum multiplied by a constant, $C$, so as to give quantities of the same order of magnitude as the measured values. These results indicate that the application of the $\mathrm{ROH}$ spectrum to the calculation of the displacement production rate for this position in the Graphite Reactor is reasonably well justified.

The positions where samples were irradiated are shown in Fig. 18.3. Particularly for the two samples located in the reflector region of the reactor (Positions 5 and 6 ) where the ratio of thermal to fast flux is high, it was necessary to take in to account the displacements produced as a result of $(n, \gamma)$ recoil events. ${ }^{15,16}$ The displacement production rate due to $(n, \gamma)$ recoil is

$$
K(n, y)=\phi_{\mathrm{th}} \sigma_{\mathrm{th}} \frac{\bar{T}_{R}}{2 T_{d}},
$$

\footnotetext{
${ }^{14} \mathrm{M} . \mathrm{T}$. Robinson, O. S. Oen, and D. K. Holmes, Nucl. Sci. Eng. 10, 61 (1961).

${ }^{15}$ R. M. Walker, J. Nucl. Mater. 2, 147 (1960).

${ }^{16} \mathrm{R}$. R. Coltman et al., "Reactor Damage in Pure Metals," to be published.
}

Table 18.3. Thermal Cross Sections and Activation Integrals Above Cadmium Cutoff Energles for Several Reactions in $1 / v$ Detectors

\begin{tabular}{|c|c|c|c|c|c|}
\hline \multirow[b]{2}{*}{ Reaction } & \multirow{2}{*}{$\begin{array}{c}\sigma_{\text {th }} \text {, Thermal } \\
\text { Cross Section } \\
\text { (barns) }\end{array}$} & \multirow{2}{*}{$\begin{array}{c}l_{r} \\
\text { (barns) }\end{array}$} & \multirow{2}{*}{$\begin{array}{l}\text { References } \\
\text { for } I_{r}\end{array}$} & \multicolumn{2}{|c|}{$E_{\mathrm{Cd}}=0.53 \mathrm{ev}, \mathrm{Cd}$ Thickness $=0.027 \mathrm{in}}$. \\
\hline & & & & $\begin{array}{l}l_{1 / \nu} \\
\text { (barns) }\end{array}$ & $\begin{array}{c}I_{T}=I_{r}+I_{1 / v} \\
\text { (barns) }\end{array}$ \\
\hline $\mathrm{Co}^{59}(n, y) \mathrm{Co}^{60}$ & 37.0 & $57 \pm 4$ & $b, c$ & $16.6 \pm 0.5$ & $74 \pm 4$ \\
\hline $\mathrm{Mn}_{\mathrm{n}} 55(n, \gamma) \mathrm{M}_{\mathrm{n}} 56$ & 13.2 & $7.9 \pm 0.5$ & $b, d, e$ & $5.8 \pm 0.2$ & $13.7 \pm 0.5$ \\
\hline $\mathrm{Na}^{23}(n, y) \mathrm{Na}^{24}$ & 0.54 & $0.07 \pm 0.01$ & $b$ & $0.23 \pm 0.01$ & $0.30 \pm 0.02$ \\
\hline
\end{tabular}

${ }^{a}$ D. J. Hughes and R. B. Schwartz, Neutron Cross-Sections, BNL-325 (1958).

${ }^{b}$ R. Dahlberg, K. Jirlow, and E. Johansson, J. Nucl. Energy: Pt. A \& B 14, 53 (1961).

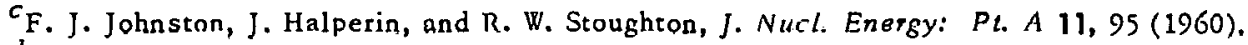

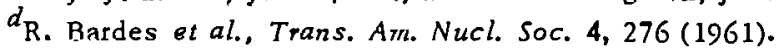

${ }^{e}$ W. H. Walker, C. H. Westcott, and T. K. Alexander, Can. J. Pbys. 38, 57 (1960). 


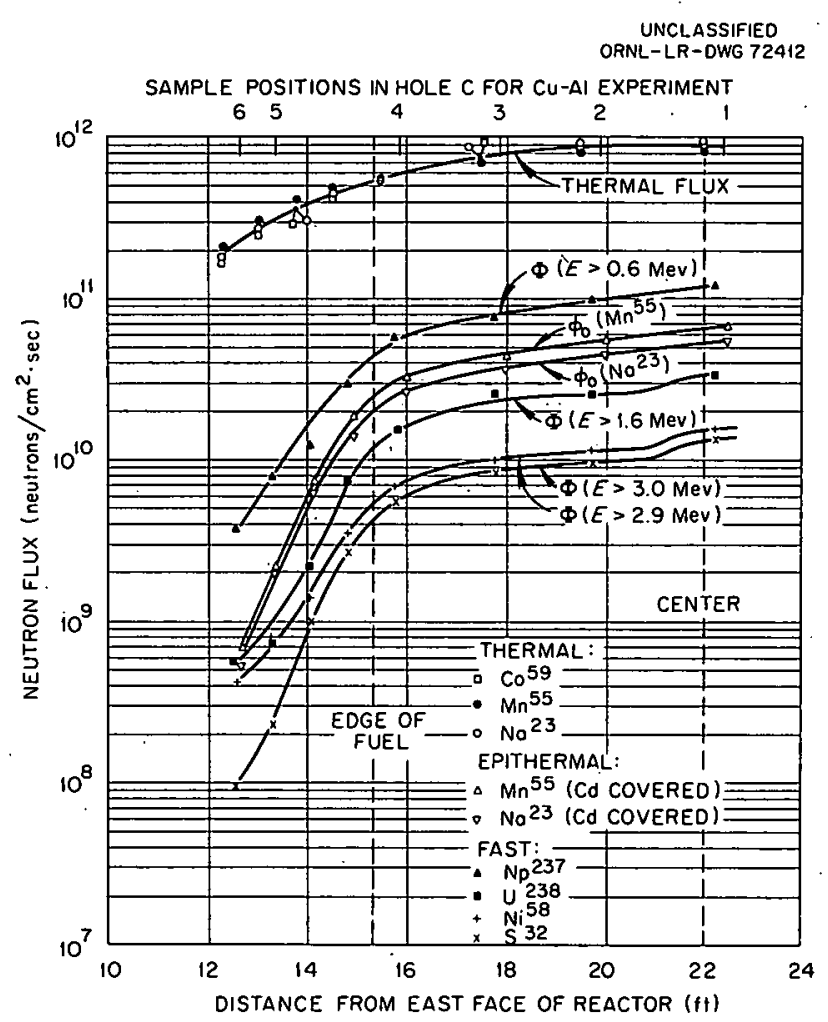

Fig. 18.3. Neutron Flux vs Distance into Hole C, ORNL Grophite Reactor. where $\phi_{\text {th }}$ is shown in Fig. 18.3. The average recoil energy is given by

$$
\bar{T}_{R}=\frac{\overline{E_{\gamma}^{2}}}{2 M c^{2}}
$$

where $E_{\gamma}$ is the energy of the capture gamma ray and $\overline{E_{\gamma}^{2}}$ is a weighted average of $E_{\gamma}^{2}$ over the capture gamma-ray energy spectrum. The calculation of $K(n, y)$ was made for pure copper, using the capture gamma-ray spectrum given by Troubetskoy and Goldstein ${ }^{17}$ and the values $\sigma_{\text {th }}=3.8$ barn s $^{18}$ and $T_{d}=25 \mathrm{ev}$ for the thermal neutron absorption cross section and the displacement energy respectively.

As concerns the calculation of $K$ (fast), the contribution to the displacement production rate made by fast neutron collision events, the relative values of $K$ (fast) as a function of position in Hole $C$ may be estimated from the integral flux curves in Fig. 18.3. The integral flux

${ }^{17} \mathrm{E}$. Troubetskoy and $\mathrm{H}$. Goldstein, Nucleonics 18(11), 171 (1960); see also $A$ Compilation of Informa. tion on Gamma-Ray Spectra Resulting from ThermalNeutron Capture, ORNL-2904 (May 17, 1960).

${ }^{18} \mathrm{D}$. J. Hughes and R. B. Schwartz, Neutron Cross Sections, BNL-325 (July i, 1958).

Table 18.4. Comparison of Calculated and Observed Fluxes for a Position 19 ft 10 in. into Hole C, Grophite Reactor

\begin{tabular}{|c|c|c|c|c|c|}
\hline No. & Monitor & Measured Quantity & $\begin{array}{c}\Phi_{\text {meas }}^{a} \\
\text { (neutrons } \mathrm{cm}^{-2} \mathrm{sec}^{-1} \text { ) }\end{array}$ & $\begin{array}{c}\Phi_{\text {theor }}{ }^{b} \\
\text { (neutrons } \mathrm{cm}^{-2} \mathrm{sec}^{-1} \text { ) }\end{array}$ & $\frac{\Phi_{\text {meas }}}{\Phi_{\text {theor }}}$ \\
\hline & & & $\times 10^{10}$ & $\times 10^{10}$ & \\
\hline 1 & $\mathrm{Mn}^{55}$ & $\Phi_{0}$ & 5.5 & 4.2 & 1.3 \\
\hline 2 & $\mathrm{Na}^{23}$ & $\Phi_{0}$ & 4.4 & 4.2 & 1.0 \\
\hline 3 & $\mathrm{~Np}^{237}$ & $\Phi(E>0.6 \mathrm{Mev})$ & 9.5 & 9.1 & 1.0 \\
\hline 4 & $U^{238}$ & $\Phi(E>1.6 \mathrm{Mev})$ & 2.6 & 3.9 & 0.67 \\
\hline 5 & $\mathrm{Ni}^{58}$ & $\Phi(E>2.9 \mathrm{Mev})$ & 1.1 & 1.4 & 0.78 \\
\hline 6 & $s^{32}$ & $\Phi(E>3.0 \mathrm{Mev})$ & 0.97 & 1.3 & 0.75 \\
\hline
\end{tabular}

${ }^{\text {a }}$ ead from graph in Fig. 18.3.

${ }^{b}$ For Nos. 1 and $2, \Phi_{\text {the or }}=\Phi_{0}=C\left[\lim _{E \rightarrow 0} E \phi(E)\right]$;

for Nos. 3 through $6, \Phi_{\text {theor }}=C \int_{E_{i}}^{\infty} \phi(E) d E$, where $\phi(E)=$ ROH spectrum. 
above $0.6 \mathrm{Mev}$ was used as determined by the $\mathrm{Np}^{237}$ fission threshold detector. In order to set the absolute magnitudes of the values of $K$ (fast), we have relied on the results of Colman et al., ${ }^{16}$ who observed that $K($ fast $)=4 K(n, \gamma)$ for copper irradiated at $4 \% \mathrm{~K}$ near the center of the reactor. Using this relation and Eq. (1) for our experiment at the center of the reactor, we may calculate $K$ (fast) for this position and, thus, for all positions in Hole $C$. Finally, $K$ is set equal to $K=K$ (fast) $+K(n, \gamma)$, which assumes that no distinction is to be made between the effects of displacements produced by neutron collisions and those resulting from $(n, \gamma)$ events.

\section{Resistivity Measurements and Discussion}

The curves of resistivity vs time of irradiation are given in Fig. 18.4. The change in resistivity is shown normalized to the total decrease, which was approximately the same for each run. The time for half-completion, $\tau_{1 / 2}$, corresponds to $f=0.5$. The curves in Fig. 18.4 refer to freshly prepared samples which were annealed at $750^{\circ} \mathrm{C}$ followed by cooling to room temperature at $15^{\circ} \mathrm{C} / \mathrm{hr}$. We shall designate these runs as Series
I. Referring to Fig. 18.2, we see that each of the runs in Series $I$ corresponds to a decrease in resistivity from $B$ to $C$ where $T_{0}=100^{\circ} \mathrm{C}$.

For the runs of Series II, the samples were warmed, following the decrease from $B$ to $C$ (Fig. 18.2), along $C D$ with the reactor on and held at $T_{2}\left(210^{\circ} \mathrm{C}\right)$ where the original value at $A$ was achieved. Then, with the reactor off, the samples were cooled along $A B$ to $T_{0}=100^{\circ} \mathrm{C}$. The initial resistivity at $B$ was recovered to within 0.01 $\mathrm{microhm}-\mathrm{cm}$ on the average, which is about $5 \%$ of the total decrease or $0.1 \%$ of the absolute resistivity. We may represent the irradiation and thermal cycling treatment as follows (letters refer to points in Fig. 18.2):

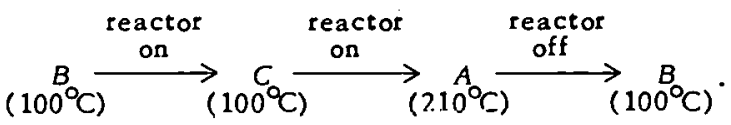

The results for repeated runs at position 4 are shown in Fig. 18.5. The first run (Run 4-I) is for a freshly prepared sample. Following Run 4-I, the sample was given the irradiation and thermal cycling treatment of Eq. (2) and Run 4-II was conducted. Then, similar treatments were used for Runs 4-III and 4-IV. For each of the

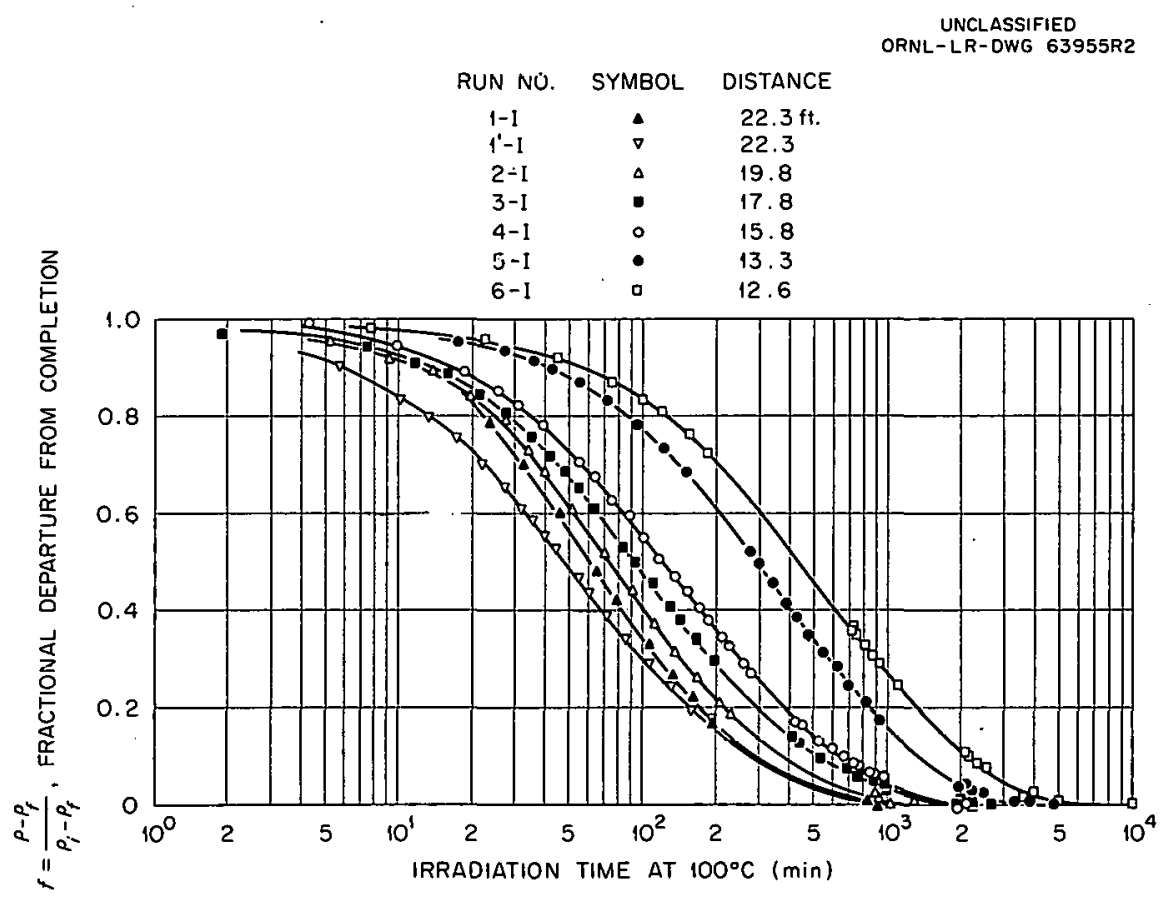

Fig. 18.4. Fractional Departure from Completion of the Decrease in Resistivity of Cu-Al (15 at. \% Al) vs Time of Irradiation at $100^{\circ} \mathrm{C}$ for Samples at Various Distances into Hole C, ORNL Graphite Reactor. 


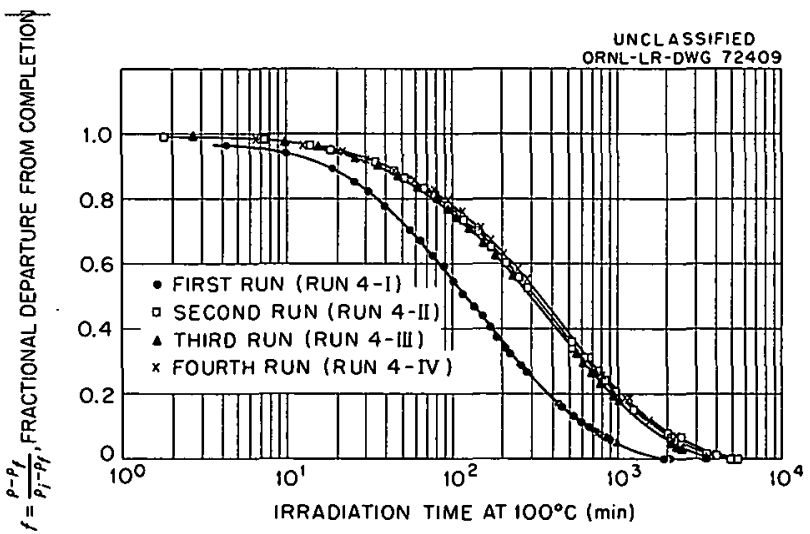

Fig. 18.5. Fractional Departure from Completion of the Decrease in Resistivity of Cu-Al (15 at. \% Al) vs Time of Irradiation for Repeated Runs at Position 4, Hole C, ORNL Graphite Reactor.

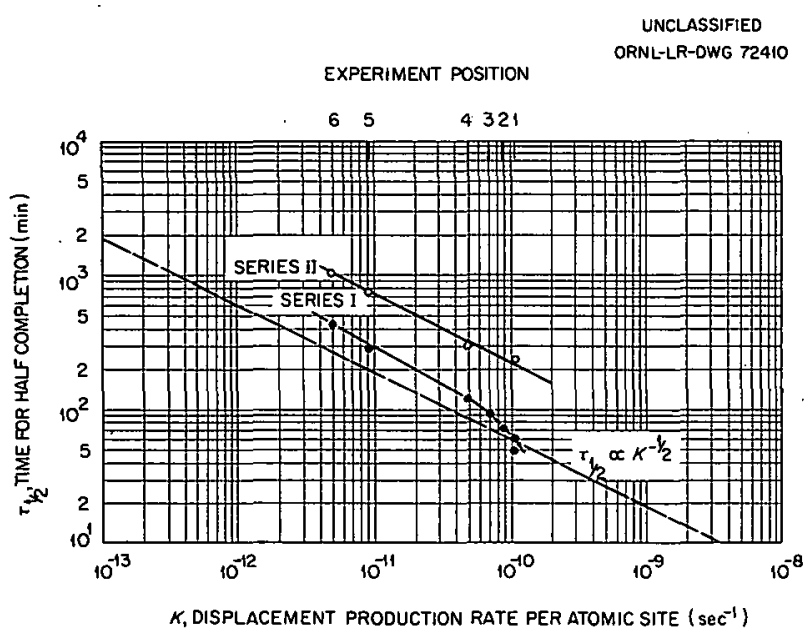

Fig. 18.6. Time for Half-Completion of the Decrease in Resistivity as a Function of the Defect Production Rate in Cu-Al (15 at. \% Al). The dashed line was obtained from previous electron and gamma irradiation experiments (refs 8,10 ).

runs, the magnitude of the decrease in resistivity was about the same. However, as Fig. 18.5 shows, the effect of the cycling treatment is to increase $\tau_{1 / 2}$ for Run 4 -II by a factor of 2.5 over that for Run 4-I. The repeated cycling treatment for $\mathrm{R}$ uns 4-III and 4-IV did not appreciably increase $\tau_{1 / 2}$ further.

In Fig. 18.6 the values of $\tau_{1 / 2}$ are plotted vs $K$ for Series I and II. For both series, $\tau_{1 / 2}$ appears to be proportional to $K^{-1 / 2}$. It might be mentioned that $K$ was also evaluated using the Robinson, Oen, and Holmes ${ }^{14}$ neutron spectrum for graphitemoderated reactors for the calculation of $K$ (fast). In this case, the slopes of the lines in Fig. 18.6 are unchanged, but the values of $K$ are increased by a factor of about 3 . The dashed curve labeled

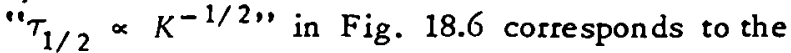
line previously determined from electron and gamma irradiation experiments. ${ }^{8,10}$

The slopes of the lines in Fig. 18.6 indicate that the mechanism which operates in $\mathrm{Cu}-\mathrm{Al}$ corresponds to $p=-1 / 2$ in Table 18.1. Also, the results of Blewitt et al., ${ }^{19}$ on $\mathrm{Cu}-13$ at. \% $\mathrm{Zn}$. suggest that the diffusion-enhancing defect is the vacancy ${ }^{20}$ and, as we have stated, the previous temperature-dependence experiment on freshly prepared samples, such as those used in the Series I experiments, indicates that the appropriate activation energy, $E$ in Table 18.1 , is $1 / 2 E_{\mathrm{mv}}$. However, we are not able at this time to explain the longer $\tau_{1 / 2}$ for the runs of Series II (Fig. 18.6), and it is felt that any further comment on the mechanism of radiation-enhanced diffusion in $\mathrm{Cu}-\mathrm{Al}$ should await more understanding on this point.

\subsection{STRESS RELAXATION IN IRON ALLOYS CONTAINING CARBON AND/OR NITROGEN}

\section{J. T. Stanley}

Present knowledge of the effect of radiation damage on iron-carbon and iron-nitrogen alloys is quite limited. It has been shown that neutron irradiation can markedly increase the rate of decomposition of the supersaturated solid solution of carbon in iron. ${ }^{21}$ The exact mechanism by which the carbon is removed from solution in irradiated alloys is uncertain. Since carbon and

${ }^{19} \mathrm{~T}$. H. Blewitt et al., "Mechanism of Annealing in Neutron Irradiated Metals," p 84 in Creep and Recovery, American Society for Metals, Metals Park, Ohio, 1957.

${ }^{20} \mathrm{M}$. S. Wechsler, "Fundamental Aspects of Radiation Effects on Diffusion-Controlled Reactions in Alloys," Symposium on Radiation Effects on Metals and Neutron Dosimetry, ASTM, Los Angeles, Oct. 1-5, 1962.

${ }^{2} \mathrm{l}_{\mathrm{H}}$. Wagenblast and A. C. Damask, Phys. Chem. Solids 23, 221 (1962). 
nitrogen occupy interstitial lattice positions in the solid solution, it is unlikely that the lattice defects produced by irradiation can change the rate of diffusion of these elements in the lattice. Thus the radiation-damage effects observed in iron-carbon alloys are expected to be somewhat different from those observed in substitutional alloys such as copper-aluminum.

If the damping capacity of an iron-carbon specimen vibrating at $1 \mathrm{cps}$ is measured as a function of temperature, a sharp maximum is found at $40^{\circ} \mathrm{C}$. The height of this damping peak is proportional to the amount of carbon in solution. ${ }^{22}$ Carbon atoms associated with other impurity atoms or dislocations or in precipitates do not contribute to this damping peak, but may give rise to damping peaks at other temperatures. ${ }^{22,23}$ In a similar way, the magnitude of stress relaxation (at constant strain) is proportional to the amount of carbon in solid solution. Also, the time constant of the stress relaxation at a given temperature is a measure of the jump frequency of the carbon atoms.

Wagenblast and Damask ${ }^{21}$ made measurements of the damping capacity of iron-carbon wires after irradiating them in the reactor and removing them for measurements. It seems worthwile to attempt making both stress-relaxation and damping-capacity measurements during reactor irradiation. There are two advantages of making stress-relaxation measurements on these alloys in addition to damping measurements. First, the stress-relaxation measurements can be made at lower temperatures than damping measurements. This is important because carbon and nitrogen diffuse at appreciable rates at room temperature and some of the defects introduced by irradiation diffuse rapidly even below room temperature. Second, it is sometimes possible to separate the relaxation data into several superimposed relaxations. Thus, it has been shown that the relaxation curve for iron-nitrogen alloys can be analyzed in terms of four separate relaxation processes. ${ }^{24}$ Hence, by stress-relaxation measurements, it may be possible to observe

\footnotetext{
${ }^{22}$ J. L. Snoek, Pbysica 8, 711 (1941).

${ }^{23} \mathrm{~L}$. J. Dijkstra and R. J. Sladek, Trans. AIME 5, 69 (1953).

7.4D. Keefer and C. Wert, AROD 2085:4, AROD $2085: 3$.
}

relaxations due to carbon-vacancy or carboninterstitial pairs induced by irradiation.

During the past year, equipment has been designed for measuring both stress relaxation and damping capacity in the reactor. The stress-relaxation apparatus has been built and tested. In addition, heat treating equipment for introducing various amounts of carbon and nitrogen into iron has been built. The stress relaxation is detected by measuring the resonant frequency of a stretched flexible wire. For a perfectly flexible string, the resonant frequency is proportional to the square root of the stress. In the present case, the strain on the wire should not exceed $5 \times 10^{-4}$, in order to prevent plastic flow. It was calculated that, in order to approach the flexible-string case, the wires should be $\leqq 0.005 \mathrm{in}$. in diameter and $4 \mathrm{in}$. long. The space available for the apparatus in the reactor facility is quite small. Because of this limited space it seemed that only capacitive methods of driving and detecting vibrations could be used.

Figure 18.7 is a schematic diagram of the specimen, the apparatus used to stress the specimen, and the capacitance plates for detecting and driving oscillations. Three small metal bellows are used to stress the specimen, to lock the specimen at constant strain, and to release the stress. The driving electrode is positioned at the midpoint of the specimen so that, when voltage is applied, the specimen is forced to move in a plane normal to the plane which passes through the two pickup electrodes. The specimen acts as a grounded shield between the two electrodes, and as it moves between them, it changes their capacitance.

Figure 18.8 is a block diagram showing the electronic equipment used in driving the specimen and measuring its resonant frequency. The change in capacitance of the pickup electrodes is detected by a bridge operating at $100 \mathrm{kc}$. The bridge contains a two-stage amplifier tuned to $100 \mathrm{kc}$ and a diode detector to give a de voltage which is proportional to the change in capacitance unbalance. The time constant of the detector is short compared with the period of the specimen oscillation. The output of the bridge is further amplified and fed into the driving electrode. A phase shift control is provided so that the phase anglc of the driving voltage with respect to the detected signal can be adjusted until resonance occurs. The resonant frequency is then measured 
UNCLASSIFIED
ORNL-LR-DWG $72406 R$

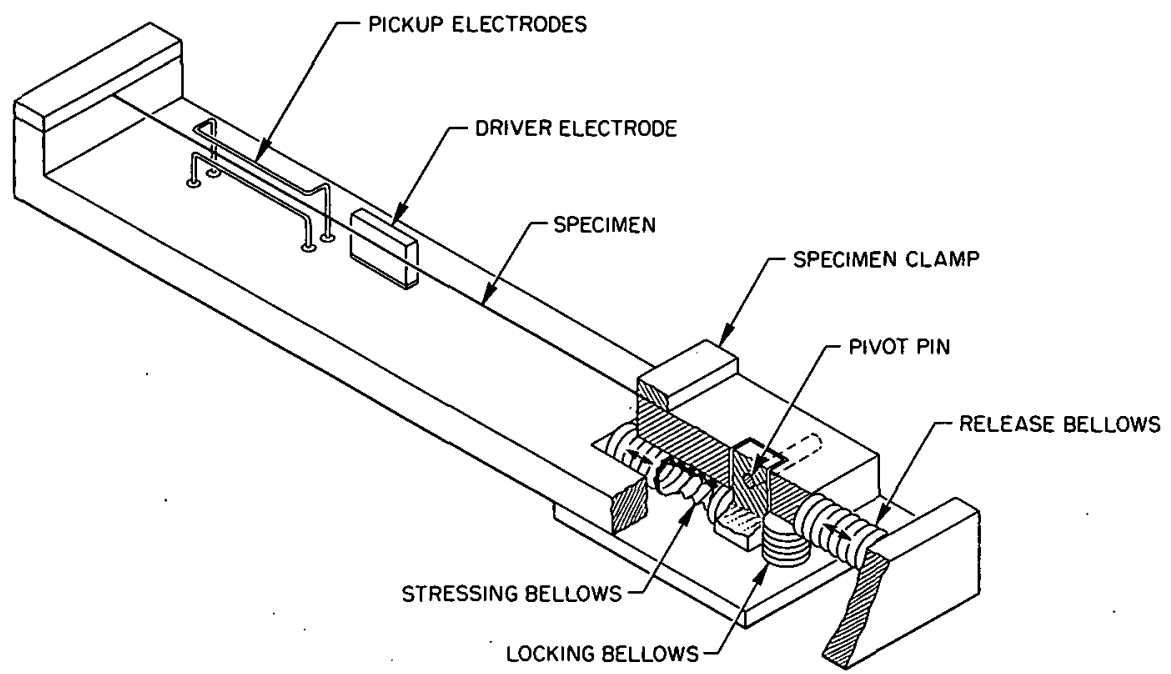

Fig. 18.7. Schematic Drawing of Stress-Relaxation Apparatus.

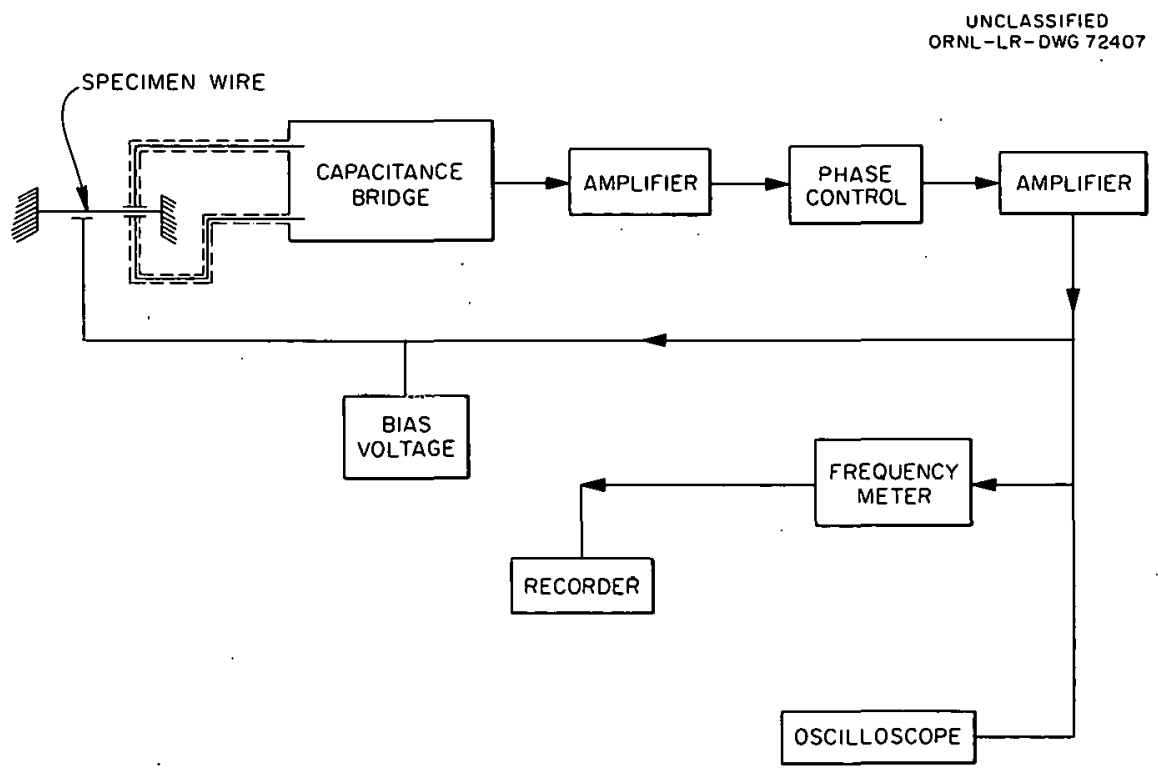

Fig. 18.8. Block Diagram of Components for Stress-Relaxation Measurements.

and recorded continuously as the specimen relaxes.

A second apparatus under construction is a flexure pendulum. In this apparatus, Fig. 18.9, a specimen about $1 / 4 \times 1 \times 0.003$ in. is clamped at one end and is vibrated in flexure. A vane; attached to the free end, moves between two capacitance plates. The change in capacitance induced by the moving vane is detected and re: corded. The pendulum is set in motion by applying a voltage pulse between the specimen and a plate parallel to the specimen. The rate of decay of the vibrations so induced gives a measure of the damping capacity. This apparatus will be used to measure the change in the amount of carbon or nitrogen in solid solution as speci- 


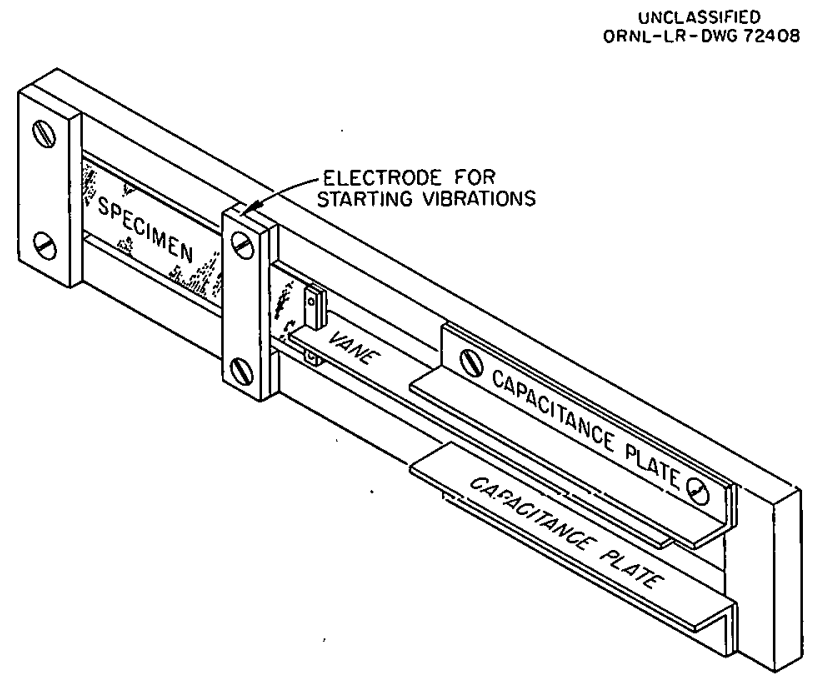

Fig. 18.9. Flexure Pendulum.

mens are irradiated in the reactor at slightly above reactor ambient temperatures. For a frequency of $20 \mathrm{cps}$ the nitrogen peak in iron occurs at $60^{\circ} \mathrm{C}$. By adjusting the specimen to vibrate at $20 \mathrm{cps}$ and maintaining the specimen at $60^{\circ} \mathrm{C}$, the change in the nitrogen peak during irradiation can be observed. This apparatus will also be suitable for electron bombardment experiments, because of the thin specimens which can be used.

\subsection{ATOMIC REARRANGEMENTS IN DEFORMED Cu-Si AND Cu-Si-Mn ALLOYS ${ }^{25}$}
J. M. Williams
M. S. Wechsler
H. M. Otte 26

The effect of room temperature deformation and subsequent isochronal annealing on the electrical resistivity, the $x$-ray diffraction pattem, lattice parameter, stacking fault probability, microhardness, yield stress, and microstructure have been recorded for $\mathrm{Cu}-6.7$ at. \% Si (alloy $A$ ) and $\mathrm{Cu}-$ 6.7 at. \% Si-1.3 at. \% Mn (alloy B). The high stacking fault density produced by deformation is accompanied by a measurable change in the other physical and mechanical properties. Both the stacking faults and residual elastic strains become zero as the temperature is raised to $350^{\circ} \mathrm{C}$, but solute clustering (or possibly the formation of an as yet undctected precipitate) reaches a maximum. Between 350 and $550^{\circ} \mathrm{C}$, the solute dissolves on increasing the temperature and re-forms on decreasing the temperature. Once recrystallization has occurred, the effect is no longer reversible. The deformation is considered to en$h$ ance the redistribution of solute by providing nucleation sites rather than point defects.

\footnotetext{
25 Abstract of paper presented by $H$. M. Otte at the International Conference on Crystal Lattice Defects, Kyoto, Japan, Sept. 7-12; 1962.

${ }^{26}$ RIAS, Baltimore, Md.
} 


\section{Engineering Properties}

\subsection{IN-PILE STRESS RUPTURE EXPERIMENTS}
W. E. Brundage
N. E. Hinkle
W. W. Davis ${ }^{1}$
O. Sisman 2,3
J. C. Zukas. ${ }^{1}$
A. L. Johnson ${ }^{2}$

The interest in high-temperature nuclear reactors for power production has created a reed for information about the effect of reactor environment on the mechanical properties of structural materials at elevated temperatures, especially when these are selected for such critical applications as fuel element containment. Tests of several alloys have been operated in the poolside facility of the Oak Ridge Research Reactor. The tests were conducted on tubular specimens, internally pressurized to the desired tangential stress level, and maintained at the selected temperature during reactor operation until failure occurred. The times-to-rupture were then compared with the times for similar specimens tested out of the reactor. Postirradiation examination of the specimens included measurements of dimensional changes and metallographic studies.

The recent work includes tests on Inconel, type 304 stainless steel, Zircaloy-2, and niobium-1\% zirconium alloy. The irradiation methods for these tests have been previously described. ${ }^{4}$

\section{Inconel}

Creep-rupture experiments in the ORR at $1500^{\circ} \mathrm{F}$ with air atmosphere on two commercial heats of Inconel, heat A (INCO No. NX 8962) and heat B (INCO No. NX 5757), were previously reported. ${ }^{4,5}$

\footnotetext{
${ }^{1}$ Now with Metals and Ceramics Division.

${ }^{2}$ Now with Reactor Chemistry Division.

${ }^{3}$ Section Head until July 1, 1962.

${ }^{4}$ W. E. Brundage et al., Solid State Div. Ann. Progr. Rept. Aug. 31, 1961, ORNL-3213, Pp 124-33.

${ }^{5}$ N. E. Hinkle et al.. Solid State Div. Ann. Progr. Rept. Aug. 31, 1960, ORNL-3017, p 120.
}

Both heats showed a decreased rupture life due to the irradiation. Also, the deformation at fracture for heat $A$, as indicated by the increase in diameter, was observed to be smaller for the irradiated than for the control samples. Recently, however, similar measurements for heat-B samples showed the opposite effect, that is, about two times greater deformation at rupture for the inpile specimens.

To investigate the matter further, an in-pile experiment, again in air and at $1500^{\circ} \mathrm{F}$, was operated to determine the amount of deformation of heat $A$ after predetermined creep times at selected stress levels. The results, shown in Table 19.1, indicate no gross effect of the irradiation on the creep rate for this heat.

Postirradiation examination of irradiated specimens from the two heats showed that intergranular separation was localized in heat: A (Fig. 19.1) and general in heat B (Fig. 19.2).

Specimens fabricated from six special laboratory heats of Inconel, which contained varying amounts of either natural or isotopically-enriched boron, were examined after the previously reported ${ }^{4}$ stress-rupture tests in the ORR. The tests were performed to determine whether the reduction in the high-temperature stress-rupture strength previously noted ${ }^{5-7}$ was due to the $\mathrm{B}^{10}(n, a) \mathrm{Li}^{7}$ reaction, sin ce Inconel contains 30 to $100 \mathrm{ppm}$ natural boron. These results did not show a clear correlation between the reduction in strength and the increasing $\mathrm{B}^{10}$ content.

It now seems unlikely that a confusion of specimen material had occurred, as was at first suspected. Metallographic examination of both the unirradiated and irradiated specimens did, however, present evidence of considerable variation

\footnotetext{
${ }^{6} \mathrm{~J}$. C. Wilson et al., Solid State Div. Ann. Progr. Rept. Aug. 31, 1958, ORNL-2614, pp 106-11.

${ }^{7}$ N. E. Hinkle et al. Solid State Div. Ann. Progr. Rept. Aug. 31, 1959, ORNL-2829, pp 214-18.
} 
Table 19.1. Effect of Neutron Bombardment on the Creep Rate of Inconel Heat A (INCO NX 8962 ) in Air at $1500^{\circ} \mathrm{F}$

Specimen No.

$\begin{array}{cr}\text { Stress } & \text { Time } \\ \text { (psi) } & (\mathrm{hr})\end{array}$

Maximum

Transverse Strain

Rate

(\%)

$(\% / \mathrm{hr})$

Irradiated

41-3

5000

85

0.6

0.0071

41-7

5000

95, ruptured

1.0

0.0105

Control

C-279

5000

45

0.4

0.0089

90

0.0121

200 , ruptured

1.1

0.0350

C -291

5000

53

7.0

0.0567

125

0.3

0.0096

240 , ruptured

1.2

0.0208

Irradiated

$\begin{array}{lll}41-1 & 4000 & 130 \\ 41-5 & 4000 & 225 \\ 41-8 & 4000 & 224, \text { ruptured }\end{array}$

0.4

0.0032

0.5

0.0022

Control

C-12

4000

110

0.7

0.0064

150

1.6

0.0107

245 , ruptured

7.0

0.0286

C-281

4000

115

0.15

0.0013

280

0.5

0.0018

630 , ruptured

3.0

0.0048

Irradiated $^{a}$

$\begin{array}{lll}41-2 & 3000 & 33 \\ 41-4 & 3000 & 530 \\ 41-10 & 3000 & 89\end{array}$

330

530

890
0.35

0.0106

0.4

0.0008

0.95

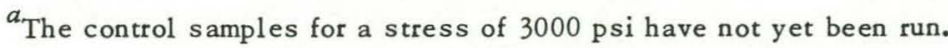

between the different heats in grain size, precipitate concentration, and stringer-type inclusions. A rather large variation in the stress-rupture properties of the materials was also noted in the out-of-reactor tests. These points seem to indicate that the stress rupture strength during irradiation was not directly related to the $B^{10}$ con- tent of the alloy, but was more dependent on other material variables. One complicating factor is that evidence exists which indicates that the boron was not evenly distributed in a given heat. Figure 19.3 is a transverse section through the wall of one specimen. The void (which contained an inclusion lost in the final polishing) appears 


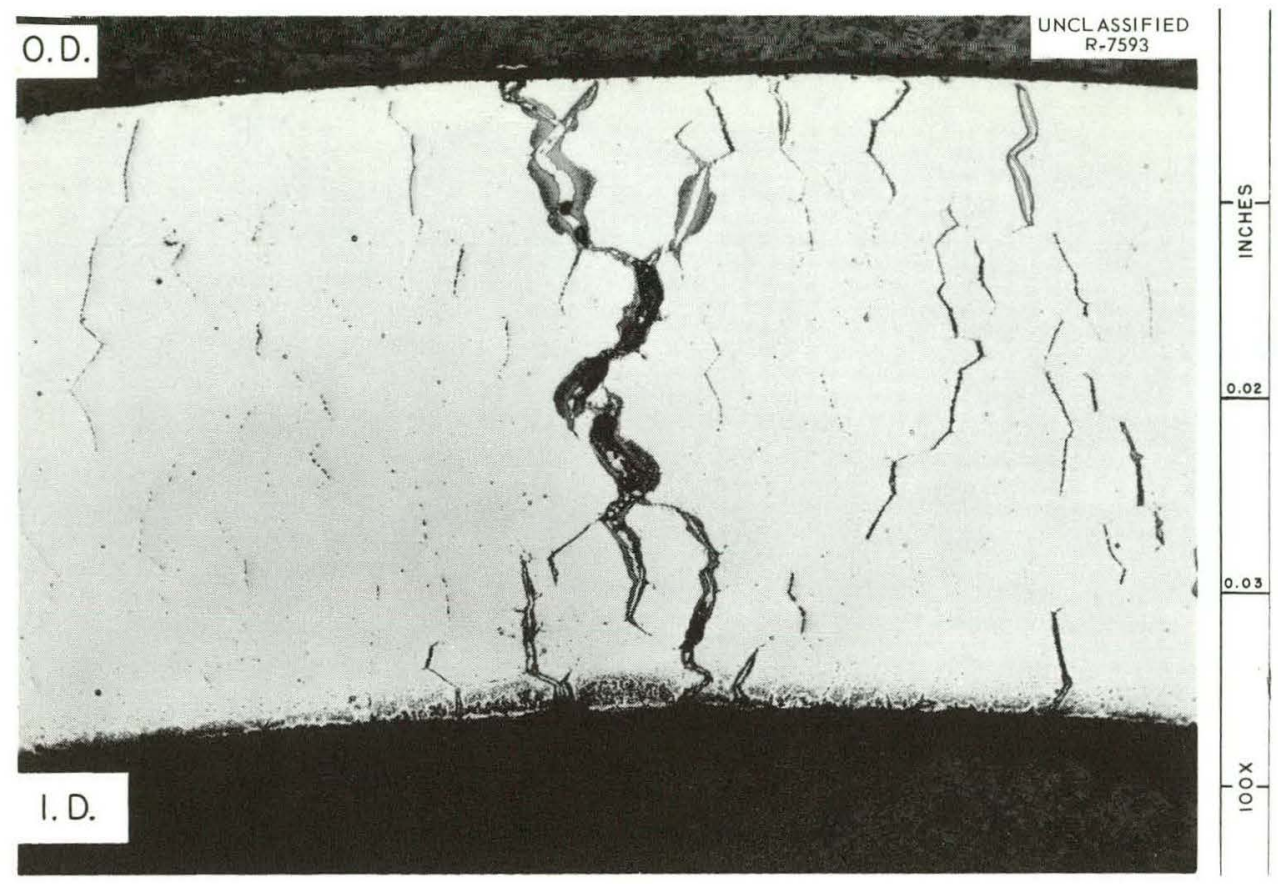

Fig. 19.1. Transverse Section of In-Pile Tube-Burst Specimen of Inconel Heat A (INCO No. NX-8962). Note the localized areas of intergranular separation. Test temperature, $1500^{\circ} \mathrm{F}$; stress, 5000 psi; rupture time, $91 \mathrm{hr}$. As polished. 100X. Reduced 23.5\%.

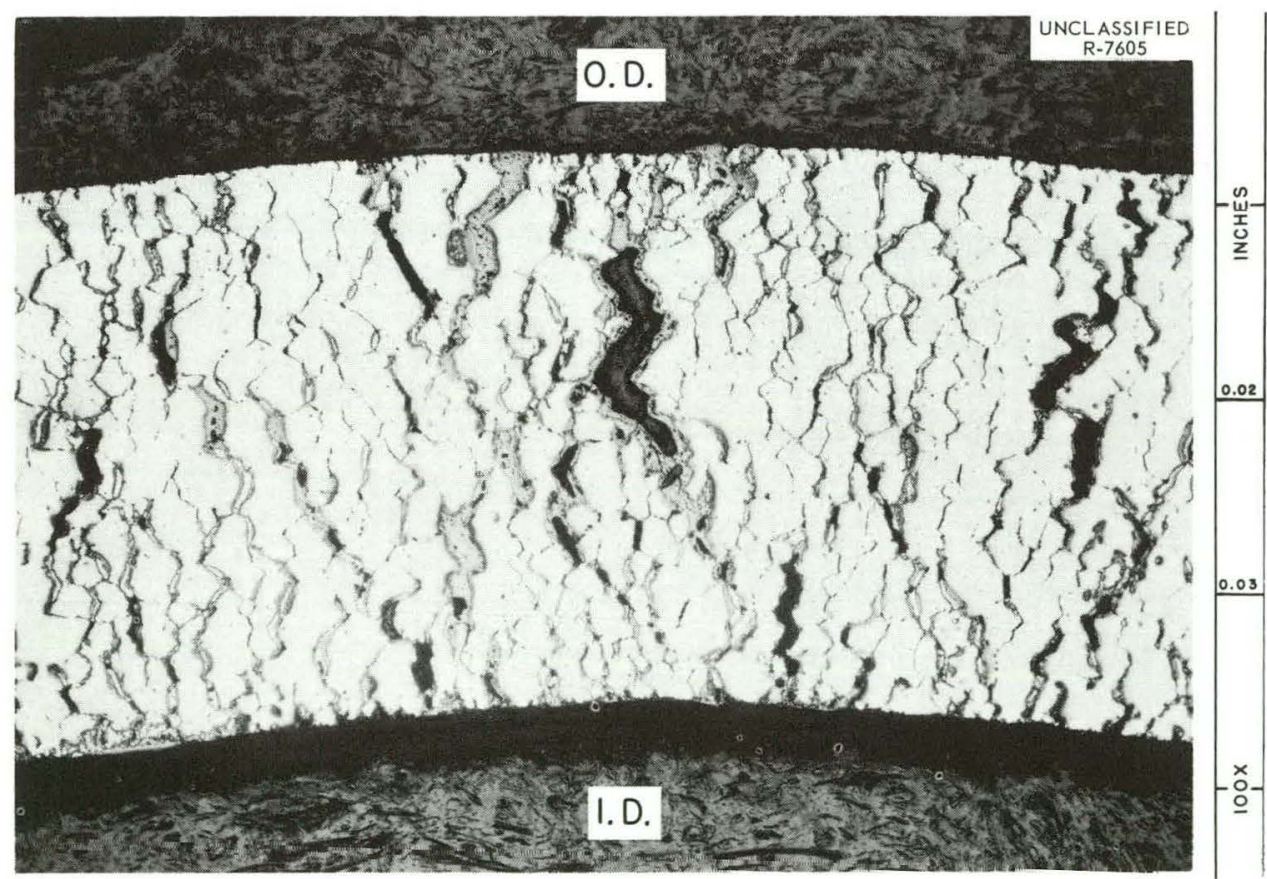

Fig. 19.2. Transverse Section of In-Pile Tube-Burst Specimen of Inconel Heat B (INCO No. NX-5757). Note that the intergranular separation is general throughout the wall. Test temperature, $1500^{\circ} \mathrm{F}$; stress, 5000 psi; rupture time, $66 \mathrm{hr}$. As polished. 100X. Reduced $23.5 \%$. 


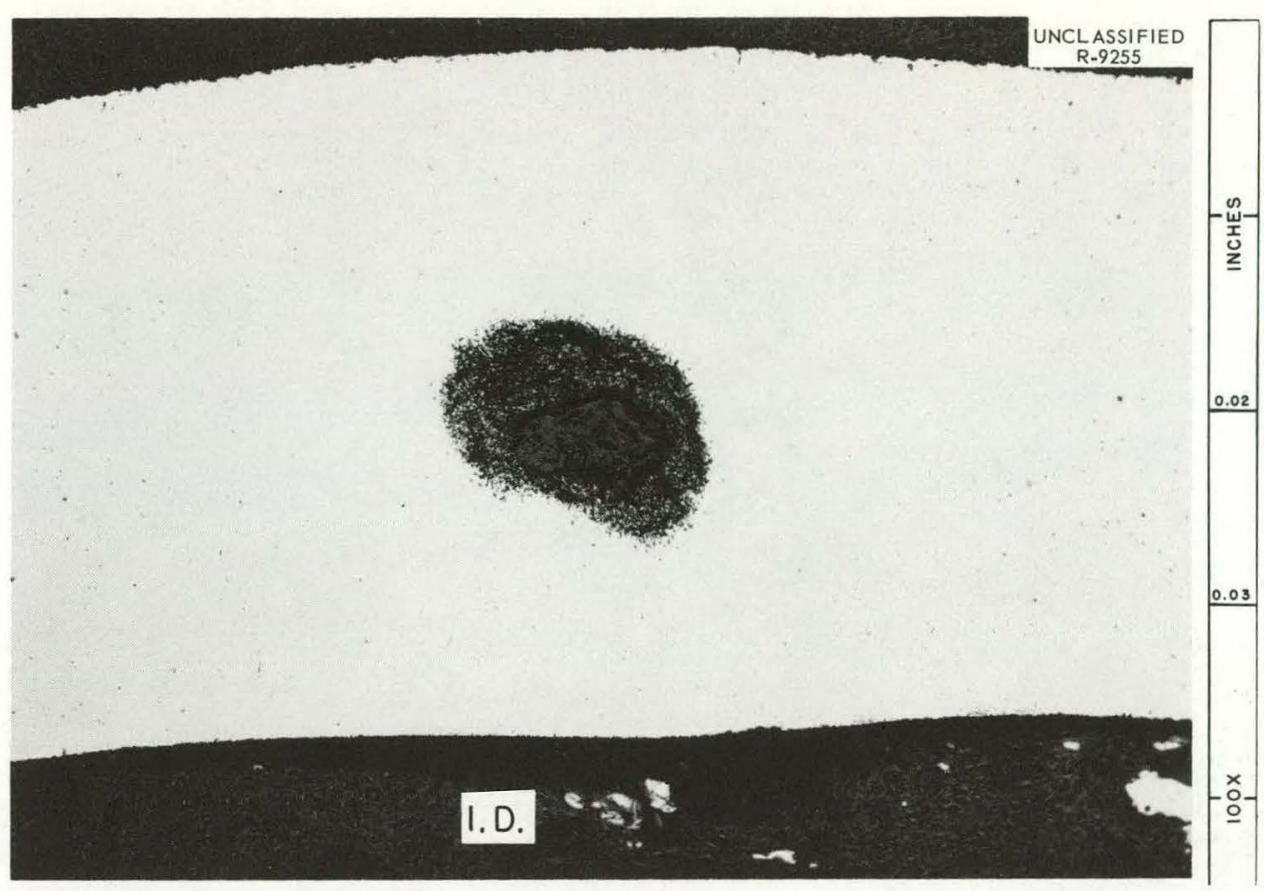

Fig. 19.3. Transverse Scction Through the Wall of a Specimen of Inconel OB Tested in the Reactor. Note the change in wall thickness associated with the hole. The void was apparently pressurized during part of the test. 100X. Reduced 23.5\%.

to have been pressurized during the test, causing a deformation of the specimen wall. Boron anal$y$ sis of samples from different portions of the material also indicated that the boron content of the alloys varied considerably. Nonhomogeneity of the boron content in some or all of the alloys could have destroyed the trend expected from the helium production. This, however, should also have caused a large scatter in the tests on irradiated samples, which was not observed.

The transverse deformation at fracture for irradiated Inconel, obtained from measurements of the maximum diameter increase of the irradiated specimens, was much smaller than that of the control specimens. These data are plotted in Fig. 19.4, in which information is also given of the stress-rupture life and the measured boron content.

The fractures were due to the intergranular separation often observed in high-temperature creep, as shown in Fig. 19.5. The fracture is localized, and a pattern of spaced voids along some grain boundaries can be seen. A typical out-of-pile specimen section is shown in Fig. 19.6, in which more regions of grain boundary separation can be observed. The spaced voids along the grain boundaries were not observed in the control specimens.

\section{Type 304 Stainless Steel}

Tube-burst tests were continued on the type 304 stainless steel tubing selected for EGCR fuel-element cladding. The tubing had a diameter of $0.750 \mathrm{in}$. and a wall thickness of $0.020 \mathrm{in}$.

The results reported in the previous report ${ }^{4}$ were thought to refer to a single heat of type 304 stainless steel. More recently it was found that two separate heats were included. Consequently, the previous data have been separated according to heats and plotted along with the recent results ${ }^{8,9}$ in Figs. 19.7 and 19.8. The

${ }^{8} \mathrm{~J}$. T. Venard, Gas-Cooled Reactor Program Ouart. Progr. Rept. March 31, 1962, ORNL-3302, p 232.

${ }^{9} \mathrm{~J} . \mathrm{T}$. Venard, unpublished data. 
UNCLASSIFIED

ORNL-LR-DWG 63801R2

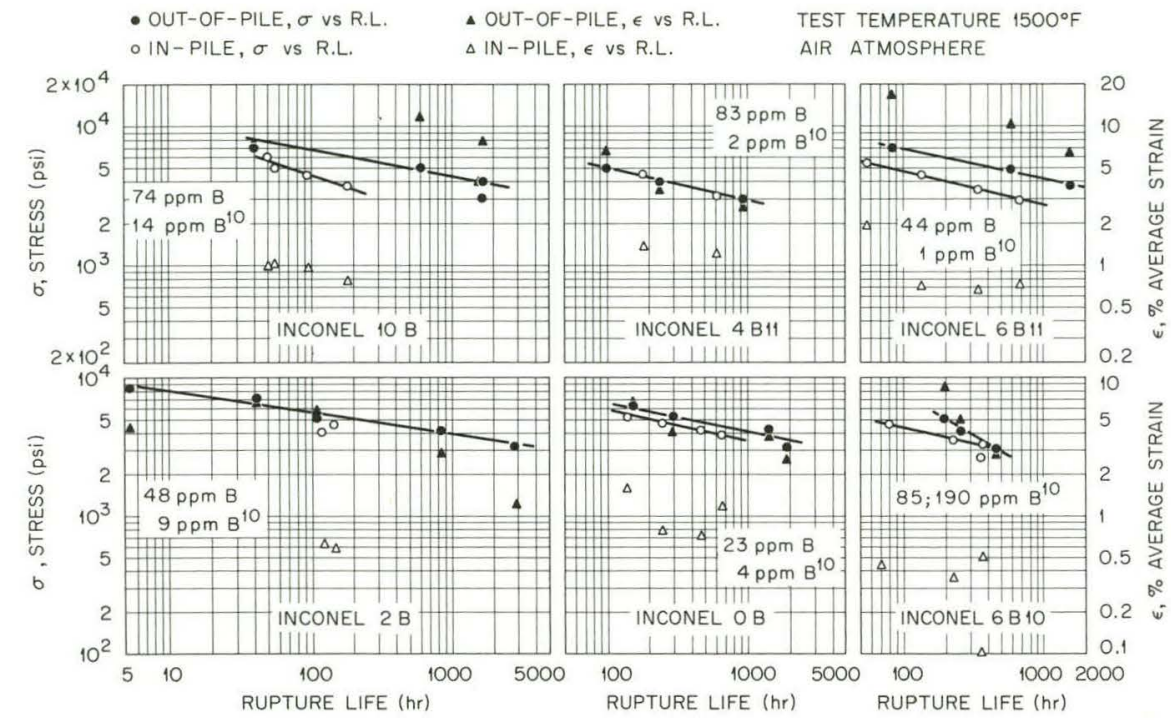

Fig. 19.4. Effect of Neutron Bombardment on the Stress Rupture Properties of the Boron-Adjusted Heats of Inconel.

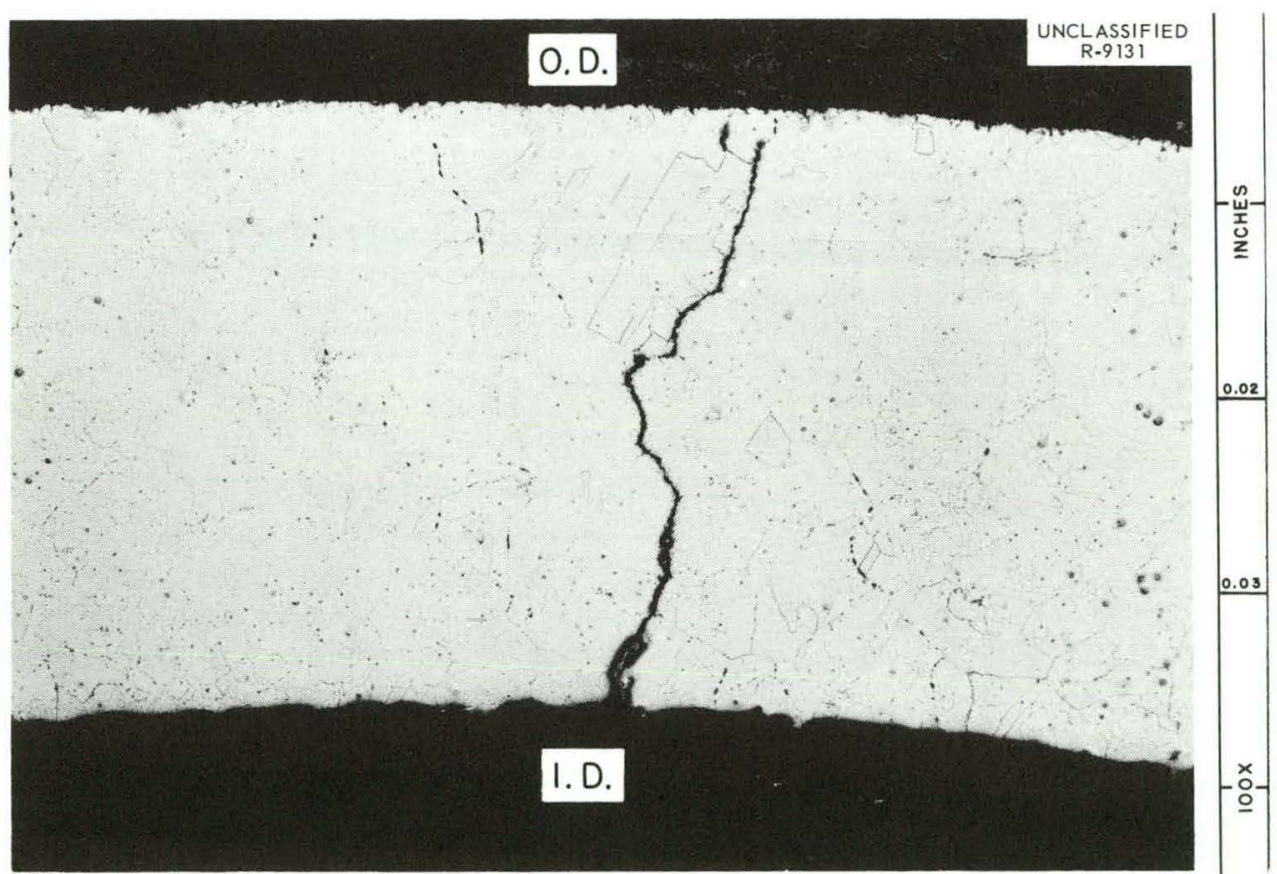

Fig. 19.5. Transverse Section of an In-Pile Tube-Burst Specimen of Inconel 6B11 Showing the Mode of Failure. Note the concentration and shape of the grain-boundary voids. Test temperature, $1500^{\circ} \mathrm{F}$; stress, 3000 psi; rupture time, $684 \mathrm{hr}$. Etchant, aqua regia. $100 \mathrm{X}$. Reduced $23.5 \%$. 


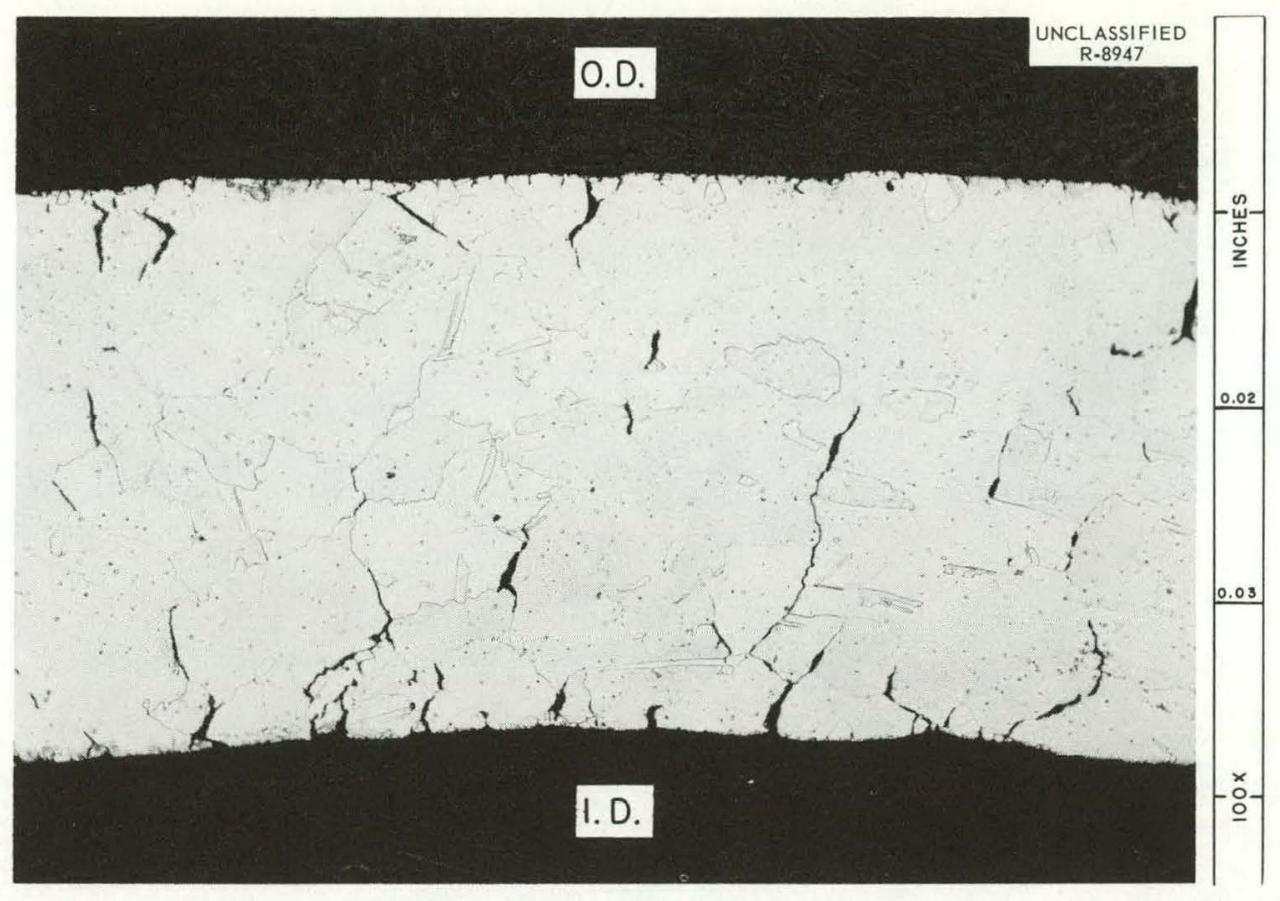

Fig. 19.6. Transverse Section of an Out-of-Pile Tube-Burst Specimen of Inconel 6B11 Showing the Type of Intergranular Separation. Note the absence of the fine, intergranular voids seen in Fig. 19.5. Test temperature, $1500^{\circ} \mathrm{F}$; stress, $5000 \mathrm{psi}$; rupture time, $585 \mathrm{hr}$. Etchant, aqua regia. 100X. Reduced 23.5\%。

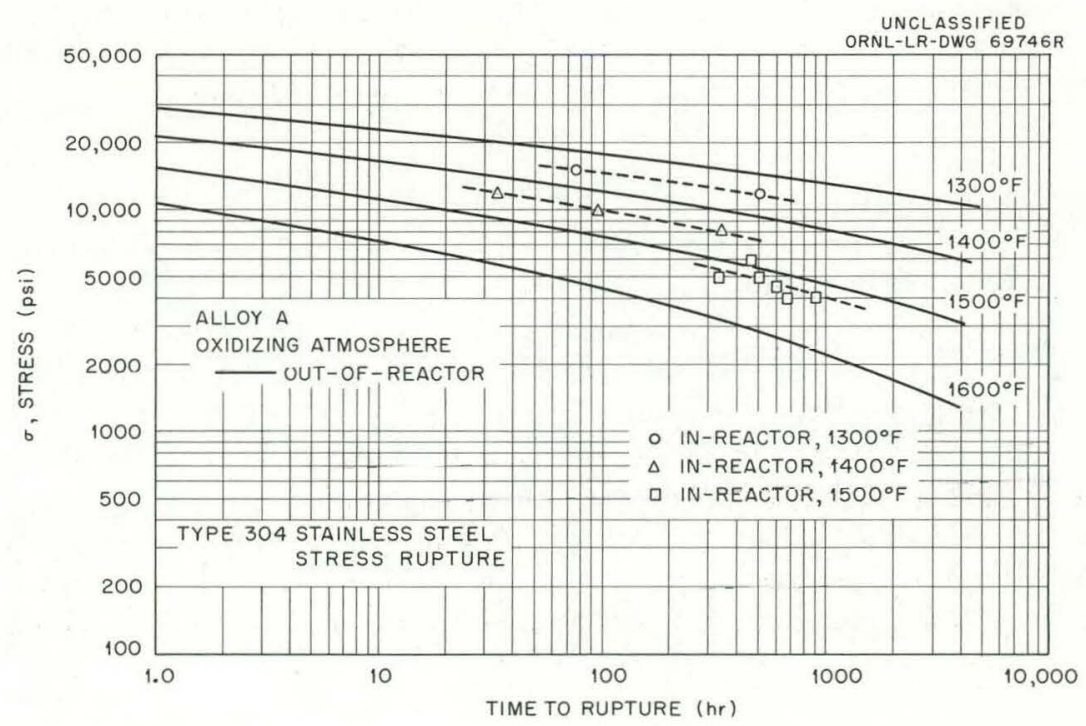

Fig. 19.7. Effect of Neutron Bombardment on the Stress-to-Rupture of Type 304 Stainless Steel Alloy A. The out-of-reactor curves are taken from ref 8 . 


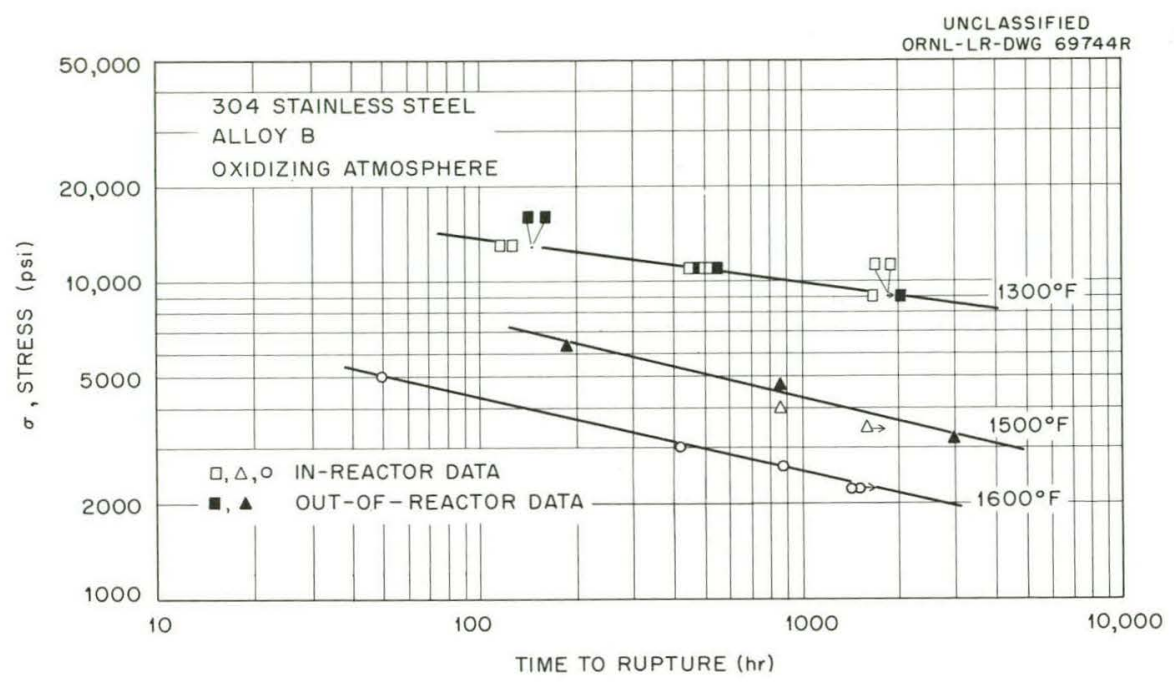

Fig. 19.8. Effect of Neutron Bombardment on the Stress-to-Rupture of Type 304 Stainless Steel Alloy B. The out-of-reactor data were taken from ref 9.

rupture strength of heat $\mathrm{A}$ is reduced about $20 \%$ at 1300 and $1400^{\circ} \mathrm{F}$ and somewhat less at $1500^{\circ} \mathrm{F}$. In contrast, the radiation has little or no effect on the rupture strength of heat $\mathrm{B}$ at $1300^{\circ} \mathrm{F}$. No control data are available for heat $\mathrm{B}$ at $1600^{\circ} \mathrm{F}$.

Precipitates were observed in irradiated specimens tested with air as the pressurizing gas. These precipitates, shown by the Widmanstätten structure in Fig. 19.9, are believed to be $\mathrm{CrN}$ and $\mathrm{CrN}_{2}$. No evidence of a precipitate was observed for samples pressurized with helium.

\section{Zircaloy-2}

The Zircaloy-2 tube-burst tests, previously described, ${ }^{4}$ were continued. The data indicate that reactor radiation has little, if any, effect on the stress-rupture properties at 700 and $900^{\circ} \mathrm{F}$ as shown in Fig. 19.10.

Two specimens of Zircaloy-2 were irradiated and tested in the reactor in order to investigate the effect of neutron bombardment on a strainaging phenomenon observed ${ }^{10}$ in this alloy. One specimen, strain-aged under irradiation at $500^{\circ} \mathrm{F}$ at $40,000 \mathrm{psi}$ for $860 \mathrm{hr}$, ruptured at 51,600 psi when the stress was subsequently raised.

\footnotetext{
${ }^{10} \mathrm{~J} . \mathrm{J}$. Holmes, The Activation Energies for Creep of Zircaloy-2, HW-70151 (March 1962).
}

The second specimen was strain-aged and irradiated at the same stress for $1500 \mathrm{hr}$ and ruptured when the stress was increased to 55,500 psi. These rupture stresses are more than $25 \%$ greater than the corresponding value of 43,000 psi observed ${ }^{11}$ without strain aging and irradiation. Thus, these results indicate an increase in rupture strength with time of strain aging and irradiation at $500^{\circ} \mathrm{F}$ and $40,000 \mathrm{psi}$. Since duplicate out-of-reactor experiments at $500^{\circ} \mathrm{F}$, using the same aging stress and time, have not yet been conducted, it is not yet possible to specify the effect of irradiation on the strain-aging phenomenon.

\section{Niobium-1\% Zirconium}

It was previously reported ${ }^{4}$ that the rupture life of niobium-1\% zirconium samples, stressed at $2000^{\circ} \mathrm{F}$, was reduced upon irradiation. Recent hot-cell examination has shown, however, that this conclusion was incorrect, in that the failure occurred in the brazed joint rather than in the specimen. Therefore, the reported data represent only a limit for any radiation-induced weakening of this alloy at $2000^{\circ} \mathrm{F}$.

\footnotetext{
${ }^{11} \mathrm{C} . \mathrm{R}$. Kennedy, Metals and Ceramics Div. Ann. Progr. Rept. May 31, 1962, ORNL-3313, p 45.
} 


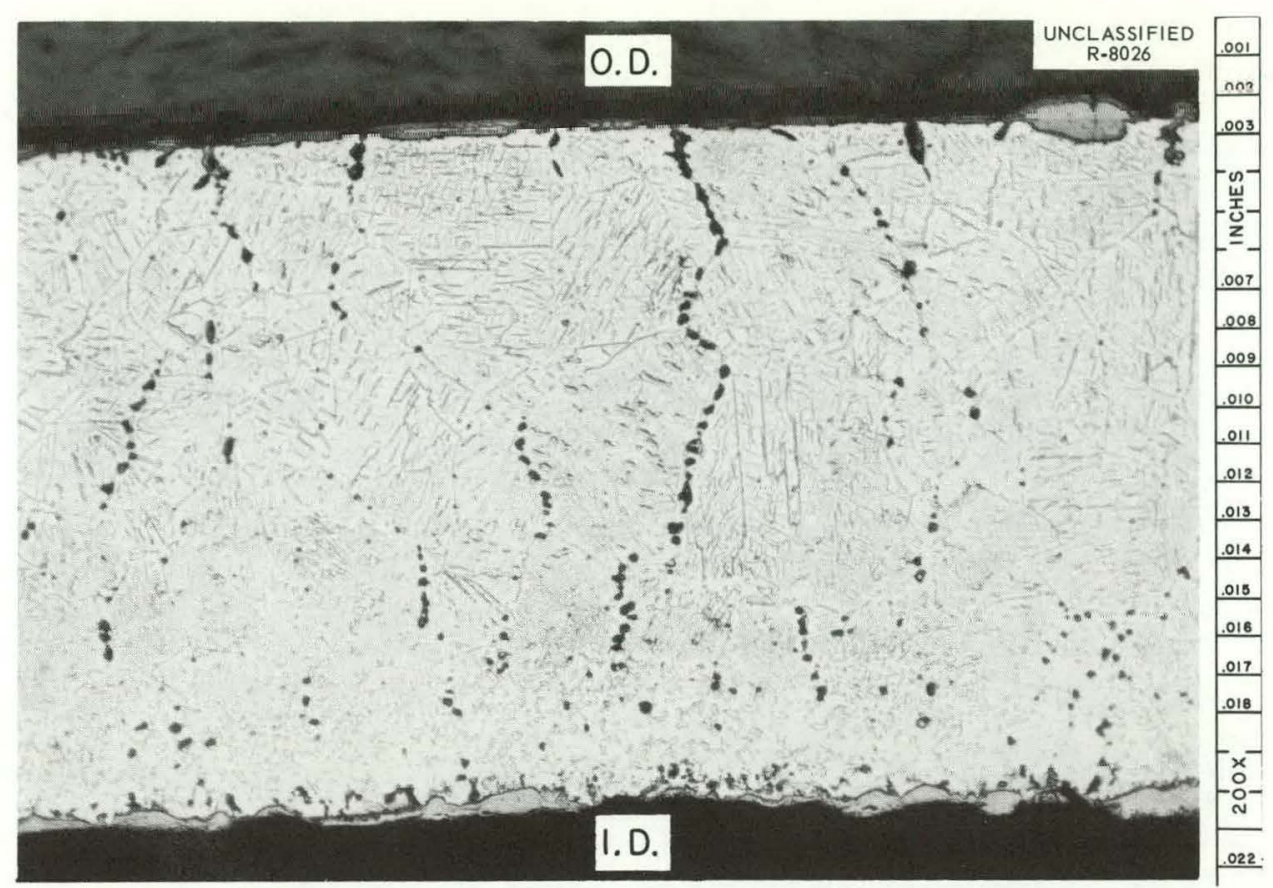

Fig. 19.9. Transverse Section of an Irradiated Tube-Burst Specimen of Type 304 Stainless Steel Alloy B Tested at $1600^{\circ} \mathrm{F}$ and Ruptured After $1430 \mathrm{hr}$ at $2200 \mathrm{psi}$. Etchant, aqua regia. 200X. Reduced $23.5 \%$.

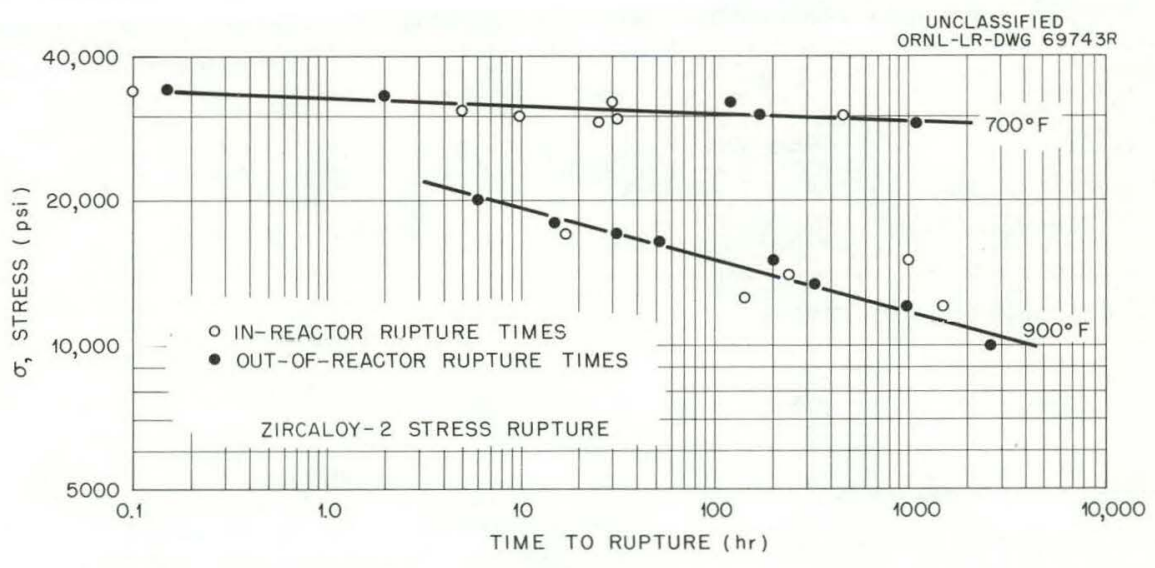

Fig. 19.10. Effect of Neutron Bombardment on the Stress-to-Rupture of Zircaloy-2 at 700 and $900^{\circ} \mathrm{F}$ in Helium.

Vacuum fusion analyses on samples of two irradiated specimens stressed at $1800^{\circ} \mathrm{F}$ indicated slight increases in the concentrations of hydrogen, nitrogen, and oxygen. This represents reasonably good atmosphere control, and the increase in the amount of impurities is not believed to have a significant effect on the high-temperature stressrupture properties.

\section{Neutron Flux Monitoring}

Neutron flux monitors have been included in each assembly of tube-burst specimens irradiated in the ORR poolside. Monitors of cobalt, sulfur, $\mathrm{Np}^{237}$, and $\mathrm{U}^{238}$ were irradiated during the entire time of the test. The disintegration rates of the neutron-reaction products were determined after 
removal from the reactor, and the average neutron flux was calculated.

The results of the neutron flux monitoring tests in the ORR poolside facility positions P-5 and P-6 are listed in Table 19.2. The ranges of values listed for each entry are probably due to such factors as the variations in the fuel loading of the reactor and the relative position of the monitors in the experiment cans.

A source of error in the use of the $\mathrm{Np}^{237}(n$, fission) reaction as a threshold monitor has been found. The $\mathrm{Np}^{237}(n, \gamma) \mathrm{Np}^{238}$ reaction for thermal neutrons, leading to a buildup of $\mathrm{Np}^{238}$ and $\mathrm{Pu}^{238}$ which yield fission by thermal neutrons, had been overlooked. For short irradiations or low flux the error introduced is negligible. However, for irradiation times exceeding a few days at a flux of $10^{13}$ neutrons $\mathrm{cm}^{-2} \mathrm{sec}^{-1}$ or greater, the fission products produced by thermal-neutron reactions cause an appreciable error in the calculated fast flux. Since this error is very large for the conditions encountered in these monitoring experiments, the flux measurements with $\mathrm{Np}^{237}$ are not considered reliable. The neutron flux determinations using $\mathrm{U}^{238}$ are also considered unreliable, possibly because of the $U^{235}$ impurity.

In the future, the $\mathrm{Np}^{237}$ and $\mathrm{U}^{238}$ monitors will be shielded with cadmium to reduce the effect of the thermal flux. Cadmium-shielded cobalt monitors will also be added, to enable the separate determination of the thermal and epithermal fluxes.

Table 19.2. Neutron Flux Measurements in the ORR Poolside Facility Positions P.5 and P.6

\begin{tabular}{llcc}
\hline \multirow{2}{*}{ Monitor } & $\begin{array}{c}\text { Energy } \\
\text { Range }\end{array}$ & \multicolumn{2}{c}{ Flux } \\
\cline { 3 - 3 } & & $\times 10^{13}$ & $\times 10^{13}$ \\
Cobalt & Thermatrons $\mathrm{cm}^{-2} \mathrm{sec}^{-1}$ ) \\
$\mathrm{Np}$ & $3-7$ & $5-8$ \\
$\mathrm{U}^{238}$ & $>0.6 \mathrm{Mev}$ & $3-9$ & $10-35$ \\
$\mathrm{~S}^{32}$ & $>1.5 \mathrm{Mev}$ & $1.5-15$ & $20-50$ \\
\hline
\end{tabular}

\section{Discussion}

The occurrence of spherical voids arranged along grain boundaries and separated grains with "scalloped" edges in irradiated Inconel specimens was first thought to be due to helium production from the fissioning of the $B^{10}$ impurity. ${ }^{7}$ The helium, precipitating out at grain boundaries, would act to increase the local stress due to the internal pressure and to form stress-raisers by reducing the area of the grain boundary. This offers a possible explanation of the observed reduction in rupture strength. To test this hypothesis, samples from special heats of Inconel, containing varying amounts of $\mathrm{B}^{10}$ and $\mathrm{B}^{11}$, were tested in the reactor. The expected correlation between reduced rupture strength and the $B^{10}$ concentration was not observed, although the $\mathrm{B}^{10}$ concentration was varied by a factor of about 100 .

The lack of supporting evidence of the effect of boron for the Inconel probably rules out this effect in the stainless steel, since the concentration of boron in the stainless steel was lower by a factor of 5 than in the Inconel.

Another point of interest is that the reduction in the rupture strength of these materials during irradiation is accompanied by a decrease in the deformation at rupture in the stainless steel and all but one of the heats of Inconel.

The high-temperature property changes induced by irradiation appear to be connected with an increased grain-boundary-void nucleation or growth. Several models have been proposed to explain voids formed in grain boundaries transverse to the major stress direction during creep. One of the mechanisms proposed by Greenwood et $a l .{ }^{12}$ is that the voids grow as a result of the condensation of vacancies generated during creep. Also, Balluffi and Seigle ${ }^{13}$ postulate that voids, nucleated at grain boundaries, grow by migration of vacancies from the boundaries in to the voids. If the precipitation of vacancies is important in the growth of intergranular voids, the dynamic vacancy supersaturation existing during neutron bombardment would accelerate the void

\footnotetext{
${ }^{12} \mathrm{~J}$. N. Greenwood, D. R. Miller, and J. W. Suiter, Acta Met. 2, 250 (1954).

${ }^{13}$ R. W. Balluffi and L. L. Seigle, Acta Met. 5, 449 (1957).
} 
growth and permit failure to occur sooner and at lower strains than in the absence of radiation.

The intergranular cavitation was not observed in the tests on Zircaloy-2 and the niobium-1\% zirconium alloy. This was probably due to the fact that these alloys were not investigated in the high-temperature creep range.

It is unlikely that the mechanisms of the void formation and growth will be determined by testing commercial alloys. The mechanisms are probably very dependent upon such material variables as impurities, precipitates, grain size, and relative grain orientation. Sufficient control over these parameters is not easily obtained in these alloys.

\subsection{IMPACT TESTS ON IRRADIATED PRESSURE-VESSEL STEELS}
R. G. Berggreit
L. D. Schaffer ${ }^{14}$
M. S. Wechsler
T. N. Jones

Surveillance samples of ASTM A212, Grade B, steel from the pressure vessel of the SM-1 reactor

\footnotetext{
14 Reactor Division,
}

at Fort Belvoir, Virginia, were placed in the reactor at the initial startup in April 1957. Eight capsules containing impact bars were located on the core support plate 15.0 in. from the vertical center line of the reactor, with the bottom ends of the impact bars 15.0 in. from the core center. Each capsule contained three subsize Izod impact specimens with six notches each. Since there is a steep fast-neutron flux gradient at this position, each notch was exposed to a different fastneutron flux. The exposure temperature of the capsules was about that of the exit coolant $\left(450^{\circ} \mathrm{F}\right)$.

Thus far, two capsules have been removed; the first after $16.4 \mathrm{Mwyr}$ of operation and the second after $20.8 \mathrm{Mwyr}$. The impact tests were conducted at the Oak Ridge National Laboratory in Hot Cell 4 of Building 3025. Unirradiated specimens from the same steel plate were tested in the hot cell along with the irradiated surveillance specimens. The results of the impact tests are shown in Figs. 19.11 and 19.12. Since only three tests were possible for each notch location and exposure, the impact curves are not very well defined. Therefore, an estimate of an effective brittle-ductile transition temperature is rather

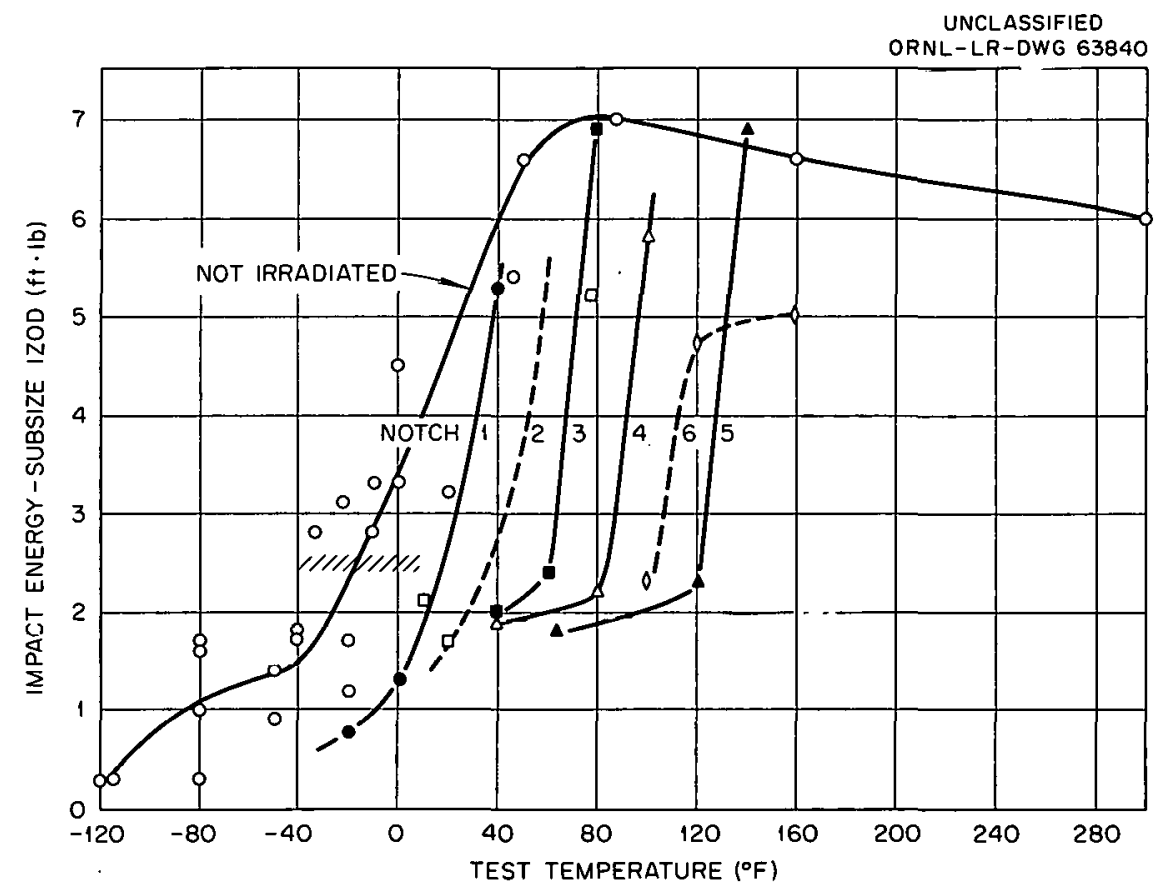

Fig. 19.11. Impact Energy vs Test Temperature for Surveillance Specimens of ASTM A212, Grade B Steel from SM-1 Pressure.Vessel Material After 16.4 Mwyr of Reactor Operation. 


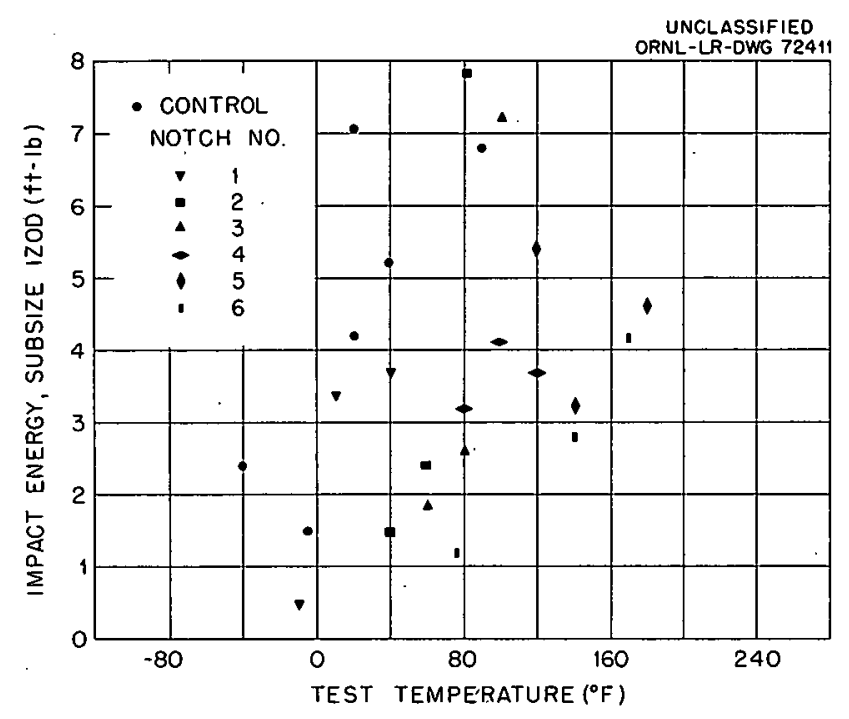

Fig. 19.12. Impact Energy vs Test Temperature for Surveillance Specimens of ASTM A212, Grade B Steel from SM-1 Pressure.Vessel Material After 20.8 Mwyr of Reactor Operation.

difficult. However, the following procedure was adopted. A transparent overlay was constructed, which defined the scatter-band for the unirradiated samples. The overlay was then translated along the temperature axis so that the three points for a given notch were best fitted by eye inside of the scatter band. The amount of translation (in ${ }^{\circ} \mathrm{F}$ ) thus determined was taken to be the increase in transition temperature. The results of this analysis for the two capsules are given in Table 19.3 .

It is current practice, particularly as concems design and operating criteria for reactor pressure vessels, to use the Charpy V-notch impact sample in the determination of transition temperatures. It has been found ${ }^{15}$ that the irradiation-induced increase in transition temperature for the Charpy $V$-notch sample is roughly $25^{\circ} \mathrm{F}$ higher than for the subsize Izod sample. On this basis, the corresponding Charpy V-notch transition temperature increases are also given in Table 19.3.

${ }^{15} \mathrm{R}$. G. Berggren, USAEC Conference on the Status of Radiation Effects Research on Structural Materials and the Implications to Reactor Design. TID-7588, p 91 (October 1960).
A study of the neutron flux distribution has been reported ${ }^{16}$ for a mockup of the SM-1, containing mockups of the themal shield and pressure vessel: The neutron flux measured with sulfur detectors was found to be $4.8 \times 10^{2}$ neutrons $\mathrm{cm}^{-2} \mathrm{sec}^{-1} \mathrm{w}^{-1}$ at the pressure vessel and $13.9 \times 10^{2}$ neutrons $\mathrm{cm}^{-2} \mathrm{sec}^{-1} \mathrm{w}^{-1}$ at a position corresponding to notch 5 of the surveillance samples. Thus, for notch 5 of capsule I (16.4 Mwyr) and capsule II (20.8 Mwyr), we calculate sulfur doses of $6.9 \times 10^{17}$ and $8.7 \times 10^{17} \mathrm{neu}$ tron $\mathrm{s} / \mathrm{cm}^{2}$, respectively. A formula relating transition temperature increase, $\Delta T T$, and neutron dose has recently been given by $\mathrm{Nichols}$ and Harries ${ }^{17}$

$$
\Delta T T=49 \Phi(\mathrm{Ni})^{1 / 2},
$$

\footnotetext{
${ }^{16} \mathrm{~K}$. C. Sontheimer et al., Fast Neutron Flux Meas. urements and Analysis in $S M-1$ and $P M-2 A$ Core and Vessel Mock-ups, APAE-95 (March 31, 1962).

${ }^{17}$ R. W. Nichols and D. R. Harries, "Brittle Fracture and Irradiation Effects in Ferritic Pressure Vessel Steels," Symposium on Radiation Effects on Metals and Neutron Dosimetry, ASTM, Los Angeles, October $1-5,1962$.
}

Table 19.3. Increase in Brittle-Ductile Transition Temperature for the SM-I Surveillance Samples

\begin{tabular}{|c|c|c|}
\hline \multirow{3}{*}{$\begin{array}{l}\text { Notch } \\
\text { Number }\end{array}$} & \multicolumn{2}{|c|}{$\begin{array}{l}\text { Subsize Izod Transition } \\
\text { Temperature Increase }\left({ }^{\circ} \mathrm{F}\right) \\
\text { rpy V } \Delta T T \text { Values }{ }^{b} \text { in Parentheses }\end{array}$} \\
\hline & Capsule I & Capsule II \\
\hline & $16.4 \mathrm{Mwyr}$ & $20.8 \mathrm{Mwyr}$ \\
\hline 1 & $45(70)$ & $51(76)$ \\
\hline 2 & $58(83)$ & $67(92)$ \\
\hline 3 & $69(94)$ & $76(101)$ \\
\hline 4 & $88(113)$ & $109(134)$ \\
\hline 5 & $120(145)$ & $141(166)$ \\
\hline 6 & $130(155)$ & $150(175)$ \\
\hline
\end{tabular}

$a_{\text {Notches numbered in order of decreasing height (de- }}$ creasing distance from horizontal core midplane).

${ }^{b}$ Calculated according to $\Delta T T$ (Charpy $\left.\mathrm{V}\right)=\Delta T T$ (Subsize Izod) $+25^{\circ} \mathrm{F}$. (See ref 15.) 
where $\Phi(\mathrm{Ni})$ is integrated flux obtained with a nickel threshold detector (threshold energy, 2.9 Mev) in units of $10^{18}$ neutron s/ $\mathrm{cm}^{2}$. The sulfur flux should be about the same as for nickel, since the threshold energy for sulfur is $3.0 \mathrm{Mev}$. Equation (1) was determined for Charpy V-notch samples irradiated at $300^{\circ} \mathrm{F}$ at much higher fluxes than the SM-1 surveillance samples. If now we substitute into Eq. (1) the integrated fluxes, $6.9 \times 10^{17}$ and $8.7 \times 10^{17}$ neutrons $/ \mathrm{cm}^{2}$ given above for the surveillance samples, we find that the predicted $\Delta I^{\prime \prime} I$ values are 40 and $45^{\circ} \mathrm{F}$ for notch 5 of the capsules I and II, respectively, as compared to the values 145 and $166^{\circ} \mathrm{F}$ (Table 19.3) obtained from our impact tests on the surveillance samples.

The discrepancy between the transition temperature increases observed for the surveillance samples ( 145 and $166^{\circ} \mathrm{F}$ ) and those predicted on the basis of Eq. (1) ( 40 and $45^{\circ} \mathrm{F}$ ) may be due to the following reasons:

1. Lack of precision in the impact tests.

2. Errors in neutron dosimetry.

3. Differences in neutron spectrum.
4. The higher exposure temperature of the surveillance specimens $\left(450^{\circ} \mathrm{F}\right)$ as compared with that of the samples on which Eq. (1) is based $\left(300^{\circ} \mathrm{F}\right)$. This may be a factor, since some steels have shown a maximum between 300 and $500^{\circ} \mathrm{F}$ in the curve relating embrittlement to irradiation temperature.

5. The higher flux, by a factor of about 10 , for the accelerated-irradiation samples as compared with that for the surveillance samples. It has been pointed out ${ }^{18,19}$ that, if the changes in properties of pressure vessel steels upon irradiation are associated with radiation-enhanced diffusion, then larger effects are to be expected in certain cases when a given dose is received at a lower flux.

\footnotetext{
${ }^{18} \mathrm{M}$. S. Wechsler and R. G. Berggren, "The Radiation Embrittlement of Reactor Pressure Vessels. I. Fracture and Radiation Damage," to be published.

${ }^{19} \mathrm{M}$. S. Wechsler, "Fundamental Aspects of Radiation Effects on Diffusion-Controlled Reactions in Alloys," Symposium on Radiation Effects on Metals and Neutron Dosimetry, ASTM, Los Angeles, October $1-5,1962$.
} 
THIS PAGE

WAS INTENTIONALLY LEFT BLANK 


\section{Part VI. Reactor Materials \\ 0. Sisman}


THIS PAGE WAS INTENTIONALLY LEFT BLANK 


\title{
20. Fuel Materials
}

\author{
O. Sisman
}

\subsection{POSTIRRADIATION EXAMINATION OF GRAPHITE MATRIX FUEL}
J. G. Morgan
E. L. Long, Jr. ${ }^{1}$
M. F. Osborne
P. E. Reagan

\section{MTR-Irradiated Fueled Graphite}

Examination of the six MTR-irradiated fueled graphite experiments is nearly complete. Results from the first three experiments in this series were reported previously. ${ }^{2,3}$ As shown in Table 20.1, three different types of fuel were used. Experiment MTR-48-4 contained four fuel pellets sealed in a low-permeability graphite can, while experiments -5 and -6 contained single fuel pellets and were unsealed. All elements were purged with a slow flow of helium, which facilitated fission-gas release measurements during irradiation.

\footnotetext{
${ }^{1}$ Metals and Ceramics Division.

${ }^{2}$ O. Sisman et al., Solid State Div. Ann. Progr. Rept. Aug. 31, 1960, ORNL-3017, pp 113-14.

${ }^{3}$ O. Sisman et al., Solid State Div. Ann. Progr. Rept. Aug. 31, 1961, ORNL-3213, pp 110-11.
}

The irradiation effects on experiment -4 were similar to those of previous experiments. There were small decreases in fuel-pellet dimensions, both length and diameter, for experiments -4 and -5 . No dimensional changes were apparent for the -6 fuel pellet, which contained coated $(\mathrm{U}, \mathrm{Th}) \mathrm{C}_{2}$ particles. The appearances of the three types of fuel pellets are compared in Fig. 20.1. The -4 fuel pellets, which contained uncoated fuel particles, were coated with pyrolytic carbon. With the exception of two small cracks in the -5 fuel pellet (one of which is apparent in Fig. 20.1b), there were no changes in appearance caused by the irradiation.

Samples of the fuel pellets and graphite cans were analyzed to determine the extent of uranium and fission-product migration. As was the case in earlier experiments, significant migration was detected, and is illustrated in Fig. 20.2. The original uranium concentration in the -5 fuel pellet was $0.0904 \mathrm{~g}$ of $\mathrm{U}$ per $\mathrm{cm}^{3}$, or $2.3 \times 10^{17}$ atoms of $U$ per $\mathrm{mg}$. The uranium concentration in the graphite can after irradiation ranged from about $4 \%$ of that in the fuel near the inside surface to

Table 20.1. Data Summary for MTR-48 Experiments

\begin{tabular}{|c|c|c|c|c|}
\hline $\begin{array}{l}\text { Experiment } \\
\text { No. }\end{array}$ & $\begin{array}{l}\text { Type of Fuel } \\
\text { Particle in } \\
\text { Graphite Matrix }\end{array}$ & $\begin{array}{l}\text { Fucl Loading } \\
\left(\mathrm{g} \text { of } \mathrm{U}^{235} \text { per } \mathrm{cm}^{3} \text { ) }\right.\end{array}$ & $\begin{array}{c}\text { Maximum Fuel } \\
\text { Temperature } \\
\left({ }^{\circ} \mathrm{F}\right)\end{array}$ & $\begin{array}{c}\text { Burnup } \\
\left(\text { at. } \% \mathrm{U}^{235} \text { ) }\right.\end{array}$ \\
\hline MTR-48-4 & Uncoated $\mathrm{UC}_{2}$ & $\begin{array}{l}0.169 \text { to } \\
0.517\end{array}$ & $\begin{array}{l}2500 \text { to } \\
2800\end{array}$ & $\begin{array}{l}3.5 \text { to } \\
0.9\end{array}$ \\
\hline MTR-48-5 & $\begin{array}{l}\text { Pyrolytic-carbon- } \\
\text { coated } \mathrm{UC}_{2}\end{array}$ & 0.0841 & 2600 & 17 \\
\hline MTR-48-6 & $\begin{array}{l}\text { Pyrolytic-carbon- } \\
\text { coated }(\mathrm{U}, \mathrm{Th}) \mathrm{C}_{2}\end{array}$ & 0.088 & 2000 & $a$ \\
\hline
\end{tabular}

\footnotetext{
${ }^{a}$ Analysis incomplete, probably $\sim 2.3$.
} 

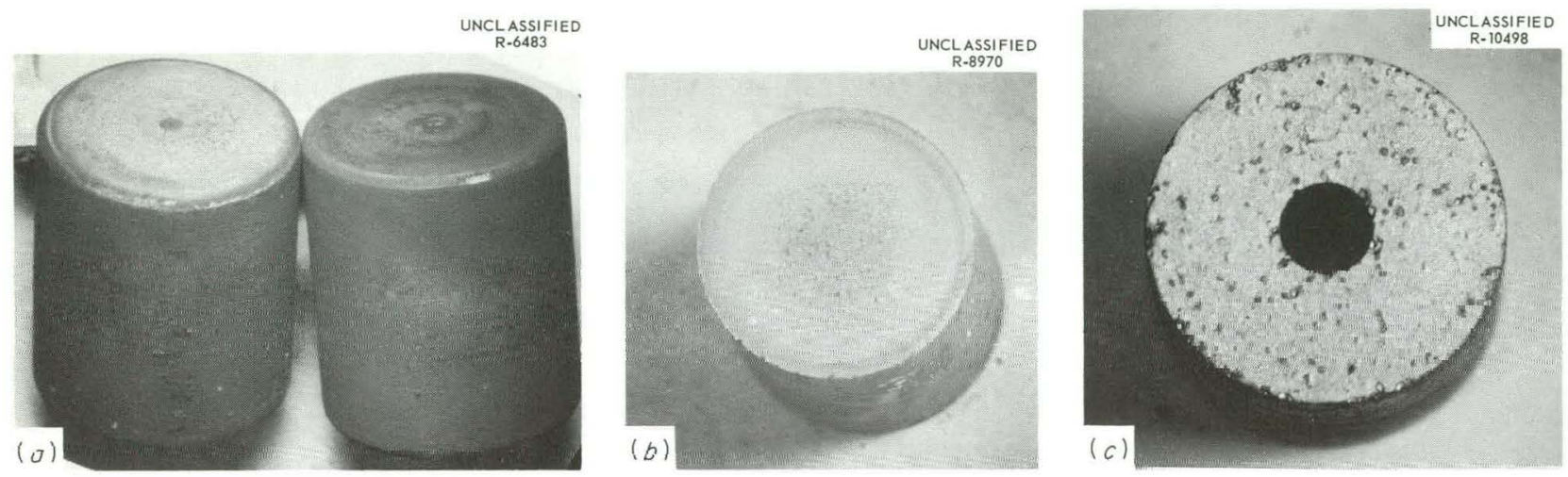

Fig. 20.1. Appearance of Irradiated Fuel Pellets from MTR-48 Experiments. Reduced $36 \%$.

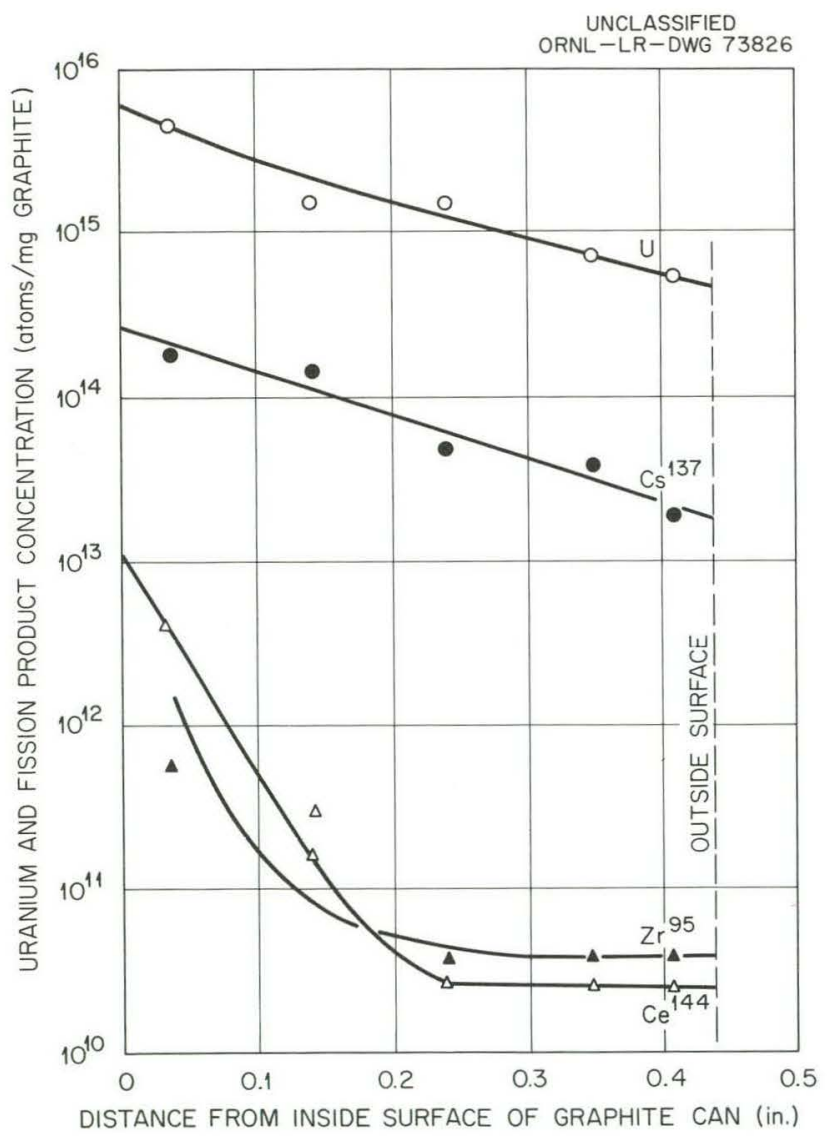

Fig. 20.2. Distribution of Uranium and Fission Products Across A Graphite-Can Wall, MTR-48-5.

about $0.4 \%$ near the outside surface. The fissionproduct concentrations found in the can represent a significant fraction of the total formed - about $30 \%$ for $\mathrm{Cs}^{137}$.
Metallographic examination of the fuel pellets indicated that the structure of the uncoated fuel particles was sensitive to uranium burnup. Fuel particles from pellets $A$ and $D$, experiment -4 , are shown in Fig. 20.3. There was no visible change in the structure of particles from pellet D (0.85 at. $\% U^{235}$ burnup), but particles from pellet $A$ (3.5 at. $\% \mathrm{U}^{235}$ burnup) had undergone considerable change. These particles had become porous and amorphous in appearance, and part of the fuel had migrated into the surrounding graphite. The central temperatures were about 2500 and $2800^{\circ} \mathrm{F}$ for fuel pellets $D$ and $A$ respectively.

All the fuel-particle coatings observed during the examination of the -5 fuel pellet were broken. There were no differences in the appearances of particles from different areas, that is, the center of the fuel pellet as compared with regions near the outside surface. As shown in Fig. 20.4, the fuel particles had lost their well-defined microstructure.

\section{ORR-B9-6 Experiment}

Postirradiation examination has been completed on capsule B9-6, which contained pyrolytic-carboncoated uranium carbide particles dispersed in a graphite matrix. The graphite body was coated with siliconized SiC. This specimen was irradiated at $2000^{\circ} \mathrm{F}$ for $362 \mathrm{hr}$ to a burnup of $3000 \mathrm{Mwd}$ per metric ton of uranium. Postirradiation examination revealed that the capsule had broken circumferentially about $\frac{1}{8}$ in. from the top, and the top section was in several wedge-shaped pieces. The center of the specimen was a loose mixture of coated particles and small graphite chunks. When 

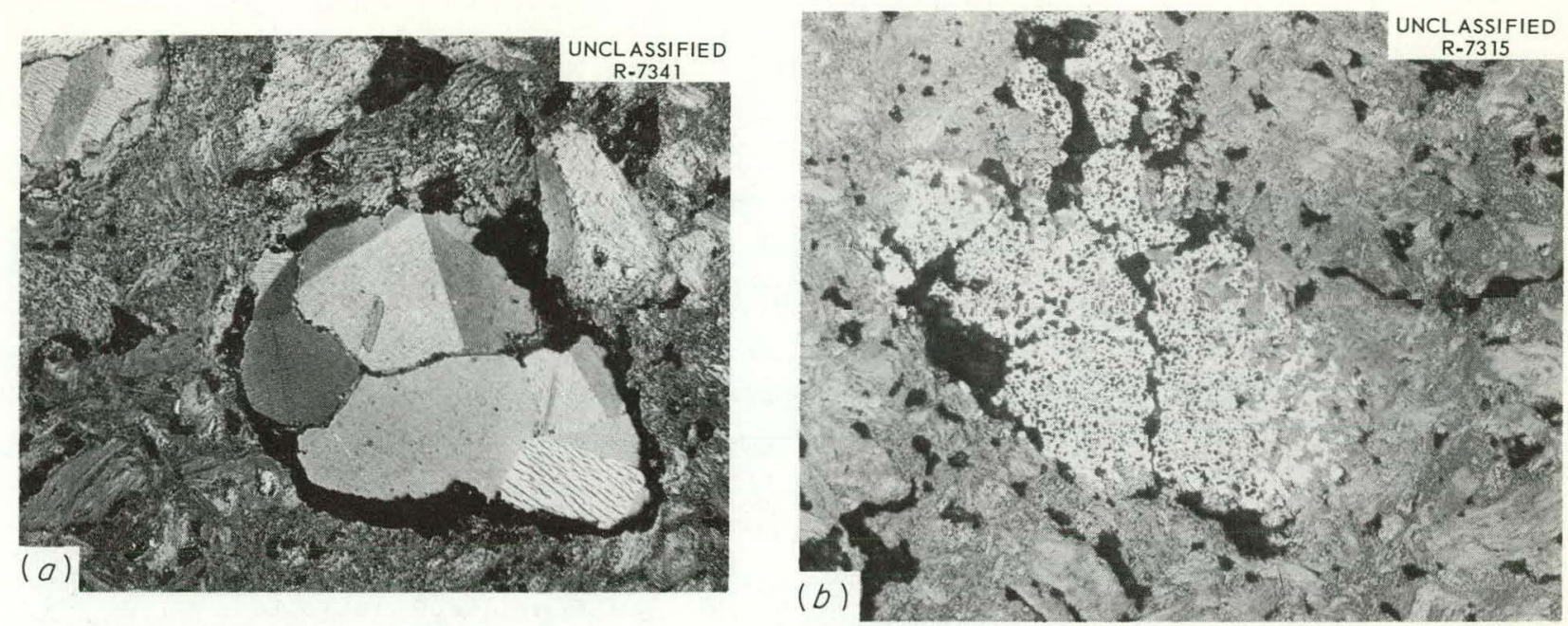

Fig. 20.3. Representative Fuel Particles from MTR-48-4, Illustrating Microstructural Differences Associated with Different Burnup Levels. Etched. 250X. (a) Particle from fuel pellet D, 0.85 at. \% U 235 burnup. Similar to unirradiated particle. (b) Particle from fuel pellet A, 3.5 at. $\% U^{235}$ burnup. Note porosity in fuel particle. Reduced $15 \%$.

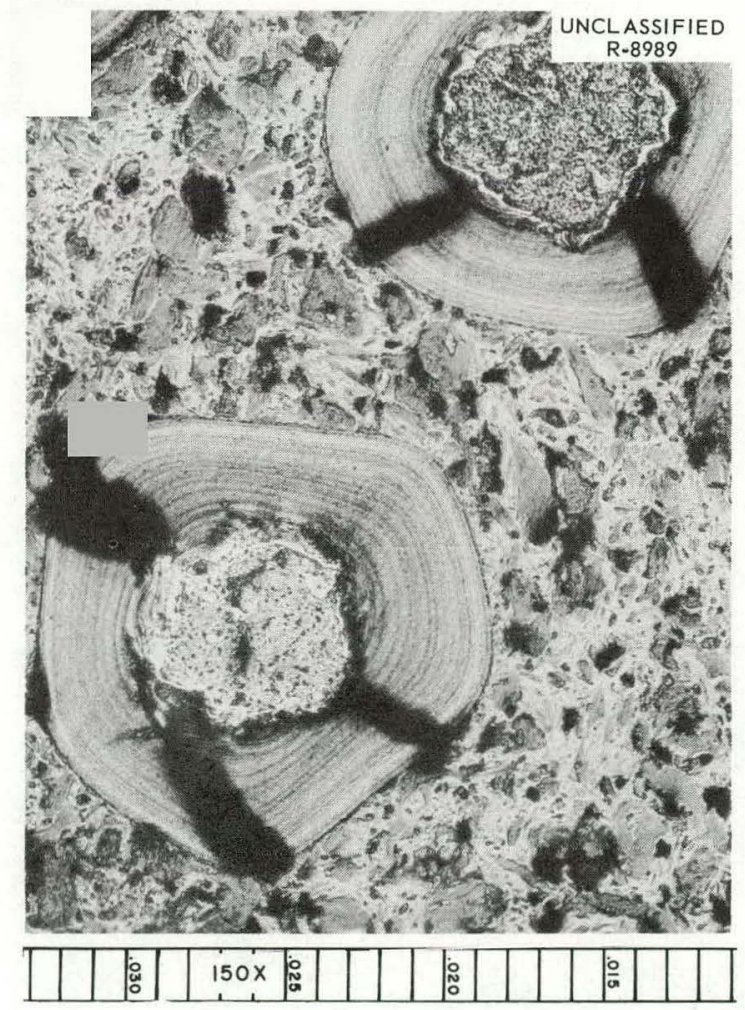

Fig. 20.4. Representative Fuel Particles from MTR48-5. Note fractures in pyrolytic-carbon coatings and absence of a well-defined microstructure in uranium carbide particles. Etched. 150X. Reduced $16 \%$. the loose material was removed, a cavity roughly $3 / 8$ in. in diameter extended to within about $\frac{1}{8}$ in. of the bottom of the specimen. Pieces of the top section and loose particles that were removed from the cavity are shown in Fig. 20.5, and the cavity is shown in Fig. 20.6. Since air leaked into the helium sweep system during part of the irradiation period, it is thought that the cavity may have been caused by selective oxidation in the central and hottest part of the specimen.

Most of the coated particles removed from the cavity had retained their smooth surface, but about 3 to $5 \%$ of them were broken. Several particles were covered with bits of silicon from the graphitebody coating. Smooth and silicon-flecked particles removed from the specimen center are shown in Fig. 20.7. Typical unbroken particles are shown in Figs. 20.8 and 20.9. All unbroken particles examined showed migration of the uranium carbide outward into the laminar coating. Bits of the carbide can be seen in the particle coating shown in Fig. 20.8. The migration did not extend completely through the coating, and thus it appears that migration does not occur at the temperature of the outer part of the coating. It is possible, but perhaps less likely, that the distance of the migration is a function of time.

An unirradiated particle from the same batch is shown in Fig. 20.10. This particle shows no 
Fig. 20.5. Broken Top Pieces of Irradiated Capsule B9-6 and Pyrolytic-Carbon-Coated Uranium Carbide Particles Removed from Capsule Center. $2.2 x$.

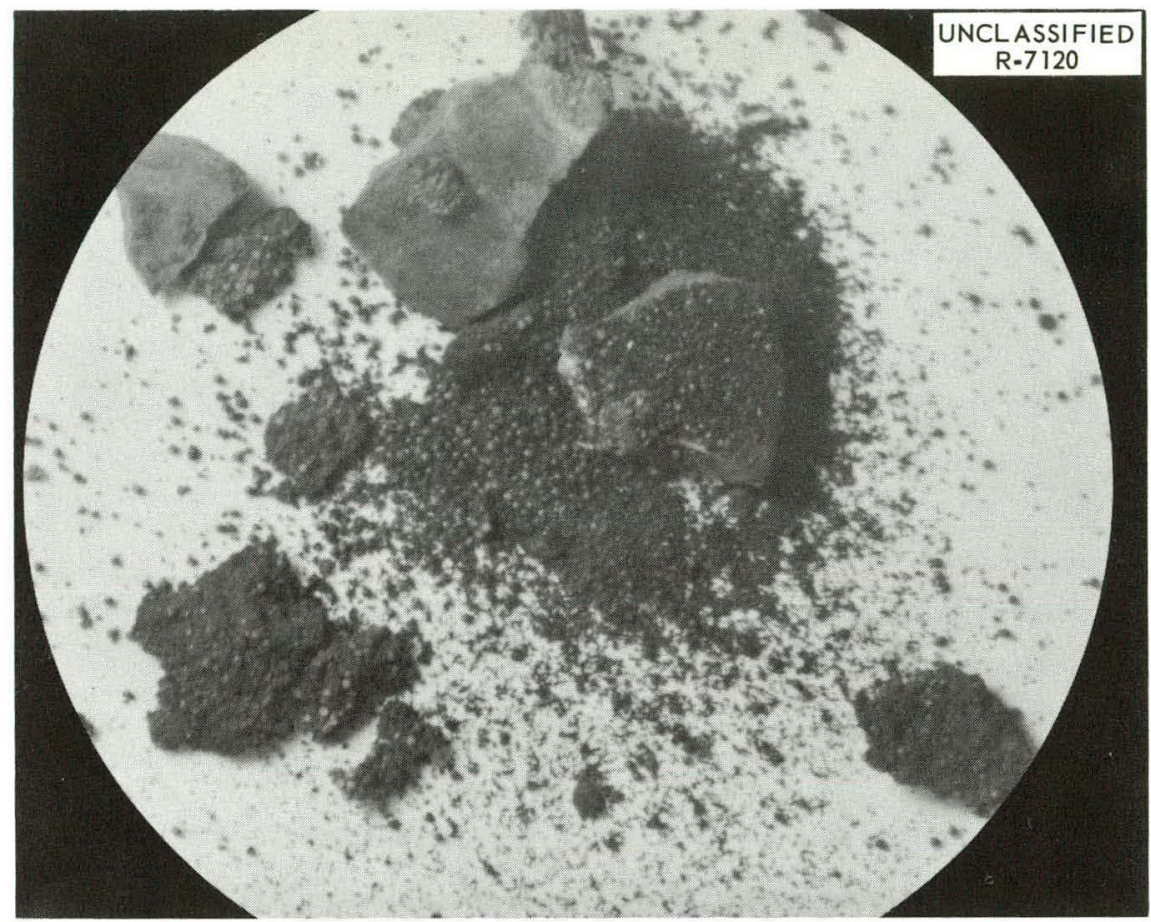

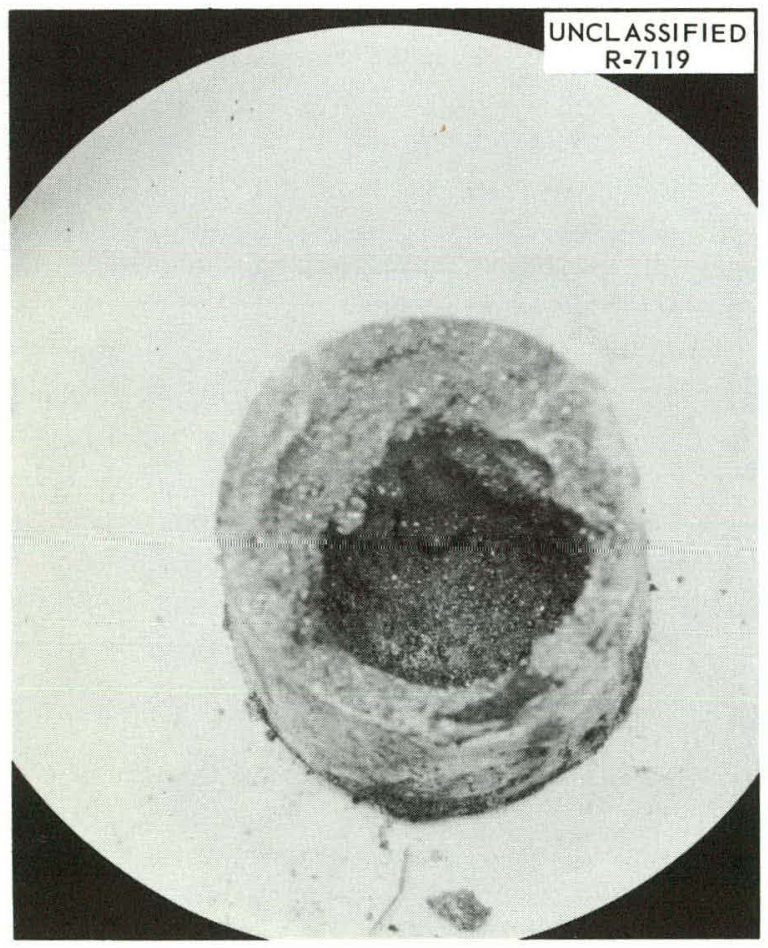

Fig. 20.6. Irradiated Capsule B9-6, Showing Cavity That Remained After Removal of Material Shown in Fig. 20.5. $2 X$.

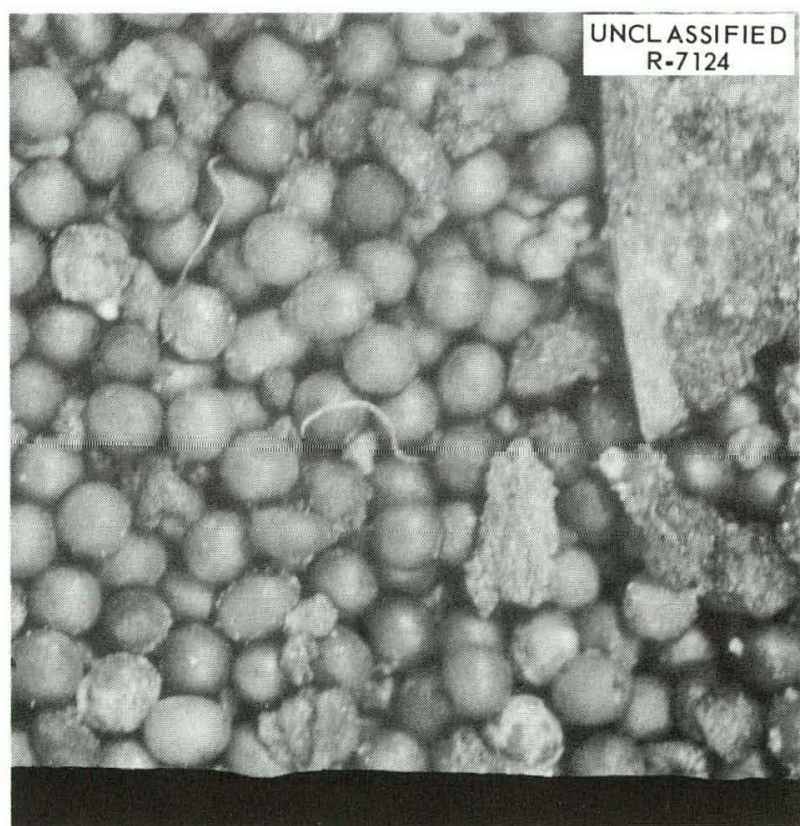

Fig. 20.7. Pyrolytic-Carbon-Coated Uranium Carbide Particles from Capsule B9-6. Some particles are flecked with silicon. $20 X$. 
migration of uranium carbide. It may be noted, however, that the siliconized $\mathrm{SiC}$ penetrated the graphite specimen body and surrounded the coated particle.

Metallographic examination of broken sections showed no relation between cracks and fuelmigration areas. A typical broken particle is shown in Fig. 20.11. The cracks may have passed through these areas at other places, but were not connected at any of the sectioned planes. Metallographic examination of the fuel particle revealed an integral grain structure; however, an unidentified second phase was observed in some of the coated particles. The second phase existed mainly in the grain boundaries.

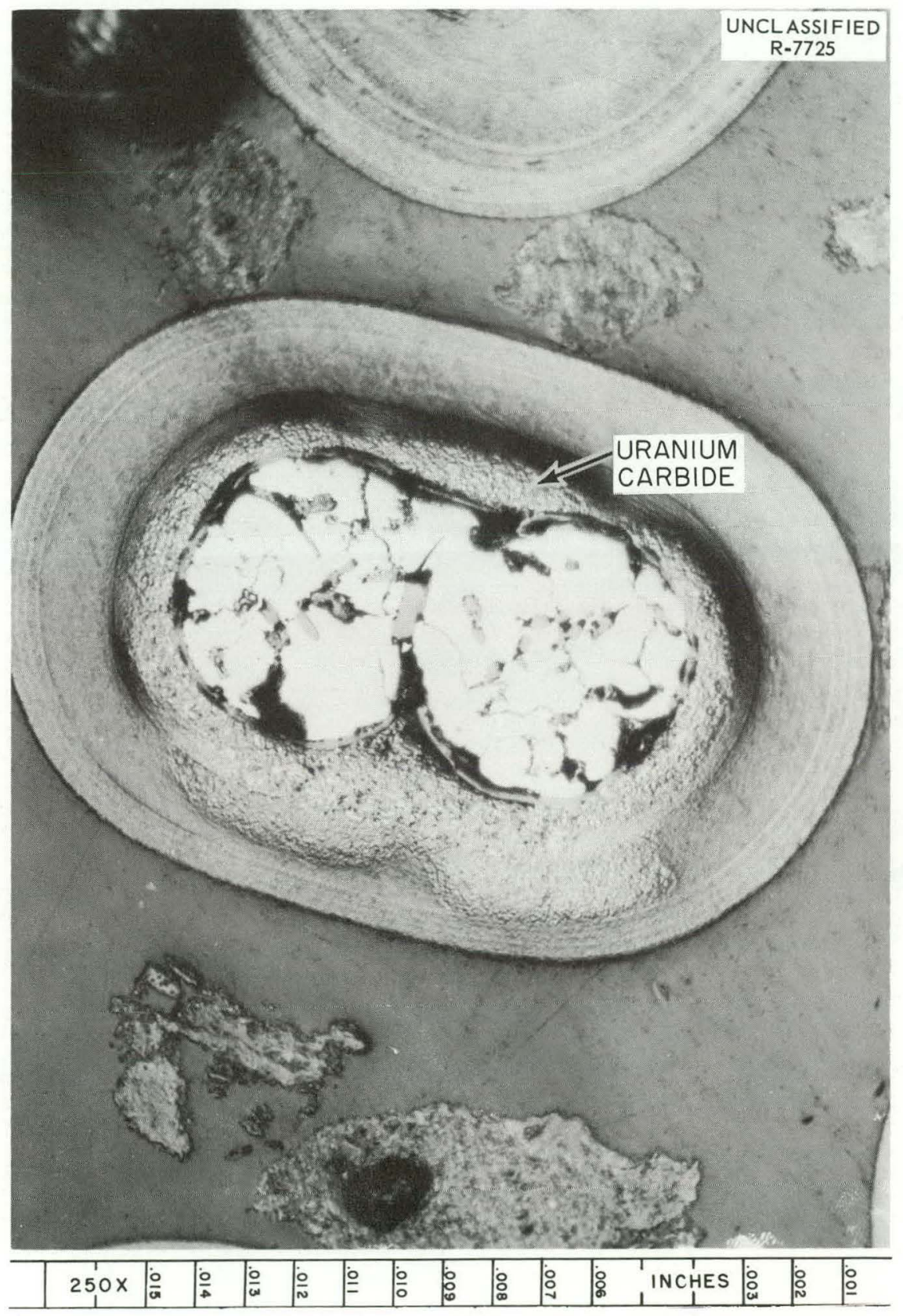

Fig. 20.8. Double-Centered Pyrolytic-Carbon-Coated Uranium Carbide Particle from Irradiated Capsule B9-6, Showing Bits of Uranium Carbide That Have Migrated into the Coating. 250X. 


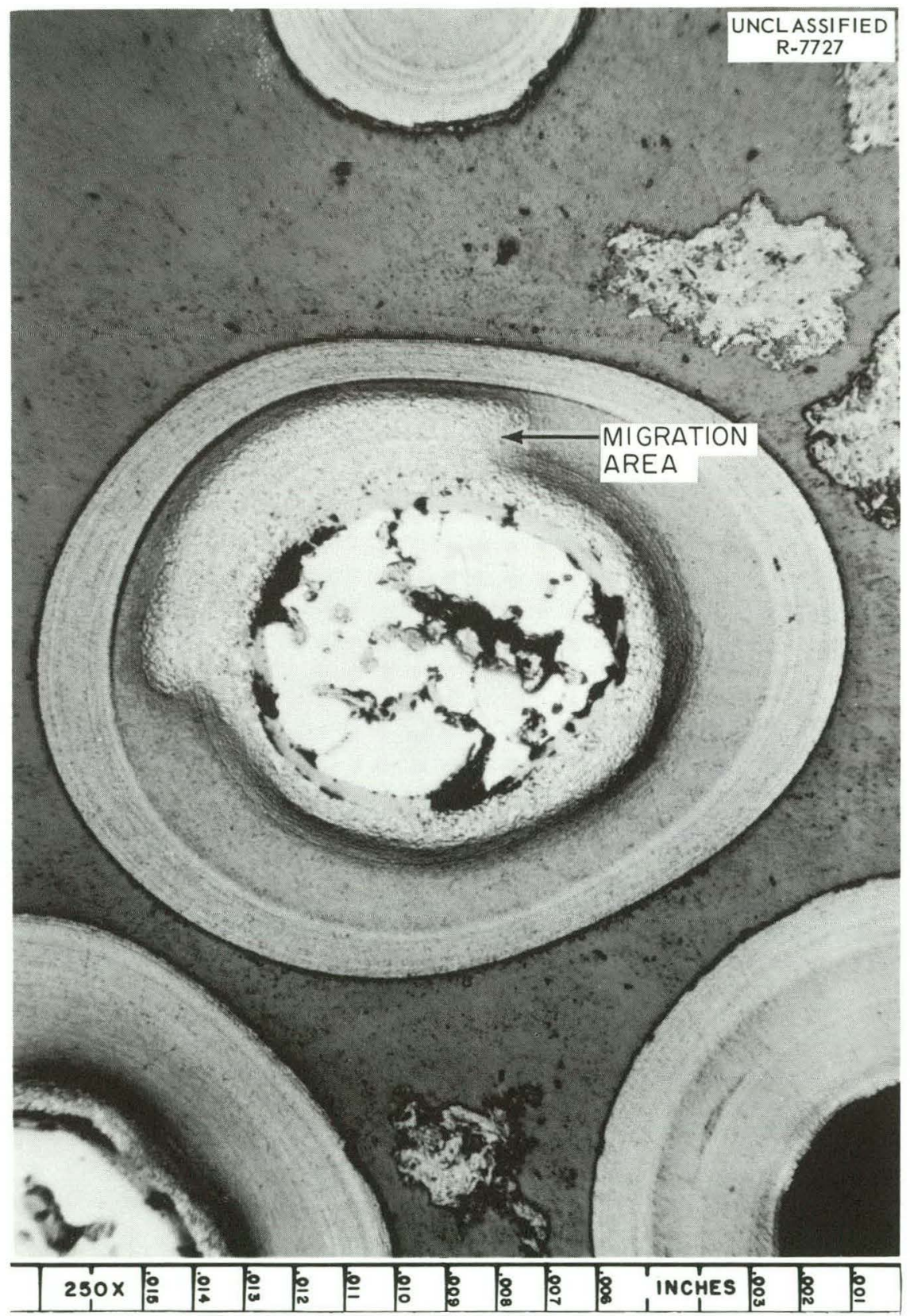

Fig. 20.9. Typical Unbroken Particle from Irradiated Capsule B9-6, Showing Uranium Carbide Migration Outward Through Part of the Pyrolytic-Carbon Coating. 250X. 


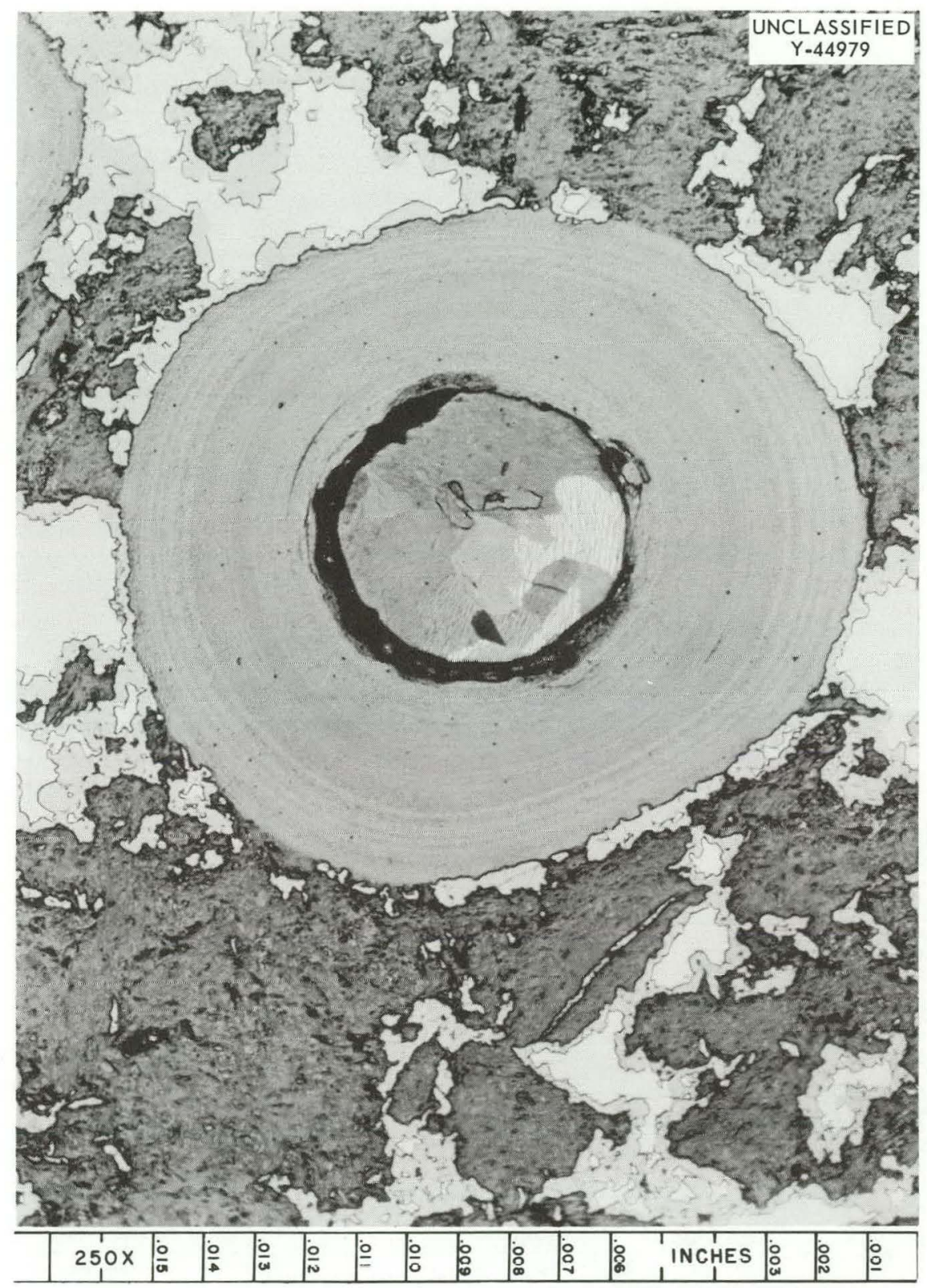

Fig. 20.10. Unirradiated Pyrolytic-Carbon-Coated Particle from the Same Batch as Capsule B9-6 Particles. Siliconized $\mathrm{SiC}$ penetrated the graphite body and surrounded the particle. $250 \mathrm{X}$. 


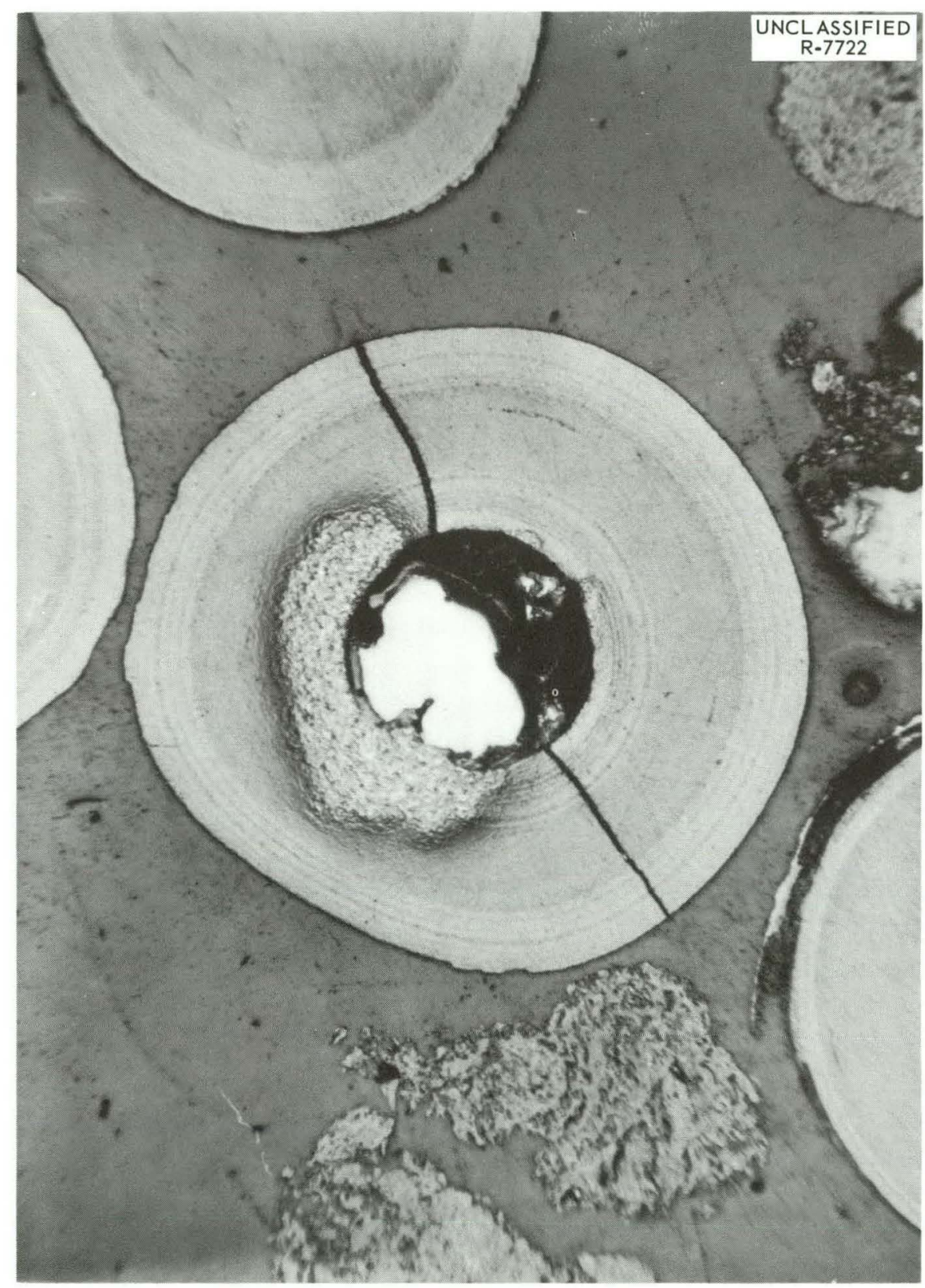

Fig. 20.11. Typical Broken Pyrolytic-Carbon-Coated Particle from Irradiated Capsule B9-6. 250X. 


\subsection{COATED FUEL PARTICLES}

\author{
R. M. Carroll \\ M. F. Osborne \\ J. G. Morgan \\ P. E. Reagan
}

T. W. Fulton

\section{Postirradiation Examination of Capsules LCP. 1 and LCP.2}

Uranium carbide fuel particles coated with pyrolytic carbon were irradiated in air-cooled core facilities in the LITR. ${ }^{4}$ Two types of coatings were tested: capsule LCP-1 contained particles with a columnar coating, supplied by High Temperature Materials, Inc., and capsule LCP-2 contained particles with a laminar coating supplied by the $3 \mathrm{M}$ Company. Both types of coatings were approximately $82 \mu$ thick. Each capsule consisted of two separate sections, denoted "a" (top) and "b" (bottom) as shown in Fig. 20.12. The fuel particles were contained in tantalum cans, which were sealed inside and insulated from the Inconel irradiation capsules. The capsule fill gas was purified helium.

The capsules were first pierced for fission-gas analysis. The results, along with irradiation conditions, are shown in Table 20.2. The low fissiongas release values for both " $a$ " sections, compared with the " $b$ " sections, indicate leakage. The appearance of the internal components from the two " $a$ " sections, especially the tantalum-clad center thermocouple, was quite different. There was no obvious oxidation of the tantalum in LCP$1 \mathrm{a}$, indicating that leakage in this capsule occurred after the irradiation. Conversely, the interior of LCP-2a was badly oxidized, indicating leakage during irradiation, that is, at elevated temperature. There was no evidence of oxidation in either LCP$1 \mathrm{~b}$ or LCP-2b.

The appearance of the fuel particles had not changed markedly during irradiation. A few particles with cracked coatings were found in all four sections. Most of the HTM-type coatings which cracked were those on "doublet" particles, illustrated in Fig. 20.13. The particles from LCP-2a were duller in appearance and exhibited more cracked coatings than those from LCP- $2 b$, probably due to oxidation of the carbon coatings in the former. In addition, as shown in Table

\footnotetext{
${ }^{4}$ The irradiations were performed by the Reactor Division.
}

20.2, acid leaching of batches of the fuel particles indicated that a larger fraction of the coatings failed in the " $a$ " sections of the capsules.

Samples of the fuel particles from both sections of each capsule were examined metallographically. Most of the coatings showed some damage

$$
\text { UNCLASSIFIED }
$$

ORNL - LR - DWG $71660 R$

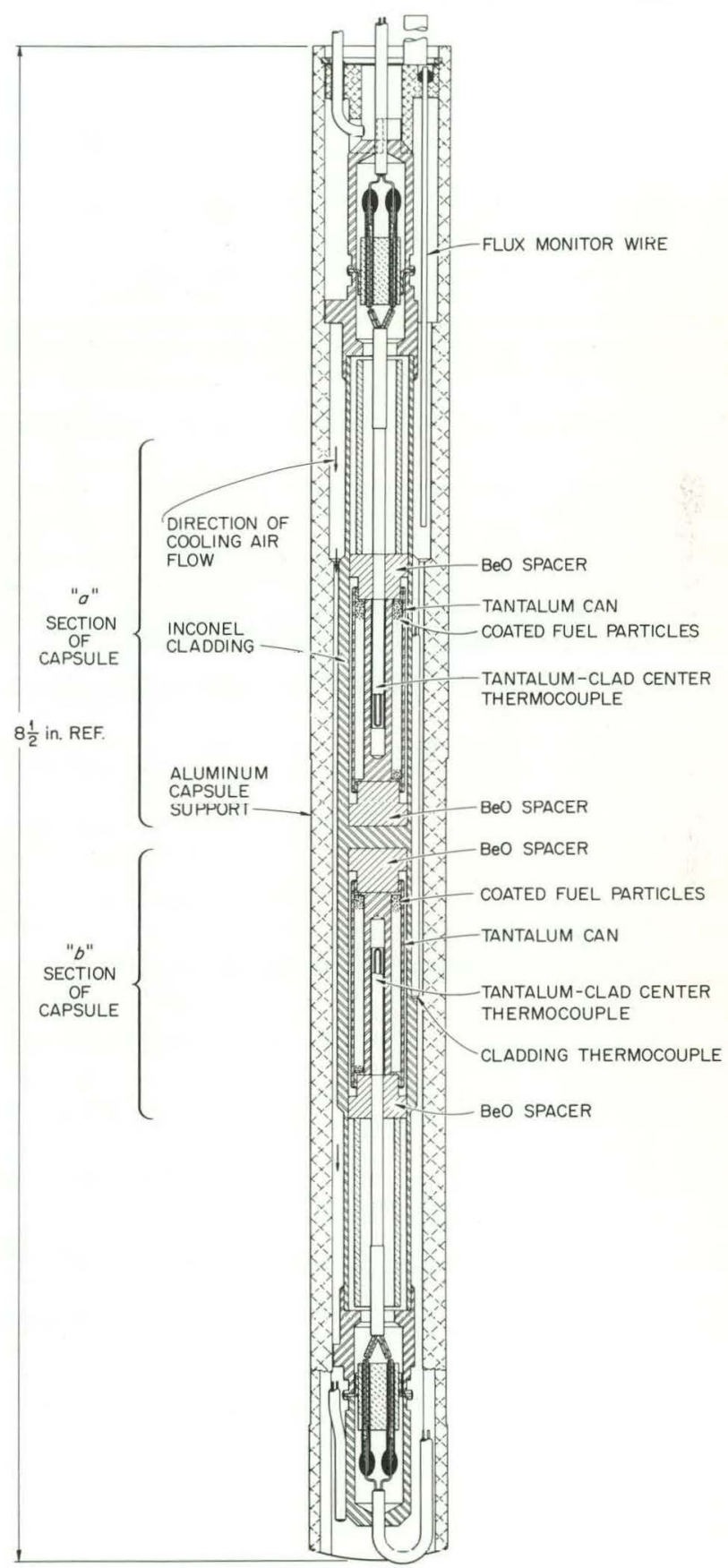

Fig. 20.12. Diagram of Coated-Particle Irradiation Capsule. 
Table 20.2. Results of Examination of Irradiated Coated Particles

\begin{tabular}{|c|c|c|c|c|c|}
\hline \multirow{2}{*}{$\begin{array}{c}\text { Capsule } \\
\text { No. }\end{array}$} & \multirow{2}{*}{$\begin{array}{c}\text { Central } \\
\text { Temperature } \\
\left({ }^{\mathrm{O}} \mathrm{F}\right)\end{array}$} & \multicolumn{2}{|c|}{ Uranium Burnup } & \multirow{2}{*}{$\begin{array}{c}\mathrm{Kr}^{85} \\
\text { Fission Gas Released } \\
(\%)\end{array}$} & \multirow{2}{*}{$\begin{array}{c}\text { Uranium Removed } \\
\text { by Leaching }{ }^{b} \\
(\%)\end{array}$} \\
\hline & & $\mathrm{Mwd} /$ metric ton & At. $\%$ & & \\
\hline LCP-1a & 1950 & 45,000 & 4.7 & 0.01 & 22 \\
\hline LCP-1b & 2100 & 45,000 & 4.7 & 0.1 & 2.0 \\
\hline $\mathrm{LCP}-2 \mathrm{a}$ & $2400^{C}$ & $70,000^{d}$ & 7 & $e$ & 10 \\
\hline $\mathrm{LCP}-2 \mathrm{~b}$ & $2300^{f}$ & $70,000^{d}$ & 7 & 0.2 & 0.62 \\
\hline
\end{tabular}

\footnotetext{
${ }^{a}$ Expressed as percentage of that produced.

${ }^{b}$ Expressed as percentage of total uranium.

${ }^{c}$ Thermocouple indicated a temperature rise to $3200^{\circ} \mathrm{F}$ and then failed.

${ }^{d}$ Estimated.

${ }^{e}$ Krypton-85 was detected but could not be counted; less than $0.1 \%$ of Xe ${ }^{133}$ was released.

${ }^{f}$ Thermocouple failed two days after startup; temperature was probably higher than in capsule LCP-2a.
}

resulting from the irradiation. Examples of these effects on the HTM coatings (LCP-1) are shown in Figs. 20.13 and 20.14. There appeared to have been some reaction at the core-to-coating interfaces; however, most of the detail in these regions was lost during sample preparation due to the loose nature of the material.

The coated particles from LCP- 2 were somewhat angular, rather than spherical, as illustrated in Fig. 20.15. The effect of oxidation is illustrated by Fig. 20.16, which shows typical particles from LCP-2a and LCP-2b. This figure also shows evidence of some reaction at the core-to-coating interfaces. The pyrolytic carbon in this region appeared to be less dense, and numerous "spearhead" fractures had begun to penetrate the coating. A typical fractured particle is shown in Fig. 20.17. The fuel cores in both types of particles had changed in appearance, and in most cases the microstructure was less well defined after irradiation. Fine porosity and a small amount of secondphase material were observed in the fuel cores from LCP-2.

\section{Instantaneous Fission-Gas Release}

In-pile tests have continued in the ORR B9 and $\mathrm{C} 1$ facilities to determine fission-gas release from pyrolytic-carbon-coated uranium carbide particles. These particles are unsupported; that is, they are not contained in a graphite matrix, but are held in a cylindrically shaped monolayer in a graphite holder. The holder, shown in Fig. 20.18, is vented to allow fission gas released from the particles to be swept from the capsule in a helium stream. Fission-gas release rates from three basically different types of pyrolytic carbon coatings are being studied. Also, the release rate from bare uranium carbide particles was measured to determine how much of the fission-gas retention could be attributed to a coating. The three coating types are laminar, columnar, and duplex; the duplex coating consists of a laminar inner layer and a columnar outer coating. Sectioned particles of the three coating types and bare uranium carbide are shown in Fig. 20.19. Particle size, coating thickness, manufacturer, and fuel loading are given in Table 20.3.

Experiment B9-7. - The B9-7 capsule, containing HTM-1 columnar-type coated particles, was irradiated for 58 days. The particles were held at $1500^{\circ} \mathrm{F}$ in a thermal-neutron flux of $3.7 \times 10^{13}$ $n v$ for 43 days. The average fractional release of fission gas during this period is shown in Fig. 20.20. The temperature was subsequently increased to $1650^{\circ} \mathrm{F}$ by inserting the capsule into a higherflux region of $5.5 \times 10^{13} n v$, and irradiation was continued for 15 days.

The fractional release rates of $\mathrm{Xe}^{133}, \mathrm{Xe}^{135}$, $\mathrm{Kr}^{85 m}, \mathrm{Kr}^{87}$, and $\mathrm{Kr}^{88}$ were found to be temperature dependent. When the temperature was decreased from $1500^{\circ} \mathrm{F}$ to $1370^{\circ} \mathrm{F}$ with the neutron 

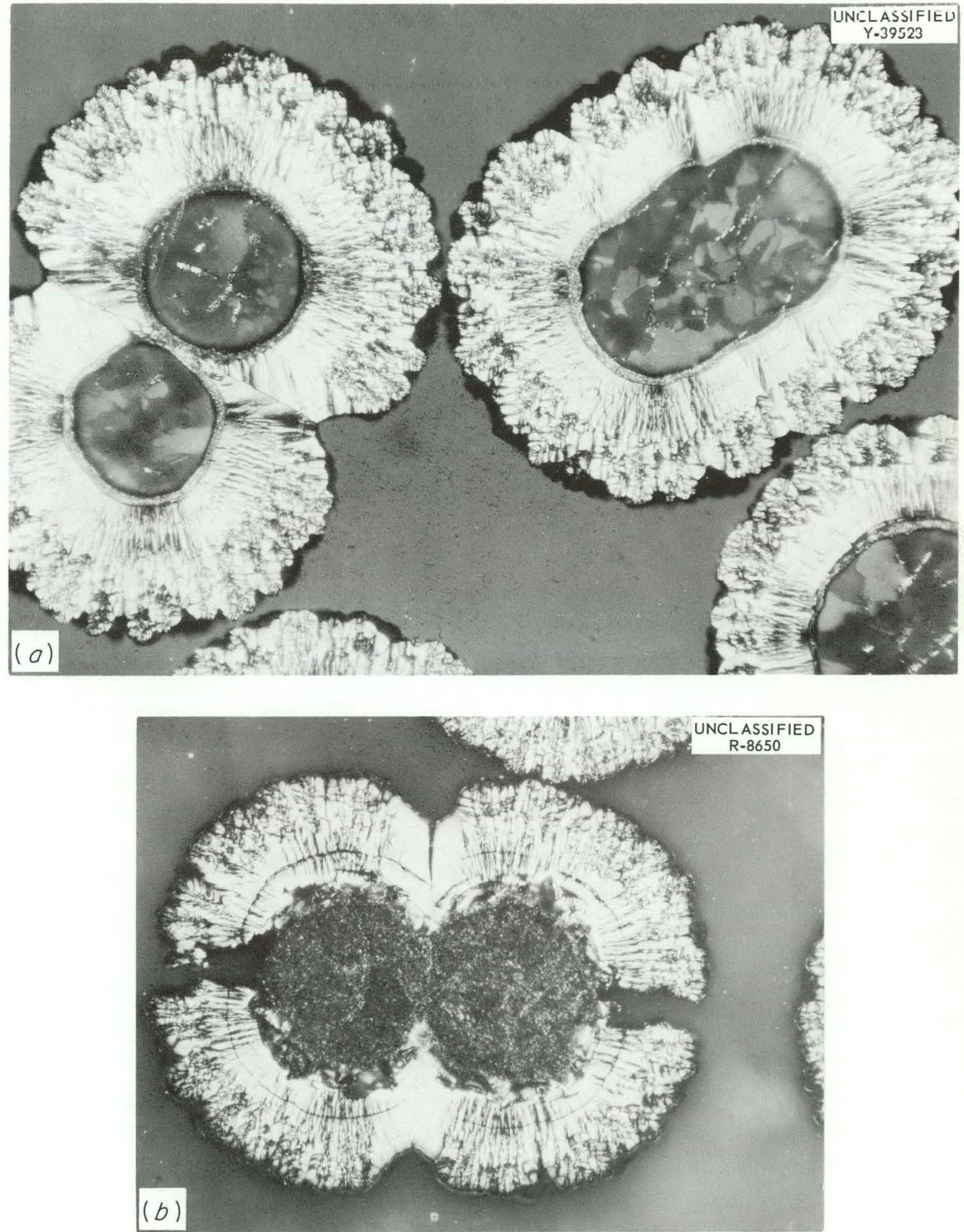

Fig. 20.13. Pyrolytic-Carbon-Coated Uranium Carbide Particles from Batch HTM-1. (a) Unirradiated "doublet." As polished. Polarized light. 150X. (b) Broken "doublet" from section "b" of LITR capsule LCP-1. Etched. Polarized light. 150X. 

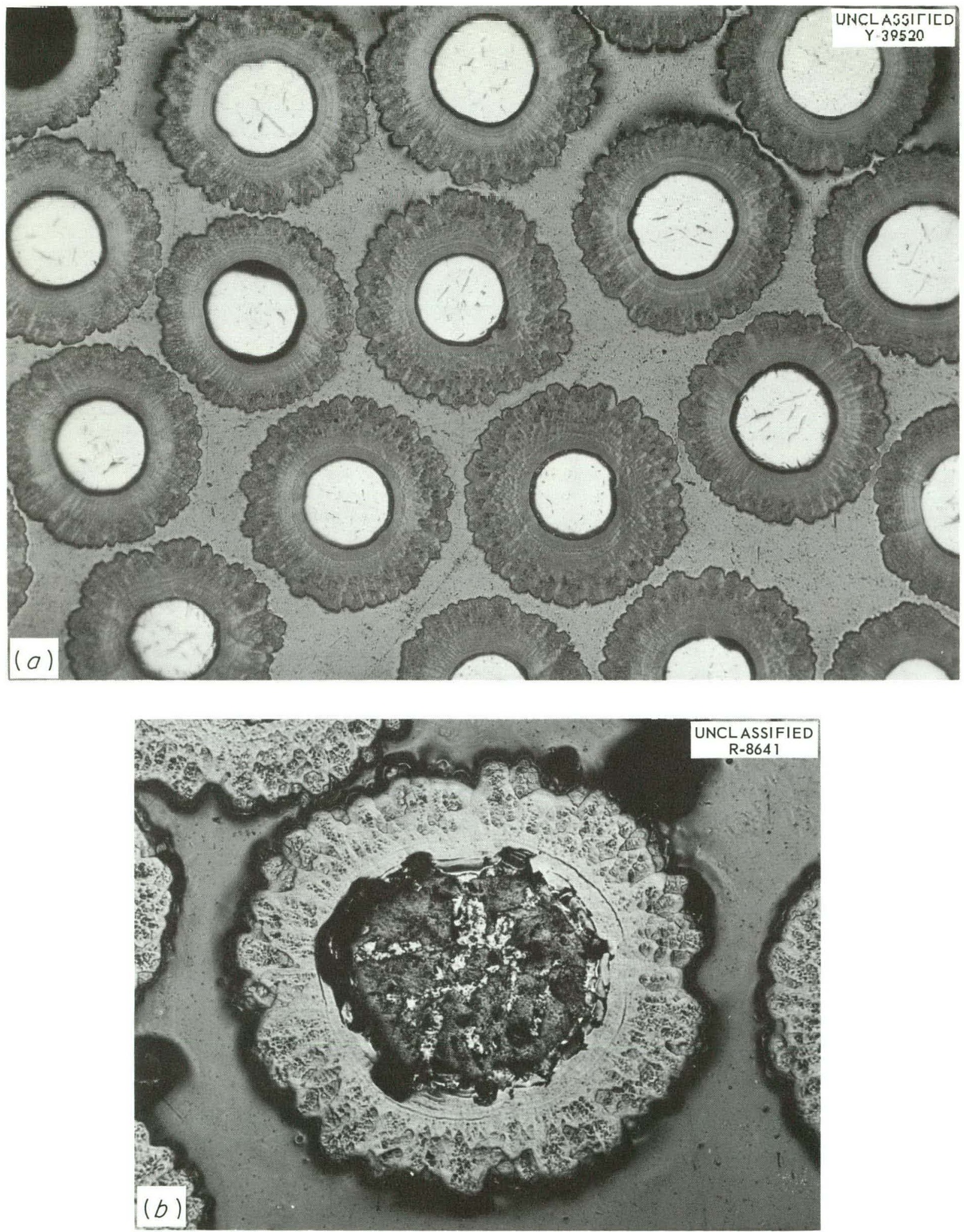

Fig. 20.14. Typical Coated Particles from Batch HTM-1. (a) Unirradiated. Etched. 75X. (b) Irradiated. Etched. 150X. Note basal-plane fractures and reaction area at core-to-coating interface. 


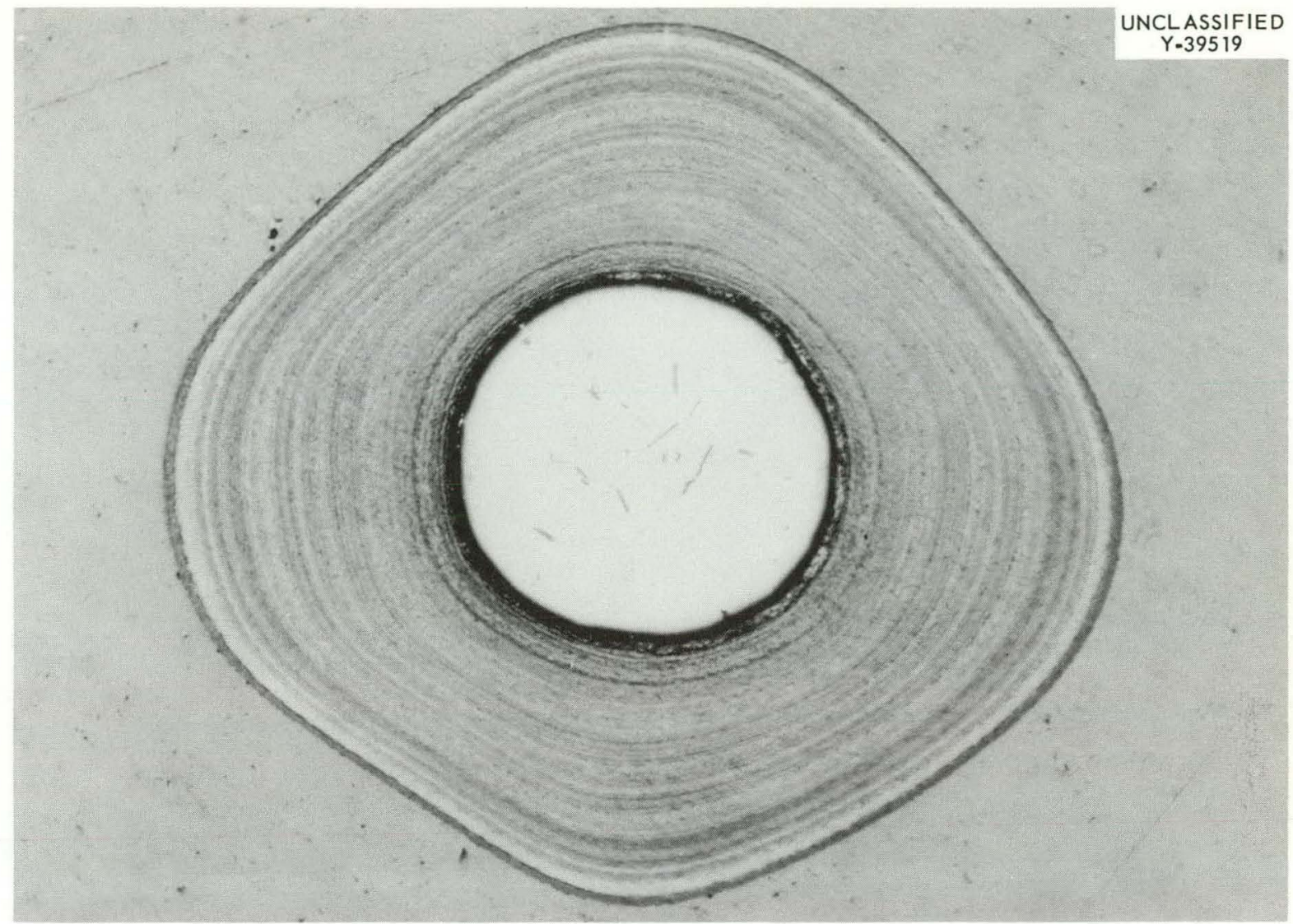

Fig. 20.15. Typical Cross Section of Unirradiated Particle from Lot 3M-SP-2. Used in LITR capsule LCP-2. As polished. $250 X$.

flux remaining constant, the fractional release rates for all the isotopes, except $\mathrm{Xe}^{133}$, decreased by a factor of approximately 3 ; the release rate for $\mathrm{Xe}{ }^{133}$ decreased by a factor of 5. A total of $12.1 \%$ uranium burnup was attained.

Experiment Cl-7. - The 3M-SP-2 laminar-type coated particles were irradiated in capsule C1-7 at $1550^{\circ} \mathrm{F}$ for 33 days at a thermal-neutron flux of $3.1 \times 10^{13} n v$. The specimen released fission gas as soon as it was inserted into the reactor. The gas release rate slowly increased until the isotopes generated within the fuel reached a production-decay equilibrium after about 14 days of irradiation. The release rates were reasonably constant for the remainder of the 33-day irradiation period. The capsule was then rapidly cooled by withdrawing it from the reactor flux, and the fissiongas release was measured during the cooling period. The steady-state fractional gas release after 33 days of irradiation is given in Fig. 20.20.

The steady-state fission-gas release rates and the fission-gas release rates during the cooling period are plotted in Fig. 20.21. The release rates are given in atoms per second for the isotope emerging from the graphite container. The first two points show the steady-state release. As the specimen cooled, the fission-gas release decreased abruptly until the krypton release was no longer detectable. This was caused by a decrease in the gas pressure in the graphite holder when it cooled, which allowed gas to flow into the holder until the pressure was equalized with the external gas pressure. Xenon was always detectable because a large portion of the total xenon is born from iodine that has escaped from the capsule and deposited on a cooler portion of the sweep system. The 

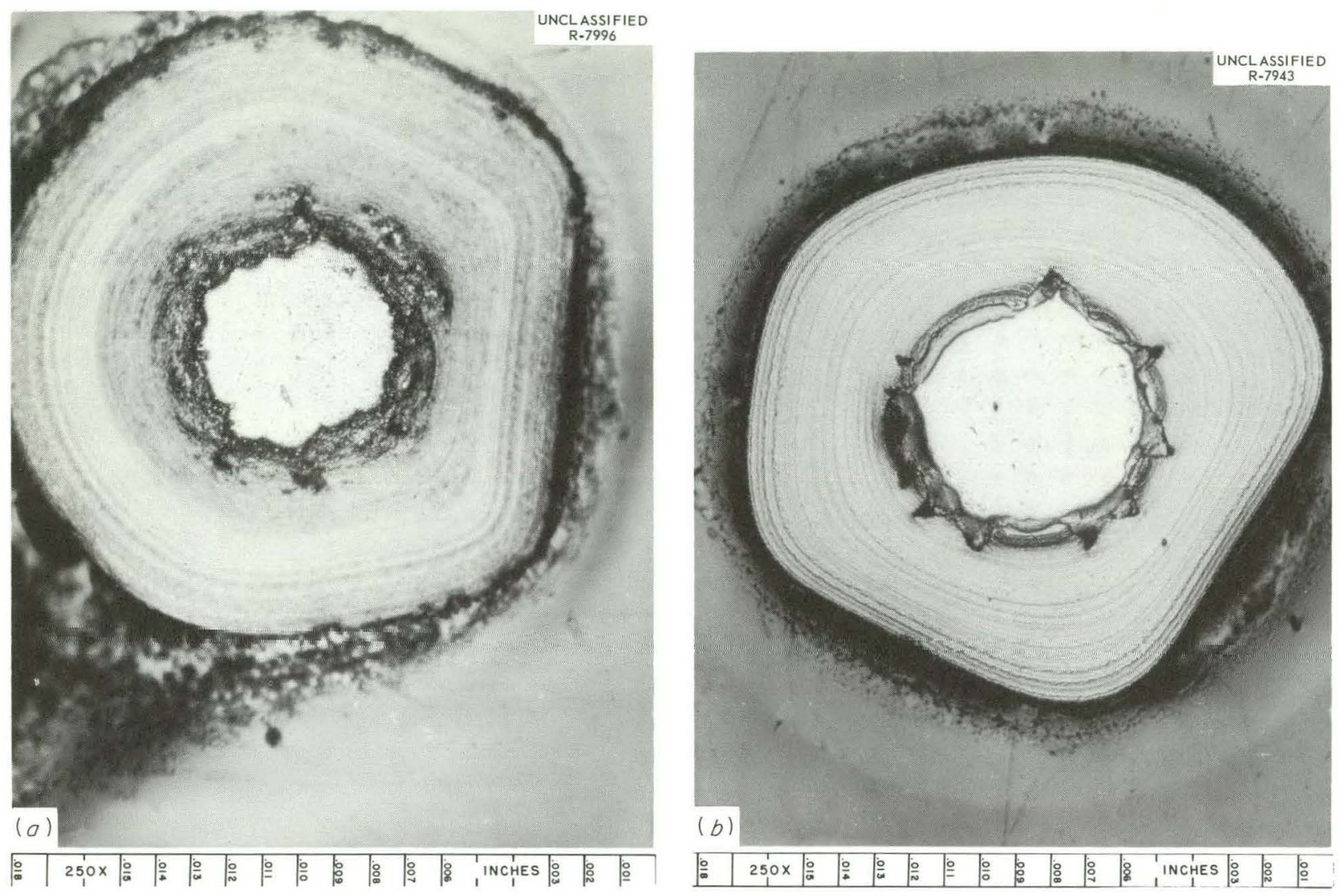

Fig. 20.16. Typical Irradiated Particles from Capsule LCP-2. As polished. 250X. (a) Particle from LCP-2a showing oxidation of the coating. (b) Particle from LCP-2b, no oxidation. Reduced $31 \%$.

krypton isotopes have no long-lived precursors; so their total emission is from the fuel particle. The minimum in the xenon curve probably represents the steady-state release rate of iodine from the particles. These rates indicate release of $25 \%$ of the total $\mathrm{Xe}^{133}$ and $82 \%$ of the total $\mathrm{Xe}^{135}$ from the coated particle in the form of iodine.

After the cooling dip, shown in Fig. 20.21, the fission gas surged out of the graphite holder. The amount of $\mathrm{Xe}^{133}$ released in this surge demonstrated that stored gas was released from within the particle. Stored gas contains relatively large amounts of long-lived gases. The graphite holder would tend to adsorb greater amounts of xenon as it cooled, and thus the graphite could not be the source of the burst. This burst of stored gas is also noticeable in the krypton release, where the outward surge of $\mathrm{Kr}^{85 \mathrm{~m}}$ was considerably greater than that of $\mathrm{Kr}^{87}$ although the steady-state release of $78-\mathrm{min}^{\mathrm{K} r}{ }^{87}$ was approximately equal to that of 4. $4-\mathrm{hr} \mathrm{Kr}^{85 m}$.
After the cooling burst, the fission-gas release for all isotopes slowly declined. Xenon release from deposited iodine is natural under these conditions, but krypton release usually terminates at once. This effect is probably caused by fission gas diffusing through the spongy layer between the particle and its coating until the gas reaches a break in the coating. The spongy layer is the dark inner ring shown on 3M-SP-2 in Fig. 20.19.

When the specimen was reinserted into the reactor after the cooling burst, the fission-gas release rates were erratic and, in general, higher than for the first month of irradiation. The specimen temperature was then lowered while at constant power so that the temperature dependence of the gas release could be studied. No temperature dependence was observed over a temperature range of $670^{\circ} \mathrm{F}$.

The specimen was then irradiated at an increased flux of $5.2 \times 10^{13}$ thermal neutrons $\mathrm{cm}^{-2} \mathrm{sec}^{-1}$. Fission-gas release continued to be erratic, 


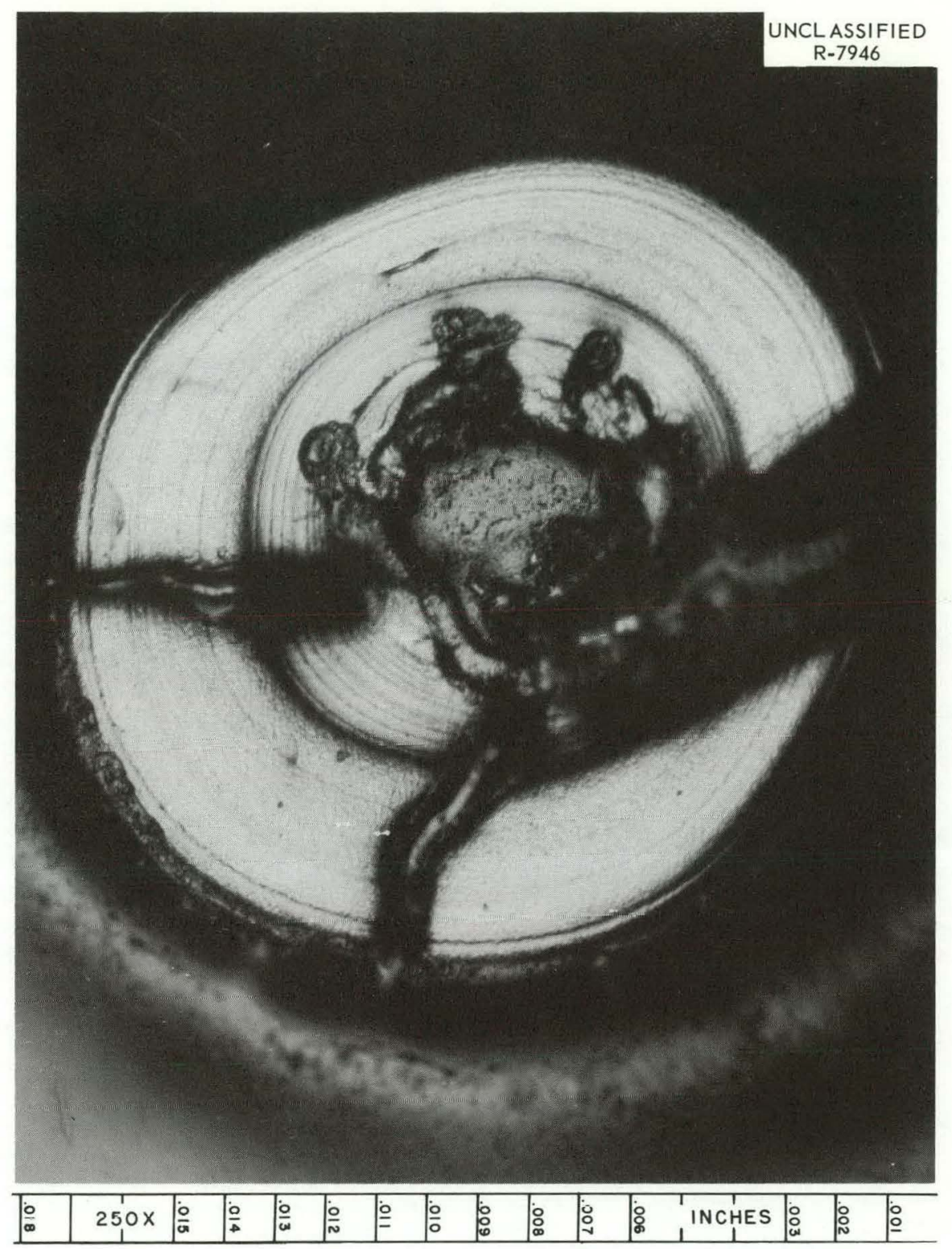

Fig. 20.17. Fractured Particle from Capsule LCP-2b. Etched. 250X.

especially the $\mathrm{Xe}^{133}$ release, which fluctuated by large amounts for no apparent causc. For the next 30 days the capsule was irradiated at $1815^{\circ} \mathrm{F}$ with little effect on the steady-state fission-gas release. There were no significant changes in fractional fission-gas release rates as a function of power level, burnup, or temperature.

When capsule C1-7 was being prepared for irradiation at the higher flux of $5.2 \times 10^{13}$ thermal neutrons $\mathrm{cm}^{-2} \mathrm{sec}^{-1}$, the temperature of the specimen was increased to $1700^{\circ} \mathrm{F}$ for about 30 min. This resulted in a burst of activity that was two orders of magnitude higher than any previous release. The gamma-emission spectrum of the fission gas appeared normal in every respect except for the magnitude. Since a burst of stored gas would be rich in long-lived isotopes, it appears that the gas released in this burst was the result of accelerated diffusion. Later the capsule tempcrature was raised to $1815^{\circ} \mathrm{F}$, but no abnormal release rates were noted. The capsule had been irradiated for about a month at $1550^{\circ} \mathrm{F}$, and the 


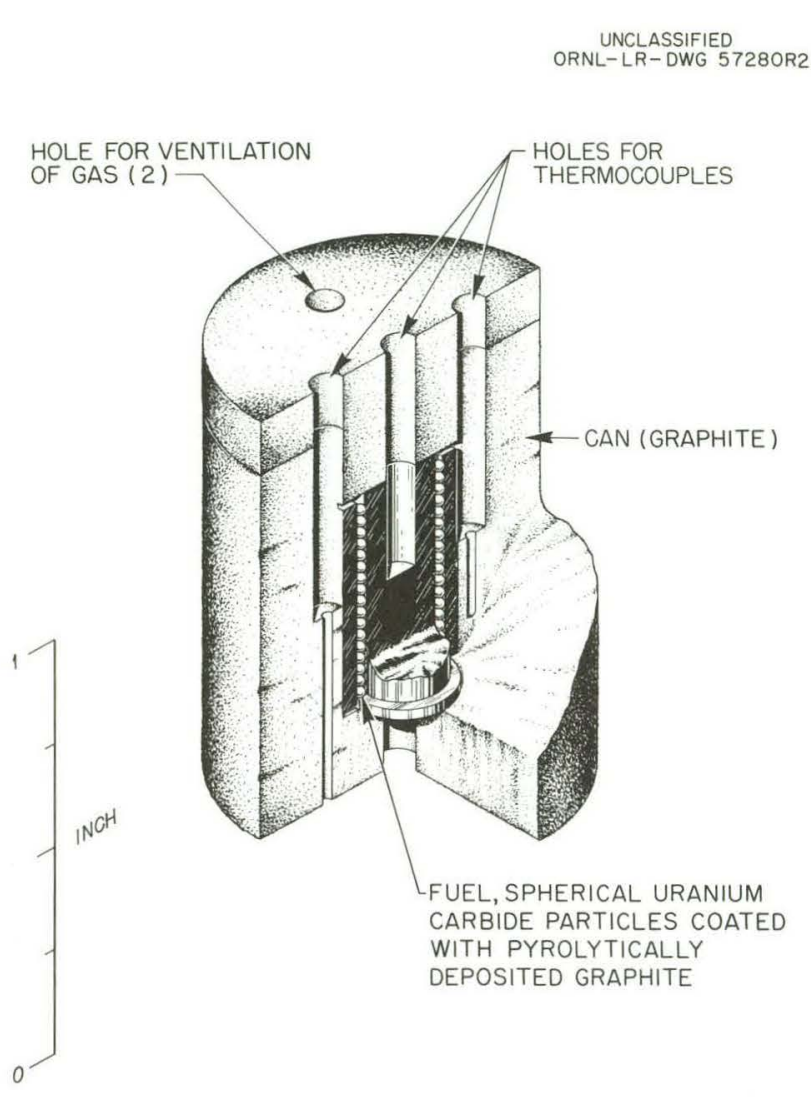

Fig. 20.18. Coated-Particle Holder for ORR C-1 and B-9 Facilities.

burst was produced when the temperature was first increased to $1700^{\circ} \mathrm{F}$. This burst did not signify fuel damage since no significant change of steady-state fission-gas release resulted. Subsequent attempts to duplicate the heating burst were not successful.

Experiment B9-8. - The B9-8 capsule, containing coated particles of the duplex type from batch NCC-AD, was irradiated for 88 days to a $30 \%$ uranium burnup. A sectioned duplex-type particle is shown as NCC-AD in Fig. 20.19. The average fractional fission-gas release rates, at temperatures of 600 to $1700^{\circ} \mathrm{F}$, are given in Fig. 20.22. The fission-gas release rate during the first six days was below the detectable range. On the seventh day the release rate increased, reached a steady-state condition after a few days, and remained steady for the $1500^{\circ} \mathrm{F}$ irradiation period. The average fractional release rates at $1500^{\circ} \mathrm{F}$ are compared in Fig. 20.20. with those from other tests.
After a $20 \%$ bumup had been reached, activity bursts were observed. These bursts continued at irregular intervals throughout the remainder of the $1700^{\circ} \mathrm{F}$ irradiation period, but did not appear after the temperature was reduced to 800 or to $600^{\circ} \mathrm{F}$. The fission-gas release rates showed a marked dependence on temperature during the first few weeks of irradiation, but this dependence decreased as burnup increased. This means that fuel was within the recoil range of the outer particle surface - probably due to both broken coatings and fuel migration into the coating.

Experiment C1-8. - The C1-8 capsule, containing bare uranium carbide particles from batch 3M-101-U, was irradiated for 41 days at $1500^{\circ} \mathrm{F}$ and at $4 \times 10^{13} n v$ to a $4.3 \%$ uranium burnup. While at constant flux, the fission-gas release rates remained unchanged after the first seven days of irradiation. Average fractional release rates are given in Fig. 20.20. No temperature dependence for fission-gas release was found for temperatures up to $1500^{\circ} \mathrm{F}$.

The bare uranium carbide particles actually had a layer of porous graphite of about $15-\mu$ thickness (see Fig. 20.19). The bare particles did not release the sudden bursts during steady-state irradiation that were noted from B9-7, B9-8, and C1-7 capsules, strengthening the argument that the bursts indicate cracking of the coatings. The bare particles do release heating bursts during temperature increases. Contrasted to the laminarcoated particles in the C1-7 specimen, the bare particles did not release bursts while cooling. This implies that the cooling burst was caused by a cracking or contraction of the coating.

Activity Bursts Not Due to Temperature Change. - Activity bursts were noted during the irradiation of all three types of coated particles, but not from the bare uranium carbide particles. These bursts occurred while power level and temperature were at a steady state and at irregular intervals. As shown in Fig. 20.23, the steady-state activity level increased for a few hours and then gradually returned to the previous level. A gas sample taken during a burst was abnormally rich in 5-day Xe ${ }^{133}$, indicating the release of trapped gas.

It appears that activity bursts are caused by the coating rupture of a single particle. The bursts contain about $1.2 \times 10^{-5}$ curie of gas, whereas after 30 days of irradiation time a coated particle contains about $1.1 \times 10^{-4}$ curie of gas. Irradiation of bare particles demonstrated that most of 
UNCLASSIFIED $\mathrm{R}-9092$

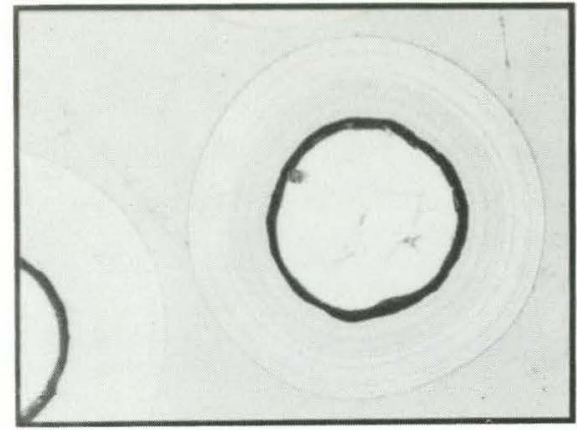

$3 M-S P-2$

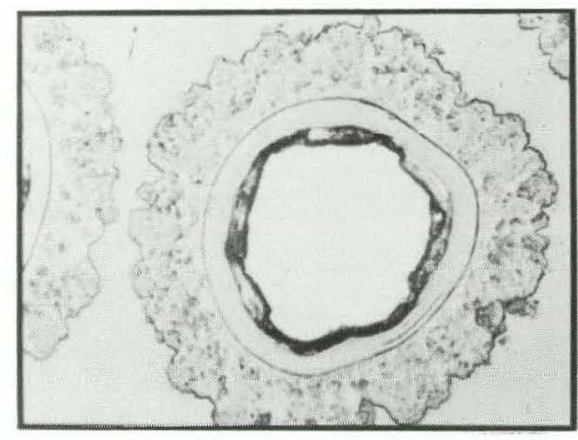

NCC - AD

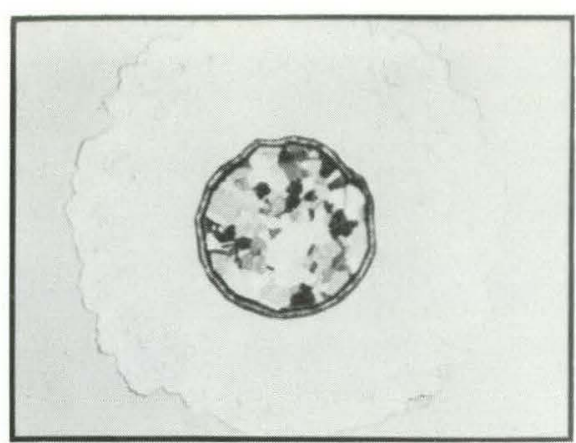

HTM-1

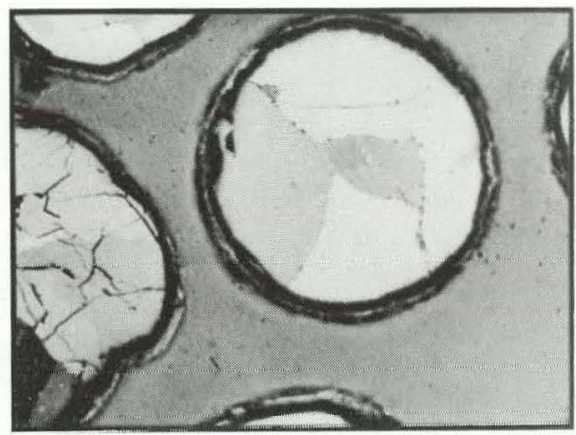

UNCOATED URANIUM CARBIDE

Fig. 20.19. Pyrolytic-Carbon-Coated Uranium Carbide Particles. 100X.

Table 20.3. Three Basically Different Types of Pyrolytic-Carbon Coatings

\begin{tabular}{|c|c|c|c|c|c|}
\hline \multirow{2}{*}{$\begin{array}{l}\text { Capsule } \\
\text { No. }\end{array}$} & \multirow{2}{*}{$\begin{array}{l}\text { Coating } \\
\text { Type }\end{array}$} & \multirow{2}{*}{$\begin{array}{c}\text { Manufacturer and } \\
\text { Batch No. }\end{array}$} & \multicolumn{2}{|c|}{ Particle Size $(\mu)$} & \multirow{2}{*}{$\begin{array}{c}\mathrm{U}^{235} \\
(\mathrm{~g})\end{array}$} \\
\hline & & & $\begin{array}{c}\text { Average } \\
\text { Overall Diameter }\end{array}$ & $\begin{array}{c}\text { Coating } \\
\text { Thickness }\end{array}$ & \\
\hline C1-7 & Laminar & $\begin{array}{l}\text { 3M Co. } \\
\text { 3M-SP-2 }\end{array}$ & 414 & 82 & 0.087 \\
\hline B9-7 & Columnar & $\begin{array}{c}\text { HTM, Inc. } \\
\text { HTM-1 }\end{array}$ & 364 & 82 & 0.063 \\
\hline B9-8 & Duplex & $\begin{array}{l}\text { National Carbon } \\
\text { NCC-AD }\end{array}$ & 460 & 80 & 0.086 \\
\hline C1-8 & Bare & $\begin{array}{l}\text { 3M Co. } \\
3 \mathrm{M}-101-\mathrm{U}\end{array}$ & 228 & No coating & 0.0045 \\
\hline
\end{tabular}




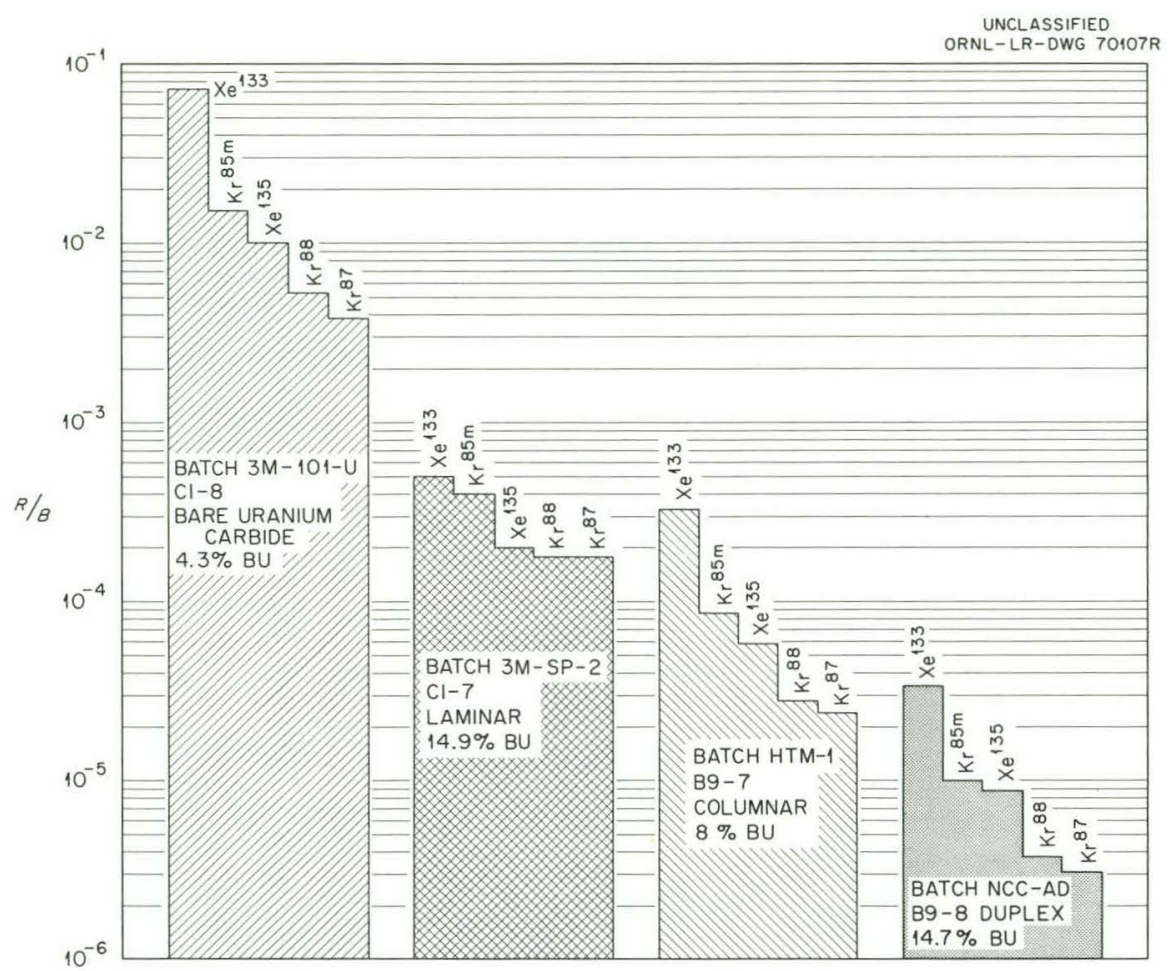

Fig. 20.20. Average Fractional Fission-Gas Release, $R / B$ at $1500^{\circ} \mathrm{F}$.

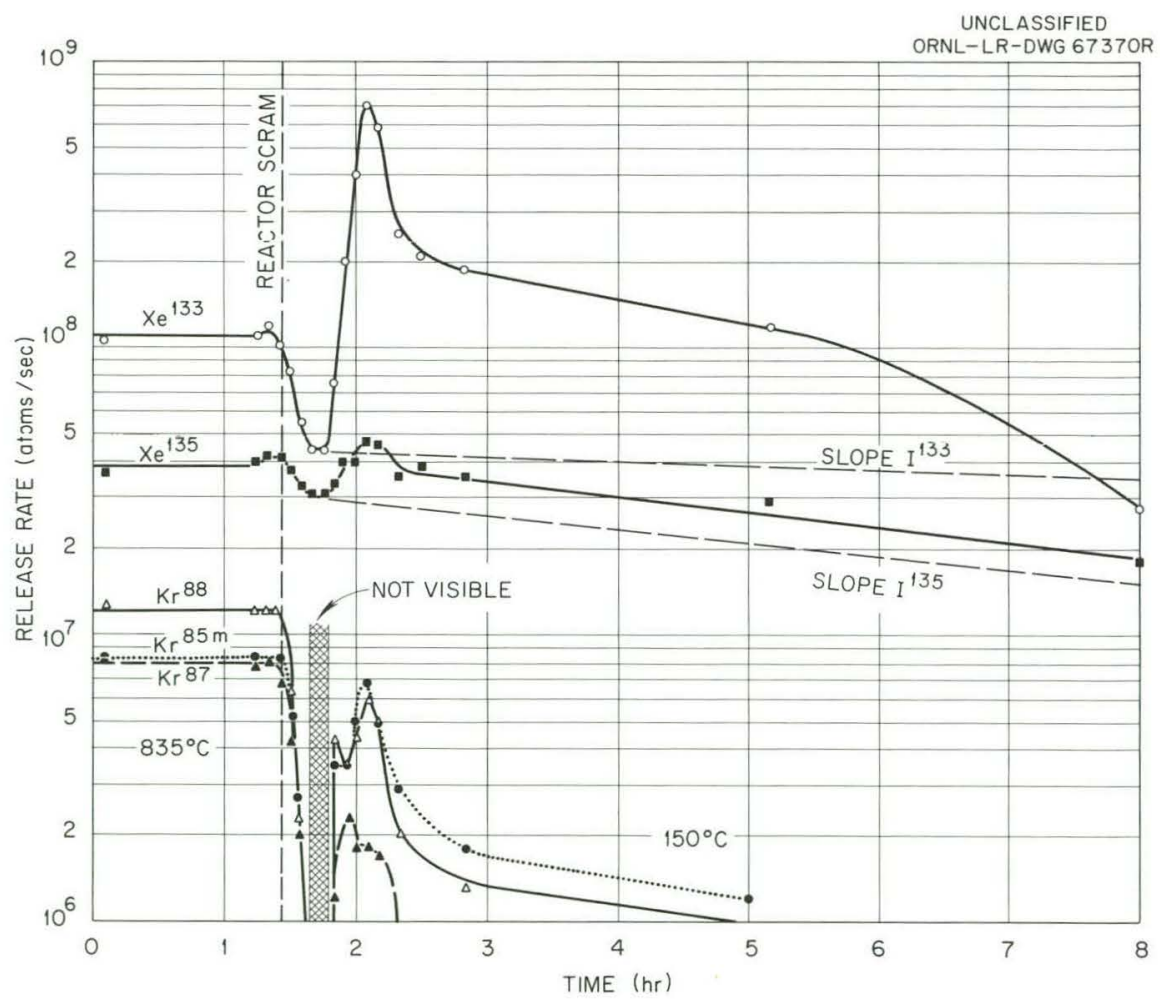

Fig. 20.21. Fission-Gas Release Rates of Batch from 3M-SP-2 Coated Particle During Steady-State Irradiation, Reactor Scram, and Cooling of Capsule Cl-7. 


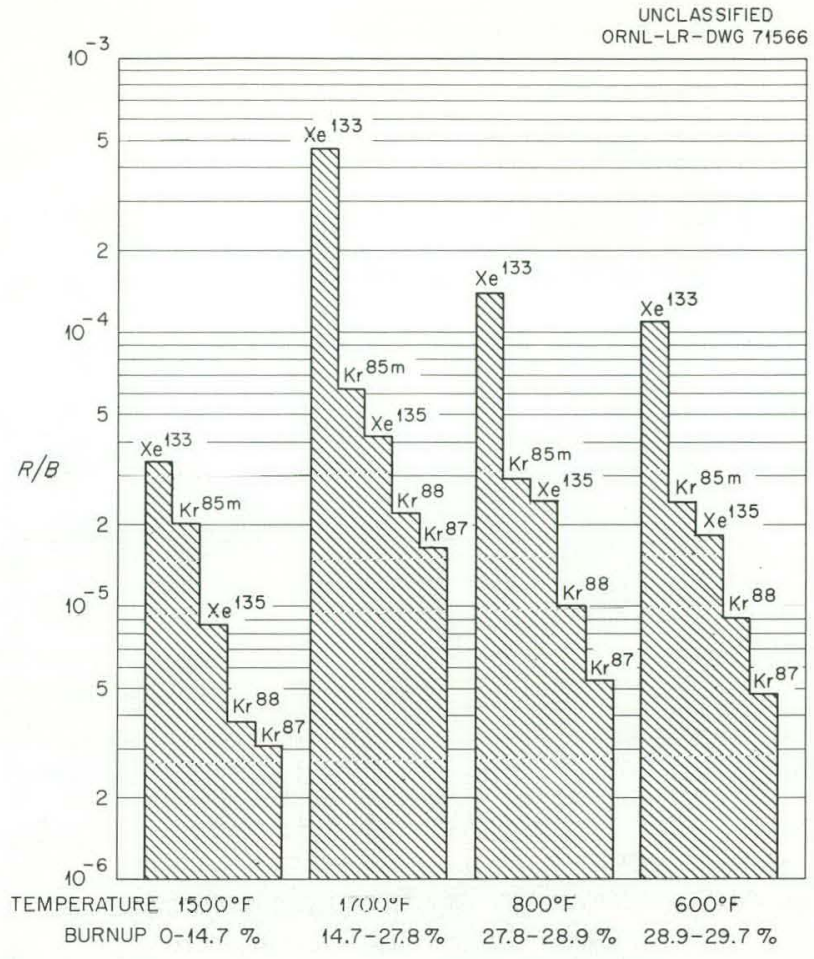

Fig. 20.22. Fractional Fission-Gas Release Rates from Pyrolytic-Carbon-Coated Uranium Carbide. B9-8 MCC-AD Duplex.

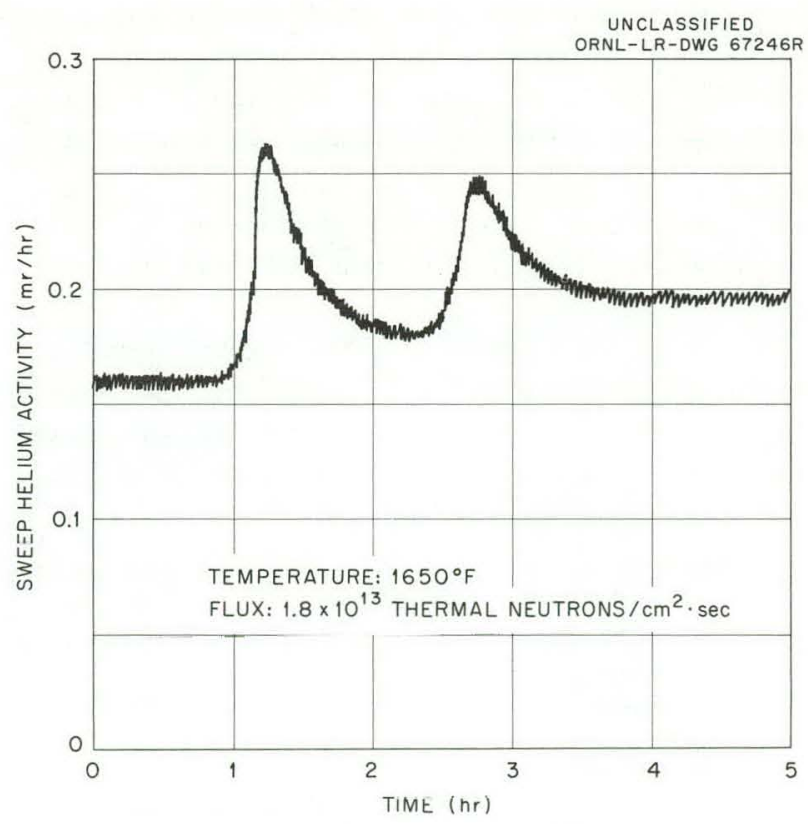

Fig. 20.23. Typical Activity Burst During Irradiation of Pyrolytic-Carbon-Coated UC 2 Particles. the generated gas is held in the uranium carbide at temperatures up to $1500^{\circ} \mathrm{F}$. If a particle should suddenly release one-tenth of its gas, this would cause the observed burst.

Coating Efficiency. - In evaluating coatings, the efficiency of a coating could be expressed as a relationship between the fractional release rate from coated fuel particles compared with the fractional release rate from uncoated fuel particles. This relationship is given in Table 20.4 as an efficiency factor. It should be noted that this factor for each isotope from a given coating is in close agreement except for the $3 M$ Company laminar coating. This is because the so-called bare particles have a thin, spongy graphite coating that may act as a recoil barrier, while on a broken 3M coating this thin barrier was probably removed along with the laminar shell.

Conclusions. - Results indicate that the failure of the laminar coatings ( $3 \mathrm{M}$ Company) and the columnar coatings (High Temperature Materials, Inc.) occurred as soon as fission heating began. These failures were probably due to differential thermal expansion between the particle and coating, and only a very small percentage of the particles were broken in this way. The NCC duplex coating did not begin to break until $20 \%$ burnup had been attained. The small fission-gas release rates up to this bumup were by diffusion through intact coatings.

The particles with the $3 \mathrm{M}$ laminar coatings appeared to release fission gas with less holdup than the particles with the HTM columnar or the NCC duplex coatings. This was determined by the relatively larger proportion of long-lived fission gas in the steady-state emission from the HTM and NCC coated particles. Also, the gas release from the $3 \mathrm{M}$ coated particles was not temperature dependent, whereas the release from the HTM and NCC coated particles was highly temperature dependent. From these observations it is concluded that the fission-gas release from the $3 \mathrm{M}$ coated particles was primarily by recoil from broken and peeled particles and that the initial release from the HTM and NCC particles was primarily by diffusion through the coating. 
Table 20.4. Efficiency Factors for Pyrolytic-Carbon Coatings at $1500^{\circ} \mathrm{F}$

\begin{tabular}{|c|c|c|c|c|c|}
\hline \multirow{2}{*}{$\begin{array}{l}\text { Capsule No. } \\
\text { and Coating }\end{array}$} & \multicolumn{5}{|c|}{ Ratio of $R / B^{a}$ for Bare Particles to $R / B$ for Coated Particles } \\
\hline & $\mathrm{Xe}^{133}$ & $\mathrm{Xe}^{135}$ & $\mathrm{Kr}^{85 m}$ & $\mathrm{Kr}^{88}$ & $\mathrm{Kr}^{87}$ \\
\hline $\begin{array}{l}\text { 3M-SP-2 } \\
\text { Laminar }\end{array}$ & 140 & 50 & 37 & 28 & 20 \\
\hline $\begin{array}{l}\text { HTM-1 } \\
\text { Columnar }\end{array}$ & 200 & 160 & 200 & 250 & 250 \\
\hline $\begin{array}{r}\text { NCC-AD } \\
\text { Duplex }\end{array}$ & 2000 & 1100 & 1500 & 1250 & 1300 \\
\hline
\end{tabular}

$a_{\text {Release rate/birth rate. }}$

\subsection{IRRADIATION EFFECTS ON METAL- CLAD FUELS}
M. T. Morgan
C. D. Baumann
D. F. Toner
J. G. Morgan
E. L. Long, Jr. ${ }^{5}$
M. F. Osborne
H. E. Robertson
J.W. Gooch

A. L. Johnson

\section{ORR Prototype Capsules (Group IV)}

Six stainless-steel-clad UO ${ }_{2}$, EGCR prototype, fuel elements were irradiated in the ORR poolside facility $^{6}$ at temperatures of 1300 to $1600^{\circ} \mathrm{F}$ to burnups of 1000 to $3000 \mathrm{Mwd}$ per metric ton of uranium. Capsules $\mathrm{O} 1 \mathrm{C}$ and $\mathrm{O} 8 \mathrm{~B}$ were approximately 7 in. long $\times 3 / 4$ in. OD and were fitted with six clad thermocouples and one central thermocouple. These capsules were intended to serve as proof tests for EGCR instrumented fuel assemblies, to evaluate central thermocouple weld materials, and to study the effect of initial grain size of the UO ${ }_{2}$ fuel pellets on fission-gas release. Of the four remaining capsules in group IV, O3, $\mathrm{O} 4$, and $\mathrm{O} 7$ were fabricated by swaging at $800^{\circ} \mathrm{C}$, and $\mathrm{O} 2$ was fabricated by hand-tamping $\mathrm{UO}_{2}$ powder into a $1 / 2$-in. type 304 stainless steel tube. The purpose of these irradiations was to evaluate the irradiation behavior of bulk $\mathrm{UO}_{2}$ under EGCR conditions in the form of both hot-swaged and tamp-packed fuel capsules.

\footnotetext{
${ }^{5}$ Metals and Ceramics Division.

${ }^{6}$ Irradiation experiments performed by the Reactor Division.
}

The postirradiation examination of the first two capsules has previously been reported in detail. ${ }^{7}$ Examination revealed that $\mathrm{O} 1 \mathrm{C}$ and $\mathrm{O} 8 \mathrm{~B}$ failed during irradiation. Cracks were found in the GE-81 braze joint in both capsules. Separation of the braze from the thermocouple sheath and the cracking of the braze material itself were evident. Severe void formation was found in the cladding adjacent to a missing piece of $\mathrm{UO}_{2}$ pellet (see Fig. 20.24). Circumferential ridges at pellet-topellet interfaces were observed in the cladding. Voids seen in these ridges are believed to be the result of shear rather than hoop stresses. This was ascertained by determining metallographically the size and shape of the voids in both longitudinal and transverse directions of the clad, which showed the intergranular voids to be essentially of the same shape and size in both planes. If hoop stresses had been prevalent during clad deformation, the resultant voids would not have been alike in character in the two sections examined. Sigma phase was found in some areas of the cladding of both fuel elements. Since the sigma phase occurred only in a small, spherical precipitate, it was concluded that sigma phase does not cause the deleterious effect on the cladding during irradiation.

The swaged and tamp-packed fuel elements ${ }^{8}$ were irradiated to clad temperatures of $1300^{\circ} \mathrm{F}$

${ }^{7}$ D. F. Toner et al., Gas-Cooled Reactor Program Quart. Progr. Rept. Mar. 31, 1962, ORNL-3302, PP 67-76.

${ }^{8}$ D. F. Toner et al., Gas-Cooled Reactor Program Quart. Progr. Rept. Mar. 31, 1962, ORNL-3302, pP 76-83, and J. G. Morgan et al., "Examination of Irradiated Capsules," GCR Semiann. Progr. Rept. Sept. 30,1962 (now in process). 


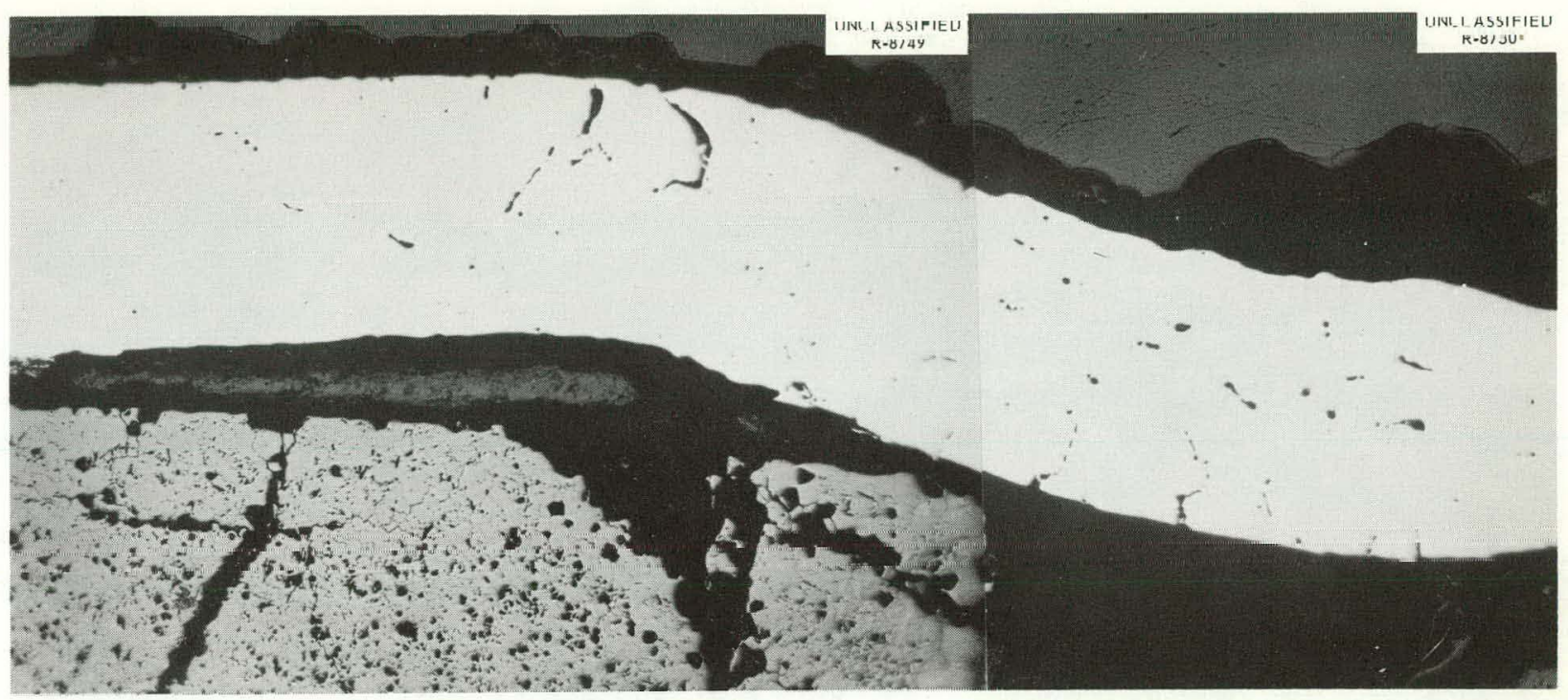

Fig. 20.24. Longitudinal Section Through Depression in the Cladding of ORR Capsule OIC-4. Depression occurred at a pellet-to-pellet interface. Severe deformation resulted in intergranular voids in the type 304 stainless steel cladding. As polished. $75 \mathrm{X}$. Reduced $33 \%$.

and linear heat ratings of 8,000 and 15,000 Btu $\mathrm{hr}^{-1} \mathrm{ft}^{-1}$. 9 Postirradiation examination revealed that none of the fuel elements failed during reactor operation. The tamp-packed capsule 02-4 contained $\mathrm{UO}_{2}$ packed to $59 \%$ of theoretical density. The cladding showed no evidence of potential failure, even though a fine precipitate was observed throughout the cladding. Metallographic examination of the $\mathrm{UO}_{2}$ showed that no sintering had occurred during operation. The stainless steel tube collapsed under the irradiation conditions, but this was in accordance with previous predictions. ${ }^{10}$

The examination of $\mathrm{O} 3-4$, swaged at $800^{\circ} \mathrm{C}$ and irradiated to a linear heat rating of $15,000 \mathrm{Btu}$ $\mathrm{hr}^{-1} \mathrm{ft}^{-1}$, revealed that $\mathrm{UO}_{2}$ had been forced into the cladding to a depth of approximately $1 / 2 \mathrm{mil}$. Metallographic examination revealed evidence of sintering in the center of the fuel column, whereas there was no evidence of sintering at the outer surface.

${ }^{9}$ W. C. Thurber, "Compact Powder Fuel Irradiation Studies at Oak Ridge National Laboratory," presented at the Symposium on the Powder Packed UO, Fuel Element, Windsor, Connecticut, Nov. 30-Dec. 1, 1961.

${ }^{10}$ C. R. Kennedy and J. T. Venard, Collapse of Tubes by External Pressure, ORNL TM-166 (Apr. 17, 1962).
The examination of $06-4$, swaged at $800^{\circ} \mathrm{C}$ and irradiated to a linear heat rating of 15,000 Btu $\mathrm{hr}^{-1} \mathrm{ft}^{-1}$, showed that a central void was present in the fuel column (see Fig. 20.25). Columnar grains began at the central void and extended past the midradius. A two-phase area, which has not yet been identified, was found at the outer surface of the $\mathrm{UO}_{2}$ adjacent to the clad. The central void, present in this capsule and not in $\mathrm{O} 3-4$, is thought to have been caused by the poorer thermal conductivity and resultant higher fuel temperature due to a lower density $\mathrm{UO}_{2}$ and a higher $\mathrm{O}$ to $\mathrm{U}$ ratio.

The postirradiation examination of $07-4$, swaged at $800^{\circ} \mathrm{C}$ and irradiated to a linear heat rating of $8000 \mathrm{Btu} \mathrm{hr}^{-1} \mathrm{ft}^{-1}$, showed that a considerable amount of sigma-phase material was present at the inner surfaces of the cladding to approximately half-way across the wall. A shell surrounded the particles of $\mathrm{UO}_{2}$ adjacent to the outer surface. This shell is thought to have been caused by excess oxygen present during the swaging of the fuel element. No evidence of sintering was observed.

Two important conclusions which may be drawn from this examination are that the sigma phase which has been observed in capsule claddings is not significant with respect to their irradiation 


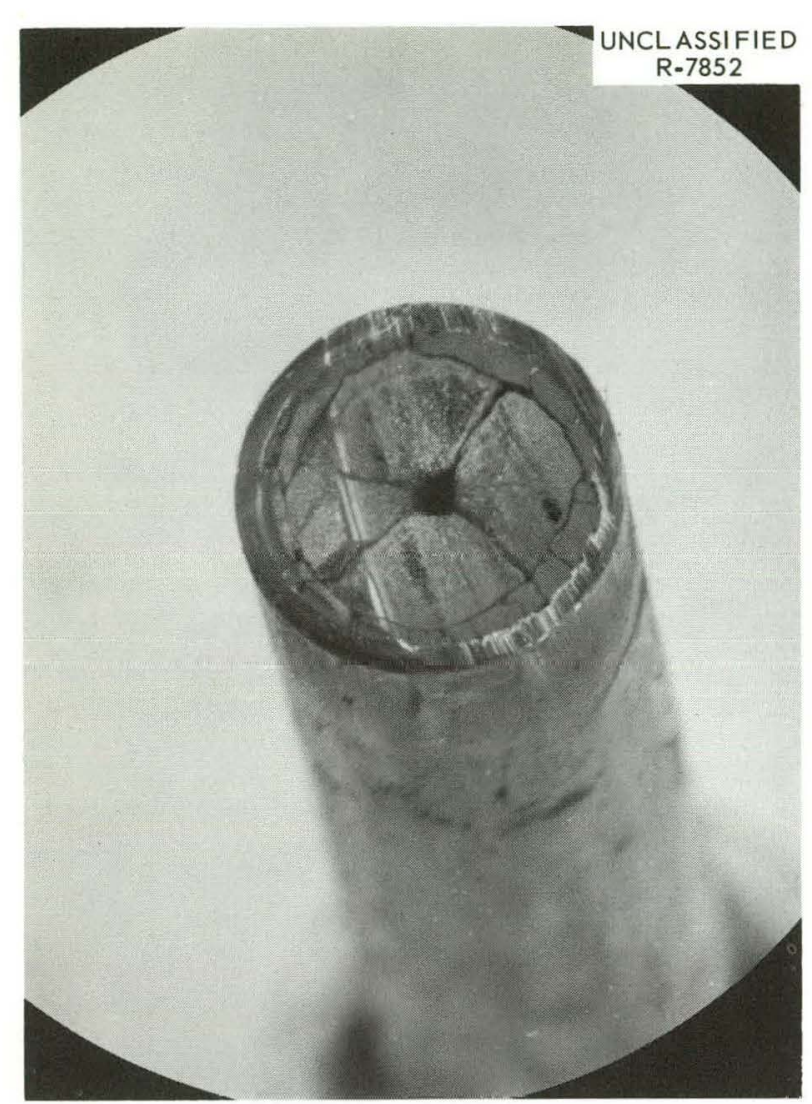

Fig. 20.25. Cross Section of Capsule 06-4, Showing a Central Void in the $\mathrm{UO}_{2}$.

integrity, and that the method of hot swaging and tamp-packing of $\mathrm{UO}_{2}$ has proven to be acceptable for fuel elements irradiated to a burnup of 2500 Mwd per metric ton of uranium at a clad temperature of $1300^{\circ} \mathrm{F}$.

Loop 1 Capsules. - Test elements 19 in. long and 0.75 in. in diameter containing hollow $\mathrm{UO}_{2}$ fuel pellets clad in type 304 stainless steel were fabricated and were irradiated in the GCR-ORR loop No. 1 for periods of from 7 to 14 weeks at clad temperatures between 1300 and $1500^{\circ} \mathrm{F} 。{ }^{11}$ This facility is operated by the Reactor Division. Elements 5, 6, 7A, and 7B were irradiated for 7 weeks and element $7 \mathrm{C}$ was irradiated for 14 weeks

${ }^{11} \mathrm{~J}$. K. Franzreb, Gas-Cooled Reactor Program Quart. Progr. Rept. Mar. 31, 1962, ORNL-3302, p 66; D. F. Toner et al., Gas-Cooled Reactor Program Quart. Progr. Rept. Mar. 31, 1962, ORNL-3302, p 67; J. G. Morgan et al., "Examination of Irradiated Capsules," GCR Semiann. Progr. Rept. Sept. 30, 1962 (now in process). at a linear heat rating of between 24,000 and

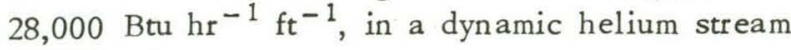
at 300 psi.

Postirradiation examination, which has been previously reported, ${ }^{12}$ has shown that under the EGCR designed operating conditions the $3 / 4-$ in. hollow-pellet stainless-steel-clad fuel elements are satisfactory. In addition, it has been demonstrated that the cored pellets do not disintegrate even though cracked during irradiation, that the fuel element maintains dimensional stability, and that no reactions occur during irradiation between the $\mathrm{UO}_{2}$ and the stainless cladding. However, a peculiar corrosion effect on the type 304 stainless steel shroud, clad, and lead tube has been noted.

The lead tube, which is immediately above the fuel element, and the shroud, which surrounds the fuel element, on experiment No. 5 were rusty and very brittle. Subsequent metallographic examination of these accessory parts of the experiment showed an intergranular fracture which penetrated the wall of both parts. A typical microstructure is shown in Fig. 20.26. At higher magnification it was seen that a corrosion product, probably an oxide (see Fig, 20.27), was present in the grain boundaries. Analysis of the rust showed that $\mathrm{Fe}, \mathrm{Cr}$, and $\mathrm{Ni}$ were the principal constituents and that smaller quantities of $\mathrm{Bi}, \mathrm{Zn}, \mathrm{Au}, \mathrm{Ag}, \mathrm{Mn}$, $\mathrm{Mo}, \mathrm{Cu}$, and $\mathrm{Si}$ were present. Structurally the rust was the gamma $\mathrm{Fe}_{2} \mathrm{O}_{3} \cdot \mathrm{H}_{2} \mathrm{O}$. Similarly, rust was observed on the insert of element $7 \mathrm{~A}$ and on the shroud of element 7C.

Analysis of the temperatures and of the helium purities under which these experiments were operated indicated that the stainless steel which was attacked was between 1000 and $1200^{\circ} \mathrm{F}$ and that up to $800 \mathrm{ppm}$ of hydrogen and $500 \mathrm{ppm}$ of $\mathrm{H}_{2} \mathrm{O}$ were present in the helium coolant stream. After the fuel elements had been removed from the reactor, they were encapsulated in a loading tube under 20 psig of nitrogen and stored in the ORR pool. On at least two occasions water was observed in the loading tube when the fuel element was brought into the hot cell. It is suggested that during reactor operation a slight sensitization of the stainless steel occurred in the temperature range from 1000 to $1200^{\circ} \mathrm{F}$. Subsequently, when

${ }^{12} \mathrm{D}$. F. Toner et al., Gas-Cooled Reactor Program Quart. Progr. Rept. Mar. 31, 1962, ORNL-3302, pP 83-96. 


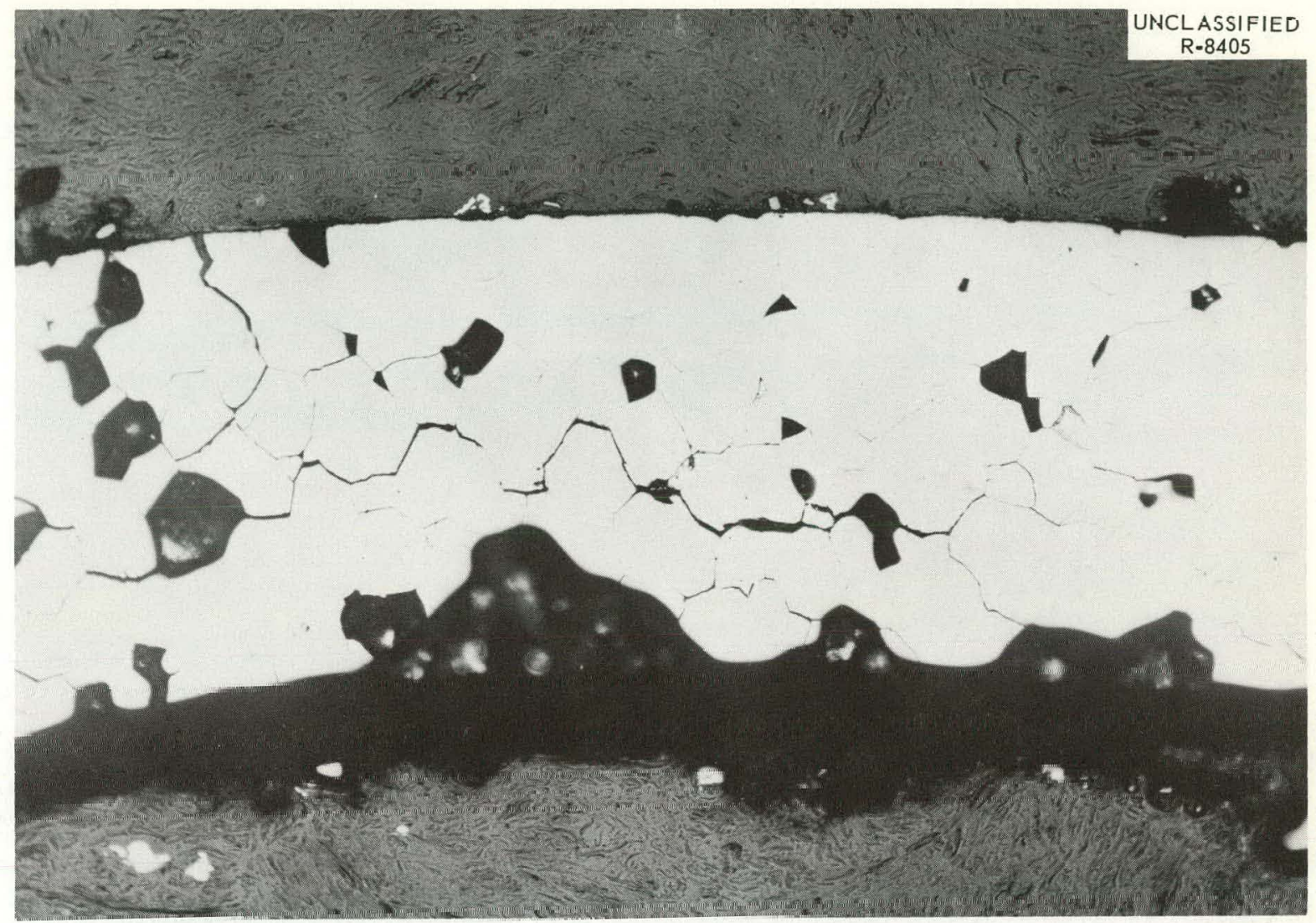

Fig. 20.26. Transverse Sample Taken from Near One of the Breaks in the Type 304 Stainless Steel Lead Tube from Element 5. Friability of the tubing was demonstrated when whole grains loosened during mounting and fell out during polishing. As polished. 100X.

the fuel element was put into the loading tube, water leaked in through the valve and came in contact with the fuel element. Gamma activity from the fuel element could cause dissociation of nitrogen and water to form nitric acid, and the nitric acid could then attack the sensitized areas of the shroud, lead tube, and fuel element that were in the temperature range indicated above. Stobbs and Swallow ${ }^{13}$ observed that a pressurizedwater loop with an air-cooled annulus surrounding it was attacked by a concentrated nitric acid in the Materials Testing Reactor.

Further investigation of this corrosion problem is in progress.

\footnotetext{
${ }^{13}$ Stobbs and Swallow, Met. Rev. 7, 25 (1962).
}

\section{ETR Prototype Capsules}

Three ETR-irradiated capsules ${ }^{14}$ were examined. Fabrication and irradiation data are summarized in Table 20.5. The results of one experiment (E-12) were similar to those reported previously for ORR-irradiated capsules. ${ }^{15}$ There were no major changes in the appearance or structure of this capsule. However, the other two capsules were found to have suffered gross fractures in the stainless steel cladding, shown in Figs. 20.28 and 20.29. Dimensional measurements showed

\footnotetext{
${ }^{14}$ The capsules were fabricated by the Metals and Ceramics Division. The experimental assemblies were built and the operation was supervised by the Reactor Division.

${ }^{15}$ J. G. Morgan et al., Solid State Div. Ann. Progr. Rept. Aug. 31, 1961, ORNL-3213, Pp 105-106.
} 


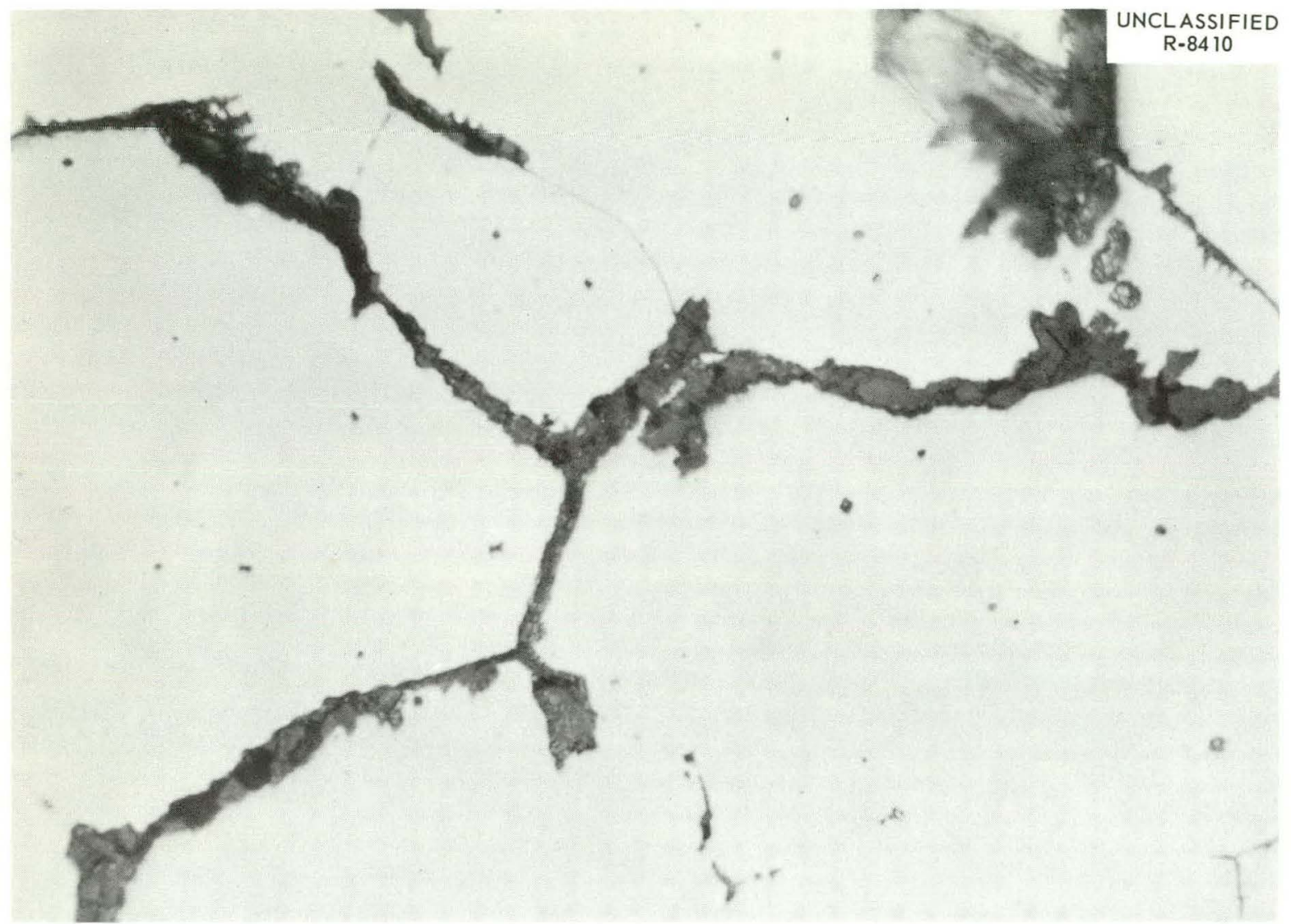

Fig. 20.27. Corrosion Product Found in Fractures in Type 304 Stainless Steel Lead Tubes of Element 5. As polished. $1000 x$.

diameter increases up to about 0.060 in.: or about $8 \%$ (capsule E-4). As a result of the fractures, no fission-gas release data were obtained, and $\mathrm{NaK}$ from the surrounding heat transfer baths had completely filled both capsules. When the capsules were disassembled, longitudinal voids were found in the centers of the originally solid fuel columns, and extensive columnar grain growth around these voids was apparent (Figs. 20.30 and 20.31). These phenomena are typical of sintered $\mathrm{UO}_{2}$ which has been heated to very high temperatures in a steep thermal gradient. ${ }^{16,17}$

Metallographic examination of fuel and cladding samples from capsule E-1A has been completed.

\footnotetext{
${ }^{16}$ V. B. Lawson and J. R. MacEwan, Thermal Simulation Experiments with a UO $\mathrm{U}_{2}$ Fuel Rod Assembly, CRFD-915 (March 1960).

${ }^{17} \mathrm{~J}$. L. Bates, Nucleonics 19, 6 (June 1961).
}

There was no evidence that any portion of the $\mathrm{UO}_{2}$ had been molten during irradiation. Since the region of columnar grain growth extended from the central void to about 0.15 in. from the outside surface of the fuel pellet, it is obvious that the maximum design temperature of $3700^{\circ} \mathrm{F}$ was exceeded. The $\mathrm{UO}_{2}$ structure indicates that the maximum central temperature was $\sim 5000^{\circ} \mathrm{F}$. In addition, the results of burnup analysis (Table 20.5) indicate that the capsule was overpowered more than $50 \%$. The measured burnup was 5050 Mwd per metric ton of uranium compared with a design value of $3350 \mathrm{Mwd}$ per metric ton of uranium. There was a slight reduction in the thickness of the cladding at the fractures. As shown in Fig. 20.32, a heavy precipitate was found along the inner surface and along the intergranular fractures. This precipitate, which extended from the inner surface of the cladding to about midwall throughout the capsule, appeared to be nitrides of 
Table 20.5. Data Summary for ETR-Irradiated Prototype Capsules

\begin{tabular}{clcccc}
\hline $\begin{array}{c}\text { Capsule } \\
\text { No. }\end{array}$ & $\begin{array}{c}\text { Type of } \\
\text { Fuel Pellet }\end{array}$ & $\begin{array}{c}\mathrm{U}^{235} \\
\text { Enrichment } \\
(\%)\end{array}$ & $\begin{array}{c}\text { Design } \\
\text { Heat Flux } \\
\left(\mathrm{Btu} \mathrm{hr}^{-1} \mathrm{ft}^{-2}\right)\end{array}$ & $\begin{array}{c}\text { Average Clad } \\
\text { Temperature } \\
\left({ }^{\circ} \mathrm{F}\right)\end{array}$ & $\begin{array}{c}\text { Uranium } \\
\text { Burnup }\end{array}$ \\
\hline E-1A & Solid & 0.71 & 159,000 & 1290 & 5050 \\
E-4 & Solid & 0.71 & 159,000 & 1320 & 8300 \\
E-12 & $\begin{array}{c}\text { Hollow-BeO } \\
\text { bushing }\end{array}$ & 1.0 & 153,000 & 1500 & 1140 \\
\hline
\end{tabular}

${ }^{a}$ Based on $\mathrm{Ce}^{144} / \mathrm{U}$ ratio.

stainless steel. The probable source of the nitrogen was an abundance of $\mathrm{UN}_{2}$ in the outer regions of the $\mathrm{UO}_{2}$ fuel pellets.

Thus it is concluded that the primary causes of cladding failure in capsule E-1A were (1) formation of nitrides in the inner surface region of the cladding and (2) overheating of the $\mathrm{UO}_{2}$ fuel. The presence of the nitrides tended to weaken and embrittle the cladding, making it more susceptible

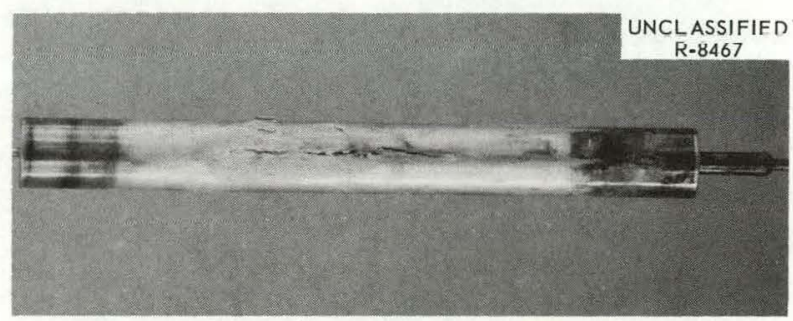

Fig. 20.28. Overall View of Capsule E-1A, Showing Clad Fracture. 0.45X. Reduced $19 \%$. to fracture when subjected to the unusual stress from the overheated fuel. The examination of capsule E- 4 is incomplete.

\section{Examination of Beryllium-Clad $\mathrm{UO}_{2}$ Capsules}

The examination of eight beryllium-clad $\mathrm{UO}_{2}-$ fueled capsules, which was partially reported previously, ${ }^{18,19}$ has been completed. The United Kingdom AEA and the French CEA cooperated with ORNL in the preparation of these experiments. The work at ORNL was sponsored by the Gas-Cooled Reactor Program.

The capsules were irradiated in the ORR at a nominal clad temperature of $1100^{\circ} \mathrm{F}$ to uranium

${ }^{18} \mathrm{~J}$. G. Morgan et al., Solid State Div. Ann. Progr. Rept. Aug. 31, 1961, ORNL-3213, pp 106-10.

${ }^{19}$ M. F. Osborne et al., Trans. Am. Nucl. Soc. 5, 43 (1962).

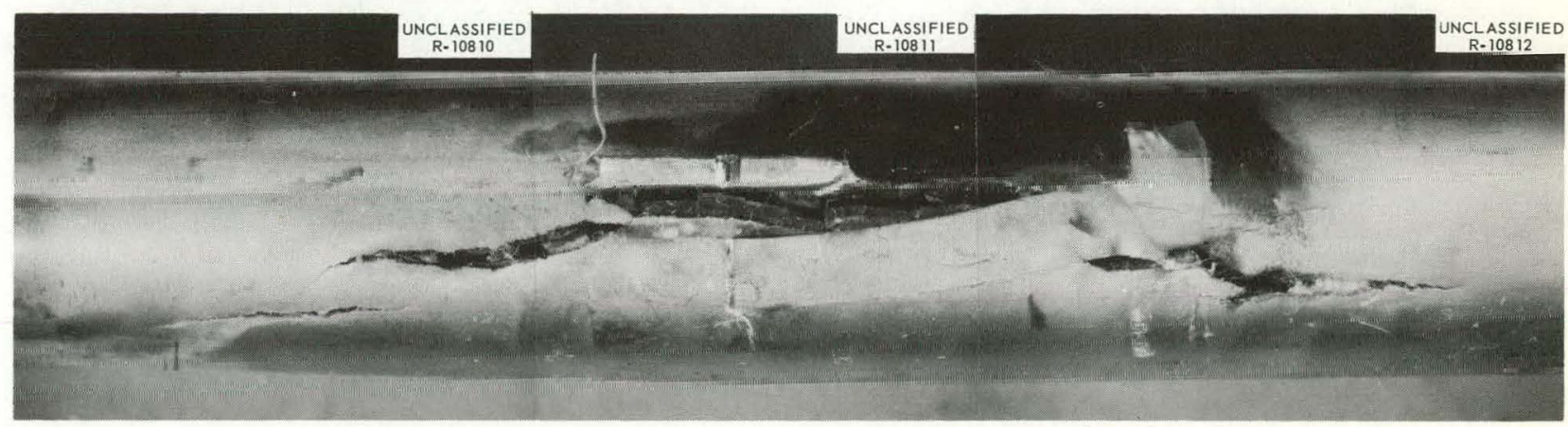

Fig. 20.29. Closeup View of Clad Fracture, Capsule E-4. Note pieces of sheathed thermocouple and foil thermocouple strap adhered to clad. 2.5X. Reduced $34 \%$. 


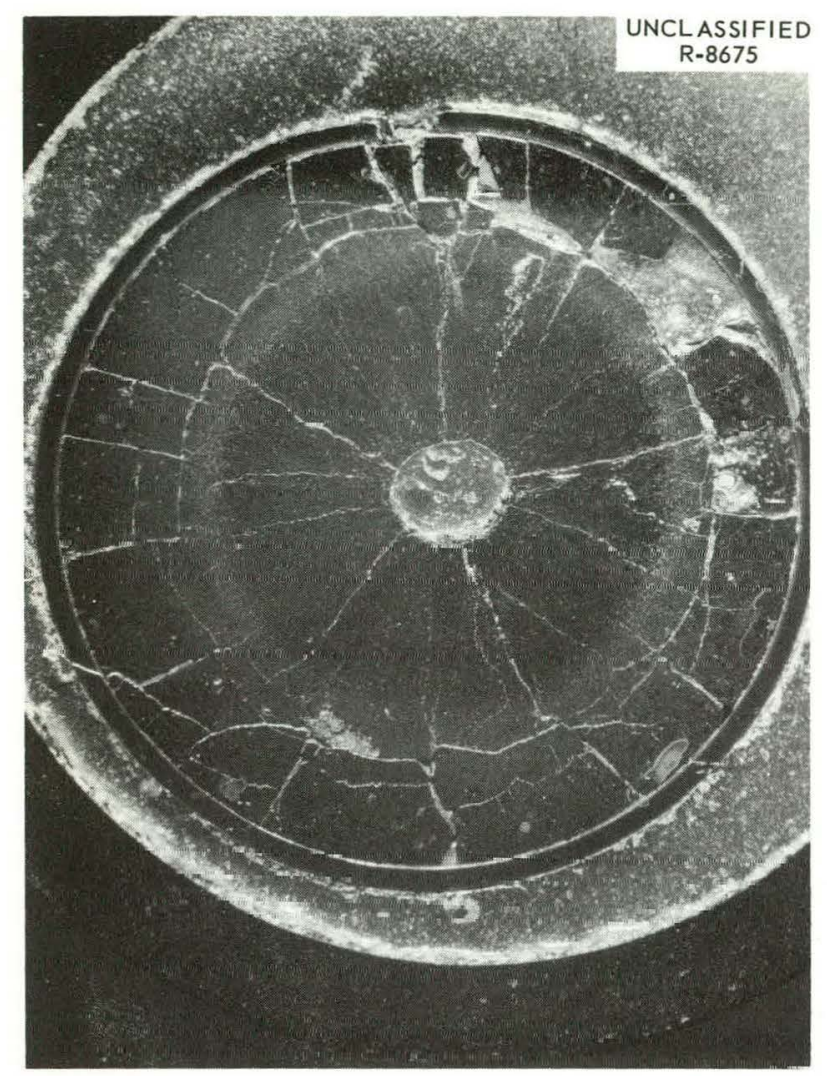

Fig. 20.30. Transverse Section of Capsule E-1A, Showing Clad Fracture and Central Void in $\mathrm{UO}_{2}$. $4.3 \mathrm{X}$. Reduced $7 \%$.

burnups of from 1000 to $4000 \mathrm{Mwd}$ per metric ton, or about 0.1 to 0.4 at. \%, of uranium. The results of the last two experiments were similar to those of the first six. Clad fractures during operation occurred only in the two capsules which were known to have substandard welds. Two other capsules, which had been subjected to the highest external pressure during irradiation (up to $725 \mathrm{psi}$ ), suffered stress-relief fracturing during disassembly in the hot cell.

No significant changes in the microstructure of the $\mathrm{UO}_{2}$ were found during metallographic examination. However, there were deleterious effects on the beryllium cladding. There was general subsurface void formation in all interior regions which were in contact with the $\mathrm{UO}_{2}$. These voidshad penetrated to a maximum depth of about 20 mils, more than half the wall thickness. No voids were found in the cladding adjacent to $\mathrm{MgO}$ end spacers. There was a reaction area at the interface between the $\mathrm{UO}_{2}$ pellet and the beryllium end plug in one of the capsules which did not contain $\mathrm{MgO}$ end spacers. This reaction had consumed about 15 mils of the beryllium end plug and had penetrated the grain boundaries in the $\mathrm{UO}_{2}$. The reaction product appeared to be $\mathrm{UBe}_{13}$. Other investigators have not found such an extensive reaction at this temperature in out-of-pile tests. ${ }^{20}$ On the basis of these relatively short-term experiments, beryllium docs not appear to be a promising material for a high-tempcrature $\mathrm{UO}_{2}$-containing reactor fuel element.

\section{Irradiation Effects on Fueled $\mathrm{BeO}$}

The dimensional stability of $\mathrm{BeO}-\mathrm{UO}_{2}-\mathrm{ThO}_{2}$ was found to be somewhat hetter than that of

\footnotetext{
${ }^{20}$ A. G. Knapton and K.B.C. West, J. Nucl. Mater. 3(2), 239 (1961).
}

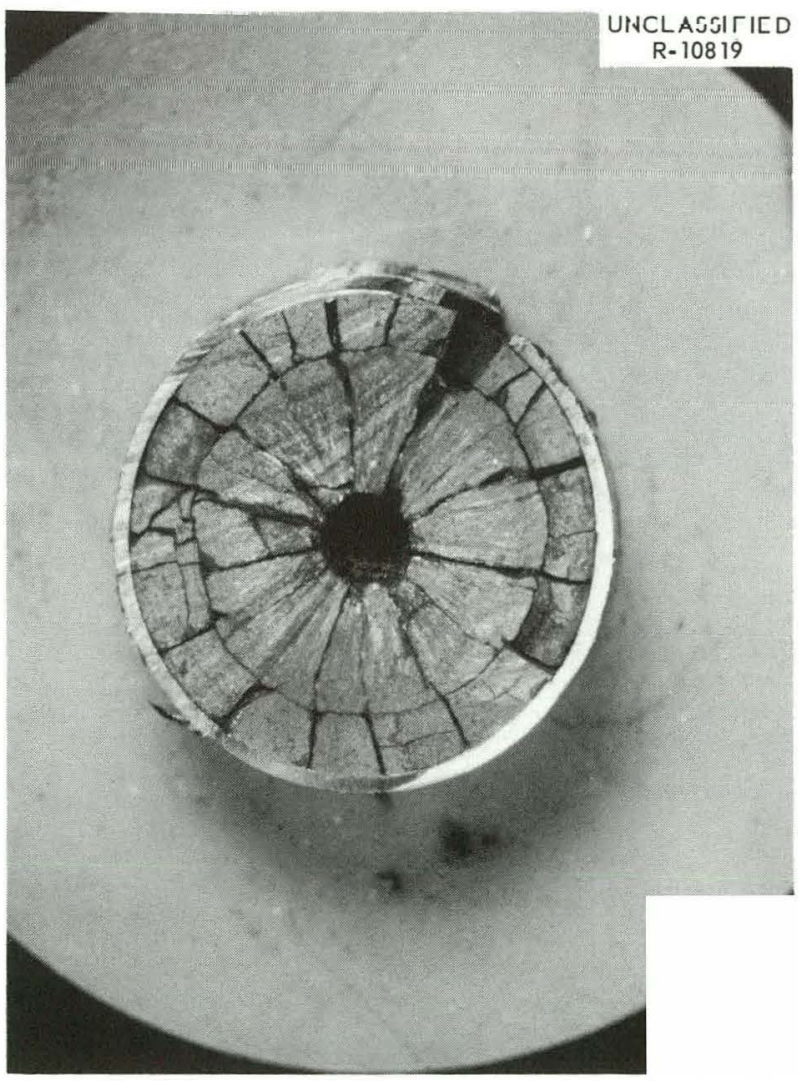

Fig. 20.31. Transverse Section of Capsule E-4, Showing $\mathrm{Clad}$ Fracture and Central Void and Circumferential Crack in $\mathrm{UO}_{2} .2 .8 \mathrm{X}$. Reduced $7 \%$. 


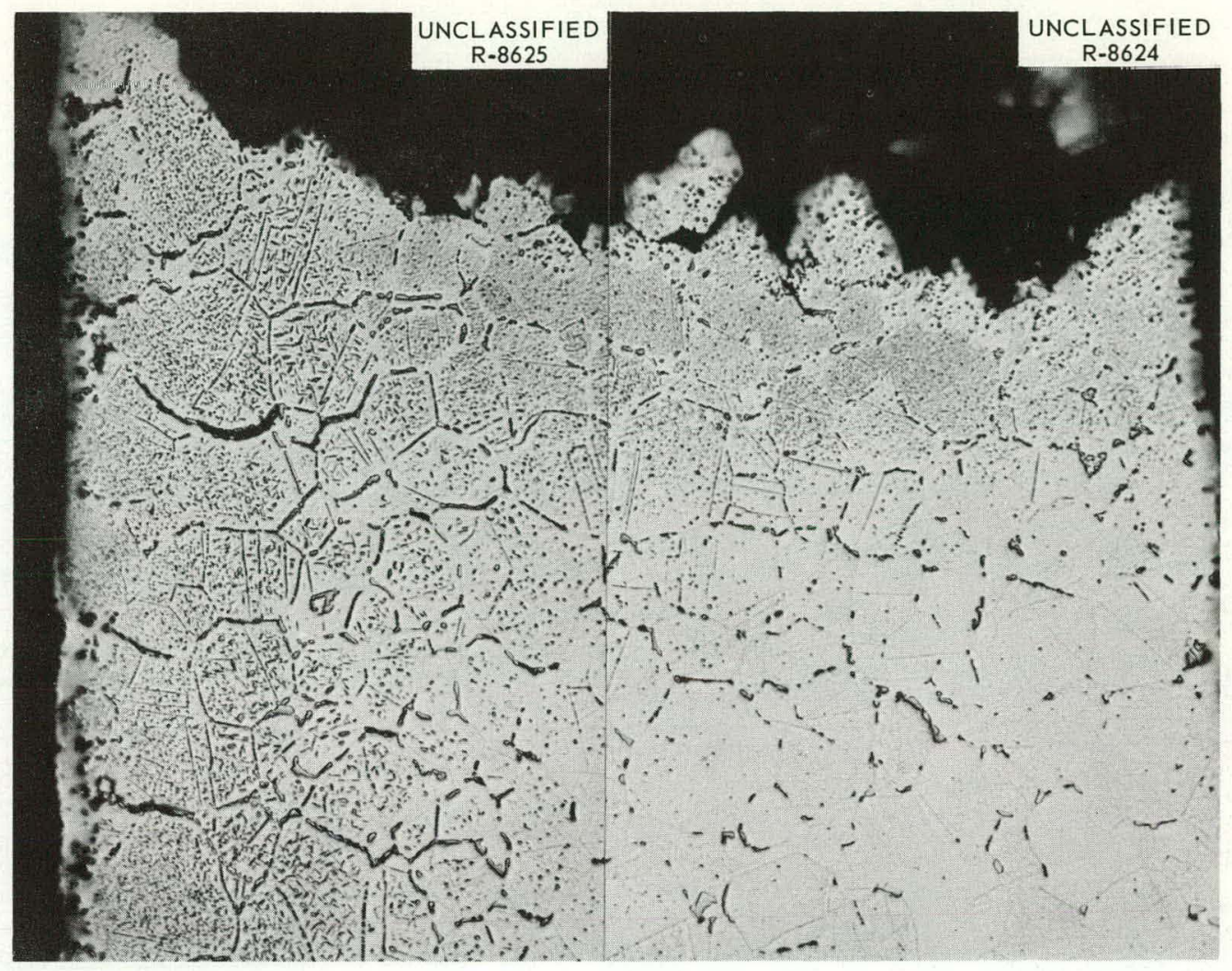

INNER SURFACE

OUTER SURFACE

Fig. 20.32. Fracture in Cladding of Capsule E-1A. Note heavy precipitate along inner surface and fracture region. Etched. $250 \mathrm{X}$.

BeO-UO ${ }_{2}$ for irradiations to about 4 at. $\%$ of uranium plus thorium burnup at about $2000^{\circ} \mathrm{F}$. The dimensional increase for the former was about $0.5 \%$; for the latter the average increase was about $2 \%$, with a maximum of $3.5 \%$. With the exception of those capsules which leaked during irradiation, all fuel pellets were removed intact and none were broken during handling in the hot cell. Fission-gas release was low, 0.1 to $0.3 \%$ of the $\mathrm{Kr}^{85}$ formed. There was no evidence of any chemical reactions in capsules which had inert atmospheres. There appeared to be considerable fission-fragment damage to the microstructure of the $\mathrm{BeO}$ matrix material in both types of fuel. Apparently, the finely dispersed fuel particles tended to maximize this damage and possibly to maximize physical swelling of the fuel pellets. These results indicate that dispersions containing larger fuel particles $(\sim 100 \mu)$ might be more stable dimensionally. With the exception of dimensional stability, the radiation stability properties of these dispersion fuels are equal to or superior to those of bulk $\mathrm{UO}_{2}$.

These experiments have been reported in detail previously. ${ }^{21}$

\subsection{CONTINUOUS RELEASE OF FISSION GAS DURING IRRADIATION OF $\mathrm{UO}_{2}$}
R. M. Carroll
P. E. Reagan

T. W. Fulton

An in-pile facility is in operation for the study of the factors controlling the fission-gas release from ${ }^{21} \mathrm{~J}$. G. Morgan and M. F. Osborne, Radiation Effects
on Fueled BeO, ORNL TM-311 (Oct. 4, 1962). 
$\mathrm{UO}_{2}$ during irradiation. ${ }^{22}$ The fuel specimen is heated by its own fission power. The power output is regulated by moving the fuel into or out of the reactor neutron flux. At constant fission power the temperature of the specimen is controlled by air cooling. Fission gas from the $\mathrm{UO}_{2}$ fuel is entrained in an inert sweep gas (normally helium) and carried outside the reactor for analysis. The neutron flux for any power level is found by using argon as the sweep gas and measuring the argon activation. ${ }^{23}$ To prevent oxidation of $\mathrm{UO}_{2}$ specimens from trace amounts of oxygen in the purified sweep gas, $3 \%$ hydrogen is added to the argon and helium.

\section{Thin-Plate Fused-Crystal $\mathrm{UO}_{2}$}

Two thin plates of fused-crystal $\mathrm{UO}_{2}$ were sandwiched together with thermocouples between them and with thermocouples also pressed against the outer surface. These specimens, obtained by slicing a lump of fused $\mathrm{UO}_{2}$, had very large grains. The fission-gas release from the fused-crystal specimen was similar to that from a sintered specimen under the same conditions. This demonstrated either that the surface area of the high-density sintered specimen was almost equal to the geometric surface area of the polished fused

\footnotetext{
${ }^{22}$ R. M. Carroll and C. D. Baumann, Experiment on Continuous Release of Fission Gas During Irradiation, ORNL-3050 (Feb. 9, 1961).

${ }^{23}$ R. M. Carroll, "Argon Activation Measures Irradiation Flux Continuously," Nucleonics 20(2) (1962).
}

specimen or that total surface area is not the dominating factor for fission-gas release.

The steady-state fission-gas release from this specimen was measured over a series of different temperature ranges. These are summarized in Table 20.6. The last two measurements, after about nine weeks of irradiation, showed a definite increase of release rate. When the capsule was removed from the reactor and examined to determine the cause of this increase, it was found that the specimens were broken, apparently along the grain boundaries. The increased fission-gas release was probably caused by the increased surface area of the specimen.

Iodine release measurements are obtained by withdrawing the specimen from the reactor flux after an equilibrium release rate has been obtained. The continued production of $\mathrm{Xe}^{133}$ and $\mathrm{Xe}^{135}$ was from the decay of iodine that emerged from the $\mathrm{UO}_{2}$ during irradiation and deposited within the sweep system. At steady-state operation, the escape of iodine from the $\mathrm{UO}_{2}$ is equal to the production of xenon from the deposited iodine. The steady-state production of xenon can be determined (see Fig. 20.33) by extrapolating the measured release rate to the moment the sample is withdrawn.

Iodine was found to diffuse from the fused-crystal specimen with the same temperature dependence as xenon and krypton. This identical temperature dependency was also found for all previous specimens, regardless of composition. For the gas that was released, $48 \%$ of the 133 isobar and $75 \%$ of the 135 isobar were released as iodine. This same ratio of xenon-to-iodine release was found for a

Table 20.6. Fractional Fission-Gas Release from Fused-Crystal UO 2 Specimen [(Release Rate/Production Rate) $\left.\times 10^{4}\right]$

\begin{tabular}{cccccccc}
\hline $\begin{array}{c}\text { Temperature } \\
\left({ }^{\circ} \mathrm{C}\right)\end{array}$ & $\mathrm{Xe}^{133}$ & $\mathrm{Xe}^{135}$ & $\mathrm{I}^{133}$ & $\mathrm{I}^{135}$ & $\mathrm{Kr}^{85 m}$ & $\mathrm{Kr}^{88}$ & $\mathrm{Kr}^{87}$ \\
\hline 850 & 13 & 2.9 & 7.3 & 8.5 & 17 & 6.5 & \\
912 & 16 & 3.9 & 14 & 16 & 23 & 7.8 & \\
765 & 9.1 & 1.2 & 10 & 7 & 14 & 7.4 & 6.1 \\
985 & 40 & 8.4 & 43 & 33 & 40 & 19 & 18 \\
812 & 22 & 2.3 & 12 & 10 & 22 & 13 & 8.1 \\
955 & 47 & 9.7 & 59 & 54 & 70 & 33 & 26 \\
\hline
\end{tabular}




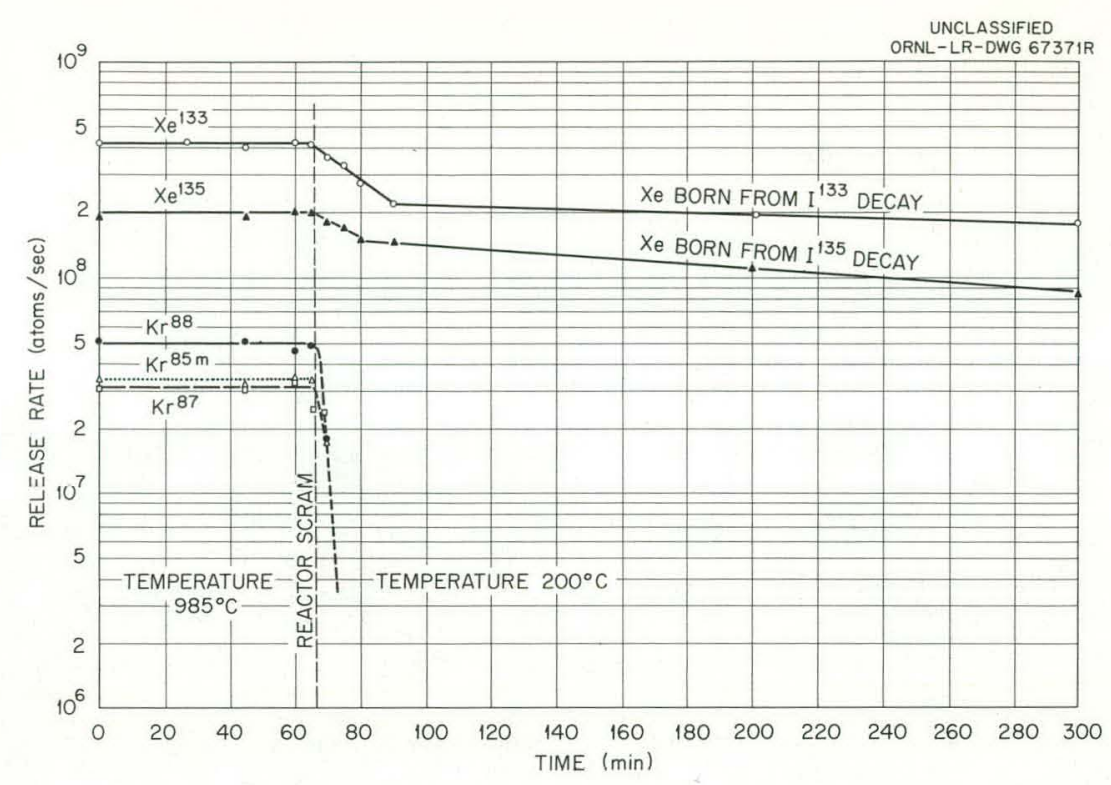

Fig. 20.33. In-Pile Fission-Gas Release from Fused Crystal of UO 2 During Reactor Scram; Cl-7 Experiment.

sintered nonstoichiometric specimen, ${ }^{24}$ although the absolute release rate was much greater for the sintered specimen. Apparently, anything affecting the release of xenon affects the iodine release in the same manner.

The fused-crystal specimen had a low $(0.3 \%)$ enrichment and was irradiated at a neutron flux of $3.3 \times 10^{13} n v$ to produce the desired fission rate. This flux was a factor of 10 higher than for the nonstoichiometric sintered specimen. Because of the difference in flux, it was expected that the $2.7 \times 10^{6}$ barn cross section of $\mathrm{Xe}^{135}$ would cause the $\mathrm{Xe}^{133} / \mathrm{Xe}^{135}$ ratio to increase with increasing flux. The ratio, however, remained the same for both specimens. ${ }^{25}$ The only explanation, thus far, for this phenomenon is that the iodine precursor of the xenon dominates the diffusion of the iodinexenon isobar. Thus the iodine might diffuse to

\footnotetext{
${ }^{24}$ R. M. Carroll, "Continuous Release of Fission Gas from $\mathrm{UO}_{2}$ During Irradiation," Symposium on Radiation Effects in Refractory Fuel Compounds, ASTM Special Technical Publication No. 306.

${ }^{25}$ F. R. McQuilkin, Gas-Cooled Reactor Program Quart. Progr. Rept. Mar. 31, 1962, ORNL-3302, p 62.
}

the surface of the $\mathrm{UO}_{2}$, and then, after the iodine decays to xenon, the xenon is released close to the surface with very little time for decay.

The activation energy for the fused-crystal specimens was the same as for the previous specimens, 30 to $40 \mathrm{kcal} / \mathrm{mole}$. This was somewhat unexpected because of the different composition of the fused-crystal specimen.

Thin-plate specimens of sintered $\mathrm{UO}_{2}$, containing excess oxygen, released bursts of fission gas when the specimens were heated or cooled. ${ }^{24}$ The fission-gas release from the fused-crystal specimen was also observed during temperature fluctuations to see if similar bursts were produced. Heating bursts were always produced, but no cooling bursts were found (Fig. 20.33).

\section{Thin-Plate Single-Crystal $\mathrm{UO}_{2}$}

Thin-slab specimens of high-quality singlecrystal $\mathrm{UO}_{2}$ have been obtained from Hanford. These were sandwiched together with thermocouples between them and pressed against the outer surfaces as described above. The thermocouples served the added function of electrical 
probes to measure the electrical conductivity of the $\mathrm{UO}_{2}$ during irradiation ${ }^{26}$ (see Fig. 20.34). The electrical resistivity of crystalline $\mathrm{UO}_{2}$ was decreased by a factor of 200 as soon as irradiation began. This decrease was due to ionizing irradiation rather than fission-product impurities since, in the initial portion of irradiation, the resistivity could be increased by removing the specimen from the flux. After $24 \mathrm{hr}$ of irradiation, the change of resistivity was irreversible.

The activation energy for electrical resistivity changed dramatically from $11.3 \mathrm{ev}$ (preirradiation) to $1.6 \mathrm{ev}$ during the first few hours of irradiation, then stabilizing at $0.4 \mathrm{ev}$ for subsequent irradiation. The values of resistivity and activation energy are compared in Table 20.7.

From these data we conclude that irradiation lowers the electrical resistivity by providing carriers for the electrical current. The activation energy is decreased because of irradiation-induced

${ }^{26}$ M. D. Karkhanavala and R. M. Carroll, In-Pile Measurement of the Electrical Resistivity and Thermoelectric Power of Sintered $\mathrm{UO}_{2}$, ORNL-3093 (April 1961). disorder in the $\mathrm{UO}_{2}$ structure. This effect is more pronounced in the case of a highly ordered structure such as the single-crystal specimen.

Fission-gas release decreased slightly after the first two weeks of irradiation. This was probably caused by reduction of the oxygencontaminated surface by the hydrogen in the sweep gas. The fractional fission-gas release in the $700^{\circ} \mathrm{C}$ range is compared in Table 20.8 for a sintered fine-grain specimen, a fused-crystal largegrain specimen, and the single-crystal specimen.

The activation energy for $\mathrm{Kr}^{88}$ release is 46 to $50 \mathrm{kcal} / \mathrm{mole}$ for the single-crystal specimen. This is higher than the $30-$ to $40-\mathrm{kcal} / \mathrm{mole}$ activation energy for all previous specimens. The temperature-dependent gas release for the singlecrystal specimen begins at about $630^{\circ} \mathrm{C}$. This is about $100^{\circ} \mathrm{C}$ higher than for the sintered and the fused specimens.

The single-crystal specimen emits heating bursts, thus demonstrating that the heating burst is not a grain-boundary phenomenon. No cooling bursts have been emitted by this specimen.
UNCLASSIFIED

ORNL-LR-DWG $55090 R$

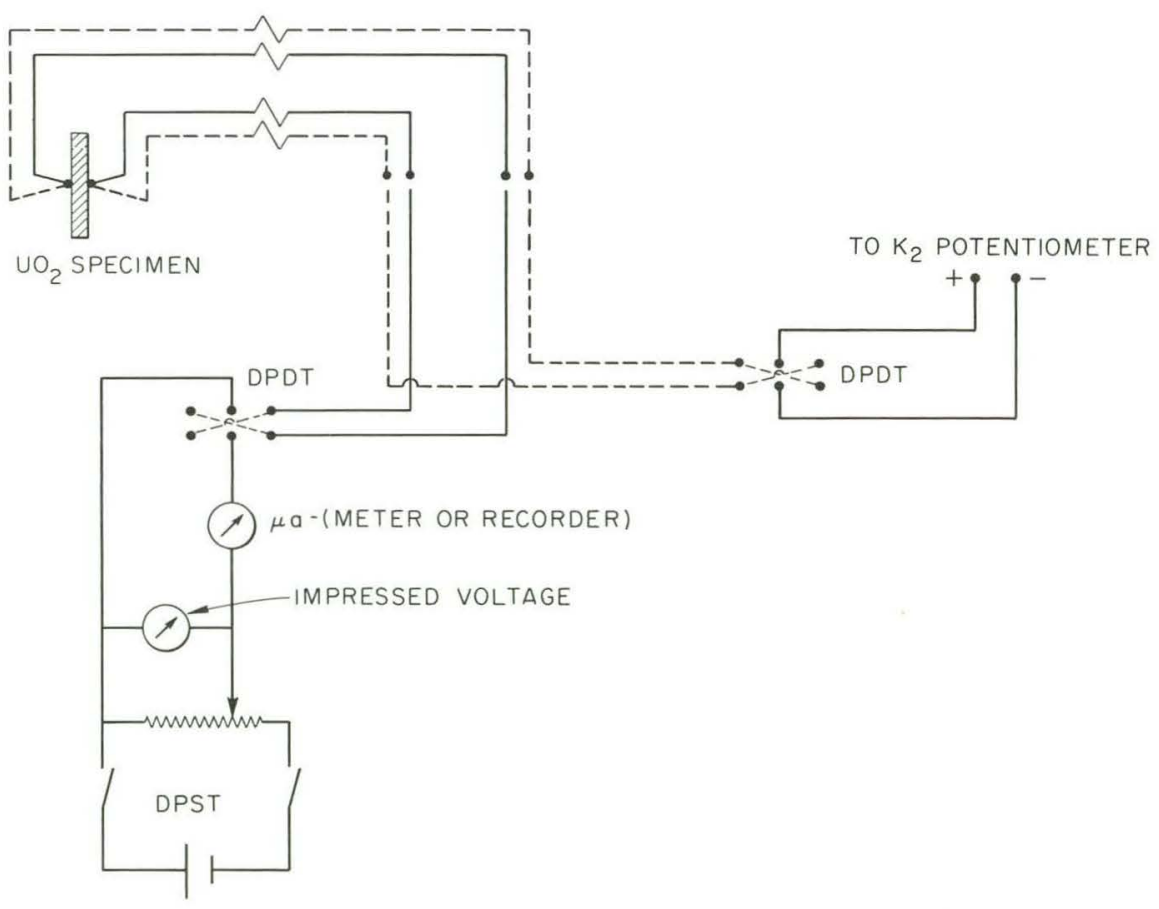

Fig. 20.34. Schematic of Arrangement Used for Measurement of Electrical Conductivity and Thermoelectric Power. 
Table 20.7. Comparison of Resistivity and Activation-Energy Values

\begin{tabular}{|c|c|c|c|c|}
\hline \multirow[b]{2}{*}{ Specimen } & \multicolumn{2}{|c|}{ Resistivity at $700^{\circ} \mathrm{C}$ (ohm-cm) } & \multicolumn{2}{|c|}{ Activation Energy (ev) } \\
\hline & $\begin{array}{c}\text { Before } \\
\text { Irradiation }\end{array}$ & $\begin{array}{c}\text { After } \\
\text { Irradiation } \\
\text { to } 10^{18} n v t\end{array}$ & $\begin{array}{c}\text { Before } \\
\text { Irradiation }\end{array}$ & $\begin{array}{c}\text { After } \\
\text { Irradiation } \\
\text { to } 10^{18}{ }_{n v t}\end{array}$ \\
\hline $\begin{array}{l}\text { Sintered, fine } \\
\text { grain }\end{array}$ & 2.0 & 0.8 & 0.65 & 0.50 \\
\hline $\begin{array}{l}\text { Fused crystal, } \\
\text { very large grain }\end{array}$ & 1.7 & & 0.52 & \\
\hline Single crystal & $\sim 500$ & 2.5 & 11.3 & 0.4 \\
\hline
\end{tabular}

Table 20.8. Steady-State Fractional Fission-Gas Release in $700^{\circ} \mathrm{C}$ Range $(R / B)^{a}$

\begin{tabular}{lccccc}
\hline Specimen & $\mathrm{Kr}^{85 m}$ & $\mathrm{Kr}^{88}$ & $\mathrm{Kr}^{87}$ & $\mathrm{Xe}^{135}$ & $\mathrm{Xe}^{133}$ \\
\hline $\begin{array}{l}\text { Fine grain, sintered; } \\
730^{\circ} \mathrm{C}\end{array}$ & $8.3 \times 10^{-4}$ & $7.5 \times 10^{-4}$ & & $5.2 \times 10^{-4}$ & $1.5 \times 10^{-3}$ \\
$\quad \begin{array}{l}\text { Large grain, fused; } \\
\quad 765^{\circ} \mathrm{C} \\
\quad \text { Single crystal; } \\
\quad 712^{\circ} \mathrm{C}\end{array}$ & $14 \times 10^{-4}$ & $7.4 \times 10^{-4}$ & $6.1 \times 10^{-4}$ & $8.2 \times 10^{-4}$ & $1.9 \times 10-3$ \\
& $1.3 \times 10-4$ & $5.2 \times 10^{-5}$ & $3.7 \times 10^{-5}$ & $1.9 \times 10^{-4}$ & $3.1 \times 10^{-4}$ \\
\hline
\end{tabular}

$a_{\text {Release rate/birth rate. }}$

Thermocouple Comparisons: W $-5 \%$ Re vs W $-26 \%$ Re with Pt vs Pt-10\% Rh. - A hydrogen-trace atmosphere must be used in these sweep experiments to avoid oxidizing the $\mathrm{UO}_{2}$ specimen. A disadvantage in using hydrogen additions is that the hydrogen will react with the high-temperature junctions of the Pt vs Pt-10\% Rh thermocouples, causing them to become brittle. Early failure of bare $\mathrm{Pt}$ vs $\mathrm{Pt}-10 \% \mathrm{Rh}$ thermocouples is to be expected under these conditions. Nevertheless, the electrical resistivity tests described above require that bare-wire $\mathrm{Pt}$ vs $\mathrm{Pt}-\mathrm{Rh}$ thermocouples be used.

For the single-crystal $\mathrm{UO}_{2}$ specimens, three types of thermocouples are used: bare-wire Pt vs $\mathrm{Pt}-10 \% \mathrm{Rh}, \mathrm{Pt}$ vs $\mathrm{Pt}-10 \% \mathrm{Rh}$ clad in Inconel, and bare-wire $\mathbb{W}-5 \% \operatorname{Re}$ vs $\mathbb{W}-26 \%$ Re. For the preirradiation electrical conductivity test the assembled capsule (in a reducing atmosphere) was heated in a furnace before being inserted into the reactor. At this time in the reducing atmosphere all thermocouples indicated the same temperature. During irradiation the $\mathbb{W}-5 \%$ Re vs $\mathbb{W}-26 \%$ Re thermocouples have indicated about 10\% lower temperatures than the $\mathrm{Pt}$ vs $\mathrm{Pt}-10 \% \mathrm{Rh}$. This difference started immediately and has not changed appreciably. The amount of shift is temperature dependent, being least (about $6 \%$ ) at 700 to $800^{\circ} \mathrm{C}$ and increasing at higher and lower temperatures. The specimen has not been irradiated at temperatures higher than $1000^{\circ} \mathrm{C}$, and the platinum thermocouples, both bare and clad, indicated $13 \%$ higher values for this temperature than did the tungsten thermocouples.

In view of the identical temperatures from all thermocouples during bench testing in the hydrogen atmosphere, we must conclude that irradiation lowcred the emf output of the tungsten thermocouples. 


\subsection{MARITIME REACTOR FUEL}

$$
\text { J. G. Morgan M. F. Osborne }
$$

Four additional maritime reactor fuel bundles, similar to those reported previously, ${ }^{27}$ have been examined. Each of these fuel-rod bundles had been irradiated in the pressurized-water loop facility in the ORR. ${ }^{28}$ Each bundle consisted of three fuel rods $18 \mathrm{in.} \mathrm{long} \mathrm{and} 0.5 \mathrm{in}$. in diameter, as shown in Fig. 20.35. Each rod was made of type 304L stainless steel tubing containing fused and ground $\mathrm{UO}_{2}$.

The fuel rods were fabricated by two processes: (1) 0.75 -in.-OD tubing containing $\mathrm{UO}_{2}$ powder was swaged to the finished diameter and (2) 0.5-in.-OD tubes were filled with vibratorily compacted $\mathrm{UO}_{2}$. Uranium dioxide densities of about $90 \%$ of theoretical were attained by both methods. ${ }^{29}$ Fuel enrichment ranged from 0.80 to $2.83 \% \mathrm{U}^{235}$.

The rods were cooled by high-purity water at $1750 \mathrm{psi}$ and $500^{\circ} \mathrm{F}$, flowing at about $10 \mathrm{fps}$. The experiments were irradiated to burnups of up to 4600 Mwd per metric ton of uranium, with centerfuel temperatures estimated to be as high as $5000^{\circ} \mathrm{F}$ and a maximum heat flux of 400,000 Btu $\mathrm{hr}^{-1} \mathrm{ft}^{-2} \cdot 30$

${ }^{27}$ J. G. Morgan, M. F. Osborne, and H. E. Robertson, Solid State Div. Ann. Progr. Rept. Aug. 31, 1961, ORNL3213, P 112; J. G. Morgan and M. F. Ósborne, Solid State Div. Ann. Progr. Rept. Aug. 31, 1961, ORNL-3213, p 113.

${ }^{28} \mathrm{~J}$. A. Colins, J. K. Franzreb, and D. E. Tidwell, Maritime Reactor Program Ann. Progr. Rept. Nov. 30, 1961, ORNL-3238, P 41.

${ }^{29}$ J. T. Lamartine, Preirradiation Data: Maritime $O R R$ Loop Experiments 4, 5, and 6, ORNL CF-62-3-88 (Mar. $28,1962)$.

${ }^{30}$ V. O. Haynes, ORR Pressurized-Water Loop Experiments: General Description and Operation Information for Experiments 4, 5, and 6, ORNL CF-61-2-92.
There was no change in the exterior appearance of the irradiated rods except for the accumulation of a dark oxide film. Radiochemical analysis of this film showed that the principal components were stainless steel corrosion products: $\mathrm{Cr}, \mathrm{Fe}$, $\mathrm{Mn}$, and Co. The bundles and the individual rods were measured for diameter changes and bowing. No significant rod-bowing changes were found. Diameter measurements indicated that one fuel rod was 0.007 to 0.010 in. smaller after irradiation, and the diameter change of another rod varied from +0.011 to -0.005 in. Diameter changes for the other ten rods were within experimental uncertainty.

Samples of the gas from each rod were analyzed for both stable and radioactive components. The fractions of the $\mathrm{Kr}^{85}$ released from the fuel, based on measured burnup, are shown in Table 20.9. As expected, the fission-gas release increased with irradiation temperature and burnup, reaching a maximum of $23 \%$. Gamma scans of the fuel rods were similar to that reported previously; ${ }^{27}$ no unusual distributions of radioactivity were found. The ratios of peak-to-average radioactivity varied from 1.14 to 1.23 , indicating relatively uniform burnup along the length of the rod.

The fuel rods were sectioned transversely and longitudinally for examination of the fuel. The appearance of the fuel in a typical rod from experiment No. 3 is shown in Fig. 20.36. Transverse cracks in the fuel are correlated with irregularities in the gamma scan. One fuel rod, denoted 601, contained a void in the center of the fuel column (Fig. 20.37). The void was about 6 in. long, and its maximum diametei was about $0.04 \mathrm{in.}$ This type of central void, with the accompanying growth of columnar grains around it, is formed in $\mathrm{UO}_{2}$ when steep thermal gradients exist at temperatures

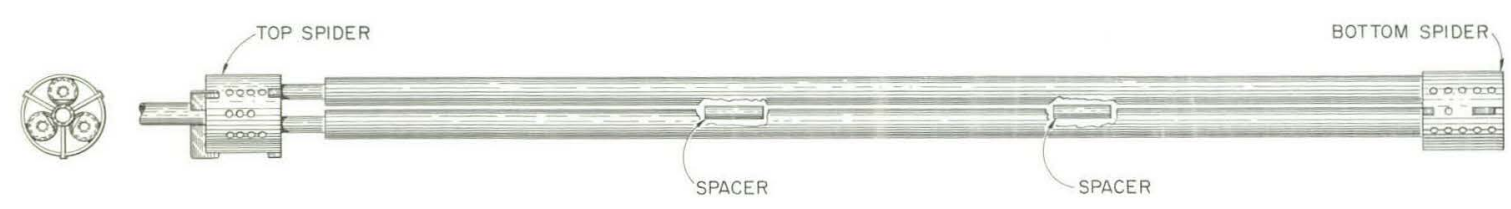

Fig. 20.35. Schematic of Experimental Fuel-Rod Bundle, Maritime Reactor Program. 
Table 20.9. Data Summary for Maritime Reactor Fuel Examinations

\begin{tabular}{|c|c|c|c|c|c|c|c|}
\hline $\begin{array}{c}\text { Experiment } \\
\text { No. }\end{array}$ & $\begin{array}{l}\text { Rod } \\
\text { No. }\end{array}$ & $\begin{array}{c}\text { Fabrication } \\
\text { Method }\end{array}$ & $\begin{array}{c}\text { Maximum } \\
\text { Heat Flux } \\
\left(\text { Btu hr }{ }^{-1} \mathrm{ft}^{-2}\right)\end{array}$ & $\begin{array}{l}\text { Maximum Fuel } \\
\text { Temperature }{ }^{a} \\
\quad\left({ }^{\circ} \mathrm{F}\right)\end{array}$ & $\begin{array}{c}\text { Maximum }_{\text {Burnup }}^{b} \\
\text { (Mwd per metric ton of uranium) }\end{array}$ & $\begin{array}{c}\mathrm{Kr}^{85} \\
\text { Release } \\
\quad(\%)\end{array}$ & $\begin{array}{c}\text { Plutonium } \\
\text { Concentration } \\
(\mathrm{mg} \text { of } \mathrm{Pu} \text { per } \mathrm{g} \text { of } \mathrm{U})\end{array}$ \\
\hline \multirow[t]{6}{*}{3} & $3 \mathrm{~A} 1$ & Cold swaged & 100,000 & 2200 & 2480 & 3.7 & \\
\hline & $3 \mathrm{~B} 1$ & Cold swaged & 100,000 & 2700 & 3030 & 3.4 & \\
\hline & $3 C 1$ & Cold swaged & 100,000 & 2700 & 3320 & 7.6 & 1.74 \\
\hline & $3 N 1$ & Cold swaged & 100,000 & 2200 & 2950 & 3.3 & \\
\hline & 301 & Hot swaged & 120,000 & 2200 & $29 C 0$ & 3.1 & \\
\hline & 3P 1 & Hot swaged & 120,000 & 2700 & 3820 & 3.2 & 3.52 \\
\hline \multirow[t]{3}{*}{4} & $4 \mathrm{~N} 1$ & Cold swaged & 200,000 & 3800 & 3120 & 4.1 & 1.65 \\
\hline & 401 & Vibratorily compacted & 275,000 & 2900 & 2870 & 2.2 & \\
\hline & $4 \mathrm{P} 1$ & Vibratorily compacted & 275,000 & 2900 & 2440 & 2.9 & 1.87 \\
\hline \multirow[t]{3}{*}{6} & $6 \mathrm{~N} 1$ & Vibratorily compacted & 400,000 & 4500 & 3750 & 4.7 & 1.34 \\
\hline & 601 & Vibratorily compacted & 400,000 & 4500 & 4660 & 23.4 & \\
\hline & 6P1 & Vibratorily compacted & 400,000 & 5000 & 4150 & 10.5 & 1.58 \\
\hline
\end{tabular}

${ }^{a}$ Calculated.

${ }^{b}$ Based on $\mathrm{Ce}^{144} / \mathrm{U}$ ratio.

${ }^{c}$ Based on average burnup. 

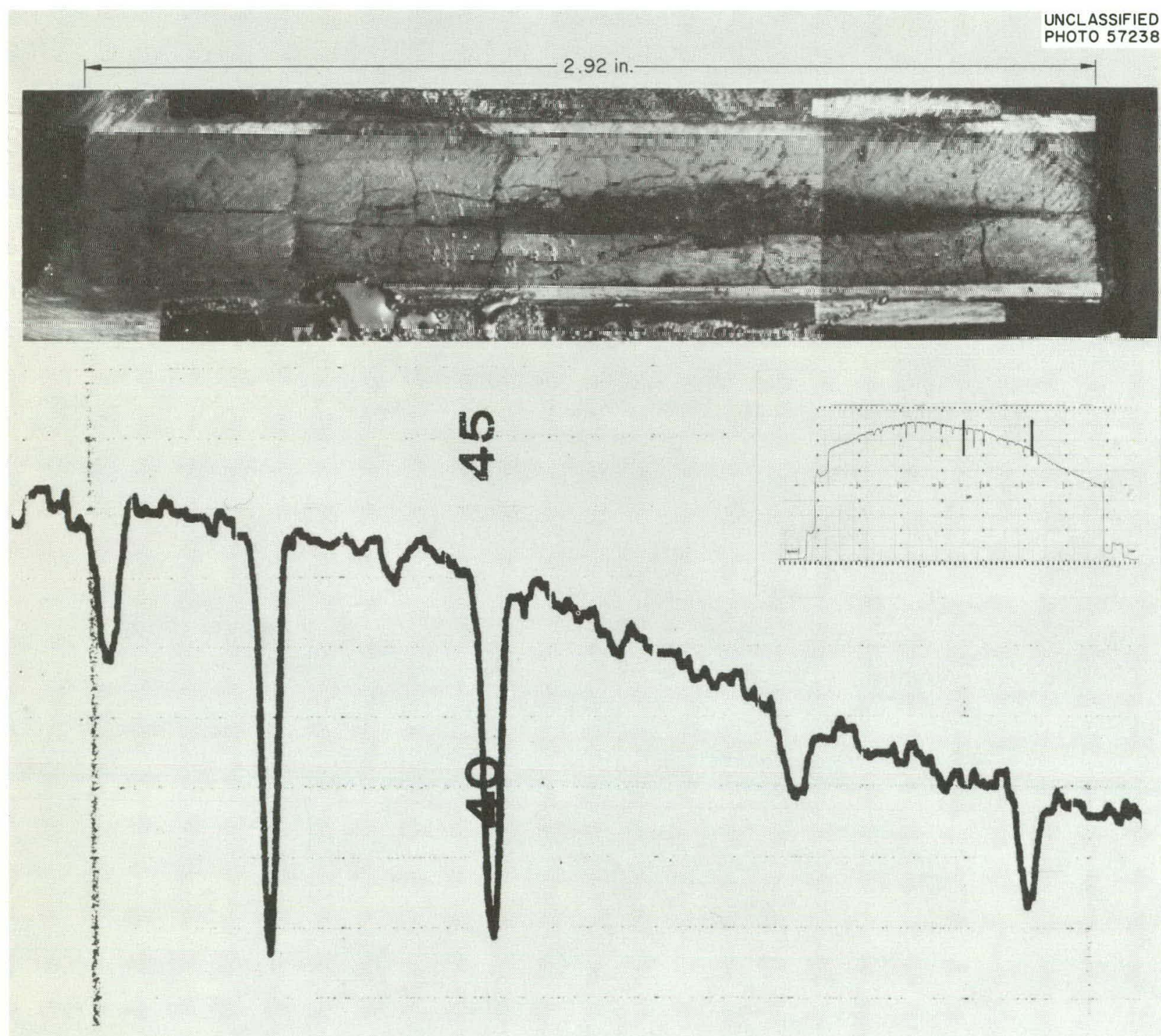

Rod 3-P-1 Hot Swaged (-20 Mesh $\mathrm{UO}_{2}$ )

Fig. 20.36. Correlation of Gamma Radioactivity Scan with Section of Maritime Fuel Rod. Note dips in gamma scan adjacent to fractures in $\mathrm{UO}_{2}$ fuel; section of fuel rod was cut longitudinally to expose fuel. 


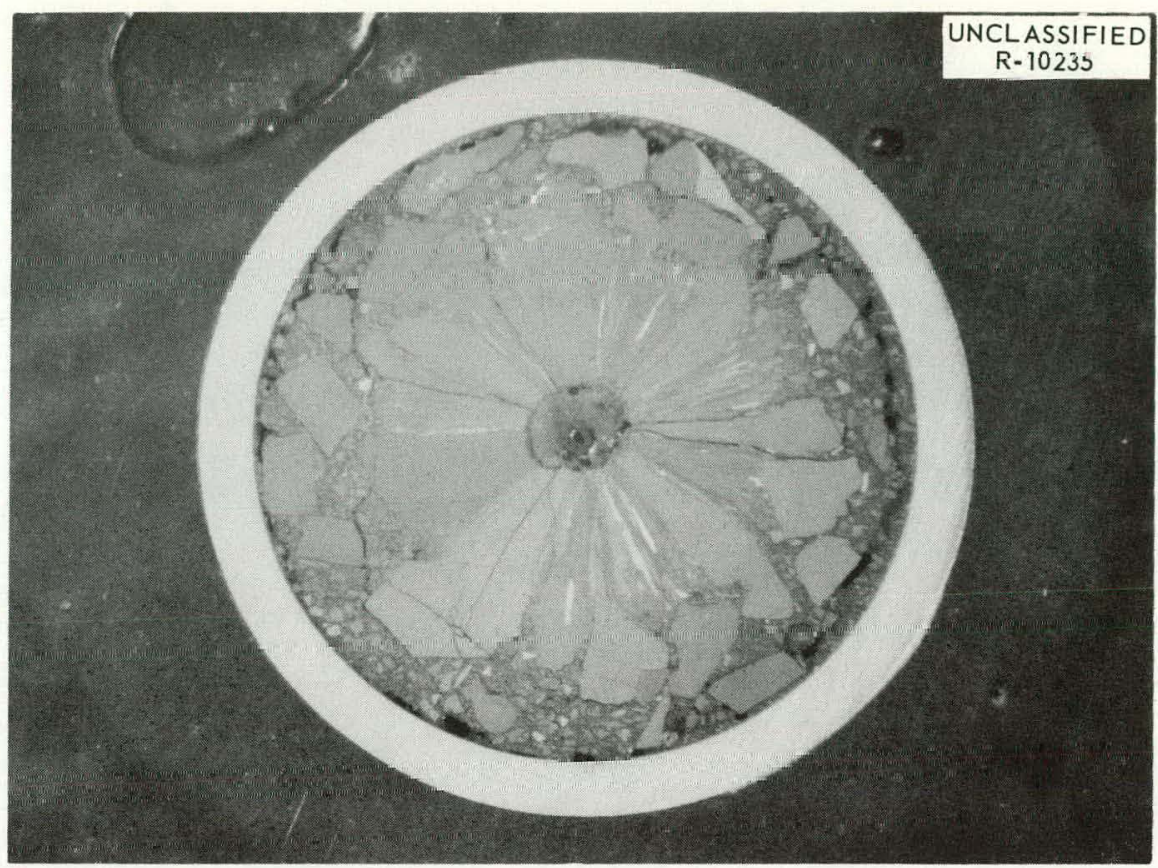

Fig. 20.37. Transverse Section of Maritime Rod 601, Showing Central Void and Columnar Grain Growth. Original appearance of fuel was similar to that near clad.

greater than $3000^{\circ} \mathrm{F} .{ }^{31,32}$ Partial sintering of the $\mathrm{UO}_{2}$ was observed in several other fuel rods which experienced temperatures greater than $3000^{\circ} \mathrm{F}$.

In order to evaluate the breeding efficiency of this fuel, some samples were analyzed by mass spectrometry for the plutonium content. These results, shown in Table 20.9, agreed fairly well with values calculated from the neutron flux.

\subsection{FUEL CYCLE PROGRAM ${ }^{33}$}

\section{J. G. Morgan $\quad$ M. F. Osborne}

\section{Postirradiation Examination}

The primary objective of the Fuel Cycle Program is the development of suitable techniques for remote reprocessing and fabrication of reactor fuel containing $\mathrm{U}^{233} .34$ 'Test fuel rods of vibratorily

\footnotetext{
${ }^{31}$ V. B. Lawson and J. R. MacEwan, Thermal Simulation Experiments with a UO ${ }_{2}$ Fuel Rod Assembly, CRFD915 (March 1960).

${ }^{32}$ J. L. Bates, Nucleonics 19, 6 (June 1961).

${ }^{33}$ Administered by the Metals and Ceramics Division.

${ }^{34}$ S. A. Rabin, Stalus of the Fuel Cycle Irradiation Program at ORNL, ORNL CF-62-7-55.
}

compacted $\mathrm{UO}_{2}-\mathrm{ThO}_{2}$ powder in type 304 stainless steel tubing (Fig. 20.38) have been irradiated, and examinations are in process. Fuel prepared by two differcnt processes is being studied. Fused and ground 4.5 wt $\% \mathrm{UO}_{2}$ fuel was obtained from Spencer Chemical Company, and material of the same composition was fabricated at ORNL by the "sol-gel E" process, which produces the oxide by chemical derivation. All uranium was fully enriched, that is, about $93 \% \mathrm{U}^{235}$.

Preliminary postirradiation data have been obtained $\mathrm{fcr}$ three rods irradiated in the MTR. These data are summarized in Table 20.10. One rod, designated MTR-43-41, contained sintercd fuel pellets for direct comparison with the powder fuels. There was no significant difference in rod appearance after irradiation, and dimensional changes were small. The maximum bowing change was equal to the maximum bow of the rod before irradiation. The maximum diameter change was an increase of about 0.003 in.

Gamma scans indicated a burnup gradient along the rods, as shown in Fig. 20.39. Samples of the fission gas were analyzed by mass and gamma-ray spectrometry. Fractional release values for $\mathrm{Kr}^{85}$ were low, as shown in Table 20.10. 
UNCLASSIFIED ORNL-LR-DWG $64168 C$
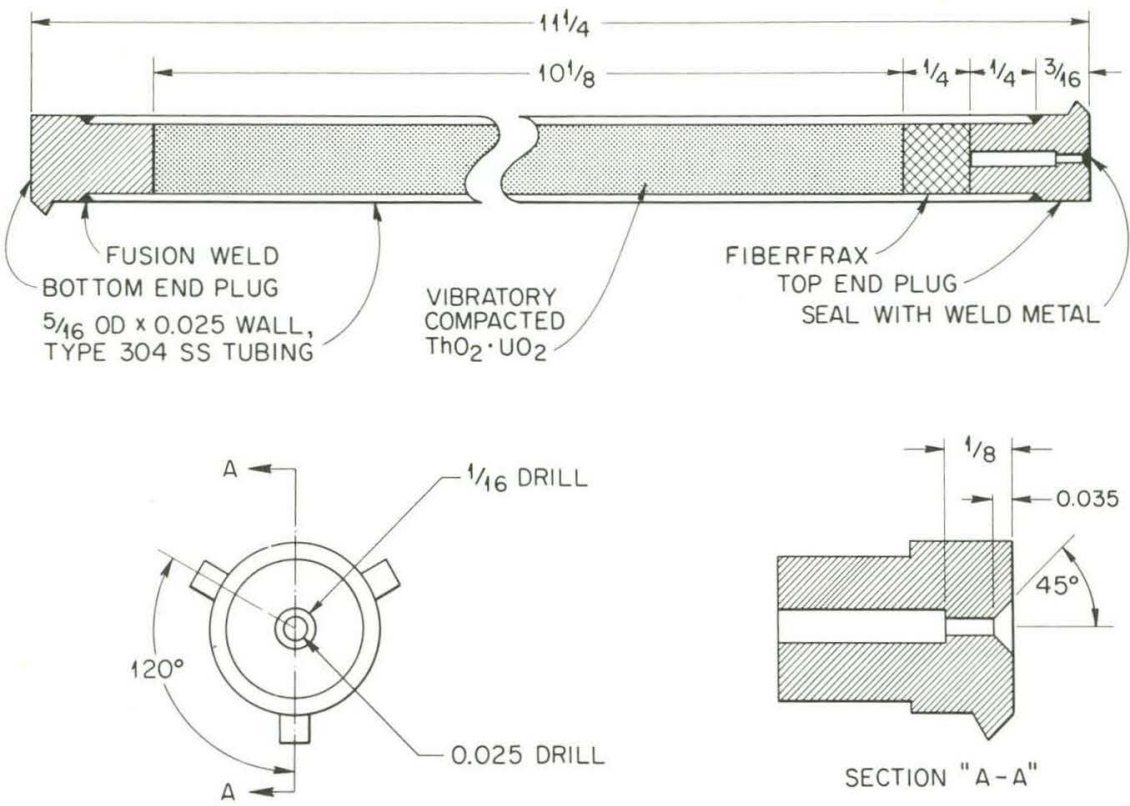

SECTION "A-A"

ALL DIMENSIONS IN INCHES

Fig. 20.38. Design of $\mathrm{ThO}_{2}-\mathrm{UO}_{2}$ Fuel-Cycle Capsule for MTR Group I Irradiations.

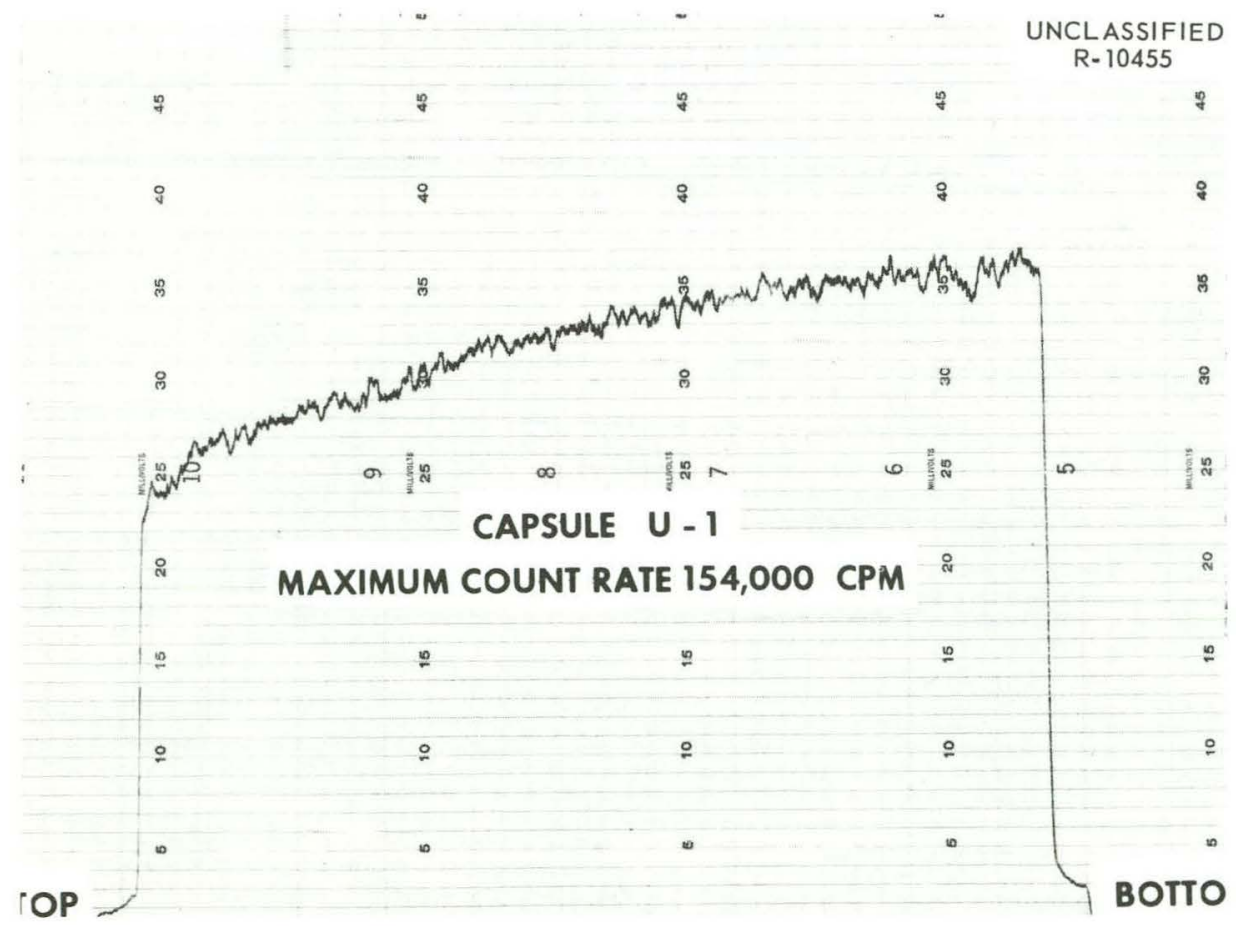

Fig. 20.39. Gamma Radioactivity Scan for Fuel-Cycle Rod MTR-43-59. 
Each rod was sectioned for fuel examination. No change in the appearance of the vibratorily compacted fuel was seen. As expected, the sintered fuel pellets were severely cracked. Samples of the fuel were analyzed for uranium and thorium burnup by radiochemical and mass spectrometric techniques. [Thorium is consumed by the process $\operatorname{Th}^{232}(n, y) \mathrm{Th}^{233} \stackrel{\beta^{-}}{\longrightarrow} \mathrm{Pa}^{233} \stackrel{\beta^{-}}{\longrightarrow}$ $\mathrm{U}^{233}(n, f)$.] The fuel is being examined metallographically.

Table 20.10. Data Summary for Fuel Cycle Rods

\begin{tabular}{clccccc}
\hline $\begin{array}{c}\text { Experiment } \\
\text { No. }\end{array}$ & \multicolumn{1}{c}{ Type of Fuel } & $\begin{array}{c}\text { Uranium } \\
\text { Content } \\
(\text { wt \%) }\end{array}$ & $\begin{array}{c}\text { Fuel Density } \\
(\% \text { of Theoretical) }\end{array}$ & $\begin{array}{c}\text { Heat Flux } \\
\left(B t u \mathrm{hr}^{-1} \mathrm{ft}^{-2} \text { ) }\right.\end{array}$ & $\begin{array}{c}\text { Burnup } \\
\text { (Mwd per metric } \\
\text { ton of metal) }\end{array}$ & $\begin{array}{c}\mathrm{Kr}^{85} \text { Release } \\
(\% \text { of Total) }\end{array}$ \\
\hline MTR-43-41 & Solid pellet & 3.88 & 92.5 & 625,000 & 33,000 & $c$ \\
MTR-43-59 & Fused and ground & 3.96 & 85.7 & 570,000 & 11,000 & 2 \\
MTR-43-63 & Sol-gel & 4.0 & 86.2 & 570,000 & 13,000 & $<1$ \\
\hline
\end{tabular}

${ }^{a}$ Calculated.

${ }^{b}$ Based on mass spectrometer measurements.

${ }^{c}$ Gas sample lost. 


\section{Polymers}

\author{
W. W. Parkinson \\ R. M. Keyser
}

\section{Differences in the Effects of Gamma and Reactor Radiation on Polystyrene}

The previous report ${ }^{1}$ presented measurements demonstrating that, for equal energy deposition, the mixed gamma radiation and neutrons of the ORNL Graphite Reactor produced 2.4 times as many cross-links in polystyrene as did gamma radiation alone. Other workers have found that energetic particles and gamma radiation produce about the same effects in simple aliphatic hydrocarbons. ${ }^{2,3}$ Recent evidence indicates that in aromatic liquids, product yields differ for heavy particles (e.g., neutrons and alphas) and gamma radiation. 4,5 Therefore it is desirable to investigate the differences in molecular structure and in the volatile radiolysis products from polystyrene resulting from irradiation in a gamma source and in a reactor. Radiation-induced changes in the molecular structure of polystyrene are indicated by infrared spectra before and after irradiation. The volatile radiolysis products are being analyzed by gas chromatographic methods.

Infrared Analysis. - For infrared measurements, polystyrene films about $0.001,0.002$, and 0.004 in. thick have been irradiated up to doses of $2.5 \times 10^{23}$ $\mathrm{ev} / \mathrm{g}$ in a water-cooled $\mathrm{Co}^{60}$ gamma source and in

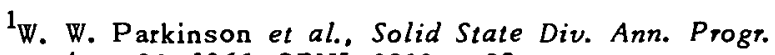
Rept. Aug. 31, 1961, ORNL-3213, p 92.

${ }^{2} \mathrm{H}$. A. Dewhurst and R. H. Schuler, J. Am. Chem. Soc. 81,3210 (1959).

${ }^{3}$ A. E. DeVries and A. O. Allen, J. Phys. Chem. 63, 879 (1959).

${ }^{4}$ W. G. Burns, W. Wild, and T. F. Williams, Proc. U.N. Interm. Con/. Peaceful Uses At. Energy, 2nd, Geneva, 1958 29, 266 (1958).

${ }^{5}$ T. Gaumann and R. H. Schuler, J. Phys. Chem. 65, 703 (1961).
}
W. K. Kirkland
O. Sisman

the ORNL Graphite Reactor. The spectra show that there is a loss of phenyl groups as indicated by the reduction in the $\mathrm{C}-\mathrm{H}$ stretching bands of the aromatic ring at 3088 and $3065 \mathrm{~cm}^{-1}$ and by the reduction in aromatic $\mathrm{C}-\mathrm{H}$ bending modes at 1030 and $905 \mathrm{~cm}^{-1}$. The reduction in these bands after $2.5 \times 10^{23} \mathrm{ev} / \mathrm{g}$ amounts to about $10 \%$ of the original optical density, but is not large enough to show conclusively differences between reactor irradiation and gamma irradiation.

New bands appear at 865,820 , and $675 \mathrm{~cm}^{-1}$, and these peaks. show a greater increase for reactor irradiation than for gamma. The 865 and 820 bands have not been identified with a definite molecular species, but the 675 band corresponds very closely to the $671-\mathrm{cm}^{-1}$. band for out-of-plane $\mathrm{C}-\mathrm{H}$ bending in benzene. ${ }^{6}$ This band also has about the same frequency as a similar bending mode $\left(680\right.$ to $\left.700 \mathrm{~cm}^{-1}\right)$ in cis olefin groups. The narrow width of the band, however, is much more similar to the sharp benzene band than to the broad band of the olefin group.

The reduction in phenyl groups could result from rupture of the aromatic ring to form olefinic side chains. Reduction of phenyl groups could also result from breakage of the bond between the main chain and the benzene ring, followed by the evolution of benzene. The latter possibility will be studied by observation of the 675- and 1030$\mathrm{cm}^{-1}$ bands when irradiated samples are heated under vacuum. The latter band is assigned to corresponding $\mathrm{C}-\mathrm{H}$ bending modes of both the benzene ring and the polystyrene aromatic ring.

A marked difference between gamma- and reactor-irradiated samples is the rate of oxidation

\footnotetext{
${ }^{6}$ N. Herzfeld et al. J. Chem. Soc. 1946, 272.
} 
upon exposure of the samples to air after irradiation. The growth of infrared bands at 3400 and $1710 \mathrm{~cm}^{-1}$, characteristic of the $-\mathrm{OH}$ and $>\mathrm{CO}$ groups, respectively, is an indication of oxidation rate. These bands are negligible in samples which have received $2.5 \times 10^{23} \mathrm{ev} / \mathrm{g}$ of gamma radiation. In contrast, samples receiving equivalent doses of mixed reactor radiation show absorptivities in these bands of 3 to $4 \mathrm{~cm}^{-1}$ after 45 days in air.

Gas Chromatographic Analysis. - Samples of polystyrene were irradiated up to doses of $1.8 \times 10^{23} \mathrm{ev} / \mathrm{g}$ in a water-cooled $\mathrm{Co}^{60}$ source having a dose rate of $3 \times 10^{20} \mathrm{ev} \mathrm{g}^{-1} \mathrm{hr}^{-1}$. The retention characteristics of the gas chromatographic columns made it necessary to separate the volatile products into condensable and noncondensable fractions before analysis. This was accomplished by transferring the products through a detachable U-shaped trap immersed in liquid nitrogen and then into a l'oepler pump. After irradiation all samples were annealed for $4 \mathrm{hr}$ at $100^{\circ} \mathrm{C}$ before being opened and were maintained at this temperature during transfer of radiolysis products to reduce the solubility of gaseous products in the polystyrene.

The noncondensable fraction was collected in the Toepler pump, where its volume was measured. Gas chromatographic analysis, using a PerkinElmer model 154 vapor fractometer with a thermal conductivity detector and a silica gel column, revealed that the noncondensable fraction consisted essentially of hydrogen with trace amounts of methane too small to measure. The Toepler pump therefore afforded a convenient means of measuring the amount of radiation-produced hydrogen in each sample.

The condensable fraction was introduced into the chromatograph and was found to consist entirely of benzene. The identification was carried out by comparing the retention times of benzene with those of the unknown from two separate runs: one on a column of didecyl phthalate on Celite and the other on a column of triphenyl phosphate on Celite. Measurement of the height of the benzene peak allowed a quantitative determination of benzene once the response of the chromatograph had been calibrated in terms of the amount of benzene required to produce a given peak height.

In order to calibrate the chromatograph for benzene, the system ${ }^{7}$ shown in Fig. 21.1 was assem-

\footnotetext{
${ }^{7}$ P. S. Rudolph, private communication.
}

bled for introducing small known amounts of benzene vapor into the chromatograph. The apparatus consisted of a reservoir of benzene maintained at a temperature of $20.00 \pm 0.05^{\circ} \mathrm{C}$, a gas burette, a mercury manometer, and a detachable U-shaped trap. Benzene vapors were allowed to expand into a known volume in the gas burette, the vapor pressure was measured, and the vapor was condensed into the trap with liquid nitrogen. The trap was then removed from the system, attached to the chromatograph, and the contents were flushed onto the column with the carrier gas.

Considerable difficulty was encountered in choosing a suitable stopcock grease for use in contact with benzene vapor. Various fluorocarbon, silicone, polyethylene, and other hydrocarbon greases were tried and found unsatisfactory due to absorption of benzene vapor. A water-soluble grease (Phynal, made by Podbielniak, Inc., Chicago, Illinois) was found to be quite satisfactory, provided traces of water were removed from the grease prior to use. With the use of this grease and the system described, it was possible to introduce known amounts of benzene into the chromatograph with a reproducibility of $2 \%$ or better in the range of 0 to $15 \times 10^{-6}$ mole of benzene.

The analytical results for several gamma-irradiated polystyrene samples are presented in Table 21.1. The values of $G\left(\mathrm{H}_{2}\right)$ are in relatively good agreement with the value of 0.0223 found by Wall and Brown, who studied gas production from polystyrene and deuterated polystyrenes subjected to gamma radiation. ${ }^{8}$

With regard to benzene formation, Wall and Brown concluded that what benzene they observed arose from residual traces of benzene dissolved in the polymer prior to irradiation because their polystyrene samples were purified by precipitation from benzene solution. As a result of the present investigation, however, it appears that benzene production may indeed be radiation induced. The facts that benzene formation increases with dose and that blank runs, in which unirradiated samples were treated in exactly the same fashion as irradiated ones, yielded no detectable amounts of benzene tend to support this contention. Experiments involving samples irradiated to higher doses are in progress to clarify this point.

${ }^{8}$ L. A. Wall and D. W. Brown, J. Phys. Chem. 61, 129 (1957). 
UNCLASSIFIED

ORNL-LR-DWG 73827

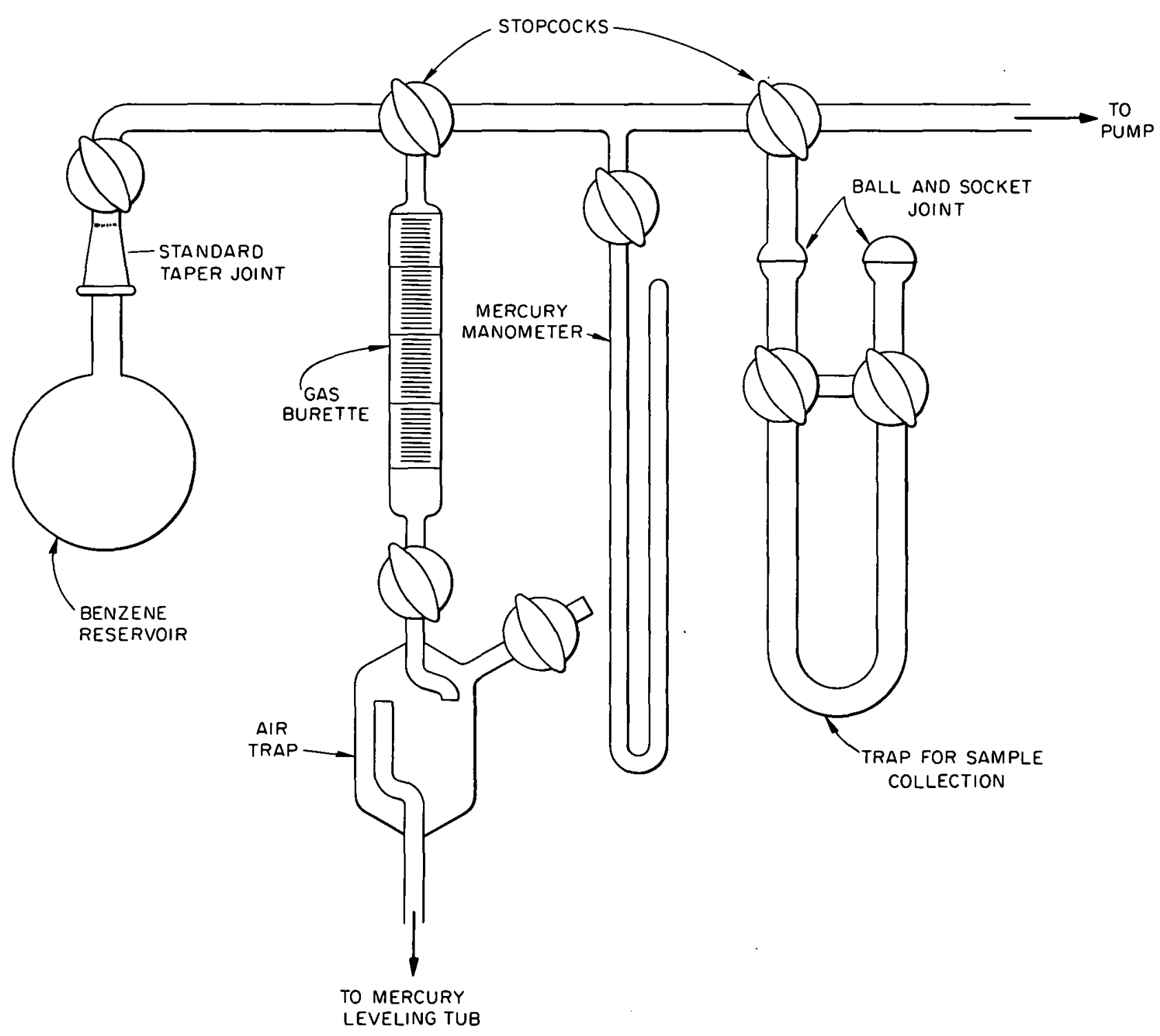

Fig. 21.1. Apparatus for Transferring Known Amounts of Benzene Vapor. 
A Versatile Gamma-Irradiation Facility. - In order to irradiate specimens of plastics and rubbers while mechanical properties are being measured over a broad range of temperatures, a source is required which has an intensity of at least $10^{6}$ $\mathrm{r} / \mathrm{hr}$, uniform over a region of about $2 \mathrm{in}$. in diameter and 2 in. in height, and with maximum accessibility above and around the intense region. To meet these requirements a facility comprised of a cobalt source in a shielded compartment $7 \times 10$ $\mathrm{ft}$ and $7 \mathrm{ft}$ high has been designed. The shielded comparment is designed with concrete walls adequate for 20,000 curies of cobalt.

The source itself is composed of cobalt pellets enclosed in tubcs to form cylindrical source assemblies. These assemblies are arranged vertically around a circle of $27 / 16$ in. in diameter to give a cylindrical source array. The source array is mounted on a screw jack by which the source can be retracted into a hole in the floor of the shielded compartment. A lead shielding plug is moved horizontally to close the storage hole when the source is withdrawn, thus permitting the positioning of specimens and apparatus in the compartment for irradiation.

Toble 21.1. Gos Production

from Gamma-Irradiated Polystyrene

\begin{tabular}{ccccc}
\hline $\begin{array}{c}\text { Dose } \\
(\mathrm{ev} / \mathrm{g})\end{array}$ & $\begin{array}{c}\mathrm{H}_{2} \\
(\mathrm{~mole} / \mathrm{g})\end{array}$ & $\begin{array}{c}\mathrm{C}_{6} \mathrm{H}_{6} \\
(\mathrm{~mole} / \mathrm{g})\end{array}$ & $G\left(\mathrm{H}_{2}\right)$ & $G\left(\mathrm{C}_{6} \mathrm{H}_{6}\right)$ \\
\hline$\times 10^{22}$ & $\times 10^{-6}$ & $\times 10^{-7}$ & $\times 10^{-2}$ & $\times 10^{-4}$ \\
2.16 & 6.36 & 1.65 & 1.77 & 4.60 \\
2.16 & 6.23 & 1.44 & 1.74 & 4.01 \\
2.15 & 6.38 & 1.31 & 1.79 & 3.67 \\
4.86 & 12.6 & 5.29 & 1.56 & 6.54 \\
4.97 & 12.7 & 5.73 & 1.54 & 6.94 \\
8.47 & 23.1 & 10.0 & 1.64 & 7.11 \\
9.91 & 24.6 & 9.58 & 1.49 & 5.82 \\
18.1 & 37.3 & 12.3 & 1.24 & 4.09 \\
\hline
\end{tabular}




\title{
22. Ceramics
}

\author{
C. D. Bopp \\ O. Sisman
}

\section{R. L. Towns}

\section{Thermal Analysis Apparatus}

Equipment has been developed which is suitable for thermal analysis of small samples $(0.01$ to $10 \mathrm{~g})$ of a poor thermal conductor. The method is similar to that of Allison; ${ }^{1}$ however, modifications have been introduced with respect to the places of measurement of temperature and to the method of calibration. A portion of the heat shield (Fig. 22.1) is removable to facilitate the loading of the crucible. After a sample is loaded, the heat shield is placed in a furnace, which is heated at a uniform rate, and curves are taken of both the temperature difference between the crucible and the heat shield, $\delta$, vs time, $t$, and the temperature of the crucible vs time.

A convenient method of calibration ${ }^{2}$ employs Peltier power which is obtained by passing a given value of direct current for a given time through a thermocouple junction attached to the crucible. The area under the resulting peak in the $\delta$ vs $t$ curve is determined; the current is reversed, and the procedure repeated. The difference between the two areas equals twice the area equivalent to the Peltier power. In this method of calculation, the Joule power is eliminated by cancellation. If the Peltier current is applied for a time sufficiently long to reach a steady state, the labor of computing the area under the peaks is avoided. To a good approximation the area is proportional to energy if $\delta$ does not exceed a few degrees.

The Peltier method of calibration was tested by determining the heat of fusion of $\mathrm{NaCl}$. A 50-mg

\footnotetext{
${ }^{1}$ E. B. Allison and (Mrs.) J. Taylor, Trans. Brit. Ceram. Soc. 54, 677 (1955).

${ }^{2}$ W. Steiner and 0 . Krisement, Arch. Eisenbuettenw. 32, 701 (1961).
}

sample was sealed in a silica glass capillary and loaded into the crucible. The peak in the $\delta$ vs $t$ curve was recorded when the fumace was heated uniformly past the fusion temperature $\left(804.3^{\circ} \mathrm{C}\right)$ at a uniform rate of about $2^{\circ} \mathrm{C}$ per minute. The fumace temperature was then held steady at the average temperature of the fusion peak, and the apparatus was calibrated by the Peltier method. The area under the fusion peak, within the reproducibility of repeated trials (about 5\%), was equivalent to the accepted value of the heat of fusion $(124 \mathrm{cal} / \mathrm{g})$.

If it is desired to obtain the specific heat or the heat of a reaction which does not give a sharp peak, a more extensive procedure is required. At the same rate of heating, $\delta$ vs $t$ curves are obtained for the crucible plus the unknown and for the empty crucible. From a plot of the Peltier calibration vs temperature, the difference between the two curves may be interpreted in terms of the apparent specific heat of the unknown.

Nickel proved superior to copper or Monel as the material of construction of the heat shield and crucible, because oxidation of the metal surfaces and the formation of metallic deposits on the insulators were minimized. A vacuum furnace is used to reduce oxidation of the apparatus. At $25^{\circ} \mathrm{C}$ a pressure of less than about $0.1 \mu$ was required to eliminate pressure dependence of the calibration (from heat transfer by convection). The calibration remained constant within the reproducibility of about $5 \%$ except on heating above about $900^{\circ} \mathrm{C}$. By heating to above this temperature and then cooling to a lower temperature, the calibration (in terms of energy per unit area) was increased, but returned to the former value on standing at low temperature. 


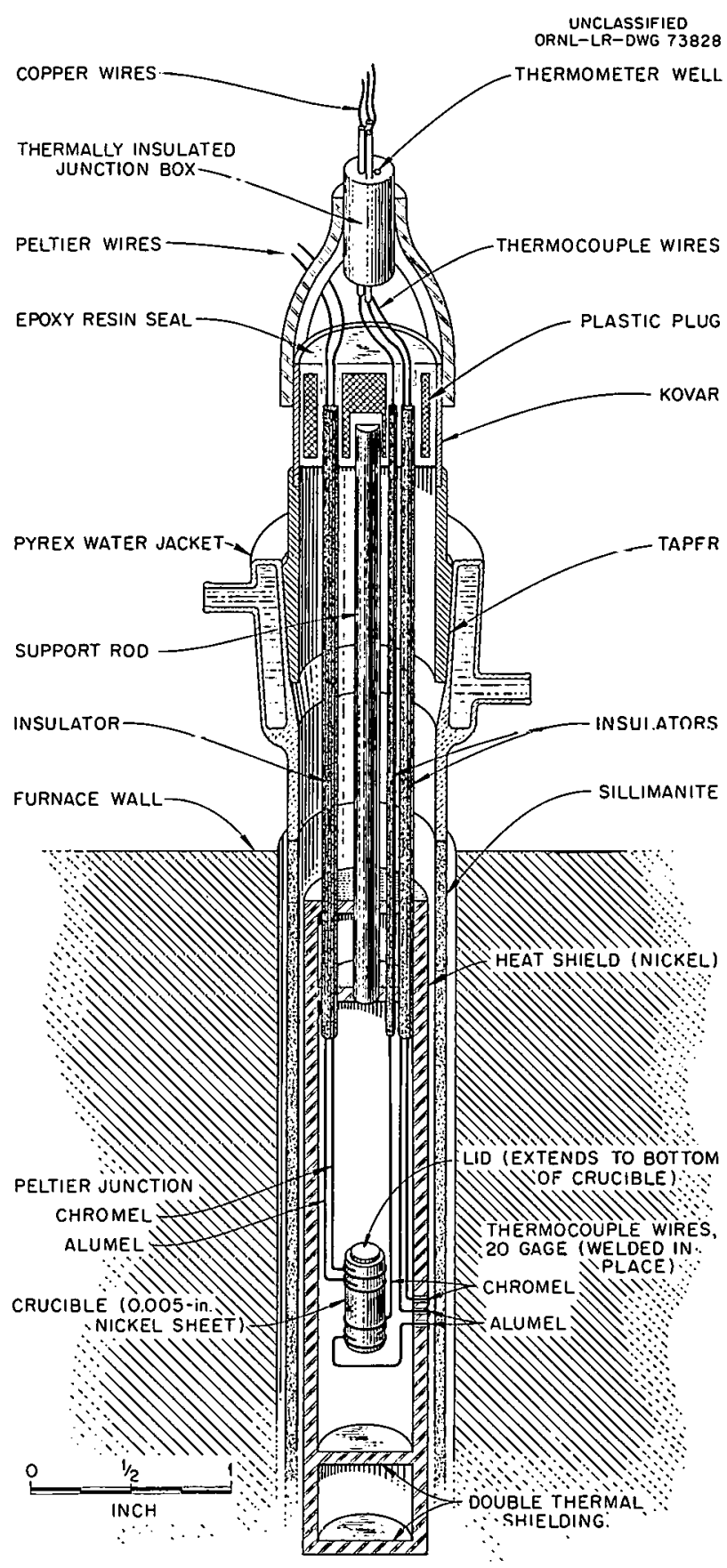

Fig. 22.1. Thermal Analysis Apparatus.

\section{Radiation Stability of a Zircon Ceramic}

Irradiation-induced changes in density, thermal conductivity, and Young's modulus for a zircon ceramic were previously described. ${ }^{3}$ Thermal analysis and $\mathrm{x}$-ray diffraction ${ }^{4}$ were employed in a further study. Reactor irradiation causes the material to resemble metamict zircon ${ }^{5-8}$ in the effects produced by heating. Heating produces partial annealing at relatively low temperature, decomposition at higher temperature, and re-formation of zircon at still higher temperature. According to the degree of irradiation, not all of these processes may occur.

For the range of radiation exposure studied (Table 22.1), the $x$-ray diffraction patterns of all but the sample with the lowest exposure were completely amorphous before heating. The change in the hydrostatically measured density is used to rank the exposures listed in Table 22.1, since this property is easy to measure and the accuracy is probably better than that for the flux estimates. The two highest exposures, for which the density change was saturated, are designated by listing the density change followed by "a" or "b." The density change correlates well with the themal analy sis data, as will be seen. The flux estimates are more accurate for the irradiation facility in the graphite reactor, since the variation with position and the variation during irradiation (from burnup of

\footnotetext{
${ }^{3}$ O. Sisman, C. D. Bopp, and R. L. Towns, Solid State Div. Ann. Progr. Rept. Aug. 31, 1957, ORNL-2413, p 80 .

${ }^{4}$ Debye-Schetrer method by M. C. Wittels and F. A. Sherrill.

${ }^{S}$ M. V. Akhmanova and L. L. Leonova, Geokbimiya $1961,401$.

${ }^{6}$ H. D. Holland and D. Gottfried, Acta Cryst. 8, 291 (1955).

${ }^{7}$ M. V. Stackelberg and K. Chudoba, Z. Krist. 97, 252 (1937).

${ }^{8} \mathrm{~J}$. Orcel, Compt. Rend. 236, 1052 (1953).
}

Table 22.1. Irradiation of Zircon

\begin{tabular}{clcc}
\hline $\begin{array}{c}\text { Density } \\
\text { Decrease } \\
(\%)\end{array}$ & $\begin{array}{c}\text { Reactor } \\
\text { Type }\end{array}$ & $\begin{array}{c}\text { Integrated Neutron Flux } \\
\text { (neutrons } / \mathrm{cm}^{2} \text { ) }\end{array}$ \\
\cline { 3 - 4 } & & $\times 10^{20}$ & $\times 10^{20}$ \\
6.7 & Light water & 5 & 0.5 \\
7.8 & Graphite & 1.3 & 1.0 \\
$10 \mathrm{a}$ & Graphite & 2.0 & 1.6 \\
$10 \mathrm{~b}$ & Light water & 30 & 3 \\
\hline
\end{tabular}


fuel) are less. The temperature of irradiation was less than $100^{\circ} \mathrm{C}$.

The kinetic energy of fission fragments from thermal-neutron-induced fission of trace elements (uranium, $0.022 \%$; thorium, $0.035 \%)^{9}$ is severalfold greater than energy from fast-neutron scattering, but, since the relative efficiency of the two types of radiation is unknown, it is uncertain whether or not fission fragments cause the major portion of the radiation change. It appears from Table 22.1 that the density change may be corre- lated with the epithermal flux. However, the epithermal fluxes in the two irradiation facilities are not directly comparable, since the average energy is higher in the facility in the light-water reactor.

Three exothermic peaks were found in the apparent specific heat of the irradiated zircon (Table 22.2). All three were absent when a sample was

9'Uranium by delayed-neutron activation analysis, $F$. F. Dyer; thorium by activation analysis, W. T. Mullins.

Table 22.2. Thermal Analysis of Irradiated Zircon

\begin{tabular}{|c|c|c|c|}
\hline & $\begin{array}{l}\text { Threshold Temperature of Peak } \\
\qquad\left({ }^{\circ} \mathrm{C}\right)\end{array}$ & $\begin{array}{l}\text { Irradiation-Induced } \\
\text { Density Decrease } \\
\text { (\%) }\end{array}$ & $\begin{array}{l}\text { Energy Under Peak } \\
(\mathrm{cal} / \mathrm{g})\end{array}$ \\
\hline & $200 \pm 30$ & 6.7 & $0.5 \pm 0.3$ \\
\hline & $200 \pm 30$ & $10 \mathrm{a}$ & 0.03 \\
\hline & (Low-temperature peak not detected) & $10 \mathrm{~b}$ & \\
\hline & $880 \pm 10$ & 6.7 & 15 \\
\hline & $880 \pm 10$ & 7.8 & 15 \\
\hline & $880 \pm 10$ & $10 \mathrm{a}$ & 16 \\
\hline & $880 \pm 10$ & $10 \mathrm{~b}$ & 16 \\
\hline & 1090 & 6.7 & $25 \pm 10$ \\
\hline & $\begin{array}{l}\text { (High-temperature peak not detected } \\
\text { below } 1200^{\circ} \mathrm{C} \text { ) }\end{array}$ & 7.8 & \\
\hline- & $\begin{array}{l}\text { (High-temperature peak not detected } \\
\text { below } 1200^{\circ} \mathrm{C} \text { ) }\end{array}$ & $10 \mathrm{a}$ & \\
\hline
\end{tabular}

Table 22.3. X-ray Diffraction Patterns of Irradiated Zircon ${ }^{a}$

Heat Treatment

None

6.7.

None

7.8

After heating to $400^{\circ} \mathrm{C}$

6.7

After heating to $900^{\circ} \mathrm{C}$

$10 \mathrm{~b}$

After heating to $1100^{\circ} \mathrm{C}$
6.7
Irradiation-Induced

(\%)
Description
Extremely broad lines

Amorphous

Moderately broad zircon lines; no zirconia lines

Moderately broad zirconia lines; no zircon or quartz lines

Sharp zircon lines; no zirconia lines

\footnotetext{
${ }^{a}$ See ref. 4.
} 
heated a second time. The threshold temperatures of the peaks are 200,880 , and $1090^{\circ} \mathrm{C}$. The decrease in line breadth (Table 22.3) associated the the $200^{\circ} \mathrm{C}$ peak with annealing of a part of the radiation change. This peak was absent in the most highly irradiated sample. The $880^{\circ} \mathrm{C}$ peak is identified with the decomposition of zircon to zirconia (however, both the zircon and zirconia patterns show broadened lines). Decomposition was undergone by all the samples studied, and there was small effect of the degree of irradiation on the temperature or energy of the reaction. The $1090^{\circ} \mathrm{C}$ peak, which is identified with the formation of zircon from zirconia and silica, was found only for the lowest radiation exposure. The absence of this peak in the more highly irradiated samples is probably related to the lower density of such samples (Table 22.4).

Table 22.4. Density (at $25^{\circ} \mathrm{C}$ ) After Decomposition

\begin{tabular}{ccc}
\hline $\begin{array}{c}\text { Irradiation-Induced } \\
\text { Density Decrease } \\
(\%)\end{array}$ & $\begin{array}{c}\text { Density } \\
\text { Before } \\
\text { Heating } \\
\left(\mathrm{g} / \mathrm{cm}^{3}\right)\end{array}$ & $\begin{array}{c}\text { Density } \\
\text { After } \\
\text { Heating } \\
\text { to } 900^{\circ} \mathrm{C} \\
\left(\mathrm{g} / \mathrm{cm}^{3}\right)\end{array}$ \\
\hline Unirradiated & 3.70 & 3.76 \\
7.8 & 3.44 & 3.62 \\
$10 \mathrm{a}$ & 3.36 & 3.47 \\
$10 \mathrm{~b}$ & 3.38 & 3.39 \\
\hline
\end{tabular}




\section{Radiation Dosimetry}

C. D. Bopp
W. K. Kirkl and

The use of mixtures of a hydrocarbon and halogenated compounds to measure radiation dose by means of hydrogen ion production was described earlier. ${ }^{1}$ By selection of mixtures having the minimum and maximum hydrogen content, it should be possible to prepare dosimeters sensitive only to gamma radiation in one case and sensitive to both gammas and fast neutrons in the other case.

A solution of low hydrogen content consisting of 0.3 we $\% \mathrm{C}_{10} \mathrm{H}_{22}$ and 0.3 we $\% \mathrm{C}_{2} \mathrm{H}_{2} \mathrm{Cl}_{4}$ in $\mathrm{C}_{8} \mathrm{~F}_{16}$ would receive no more than $5 \%$ of its total dose from interaction with neutrons when exposed to the flux of most reactors. This mixture gave hydrogen ion yields of poor reproducibility in a series of irradiations in a gamma source. The variation in yields appears to have resulted from variations in moisture condensed in the solution by the chilling process employed during the evacuation and sealing of the containers. Efforts to reduce water contamination did not improve the reproducibility of yields, but the introduction of a slight excess of water over saturation resulted in consistent yields. Since the solubility of water in the mixture is low, the added water does not increase the interaction with fast neutrons appreciably. The acid produced in wet dosimeter mixtures having about $0.002 \mathrm{~g}$ of water per gram of solution is plotted in Fig. 23.1.

For a dosimeter solution sensitive to both fast neutrons and gamma radiation, solutions of $0.3 \mathrm{wt}$ $\% \mathrm{C}_{2} \mathrm{H}_{2} \mathrm{Cl}_{4}$ in decane $\left(\mathrm{C}_{10} \mathrm{H}_{22}\right)$ were prepared. Specimens were irradiated in gamma sources at room temperature and at $80^{\circ} \mathrm{C}$ and at two intensities to measure the linearity and the dependence on temperature and intensity. The hydrogen ion or acid yields are shown in Fig. 23.2. The high

${ }^{1}$ C. D. Bopp et al., Solid State Div. Ann. Progr. Rept. Aug. 31, 1961, ORNL-3213, p 101.
O. Sisman

W. W. Parkinson

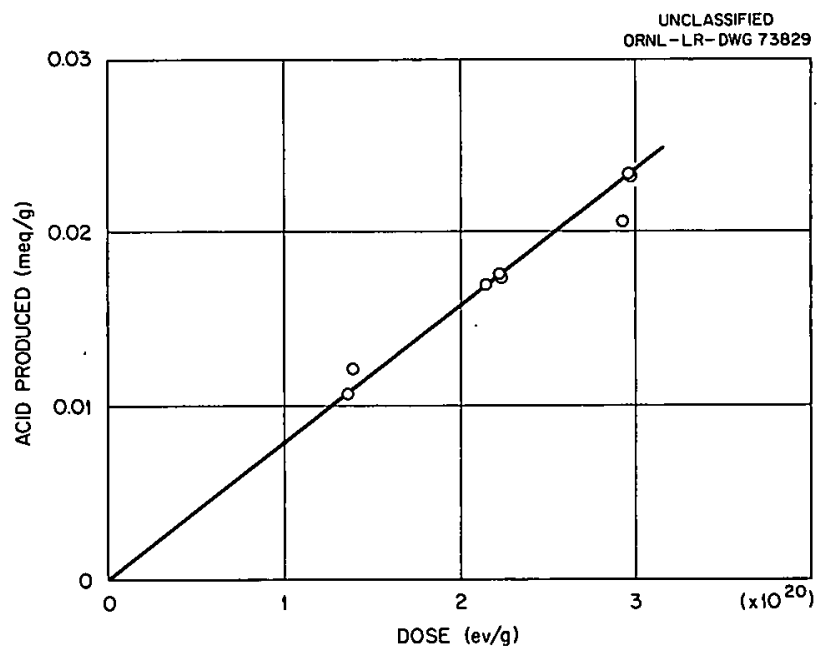

Fig. 23.1. Acid Production in Wet $\mathrm{C}_{8} \mathrm{~F}_{16}-\mathrm{C}_{10} \mathrm{H}_{22}$ $\mathrm{C}_{2} \mathrm{H}_{2} \mathrm{Cl}_{4}$.

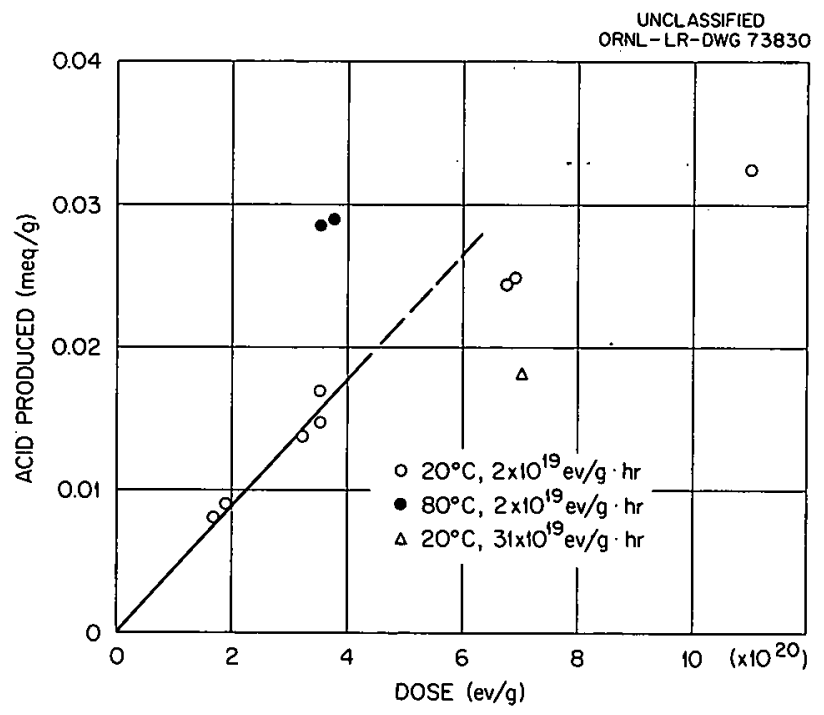

Fig. 23.2. Acid Production in $\mathrm{C}_{10} \mathrm{H}_{22}-\mathrm{C}_{2} \mathrm{H}_{2} \mathrm{Cl}_{4}$. 
yields at $80^{\circ} \mathrm{C}$ indicate that in the reaction mechanisms of acid production, there is at least one ratecontrolling process which is temperature dependent, perhaps a diffusion process or atomic abstraction step. The lower yield at high intensity suggests that in the overall reaction there is a step involving the disappearance of an active species, without producing hydrogen ions, which is bimolecular and, hence, inten sity dependent.

These intensity- and temperature-dependent steps are probably inherent in the acid production re- actions in a hydrocarbon solvent. The possibility of altering the acid production reactions was investigated by adding water and ethanol to the dosimeter solution. Ethanol was added to make up 21.1 wt \% to permit dissolving 0.21 wt \% water. Irradiations in progress indicate that dependence on temperature may be reduced, but not eliminated. Figure 23.3 presents the acid production as a function of dose for dosimeter solutions composed of 78.4 wt \% $\mathrm{C}_{10} \mathrm{H}_{22}, 21.1$ wt $\% \mathrm{C}_{2} \mathrm{H}_{2} \mathrm{OH}, 0.24$ wt $\% \mathrm{C}_{2} \mathrm{H}_{2} \mathrm{Cl}_{4}$, and 0.21 we $\% \mathrm{H}_{2} \mathrm{O}$.

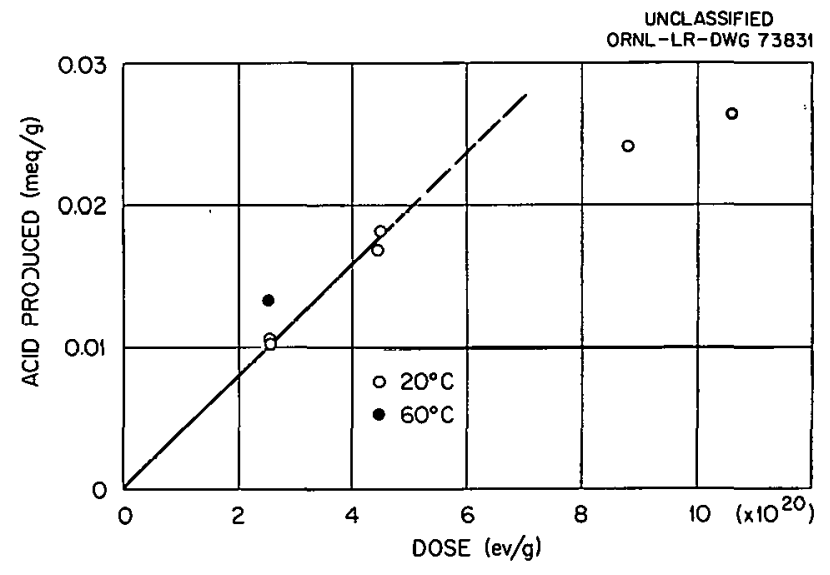

Fig. 23.3. Acid Production in $\mathrm{C}_{10} \mathrm{H}_{22}-\mathrm{C}_{2} \mathrm{H}_{5} \mathrm{OH}$ $\mathrm{C}_{2} \mathrm{H}_{2} \mathrm{Cl}_{4}-\mathrm{H}_{2} \mathrm{O}$. 
THIS PAGE

\section{WAS INTENTIONALLY LEFT BLANK}


Publications and Papers 


\section{THIS PAGE}

\section{WAS INTENTIONALLY \\ LEFT BLANK}




\section{Publications and Papers}

R. F. Bass, J. W. Cleland, and J. H. Crawford, Jr.

"Radiation Effects on Deep-Level Impurity States in Gc," Bull. Am. Phys. Soc. 6, 461 (1961).

C. Bassani, M. S. Wechsler, J. M. Williams, J. H. Barrett, and R. H. Kernohan

"The Dependence of Radiation-Enhanced Diffusion in Cu-Al on Neutron Flux and Energy Spectrum," Bull. Am. Phys. Soc. 7, 170 (1962).

R. G. Berggren

"Critical Factors in the Interpretation of Radiation Effects on the Properties of Metals," presented at 1962 Nuclear Congress, New York, June 1-T, 1962.

D. S. Billington and D. O. Thompson

"Radiation Damage Research in Low-Power Nuclear Reactors," presented at Intemational Atomic Energy Agency Symposium on the Programming and Utilization of Research Reactors, Vienna, Austria, Oct. 16-20, 1961.

T. H. Blewitt and R. R. Coltman

"Cryogenic Techniques for Irradiation Studies in Nuclear Reactors," p 274 in Experimental Cryophys. ics (ed. by F. E. Hoare et al.), Butterworths, London, 1961.

T. H. Blewitt, R. R. Coltman, C. E. Klabunde, and D. L. McDonald

"Low Temperature Resistivity of Sodium and the Effects of Nuclear Irradiation," J. Appl. Phys. 33, 1612 (1962).

C. D. Bopp and R. L. Towns

"Calorimetric Measurement of Nuclear Heating in a Reactor," Nucl. Sci. Eng. 13, 245 (1962).

R. H. Busey and E. Sonder

"Magnetic Susceptibility of Potassium Hexachlororhenate (IV) and Potassium Hexabromorhenate (IV) from 5 to $300^{\circ} \mathrm{K} ", J$. Chem. Phys. 36, 93 (1962).

R. M. Carroll

"Argon Activation Measures Irradiation Flux Continuously," Nucleonics 20(2), 42 (1962).

"Continuous Release of Fission Gas from UO, During Irradiation," p 110 in Symposium on Radiation Effects in Refractory Fuel Compounds, ASTM-STP-306, 1961.

J. G. Castle, Jr., D. W. Feldman, and R. A. Weeks

"Spin-Lattice Relaxation of $E_{1}^{\prime}$ Centers in SiO 2 ," Bull. Am. Phys. Soc. 7, 188 (1962).

J. W. Cleland, R. F. Bass, and J. H. Crawford, Jr.

"The Nature and Yield of Radiation-Induced Defects in Semiconductors," Bull. Am. Pbys. Soc. 7, 186 (1962).

R. R. Coltman

"Reactor Irradiation Studies at $4{ }^{\circ} \mathrm{K}$," presented at Intemational Atomic Energy Agency Symposium on Radiation Damage in Solids and Reactor Materials, Venice, Italy, May 7-11, 1962.

"Thermal and Fast Neutron Damage in Metals," presented at Southeastern Section :Meeting, American Physical Society, Tallahassee, Florida, Apr. 5-7, 1962. 
T. F. Connolly

Operation of Research Materials Information Center, ORNL-TM-295 (July 30, 1962).

J. H. Crawford, Jr.

"Radiation Stability of Nonmetallic Structures," Progr. Nucl. Energy, Ser. V, 3, 371 (1961).

J. H. Crawford, Jr., and J. W. Cleland

"Transmutation Doping and Recoil Effects in Semiconductors Exposed to Thermal Neutrons," p 269 in Radioisotopes in the Pbysical Sciences and Industry, Intemational Atomic Energy Agency, Vienna, 1962.

D. L. Curtis, Jr.

"Effect of a Phase Transformation on the Vapor Phase Growth of Single-Crystal Igৎ," J. Appl. Phys. 33, 2461 (1962).

o. L. Curtis, Jr., and J. H. Crawford, Jr.

"Carrier Recombination and Trapping Processes in Irradiated Germanium," Discussions Faraday Soc. 31, 107 (1961).

"Radiation-Induced Recombination and Trapping Centers in Germanium. I. The Nature of the Recombination Process," Phys. Rev. 124, 1731 (1961).

"Radiation-Induced Recombination and Trapping Centers in Germanium. II. Annealing in GammaIrradiated, Antimony- and Arsenic-Doped Material," Phys. Rev. 126, 1342 (1962).

R. Gwin

"Studies of the Scintillation. Process in CsI(Tl)," thesis, submitted to the Graduate Council of the University of Tennessee in partial fulfillment of the requirements for the Ph.D. degree, 1962.

R. Gwin and R. B. Murray

"Studies of the Scintillation Process in CsI(Tl)," IRE Trans. Nucl. Sci. NS.9, 28 (1962).

D. K. Holmes

"Approximate Methods for Treating Annealing Processes which Involve a Spectrum of Activation Energies," p 449 in Cbemical Effects of Nuclear Transformations, vol I, Intemational Atomic Energy Agency, Vienna, 19.61.

"The Ranges of Energetic Atoms in Solids," presented at International Atomic Energy Agency Symposium on Radiation Damage in Solids and Reactor Materials, Venice, Italy, May 7-11, 1962.

D. K. Holmes, J. W. Corbett, R. M. Walker, J. S. Koehler, and Fredrick Seitz

"On the Interpretation of Radiation Effects in Noble Metals," Progr. Nucl. Energy, Ser. V, 3, 178 (1961).

D. K. Holmes and D. O. Thompson

"The Investigation of Radiation Damage Using !-jechanical Vibrations," P IX-1 in Resonance and Relaxation in Metals, American Society for Metals, 1962.

L. D. Hulett, Jr.

"Dissolution of Single Crystals of Copper in Aqueous Systems," presented at Southeastem Section American Chemical Society Meeting, New Orleans, Louisiana, Dec. 7-9, 1961.

L. H. Jenkins and J. O. Stiegler

"El ectrochemical Dissolution of Single Crystalline Copper," J. Electrocbem. Soc. 109, 467 (1962).

C. E. Klabunde, R. R. Coltman, and D. L. McDonald

"Low. Temperature $n, y$ Recoil Damage in Cadmium," Bull. Am. Pbys. Soc. 7, 171 (1962).

G. Leibfried

"Calculation of Averages for Primary Recoil Distributions," J. Appl. Phys. 33, 1933 (1962).

G. Leibfried and O. S. Oen

"A Simple Approximation for Classical Scattering at Large Angles," J. Appl. Phys. 33, 2257 (1962). 
E. Lell and R. A. Weeks

"Dielectric Measurements in Some Irradiated Glasses," presented at Conference on Irradiation Effects in Simple Glasses and Crystals, Rochester, New York, Apr. 12-13,1962.

D. I.. McDonald, R. R. Coltman, and C. E. Kläbunde

"Contribution of Slow Neutrons to Reactor Damage in Metals," Bull. Am. Pbys. Soc. 7, 171 (1962).

R. B. Murray and A. Meyer

"Effect of Delta Rays on the Response of Inorganic Scintillators to Heavy Particles," IRE Trans. Nucl. Sci. NS-9, 33 (1962).

T. S. Noggle and J. O. Stiegler

"Fission Fragment Tracks in Metal Films," J. Appl. Pbys. 33, 1726 (1962).

"Fission Fragment Tracks in Thin Films of UO ," PP 47-63 in Symposium on Radiation Effects in Refractory Fuel Compounds, ASTM-STP-306, 1961.

O. S. Den, M. T. Robinson, and D. K. Holmes

"The Ranges of Energetic Atoms in Solids. I. Random Models," Bull. Am. Phys. Soc. 7, 171 (1962).

M. F. Osborne, A. E. Goldman, E. I. Long, Jr., F. R. McQuilkin, and J. G. Morgan

"Beryllium Sheathed UO 2 Fuel Element Irradiation Tests," Trans. Am. Nucl. Soc. 5, 43 (1962).

M. F. Osbome and J. G. Murgan

Post-Irradiation Examinations of Uranium Monocarbide, ORNL CF-62-8-15 (Aug. 9, 1962).

V. K. Paré and D. O. Thompson

"Temperature Dependence from $250^{\circ} \mathrm{K}$ to $370^{\circ} \mathrm{K}$ of Dislocation Pinning in Copper Single Crystals by Radiation Defects," Acta Met. 10, 327 (1962).

P. E. Reagan

"Fission Gas Release from Coated Particles Irradiated in ORR Facilities B9 and C1," presented at Third Meeting of Coated Particle Work Group, Oak Ridge National Laboratory, Oak Ridge, Tennessee, May 7-8, 1962.

L. D. Roberts, H. Pomerance, J. O. Thompson, and C. F. Dam

"A Mössbauer Study of a Series of Gold Alloys and Compounds," presented at Southeastem Section Meeting, American Physical Society, Tallahassee, Florida, Apr. 5-7, 1962.

M. T. Robinson, D. K. Holmes, and O. S. Oen

"Monte Carlo Calculations of the Ranges of Energetic Atoms in Solids," presented at Intemational Colloquium on Ion Bombardment, Bellevue, France, Dec. 4-8, 1961.

"The Ranges of Energetic Atoms in Solids. II. Lattice Model," Bull. Am. Phys. Soc. 7, 171 (1962).

H. C. Schweinler

"Distribution in Kinetic Energy of Daughter Nuclei," p 63 in Chemical Effects of Nuclear Transformations, vol I, International Atomic Energy Agency, Vienna, 1961.

H. C. Schweinler and J. W. Miller

"Trapping and Recombination Probabilities in Nonmetals," Bull. Am. Pbys. Soc. 7, 495 (1962).

E. Sonder

"?.1agnetic Properties of $F$ and $M$ Centers in Potassium Choride," Bull. Am. Phys. Soc. 7, 37 (1962).

"Magnetism of KCl," Phys. Rev. 125, 1203 (1962).

E. Sonder and S. T. Sekula

"Effects of Gaseous Impurities on the Magnetic Properties of Copper," J. Phys. Cbem. Solids 36, 93 (1962).

E. Sonder and W. A. Sibley

"The Relation Between $F$ and $M$ Centers in $\mathrm{KCl}$ at Room Temperature," presented at Intemational Symposium on Color Centers, Stuttgart, Germany, Aug. 21-24, 1962. 
J. O. Stiegler and T. S. Noggle

"Nitrogen Ion Bombardment of Thin Pt Films," J. Appl. Pbys. 33, 1894 (1962).

D. O. Thompson

"A Thermally Activated Internal Friction Spectrum in $\mathrm{Cu}$.Following Neutron Irradiation," Acta. Met. 10, 327 (1962).

D. O. Thompson and V. K. Paré

"Effect of Fast Neutron Irradiation on Dislocation Intemal Friction in Pure Copper at Megacycle Frequencies," Bull. Am. Pbys. Soc. 7, 171 (1962).

"Measurements of Fast Neutron Produced Defect Motion in Copper from $250^{\circ} \mathrm{K}$ to $370^{\circ} \mathrm{K}$ Using Internal Friction Techniques," presented at Diskussionstagung uber Strahlungschaden und Plastische Verformung, Gottingen, Germany, Oct. 9-13, 1961.

D. F. Toner

"LITR Static Capsule Tests for Coated UC, Particles," presented at Third Meeting of Coated Particle Work Group, Oak Ridge National Laboratory, Oak Ridge, Tennessee, May 7-8, 1962.

"Post-Irradiation Examination of MTR-48 Fuel Bodies," presented at Third Meeting of Coated Particle Work Group, Oak Ridge National Laboratory, Oak Ridge, Tennessee, May 7-8, 1962.

M. S. Wechsler and R. H. Kemohan

"The Effect of Radiation on Diffusion-Controlled Reactions in Copper-Base Alloys," presented at International Atomic Energy Agency Symposium on Radiation Damage in Solids and Reactor Materials, Venice, Italy, May 7-11, 1962.

M. S. Wechsler, J. M. Williams, and H. M. Otte

"The Annealing of Cold-Worked Silicon Bronze," J. Metals 14, 81 (1962).

R. A. Weeks

"Paramagnetic Properties of $E_{2}^{\prime}$ Centers in Crystalline Quartz," presented at 1962 Conference on Radiation Effects on Glass, Irradiation Effects in Simple Glasses and Cry stals, Rochester, New York, Apr. 12-13, 1962.

"The Interaction of $\mathrm{O}-\mathrm{H}$ in Silica and Its Effect on the $E_{1}$ Center," preserted at Fourteenth Annual Pacific Coast Regional Meeting, American Ceramic Suciety, San Francisco, California, Oct. 25-27, 1961.

R. A. Weeks and E. Sonder

"The Relation Between the Magnetic Susceptibility, Electron Spin Resonance, and the Optical Absorption of the $E_{1}$ Center in Fused Silica," presented at First Intemational Conference on Paramagnetic Resonance, Jerusalem, Israel, July 16-20, 1962.

J. E. White, W. W. Parkinson, D. Binder, and C. D. Bopp

"A Comparison of Reactor Neutrons to Gamma Radiation in Crosslinking Polystyrene," presented at 2nd Conference on Nuclear Reactor Chemistry, Gatlinburg, Tennessee, Oct. 10-12, 1961.

M. C. Wittels

"Stored Energy in the Dak Ridge Graphite Reactor - 1960," J. Nucl. Energy, Pts. A \& B 15, 185 (1961).

M. C. Wittels, J. O. Stiegler, and F. A. Sherrill

"Au ${ }_{0.82} \mathrm{Hg}_{0.18}$ : A Transmutation Alloy," J. Appl. Phys. 33, 241 (1962).

"Radiation Effects in Uranium-Doped Zirconia," J. Nucl. Energy, Pts. A \& B 16, 237 (1962).

F. W. Young, Jr.

"Elastic-Plastic Transition in Copper Crystals as Detemined by an Etch Pit Technique," J. Appl. Phys. 32, 1815 (1961).

"Etching of Irradiated Copper," J. Appl. Phys. 33, 749 (1962). 
F. W. Young, Jr.

"On the Formation of Dislocations Around Precipitate Particles of $\mathrm{Cu}_{2} \mathrm{O}$ in $\mathrm{Cu}, " \mathrm{p} 103$ in Direct Observation of Imperfections in Crystals (ed. by J. B. Newkitk and J. H. Wemick), Interscience, New York, 1962.

"On the Growth of Copper Crystals of Low Dislocation Density," Bull. Am. Pbys. Soc. 7, 215 (1962).

"On the Yield Stress of Copper Crystals," J. Appl. Phys. 33, 963 (1962).

\section{SOLID STATE SEMINARS AT ORNL}

The following speakers gave seminars to members of the Solid State Division during the period covered by this report:

1961

September 12

September 25

Octuber 10

October 19

October 26

November 6

Decomber 7

December 14

1962

February 1

February 2

February 16

March 14,15

April 6

April 18

June 26
H. M. Otte, RIAS

"Lattice Parameter Changes Associated with Solute Clustering in Alloys"

A. A. Johnson, Imperial College, Iniversity of London

"Radiation Damage in bcc Transition Metals"

P. Baruch, Ecole Normale Superieure, Paris

"Studies of Irradiation F.ffects in Semiconductors at Ecole Normale Superieure"

J. J. Markham, Armour Research Foundation

"The Absorption and Luminescent Properties of the F-Center"

F. Bassani, Argonne National T.aboratory

"Band Structure in Compound Semiconductors"

G. Watkins, General Electric Research Laboratory

"Radiation-Produced Defects in Silicon"

R. Smoluchowski, Princeton University

"Present Status of the Mechanism of Defect Formation in Ionic Crystals"

R. Warren, Westinghouse Research Laboratories

"Purification of Alkali Halides"

H. J. Stoecker, EUR A TOM

"Structures of Zirconia Solid Solutions Containing Various Oxides"

R. K. Swank, University of Illinois

"Lifetimes of Excited States in Color Centers"

H. Rabin, Naval Research Laboratory

"Equilibria of $F$ and $M$ Centers"

R. S. Knox, University of Rochester

"Theory of Excitons"

T. J. Koppenaal, Armour Research Foundation

"The Solid Solution Strengthening in Alpha Cu-Al Single Crystals"

E. W. J. Mitchell, University of Reading

"Recent Work on Radiation Damage in Solids at Reading University"

M. M. Saffren, General Electric Research Laboratory

"The Order of Lcvels of Symmetric Hamiltonians" 
July $12^{\circ}$ J. Silcox, Cornell University

"Dislocations and Magnetic Domain Walls in Thin Films of Cobalt and Nickel"

August 15

C. Walker, University of Chicago

"A Neutron Diffraction Study of Short Range Order in Beta-Brass"

During June and July, 1962, W. H. Young of Cornell University gave a series of lectures on "The Theory of Supercon ductivity."

\section{EDUCATIONAL LECTURES AND SEMINARS}

Four speakers from the Solid State Division participated in the University Traveling Lecture Program sponsored by ORINS. They presented the following lectures under the auspices of this program.

J. H. Barrett

O. L. Curtis, Jr.

W. W. Parkinson

M. T. Robinson

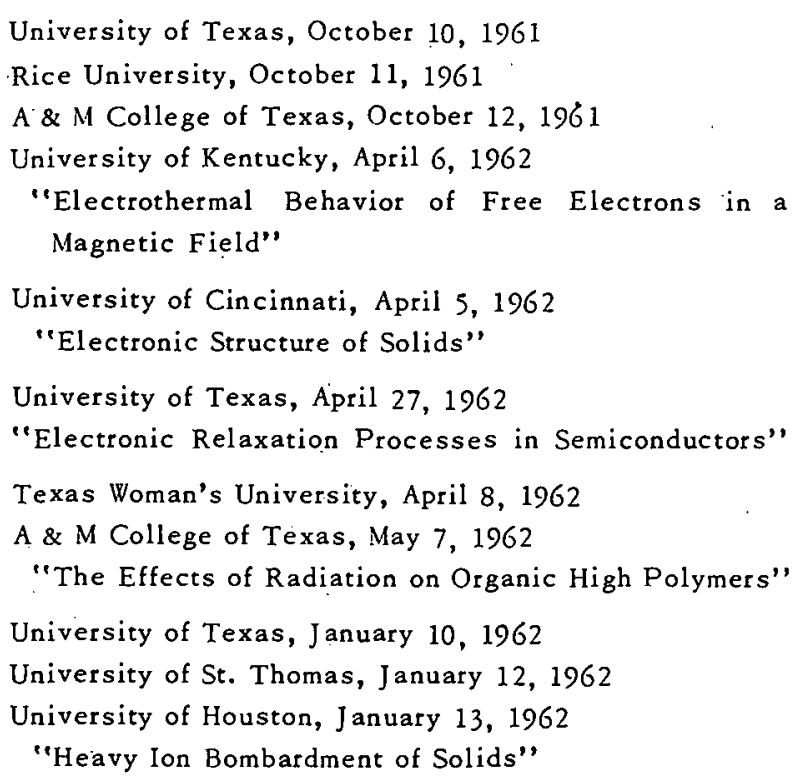

University of Texas, October 10, 1961

A \& M College of Texas, October 12, 1961

University of Kentucky, April 6, 1962

'Electrothermal Behavior of Free Electrons in a

niversity of Cincinnati, April 5, 1962

"Electronic Structure of Solids"

University of Texas, April 27, 1962

Texas Woman's University, April 8, 1962

A \& M College of Texas, May 7, 1962

"The Effects of Radiation on Organic High Polymers"

University of Texas, January 10,1962

University of St. Thomas, January 12, 1962

"Heavy Ion Bombardment of Solids"

Other educational lectures were presented by members of the Division. A series uf lectures on "Radiation Damage Research in University Reactors" was given at Georgia Institute of Technology from April 20 to May 25:

D. S. Billington

J. W. Gleland

D. K. Holmes

D. O. Thompson

M. C. Wittels

\footnotetext{
"Radiation Damage Research at Nuclear Reactors," April 20, 1962

"Radiation Damage Investigations on Semiconductors Using Nuclear Reactors," May 4, 1962

"Theory of the Radiation Damage Process in Solids" and "Calculation of Fast Neutron Fluxes," May 25, 1962.

"Experiments in Metallic Inelasticity Using Nuclear Reactors," May 11, 1962

"X-Ray. Diffraction Studies of Neutron Irradiated Solids," April 13, 1962
} 
The Division also assisted in the program on graduate nuclear engineering education sponsored by General Electric Company, Richland, and Washington State University, held at Richland, Washington, during the month of August:

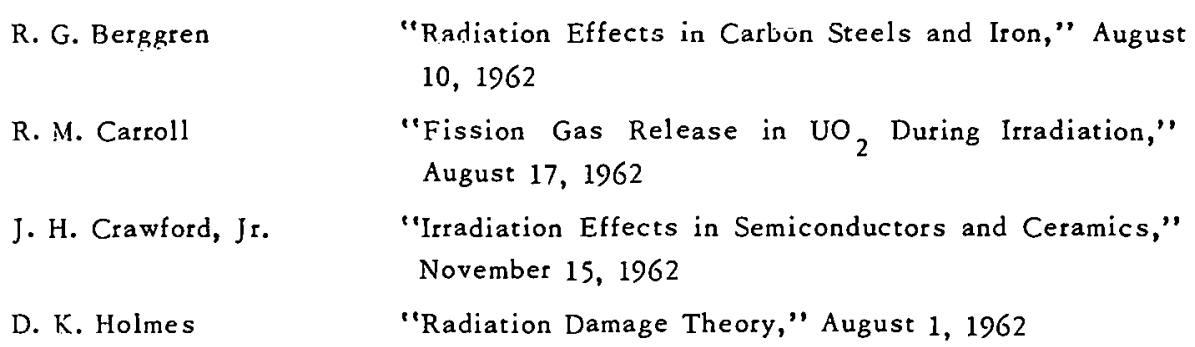

H. C. Schweinler was one of the principal lecturers at the ORINS-AEC Summer Institute for Physics Teachers from Small Colleges, held at Oak Ridge July 23 - August 17. He gave a series of 20 lectures on "Quantum Mechanics and Solid State Physics." He also presented a series of five lectures on "Solid State Physics" at a summer school devoted to physics, held at Beloit College, Beloit, Wisconsin, July 11-13, 1962.

A number of other seminars and lectures were presented during the year by members of the Division, both in this country and ahroad:
R. M. Carroll
General Electric Company, San Jose, California, May 1, 1962.
"Fission Gas Release Studies"
R. R. Coltman
Argonne National Laboratory, Argonne, Illinois, March
1,1962
"Thermal and Fast Neutron Damage in Metals"
J. H. Crawford, Jr.
Arizona State University, Tempe, Arizona, November
15,1961
"Radiation Effects in Semiconductors"
O. L. Curtis, Jr.
Arizona State University, Tempe, Arizona, January 8,1962
Sandia Corporation, Albuquerque, New Mexico, May 2, 1962
"Electronic Relaxation Processes in Semiconductors"
Lockheed Missile and Space Corporation, Palo Alto, California, May 15, 1962
"Recombination and Trapping in Co ${ }^{60} y$-Irradiated $\mathrm{Ge} "$

G. Leibfried

University of Illinois, Urbana, Illinois, March 2, 1962

Argonne National Laboratory, Argonne, Illinois, March 5, 1962

University of Virginia, Charluttesville, Virginia, March 23, 1962

"Slowing Down of Primary Recoils in Solids"

R. B. Murray

University of Tennessee, Knoxville, Tennessee,
December 5, 1961
University of Delaware, Newark, Delaware, June 27,
1962
"Scintillation Process in Alkali Halide Crystals",

O. S. Oen

Neutron Physics Division, ORNL, November 15, 1961

"Monte Carlo Calculations of the Ranges of Energetic Atoms in Solids" 
V. K. Paré

M. T: Robinson

H. C. Schweinler

E. Sonder

D. O. Thompson

M. S. Wechsler

R. A. Weeks

M. C: Wittels .

F. W. Young, Jr.
CEN, Mol, Belgium, April 24, 1962

"Delayed Pinning Effects in Copper"

The University, Reading, England, December 11, 1961 General Dynamics/Astronautics, San Diego, California, August 14, 1962

"Heavy Ion Bombardment of Solids"

Sandia Corporation, Albuquerque, New Mexico, August 22, 1962

"Clean Experiments in Solid State Physics"

Franklin Institute, Philadelphia, Pennsylvania, December 18,1961

"Magnetism of Color Centers in Alkali Halides"

Institut fuer Metallphysik, Gottingen, Germany, October 11,1961

"Radiation Damage Measurements and Defect-Dislocation Interactions in Copper"

C.C.R. EURA TOM, Ispra, Italy, October 26, 1961 "Defect Mobility in Copper from $280^{\circ} \mathrm{K}$ to $37.5^{\circ} \mathrm{K}$ as Measured by Internal Friction Techniques"

C.C:R. EURA TOM, Ispra, Italy, May 16, 1962

Imperial College, London, England, May 1, 1962

"The Effect of Radiation on Copper-Aluminum Alloys"

Argonne National Laboratory, Argonne, Illinois, October 23,1961

"The Relationship of the E' Centers and O-H Radicals in Silica"

Series of lectures at Summer. School sponsored by.NATO and Greek AEC, Rhodes; Greece, July 12-1.3, 1962

"The Structure of Paramagnetic Defects in Crystals"

Argonne National Laboratory, Argonne, Illinois; April 13,1962

"Some. X-Ray Diffraction and. Thermal Studies of Radiation Damage".

Yale University, New Haven, Connecticut, November 9,1961

University of Virginia, Charlottesville, Virginia, November 10, 1961

Technische Hochschule, Stuttgart, Germany, July 6 , 1962

"On the Yield Stress of Copper"

Ford Motor Company, Detroit, Michigan, January 24, 1962

Michigan State University, East Lansing, Michigan, January 26, 1962

"Mechanical Properties of Dislocations in Copper"

University of Illinois, Urbana, Illinois, May 25, 1962

"Deformation of Copper Crystals"

Institute for Physical Chemistry, Acad. Nauk, Moscow, USSR, July 6, 1962

"Crystal Imperfections and Chemical Reactivity" 
ORNL-3364

UC-34 - Physics

TID.4500 (18th ed.)

INTERNAL DISTRIBUTION

1. J. H. Barrett

2. R. F. Bass

3. C. D. Baumann

4. P. R. Bell

5. W. T. Berg

6. R. G. Berggren

7. U. Bertocci

8. J. O. Betterton, Jr.

9. D. S. Billington

10. E. P. Blizard

11. A. L. Boch

12. E. G. Bohlmann

13. C. D. Bopp

14. C. J. Borkowski

15. G. E. Boyd

16. M. A. Breazeale (consultant)

17. M. A. Bredig

18. R. B. Briggs

19. H. Brooks (consultant)

20. F. R. Bruce

21. W. E. Brundage

22. C. T. Butler

23. N. Cabrera (consultant)

24. R. M. Carroll

25. R. O. Chester

26. J. W. Cleland

27. R. G. Cleland (consultant)

28. T. F. Connolly

29. R. R. Coltman

30. J. A. Cox

31. J. H. Crawford, Jr.

32. F. L. Culler

33. J. E. Cunningham

34. O. L. Curtis

35. W. W. Davis

36. J. L. Fowler

37. J. H. Frye, Jr.

38. J. L. Gabbard

39. B. R. Gossick (consultant)

40. R. J. Gray

41. W. R. Grimes

42. D. E. Harrison (consultant)

43. R. F. Hibbs (Y-12)

44. N. E. Hinkle

45. A. Hollaender

46. D. K. Holmes

47. A. S. Householder
48. L. D. Hulett

49. H. B. Huntington (consultant)

50. J. T. Howe

51. L. H. Jenkins

52. L. K. Jetter

53. R. W. Johnson

54. R. J. Jones

55. W. H. Jordan

56. C. E. Klabunde

57. G. W. Keilholtz

58. M. T. Kelley

59. R. H. Kernohan

60. E. M. King

61. J. A. Krumhansl (consullant)

62. E. J. Lee

63. C. Lehmann

64. G. Leibfried

65. H. Leidheiser, Jr. (consultant)

66. A. B. Lewis (consultant)

67. T. A. Lewis

68. T. A. Lincoln

69. S. C. Lind

70. R. S. Livingston

71. H. G. MacPherson

72. D. L. McDonald

73. C. J. McHargue

74. W. D. Manly

75. A. Meyer

76. A. J. Miller

77. E. C. Miller

78. J. W. Mitchell (consultant)

79. J. G. Morgan

80. K. Z. Morgan

81. M. T. Morgan

82. J. P. Murray (K-25)

83. R. B. Murray

84. C. M. Nelson (consultant)

85. M. L. Nelson

86. T. S. Noggle

87. O. S. Oen

88. M. F. Osborne

89. V. Paré

90. W. W. Parkinson

91. J. C. Pigg

92. H. Pomerance

93. M. E. Ramsey

94. T. A. Read (consultant) 
95. P. E. Reagan

96. J. K. Redman

97. H. E. Robertson

98. C. C. Robinson

99. M. T. Robinson

100. A. F. Rupp

101. O. E. Schow

102. H. C. Schweinler

103. H. E. Seagren

104. F. Seitz (consultant)

105. S. T. Sekula

106. F. A. Sherrill

107. E. D. Shipley

108. W. A. Sibley

109. R. H. Silsbee (consultant)

110. O. Sisman

111. M. J. Skinner

112. L. Slifkin (consultant)

113. G. P. Smith

114. M. J. Smith

115. A. H. Snell

116. E. Sonder

117. A. L. Southern

118. R. L. Sproull (consultant)

119. J. T. Stanley

120. E. E. Stansbury

121. W. J. Steizman

122. C. D. Susano
123. J. A. Swartout

124. E. H. Taylor

125. W. E. Taylor (consultant)

126. L. C. Templeton

127. D. O. Thompson

128. David Turnbull (consultant)

129. D. Walton

130. M. S. Wechsler

131. R. A. Weeks

132. A. M. Weinberg

133. E. P. Wigner (consultant)

134. M. K. Wilkinson

135. J. M. Williams

136. W. R. Willis (consultant)

137. M. C. Wittels

138. E. O. Wollan

139. R. F. Wood

140. F. W. Young

141. J. C. Zukas

142. Biology Library

143-145. Central Research Library

146-275. Laboratory Records Department

276. Laboratory Records, ORNL R.C.

277. Laboratory Shift Supervisor

278-279. ORNL - T.12 Technical Library, Document Reference Section

280. Reactor Division Library

\section{EXTERNAL DISTRIBUTION}

281. W. W. Shaver, Corning Glass Works, Corning, New York:

282. R. W. McNamee, Union Carbide Corporation, New: York

283. Boeing Airplane Company

284. Division of Research and Development, AEC, ORO

285. J. R. Johnson, Minnesota Mining and Manufacturing Co., St. Paul

286. A. E. Ruark, AEC, Washington

287. G. L. Stiehl, Convair Division of General Dynamics Corp., San Diego

288. R. O. Bolt, California Research Corp., Richmond

289. Benjamin Lax, Lincoln Laboratory, Lexington, Massachusetts

290. George H. Wagner, Linde Air Products Company, Tonawanda Laboratory, East Park Drive and Woodward Avenue, Tonawanda, New York

291. D. K. Stevens, Materials and Metallurgy Branch, Division of Research, U.S. Atomic Energy Commission, Washington, D.C.

292. R. I. Leininger, Battelle Memorial Institute, 505 King Avenue, Columbus, Ohio

293. Westinghouse Electric Corp., Research Laboratories, Pittsburgh, Pennsylvania

294. J. G. Castle, Jr., Physics Dept., Westinghouse Electric Corp., Research Laboratories, Pittsburgh, Pennsylvania

295. C. Kikuchi, University of Michigan, Willow Run Laboratory, Ann Arbor, Michigan

296. N. R. Beaudry, Office of Ordnance Research, Duke Station, Durham, North Carolina

297. J. Hitch, Office of Isotope Development, AEC, Washington, D.C.

298. P. W. McDaniel, Division of Research, USAEC, Washington, D.C. 
299. H. Y. Fan, Solid State Physics Dept., Purdue University, Lafayette, Indiana

300. T. J. Turner, Department of Physics, Wake Forest College, Winston-Salem, North Carolina

301. J. B. Trice, Military Space Vehicle Department, General Electric Co., Philadelphia, Pennsylvania

302. T. H. Blewitt, Argonne National Laboratory, Chicago, Illinois

303. R. A. Charpie, Union Carbide Corporation, New York, New York

304. L. B. Emlet, Union Carbide Corporation, New York, New York

305. C. D. Yost, ARPA, Pentagon, Washington, D.C.

306. D. F. Cope, Reactor Division, Oak Ridge Operations Office

307. J. M. Simmons, Division of Reactor Development, AEC, Washington, D.C.

308. H. Shulman, Diamond Ordnance Fuse Laboratory, Washington, D.C.

309. G. D. Watkins, General Electric Research Laboratories, Schenectady, New York

310-938. Given distribution as shown in TID-4500 (18th ed.) under Physics category (75 copies - OTS)

Reports previously issued in this series are:

\begin{tabular}{|c|c|}
\hline ORNL-1025* & Period Ending January 31, 1951 \\
\hline ORNL-1095 & Period Ending April 30, 1951 \\
\hline ORNL-1128* & Period Ending July 31, 1951 \\
\hline ORNL-1214* & Period Ending October 31, 1951 \\
\hline ORNL-1261* & Period Ending January 31, 1952 \\
\hline ORNL-1301* & Period Ending May 10, 1952 \\
\hline ORNL-1359* & Period Ending August 10, 1952 \\
\hline ORNL-1429* & Period Ending November 10, 1952 \\
\hline ORNL-1506* & Period Ending February 10, 1953 \\
\hline ORNL-1606 & Period Ending August 31, 1953 \\
\hline ORNL-1677 & Period Ending February 28, 1954 \\
\hline ORNL-1762* & Period Ending August 30, 1954 \\
\hline ORNL-1851* & Period Ending February 28, 1955 \\
\hline ORNL-1852 & Period Ending February 28, 1955 \\
\hline ORNL-1944 & Period Ending August 30, 1955 \\
\hline ORNL-1945 & Period Ending August 30, 1955 \\
\hline ORNL-2051 & Period Ending February 29, 1956 \\
\hline ORNL-2052* & Period Ending February 29, 1956 \\
\hline ORNL-2188 & Period Ending August 30, 1956 \\
\hline ORNL-2189* & Period Ending August 30, 1956 \\
\hline ORNL-2413 & Period Ending August 31, 1957 \\
\hline ORNL-2614 & Period Ending August 31, 1958 \\
\hline ORNL-2829 & Period Ending August 31, 1959 \\
\hline ORNL-3017 & Period Ending August 31, 1960 \\
\hline ORNL-3213 & Period Ending August 31, 1961 \\
\hline
\end{tabular}




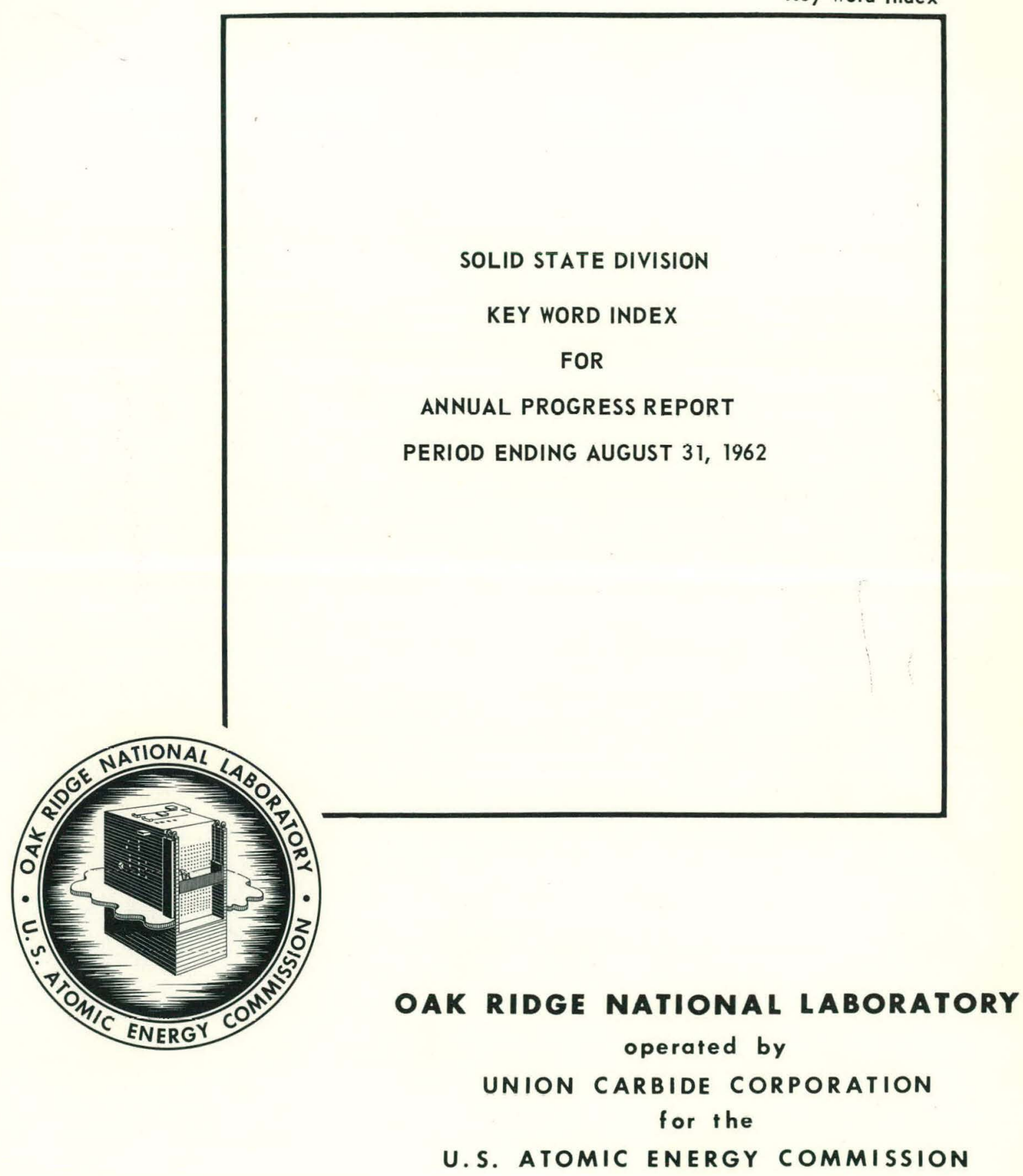


ORNL-3364

Key Word Index

Contract No. W-7405-eng-26

SOLID STATE DIVISION

KEY WORD INDEX FOR ANNUAL PROGRESS REPORT

PERIOD ENDING AUGUST 31, 1962

\author{
OAK RIDGE NATIONAL LABORATORY \\ Oak Ridge, Tennessee \\ operated by \\ UNION CARBIDE CORPORATION \\ for the \\ U. 5. ATOMIC ENERGY COMMISSION
}


THIS PAGE

\section{WAS INTENTIONALLY LEFT BLANK}


CONTENTS

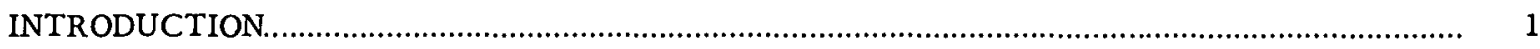

KEY WORD INDEX

TITLE INDEX

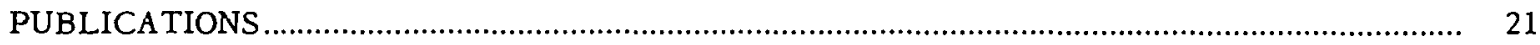

AUTHOR INDEX 


\section{INTRODUCTION}

The ORNL-3364 Key Word Index provides an insight to the contents of the Solid State Division Annual Progress Report for the Period Ending August 31, 1962 (ORNL-3364). The Index contains four parts: (1) Key Word Index, (2) Title Index, (3) Publications, and (4) Author Index.

The Key Word Index section is a combined alphabetical listing of the Title Index and the publications. If it is necessary to know a complete title, use the identification number and refer to the Title Index or the Publications section.

The Title Index is a listing of chapter titles and their primary subordinate headings, with the addition of key words to further aid in the understanding of the work reported. An example of the numbering system is 62-III-11-2, where 62 indicates the year of publication, III is the part number (or major division), 11 is the chapter, and 2 is the number of the primary heading within the chapter. Example:
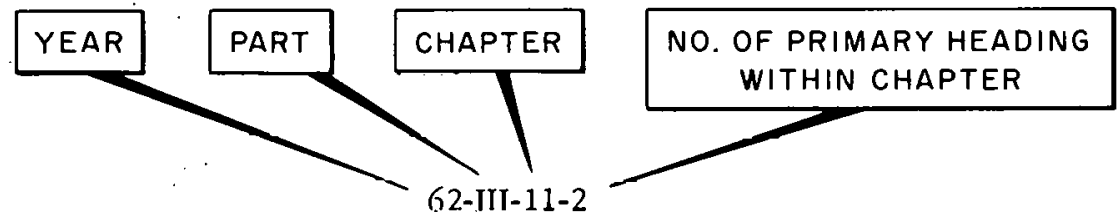

The Publications section is a listing of published work by Division members for the period August 31, 1961, to August 31, 1962. (Papers presented at technical meetings and articles submitted for publication are not included.) An example of the numbering system is 62-PUB-42, where 62 shows the year in which the publication was first reported as such, PUB identifies the work as having been published, and the last number was assigned to clearly identify a specific publication. Example:

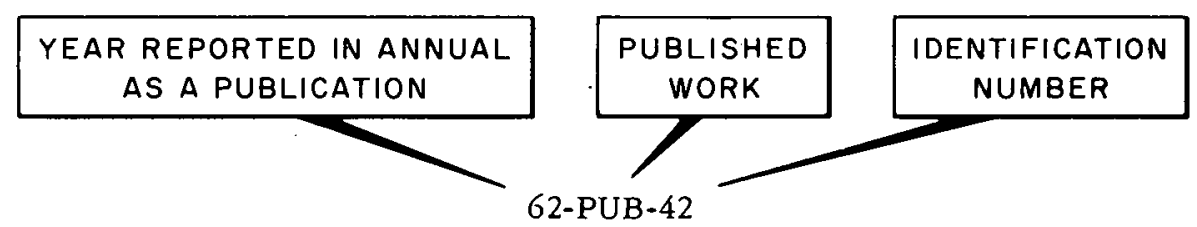

The Author Index is an alphabetical listing of the names of authors, followed by the numbering systems used in the Title Index and the Publications. This list shows reported work to which each author contributed.

To locate specific work in the Annual Report, it is necessary to use only the last two groups of numbers, since chapters are numbered consecutively.

The coinputer used for this program does not permit the use of lower-case type, superscript and subscript numbers, and certain specific punctuation marks. Therefore, to avoid confusion, Greek letters, chemical symbols, formulas, and names of elements and compounds are written out. When a title is too long to be given on one line, a slant, line $(/)$ indicates the title break. 
THIS PAGE

\section{WAS INTENTIONALLY LEFT BLANK}


R BOMBARDMENT WITH 4-KEV ARGON IONS SPUTTERING YIELD FOR $(I I I)$ - ALUMINUM PLANE IOF METAL SURFACES COPPE 62-III-I4 KINETICS OF EICH PIT GROWIH AND ASSOCIATED PHENOMENA ON (III) - COPPER SURFACES IN ACID HALIDES ITIC SUSCEPIIBILITY, ELECIRON SPIN RESONANCE. AND OPIICAL ABSORPIION OF THE E-PRIME-ONE CENTER IN FUSED S/ G2-II-9-I OOA AND GOODA

OPIICAL FISSICN FRAGMENT TRACKS IN THIN FILMS OF URANIUM-DIOXIDE RANGES OF ENERGEIIC ATOMS IN SOLIDS

CALCULATION OF AVERAGES FOR PRIMARY RECOIL DISTRIBUTIONS APPROXIMATION FOR CLASSICAL SCATTERING AT LARGE ANGLES ARLO CALCULATIONS OF RANGES OF ENERGETIC ATOMS IN SOLIDS ANNELING EFFECTS IN IRRADIATED CRYSTALS COPPER CRYSTALS BOMBAROMENT OF THIN PLATINUM FILMS 25-MEV NITROGEN IONS CINTILLATION PROCESS IN THALLIUM-ACIIVATEO CESIUM-IODIDE ANSITION TEMPERATURE OF PURIFIED TECHNETIUM $8.22 \mathrm{~K}$ VALUE GLE CRYSTAL MANANOUS-CHLORIDE IN APPLIED MAGNETIC FIELOS ON IN THERMAL NEUTRON SHIELDED MATERIALS SEMICONDUCTORS ION CN THE VAPOR-PHASE GROWTH OF SINGLE MERCURIC-SULFIDE RICTICN SPECTRUM IN COPPER FOLLOWING NEUTRON IRRADIATION PLASTICITY OPIICAL AND ELECIRON MICROSCOPE OBSERVATIONS COPPER - SILICON AND COPPER - SILICON - MANGANESE ALLOYS TRONS ON SCINTILLATION PROCESS IN ALKALI HALIDE CRYSTALS O CENTERS IA CRYSTALLINE OUARTZ ELECIRON SPIN RESONANCE CED DEFECTS ANO MAGNETIC STRUCTURE IRON - NICKEL ALLOYS EHAVICR OASERVATIONS OF RADIATION-INOUCED RECOMBINATION N PIANING IN COPPEP SINGLE CRYSIALS BY RADIATION DEFECTS CENTER PRODUCTION IN GAMMA-IRRAOIATED POTASSUIM-CHLORIDE $Y$ BENDING I. ANNEALED CRYSTALS II. IRRADIATED CRYSTALS COPPER THREE - GOLD, AND GERMANIUM-MELT CRYSTALLIZATION C TO FACE-CENTERED-CUBIC TRANSITION IN ZIRCONIUM-DIOXIDE E-PRIME-ONE CENTER IN FUSED SILICA OSCILLATOR STRENGTH TH AND ASSOCIATED PHENOMENA ON $(111)$ - COPPER SURFACES IN OLLOWINC NEUTRON IRRADIATION ABSTRACT OLLOWING NEUTRON IRRADIATION HIGHER-ORDER AVERAGES OF PRIMARY RECOIL OISTRIBUTIONS DIFFRACTION TOPOGRAPHS AND SINGLE CRYSIIAL ROCKING CURVES ANE IN A FLUOR/ RADIATION DOSIMETRY IRRADIATION PRODUCED

ABSORPTION STUDIES ON CUPROUS-OXIDE BETWEEN 250 ABSTRACT

ABSTRACT

ABSTRACT

ABSTRACT

ABSTRACT

ABSTRACT

ABSTRACT

ABSTRAC T

ABSTRACT

ABSTRACT

ABSTRACT

ABSTRACT

ABSTRACT

ABSTRACT

ABSTRACT

ABSTRACT

ABSTRACT

ABSTRACT

ABSTRACT

ABSTRACT

RECOMBINATI ON PROCESS 11 . ANNEALING B 62-IV-15-1

ABSTRACT /NDENCE FROM 250K TO 370K OF DISLOCATIO 62-II1-13-3

ABSTRACT /NL - - STRUCTURE SENSITIVITY OF COLOR 62-1V-16-3

ABSTRACT IOCATIONS IN COPPER CRYSTALS DEFORMED B 62-111-11-5

ABSTRACT IS GOLD, COPPER, SILVER, LEAD, INDIUM, 62-11-7-1

ABSTRACT IS IN URANIUM-DOPED ZIRCONIA MONOCLINI 62-11-7-2

ABSTRACT / SONANCE, AND OPTICAL ABSORPTION OF THE 62-11-9-1

ACID CONCENTRATIONS OF TETRACHLOROETHANE ANO DEC 62-VI-23

KINETICS OF ETCH PIT GROW 62-1II-11-3

ACTIVATED INTERNAL FRICTICN SPECTRUM IN COPPER F 62-III-13-4

ACTIVATED INTERNAL FRICTION SPECTRUM IN COPPER F 62-PUB-4I ACTIVATION ENERGIES APPROXIMATE METHODS FOR ALTIVATION MEASURES IRRAOIATION FLUX CONTINUOUSL LUMINESCENCE OF ALKALI HALIDES GAMMA IRRADIATION

OF COLOR CENTER PROOI INVESTIGATIONS OF COLOR CENTERS IN GOLD(O.82) - MERCURY(C. (B) - A TRANSMUTATION ALLOY

EFORMED COPPER - SILICON AND COPPER - SILICON - MANGANESE ALLOYS

ION-INOUCED DEFECTS AND MAGNETIC STRUCTURE IRON - NICKEL

RATUS FOR LOW FREOUENCY INTERN/ STRESS RELAXATION IN IRON

/ SUPERCONDUCTING CRITICAL CURRENT OF NIOBIUM - ZIRCONIUM

ITY OF GLASSES LEAD-SILICATE GLASS POLARIZATION AND CHAI

EFFECT OF RADIATION ON ATOMIC REARRANGEMENTS IN COPPER -
HE DEPENDENCE OF RADIATION-ENHANCED DIFFUSION IN COPPER DMENT WITH $4-K E V$ ARGON IONS SPUTTERING YIELD FOR $(111)$ EFFECTS ON POLYSTYRENE INFRARED AND GAS CHROMATOGRAPHIC YCLE FREOUENCIES OSCILLATORY DISLOCATION MOTION

A SIMPLE APPROXIMATION FOR CLASSICAL SCATTERING AT LARGE APPROXIMATION FOR CLASSICAL SCATIERING AT IARGF
CHAINS COI EXAMINATION OF IHEORETICAL MODEL OF AESTRACT
ABSTRACT ALLOYS ALLOYS ON MAGNET CCARBON AND ALTERNATING CURRENT AND DIRECT CURRENT CONDUCTIV ALUMINUM FLUX DEPENDENCE ALUMINUM ON NEUTRON FLUX AND ENERGY SPECTRUM T $\begin{aligned} & 62-V-18-2 \\ & 62-P U B-2\end{aligned}$ ALUMINUM PLANE /OF METAL SURFACES COPPER BOMBAR 62-III-14 ANALYSIS POLYMERS GAMMA AND REACTOR RADIATION 62-VI-2 ANALYSIS OF INTERNAL FRICTION IN COPPER AT MEGAC 62-III-I3-2 ANGLES

ANGI.ES ARSTRACT 62-PU8-25 $62-1-1-7$ FOCUSING CHAINS CO/ EXAMINATION OF IHEORETICAL MODEL OF ANISOTROPIC SPUTTERING AT HIGH ENERGIES BASED ON 62-I-2-2 I DISLOCATIONS IN COPPER CRYSTALS DEFORMED BY BENDING I. ANNEALED CRYSTALS II. IRRADIATED CRYSTALS ABSI G2-III-II-5 IGERMANIUM. I. THE NATURE OF THE RECOMBINATION PROCESS II. ANNEALING BEHAVIOR OBSERVATIONS OF RADIATION-I/ O2-IV-IS- I ICED RECOMBINATION AND TRAPPING CENTERS IN GERMANIUM II ANNEALING IN GAMMA-IRRADIATED, ANTIMONY-DOPED AI G2 -PUB-IT IN-INDUCEC CAMAGE IN GERMANIUM ISOCHRONAL AND ISOTHERMAL ANNEALING OF ANTIMCNY-DOPED GERMANIUM GAMMA-RA/ GO THE ANNEALING OF COLD-WORKED SILICON BRONZE

$62-1 V-15-2$ $62-P \cup B-43$

NIUM ISOCHRONAL AND ISOTHERMAL ANNEALING OF ANTIMONY-DO/ ACTIVATION ENERGIES APPROXIMATE METHODS FOR TREATING A TIONS ETCH PITS AT DISLOCATIONS

NING COPPER IONS ORIENTATION EFFECTS

CENTERS IN GERMANIUM II. ANNEALING IN GAMMA-IRRADIATED, IAGE IN GERMANIUM ISOCHRONAL AND ISOTHERMAL ANNEALING OF ATION IN IRON ALLOYS CONTAININC CARBON AND $/$ OR NITROGEN LCULATIONS

ANGLES
SFER

NUOUSLY

A SIMPLE HARD SPHERE SCATTERING ANNEALING OF IRRADIATION-INDUCED DAMACE IN GERMA ANNEALING PROCESSES WHICH INVOLVE A SPECTRUM OF 62-PUB-19 ANODIC DISSOLUTION OF COPPER IN COPPER-FREE SOLU 62-I11-11-2 ANODIC DISSOLUTION OF COPPER IN SOLUTIONS CONTAI 62-11I-11-1 ANTIMONY-DOPED AND ARSENIC-DOPED MATERIAL /PPING 62-PUB-17 ANTIMONY-DOPED GERMANIUM GAMMA-RAY IRRADIATION/ 62-IV-15-2 APPARATUS FOR LOW FREQUENCY INTERNAL FRICTION $/ \times 62-V-18-3$ APPROXIMATION BOHR POTENTIAL. ANE MONTE CARLO CA 62-I-1-1 APPROXIMATION FOR CLASSICAL SCATTERING AT LARGE 62-PUB-25 ARGON ACTIVATION MEASURES IRRADIATION FLUX CONTI 62-PUB-7 IARDMENT OF METAL SURFACES COPPER BOMBARDMENT WITH 4-KEV ARGON IONS SPUTTERING YIELD FOR IIIII - ALUMIN/ 62-III- I4
COPPER BONBARCMENT WITH 4-KEV ARGON IONS SPUTTERING Y/ ARGON-PLUS-ONE ION BOMBARDMENT OF METAL SURFACES O2-III- I4 NOUCTIVITY TRAPPING CENTERS IN ARSENIC-DOPED GERMANIUM DIRECT CURRENT PHOTO CO 62-IV-IS-3 UM II. ANNEALING IN GAMMA-IRRADIATED, ANTIMONY-DOPED AND ARSENIC-DOPED MATERIAL IPPING CENTERS IN GERMANI G2-PUB-I7 NG IBM-7090 FORTRAN PROCRAM ELECTRON CROSS SECTIONS FOR ATOMIC DISPLACEMENTS MOTT SERIES EVALUATION USI O2-I-6 DEPENDENCE ON ANC COPPER - SILICON - MANGANESE ALLOYS ABSTRACT ATOMIC REARRANGEMENTS IN COPPER - ALUMINUM FLUX 62-V-18-2 ATOMIC REARRANGEMENTS IN DEFORMED COPPER - SILIC 62-V-18-4 TER PROGRAM FOR MCNTE CARLO CALCULATIONS OF RANGES OF ENERGETIC ATOMS IN SOLIDS ABSTRACT RANGES OF ENERGETIC ATOMS IN SOLIDS ABSTRACT THE RANGES OF ENERGETIC ATOMS IN SOLIDS THE RANGES OF ENERGETIC ATOMS IN SOLIDS ABSTR ACT I. RANDOM MODEL $62-1-1-4$ $62-1-1-5$ $62-P \cup B-30$ $62-P U B-34$ RANGE-II COMPUTER PROGRAM FOR L $62-I-1-2$ ATIICE / MACHINE STUDIES OF THE SLOWING DOHN OF ENERGETIC ATOMS IN SOLIDS RANGE-II COMPUTER PROGRAM FOR L G2-I-I-2 STUDIES OF PENETRATION OF MOVING ATOMS IN SOLIDS ANC INTERATOMIC POTENTIALS
CALCULATION OF AVERAGES FOR PRIMARY RECOIL DISTRIBUTIONS CALCULATION OF AVERAGES FOR PRIMARY RECOIL DISTRIBUTIONS
CALCULATION OF AVERAGES FOR PRIMARY RECOIL DISTRIBUTIONS ABSTR $62-1-1-6$ HIGHER-ORDER AVERAGES OF PRIMARY RECOIL DISTRIBUTIONS ABSTRA $62-1-1-8$ $\begin{array}{lr}\text { ACT } & \text { CALCULATION HIGHER-ORDER AVERAGES } \\ \text { CT } & \text { HLSTERING IN DISLOCATIONS IN COPPER DISLOCATION PINNING DCHAVIOR }\end{array}$ EVIDENCE FOR VACANCY $62-111-13-1$ 1I. THE NATURE OF THE RECOMBINATION PROCESS II. ANNEALING BEHAVIOR OBSERVATIONS OF RADIAIION-INOUCED RECI G2-IV-15-1 IT STUDIES OF DISLOCATIONS IN COPPER CRYSTALS DEFORMED BY BENDING I. ANNEALED CRYSTALS II. IRRADIATED CI G2-III-II-5 I FUELS URANIUM-DIOXIDE PELLETS CLAD IN STAINLESS STEEL IRRADIATION TESTS
BENDING I ANNEALED CRYSTALS II : IRRADIATED C/ $62-I I I-11-5$
BERYLLIUM CANNED URANIUM-DIOXIDE AND FUELED BER/ $62-V I-20-3$ BERYLLIUM SHEATHED URANIUM-DIOXIDE FUEL ELEMENT O2-PUB-3। 
INLESS STEEL BERYLLIUM CANNED URANIUM-DIOXIDE AND FUELED BERYLLIUM-OXIDE EVALUATIONS /PELLETS CLAD IN STA 62-VI-20-3 IONESS STEEL BERYLLIUM CANMED URANIUM-DIOXIDE AND FUELED BERYLLIUM-OXIOE EVALUATI H BISMUTH SELF DIFFUSION Y OF EFFECTS OF COBALT-60 GAMMA-RADIATION UPON EXIRINSIC. BISMUTH-IELLURIDE ELECIRICAL PROPERTIES MAGNEI O2-IV-I5-8 ING AT HISH TWITH L WKEV ENERGIES BASED ON FOCUSING CHAINS COPPER ION BOMBARDME WITH L KEV ARGON IONS SPUTTERING YI ARGON-PLUS-ONE ION BOMBARDMENT OF METAL SURFACES COPPER BOMBARDMEN 62-III-IL GEN IONS ABSTRACT NITROGEN ION BOMBARDMENT OF THIN PLAIINUM FILMS COP 25-MEV NITRO $62-I 1-B-3$ OF COPPER CRYSTALS WITH LOW DISLOCATION DENSITY MODIFIED BKIDGMAN TECHNIQUE CRITICAL SIMPLICES IN BRILLOUIN ZONES

IMPACT TESTS ON IRRADIATED PRESSURE VESSEL STEELS BRITTLE-DUCTILE TRANSITION TEMPERATURE $62-I I I-14$ IMPACT TESTS ON IRRADIATED PRESSURE VESSEL STEELS BRITTLE
THE ANNEALING OF COLD-WORKED SILICON BRONZE

NEW LOW TEMPERATURE IRRADIATION FACILITY FOR THE BULK SHIELDING REACTOR $4 K$ FACILITY $62-1-4$ $62-V-19-2$ $62-P \cup B-43$ LOW TEMPERATURE NEUTRON, GAMMA RECOIL DAMAGE IN CADMIUM $62-I I I-12-3$ $62-P \cup B-23$ INTENSE DAMAGE IN CADMIUM SUPPRESSION OF ISOCHRONAL R
IMATE OF DEFECT CONCENTRATION

ADIATION OF DOPED POTASS IUM-CHLORIDE CALCIUM, STRONTIUM.

ITIES

CADMIUM, AND MAGNESIUM IMPUR ITIES

CALCIUM, STRONTIUM, CADMIUM, AND MI
REACTOR

$$
\begin{aligned}
& \text { A VERSATILE LANG CAMERA } \\
& \text { STEEL BERYLLIUM CANNED }
\end{aligned}
$$

CANNED URANIUM-DIOXIDE AND FUELED BERYLLIUM-OXI/ FERROUS-SULFATE OXIDATION MONITORING TECHNIQUES FOR DENSITY FUNCT MODIFYING MINORITY CARRIER UENCY INTERN' STRESS PELAXAYION IN IRON ALLOYS CONTAINING CAF ON GAS REIENTION $S$ FOR HARD-SPHERE
IN SOLIDS ABSTRACT IN SOS COATED FUEL PARIICLES PYROLYTIC
IMATION BOHR POTENTIAL AND MONTE CA

IRRADIATED GERMANIUM DENSITY FUNCTION MOOIFYING MINORITY CANNED URANIUM-DIOXIOE ANO FUELED BERYLLIUM-OS CAPTURE PROBABILITY IN NONMETALLIC CRYSTALS ARBON ANO $/$ OR NITROGEN APPARATUS FOR LOW FREQ
ARBON COATED URANIUM-DICARBIDE PARTICLES FISSI STOPPING POWER AS A BASI

$62-11 \mathrm{I}-12-2$ RESEARCH MATERIALS INFORMATION CENTER
OPERATION OF RESEARCH MATERIALS INFORMATION CENTER
, AND OPT ICAL ABSORPTION OF THE E-PRIME-ONE CENTER

CARRIER CAPTURE PROBABILITY IN NONMETALLIC CRYST ARRIER RECOMBINATION AND TRAPPING PROCESSES IN
IN 作 SENSIIIVIIY OF COLOR CENTER PROD/ INVESTIGATIONS OF COLOR CENTERS IN ALKALI HALIDES AT ORNL - - STRUCTURE OR NT PHCTO CONDUCTIVIIY ONANCE ABSTRACT PARAMAGNETIC SPECTRA OF E-PRIME-TWO CENTERS IN CRYSTALLINE QUIARTZ ELECTRON SPIN RES OR BINATION PRI RADIATION-INDUCED RECOMBINATION AND TRAPP.ING CENTERS IN GERMANIUM I. THE NATURE OF THE RECOM OL INATICN PRO/ RADIATION-INOUCED RECOMBINATION AND TRAPPING CENTERS IN GERMANIUM I THE NATURE OF THE RECOMB O SPIN-LATTICE RELAXATION OF E-PRIME-ONE CENTERS IN SILICON-DIOXIDE CERAMICS PELTIER POWER CALIBRATION ZIRCON CERAMIC RADIATION STABILITY.

CERAMIC RADIATION STABILITY. MIC RADIATION STABILITY ATION MCNITORING TECHNIOUES FOR CAPTURE CAMMA RAYS CERIC-SULFATE
TUDIES OF THE SCINTILLATION PROCESS IN THALLIUM-ACTIVATED CESIUM-IODIDE TUDIES OF THE SCINTILLATION PROCESS IN THALLIUM-ACT IVATED CESIUM-IODIDE SOLIOS RANGE-IICOMPUTER PROGRAM FOR LATTICE SYMMETRY CHANNELED DIRECTIONS OF ATOMS /ENERGETIC ATOMS I G2-I-1-2 R CRYSTALS ABSTRACT CONG-RANGE CHANNELING EFFECTS IN IRRADIATED CRYSTALS COPPE G2-I-I-9 UCTIVITY OF GLASSES LEAD-SILICATE GLASS POLARIZATION AND CHARGE SIORAGE $/$ CURRENT AND DIRECT CURRENT COND G2-II-9-3 EACTOR RADIATION EFFECTS ON POLYSTYRENE INFRARED AND GAS CHROMATOGRAPHIC ANALYSIS POLYMERS GAMMA AND R G2-VI-2I NLESS STEEL BERYLLIUM CANN/ IRRADIATION EFFECTS ON METAL CLAD FUELS URANIUM-DIOXIDE PELLETS CLAD IN SIAI 62-VI-20-3 IION EFFECTS ON METAL CLAD FUELS URANIUM-DIOXIDE PELLETS CLAD IN STAINLESS STEEL BERYLLIUM CANNED URANIV G2-VI-20-3 A SIMPLE APPROXIMATION FOR CLASSICAL SCATIERING AT LARSE ANGLES LARGE ANGLES APPROXIMATION FOR CLASSICAL SCATTERING AT LARGE ANGLES ABSTRACT O2-1-I-7 N PINNING BEHAVIOR EVIDENCE FOR VACANCY
PRODUCTION OF DEFECT HOLD IRRADIATION ENERGY REQUIRED FOR PRODUCTIONOR OETAETY E POSTIRRADIATION EXAMINATION OF GRAPHITE MATRI RANIUN-DICARBIDE PARTICLES FISSION GAS RETENTION RETENT ION PARTICLES FISSION GAS RETENTION CLUSTERINO CLUSTERS
COATED ANO UNCOATED URANIUM-DICARBRIDE IN GRAPHIT COATED FUEL PARTICLES PYROLYTIC CARBON COATED U - TELLURIDE ELECTRICAL PROPERTIES MI STUDY OF EFFECTS OF COBALT-GO GAMMA-RADIATION UPON EXTRINSIC BISMUTH EFFECT OF DISLOCATIONS ON COBALT-60 RADIATION-INDUCED DEFECTS IN GERMANIUM G2
OWER IN GERMANIUM SEEBECK COEFFICIENT GAMMA-RAY IRRADIATION IDE THERMOELECTRIC POWER IN GERMAN THE ANNEALING OF COLO-WORKED SILICON BRONZE

IS IN ALKALI HALIDES AT ORNL - - STRUCTURE SENSITIVITY OF COLOR CENTER PRODUCTION IN GAMMA-IRRADIATED POT/ G2 CTURE SENSIIIVIIY OF COLOR CENTER PRODI INVESTIGATIONS OF COLOR CENTERS IN ALKALI HALIOES AT ORNL - STRU
SIUM-CHLORICE. MARITI I STATISTICAL MECHANICS OF SEMICONDUCTORS COMPACTED URANIUM-DIOXIDE

STATISTICAL MECHANICS OF SEMICONDUCTORS COMPUTATION OF THE FERMI LEVEL IN NON METALS

1. THE SLOWING DOWN OF ENERGETIC ATOMS IN SOLIDS RANGE-II COMPUTER PROGRAN FOR LATTICE SYMMETRY CHANNELE/ OZ INTENSE DAMACE INANALE/ O2-1-1-2 M. SUPPRESSION OF ISOCHRONAL RECOVERY ESTIMATE OF DEFECT CONCENTRATION INTENSE DAMACE IN CADMIU $62-$ III-12-2 $N$ A FLUOR/ RADIATION DOSIMETRY IRRADIATION PRODUCED ACID CONCENTRATIONS OF TETRACHLOROETHANE AND DECANE I G2-VI-23 CENTERS IN ARSENIC-DOPED GERMANIUM DIRECT CURRENT PHOTO CONDUCTIVITY ARIZATION AND CHAI ALTERNATING CURRENT AND DIRECT CURRENT CONDUCIIVITY OF GLASSES LEAD-SILICATE GLASS POL 6 ARGON ACT IVATION MEASURES IRRADIATION FLUX CONTINUOUSLY ELECTROCHEMICAL OISSOLUTION OF. SINGLE CRYSTALLINE COPPER ETCHING OF IRRADIATED COPPER FECTS OF GASEOUS IMPURITIES ON IHE MAGNETIC PROPERTIES OF COPPER OCATIONS AROUND PRECIPITATE PARTICLES OF CUPROUS-OXIDE IN COPPER EVIDENCE FOR VACANCY. CLUSTERING IN DISLOCATIONS IN COPPER IGIES BASED ON FOCUSING CHAINS COPPER ION BOMBARDMENT OF COPPER EFFECT OF RADIATION ON ATOMIC REARRANGEMENTS IN COPPER CIRUM THE DEPENDENCE OF RADIATION-ENHANCED DIFFUSION IN COPPER ATCMIC REARRANGEMENTS IN DEFORMED COPPER - SILICON AND COPPER E ALLCYS ABSTRACT IONS ATOMIC REARRANGEMENTS IN DEFORMED COPPER

LEAD-SILICATE GLASS POL
$62-1 V-15-3$ 62-PUB-7 $62-P \cup B-22$ $62-P \cup B-48$ EF $62-P \cup B-39$ ON THE FORMATION OF DISL 62-PUB-49 OISLOCATION PINNING BEHAVIOR SPUTIERING YIELDS AS A FUNCTION OF ORIE/ 62-I-2-2 $\begin{array}{ll}\text { - ALUMINUM FLUX DEPENDENCE } & 62-V-18-2 \\ - & \end{array}$ $62-V-18-2$ $\begin{array}{ll}\text { ALUMINUM ON NEUTRON FLUX AND ENERGY SPE } & 62-P U B-2 \\ \text { SILICON - MANGANESE ALLOYS ABSTRACT } & 62-y-1.8-4\end{array}$ GEOMETRICAL MODEL OF MONOCRYSTAL SPUTTERING COPPER AND GERMANIUM SPUTTERING WITH 5-KEV ARGON O2-I-2-1 LOCATION MOTION ANALYSIS OF INTERNAL FRICTION IN COPPER AT MEGACYCLE FREQUENCIES OSCILLATORY OIS 62-III-I3-2 RING YI ARGON-PLUS-ONE ION BOMBARDMENT UF METAL SURFACES COPPER BOMBARDMENT WITH $4-K E V$ ARGON IONS SPUTTE G2 ON THE YIELO STRESS OF COPPER CRYSTALS

LCNG-RANGE CHANNELING EFFECTS IN HNIQUE $62-P \cup B-51$
$62-1-1-9$

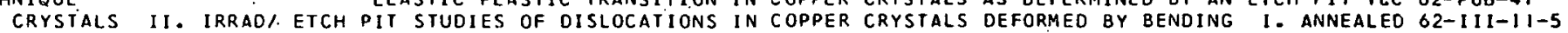


OIFIEC BRIDGMAN TECHNIOUE

A THERMALLY ACTIVATED INTERNAL
THERMALLY ACTIVATED INTERNAL

SLOCATIONS

NTATION EFFECTS

IIC SPUTTERING AT HIGH ENERGIES BASED ONODIC DISSOLUTION OF ANODIC DISSOLUITION OF COPPER IN SOLUTIONS CONTAINING PHS AND SINGLE CRYS/ X-RAY OBSERVATIONS OF NEARLY PERFECT IE DEPENDENCE FROM 250K TO $370 K$ OF DISLOCAIION PINNING IN RE DEPENOENCE FROM 250K TO $370 K$ OF DISLOCATION PINNING IN CS OF EICH PIT GROWTH AND ASSOCIATED PHENOMENA ON $(111)$ IFROM SMALL DROPLETS GOLD, COPPER, SILVER, LEAD, INDIUM,
D, ANC, SINGLE CRYSTALS GROWN FROM SMALL DROPLETS GOLD, ANODIC DISSOLUTION OF COPPER IN

N MAGNETIC FIELD ORIENTATI/ DEPENDENCE OF SUPERCONDUCTING

RIES EVALUATION USING 1 BM-7090 FORTRAN PROGRAM NUCLEAR REACTORS

ELDS ABSTRAC

ELECTRON

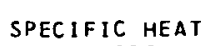

SPECIFIC HEAT

C HEAT OF SINGLE

R SINGLE CRYSTALS LANC DIFFRACTION TOPOGRAPHS AND SINGLE ELECTROCHEMICAL DISSOLUTION OF SINGLE TRACT PARANACNETIC SPECTRA OF E-PRIME-TWO CENTERS IN
ER, LEAD, INDIUM, COPPER THREE - GOLD, AND GERMANIUM-MELI INSULATING POTASSIUM-CHLORIDE SINGLE CI ON THE YIELD SIRESS OF COPPER CRYSTALS FYING MINORITY CARRIER CAPIURE PROBABILITY IN NONMETALLIC CRYSTALS G-RANGE CHANNELINC EFFECTS IN IRRADIATED CRYSTALS COPPCR CRYSTALS NDARY ELECTRONS ON SCINTILLATION PROCESS IN ALKALI HALIDE CRYSTALS DEFORMED BY BENOING I. ANNEALED CRYSTALS II. IRRADIATED CRYSTALS LONG-RANGE CHANNELING EFFECTS IN IRRADIATED CRYSTALS TIONS IN COPPER CRYSTALS DEFORMED BY BENDING I. ANNEALEU CRYSTALS CRYSI X-RAY OBSERVATIONS OF NEARLY PERFECT COPPER SINGLE CRYSTALS FROM-25CK TC $370 K$ OF OISLOCATION PINNINC IN COPPER SINGLE CRYSTALS

COPPER CRYSTALS OF LOW DISLOCATION DENSITY COPPER CRYSTALS WITH LOW DISLOCATION
COPPER FOLLOWING NEUTRON IRRADIATION COPPER FOLLOWING NEUTRON IRRADIATION ABSTRACT COPPER IN COPPER-FREE SOLUTIONS ETCH PITS AT DI 6 COPPER IN SOLUTIONS CONTAINING COPPER IONS ORIE COPPER ION BOMBARDMENT OF COPPER
COPPER IONS ORIENTATION EFFECTS

OPPER SINGLE CRYSTALS LANG DIFFRACTION TOPOGRA $62-11-7-5$ OPPER SINGLE CRYSTALS BY RADIATION DEFECTS AB/ 62-III-I3-3 OPPER SURFACES IN ACIO HALIDES KINETI 62-111-11-3 COPPER, SILVER, LEAD, INDIUM, COPPER IHREE - GOL 62-11-7-1 COPPER-FREE SOLUTIONS EICH PIIS AT DISLOCATIONS O2-III-11-2 CRITICAL CURRENT OF NIOBIUM - ZIRCONIUM

$62-11-10-1$ $62-1-4$ CROSS SECTIONS FOR ATOMIC DISPLACEMENTS MOTT SE $62-1-6$ CRYSTAL MANANOUS-CHLORIDE IN APPLIED MAGNETIC FI $62-1 \mathrm{~V}-16-7$ CRYSTAL MERCURIC-SULFIDE 62-1V-15-7 CRYSTAL ROCKING CURVES ABSTRACT, PERFECT COPPE 62-II-7-5 RYSTALLINE COPPER $62-11-7-5$
$62-P U B-22$ FROM 25CK TC 37 CK OF DISLOCATION PINNING IN COPPER SINGLE CRYSTALS BY LS II. IRRADI ETCH PIT STUOIES OF DISLOCATIONS IN COPPER CRYSTALS DEI
IFFUSION IFFUSION
- SILVER, LEAD, INDILIN, COPPER THKEE - GOLD, AND/ SINGLE CRYSTALS GRO ON THE GROWTH OF COPPER CRYSTALS OF LOW DISLOCATION DENSITY
GMENT, IRANSMISSION ELECTKON MICROSCOPE STUOIES ON SINGLE CRYSTALS OF UNANIUM-DIOXIDE SURFACE FISSION FRA BRIDGNAN TECIINIQUL GROWTH OF COPPER OPTICAL $\triangle B S O R P T I O N$ STUDIES ON FORMATION OF DISLOCATIONS ARUUND PRECIPIIMIE PAKIICLES OF
ES LEAD-SILICATE GLASS POLARIZATION AND CHAI ALTERNATING ES LEAD-SILICATE GLASS POLARIZATION AND CHAI ALTERNATING
LASS POLARIIZATION AND CHAI ALTERNATING CURRENT AND DIRECT C FIELD ORIENTATI/ DEPENDENCE OF SUPERCONDUCTING CRITICAL TRAPPINC CENTERS IN ARSENIC-DOPED GERMANIUM DIRECT $S$ LANG DIFFRACTION TOPOCRAPHS AND SINGLE CRYSTAL ROCKING CURVES PHOTO CONDUCTIVITY ECOILESS RADIATIO/ POSSIBLE EXPERIMENT TO STUDY RADIATION DAMAGE BY THE MOSSBAUER EFFECT SPECTRA OF THE R OR LOW TEMPERATURE NEUTRON, GAMMA RECOIL DAMAGE IN CADMIUM
OVERY ESTIMATE OF DEFECT C.UNCENTRATION INTENSE DAMAGE IN CADNIUM

ABSTRACT ABSTRACT ABSTRACT IOCATIONS IN COPPER CRYSTALS COPPER CRYSTALS ABSTRAC

II. IRRADIATED CRYSTALS ABSTRACT /OCA LANG DIFFRAC TION TOPOGRAPHS AND SINGLE AS DETERMINED BY AN ETCH PIT TECHNIQUE Y RADIATION DEFECTS ABSTRACT INDENCE RADIATION DEFECTS /ATURE DEPENDENCE I. ANNEALED CRYSTA TA 62 D $62-11-17-5$ $62-11-7-1$ $62-P \cup B-50$ $62-111-11-4$ ON THE $\begin{aligned} & 62-I V-17-2 \\ & 62-P U B-49\end{aligned}$ CUPROUS-OXIDE BETWEEN 250ODA AND OOOOA $\begin{array}{ll}\text { ON THE } & 62-P U B-49 \\ \text { OF GLASS } 62-11-9-3\end{array}$ OVERY ESTINATE OF DEFECT C.UNCENTRATION INTENSE DAMAGE IN CADNIUM SIJPPRESSION OF ISOCHRONAL REC
-INDUCED DAMAGE IN GERMANIUM ISOCHRONAL AND ISOTHERMAL A NNEALING OF ANTIMONY-DOI ANNEALING OF IRRADIATION-INDUCED DAMAGE IN GERMANIUI
CONTRIBUTION OF SLOW NEUTRONS TO REACTOR DAMAGE IN METALS REACTOR DAMAGE IN PURE METALS AT $4 K$ THE INVESTIGATION OF RADIATION DAMAGE USING MECHANICAL VIBRATIONS
DISTRIBUTION IN KINETIC ENERGY OF DAUGHTER NUCLEI THE INVESTIGATION OF RADIATION DAMAGE USING MECHANICAL VIBRATIONS
DISTRIBUTION IN KINETIC ENERGY OF DAUGHTER NUCLEI

ANE IN A FLUOROCARHON SOLVENT AND OF TETRACHLOROETHANE IN DECANE /CENTRATIONS OF IEIRACHLOROETHANE AND DEC G ION PRODUCED ACID CONCENTRATIONS OF TETRACHLOROETHANE AND DECANE IN A FLUOROCARBON SOLVENT AND OF TETRACH/ G RADIATION EFFECTS ON DEEP-LEVEL IMPURITY STATES IN GERMANIUM EXPERIMENTA $62-P U B-1$ L THRESHOLD IRRADIATION ENERGY REOUIRED FOR PRUDUCTION OF DEFECT CLUSTERS CADMIUM SUPPRESSION DF ISOCHRONAL RECOVERY ESTIMATE OF DEFECT CONCENTRATION INTENSE DAMAGE IN $62-111-12-2$ TERIALS SEMICONDUCTORS ABSTRACT RECOVERY ESTIMATE OF DEFECT CONCENTRATION

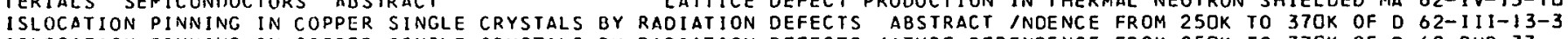
ISLOCATION PINNING IN COPPER SINGLE CRYSTALS BY RADIATION DEFECTS /ATURE OEPENDENCE FROM $250 K$ TO 37 TK OF D G2-PUB-33 LOYS ABSTRACT INTERACTION BETWEEN IRRADIATION-INDUCED DEFECTS AND MAGNETIC STRUCTURE IRON - NICKEL AL G2-II-IO-5 EFFECT OF DISLOCATIUNS ON COBALT-60 RADIATION-INDUCED DEFECTS IN GERMANIUM THE NATURE AND YIELD OF RADIATION-INDUCED DEFECTS IN SEMICONCUCTORS

RRAD/ ETCH PIT STUDIES OF DISLOCATIONS IN COPPER CRYSTALS DEFORMED BY BENDING I. ANNEALED CRYSTALS II. I G2 MANGANESE ALLOYS ABSTRACT MTOMIC REARRANGEMENTS IN DEFORMED COPPER - SILICON AND COPPER - SILICON - G
ATORS TO HEAVY PARTICLES
EFFECT OF DELTA RAYS ON THE RESPONSE OF INORGANIC SCINTILL G

ON THE GROWTH OF COPPER CRYSTALS OF LOW DISLOCATION DENSITY

GROWTH OF COPPER CRYSTALS WITH LOW DISLOCATION DENSITY MODIFIED BRIDGMAN TECHNIQUE URE PROBABILITY IN NONMETALLIC CRYSTALS

DENSITY FUNCT
X-RAY DIFFRACTION /ERVATIONS OF NEARLY PERFECT COPPER SINGLE CRYSTALS LANG DIFFRACTION
H-SELENIDE CRYSTALS DURING AND AFTER GROWTH BISMUTH SELF DIFFUSION NO ENERGY SPECTRUM THE DEPENDENCE OF RADIATION-ENHANCED RANGE-II CONPUTER PROGRAM, FOR LATTICE SYMMETRY CHANNELED
ON THE GROWTH OF COPPER CRYSTALS OF LOW ON THE GROWTH OF COPPER CRYSTALS OF LOW
GROWTH OF COPPER CRYSTALS WITH LOW

MEGACYCLE FREQUENC/ EFFECT OF FAST NEUTRON IRRADIATION ON VIDCNCE FOR VACANCY CLUSIERINC IN UISLOLATIONS IN COPPER RADIATION C/ TEMPERATURE DEPENOENCE FROM $250 \mathrm{~K}$ TO $370 \mathrm{~K}$ OF RADIATION OI TEMPERATURE DEPENDENCE RADIATION CI TEMPERATURE DEPENDENCE FROM 2SOK TO 37OK OF DISLOCATION PIN SOLUTION OF COPPER IN COPPER-FREE SOLUTIONS EICH PIIS AT OISLOCATIONS
ROUS-OXIDE IN COPPER ON THE FORMATION OF DISLOCATIONS VIOR

ING I ANNEALEC CRYSTALSIDENCE FOR VACANCY CLUSTERING IN ING I. ANNEALEC CRYSTALS II IRRAD/ ETCH PIT STUDI
ICAL AND ELECTRON MICROSCOPE OBSERVATIONS ABSTRACT
DIFFUSION ON OISPLACEMENT PRODUCTION RATE ANO IR DIRECTIONS OF ATOMS
DISLOCATION DENSITY DISLOCATION DENSITY MODI
DISLOCATION INTERACTIONS $62-|\mathrm{V}-| 5-1\}$ $62-P \cup B-10$ $62-111-11-5$ $62-V-18-4$ $62-P U B-27$
$62-P U B-50$ $62-$ III-II-4 $62-1-3-2$ $62-11-7$ $62-I I-7-5$
$62-1 V-17-5$ 62-PUB-2 $62-v-18-1$ $62-1-1-2$ 62-PUB-50 $62-1 \mid I-11-4$ $62-I I I-13$ DISLOCATION INTERNAL FRICTION IN PURE COPPER AT 62-PUB-42 ANALYSIS OF INTERNAL 62-III-13-2 E $62-111-13-1$ INNING IN COPPER SINGLE CRYSTALS BY $62-111-13-3$
PNNING IN COPPER SINGLE CRYSTALS BY $62-P U B-33$ ANDNIC. OIS $62-111-11-2$ 作 DISLOCATIONS IN COPPER CRYSTALS DEFORMED BY BEND $62-111-11-5$
DISLOCATIONS IN URANIUM-DIOXIDE PLASTICITY OPT $62-11-8-5$ 
CTS IN GERMANIUM

THEORY OF DEPENDE 700 FORTRAN PROGRAM FORTRAN PROGRAM

ETCH PITS AT DISLOCATIONS

PPER IONS, ORIENTATION EFFECTS

EFFECT OF OISLOCATIONS ON COBALT-60 RADIATION-INDUCED DEFE 62-IV-15OF RADIATION-ENHANCED OIFFUSION ON DISPLACEMENT PRODUCTION RATE AND IRRAOIATION TEM $62-V-I 8-1$ -
OLECTRON CROSS SECTIONS FOR ATOMIC OISPLACEMENTS MOTT SERIES EVALUATION USING IBM- O2-I-6 ANODIC OISSOLUTION OF COPPER IN COPPER-FREE SOLUTIONS 62-III-11-2 ANODIC OISSOLUTION OF COPPER IN SOLUTIONS CONTAINING CO $62-111-11-1$ ELECTROCHEMICAL DISSOLUTION OF SINGLE CRYSTALLINE COPPER

PLE CRYSTALLINE COPPER 62-PUB-22

CALCULATION OF AVERAGES FOR PRIMARY RECOIL DISTRIBUTIONS

CALCULATION OF AVERAGES FOR PRIMARY RECOIL DISTRIBUTIONS

ABSTRACT

$62-P \cup B-24$ 62-iI- I-6 $62-I-1-8$

OMIUM, AND MAGNESIUM IMPURITIES GAMMA IRRA

CALCIUM, STRONTIUM, CA 62-IV-16-2 SED TO THERMAL NEUTRONS

TRANSMUTATION DOPING AND RECOIL EFFECTS IN SEMICONDUCTORS EXPO $62-I V-16-2$
$62-0 U B-13$ ONS OF TETRACHLOROETHANE AND DECANE IN. A FLUOR/ RA PPER THREE - GOLD, AND ' SINGLE CRYSTALS GROWN FROM SMALL DROPLETS GOLC, COPPER, SILVER, LEAD, INDIUM, CO 62-II-7-1
1 , ELECTRON SPIN RESONANCE, AND OPTICAL ABSORPTION OF THE E-PRIME-ONE CENTER IN FUSED SILICA OSCILLATOR / G2-II-9-1

RON SPIN RESONANCE ABSTRACT SPIN-LATTICE RELAXATION OF DETERMINED QY AN ETCH PIT TECHNIQUE

BALT-6D GAMMA-RADIATION UPON EXTRINSIC BISMUTH-TELLURIDE E COPPER

MOTT SERIES EVALLIATION USING IBM-7D9D FORTRAN PROGRAM

DISLOCATIONS IN URANIUM-OIOXIDE PLASTICITY OPTICAL AND

F UNANIUM-DIOXIDE SURFACE'FISSION FRAGMENT/ TRANSMISSION

TIC SPECTRA OF E-PRIME-TWO CENTERS IN CRYSTALLINE OUARTZ OF IHE E-PRIMEI RELATION BETWEEN MAGNETIC SUSCEPTIBILITY, IDE CKYSTALS ABSTRACT E-PRIME-ONE CENTERS IN SILICON-DIOXIDE $62-$ PUB-9 ERIME-TWO CENTERS IN CRYSTALLINE QUARTZ ELECT 62-1!-9-2 ELASTIC-PLASTIC TRANSITION IN COPPER CRYSTALS AS 62-PUB-47 ELECTROCHEMICAL DISSOLUTION OF SINGLE CRYSTALLIN $62-P U B-22$ ELECTRON CROSS SECTIONS FOR ATOMIC OISPLACEMENTS $62-1-6$ ELECTRON MICROSCOPE OBSERVATIONS ABSTRACT O2-11-8-5 ECTRON MICROSCOPE ELECTRON MICROSCOPY $62-11-8-4$ ELECTRON SPIN RESONANCE. ABSTRACT 62-II-8 LECTRONS ON SCINTILLATION PROCESS IN ALKALI HAL MONTE CARLO CALCULATIONS OF RANGES OF ENERGETIC ATOMS IN SOL IOS ABSTRACT RANGES OF ENERGETIC ATOMS IN SOL IOS ABSTRAC

THE RANGES OF ENERGETIC ATOMS IN SOL IOS THE RANGES OF ENERGETIC ATOMS IN SOL IDS

GRAM FOR LATTICE, MACHINE STUDIES OF THE SLOWING DOWN OF ROCESS IN ALKALI HALIDE CRYSTALS ABSTRACT EFFECT OF

I. RANDOM MODEL

II. LATTICE MODEL ENERGETIC ATOMS IN SOLIDS RANGE-II COMPUTER PRO $62-1-1-2$
ENERGETIC SECONDARY ELECTRONS ON SCINTILLATION P $62-I V-16-5$ APPROXIMATE METHODS FOR TREATING A 62-PUB-19 IN OF THEORETICAL MODEL OF ANISOTROPIC SPUTTERING AT HIGH ENERGIES BASED ON FOCUSING CHAINS COPPER ION B/ G2-I-2-2 STORED ENERGY IN THE OAK-RIDGE GRAPHITE REACTOR - 1960 62-PUB-44 1961 POSTANNEALING OF GRAPHITE-STORED ENERGY IN THE ORNL GRAPHITE REACTOR DISTRIBUTION IN KINETIC ENERGY OF DAUGHTER NUCLEI $62-1 \mathrm{I}-7-4$ $62-I I-7-4$
$62-P \cup B-35$ EXPER IMENTAL THRESHOLD IRRADIATION ENERGY REQUIRED FOR PRODUCTION OF DEFECT CLUSTER 62-IV-I5
HANCEC DIFFUSION IN COPPER - ALUMINUM ON NEUTRON FLUX AND ENERGY SPECTRUM THE DEPENDENCE OF RADIATION-EN 62-PUB-2 EXPERIMENTAL THRESHOLD IRRADIATION ENERGY REQUIRED FOR PRODUCTION OF DEFECT CLUSTER G2-IV-IS-9 RD SPHERE SCATTERING APPROXIMATIONS IMPACT PARAMETER AND ENERGY TRANSFER ENGINEERING PROPERTIES

HA $62-1-1-3$ $62-V-19$

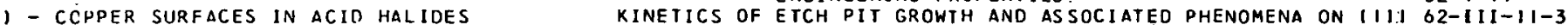

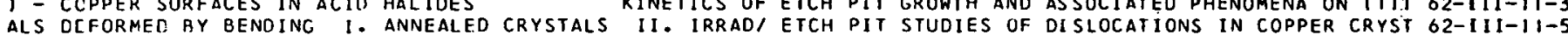
PLASTIC TRANSITION IN COPPER CRYSTALS AS DETERHINED BY

ANCDIC DISSOLUTION OF COPPER IN COPPER-FREE SOLUTIONS ETCH PITS AT DISLOCATIONS ETCHING OF IRRADIATED COPPER

ELASTIC$62-$ PUB -47 $62-1|1-1|-2$ $62-P \cup B-48$

RYLLIUM CANNED URANIUM-DIOXIDE AND FUELED BERYLLIUM-OXIDE EVALUATIONS /PELLETS CLAD IN STAINLESS STEEL BE 62-VI-2D-3 POST-IRRADIATION EXAMINATIONS OF URANIUM MONOCARBIDE

TRANSMITATION DOPING AND RECOIL EFFECTS IN SEMICONOUCTORS EXPOSEO TO THERMAL NEUTRONS ES MI STUDY OF EFFECTS OF COBALT-GI GAMMA-RADIATION UPON EXTRINSIC BISMUTH-TELLURIDE HIGH PURITY POTASSIUN-CHLORIDE EXTRINSIC BISMUTH-TELLURIDE ELECTRICAL PROPERT'I $62-P \cup B-32$ $62-P \cup B-13$ MAGNETIC PROPERTIES OF F-CENTERS AND M-CENTERS IN POTASSIUM-CHORIDE DIATION EFFECTS IN URANIUM-DOPED ZIRCONIA MONOCLINIC TO FACE-CENTERED-CUBIC TRANSIT ATISTICAL MECHANICS OF SEMICONOUCTORS COMPUTATION OF THE FERMI LEVEL IN NON METALS
IOUES FOR CAPTURE GAMMA RAYS CERIC-SULFATE REDUCTION AND FERROUS-SULFATE OXIDATION RIIICAL CURRENT OF NIOBIUM - ZIRCONIUM ALLOYS ON MAGNETIC FIELD ORIENTATION ORNL ICO - KILOGAUSS MAGNET, FISSION FRAGMENT TRACKS IN METAL FILMS

NITROGEN ION BOMBARDMENT OF THIN PLATINUM FILMS FISSION FRAGMENT TRACKS IN METAL FILMS ABSTRACT

NITROGEN ION BOMBARDMENT OF THIN PLATINUM FILMS 25-MEV NITROGEN IONS ABSTRACT FISSION FRACMENT TRACKS IN THIN FILMS OF URANIUM-DIOXIDE FISSION FRAGMENT TRACKS IN THIN FILMS OF URANIUM-DIOXIDE IE STUDIES ON SINGLE CRYSTALS OF UNANIUM-DIOXIDE SURFACE FISSION FRAGMENT TRACKS MICROCRACKING IN URANI/ FISSION FRAGMENT TRACKS IN METAL FILMS FISSION FRAGMENT TRACKS IN METAL FILMS
FISSION FRAGMENT TRACKS IN METAL FILMS ABSTRACT $62-I I-8-$ FISSION FRAGMENT TRACKS IN THIN FILMS OF URANIUM 62-II-8-2 FISSION FRAGMENT TRACKS IN THIN FILMS OF URANIUM 62-PUB-29

-DIOXIDE IABSTRACT -DIOXIDE E CONIINUOUS RELEASE OF FISSION GAS DURING IRRADIATION OF URANIUM-DIOXID 62-VI-20-4 CONTINUOUS RELEASE OF. FISSION GAS FROM URANIUM-DIOXIDE DURING IRRADIAT O2-PUB-8 ION PYROLYTIC CARBON COATED URANIUM-DICARBIDE PARTICLES FISSION GAS RETENTION LES PYROLYIIC CARBON COATED URANIUM-CICARBIOE PARTICLES FISSION GAS RETENTION AND OF TETRACHLOROETHANE I/ 62-V1-23 IATICN-ENHANCED DIFFUSION IN COPPER - ALUMINUM ON NEUTRON FLUX AND ENERGY SPECTRUM THE DEPENDENCE OF RAD G2-PUB-2 ARGON ACTIVATION MEASURES IRRADIATION FLUX CONTINUOUSLY RADIATION ON ATOMIC REARRANGEMENTS IN COPPER - ALUMINUM FLUX DEPENDENCE IODEL OF ANISOTROPIC SPIUTTERING AT HIGH ENERGIES BASED ON FOCUSING CHAINS OMIC. CISPLACEMENTS MOTT SERIES EVALUATION USING IBM-TO9O FORTRAN PROGRAM IS ON SINGLE CRYSTALS OF UNANIUM-DIOXIDE SURFACE FISSION FRAGMENT TRACKS FISSION FRAGMENT TRACKS FISSION FRAGMENT TRACKS I COPPER ION BOMBARDMENT OF COPP/ $62-1-2-2$ ELECTRON CROSS SECTIONS FOR AT $62-1-6$ MICROCRACKING IIN URANIUM-DIOXI/ 62-II-8-4 MN CROCRACKING IN URANIUM-DIOXI/ $\begin{aligned} & 62-11-8-4 \\ & 62-P U B-28\end{aligned}$
6 METAL FILMS FISSION FRAGMENT TRACKS IN METAL FILMS ABSTRACT $62-11-8-1$ FISSION FRAGMENT TRACKS IN THIN FILMS OF URANIUM-DIOXIOE 62-1I-8-2 ABSTRAC T

FISSION FRAGMENT TRACKS IN THIN FILMS OF. URANIUM-DIOXIDE $62-9 \mathrm{UB}-29$

ANALYSIS OF INTERNAL FRICTION IN COPPER AT MEGACYELE FREQUENCIES OSCILLATORY DISLOCATION MOTION $62-111-13-2$ DISLOCATION INTERNAL FRICTION IN PURE COPPER AT MEGACYCLE FREQUENCIES /ECT OF FAST NEUTRON IRRADIATION ON 62-PUB-42 YS COATAINING CARBON AND, OR NITROGEN APPARATUS FOR LOW FREOUENCY INTERNAL FRICTION IXATION IN IRON ALLO G2-V-I8-3 $N$ ANC, OR NITROGEN APPARATUS FOR LOW FREOUENCY INIERNAL FRICTION IXATION IN IRON ALLOYS CONTAINING CARBO G2-V-IB-3

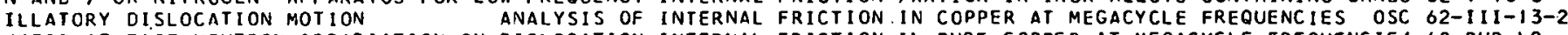
IFECT OF FAST NIEUTRON IRRADIATION ON DISLOCATION INTERNAL FRICTION IN PURE COPPER AT MEGACYCLE FREOUENCIEI G2-PUB-42 RADIATION ABSTRACI THERMALLY ACTIVATED INIERNAL FRICTION SPECTRUM IN COPPER FOLLOWING NEUTRON IR $62-111-13-4$
THERMALLY ACTIVATED INTERNAL FRICTION SPECTRUM IN COPPER FOLLOWING NEUTRON IR O2-PUB-4I RADIATION RADIATION RAPHITE

A THERMALLY ACTIVATED INIERNAL FRICTION SPECTRUM IN COPPER FOLLOWING NEUTRON IR POSTIRRADIATION EXAMINATION OF GRAPHITE. MATRIX GRAPHITE MATRIX FUEL COATED AND UNCOATED URANIUM-DICARBIDE IN G 62-VI-20-1
MARITIME REACTOR FUEL COLO SWAGED AND VIBRATORY COMPACTED URANIU 62-VI-20-5 M-OICXIDE - THORIUM-DIOXIDE
BERYLLIUM SHEATHED URANIUM-DIOXIDE FUEL CYCLE PROGRAM SINTERED AND FEN IRRADIATION TESTS 
DICARHIDE PARTICLES FISSION GAS RETENTION ABILITY IN NONMETALLIC CRYSTALS

MOSSBAUER EFFECT SPECTRA OF

COPPER ION BOMBARDMENT OF COPPER SPUTES RADIATION AS A

ANCE, AND OPTICAL ABSORPTION OF THE E-PRIME-ONE CENTER IN NE INFRARED ANO GAS CHROMATOGRAPHIC ANALYSIS POLYMERS I UMINTSC.FNC.F OF NLKALI HALIDES ALCIUN, STRCNTIUM, CADMIUM, AND MAGNESIUM IMPURITIES SULFATE OXIDATION MONITORING TECHNIOUES FOR CAPTURE LOW TEMPERATURE NEUTRON, FUNCTION OF ORIENTATION ISED ON FOCUSING CHAINS FUNDAMENTAL STUDIES
ICARHIDE PARTICLES FISSION GAS RETENTION COATED FUEL PARTICLES PYROLYTIC CARBON COATED URANIUM- O2-VI-20-2
IN STAINLESS STEEL BERYLLIUM CANNED URANIUM-DIOXIDE AND FUELED BERYLLIUM-OXIDE EVALUATIONS IPELLETS CLAD 62-VI-20-3

FUEL MATERIALS

$62-V I-20$

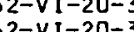
UNCTION MODIFYING MINORITY CARRIER CAPTURE PROB 62-1-3-2

FUNCTION OF INTERSTITIAL POSITION /AMAGE BY THE 62-I-5

$62-1-2-2$ $62-V-18$

GAMMA AND REACTOR RADIATION EFFECTS ON POLYSTYRE 62-VI-21

GAMMA IRRADIATION 62-IV-16-4

GAMMA IRRAOIATION OF DOPED POTASSIUM-CHLORIDE C 62-IV-I6-2

GAMMA RAYS CERIC-SULFATE REDUCTION AND FERROUS- 62-IV-I5- 12 GAMMA RECOIL DAMAGE IN CADMIUM 62-PU8-23 $L$ - - STRUCTURE SENSITIVITY OF COLOR CENTER HRODUCTION IN GAMMA-IRRADIATED POTASSUIM-CHLORIDE ABSTRACT IN O2-IV-IG-3 ITION ANO TRAPPING CENTERS IN CERMANIUM II. ANNEALING IN GAMMA-IRRADIATED, ANTIMONY-DOPED AND ARSENIC-DO/ G2-PUB-I7 ELECTRICAL PROPERTIES MI STUDY OF EFFECTS OF COBALT-60 GAMMA-RADIATION UPON EXTRINSIC BISMUTH-TELLURIOE 62-IV-I5-8 THERMOELECTRIC POWER IN GERMANIUM SEEBECK COEFFICIENT GAMMA-RAY IRRADIATION NAL AND 1 SOTHERMAL ANNEALING OF ANTIMCNY-DOPEO GERMANIUM GAMMA-RAY IRRADIATION AT 77K /GERMANIUM ISOCHRO G2-IV-IS-2 ND REACTOR RADIATION EFFECTS ON POLYSTYRENE INFRARED AND GAS CHROMATOGRAPHIC ANALYSIS POLYMERS GAMMA A G2-VI-2I CONTINUOUS RELEASE OF FISSION GAS DURING IRRADIATION OF URANIUM-DIOXIDE CONTINUOUS RELFASE OF FISSION GAS FROM URANIUM-DIOXIDE DURING IRRADIATION OLYTIC CARBON COATED URANIUM-DICARBIDE PARTICLLS FISSION GAS RETENTION DICARBIDE PARTICLES FISSION GAS RETENTION COPPER GEOMETRICAL MODEL OF MONOCRYSTAL SPUTTERING COP 62-1-2-1 RADIATION EFFECTS ON DEEP-LEVEL IMPURITY STATES IN GERMANIUM ARRIER RECONBINATION AND TRAPPING PROCESSES IN IRRAOIATED GERMANIUM OF UISLOCATIONS ON COBALT-6D RADIATION-INDUCEO DEFECTS IN GERMANIUM TRAPPING CENTERS IN ARSENIC-DOPED GERMANIUM UM ISOCHRONAL AND I SOTHFRMAI ANNFAL ING OF ANTIMONY-DOPED GERMANIUM 1 RACIATI ON-INDUCED RECONBINATION AND TRAPPING CENTERS IN GERMANIUM / RADIATION-INDUCED RECONBINATION AND TRAPPING CENTERS IN GERMANIUM F ANTIMCNY-CO/ ANNEALING OF IRRADIATION-INDUCED DAMAGE IN GERMANIUM ATION THERMOELECTRIC POWER IN GERMANIUM

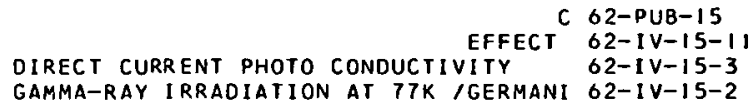
I. THE NATURE OF THE RECOMBINATION PR 62-PUB-16 11. ANNEALING IN GAMMA-IRRADIATED, AN 62-PUB-17 I SOCHRONAL AND ISOTHERMAL ANNEAL ING 0 62-IV-I5-2

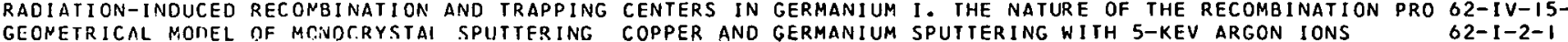
D, COPPER, SILVER, LEAD, INDIUM, COPPER THREF - GOLD, AND GERMANIUM-MELI CRYSTALLIZATION ABSTRACT /S GOL 62-II-7-I AND DIRECT CURRENT CONDUCTIVITY OF GLASSES LEAD-SILICAIE GLASS POLARIZATION AND CHARGE STORAGE / CURRENT O2-II-9-3 A' ALIFRNATIVG CURRENT ANID CIRLCT CURRENT CONDUCTIVITY OF GLASSES LEAD-SILICATE GLASS POLARIZATION AND CH G2-II-9-3 ILETS GOLD, COPPER, SILVER, LEAD, INDIUM, COPPER THREE - GOLD, AND GERMANIUM-MELT CRYSTALLIZATION ABSTR/ 62-II-7-I - GCLD, AND / SINGLE CRYSTALS GROWN FROM SMALL DROPLETS GOLD, COPPER, SILVER, LEAD, INDIUM, COPPER THREE 62-II-7-I oY

IIE MATRIX FUEL COATED AND UNCOATED URANIUM-DICARBIDE IN TRIX FIEL COATEO

DE IN CRAPHITE POSTIRRADIATION EXAMINATION OF GOLD, COPPER, SILVER, LEAD, INDIUM, COPPER THREE GRAPHITE POSTIRRADIATION EXAMINATION OF GRAPH GRAPHITE POSTIRRADIATION EXAMINATION OF GRAPH $62-V I-20-1$
GRAPHITE MATRIX FUEL COATED AND UNCOATED URANIU $62-V I-20-1$ GRAPHITE REACTOR STORED ENERGY IN THE OAK-RIDGE GRAPHITF RFACTOR - 1960 $62-11-7-4$ $62-11-7-4$

TOR 961 POSTANMEALING OF O

LEAD, INDIUM, COPPER THREE - GOLO, AND, SINGLE CRYSTALS URIFICATION OF RISMUTH-SELENIDE CRYSTALS DURING AND AFTER
R SURFACES IN ACID HALIDES KINETICS OF ETCH PIT SITY ENSITY MCDIFIED PRIDGMAN TECHNIQUE

EFFECT OF A PHASE TRANSFORMATION ON THE VAPOR-PHASE EFFECT OF A PHASE TRANSFORMATION ON IHE VAMUK HHASE IC SECONDARY ELECTRONS ON SCINTILLATION PROCESS IN ALKALI O ASSCCIATEU PHENOMENA ON $(111)$ - COPPER SURFACES IN ACID LUM INESCENCE OF ALKALI OR CENTER PROD/ INVESTIGATIONS NTE CARLO CALCULATIONS IED NAGINETIC FIELDS ABSTRAC CALUR IMETRIC MEASUREMENT OF NUCLEAR HEATING IN A REACTOR GROWTH BI SMUIH SELF DIFFUSION $62-11-7-1$ GROWTH AND ASSOCIATED PHENOMENA ON (111)- COPPE 62-111-11-3 GROWTH OF COPPER CRYSTALS OF LOW DISLOCATION DEN 62-PUB-50 GROWTH OF COPPER CRYSTALS WITH LOW DISLOCATION D GROWTH OF SINGLE CRYSTAL MERCURIC-SULFIDE GROWTH OF SINGLE MERCURIC-SULFIDE ABSTRACT

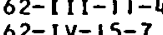
$62-1 V-15-6$ $62 \mathrm{PUB}-14$ KINETICS OF ETCH PIT GROWTH AN $2-1 \mathrm{~V}-16-5$ S AT ORNL - - SIRUCIURE SENSIIIIVITY OF COL 62-IV-I6-3 HARD SPHERE SCATTERINC APPROXIMATIONS IMPACT PA 62-1-1-3 STOPPINC POWER AS A BASIS FOR HARD-SPHERE APPROXIMATION BOHR POTENIIAL AND MO $62-I-1-1$ SPECIFIC HEAT OF SINGLE CRYSTAL MANANOUS-CHLORIDE IN APPL 62-IV-I6-7 $62-P \cup B-5$ DELTA RAYS ON THE RESPONSE OF INORGANIC SCINTILLATORS TO HEAVY PARTICLES INATION OF THEORETICAL MOIJEL OF ANISOTROPIC SPUTIERING AT HIGH ENERGIES BASEO ON FOCUSING CHAINS COPPER / 62 F-BAND AND M-BAND COLORING IN HEAVILY IRRAOIATED HIGH PURITY POTASSIUH-CHLORIDE $62-P \cup B-6$ TIONS ABSTRACT

NS FOR ATONIC DISPLACEMENTS MOTT SERIES EVALUATION USING HARD SPHERE SCATTERING $\triangle P P R O X I M A T I N N S$ S BRITTLE-CUCTILE TRANSITION TEMPERATURE SIUM-CHLORIDE CALCILIM, STRONTIUM, CADMIUM, AND MAGNESIUM

EFFECTS OF GASEOUS
RADIATION EFFECTS ON DEEP-LEVEL HIGHER-OROER AVERAGES OF PRIMARY RECOIL DISTRIBU IMPACT FORTRAN PROCRAM ELECTRON $62-1 v-16-1$ CROSS SECTIO 62-1-6 IMPUR ITIES IMPURITIES ON THE MAGNETIC PROPERTIES OF COPPER IMPUR ITIES ON THE MAGNET IC PRO
IMPURITY STATES IN GERMANIUM

OBIUM - I PERCENT ZI/ IN PILE STRESS RUPTURE EXPERIMENTS IS GROWN FROM SMALL DROPLETS GOLD, COPPER, SILVER, LEAD, OPERATION OF RESEARCH MAIERIALS MERS GAMMA AND REACTOR RADIATION EFFECIS ON POLYSTYRENE EFFECT OF DELTA RAYS ON IHE RESPONSE OF

ONAL RECOVERY ESTIMATE OF DEFECT CONCENTRAIION AND NACNETIC STRIJCTURE IRON - NICKSL ALLOYS ABSTRACT

NCONEL, 304 STAINLESS STEEL, 2IRCALOY-2, ANU NI 6 INFORMATION CENTER

INFORMAT ION CENTER

INFRARED AND GAS CHROMATOGRAPHIC ANALYSIS

INORGANIC SCINTILLATORS TO HEAVY PARIICLES

INSULATING CRYSTALS

INTENSE DAMAGE IN CADMIUM SUPPRESSION OF ISOCHR $62-1 V-16$ INTERACTION BETHEEN IRRAUIATION-INDUCED DEFECTS 62-1 $1-10-5$ DISLOCATION INTERACTIONS

STUDIES OF PENETRATION OF MOVING ATOMS IN SOLIDS AND INTERATOMIC POTENTIALS $62-111-13$ $62-1-1$ ING CARBON ANO I OR NITRIRFN APPARATUS FUR LOW FREOUENCY INTERNAL FRICTION IXATION IN IRON ALLOYS CONTAIN G2-V-I8-3 CIES OSCILLATORY DISLOCATION MOTION EQUENC/ EFFECT OF FAST NEUTRON IRRADIATION ON DISLOCATION EUTRCN IRRADIATION ABSTRACT THERMALLY ACTIVATED II A THERMALLY ACTIVATED A THERMALLY ACTIVATED
EUTRCN IRRACIATION FECT SPECTRA OF THE RECOILESS RADIATION AS A FUNCTION OF
PPROXIMATE METHODS FOR TREATING ANNEALING PROCESSES WHICH ITERINC AT hICH ENERGIES HASED ON FOCUSING CHAINS COPPER INTERNAL FRICTION IN PURE COPPER AT MEGACYCLE FR 62-PUB-42 INTERNAL FRICTION SPECTRUM IN COPPER FOLLOWING N $62-I I 1-13-4$ NTERNAL FRICTION SPECTRUM IN COPPER FOLLOWING N 62-PUB-41 INVOLVE A SPECIRUM OF ACTIVATION ENERGIES A $62-P U B-1$
ION BOMBAROMENT OF COPPER SPUTTERING YIELDS AS/ $62-1-2-2$ 
DMENT WITH 4-KEV ARGUN IONS SPUTTERING Y/ ARGON-PLUS-ONE ION BOMBAROMENT OF MEIAL SURFACES COPPER BOMBAR 62-III-14 ITROGEN IONS ABSTRACT
TTERING COPPER AND GERMANIUM SPUTTERING WITH S-KEV ARGON ION BONS NITROGEN ION BOMBARDMENT OF THIN PLATINUM FILMS N ION BOMBARDMENT OF THIN PLATINUM FILMS 2S-MEV NITROGEN IONS ODIC CISSOLUTION OF COPPER IN SOLUTIONS CONTAINING COPPER IONS ORIENTATION EFFECTS AN O2-III-IIABSTRACT GEOMETRICAL MODEL OF MONOCRYSIAL SPU $62-1-2-1$ TWEEN IRRADIATION-INDUCED DEFECTS AND MAGNETIC STRUCTURE IRON - NICKEL ALLOYS ABSTRACT INTERACTION BE 62-II-IO-5 APPARATUS FOR LOW FREQUENCY INTERNI STRESS RELAXATION IN IRON ALLOYS CONTAINING CARBON AND $/$ OR NITROGEN G2-V-I8-3 ETCHING OF IRRADIATED COPPER

R CKYSTALS DEFORMED BY HENIING I. ANNEALED CRYSTALS II. IRRADIATEO CRYSTALS ABSTRACT IOCATIONS IN COPPE 6 LONG-RANGE CHANNELING EFFECIS IN IRRADIATED CRYSTALS COPPER CRYSTALS ABSIRACT CARRIER RECOMBINATION AND TRAMPING PROCESSES IN IRRADIATED GERMANIUM F-BAND AND M-BAND COLORING IN HEAVILY IRRADIATEO HIGH PURITY PUTASSIUM-CHLORIDE LE IRANSIIION TEMPERATURE IMRACT TESTS ON IRRADIATED PRESSURE VESSEL STEELS BRITTLE-DUCTI LUMINESCENCE OF ALKALI HALIDES GAMNA IRRADIATION

INUOUS RELEASE CF FISSICN GAS FROM URANIUM-DIOXIDE DURING IRRADIATION PERATLRE RESISTIVITY OF SOUIUM AND THE EFFECTS OF NUCLEAR IRRADIATION ECTRIC POWER IN GERMANIUM SEEBECK COEFFICIENT GAMMA-RAY IRRADIATION ED INTERNAL FRICTION SPECIRUM IN COPPER FOLLOWING NEUTRON IRRADIATION ED INIERNAL FRICTION SPECIRUM IN COPPER FOLLOWING NEUTRON IRRADIATION -DIOX.IDE PELLETS CLAD IN STAINLESS STEEL BERYLLIUM CANN/ FECT CLUSTERS TOR $4 \mathrm{~K}$. FACILITY

EXPER IMENTAL IHRESHOL D IRR NEW LOW TEMPERATURE IPR
ACTIVATION MEASURES IRR IRRAOIATION EFFECTS ON METAL CLAD FUELS URANIUM 62-VI-20-3

- STRCNTIUM, CADMIUM, aNo MAGNESIUM IMPURITIES

GAMMA IRRADIATION OF DOPEO POTASSIUM-CHLORIDE CALCIUM NEUIRON IRRADIATION OF SUPERCONCUCTIING IIN

CONTINUOUS RELEASE OF FISSION GAS DURING IRRADIATION OF URANIUM-DIOXIDE $62-P \cup B-48$ $025+11-1-11-2$

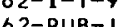
$62-1 V-16-1$ CONTINUOUS RELEASE OF FISSION GAS DUR ING ACHL COPPER ATEGACYCLE FREQUENC/ EFFECT OF FAST LOW TEMPERATURE IRRADIATION STUOIES

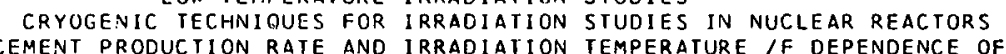
$62-I V-16-4$ LOW TEM 62-PUB-8 THERMOEL $62-1 V-15-4$

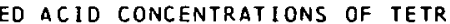

ON-ENHAINCED DIFFUSION ON DISPLACEMENT PRODUCTION RATE AND IRRADIAIION TEMPERATURE /F DEPENDENCE OF RADIATI 62 BERYLLIUM SHEATHED IIRANIUM-CIOXIDE FUEL ELEMENT IRRADIATION TESTS

NAL AND ISOTHERMAL ANNEALING OF ANTIMCNY-DO/ ANNEALING OF IRRADIAIION-INDUCED DAMAGE IN GERMANIUM ISOCHRO O2-PUB-3I RE IRON - NICKEL ALLOYS ABSTRACT INTERACTION BETWEEN IRRADIATION-INDUCEC DEFECTS AND MAGNETIC SIRUCTU O2-II-ID-5 DO/ ANNEALING OF IRRADIATION-INOUCED DAMAGE IN CERMANIUM ISOCHRONAL AND ISUTHERMAL ANNEALING OF ANTIMONY- O2-IV-IS-2 ATION INTENSE DAMAGE IN CADMIUM SUPPRESSION OF ISOCHRONAL RECOVERY ESTIMATE OF OEFECT CONCENTR O2-III- I2-2 1 IRRADIATICIN-INOUCED DAMAGE IIN GERMANIUM ISOCHRONAL AND ISOTHERMAL ANNEALING OF ANTIMONY-COPED CERMANIU/ O2-IV-15-2 IRCUNIUN ALLOYS ON MAGNETIC. FIELD ORIENTATION ORNL IOJ - KILOGAUSS MAGNET /RITICAL CURRENT OF NIOBIUM - 2 O2-IL-ID-I

MENA. CN $(111)$ - COPPER SUKFACES IN ACID HALIDES DISTRIBUTHON IN

KINETIC ENERGY OF DAUGHTER NUCLE I

KINETICS OF ETCH PIT GROWTH AND ASSOCIATED PHEND 62-PUB-35

ir OBSERVATIONS OF NEARLY PERfECT COPP.ER SINGLE CRYSSTALS LAR LANG CAMERA

$62-P U B-35$
$62-1 I I-11-3$ ELDEC MATERIALS SEMICONDUCTORS ABSTRACT THE RANGES OF ENERGETIC ATOMS IN SOLIOS 11 . LATTICE MODEL

ENERCETIC ATOMS IN SOLIDS RANGE-II COMPUTER PROCRAM FOR LA IRYSTALS GROWN FROM SMALL DROPLETS GOLD, COPPER, SILVER, LEAD, INDIUM, COPPER THREE - GOLD, AND GERMANIU/ 62-II-7-1 ITING CURRENT AND DIRECT CUIRRENT CONDUCTIVITY OF GLASSES LEAD-SILICATE GLASS POLARIZATION AND CHARGE STOI O2-II-9-3 CAL MECHANICS OF SEMICONDUCTORS COMPUTATION OF THE FERMI LEVEL IN NGN METALS STATISTI $62-1-3-1$

TALS COPPER CRYSTALS ABSTRACT LONG,-RANGE CHANNELING EFFI $62-11-7-3$

IOUE ON THE GROWTH OF COPPER CRYSTALS OF LOW DISLOCATION DENSITY ALLUYS CONTAININC CARBON AND K SHIELDING REACTOR $4 K$ TACILITY LOW TEMPERATURE IRRADIATION. FACILITY FOR THE BUL LOW TEMPERATURE IRRADIATION STUDIES

\section{CADMIUM}

FECTS OF NUICLEAR IRRADIATION

LOW TEMPERATURE RESISIVITY OF SODIUM AND THE EF $62-$ PUB--4

FCAL PFOPERTIES OF.

Y POTASSIUM-CHLORIDE LUMINESCENCE OF ALKALI HALIDES GAMMA IRRADIATIO $62-1 \mathrm{~V}-16-4$

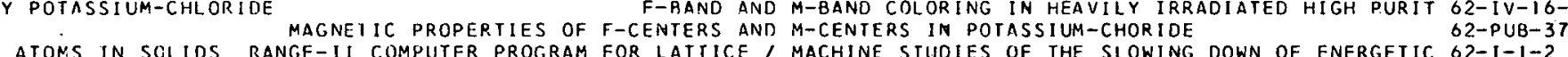
F-RAND AND LMMINESCENCE OF ALKALI HALIDES GAMMA IRRADIATIO $62-1 \mathrm{~V}-16-4$ ATORS IN SOLIDS RANGE-II COMPUTER PROCRAM FOR LATHICE MNCHINE STUD POTASSIUM CHE LLOYS ON MAGNETIC FIELD ORIENTATION ORNL IOO - KILOGAUSS MAGNET /RITICAL CURRENT OF NIOBIUM - 2IRCONIUM A G2-II-ID-I IUCTING CRITICAL CURRENT OF NIOBIUM - ZIRCONIUM ALLOYS ON MAGNEIIC FIELO ORIENTATION ORNL IOO - KILOGAUSI O2-II-IO-I

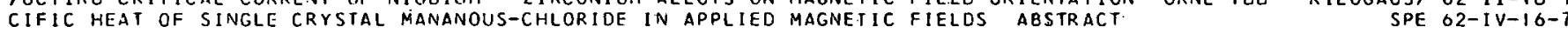
N POTASSIUM-CHORIDE EFFECTS OF GASEOUS IMPURITIES ON THE MAGNEIIC FIELDS ABSTRACT.
MAGNETIC PROPERTIES OF COPPER SPE $62-1 V-16-7$
$62-P U B-39$ MAGNETIC PROPERTIES. OF F-CENTERS AND M-CENTERS I $62-P U B-37$ CT INTERACTICN BETWEEN IRRADIATIUN-INDUCED DEFECIS AND MAGNETIC STRUCTURE IRON - NICKEL ALLOYS ABSTRA 62-II-IO-S HENATE (IV) AND POTASSIUM HEXARROMORHENATE(IV) FROM 5K TO/ MAGNETIC SUSCEPTIBILITY OF POTASSIUM HEXACHLOROR O2-PUB-6 - AND OPTICAL ABSORPTION OF THE E-PRIMEI RELATION BETWEEN MA MAGNETIC SUSCEPTIBILITY, ELECTRON SPIN RESONANCE MAGNETISM OF POTASSIUM-CHLORIDE $62-11-9-1$ UPON EXTRINSIC BISHUTH-TELLURIDE ELECTRICAL PROPERTIES MAGNETISM OF POTASSIUM-CHLORIDE S.TRACT
SPECIFIC HEAT OF SINGLE CRYSTAL MANANOUS-CHLORIDE IN APPLIED MAGNETIC FIELDS AB O2-IV-IG-7 MENTS IN DEFOPMED COPPER - SILICON ANC COPPER - SILICON - MA MANGANESE ALLOYS ABSTRACT M ATOMIC REARRANCE $62-V-18-4$.
MARITIME PREACTOR FUEL COLD SWAGED AND VIBRATORY $62-V I-20-5$ COMPACTEC LRANIUM-DIOXIDE DE IN GRAPHITE POSTIRRADIATION EXANINATION OF GRAPHITE MATRIX FUEL COATED AND UNCOAIED URANIUM-DICARBI O2-VI-2O-1 CALORIMETRIC MEASUREMENT OF NUCLEAR HEATING IN A REACTOR O2-PUB-5 ARGON ACTIVATION MEASURES IRRADIATION. FLUX CONIINUOUSLY

RCONIUM WIRE AT LOW TEMPERATURES TENSILE PROPERTIES AT, MECHANICAL PROPERTIES OF NIOBIUM - 25 PERCENT 21 62-II-ID-3 THE INVESTIGATION OF RADIATION DAMAGE USING MECHANICAL VIBRATIONS

FERMI LEVEL IN NON METALS STATISTICAL MECHANICS OF SEMICONDUCTORS COMPUTATION OF THE O2-I-3-1 OTION ANALYSIS OF INTERNAL FRICTION. IN COPPER AT MEGACYCLE FREQUENCIES OSCILLATORY DISLOCATION M O2-III-13-2 IATICN ON OISLOCAIION INTERNAL FRICTION IN PURE COPPER AT MEGACYCLE FREQUENCIES /ECT OF FAST NEUTRON IRRAD G2-PUB-42 GROWTH OF SINGLE CRYSTAL MERCURIC-SULFIDE

RANSFORMATION ON THE VAPOR PHASE GROWIH OF SINGLE-CRYSTAL MERCURIC-SULFIDE PHASE TRANSFORMATION ON THE VAPOR-PHASE GROWTH OF SINGLE MERCURIC-SULFIDE GOLDIO.82) - MERCURY(L. 18 ) -
N STAINLESS STEEL BERYLLIUN. CANN/ IRRADIATION EFFECTS ON METAL CLAD FUELS FISSION FRAGMENT TRACKS IN METAL FILMS FISSION FRAGMENT TRACKS IN METAL FILMS ABSTRACT

ABSTRACT EFFECT OF A PHASE T $62-$ PUB-14 FUELS URANIUM-DIOXIDE PELLETS CLAD I $62-V I-20-3$ INVESTIGATIONS OF METAL SURFACES

GON IONS SPUTTERING YI ARGON-PLUS-ONE ION BOMBARDMENT OF METAL SURFACES

COPPER BOMBAROMENT WITH L-KEV $62-111-11$


ON THE INTERPRETATION OF RADIATION EFFECTS IN NOBLE METALS

CONTRIBUTION OF SLOW NEUTRONS TO REACTOR DAMAGE IN METALS OF SEMICONDUCTORS COMPUTATION OF THE FERMI LEVEL IN NON METALS REACTOR DAMAGE IN PURE METALS AT $4 \mathrm{~K}$

$62-P \cup B-20$ $62-P \cup B-26$ STATISTICAL MECHANICS $62-1-3-$ TALS GF UNANIUR:DIOXIDE SURFACE FISSION FRAGMENT TRACXS MICROCRACKING IN URANIUM-DIOXIDE /ON SINGLE CRYS 62-I I-8-4 IONS IN URANIUM-OIOXIDE PLASTICITY OPTICAL AND ELECTRON MICROSCOPE OBSERVATIONS -DIOXIDE SLRFACE FISSION FRAGMENT/ TRANSMISSION ELECTRON MICROSCOPE STUDIES ON SINCLE CRYSTALS OF UNANIUM 62-II-B-4 MODIFYING MINORITY CARRIER CAPTURE PROBABILITY IN NONMETAL $62-1-3-2$

THE RANGES OF ENERGETIC ATOMS IN SOLIOS I. RANDOM MODEL

IHE RANGES OF CNERGETIC ATOMS IN SOLIDS II. LATTICE MODEL IHE RANGES OF ENLRGETIC ATOMS IN SOLIDS II. LATTICE MODEL OF ANISOTROPIC SPUTTERING AT HIGH ENERGIES O2-I-2-2
BASEC ON FOCUSINC CHAINS CO/ EXAMINATION OF THEORETICAL MODEL OF ANISOR $62-P \cup B-30$ ANIUM SPUTTERING HITH 5-KEV ARGON IONS N NONMETALLIC CRYSTALS MOOIFYING MINORITY CARRIER CAPTURE PROBABILITY I 62-1-3-2 T-IRRADIATION-SULFATE OXIDATION POST-IRRADIATION EXAMINATIONS OF URANIUM MONITORING TECHNIOUES FOR CAPTURE GAMMA RAYS CE MONOCARBIDE

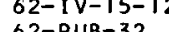
MONOCLINIC TO FACE-CENTERED-CUBIC TRANSITION IN 62-PUB-32 ZIRCONIUM-D/ RAOIATION EFFECTS IN URANIUM-DOPEO ZIRCONIA TTERING WITH 5-KEV ARGON IONS GEOMETRICAL MOOEL OF MOL
A BASIS TOR HARD-SPHERE APPROXIMATION BOHR POTENTIAL AND MO MONOCRYSTAL SPUTTERING COPPER AND GERMANIUN. SPU 62-1-2-1 A BASIS TOR HARD-SPHERE APPROXIMATION BOHR POTENTIAL AND MONTE CARLO CALCULATIONS G ATOMS IN SOLIOS ABSTRACT

TIO/ POSSIRLE EXPERIMENT TO STUDY RADIATION DAMAGE BY THE MO MONTE CARLO CALCULATIONS OF RANGES OF ENERGETIC $62-1-1-4$ SPECTRA OF THE RECOILESS RADIA $62-1-5$ OGRAM ELECTRON CROSS SECTIONS FOR ATOMIC OISPLACEMENTS MOTT SERIES EVALUATION USING IBM-7DQO FORTRAN PR G2-I-6 SEMICONOUCIORS FOR ATOMIC OISPLACEMENTS D RECCMBINATION AND TRAPPING CENTERS IN GERMANIUM I. THE NATURE OF THE RECONBINATION PROCESS ITION-INDUCE G2-PUB-IG ID RECOMBINATION AND TRAPPING CENTERS IN GERMANIUM I. THE NATURE OF THE RECOMBINATION PROCESS II. ANNEALI/ G2-IV-I5-I RACTION TOPOGRAPIIS AND SINGLE CRYSI X-RAY OBSERVATIONS OF NEARLY PLRFECT COPPER SINGLE CRYSTALS LANG DIFF O2-II-7-5 E OF RADIATION-ENHANCED DIFEUSION IN COPPER ACTIVATED INTERNAL FRICTION SPCCTRUM IN COPPER FOLLOWING NEUTRON IRRADIATION ACTIVATED INTERNAL FRICTION SPECTRUM IN COPPER FOLLOWING URON IRRADIATION ABSTRACT A THERMALLY $62-P U B-41$ NEUTRON IRRADIATION OF SUPERCONDUCTING TIN

TION IN PURE COPPER AT MEGACYCLE FREQUENCI EFFECT OF FAST NEUTRON IRRADIATION ON DISLOCATION INTERNAL FRIC O2-PUB-42 RACT LATTICE DEFECT PRODUCTION IN THERMAL NEUTRON SHIELDED MATERIALS SEMICONDUCTORS ABST 62-IV-IS-IO LOW TEMPERATURE MEUTRON GAMMA RECOIL DAMAGE IN CADMIUM 6 ANC RECOIL EFFECTS IN SEMICONDUCTORS EXPOSED TO THERMAL NEUTPONS TO REACTOR DAMACE IN METALS TONION DOPIN O2-PUB-I3 RRADIATION-INDUCED GEFECTS AND MAGNETIC STRUCTURE IRON - NICKEL ALLOYS ABSTRACT INTERACTION BETWEEN I G2-II-ID-5 ENTATI/ DEPENDENCE OF SUPERCONDUCTING CRITICAL CURRENT OF NIOBIUM - ZIRCONIUM ALLOYS ON MAGNETIC FIELD ORI G2-II-IO-I XPERIMENTS INCONEL, 304 STAINLESS STEEL, ZIRCALOY-2, AND NIORIUM - I PERCENT ZIRCONIUM / STRESS RUPTURE E 62-V-I9-1 RATURES TENSILE PROPERTIES AT / MECHANICAL PROPERT IES OF NIOBIUM IESS RELAXAIION IN IRON ALLOYS CONTAINING 25-MEV NITROGEN IONS ABSTRACT

\section{NITROGEN ION BOMBARDMENT OF THIN PLATINUM FILMS} NITROGEN ION GOMBAROMENT OF THIN PLATINUM FILMS

NITROGEN ION BOMBARDMENT OF THIN PLATINUM FILMS 25-MEV NITROGEN IONS ABSTRACT UN IHL INTERPRETATION OF RAOIAIION EFFECTS IN NOBLE METALS NICS OF SEMICONCUCTORS COMPUIATION OF THE FERMI LEVEL IN NON METALS UNCIION MODIFYING MINORI IY CARRIER CAPTURE PROBABILIIY IN NONMETALLIC CRYSTALS RADIATION STABILITY OF NONMFTALLIC STRUCTURES TRAPPING AND RECOMBINATION PROBABILITIES IN NONMFTALS

CALORIMETRIC MEASUREMENI OF NUCLEAR HEATING IN A REACTOR

LOW TEMPERATURE RESISIIVITY OF SODIUM AND THE EFFECIS OF NUCLEAR IRRADIATION CRYOGENIC IECHNIOUES FOR IRRADIATION STUOIES IN NUCLEAR REACTURS DISTRIKIIIION IN KINETIC ENERGY OF CAUGHTER NUCLEI

STORED ENERGY IN THE OAK-RIDGE GRAPHITE REACTOR - 1960 $62-11-8-3$ $62-P \cup B-40$ $62-11-8-3$ 6.2-PบB-2ก

STATISTICAL MECHA $62-1-3-1$ DENSITY F $62-1-3-2$ $62-P \cup B-12$ $62-P \cup B-36$ $62-P \cup B-5$ $62-P \cup B-4$ $62-P \cup B-3$ $62-P \cup 8-35$ $32-P \cup B-44$ IEN MAGNCTIC SUSCEPIIBILITY, ELECIRON SPIN RESONANCE, AND OPTICAL ABSORPTION OF THE E-PRIME-ONE CENTER IN/ G2-II-Q-I WEEN 25000 A AND $6000 A$

STRACT DISLOCATIONS IN URANIUM-DIOXIDE PLASTICITY OP OPTICAL AND ELECTRON MICROSCOPE OBSERVATIONS AB 62-II-8-5. BOMBARTMENT OF ISSULUTION OF CCPPER IN SOLUTIONS CONIAINING COPPER IONS ORIENTATION EFFECTS ROD/ INVESTICATIONS OF COLOR-CENTERS IN ALKALI HALIDES AT ORNL - - STRUCTURE SENSITIVITY OF COLOR CENTER P 62-IV-IG-3 1961 POSTANNEALINC OF GRAPHITE-STORED ENERGY IN THE ORNL GRAPHITE REACTOR

NIOHIUM - ZIRCONIUM ALLOYS ON MAGNETIC FIELD ORIENTATION CAL AESORPIION CF THE E-PRINE-ONE CENTER IN FUSED SILICA OF INTERNAL FRICTION IN COPPER AT MEGACYCLE FREQUENCIES E GAMMA RAYS CERIC-SULFATE REDUCTION AND FERROUS-SULFATE ORNL IOO - KILOGAUSS MAGNET IRITICAL CURRENT OF 62-11-10OSCILLATOR STRENGTH ABSTRACT ISONANCE, AND OPTI 62-II-9-1 OSCILLATORY OISLOCATION MOTION

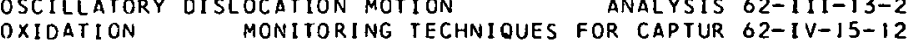
RYSTALLINE GUARTZ ELECTRON SPIN RESONANCE ABSIRACT PARAMAGNETIC SPECTRA OF E-PRIME-TWO CENTERS IN C G2-II-9-2 HARD SPHERE SCATTERING APPROXIMATIONS IMPACT PARAMETER ANO ENERGY TRANSFER

RAYS DN THE RESPONSE OF INORGANIC SCINTILLATORS TO HEAVY PARIICLES FUEL PARTICLES PYROLYTIC CARBON COATED URANIUM-DICARBIDE PARTICLES FISSION GAS RETENTION COATED 62-VI-20-2 EFFECT OF DELTA $62-1-1-3$ ON THE FORMATION OF OISLOCATIONS AROUND PRECIPITATE PARTICLES OF CUPROUS-OXIDE IN COPPER COATED $62-V 1-20-2$ IRRADIATION EFFECTS ON METAL CLAD FUELS URANIUM-DIOXIDE PELLETS CLAD IN STAINLESS STEEL BERYLLIUM CANNE O2-VI-20-3 ION STABILITY

STUDIES OF PENETRATION OF MOVING ATOMS IN SOLIDS AND INIERA G2-I-I.
TOPIC POTENTIALS CERAMICS PELTIER POWER CALIBRAIION ZIRCON CERAMIC RADIAT 62-VI-22 EFFECT OF A PHASE IRANSFORMATION ON THE VAPOR PHASE GROWTH OF SINGLE-CRYSTAL MERCURIC-SULFIDE G2-PUB-I F SINGLE-CRYSTAL MERCURIC-SULFIOE F SINGLE MERCURIC-SULFIDE ABSTRACT EFFECT OF A PHASE TRANSFGRMATICN ON THE VAPOR PHASE GROWTH 0 62-PUB-14 IDES EFFECT OF A PHASE IRANSFORMATION ON THE VAPOR-PHASE GROWTH 0 62-IV-15-6 KINETICS OF FTCH PIT GROWTH AND ASSOCIATED PHENOMENA ON 1111 - COPPER SURFACES IN ACID HAL O2-III-II-3 APPING CENTERS IN ARSENIC-DOPEO GERMANIUM DIRECT CURRENT PHOTO CONDUCTIVITY VACANCY CIUSTERING IN DISLOCATIONS IN COPPER DISLOCATION PINNING BEHAVIOR VACANCY CLUSTERING IN OISLOCATIONS IN COPPER DISLOCATION PINNING BEHAVIOR
TEMPERATURE DEPENDENCE FROM $250 K$ TO $370 K$ OF DISLOCATION PINNING IN COPPER SINGLE CRYSTALS BY RADIATION D O2-III-I3-3 1 TEMPERATURE DEPENDENCE FROM $250 K$ TO $370 K$ OF DISLOCATION PINNING IN COPPER SINGLE CRYSTALS BY RADIATION D O2-III-I3-3
1 TEMPERATURE DEPENDENCE FROM $250 \mathrm{~K}$ TO $370 K$ OF DISLOCATION PINNING IN COPPER SINGLE CRYSTALS BY RADIATION D O2-PU8-33 OPPER SURFACES IN ACID HALIDES KINETICS OF EICH PIT GROWTH AND ASSOCIATED PHENOMENA ON (III) - C G2-III-II-3 EFORMEN BY BENDING I. ANNE ALED CRYSTALS II. IRRAD/ ETCH PIT STUDIES OF DISLOCATIONS IN COPPER CRYSTALS D G2-III-II-S IC TRANSITICN IN COPPER CRYSTALS AS DETERMINED BY AN ETCH PIT TECHNIQUE OOIC CISSOLLTION OF COPPER IN COPPER-FREE SOLUTIONS ETCH PITS AT DISLOCATIONS ELASTIC-PLAST $62-P U B-47$ 114 KEV AICON IOPIS SPUTTERINC YIELD FOR 1111$)$ - 11 IIMINIIM PI.ANF /OF METAL SURFACES COPPER BOMBARDMENT HIT G2-III-I4 RVATIONS ABSTRACT DISLOCATIONS IN URANIUM-DIOXIDE PLASTICITY OPTICALANDELECTRON MICROSCOPE OBSE G2-II-8-5 NITROGEN ION BUMBARUMENT OF THIN PLATINUM FILMS

NITROGEN ION BOMBAROMENT OF THIN PLATINUM FILMS 25-MEV NITROGEN IONS ABSTRACT G2-II-8-3 RECT CURRENT CONDUCTIVITY OF GLASSES LEAD-SILICATE GLASS POLARIZATION AND CHARGE STORAGE I CURRENT AND DI G2-II-9-3 
POLYSTYRENE INFRARED AND GAS CHROMATOGRAPHIC ANALYSIS ALYSIS POLYMERS GAMMA AND REACTOR RADIATION EFFECTS ON BIDE

RNL GRAPHITE REACTOR

UEL COATED AND UNCOATED URANIUM-DICARBIDE IN GRAPHITE

IIC SUSCEPTIBILITY OF POTASSIUM HEXACHLORORHENATEIIV) AND XABROMORHENATEIIVI FROM 5K TO/ MAGNETIC SUSCEPIIBILIIY OF MAGNETISM OF

AND AND M-BAND COLORING IN HEAVILY IRRADIATEO HIGH PURITY
AND MAGNESIUM IMPURITIES GAMMA IRRADIATION OF DOPEO TASSIUM-CHLORIDE

POTASSIUM-CHLORIOE

POTASS IUM-CHLORIOE

MAGNETIC PROPERTIES OF F-CENTERS AND M-CENTERS IN POTASSIUM-CHORIDE

ENSITIVITY OF COLOR CENTER PRODUCI ION IN GAMAA-IRRADIATED POTASSUIM-CHLORIDE

PING POHER AS A BASIS FOR HARD-SPHERE APPROXIMATION BOHR POTENTIAL AN

OF PENETRATION OF MOVING ATOMS IN SOLIDS AND INTERATOMIC POTENTIALS

$$
\text { FUEL CYCLE PROGRAM SINTERED AND }
$$

STOPPING

CERAMICS PELTIER
THERMOELECTRIC

POWER AS A BASIS FOR HARD-SPHERE APPROXIMATI
POS

BOHR POTENTIAL ANO MONTE CARLO CALCULATIONS

ILI ITY
AY IRRADI ATION

ON TEMPERATURE

ON THE FORMATION OF DISLOCATIONS AROUND P IMPACT TESTS ON IRRADIATEO P CALCULATION OF AVERAGES FOR
CALCIILATION OF AVERAGES FOR HIGHER-ORDER AVERAGES OF TRAPPING AND RECOMBINATION.

DENSITY FUNCTION MODIFYING MINORITY CARRIER CAPTURE

T NEUTRON IRRADIATION ON OISLOCATION INTERNAL FRICTION IN

\section{REACTOR OAMAGE IN PURE MATERIALS}

AND AFTER GROWTH BISMUTH SELF DIFFUSION SUPERCONDUCTING TRANSITION TEMPERATURE OF

PURIFICATION OF BISMUTH-SELENIDE CRYSTALS DURING PURIFIED TECHNETIUM $8.22 \mathrm{~K}$ VALUE ABSTRACT

LES FISSION GAS RETENTION

COATED FUEL PARTICLES

PYROLYTIC CARBON COATED URANIUM-DICARBIDE PAR

ARAMAGNETIC SPECTRA OF E-PRIME-TWO CENTERS IN CRYSTALLINE OUART 2 ELECTRON SPIN RESONANCE ABSTRACT

IDAMAGE BY THE MOSSBAUER EFFECT SPECTRA OF THE RECOILESS

A OF THE RECOILESS RADIATIO/ POSSIBLE EXPERIMENT TO STUDY THE INVESTIGATICN OF RADIATION AS A FUNCTION OF INTERSTITIAL POSITIO/ 62-I-5 RADIATION DAMAGE BY THE MOSSBAUER EFFECT SPECTR 62-1-5 RADIATION DAMAGE USING MECHANICAL VIBRATIONS $370 K$ OF DISLOCATION PINNING IN COPPER SINGLE CRYSTALS BY RADIATION DEFECTS ABSTRACT INDENCE FROM 25OK TO 62-11I-I3-3 $370 K$ OF DISLOCATION PINNING IN COPPER SINGLE CRYSTALS BY RADIATION DEFECTS IATURE OEPENDENCE FROM 25OK TO O2-PUB-33 ONCENTRATIONS OF TETRACHLOROETHANE AND DECANE IN A FLUOR/ ON THE INTERPRETATION OF R

RADIATION EFFECTS IN NOBLE METALS

RADIATION EFFECTS IN URANIUM-DOPEO ZIRCONIA

OCLINIC TO FACE-CENTERED-CUBIC TRANSITION IN ZIRCONIUM-DI RADIATION EFFECTS IN URANIUM-DOPED ZIRCONIA RADIATION EFFECTS IN 2 INC-SULFIDE RADIATION EFFECTS ON DEEP-LEVEL IMPURITY STATES 62- 6 I $15-5$

IN GERMANIUM

AS CHROMATOGRAPHIC ANALYSIS

LUMINUM FLUX DEPENDENCE

POL YMERS

GAMMA AND REACTOR EFFECT OF

IATION EFFECTS ON POLYSTYRENE INFRAREO AND CERAMICS PELTIER

RADIATION ON ATOMIC RE

RADIATION ON ATOMIC
RADIATION STABILITY

RADIATION STABILITY OF NONMETALLIC STRUCTURES

$M$ ON NEUTRON FLUX ANO ENERGY SPECTRUM THE DEPENDENCE OF RADIATION-ENHANCED DIFFUSION IN COPPER - ALUMINU
OUCTION RATE ANC IRRAOIATION TEM/ THEORY OF OEPENDENCE OF RADIATION-ENHANCED DIFFUSION ON DISPLACEMENT PRO

EFFECT OF DISLOCATIONS ON COBALT-GD RADIATION-INDUCED DEFECTS IN GERMANIUM

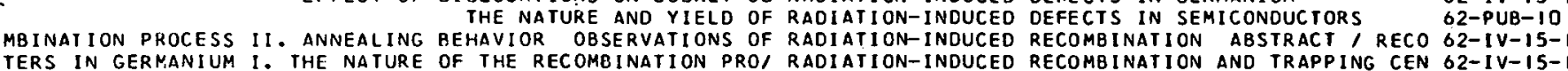

TERS IN GERMANIUM I. THE NATURE OF THE RECOMBINATION PRO/ RADIATION-INDUCED RECOMBINATION AND TRAPPING CEN O2-IV-IS-I
TERS IN GERMANIUM 1 . THE NATURE OF THE RECOMBINATION PR/ RADIATION-INDUCED RECOMBINATION AND TRAPPING CEN G2-PUB-IS

TERS IN GERMANIUM II. ANNEALING IN GAMMA-IRRADIATED, AN/ RADIAIION-INDUCED RECOMBINATION AND TRAPPING CEN G2-PUB-I7

THE RANGES OF ENERCETIC ATOMS IN SOLIDS I. RANDOM MODEL

ITUOIES OF THE SLOWING DOWN OF ENERGEIIC ATOMS IN SOLIDS RANGE-II COMPUTER PROGRAM FOR LATTICE SYMMETRY I $62-P U B-30$

RANGE-II COMPUTER PROGRAM FOR LATTICE SYMMETRY / 62-I-1-2 RANGES OF ENERGETIC ATOMS IN SOLIDS ABSTRACT $62-1-1-5$

ODEL THE RANGES OF ENERGETIC ATOMS IN SOLIDS THE RANGES OF ENERGETIC ATOMS IN SOLIDS

I. RANDOM M 62-PUB-30

II. LATTICE O2-PUB-34

F RADIATION-ENHANCED DIFFUSION ON DISPLACEMENT PRODUCTION RATE AND IRRADIATION TEMPERATURE IF DEPENDENCE O G2-VI IB-I

E OXIDATION MONITORING TECHNIQUES FOR CAPTURE GAMMA RAYS CERIC-SULFATE REDUCTION AND FERROUS-SULFAT G2-IV-I5-12

TO HEAVY PARTICLES

EFFECT OF DELTA RAYS ON
CALORIMETRIC MEASUREMENT OF NUCLEAR HEATING IN A REACTOR

TANNEALING OF GRAPHITE-STORED ENERGY IN THE ORNL GRAPHITE REACTOR

W TEMPERATURE IRRADIATION FACILITY FOR THE BULK SHIELDING REACTOR 4K FACILITY

STORED ENERGY IN THE OAK-RIDGE GRAPHITE REACTOR - 1960

CONTRIBUTION OF SLOW NEUTRONS TO REACTOR DAMAGE IN METALS

D URANIUM-DIOXIDE

REACTOR DAMAGE IN METALS
REACTOR DAMAGE IN PURE METALS AT $4 K$

62-PUB-5

1961 POS $62-11-7-4$

NEW LO $62-1$ II $1-12-3$

$62-P \cup B-44$

62-PUB-26

$62-11 \mathrm{I}-12-1$

MATIIME REACTOR FUEL COLO SWAGED AND VIBRATORY COMPACTE 62-VI-2O-5 ENCE

CRYOGENIC TECHNIQUES FOR IRRADIATION STUDIES IN NUCLEAR, REACTORS

ENCPPE

EFFECT OF RADIATION ON ATOMIC REARRANGEMENTS IN COPPER - ALUMINUM FLUX DEPEND $02-V 1-21$ $62-P \cup B-3$ E ALLOYS ABSTRACT

ATOMIC REARRANGEMENTS IN DEFORMED COPPER - SILICON AND LOW TEMPERATURE NEUTRON, GAMMA RECOIL DAMAGE IN CAOMIUM

CALCULATION OF AVERAGES FOR PRIMARY RECOIL DISTRIBUTIONS

CALCULATION OF AVERAGES FOR PRIMARY RECOIL DISTRIBUTIONS

ABSTRACT

62-PUB-23

$62-P \cup B-24$

$62-1-1-6$ $62-1-1-8$

MAL. NEUTRONS IRANSMUTATION DOPING AND RECOIL EFFECTS IN SENICONDUCTORS EXPOSED TO THER 62-PUB-1

IRADIATION DAMAGE BY THE MOSSBAUER EFFECT SPECTRA OF THE RECOILESS RADIATION AS A FUNCTION OF INTERSTITI II. ANNEALING BEHAVIOR OBSERVATIONS OF RADIATION-INDUCED RECOMBINATION ABSTRACT I RECOMBINATION PROCESS

I. THE NATURE OF THE RECOMBINAIION PRO/ RADIATION-INDUCED RECOMBINAIION AND IRAPPING CENTERS IN GERMANIUM

I. THE NATURE OF THE RECOMBINATION PR/ RADIATION-INDUCED RECOMBINATION AND TRAPPING CENTERS IN GERMANIUM

1I. ANNEALING IN GAMMA-IRRADIATED; AN/ RADIATION-INDUCED RECOMBINATION AND TRAPPING CENTERS IN GERMANIUM ED GERMANIUM CARRIER RECOMBINATION AND TRAPPING PROCESSES IN $62-1 \mathrm{I}-5-15-$ $62-1 V-15-1$ 62-PUB- 16 62-PUB-17 TRAPPING AND RECOMBINATION PROBABIL ITIES IN NONMETALS

N AND TRAPPING CENTERS IN GERMANIUM 1. THE NATURE OF THE RECOMBINATION PROCESS /TION-INDUCED RECOMBINATIO G2-PUB -16 IN AND TRAPPING CENTERS IN GERMANIUM I. THE NATURE OF THE RECOMBINATION PROCESS II. ANNEALING BEHAVIOR O/ G2-IV-IS-I

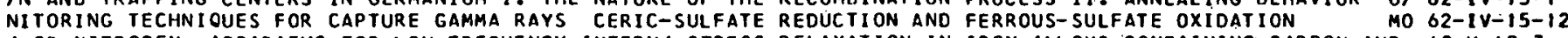
I OR NITROGEN APPARATUS FOR LOW FREQUENCY INTERN/ STRESS RELAXATION IN IRON ALLOYS CONTAINING CARBON AND G2-V-I8-3 XIDE 
E-PRIME-THO CENTERS IN CRYSTALLINE QUARTZ ELECIRON SPIN RESONANCE ABSTRACT PARAMAGNETIC SPECTRA OF 62-II-9-2 1 RELATION BETHEEN MAGNETIC SUSCEPIIBILITY, ELECIRON SPIN RESONANCE, AND OPIICAL ABSORPTION OF THE E-PRIME $62-I I-9-1$ TICLES EFFECT OF DELTA RAYS ON THE RESPONSE OF INORGANIC SCINTILLATORS TO HEAVY PAR 62-PUB-27

CRYSTALS LANG DIFFRACTION TOPOGRAPHS AND SINGLE CRYSTAL ROCKING CURVES ABSTRACT / PERFECT COPPER SINGLE 62-II-7-5 l, ZIRCAL OY-2, AND NIOBIUM - I PERCENT ZI/ IN. PILE STRESS RUPIURE EXPERIMENTS INCONEL, 304 STAINLESS STEE 62-V-19-8 ENERGY TRANSFER

HARD SPHERE SCAITERING APPROXIMATIONS IMPACT PARAMETER AND

$62-1-1-3$

$62-1-1-3$
$62-P U B-25$

A SIMPLE APPROXIMATION FOR CLASSICAL SCATIERING AT LARGE ANGLES

APPROXIMATION FOR CLASSICAL SCATTERING AT LARGE ANGLES ABSTRACT

$62-1-1-7$

ABSTRACT EFFECT OF ENERGETIC SECONDARY ELECIRONS ON SCINTILLATION PROCESS IN ALKALI HALIDE CRYSTALS $62-1 v-16-5$ UM- $10 C I D E$ ABSTRACT STUDIES OF THE SCINTILLATION PROCESS IN THALLIUM-ACTIVATED CESI 62-IV-16-6 $U M-I 0010010$ STUDIES OF THE SCINTILLATION PROCESS IN THALLIUM-ACTIVATED CESI

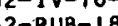
EFFECT OF DELTA RAYS ON THE RESPONSE OF INORGANIC SCINT ILLATORS TO HEAVY PARTICLES ALKALI HALIDE CRYSTALS ABSTRACT EFFECT OF ENERGETIC SECONDARY ELECTRONS ON SCINTILLATION PROCESS IN THERMOELECTRIC POWER IN GERMANIUM
ISMUTH-SELENIDE CRYSTALS DURING AND AFTER GROWTH BISMUTH SEEBECK COEFFICIENT GAMMA-RAY IRRADIATION 62-PUB-27 SELF DIFFUSION SEMICONDUCTOR STUDIES SEMICONDUCTOR THEORY

THE NATURE AND YIELD OF RADIATION-INDUCED DEFECTS IN SEMICONDUCTORS DEFECT PRODUCTION IN THERMAL NEUTRON SHIELDED MATERIALS SEMICONDUCTORS N NON METALS PURIFICATION OF B $62-18-15-4$ $62-1 v-17-5$ $62-1 V-15$ $62-1-3$ C2-PUB-10
LATIICE $62-1 \mathrm{~V}-15-10$ N NON METALS TRANSMUTATION DOPING AND RECOIL EFFECTS IN SEMICONDUCTORS EXPOSED TO THERMAL NEUTRONS IN ALKALI HAL IDES AT ORNL - - STRUCTURE SENSITIVITY OF COLOR CENTER PRODUCTION IN GAMMA/ 62-IV-16-3 $N$ TESTS LERYLLIUM SHEATHED URANIUM-DIOXIDE FUEL ELEMENT IRRADIAT
LATTICE DEFECT PRODUCTION IN THERMAL NEUTRON SHIELDED MATERIALS SEMICONDUCTORS ABSIRACT $62-1-6$ NEW LOW TEMPERATURE IRRADIATION FACILITY FOR THE BULK SHIELDING REACTOR $4 K$ FACILITY AND OPTICAL ABSORPTION OF THE E-PRIME-ONE CENTER IN FUSED SILICA OSCILLATOR STRENGTH ABSTRACT /SONANCE. 62-1II-12-3 REARRANGEMENIS IN DEFORMED COPPER - SILICON AND COPPER - SILICON - MANGANESE ALLOYS ABSTRACT ABSTRACT ATOMIC REARRANGEMENTS IN DEFORMED COPPER - SILICON AND COPPER - SILICON - MANGANESE ALLOYS IHE ANNEALING OF COLD-WORKED SILICON BRONZE $\begin{array}{ll}\text { IHE ANNEALING OF COLD-WORKED SILLICON BRONZE } & 62-P U B-43 \\ \text { SPIN-LATIIICE RELAXATION OF E-PRIME-ONE CENTERS IN SILIICON-DIOXIDE } & 62-P U B-9\end{array}$ SPIN-LAITICE RELAXATION OF E-PRIME-ONE CENTERS IN SILICON-DIOXIDE
SINGLE CRYSTALS GROWN FROM SMALL DROPLETS GOLD, COPPER, SILVER, LEAD, INDIUP, COPPER THREE - GOLD, AND G O2-II-7-

ETIC FIELDS ABSTRACT CRITICAL SIMPLICES IN BRILLOUIN ZONES $62-1-4$ SPECIFIC HEAT OF SINGLE CRYSTAL MANANOUS-CHLORIDE IN APPLIED MAGN GROWTH OF SINGLE CRYSTAL MERCURIC-SULFIDE

T COPPER SINGLE CRYSIALS LANG DIFFRACTION TOPOGRAPHS AND SINGLE CRYSTAL ROCKING CURVES ABSTRACT I PERFEC ELECTROCHEMICAL DISSOLUTION OF SINGLE CRYSTALLINE COPPER POTASSIUM-CHLORIDE SINGLE CRYSTALS

$62-11-7-5$ $62-10-17-3$ SINGLE CRYSI X-RAY OBSERVATIONS OF NEARLY PERFECI COPPER SINGLE CRYSTALS LANG DIFFRACTION TOPOGRAPHS AND 62-II-7-S NDENCE FROM 250K TO 370K OF DISLOCATION PINNING IN COPPER SINGLE CRYSTALS BY RADIATION DEFECTS ABSIRACT / O2-III-I3-3 NDENCE FROM 25OK TO 370K OF DISLOCATION PINNING IN COPPER SINGLE CRYSTALS BY RADIATION DEFECTS /ATURE DEPE 62-PUB-33 COPPER, SILVER, LEAD, INUIUM, COPPER THREE - GOLO, AND / SINGLE CRYSTALS GROWN FROM SMALL DROPLETS GOLD, O2-III-7-I ION FRAGMENT/ TRANSMISSION ELECTRON MICROSCOPE STUDIES ON SINGLE CRYSTALS OF UNANIUM-DIOXIDE SURFACE FISS O2-II-8-4 CT OF A PHASE IRANSFORMATION ON THE VAPOR-PHASE GROWTH OF SINGLE MERCURIC-SULFIDE ABSTRACT CT OF A PHASE TRANSFORMATION ON THE VAPOR PHASE GROWTH OF SINGLE-CRYSTAL MERCURIC-SIULIDIDE

DIOXIDE

FUEL CYCLE PROGRAM SINTERED AND POWDERED URANIUM-DIOXIDE - THORIUM-II COMPUTER PROCRAM FOR LATTICE / MACHINE STUDIES OF THE SLOWING DOWN OF ENERGETIC ATOMS IN SOLIOS RANGE LOH TEMPERATURE RESISTIVITY OF SODIUM AND THE EFFECTS OF NUCLEAR IRRADIATION MONTE CARLO CALCULATIONS OF RANGES OF ENERGETIC ATOMS IN SOLIDS ABSTRACT RANGES OF ENERGETIC ATOMS IN SOLIDS ABSTRACT

THE RANGES OF ENERGETIC ATOMS IN SOLIDS 1. RANDOM MODEL

THE RANGES OF ENERGETIC ATOMS IN SOLIDS II. LATTICE MODEL

$62-P \cup B-14$ $62-V I-20-6$ $62-1-1-2$ $62-P \cup B-4$ $62-1-1-4$ $62-1-1-5$ $62-P U B-30$
$62-P U B-34$

/ACHINE STUCIES OF THE SLOWING DOWN OF ENERGETIC ATOMS IN SOLIOS RANGE-II COMPUTER PROGRAM FOR LATTICE S/ STUDIES OF PENETRATION OF MOVING ATOMS IN SOLIDS AND INTERATOMIC POTENTIALS ANODIC DISSOLUTION OF COPPER IN COPPER-FREE SOLUTIONS EICH PITS AT DISLOCATIONS

$62-1-1$ $\begin{aligned} & \text { ANODIC DISSOLUTION OF COPPER IN COPPER-FREE SOLUTIONS ETCH PITS AT DISLOCATIONS } 62-111-11-2 \\ & \text { ANODIC DISSOLUTION OF COPPER IN SOLUTIONS CONTAINING COPPER IONS ORIENTATION EF O2-III-11-1 }\end{aligned}$

FECTS RATICNS OF TETRACHLOROETHANE AND DECANE IN A FLUOROCARBON SOLVENT AND OF TETRACHLOROETHANE IN DECANE ICENT 62-VI-23
ARTZ ELECTRON SPIN RESONANCE ABSTRACT
PARAMAGNETIC SPECTRA OF E-PRIME-TWO CENTERS IN CRYSTALLINE OU G2-II-9-2 IIMENT TO STUDY RADIATION OAMAGE BY THE MOSSBAUER EFFECT SPECTRA OF THE RECOILESS RADIATION AS A FUNCTIOI G2-I-5 DIFFUSION IN COPPER - ALUMINUM ON NEUTRON FLUX AND ENERGY SPECTRUM THE DEPENDENCE OF RADIATION-ENHANCED 62-PUB-2 ABSTRACT THERMALLY.ACTIVATED INTERNAL FRICTION SPECTRUM IN COPPER FOLLOWING NEUTRON IRRADIATION O2A THERMALLY ACTIVATED INTERNAL FRICTION SPECTRUM IN COPPER FOLLOHING NEUTRON IRRADIATION O2-PUB-4I METHCOS FOR TREATING ANNEALING PROCESSES WHICH INVOLVE A SPECTRUM OF ACTIVATION ENERGIES ER ANC ENERGY TRANSFER SPHERE SCATTERING APPROXIMATIONS IMPACT PARAMET 62-1-1-3 SPIN RESONANCE

$62-11-9$

RA OF E-PRIME-THO CENTERS IN CRYSTALLINE QUARTZ ELECTRON SPIN RESONANCE ABSTRACT PARAMAGNETIC SPECT 62-II-9-2 PRIMEI RELATION BETWEEN MAGNETIC SUSCEPTIBILITY, ELECIRUN SPIN RESONANCE, ANO OPTICAL ABSORPTION OF THE E- 62-II-9-1 N SILICON-DIOXIDE

THEORY OF SPINTIERING

5-KEV ARGON IONS GEOMETRICAL MODEL OF MONOCRYSTAL SPUTTERING AINS CO/ EXAMINATION OF THEORETICAL MODEL OF ANISOTROPIC CAL MCOEL OF MONOCRYSTAL SPUTTERING COPPER AND GERMANIUM METAL SURFACES COPPER BOMBARDMENT WITH 4-KEV ARCON IONS SED CN FOCUSING CHAINS COPPER ION BOMBAROMENT OF COPPER SED CN FOCUSING CHAINS COPPER ION BOMBAROMENT OF COPPER RADIATION
RADIATION
TST

ICTS ON METAL CLAD FUELS URANIUM-DIOXIDE PELLETS CLAD IN CENT ZI/ IN PILE STRESS RUPTURE EXPERIMENTS INCONEL, 304 TION OF THE FERMI LEVEL IN NON METALS

IAL CLAO FUELS URANIUM-DIOXIDE PELLETS CLAD IN STAINLESS 1 PILE STRESS RUPTURE EXPERIMENTS INCONEL, 304 STAINLESS IMPAC T TESTS ON IRRADIATED PRESSURE VES IMATION BOHR POTENTIAL AND MONTE CARLO CALCULATIONS $Y$ OF GLASSES LEAD-SILICATE GLASS POLARIZATION AND CHARGE
-1960 OSCILLATOR
OSTR
THE YIELD ON ANO, OR NITROGEN APPARATUS FOR LOW FREQUENCY INTERN
SS STEEL, ZIRCALOY-2, AND NIOBIUM - I PERCENT Z1/ IN PILE GAMPA IRRADIATION OF DOPEO POTASSIUM-CHLORIDE CALCIUM, ERACTION BETWEEN IRRADIATION-INDUCED OEFECTS ANO MAGNETIC ITIGATIONS OF COLOR CENTERS IN ALKALI HALIDES AT ORNL - -

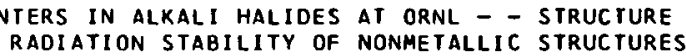
作 SPUTTERING WITH 5-KEV ARGON IONS GEOMEIRI 62-1-2-1 SPUTTERING YIELO FOR $(111)$ - ALUMINUM PLANE /OF 62-111-14 SPUTTERING YIELDS AS A FUNCTION OF ORIENTATION, $62-2-2-2$ STABILITY $\begin{array}{ll} & 62-P U 8-12 \\ \text { STAINLESS STEEL BERYLLIUM CANNED URANIUM-DIOXI/ } & 62-V I-20-3\end{array}$ TAINLESS STEEL, ZIRCALOY-2, AND NIOBIUM - I PER 62-V-19-1 STATISTICAL MECHANICS OF SEMICONDUCTORS COMPUTA $62-1-3-1$ STEEL BERYLLIUM CANNED URANIUM-DIOXIDE ANO FUEI $62-V I-20-3$ STEEL, ZIRCALOY-2, AND NIOBIUM - I PERCENT ZIRC/ 62-v-19-1 STEELS BRITTLE-DUCTILE TRANSITION TEMPERATURE $62-\mathrm{V}-19-2$ STOPPING POWER AS A BASIS FOR HARD-SPHERE APPROX 62-1-1-1 STORAGE / CURRENT AND DIRECT CURRENT CONOUCTIVIT 62-1I-9-3 STORED ENERGY IN THE OAK-RIOGE GRAPHITE REACTOR 62-PU8-44 STRESS ABSTRACT /SONANCE $62-\mid 1-9-1$ 62-PUB-51 STRESS OF COPPER CRYSTALS STRESS RELAXATION IN IRON ALLOYS CONTAINING CARB
STRESS RUPTURE EXPERIMENTS INCONEL, 304 STAINLE $2-v-18-3$ RCONIUM ALLOYS ON MAGNETIC FIELO ORIENTATI/ DEPENOENCE OF SUPERCONDUCTING CRITICAL CURRENT OF NIOBIUM - ZI G2-II-IO-1 
ED TECHNETIUM B.22K VALUE ABSTRACT

NEUTRON IRRADIATION OF SUPERCONDUCTING IIN SUPERCONDUCTING TRAR SUPERCONDUCT IVITY

DEFECT CONCENTRATION

INTENSE DAMAGE IN CADMIUM IICROSCOPE STUDIES ON SINGLE CRYSTALS OF UNANIUM-DIOXIDE

INVESIIGATIONS OF METAL NS SPUTTERING YI ARGON-PLUS-ONE ION ROMBARDMENT OF METAL TCH PIT CROWTH AND ASSOCIATED PHENUMENA ON 11111 - COPPER I AND POTASSIUM HEXABROMORHENATE (IV) FROM 5K TO/ MAGNETIC ICAL $\triangle B S O R P T I O N$ OF THE E-PRIME/ RELATION BETHEEN MAGNETIC MARITIME REACTOR FUEL COLD IC ATCMS IN SOLIDS RANGE-I 1 CONPUTER PROGRAM FOR LATTICE SUPERCONDUCTING TRANSITION TEMPERATURE OF PURIFIED DIATEC PRESSURE VESSEL STEELS BRITTLE-DUCTILE IRANSITION
DIFFUSION ON DISPLACEMENT PRODUCTION RATE AND IRRADIATION DIFFUSION ON DISPLACEMENT PRODUCTION RATE AND IRRADIATION
OCATICN PINNING IN COPPER SINGLE CRYSTALS BY RADIATION D/ OCATICN PINNING IN COPPER SINGLE CRYSTALS BY RAOIATION D IELOING REACTOR $4 K$ FACILITY 
STRESS RUPTURE EXPERIMENTS INCONEL, 304 STAINLESS STEEL, 2 IRCALOY-2, AND NIOBIUM - I PERCENT ZIRCONIUM / 62-V-19-1 CERAMICS PELTIER POWER CALIBRATION LIRCON CERAMIC RADIATION STABILITY 62-VI-22 RADIATION EFFECTS IN URANIUM-DOPED ZIRCONIA
SITION IN ZIRCONIUM-DI RADIATION EFFECTS IN URANIUM-DOPED ZIRCONIA MONOCLINIC TO FACE-CENTERED-CUBIC TRAN O2-II-7-2 304 STAINLESS STEEL, 2IRCALOY-2, AND NIOBIUM - I PERCENT ZIRCONIUM, SIRESS RUPTURE EXPERIMENTS INCONEL, 62-V $\rightarrow$ I9-1 IENDENCE OF SUPERCONDUCTING CRITICAL CURRENT OF NIOBIUM - 2 IRCONIUM ALLOYS ON MAGNETIC FIELD ORIENTATION, G2-II-IOERTIES AT / MFCHANICAL PROPERTIES OF NIOBIUM - 25 PERCENT ZIRCONIUM WIRE AT LOW TEMPERATURES TENSILE PROP 62-II-1O-3 ZIRCCNIA MCNOCLINIC TO FACE-CENTERED-CUAIC TRANSITION IN ZIRCONIUM-DIOXIDE ABSTRACT/S IN URANIUM-DOPED O2-II-7-2 CRITICAL SIMPLICES IN BRILLOUIN ZONES

INCONEL, 304 SIAINLESS STEEL, ZIRCALOY-2, AND NIOBIUM - I PERCENT ZIRCONIUM, STRESS RUPTURE EXPERIMENTS 62-V-I9-1 UM - ZIRCONIUM ALLOYS ON MAGNETIC FIELD ORIENTATION ORNL IDO - KILOGAUSS MAGNET/RITICAL CURRENT OF NIOBI O2-II-IO-I STORED ENERGY IN THE OAK-RIDGE GRAPHITE REACTOR - 1960

THE CRNL GRAPIIITE REACTOR

961 POSTANNEALING OF GRAPHITE-STORED ENERGY IN $62-P U B-44$

ENSILE PROPERTIES AT I MECHANICAL PROPER IIES OF NIOBIUM - 25 PERCENT ZIRCONIUM WIRE AT LOW TEMPERATURES T 62-II-IO-3 NITROGEN ION BOMBARDMENT OF THIN PLATINUM FILMS 25-MEV NITROGEN IONS ABSTRACT

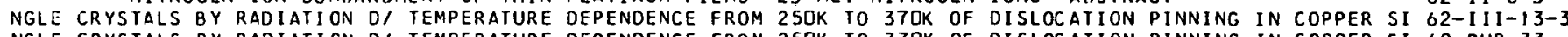
NGLE CRYSTALS BY RADIATICN D/ TEMPERATURE DEPENDENCE FROM $250 K$ TO $370 K$ OF DISLOCATION PINNING IN COPPER SI G2-PUB-33 CPTICAL ABSORPTION STUDIES ON CUPROUS-OXIDE BETWEEN 250DGA AND GOOOA

RHENATE(IV) AND POTASSIUM HEXABROMORHENATE(IV) FROM 5K TO $300 K / T I C$ SUSCEPTIBILITY OF POTASSIUM HEXACHLORO G2-IUB-6

ZIRCONIUM WIRE AT LOW TEMPERATURES TENSILE PROPERTIES. AT 3OOK, $77 K$, AND 4.2K /ES OF NIOBIUM - 25 PERCENT O2-II-1O-3

PERCENT ZI/ IN PILE STRESS RUPTURE EXPERIMENTS INCONEL, 304 STAINLESS STEEL, ZIRCALOY-2, AND NIOBIUM - I 62-V-19-1

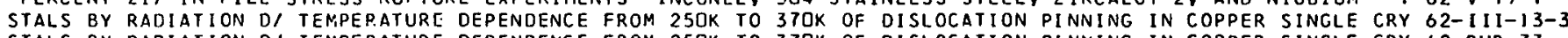
STALS BY RAOIATION D/ IENPERATURC DEPENDENCE FROM $250 K$ TO $370 K$ OF DISLOCATION PINNING IN COPPER SINGLE CRY O2-PUB-33 AT LOW.TEMPERATURES TENSILE PROPERTIES AT 3OOK, $77 K$, AND $4.2 \mathrm{~K}$ /ES OF NIOBIUM - 25 PERCENT ZIRCONIUM WIRE 62-I1-1O-3 IN BONBARDMENT OF METAL SURFACES COPPER BOMBARDMENT WITH 4-KEV ARGON IONS SPUTTERING YIELD FOR (III) - / O2-III-I4 REACTOR DANAGE IN PURE METALS AT $4 \mathrm{~K}$

TURE IRRAOIATION FACILITY FOR THE BULK SHIELOING REACTOR $4 K$ FACILITY OCRYSTAL SPUTTERING COPPER AND GERMANIUM SPUTTERING WITH 5-KEV ARGON IONS CHLORCRHENATE (IV) AND POTASSIUM. HEXABROMORHENATEIIV) FROM 5K TO $300 K$ ITIC SUSCEPTIBILITY OF POTASSIUM HEXA G2-PUB-6 L ABSCRPTION STUDIES ON CUPROUS-OXIDE BETWEEN 25000A AND 6ODOA ING CF ANIIMONY-DOPED GERMANIUM GAMMA-RAY IRRADIATION AT 77K /GERMANIUM ISOCHRONAL AND ISOTHERMAL ANNEAL G2-IV-15-2 DIOBIUM - 25 PERCENT 2IRCON 62-11-ID-3 CONDUCTING IRANSITION TEMPERATURE OF PURIFIED TECHNETIUM $8.22 \mathrm{~K} V A L U E$ ABSTRACT SUPER G2-1I-IO-2 
THIS PAGE

WAS INTENTIONALLY

LEFT BLANK 


\section{TITLE INDEX}

$62-1-1$

62-1-1-1

$62-1-1-2$

$62-1-1-3$

$62-1-1-4$

$62-1-1-5$

$62-1-1-6$

$62-1-1-7$

$62-1-1-8$

$62-1-1-9$

62-I-2

$62-1-2-1$

$62-1-2-2$

$62-1-3$

$62-1-3-1$

$62-1-3-2$

$62-1-4$

$62-1-5$

$62-1-6$

$62-11-7$

$62-11-7-1$

$62-1 I-7-2$

$62-11-7-3$

$62-I I-7-4$

STUDIES OF PENETRATION OF MOVING ATOMS IN SOLIDS AND INTERATOMIC POTENTIALS

STOPPING POWER AS A BASIS FOR HARD-SPHERE APPROXIMATION BOHR POTENTIAL AND MONTE CARLO CALCULATIONS OEN, O. S. + ROBINSON, M. T. + HOLMES, D. K.

MACHINE STUDIES OF THE SLOWIMG DOWN OF ENERGETIC ATOMS IN SOLIDS RANGE-II COMPUTER PROGRAM FOR LATTICE SYMMETRY CHANNELED DIRECTIONS OF ATOMS

ROBINSON, M. T. + OEN, O. S.

HARD SPHERE SCATTERING APPROXIMATIONS IMPACT PARAMETER AND ENERGY TRANSFER

LEE, E. J. + ROBINSON, M. T.

MONTE CARLO CALCULATIONS OF RANGES OF ENERGETIC ATOMS IN SOLIOS ABSTRACT

ROBINSON, M. T. HOLMES, D. K. O OEN, O. S

RANGES OF ENERGETIC ATOMS IN SOLIDS ABSTRACT

OEN, O. S. + HOLMES, D. K. + ROBINSON, M. T.

CALCULATION OF AVERAGES FÚR PRIMARY RECOIL OISTRIBUTIONS ABSTRACT

LEIBFRIED, G.

APPROXIMATION FOR CLASSICAL SCATTERING AT LARGE ANGLES ABSTRACT

LEIBFRIEO, G. + OEN, O. S.

HIGHER-ORDER AVERAGES OF PRIMARY RECOIL DISTRIBUTIONS ABSTRACT

LEIBFRIED， G.

LONG-RANGE CHANNELING EFFECTS IN IRRADIATED CRYSTALS COPPER CRYSTALS ABSTRACT

LEHMANN, C. + LEIBFRIED, G.

THEORY OF SPUTTERING

GEOMETRICAL MODEL OF MONOCRYSTAL SPUTIERING COPPER AND GERMANIUM SPUTIERING HITH 5-KEV ARGON IONS" ROBINSON, M. T.

EXAMINATION OF THEORETICAL MODEL OF ANISOTROPIC SPUTTERING AT HIGH ENERGIES BASED ON FOCUSING CHAINS COPPER ION BOMBARDMENT OF COPPER SPUTTERING YIELDS AS A FUNCTION OF ORIENTATION LEMMANN, $C$.

SEMICONDUCTOR THEORY

STATISTICAL MECHANICS OF SEMICONDUCTORS COMPUTATION OF THE FERMI LEVEL IN NON METALS MILLER, J. W. + SCHWEINLER, H. C.

DENSITY FUNCIION MODIFYING MINORITY CARRIER CAPTURE PROBABILITY IN NONMETALLIC CRYSTALS SCHWE INLER, H. C. + MILLER, J. W.

CRITICAL SIMPLICES IN BRILLOUIN ZONES

SCHWE INLER, H. C.

POSSIBLE EXPERIMENI TO STUDY RADIATION DAMAGE BY THE MOSSBAUER EFFECT SPECTRA OF THE RECOILESS RADIATION AS A FUNCTION OF INTERSTITIAL POSITION

BARRETT, J. H.

ELECTRON CROSS SECTIONS FOR ATOMIC DISPLACEMENTS MOTI SERIES EVALUATION USING IBM-7OSO FORTRAN PROGRAM

OEN, 0. S.

X-RAY DIFFRACTION

SINGLE CRYSTALS GROWN FROM SMALL DROPLETS GOLD, COPPER, SILVER, LEAD, INDIUM, COPPER THREE - BOLD, AND GERMANIUM-MELT CRYSTALLIZATION ABSTRACT

WITTELS, M. C. + SHERRILL, F. A. + STIEGLER, J, 0

RAOIATION EFFECTS IN URANIUM-DOPEO ZIRCONIA MONOCLINIC TO FACE-CENTERED-CUBIC TRANSITION IN

ZIRCONIUM-DIOXIDE ABSTRACT

WITTELS, M. C. + STIECLEK, J= O. + SHERRILL, F. A.

A VERSATILE LANG CAMERA

WITTELS. M. C. + SHERRILL, F. A. + FIELDER, G. E.

1961 POSTANNEALING OF GRAPHITE-STORED ENERGY IN THE ORNL GRAPHITE REACTOR

HITTELS, M. C. 


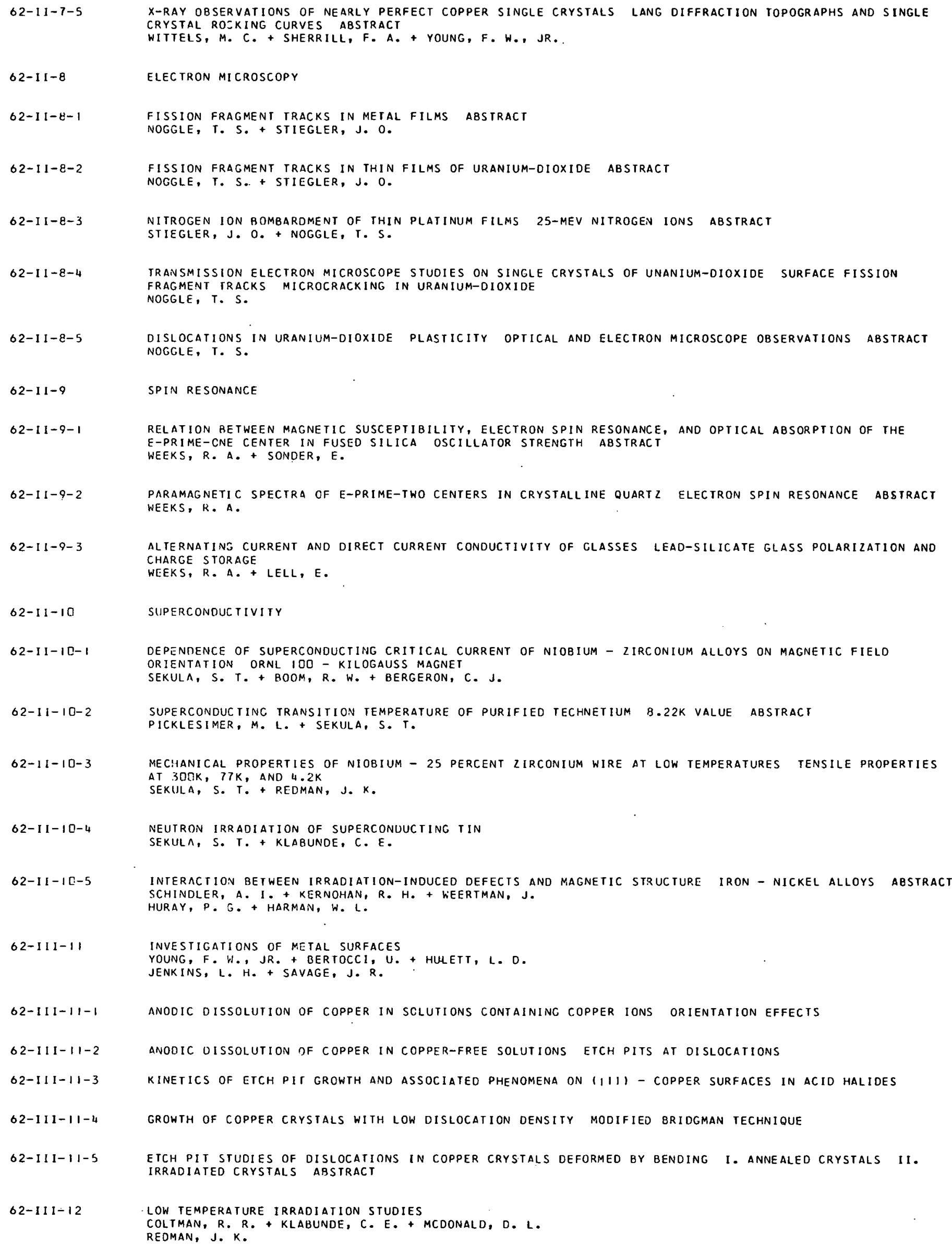




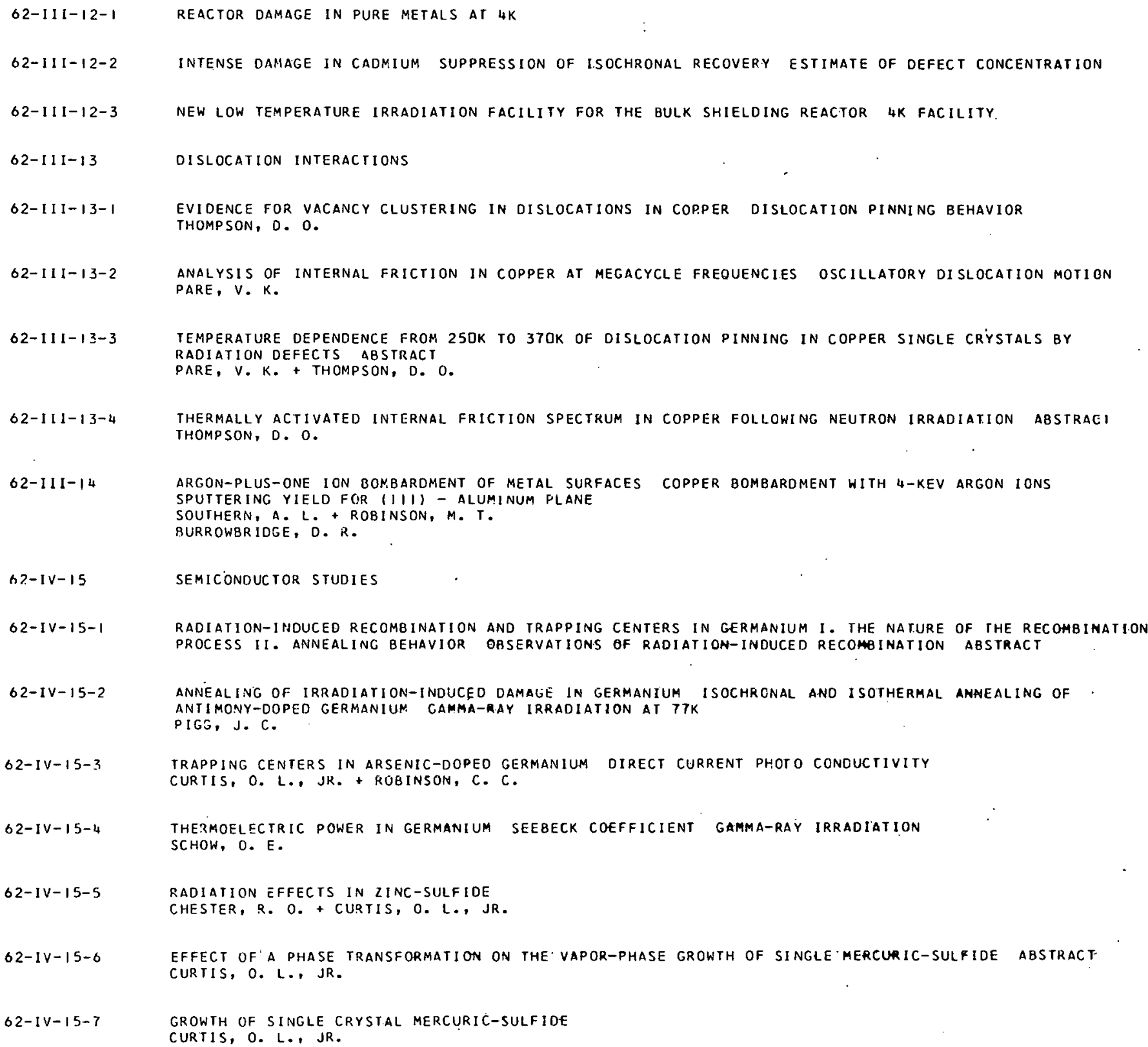




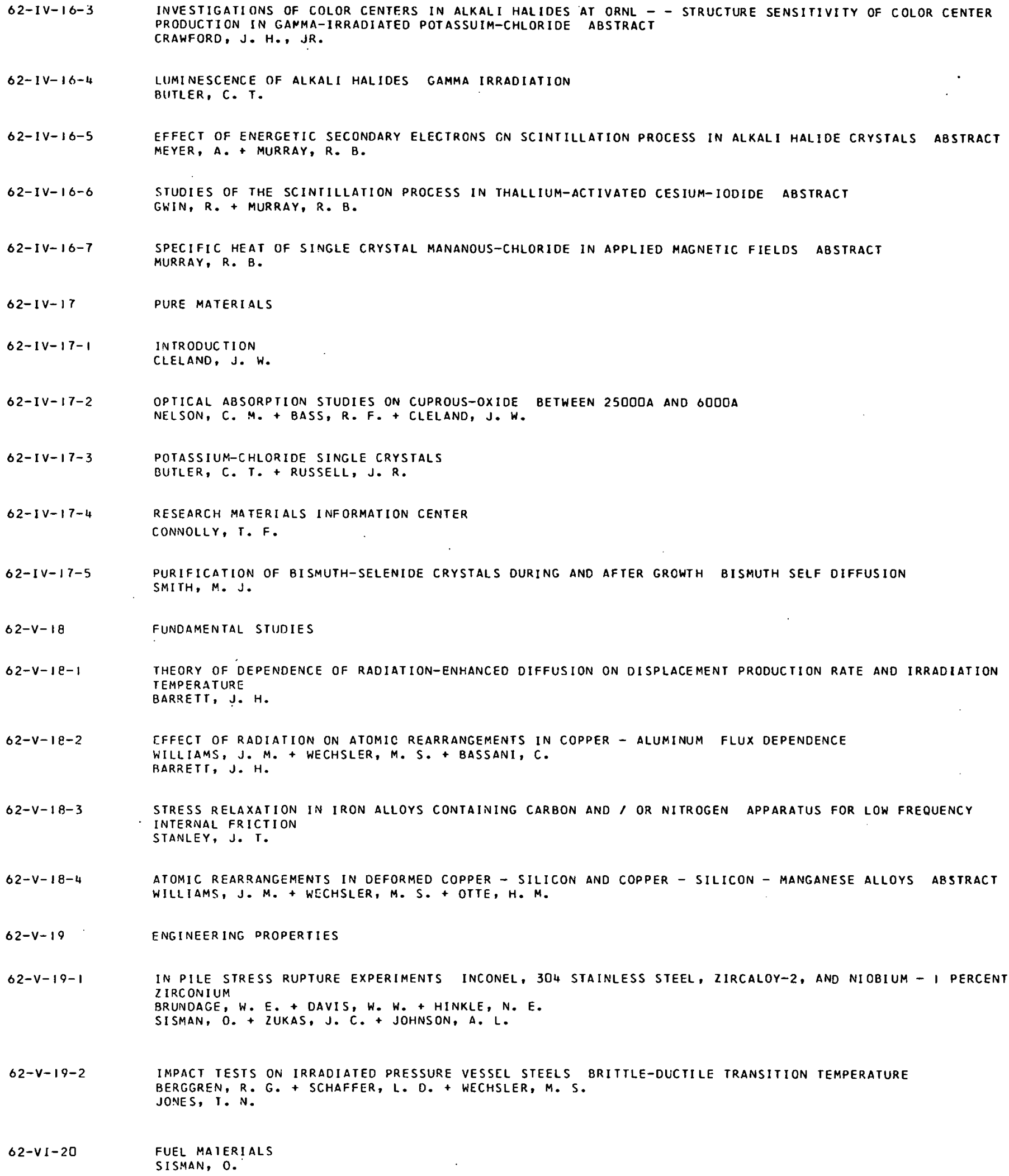


19

$62-V I-20-3$

$62-V I-20-4$

$62-V I-20-5$

$62-v 1-2 D-6$

$62-V 1-2.1$

$62-V 1-22$

$62-V I-23$
IRRADIATION EFFECTS ON METAL CLAD FUELS URANIUM-DIOXIDE PELLETS CLAD IN STA UNLESS STEEL BERYLLIUM CANNED IRAN IUM-DIOXIDE AND FUELED BERYLLIUM-OXIDE EVALUATIONS OSBORNE, M. F. + ROBERTSON, H. E. + KOCH, J. W. JOHNSON, A. L.

CONTINUOUS RELEASE OF FISSION GAS DURING IRRADIATION OF URANIUM-DIOXIDE CARROLL, R. M. + REAGAN, P. E. + FULTON, T. H.

MARITIME REACTOR FUEL COLD SWAGED AND VIBRATORY COMPACTED URANIUM-DIOXIDE MORGAN, J. G. + OSBORNE, M. F.

FUEL CYCLE PROGRAM SINTERED AND POWDERED URANIUM-DIOXIDE - THORIUM-DIOXIDE MORGAN, J. G. + OSBORNE, M. F.

POLYMERS GAMMA AND REACTOR RADIATION EFFECTS ON POLYSTYRENE INFRARED AND GAS CHROMATOGRAPHIC ANALYSIS

PARKINSON, W. W. + GEYSER, R. M. + KIRKLAND, W. K.

SI STAN, O.

CERAMICS PELTIER POWER CALIBRATION ZIRCON CERAMIC RADIATION STABILITY HOP, C. D. + SISMAN, O. + TOWNS, R. L.

RADIATION DOSIMETRY IRRADIATION PRODUCED ACID CONCENTRATIONS OF IETRACHLOROETHANE AND DECANE IN A FLUOROCARBON SOLVENT AND OF TETRACHI. OROETHANE IN DECANE BOP, C. O. + SISTMAN, O. + KIRKLAND, W. K. PARKINSON, W. W. 
THIS PAGE

WAS INTENTIONALLY

LEFT BLANK 
62-PUB-1

62-PUB-2

62-PUB-3

62-PUB-4

62-PUB- 5

62-PUB-6

62-PUB-7

62-PUB-B

62-PUB-9

$62-P \cup B-10$

62-P $18-11$

62-PUB- 12

$62-P \cup B-13$

62-PUB-14

62-PUB-15

62-PUD-16

$62-P \cup B-17$

62-PUB- 18

62-P UB- 19

62-PUB-20

RADIATION EFFECTS ON DEEP-LEVEL IMPURITY STATES IN GERMANIUM

BASS, R. F. + CLELAND, J. W. + CRAWFURD, J. H., JR.

BULL. AM. PHYS. SOC. 6,461119611

IHE DEPENDENCE OF RADIATION-ENHANCEO DIFFUSION IN COPPER - ALUMINUM ON NEUTRON FLUX AND ENERGY SPECTRUM

BASSANI, C. + WECHSLER, M. S. + WILLIAMS, J. M.

BARRETT, J. H. + KERNOHAN, R. H.

RULL. AN. PHYS. SOC. 7, $170(1962)$

CR YOCENIC TECHNI QUES FOR IRRADIAT

RLEWITT, I. H. C COLIMAN, R. R

P 274 IN EXPERIMENTAL CRYOPHYSICS (ED. BY F. E. HOARE ET AL.),

BUTTERWORTHS, LONDON, 1961

LOW TEMPERATURE RESISTIVITY OF SODIUM AND THE EFFECTS OF NUCLEAR IRRADIATION

BLEWITT, T. H. + COLINAN, R. R. + KLABUNDE, C. E.

MCDONALD, D. L.

J. APPL. PHYS. $33,1612(1962)$

CALORIMETRIC MEASUREMENT OF NUCLEAR HEATING IN A REACIOR

BOPP, C. D. + TOWNS, R. L.

NUCL. SCI. ENC. 13, $245(1962)$

MAGNETIC SUSCEPTIBILITY OF POTASSIIIM HFXACHLORORHENATE (IV) AND POTASSIUM HEXABROMORHENATE(IV) FROM SK TO 300K

BUSEY, R. H. + SONDER, E.

J. CHEM. PHYS. 36, 93 (1962)

ARGON ACTIVATION MEASURES IRRADIATION FLUX CONTINUOUSLY

CARROLL, R. M.

NUCLEONICS 20i2), $42(1962)$

CONTINUOUS RELEASE OF FISSION GAS FROM URANIUM-DIOXIDE DURING IRRADIATION

CARROLL, R. M.

P IIU IN SYMPOSIUM ON RADIATION EFFECTS IN REFRACTORY FUEL

COMPOUNDS, ASTM-STP-306, 1961

SPIN-LATTICE RELAXATION OF E-PRIME-ONE CENTERS IN SILICON-DIOXIDE

CASTLE, J. G., JR. + FELDMAN, D. W. + WEEKS, R. A.

BULL. AM. PHYS. SOC. 7. 188 (1962)

THE NATURE AND YIELD OF RADIATION-INDUCED DEFECTS IN SEMICONDUCTORS

CLELAND, J. W. + BASS, R. F. + CRAWFORD, J. H., JR.

BULL. AM. PHYS. SOC. 7, I86 (1962)

OPERATION OF RESEARCH MATERIALS INFORMATION CENTER

CONNOLLY, T. F.

ORNL TM-295 (JULY 30,1962 )

RAOIATION STABILITY OF NONMETALLIC STRUCTURES

CRAWFORD, J. H., JR.

P 371 IN PROGRESS IN NUCLEAR ENERGY, IEO. BY H. M. FINNISTON AND

J. H. HOWE), SERIES V, VOL 3, PERGAMON PRESS. NEW YORK, 196I

TRANSMUTATION DOPING AND RECOIL EFFECTS IN SEMICONDUCTORS EXPOSED TO THERMAL NEUTRONS

CRAWFORD, J. H., JR. + CLELAND, J. W.

P 269 IN RADIOISOTOPES IN THE PHYSICAL SCIENCES AND INDUSTRY,

INTERNATIONAI. ATOMIC ENERGY AGENCY, VIENNA. 1962

EFFECT OF A PHASE TRANSFORMATION ON THE VAPOR PHASE GROWTH OF SINGLE-CRYSTAL MERCURIC-SULFIDE

CURTIS, O. L., JR.

J. APPL. PHYS. 33, 2461 (1962)

CARRIER RECOMBINATION AND IRAPPING PROCESSES IN IRRADIATED GERMANIUM

CURTIS, O. L., JR. + CRAWFORD, J. H., JR.

DISCUSSIUNS FAKAOAY SOC. Ӟ1, 107 (1961)

RADIATION-INDUCED RECOMBINATION AND IRAPPING CENTERS IN GERMANIUM I. THE NATURE OF THE RECOMBINATION PROCESS

CURTIS, O. L., JR. + CRAWFORD, J. H., JR.

PHYS. REV. 124, 1731 11961

RADIATION-INDUCED PECOMBINATION AND IRAPPING CENTERS IN GERMANIUM II. ANNEALING IN GAMMA-IRRADIATED, ANT I MONY-DOPED AND ARSENIC-DOPED MAIERIAL

CURTIS, O. L., JR. + CRAWFORD, J. H., JR.

PHYS. REV. I26, 1342 (1962)

STUDIES OF THE SCINTILLATIUN PROCESS IN THALLIUM-ACTIVATED CESIUM-IODIDE

GWIN, R. + MURRAY, R. B.

IRE TRANS. NUCL. SCI. NS-9, $28(1962)$

APPROXIMATE METHODS FOR TREATING ANNEALING PROCESSES WHICH INVOLVE A SPECTRUM OF ACTIVATION ENERGIES HOLMES, D. K.

P 449 IN CHEMICAL EFFECTS OF NUCLEAR TRANSFORMATIONS, VOL I,

INTERNATIONAL ATOMIC ENERGY AGENCY, VIENNA, 1961

ON THE INTERPRETATION OF RADIATION EFFECTS IN NOBLE METALS

HOLMES, D. K. + CORBEII, J. W. + WALKER, R. M.

KOEHLER, J. S. + SEITZ, F.

P 178 IN PROGKESS IN NUCLEAR ENERGY, IED. BY H. M. FINNISTON AND

J. P. HOWE), SERIES V, VOL 3, PERGAMON PRESS, NEW YORK, 1961 


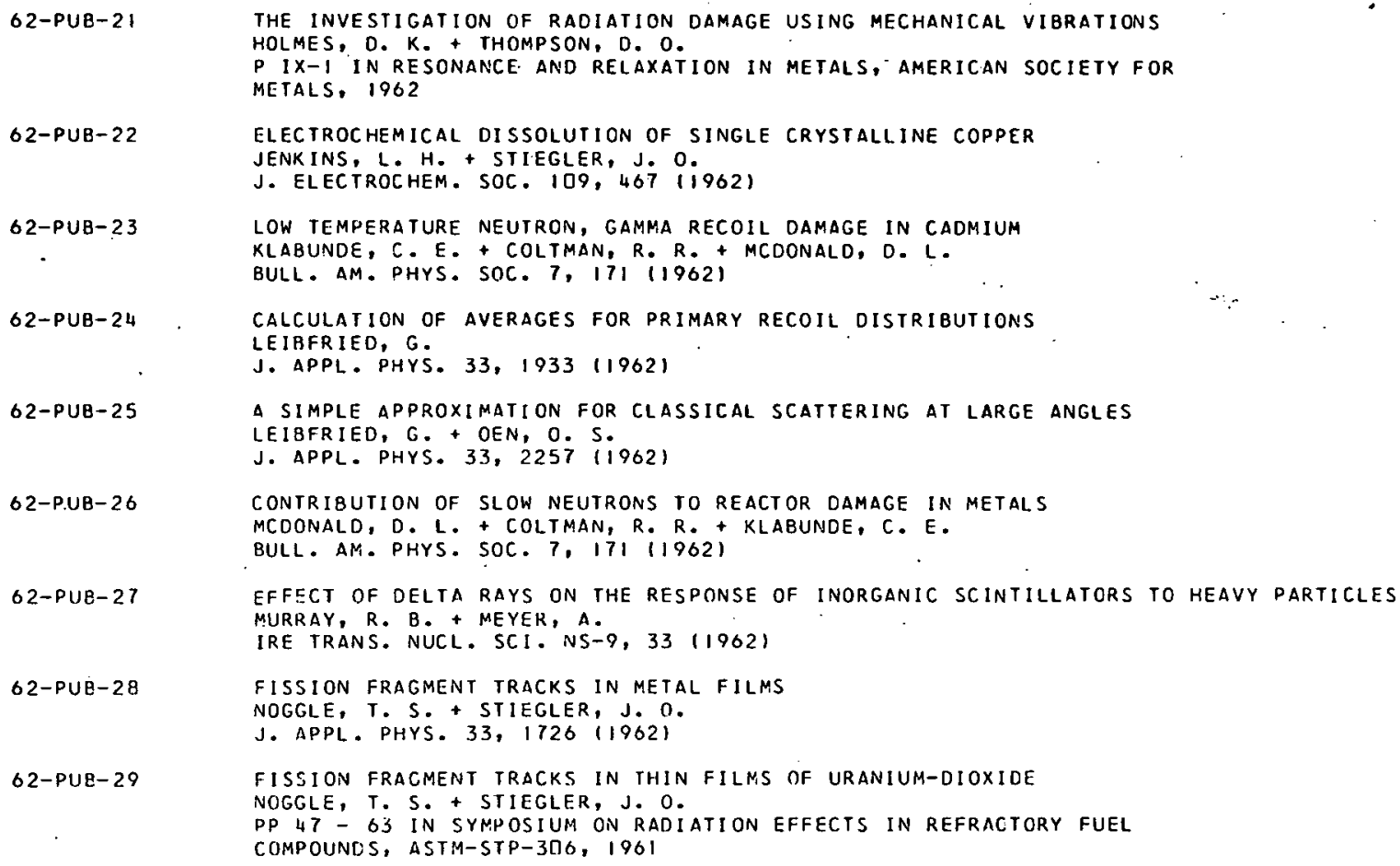

EFFECT OF FAST NEUTRON IRRADIATION ON DISLOCATION INTERNAL FRICTION IN PURE COPPER AT MEGACYCLE FREOUENCIES

THOMPSON, D. O. + PARE, V. K.

BULL. AM. PHYS. SOC. 7, 171 (1962) 
$62-P \cup B-43$

$62-P \cup B-45$

$62-P \cup B-46$

$62-P U B-47$

62-PUB-48

$62-P \cup B-49$

62-PUB-50

$62-P \cup B-51$
THE ANNEALING OF COLD-WORKED SILICON BRONZE

WECHSLER, M. S. + WILLIAMS, J. M. + OTTE, H. M.

J. METALS $14,81(1962)$

STORED ENERGY IN THE OAK-RIDGE GRAPHITE REACTOR - 1960

WITTELS, M. C.

J. NUCL. ENERGY, PTS. A AND B 15, 185 (196I)

GOLD(0.82) - MERCURY(0.18) - A TRANSMUTATION ALLOY

WITTELS, M. C. + STIEGLER, J. O. + SHERRILL, F. A.

J. $\triangle P P L$. PHYS. 33, 24$)(1962)$

RADIATION EFFECTS IN URANIUM-DOPED ZIRCONIA

WITTELS, M. C. + STIEGLER, J. $0 .+$ SHERRILL, F: A.
J. NUCL. ENERGY, PTS. A AND B $16,237(1962)$

ELASTIC-PLASTIC TRANSITION IN COPPER CRYSTALS AS DETERMINED BY AN ETCH PIT TECHNIQUE YOUNG, F: W., JR.

J. APPL. PHYS. 32, 1815119611

ETCHING OF IRRADIATED COPPER

YOUNG, F. W., JR, $749(1962)$

ON THE FORMATION OF DISLOCATIONS AROUND PRECIPITATE PARTICLES OF CUPROUS-OXIDE IN COPPER YOUNG, F. W., JR.

P IO3 IN DIRECT OBSERVATION OF IMPERFECTIONS IN CRYSTALS IED. BY

J. B. NEWKIRK AND J. H. WERNICKI, INTERSCIENCE, NEW YORK, 1962

ON THE GROWTH OF COPPER CRYSTALS OF LON DISLOCATION DENSITY

YOUIJ, F. W., JR.

BULI. AM. PHYS. SOC. 7, $215(1962)$

ON THE YIELD STRESS OF COPPER CRYSTALS

YOUNG, F. H., JR.

J. APPL. PHYS. 33, $963(19621$ 
THIS PAGE

WAS INTENTIONALLY

LEFT BLANK 
62-1-5

$62-V-18-1$

62-V-18-2

62-PUB-2

$62-I V-15-9$

62-IV-15-10

$62-I V-15-11$

62-IV-1 7-2

62-PUB-1

62-PUB-10

$62-V-18-2$

62-PUB-2

62-I I-10-

62-V-19-2

62-III-II

62-PUB-3

62-PUB-4

62-II-I I- I

$62-V_{1}-22$

$62-V I-23$

62-PUB-5

$62-v-19-1$

62-III-I 4

62-PUB-6

62-IV-16-2

$62-I V-16-4$

$62-I V-17-3$

62-VI-20-2

$62-V I-20-4$

62-PUB-7

62-PUB-8

O2-PUB-9

$62-I V-15-5$

62-IV-15-9

62-IV-15-10

$62-I V-15-11$

62-IV-15-12

$62-I v-17-1$

02-IV-17-2

62-PUB-1

62-PUB- 10

62-PUB-1 3

62-1 I I-12

62-PUB-3

$62-P \cup B-4$

O2-PUB- 23

$62-P \cup B-26$

$62-I V-17-4$

62-PUB-11

62-PUB-20

$62-I V-15-10$

62-IV-16-3

62-PUB-1

62-PUB-10

62-PUB- 12

62-PUB- 13

62-PUH- 15

62-PUB- 16

62-PUB- 17

62-IV-15-3

$62-I V-15-5$

62-IV-15-6

62-IV-15-7

62-PUB-14

62-PUB- 15

62-PUB- 16

6)-PUB- 17

62-V-19-1

62-PUB-9

$62-11-7-3$

$62-V I-20-2$

$62-\mathrm{V} 1-20-4$

$62-V I-20-3$

$62-I V-16-6$

$62-P U B-18$

62- $11-10-5$

$62-v-19-1$

$62-1-1-1$

$62-1-1-4$

$62-1-1-5$

O2-PUB-19

62-PUB-20

62-PUB-21

62-PUB-30

62-PUB-34

62-III-1 I

$62-11=10-5$

$62-\mid 11-11$

62-PUB-22

$62-V-19-1$

$62-V 1-20-3$

$62-v-19-2$

62-II-10-5.

62-PUB-2
BARRETT, J. H.

BARRETT, J. H.

BARRETT, J. H.

BARRETT, J. H.

BASS, R. F.

BASS, R. F.

BASS, R. F.

BASS, R. F.

BASS, R. F.

BASS, R. F.

BASSANI, C.

BERGERON, C.J.

BERGGREN, R. G.

BER TOCC I. U.

BLEWITT, T. H.

BOOM, R. W.

BOPP, C. D.

BOPP, C. D.

BOPP, C. D.

BRUNDAGE, W. E.

BURROWBRIDGE, D. R.

BUSEY, R. H.

BUTLER, C. T.

BUTLER, C. T.

BUTLER, C. T.

CARROLL, R. M.

CARROLL, R. M.

CARROLL, R. M.

CARROLL, R. M.

CASTLE, J. G., JR.

CHESTER, R . 0 .

CLELAND, J. W.

CLELAND, J. $\mathrm{W}$.

CLELAND, J. W.

CLELAND, J. W.

CLELAND, J. W.

CLELAND, J. W.

CLELAND, J. W.

CLELAND, J. W.

CLELAND, J. H.

COLTMAN, R. R.

COLTMAN, R. R.

COL TMAN, R. R.

COLTMAN, R. R.

COLTMAN, R. R.

CONNOLLY, T. F.

CONNOLLY, T. F

CORBETT, J. W.

CRAWFORD, J. H., JR .

CRAWFORD, J. H., JR .

CRAWFORD, J. H., JR.

CRAWFORD, J. H.J JR

CKAWFORD, J. H.. JR.

CRAWFORD, J. H. JR.

CRAWFORD, J. H., JR.

CRAWFORD, J. H., JR.

CRAWFORD, J. H., JR .

CURTIS, O. L.. JR.

CURIIS, O. L., JR.

CURTIS, O. L.JR.

CURTIS, 0.L.. JR.

CURTIS, 0. L.. JR.

CURTIS, O. L. JR.

CURTIS, 0. L., JR.

CURTIS, O: L.. JR.

DAVIS, W. W.

FELDMAN, D. W.

FIELDER, G. E.

FUL TON, T. W.

FULTON, T. W.

GOLDMAN, A. E.

GOOCH, J. W.

GWIN, R.

GWIN, R,

HARMAN, W. L.

HINKLE, N. E.

HOLMES, D. K.

HOLMES, D. K.

HOLMES, D. K.

HOLMES, D. K.

HOLMES, D. K.

HOLMES, D. K.

HOLMES, D. K.

HOLMES, D. K.

HULETT, L. D.

HURAY, P. G.

JENKINS, L. H.

JENKINS, L. H.

JOHNSON, A. L.

JOHNSON, A. 1.

JONES, T. N.

KERNOHAN, R. H.

KERNOHAN, R. H.
$62-V I-21$

$62-V I-21$

$62-V I-23$

$62-1 \mathrm{I}-10-4$

62-II I-12

$62-P \cup B-4$

62-PUB-23

62-PUB-26

62-PUB-20

$62-1-1-3$

$62-1-1-9$

62-1-1-6

$62-1-1-7$

$62-1-1-8$

$62-1-1-9$

62-PUB-24

$62-P U B-25$

62-11-9-3

$62-V 1-20-1$
$62-P \cup B-31$

$62-111-12$

62-PUB- 4

62-PUB- 23

O2-PUB-26

62-PUB-31

62-IV-16-5

$62-P \cup B-27$
$62-1-3-1$

62-I-3-2

62-PUB-36

$62-V I-20-1$

$62-V I-20-2$

$62-V I-20-5$

$62-V I-20-6$

62-PUB-31

$62-I V-16-5$

$62-I V-13-6$

$62-1 v-16-7$

$62-P \cup B-18$

ถ2-PUR-27

62-IV-17-2

$62-11-8-1$

$62-11-8-2$

$62-11-8-3$

$62-I I-8-4$

$62-1 !-8-5$

62-PUB-28

62-PUB-29

62-PUB-40

62- I-1-1

$62-1-1-2$

$62-1-1-4$

$62-1-1-5$

6

62-PUB-25

O2-PU8-30

O2-PUA-34

62-VI-20-I

$2-V I-20-2$

$62-V I-20-3$

$62-V I-20-5$

$62-V I-20-6$

$62-P \cup B-31$

62-PUB-32

$02-V-18-4$

62-I I I-13-2

62-111-13-3

62-PUB-33

62-PUB- 42

$62-V I-21$

$62-11-10-2$

62-IV-I $3-2$

$62-V 1-20-1$

62-V 1-20-2

$62-V 1-20-4$

$62-11-10-3$

$62-111-12$

$62-V 1-20-3$

$62-I V-15-3$

$62-1-1-3$

$62-1-1-4$

$62-1-1-5$

62-1-2-1

62-11 1-14

$62-1-1-1$
$62-1-1-2$

62-PUB-30

62-PUB-34

$62-1 v-17-3$

$62-11 I-\mid 1$

KEYSER, R - M.

KIRKLAND, W. K.

KIRKLAND, W. K.

KLABUNDE, C. E.

KLABUNDE, C. E.

KLABUNDE, C. E.

KLABUNDE, C. E.

KOEHLER, J. S.

LEE, E. J.

LEHMANN, C.

LEIRFR IED, G.

LEIBFRIED, G.

LEIBFR IED, G.

LEI BFR I ED, G.

LEIBFR IED, G.

LEIBFR IED, G. 